Uheverstta degu Srudi di Firanze

Dipuctimento di Stude Storict e Grocruada

DOTTORATO DI RICERCA BN STORIA MEDIEVALE

Collana Digitale

LOREnZo PUsbuCI

\title{
DAL CAUCASO AL MAR D'AZOV
}

LTMPATTO DELITNVASIONE MONGOLA IN CAUCASIA FRA NOMADISMO ESOCIETA SEDENTARIA

(1204-1295)

\author{
preseatazione $\mathrm{di}$ \\ MABCFLO GaRZAAUTI
}

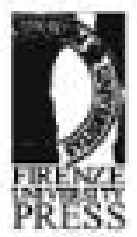




\section{Università degli Studi di Firenze \\ Dipartimento di Studi Storici e Geografici}

Dottorato di Ricerca in Storia Medievale

Collana Digitale 


\section{Dottorato di Ricerca in Storia Medievale \\ Collana Digitale}

\section{Comitato scientifico}

Giuliano Pinto, Università degli Studi di Firenze (Coordinatore)

Giulia Barone, Università di Roma "La Sapienza"

Anna Benvenuti, Università degli Studi di Firenze

Silvia Cantelli, Università degli Studi di Firenze

Sandro Carocci, Università di Roma Tor Vergata

Giovanni Cherubini, Università degli Studi di Firenze

Laura De Angelis, Università degli Studi di Firenze

Paolo Delogu, Università di Roma "La Sapienza"

Riccardo Fubini, Università degli Studi di Firenze

Isabella Gagliardi, Università degli Studi di Firenze

Antonella Ghignoli, Università degli Studi di Firenze

Jean-Claude Maire Vigueur, Università di Roma Tre

Oretta Muzzi, Università degli Studi di Firenze

Paolo Pirillo, Università di Bologna

Francesco Salvestrini, Università degli Studi di Firenze

Franek Sznura, Università degli Studi di Firenze

Andrea Zorzi, Università degli Studi di Firenze

\section{Titoli in collana}

1. Storia di un dottorato: Storia Medievale nell'Università di Firenze. Attività, ricerche, pubblicazioni (1983-2003), a cura di Andrea Zorzi, 2004 
Lorenzo Pubblici

\section{DAL CAUCASO AL MAR D’AZOV L'IMPATTO DELL'INVASIONE MONGOLA IN CAUCASIA FRA NOMADISMO E SOCIETÀ SEDENTARIA (1204-1295)}

presentazione di

Marcello Garzaniti 
Dal Caucaso al Mar d'Azov : l'impatto dell'invasione mongola in Caucasia fra nomadismo e società sedentaria (1204-1295) / Lorenzo Pubblici ; presentazione di Marcello Garzaniti. - Firenze : Firenze university press, 2007.

(Dottorato di Ricerca in Storia Medievale. Collana digitale. / Università degli Studi di Firenze, Dipartimento di Studi Storici e Geografici, 2)

http://digital.casalini.it/9788884534361

ISBN: 978-88-8453-436-1 (online)

ISBN: $978-88-8453-437-8$ (print)

947.97 (ed. 20)

Mongoli-Caucaso-Sec. 13.

Editing: Leonardo Raveggi

Impaginazione: Alberto Pizarro Fernández

(C) 2007 Firenze University Press

Università degli Studi di Firenze

Firenze University Press

Borgo Albizi, 28, 50122 Firenze, Italy

http://epress.unifi.it/

Printed in Italy 


\section{Marcello Garzaniti}

\section{PRESENTAZIONE}

Nel panorama della medievistica italiana il lavoro di Lorenzo Pubblici occupa certamente un posto assai originale. Il territorio che si sviluppa dal Mare di Azov fino al Caucaso, definito genericamente con il nome di Caucasia, appare geograficamente distante dall'Italia, o meglio dalle porzioni ristrette del nostro paese, oggetto dominante delle ricerche contemporanee, che lodevolmente stanno recuperando l'inestimabile patrimonio conservato nei nostri archivi.

L'interesse principale dell'autore, si concentra sulle popolazioni nomadi che occuparono questo territorio a cominciare dal XIII secolo, Tartari o Mongoli che dir si voglia, e cerca di analizzare le profonde trasformazioni dell'area nel contesto dei nuovi e precari equilibri che essi crearono. Si tratta dunque di uno studio di sintesi, fatica assai improba, soprattutto per un giovane studioso, e per giunta su una tematica assai complessa con una bibliografia che già di per sé rappresenterebbe un forte ostacolo a un medievista maturo. Gran parte delle opere, che si trovano citate in bibliografia non sono soltanto nelle correnti lingue occidentali, ma sono scritte in russo e sappiamo quanto sia difficile oggi trovare medievisti che conoscano questa lingua. In genere ancora oggi dobbiamo dire con rammarico: slavica non leguntur. Grazie al russo si può ancora oggi, almeno in parte sopperire, all'ignoranza delle singole lingue caucasiche, mi riferisco soprattutto all'armeno e al geogiano, la cui conoscenza, almeno elementare, sarebbe comunque auspicabile per chi si interessa dell'area. A questo si debbono aggiungere le inevitabili nozioni nell'ambito delle lingue turche, indispensabili se ci si occupa delle steppe euroasiatiche. Certo si tratta di investimenti culturali, che comportano anni di fatica, ma che aprono grandi prospettive nello studio delle fonti e della storia medievale nel suo complesso. Lattenzione agli etonimi e alle definizioni delle strutture sociali del mondo tartaro, che troviamo nel saggio, ci mostrano proprio questa fondamentale sensibilità linguistica, che vorremmo più spesso incontrare.

La tematica scelta è resa più complessa dalle implicazioni culturali, che queste riflessioni trascinano con sé a cominciare dal XX secolo conservando tutt'oggi grande attualità. L'avanzata mongola, che si arrestò solo nel cuore dell'Europa e l'imposizione della pax mongolica in gran parte del continente euroasiatico sconvolsero profondamente gli equilibri del Medio oriente, del mondo mediterraneo, ma più specificamente dell'area delle steppe eurasiatiche su cui più tardi si estenderà l'Impero russo. Accostarsi alla "questione mongola" 
significa inevitabilmente considerare le trasformazioni profonde, che l'invasione tartara provocò nel mondo slavo-orientale. Con la decadenza dell'antica città di Kiev si venne progressivamente a creare un nuovo centro di potere, Mosca, che pur patendo il dominio mongolo ha saputo alla fine attirare nella sua orbita non solo le steppe eurasiatiche, ma anche il Caucaso, la Siberia e l'Estremo oriente siberiano. La presenza e l'influsso dell'elemento "turanico" nel mondo russo, e più in generale slavo-orientale, non rappresentano dunque una questione secondaria, ma richiamano la formazione della sua identità e la fondamentale domanda sull'appartenenza della Russia all'Europa. Ravvivata dalla Rivoluzione d'ottobre e dall'ideologia eurasista negli anni venti e trenta la questione rimane oggetto di riflessione e di polemica ed ha profondamente influenzato la storiografia russa e sovietica. La formazione medievale del Canato dell'Orda d'oro rappresenta dunque l'inevitabile punto di partenza per comprendere le trasformazioni dell' intera area e preludono alle trasformazioni che hanno segnato l'epoca moderna. Non sarebbe possibile altrimenti comprendere né i rapporti della Russia con il Caucaso, né le altrettanto attuali problematiche connesse alle relazioni con l'Ucraina. E più spesso, ne siamo convinti, i politologi se lo dovrebbero ricordare.

Di grande importanza ci sembra soprattutto l'approccio metodologico dell'autore, che non si limita alla ricostruzione della storia dei Mongoli, su cui peraltro esistono già valide sintesi. L'attenzione si concentra piuttosto sulla struttura sociale con particolare riguardo al nomadismo, alla luce dei risultati dell'antropologia, dell'archeologia e della storiografia, cercando soprattutto di cogliere i rapporti fra questa realtà e il mondo sedentario conquistato, e quindi le trasformazioni e i nuovi equilibri sociali ed economici, che ne scaturirono. In questo contesto assume una diversa interpretazione il ruolo delle colonie occidentali nel Mar Nero come punto di contatto diretto fra l'impero mongolo e i mercati occidentali in un processo di globalizzazione ante-litteram, che precede (e prepara) l'apertura delle nuove grandi vie commerciali dell'Atlantico. Non è un caso, come giustamente osserva l'autore, che la merce fondamentale sia rappresentata dagli schiavi (lo sarà alla fine anche per il Nuovo Mondo), in buona parte costituita da slavi orientali (meglio questa definizione che parlare semplicemente di russi). Ci viene alla memoria, allora, come nell'Alto medioevo sempre slavi erano stati gli schiavi venduti in Occidente, a cominciare da Venezia, in quantità così rilevanti da determinare la sinonimia lessicale fra servus e l'etonimo sclavus. L'autore si concentra soprattutto sui rapporti fra nomadismo e sedentarismo, proprio parlando della colonia di Tana. Quanto fossero comuni gli interessi delle potenze marinare occidentali con il canato mongolo dell'Orda d'oro lo dimostra la presenza di mercenari occidentali, provenienti da queste colonie del Mar Nero, al fianco dei tartari nella battaglia del Campo di Kulikovo (1389), poco oltre il fiume Don, che segnò la prima vittoria della Moscovia contro il dominatore tartaro. 
Rimangono tante domande, come sempre in un'opera che tenta una nuova sintesi, molte, forse troppe, soprattutto per chi, come scrive, si occupa di storia della cultura dell'Europa orientale. Alcune sono almeno parzialmente soddisfatte, come la fondamentale questione del fattore religioso, in un'area che vede la presenza di comunità cristiane diverse, orientali e occidentali, e comunità islamiche diverse, sunniti e sciti nel contesto di una pax mongolica assai lontana dalla tolleranza religiosa di cui ancora certa storiografia favoleggia. Rimane abbastanza in ombra l'influsso sulla cultura materiale dell'impero mongolo e sulle strutture politiche ed economiche, anche se emerge già un generale quadro economico del mondo tartaro, capace di mettere in contatto diretto l'Oriente e l'Occidente e che aveva il suo centro nella due Saraj. Sarà proprio la loro scomparsa con le conquiste di Tamerlano e l'affermazione della Porta ottomana a determinare la ricerca di nuove vie commerciali per l'Oriente. Rimane da approfondire la questione della forma di feudalesimo, che si sarebbe affermata nell'impero mongolo, su cui si può discutere. Il lavoro doveva, comunque, avere dei limiti non solo cronologici, se voleva giungere al suo termine. Con particolare soddisfazione abbiamo letto le pagine sull'aspetto demografico, che pur limitatamente a determinate aree mostrano le gravissime conseguenze sul territorio provocate dalle ripetute invasioni mongole, soprattutto nella fase iniziale, con la scomparsa di numerosi insediamenti. E questo ancora una volta contraddice una storiografia contemporanea che ha cercato di sminuire l'impatto distruttivo della calata mongola, sottolineando gli aspetti positivi della conquista tartara.

Molti sarebbero gli spunti di riflessione, ma lasciamo al lettore la libertà di coglierli e di trovarne di nuovi, nella speranza che il presente lavoro sia solo l'inizio di una ricerca, che continui a valorizzare la metodologia della nostra scuola medievistica, ma allo stesso tempo sappia aprire in questi tempi di radicali trasformazioni a una visione più ampia e globale della storia. 



\section{INTRODUZIONE}

Il libro nasce da una tesi di dottorato discussa all'Università degli Studi di Firenze nel marzo 2005. Quando, nei primi giorni del 2002, ebbe inizio il lavoro mi trovai dinanzi ad alcuni problemi che ancora oggi non sono riuscito a risolvere del tutto. Era mio intendimento ripercorrere le orme di uno dei maggiori storici contemporanei che si sono occupati di Mar Nero e della sua costa orientale, George Ioan Bratianu, ${ }^{1}$ circoscrivendo i limiti geografici e cronologici e alla luce delle nuove acquisizioni storiografiche. In pratica un gigantesco lavoro di sintesi. Naturalmente peccavo di presunzione; di quella presunzione che è tipica di chi ha una grande passione, ma non le adeguate conoscenze della materia. Volevo, in poche parole, ricostruire gli oltre due secoli di storia che separarono la caduta di Costantinopoli del 1203-1204 e le campagne di Tamerlano nel Caucaso con specifico riguardo alla costa orientale del Mar Nero. Mi chiedevo cosa fosse effettivamente accaduto in quella regione così affascinante e poco indagata dagli studiosi italiani in un periodo durante il quale molte cose vennero a mutare sia da un punto di vista politico sia (ed è la cosa che più mi interessava) da un punto di vista culturale. Già questo presupponeva una serie di competenze che, da medievista formatosi in una pur prestigiosa università italiana non avevo e meritava un insieme di riflessioni che avrebbero imposto alla mia ricerca tempi enormemente superiori ai tre anni previsti per una tesi dottorale. Mi volsi allora a un ambito geografico più ristretto, il mar d'Azov e la foce del Don dove sorse, durante la grande stagione commerciale genovese e veneziana, l'emporio della Tana. Gli scambi di idee avuti con studiosi che si erano occupati di queste problematiche mi convinsero a valutare la vicenda di quell'insediamento in un contesto comparativo più ampio; vedere cioè se il caso della Tana, documentato per gli anni centrali del Trecento, costituiva un'eccezione nell'ambito dei rapporti latino-mongoli in quella regione. Quest'ultima via è quella che poi ho seguito e che ha portato alla realizzazione della tesi di cui sopra.

Pensando di ricavare un libro dal lavoro svolto nei tre anni di dottorato mi sono chiesto se fosse stato il caso di presentare al lettore un tomo di oltre cinquecento pagine che mi sono poi accorto essere stato sostanzialmente un laboratorio in itinere; un insieme di riflessioni su una materia che presenta molti,

1 Affascinato dalla lettura di quel gran bel libro che è La Mer Noire, des origines a la conquête Ottomane, München 1969. 
troppi problemi interpretativi. Il lavoro fatto sulle fonti di prima mano male si sposava con quello effettuato sulla produzione narrativa e l'insediamento della Tana risultava quasi un corpo estraneo al resto della ricerca. Ho dunque ritenuto opportuno ripensare la struttura del libro e di rimetterci mano per realizzare un'opera che potesse quanto meno fare il punto della situazione degli studi sul Caucaso nel Medioevo, presentare le più difficili questioni che lo riguardano e fornire una prima lettura di un evento complesso come l'esperienza mongola.

Nella sua introduzione al libro di W.E.D. Allen ${ }^{2}$ sir Denison Ross affermava: «nearly everything of importance on Georgia that has since been written is in Russian or in Georgian, and mostly buried in learned periodicals». L'ultima grande opera sullo stato caucasico cui anche Ross si riferiva nel suo scritto - e accessibile agli studiosi occidentali in quanto scritta in francese - era la monumentale Histoire de la Géorgie di Marie Félicite Brosset. ${ }^{3}$ A distanza di oltre settant'anni da quella pubblicazione e più di un secolo e mezzo dopo l'Histoire di Brosset lo stato della letteratura scientifica occidentale sulla Georgia è molto cambiato. ${ }^{4}$ In Russia gli studi sono andati avanti, sin dall'Ottocento, nell'esigenza di collocare il grande Paese slavo nel contesto geografico e politico mondiale e di trovare un posto fra l'Europa, interprete della civiltà dell'Occidente medievale e l'Asia, ovvero l'ambito geografico entro il quale la Russia stessa si inserisce per due terzi della sua estensione. Negli Stati Uniti molti sono gli specialisti che hanno volto con cura e grande preparazione le loro energie ad Est e sul Caucaso in particolare. In Europa si è spesso guardato al Caucaso in riferimento agli avvenimenti contemporanei. Tuttavia in Gran Bretagna, in Francia e in Germania la tradizione degli studi è assai solida. In Italia oggi manca una forte attenzione di taglio storico all'Europa centro-orientale per quanto riguarda il Medioevo.

Quello di Caucasia è un concetto in parte costruito e spesso sovrapposto all'immagine dell'Oriente; una nozione, quest'ultima, astratta, forse una categoria culturale, che acquista vigore e concretezza quando si è in grado di problematizzarla e comprenderne non solo le vicende interne, ma anche le

2 W.E.D. Allen, $A$ history of the Georgian people. From the beginning down to the Russian conquest in the Nineteenth century, London 1932.

${ }_{3}^{3}$ M.F. Brosset, Histoire de la Géorgie depuis l'antiquité jusqu'au XIX. siècle, 7 voll., Sankt Peterburg 1849-1858.

${ }^{4}$ Lo studio di questi eventi è reso possibile, fra le altre, da una delle poche fonti georgiane sopravvissute: la Cronaca. Si tratta di un documento scritto probabilmente in georgiano, ma sopravvissuto in trascrizione armena e fortemente rimaneggiato nel Settecento per volere del re georgiano Vaxtang VI. Una prima edizione in lingua francese fu data dallo stesso Brosset (Additions et éclaircissements à l'Histoire de la Géorgie, Sankt Peterburg 1851, d'ora in avanti HG). Qualche anno fa ne ha fornita una buona traduzione anche Robert Bedrosian (The Georgian Chronicle, New York 1991) basandosi sul testo armeno ripulito dalle aggiunte settecentesche; Oggi l'opere di riferimento sulle fonti georgiane medievali è S. Rapp, Studies in Medieval Georgian Historiography: Early texts and Eurasian Contexts, Lovanii 2003; si veda anche The Wellspring of Georgian historiography: the early medieval historical Chronicle, a cura di K. B. Lerner, London 2004. 
molteplici influenze che essa ebbe sulla storia occidentale. La Caucasia di cui mi sono occupato non è un concetto geografico rigido. Non si tratta della catena montuosa e della regione che la circonda o meglio, non si tratta solo di questo. Il Caucaso, che talvolta chiamo (forse impropriamente) Caucasia, è qui considerato come quell'area che dal Mar d'Azov scende verso sud fino a includere la Georgia attuale formando un'autentica barriera naturale prima di quell'Oriente che ancora nel XII secolo era, per l'uomo europeo, una pura immagine mentale. Certamente il periodo della dominazione mongola non fu la prima occasione durante la quale culture sedentarie e civiltà nomadi si trovarono a interagire; è pero in seguito a questo evento che la Caucasia subì un potere coerente da parte di un unico sistema organizzato il quale, per un certo periodo racchiuse in sé i caratteri di entrambi i modelli; la genesi di tale processo e parte dei suoi risultati costituiscono l'oggetto fondamentale del lavoro.

Geograficamente la Caucasia è un istmo che separa il Mar Nero dal Mar Caspio e l'Europa dall'Asia. È situata, da nord a sud, fra il Mar d'Azov e l'Armenia settentrionale. Nel punto più largo fra Mar Nero e Mar Caspio vi sono $500 \mathrm{~km}$. La costa del Mar Nero è complessivamente di circa $1.500 \mathrm{~km}$ compreso il Mar d'Azov (poco meno, $1.360 \mathrm{~km}$, misura la costa della Caucasia caspica). Entro questi limiti la Caucasia copre una superficie di oltre $465.000 \mathrm{~km}^{2}$. Il Mar d'Azov è un catino d'acqua che misura $42.000 \mathrm{~km}^{2}$. Il terreno ad esso circostante è prevalentemente sabbioso, la costa frastagliata. Nei mesi invernali è quasi inagibile a causa delle basse temperature e della conseguente glaciazione delle sue acque. Il Mar Nero ha una superficie d'acqua che supera i 420.000 $\mathrm{km}^{2}$. Nel punto più profondo arriva a $2.245 \mathrm{~m}$, ma mediamente non supera $\mathrm{i}$ $1200 \mathrm{~m}$. Il Mar Nero accoglie le acque di alcuni fra i fiumi più lunghi d'Europa: Dnepr, Danubio, Dnestr, Bug e, indirettamente, il Don. Diverso è il discorso per quanto riguarda il Mar Caspio. Esso è un lago chiuso la cui superficie è ampia (438.000 kmq). Accoglie il più lungo fiume d'Europa, il Volga, nel cui delta le acque sono paludose e basse. È nel mezzo della depressione uralo-caucasica. Le sue acque non sono profonde, al massimo raggiungono i 900 metri, ma oramai dovremmo aggiornare i dati con ritmo giornaliero poiché l'indiscriminato sfruttamento delle sue terre coperte per l'estrazione del petrolio ne sta letteralmente distruggendo l'ecosistema. A nord non vi sono profondità che superano i 10 $\mathrm{m}$, laddove la media complessiva è di soli $180 \mathrm{~m}$.

A differenza di altre realtà etnico-politiche inserite in contesti ambientali simili (si pensi alla Rus' o alla regione del Tibet), dove si è verificata una sorta di integrazione ideologica sovranazionale nel segno del religioso, nel Caucaso i particolarismi sono sopravvissuti e talvolta si sono addirittura accentuati. Stiamo parlando di un'area estesa, ma non più di quanto lo fosse la Rus' kieviana, né più di quanto lo siano alcuni Stati dell'Asia centrale. In una relazione tenuta a Spoleto nel 1995 Maurizio Tosi, archeologo e profondo conoscitore dell'Asia, faceva notare come nella regione del Tibet il buddismo Mahayan-Vjrayana, portato dall'India 
settentrionale attorno al VII secolo a. C., determinò una spinta integrativa per cui in quell'area si rileva ancora oggi un'omogeneità culturale straordinaria. ${ }^{5} \mathrm{Nel}$ Caucaso, nonostante la ripetuta penetrazione delle tre grandi religioni monoteiste, ciò non si è verificato. Nessuna è riuscita a prevalere. Quanto hanno influito su questo le incursioni del nomadismo e in particolare l'esperienza mongola?

I Mongoli giunsero fino alla costa adriatica durante la seconda spedizione a Ovest, ma il punto più occidentale di quell'entità organizzata che fu l'impero divenne proprio la Caucasia. Per determinare le conseguenze che su di essa ebbe l'invasione mongola sono partito da un evento che cambiò sostanzialmente gli equilibri politici della regione, favorendo l'avanzata del nomadismo: la IV crociata. L'indebolimento dell'impero bizantino non fu causato dalla crociata medesima; essa fu la conseguenza di molteplici fattori che gli specialisti di storia bizantina hanno già egregiamente illustrato. Si è cercato di indagare l'evoluzione della politica bizantina e le sue conseguenze guardando a est, mettendo l'accento sulle relazioni fra Bisanzio, la Georgia e l'Armenia, solo sfiorando le ben note vicende occidentali che portarono alla realizzazione della crociata. Si è poi deciso di affrontare il tema di quella che considero l'ultima grande ondata nomade nelle steppe russo-meridionali fino al Caucaso prima dell'esperienza mongola e rappresentata dall'insediamento della popolazione turco-nomade dei Cumani. Seguendo un ordine tematico, più che cronologico, mi sono poi soffermato (capitolo terzo) sul rapporto fra il grande regno cristiano della Georgia e il nomadismo negli anni a cavallo dell'esperienza crociata. L'indagine della società tradizionale mongola, il contesto sociale originario all'interno del quale si formò l'unione delle tribù che in Occidente venne percepita come un unico popolo sotto il nome di Tartari costituisce l'oggetto del capitolo quarto. Studiare l'organizzazione sociale originaria credo possa aiutare a comprendere molte delle dinamiche politiche, ma anche sociali e culturali che caratterizzarono l'impero mongolo dopo la sua completa formazione. Nei capitoli successivi si è cercato di dare un quadro generale delle conquiste mongole, fenomeno complesso e poco conosciuto nella sua specificità. Le conseguenze politiche furono notevoli e cercherò di darne conto; così come cercherò di ricostruire gli effetti culturali della conquista soffermandomi sul fattore religioso, la presunta tolleranza del ceto dirigente mongolo verso il culto locale e molte altre questioni legate a questo aspetto, il quale è stato spesso oggetto di fraintendimenti. Chiude il libro il capitolo ottavo nel quale vengono considerate le conseguenze demografiche dell'invasione e i fattori pregressi che ne accentuarono il carattere distruttivo in un'ottica cronologica più ampia, fino alle invasioni di Tamerlano nel 1395.

La vastità dell'area considerata e la sua eccezionale diversità etnico-culturale ha imposto alcune scelte sulla cui opportunità si potrebbe discutere a lungo. Oggi

${ }^{5}$ M. Tosi, Dalla tribù all'impero. Riflesioni sul Caucaso, le steppe e i meccanismi dell'evoluzione sociale alla luce dei dati archeologici, in Il Caucaso: cerniera fra culture dal Mediterraneo alla Persia (secoli IV-XI) (Atti della XLIII Settimana di Studi CISAM), Spoleto 1996, pp. 246-270 alla p. 248. 
più che mai si avverte la mancanza di una contestualizzazione dell'esperienza commerciale occidentale all'interno del complesso paradigma fra l'elemento etnico dominante, quello caratterizzato dal modello nomade, e l'elemento "visibile" occidentale, quello che siamo in grado di studiare meglio grazie alla memoria scritta lasciata dagli attori di quella stagione.

L'esperienza mongola è stata spesso considerata entro i limiti di un dualismo interpretativo (il giogo = rivoluzione/innovazione) che spero di aver, almeno in parte, ridimensionato.

Il motivo per cui ho scelto di occuparmi di un ambito cronologico che va dal 1204 fino al 1295 è solo apparentemente politico. Per quanto riguarda il termine di partenza ho già detto: la caduta di Costantinopoli è il simbolo di un potere straordinario come quello di Bisanzio che crolla lasciando un vuoto per certi versi incolmabile (e per quasi due secoli incolmato). Sappiamo che l'impero non cadde fra il 1203 e il 1204; il processo che ne detterminò la frantumazione fu lungo e complesso, ma queste date, storiche appunto, sono molto utili per lo studioso che deve circoscrivere cronologicamente i limiti della propria ricerca. Il 1295 è l'anno in cui l'Ilkhanato di Persia, guidato da Ghazan, prenderà definitivamente la sua via, inaugurando una serie di riforme che ne faranno uno stato in piena regola. Verrà adottato l'Islam come religione ufficiale e di fatto si consumerà la rottura con l'Orda d'Oro, peraltro già ampiamente incubata nelle guerre di confine proprio sulla linea del Caucaso (l'anno prima, 1294, era morto Quibilaj, Gran Khan).

Nel 1395 si abbatté sulla Caucasia l'invasione timuride. Gli eserciti di Tamerlano sciamarono da Bukara a Tbilisi, da Saraj alla Tana riesumando in parte la distruzione che solo un secolo e mezzo prima era stata portata da Gengis khan e dai suoi generali. Fu un evento dalle caratteristiche assai diverse. Le conquiste di Tamerlano, figura affascinante e controversa, rappresentano per certi versi il punto in cui un cerchio si chiude; l'esperienza mongola perdurerà e verrà definitivamente regolata solo nel XVII secolo, quando una famiglia di mercanti russi, gli Stroganov, aiutati da un esercito irregolare di origine contadina che combatteva sotto il nome di Cosacchi, sconfiggeranno l'esercito dell'ultimo khanato, quello di Siberia, coronando l'ascesa di Mosca e regalando allo zar (Ivan IV, il Terribile) uno Stato tricontinentale. ${ }^{6}$

Questo lavoro è pieno di limiti e d'altra parte non potrebbe essere altrimenti data la vastità dell'argomento trattato. Mi sono soffermato su quegli aspetti che ritengo possano costituire un buon esempio nella comprensione del fenomeno generale. Spero che i risultati espressi siano di compensazione laddove non sono riuscito a soddisfare appieno nemmeno le mie stesse aspettative.

Circa due secoli or sono Aleksander Puškin si volse al Caucaso per mettere in evidenza il precoce invecchiamento morale dei suoi giovani coetanei con questi versi:

${ }^{6}$ Devo questa felice definizione a Renato Risaliti che ha così intitolato il primo volume della sua Storia problematica della Russia, 8 voll. Firenze 2002-2004. 
In Russia la lontana strada mena,/Nel paese ove ardente giovinezza/Egli imprese superbo senza affanni/Ove conobbe la prima gioia/Ove amò tante cose care, Ove accolse la fiera sofferenza

e più avanti:

Fece prova degli uomini e del mondo,/Seppe il prezzo della fallace vita.

Ancora oggi, per chi ama quella terra, il prezzo della «fallace vita» è l'immagine della "prima gioia.

Putroppo.

Nei tre anni di studio trascorsi a preparare questo lavoro ho contratto parecchi debiti e non credo di poterli saldare con le poche righe che seguono. Tuttavia desidero rivolgere un sincero ringraziamento ad alcune persone senza le quali questo studio non sarebbe mai giunto alla fine né forse avrebbe avuto un inizio. Il primo pensiero va, ed è sovente andato in questi mesi, a Dino Pubblici ché da troppo non è più. Ringrazio Renato Risaliti e Giovanni Cherubini, riferimenti imprescindibili per la mia formazione scientifica. Tutta la mia gratitudine, unita a stima profonda, va a Michel Balard, Nicola Di Cosmo, Sergej Pavlovič Karpov e a Felicitas Schmieder, studiosi preparatissimi e persone squisite. Al dottorato di ricerca in Storia Medievale dell'Università degli Studi di Firenze che ha reso possibile questa pubblicazione e in particolare al suo coordinatore, Jean Claude Maire-Vigueur. Un ringraziamento speciale va a Marcello Garzaniti che ha accettato di leggere il manoscritto e mi ha dato consigli preziosi. Tutte le imprecisioni e i limiti del libro sono tuttavia da imputarsi solo al sottoscritto.

Molti altri hanno contribuito all'andare degli studi e mi hanno facilitato il lavoro; fra questi desidero ringraziare innanzitutto la mia famiglia tutta: Renato, Mara, Lorella, Stefano e Alberto; desidero inoltre ringraziare con grande stima e affetto Giovanni Canova, Enrico Faini, Ksenja V. Litvinova, Roberto Mancini, Vieri Mazzoni, Giuliano Pinto, Paolo Pirillo, Andrej L. Ponomarev, Marilia Ribeiro, Antti Ruotsala, Francesco Salvestrini, Lapo Sestan, Luigi Siciliano, Rustam M. Šukurov, Lorenzo Tanzini, Sergio Tognetti, Gabriella Uluhogian. Il personale degli Archivi di Stato di Genova e Venezia, efficiente e disponibile. Desidero inoltre rivolgere un ringraziamento del tutto particolare ad Anna Benvenuti, Sara Benzi, Andrea Zorzi e Chiara Benvenuti per il prezioso contributo offertomi e per l'affetto col quale l'hanno fatto.

Il ringraziamento più sentito va a chi in questi anni di lavoro ha sopportato le assenze e le mancanze facendo fruttare quanto più non era possibile il poco che sono stato in grado di dare: Beatrice. 


\section{Nota metodologica}

Poiché nel volume si affrontano temi legati a realtà culturali profondamente diverse fra loro la traslitterazione è un problema irrisolvibile con un sistema che sia coerente. Si è dunque deciso di adottare soluzioni non sempre rigorose privilegiando la forma più vicina alla leggibilità in italiano.

Per i nomi di origine slava si è adottata la traslitterazione scientifica dal russo adottata dagli slavisti, proposta da Giovanni Maver nell'Enciclopedia Italiana.

Per la traslitterazione dell'alfabeto armeno non ci siamo attenuti rigidamente, come rigore scientifico vorrebbe, al sistema Hübschmann-Meillet-Benveniste, in questo caso abbiamo privilegiato l'assonanza con l'italiano. Stesso discorso vale per l'alfabeto georgiano, per il quale avremmo potuto seguire i criteri seguiti dalla Revue des études géorgiennes et caucasiennes. Anche in questo caso si è privilegiato l'aspetto fonetico adottando la forma più vicina all'italiano.

Per i termini di origine araba e persiana abbiamo seguito la versione data dall'Enciclopedia dell'Islam, edizione inglese citata in bibliografia.

Quanto al mongolo ho preferito seguire i criteri accettati dalla lingua moderna. Tuttavia in alcuni casi ho utilizzato la versione comunemente adottata per i nomi più noti anche in occidente; ad esempio ho usato Gengis Khan in luogo di Chinggis Qan, Ilkhanato in luogo di Îlkhānato; Cazari in luogo di Chazari o Khazari, ecc. 

Ai miei nonni 



\section{LE PREMESSE}

Il territorio compreso fra i confini dell'odierna Georgia meridionale fino all'Ucraina occidentale è delimitato a nord dall'incrocio di due grandi vie fluviali costituite dal Don e dal Volga; a est il Mar Caspio ne costituisce una frontiera naturale. Esso non ha mai avuto una unità politico-amministrativa che ne giustifichi la trattazione a sé. Ha costituito però quella parte del bacino pontico che non è mai stata direttamente sotto l'autorità bizantina, salvo che in alcune aree e per periodi molto brevi. Ciò nonostante le vicende politiche dell'impero orientale ne hanno influenzato lo sviluppo nel corso degli anni. Esso, dal canto suo, ha fortemente suggestionato le vicende politiche bizantine. Su questa regione si sono succedute dominazioni diverse, è stata teatro di guerre e oggetto di contese violente, ha ospitato le frontiere orientali dell'impero romano, vi si sono stabilite comunità nomadi, seminomadi e ha visto l'avanzata delle popolazioni turco-mongole; è stata terreno fertile per gli scambi commerciali fra Europa occidentale e Asia, ha rappresentato la porta d'accesso all'Estremo Oriente e di tutto ciò ancora oggi se ne possono apprezzare le tracce nella fisionomia socioculturale delle popolazioni che la abitano.

Nel 1008 il Caucaso centro-meridionale era stato unificato grazie all'opera di Bagrat III, del ramo Bagratide di Georgia, il quale riuscì a mettere insieme i territori abkhazi e a imporvi la propria autorità. A nord del Mar Nero Bisanzio aveva conquistato, nella seconda metà del X secolo, Maurokastron in Crimea, che rimarrà sotto l'autorità greca per molto tempo, salvo un breve intervallo di occupazione da parte dei Variaghi cui porrà fine la pace siglata dal sovrano di quelli, Svjatoslav e Basilio II. Il sovrano bizantino pose sotto la propria protezione anche Ani e il regno Bagratide d'Armenia (1045). Dopo il 1016 tale sovranità si estese anche su Matracha, la Zichia e tutta la Khazaria. Sull'interpretazione di quest'ultimo nome è stato scritto molto. È verosimile che si tratti proprio dell'imboccatura del Don, terre un tempo sotto il dominio Cazaro e ora nelle mani di Bisanzio. ${ }^{1}$

Per avere un quadro di riferimento sulla regione in esame credo sia necessario procedere considerando gli eventi che ne determinarono la geografia politica

${ }^{1}$ In seguito alla spedizione navale che Bisanzio aveva mandato in Khazaria e che riuscì a piegare la resistenza di una delle ultime roccaforti dell'antico impero turco-giudaico nella regione che era stata il Bosporo Cimmerio dei Greci (Bratianu, La Mer Noire cit., p. 158). Bratianu cita il nome di un arconte e di uno stratega posti dall'impero per amministrare le recenti acquisizioni attorno allo stretto di Kerč. In realtà Bisanzio riaffermò la propria sovranità su questa regione. 
alla vigilia della crociata. L'XI secolo, come vedremo in seguito, ha segnato una rottura profonda nella continuità della politica estera bizantina. Le incursioni nomadi, unite alla crisi interna sofferta dall'impero ne avevano profondamente indebolite le fondamenta. Nel 1021 si verificarono le prime incursioni turche in Armenia. Fu un duro colpo inferto alla capacità dell'impero greco di rappresentare una valida difesa sul territorio. Negli stessi anni, dalle steppe dell'attuale Ucraina meridionale, dilagarono Peceneghi e Uzi. Di lì a poco la situazione precipitò. Nel 1064 i Cumani spinsero Peceneghi e Uzi oltre il Danubio. I Guzi ${ }^{2}$ (Selgiuchidi) attaccarono la Georgia meridionale. ${ }^{3} \mathrm{~A}$ est accadde, se possibile, di peggio: nel 1048 il tema bizantino d'Armenia venne invaso dai nomadi turcomanni. Nel 1054 si assistette a una nuova invasione turca contro il Caucaso e l'Armenia in particolare. Qualche anno più tardi i Guzi si spinsero nell'Anatolia centrale fino a Sivas. Sempre nel 1064 il sultano selgiuchide pose l'assedio ad Ani che, abbandonata dalla guarnigione bizantina, cadde senza potersi opporre con efficacia. Il 1071 fu l'anno della ben nota disfatta bizantina di Manzikert. È un periodo durante il quale si ridefiniscono molti equilibri politici attorno al Mar Nero e dal quale Bisanzio uscirà in parte ridimensionato, ma ancora in grado di esercitare una forte autorità sui territori a esso sottomessi. Le invasioni nomadi ne avevano evidenziati i limiti, soprattutto militari, sulle regioni periferiche.

\subsection{Fino a Manzikert. La Caucasia e il fallimento bizantino.}

La storia della costa orientale del Mar Nero - e della Caucasia in particolare - è strettamente legata alle vicende dell'impero romano sin da quando, nel

2 Sui Selgiuchidi è stato scritto molto. Per un approfondimento si vedano S.G. Agadžanov, Očerki istorii Oguzov i Turkmen Srednej Azii IX-XIII vv., Asxabad 1969; C.E. Bosworth, The Ghaznavids, Edimburgh 1963; S. Vyronis, Studies on Byzantium, Seljuks and Ottomans, Malibu 1981²; C.Cahen, The Turkish Invasion: The Selchükids, in A History of the Crusades, a cura di K.M. Setton, Madison (WI)-London 1969, I, 135-176; M.F. Koprulu-G. Leiser, The Seljuks of Anatolia: Their History and Culture According to Local Muslim Sources, Salt-Lake City (UT) 1992; L.N. Gumilev, Drevnie Tjurki, rist. Moskva 1993.

${ }^{3}$ Su questi fatti ci informa con dovizia di particolari lo storico persiano Ibn al-Athir. L'opera di Al-Athir è monumentale ed è stata pubblicata per la prima volta in occidente da C.I. Tornberg (Ibn al-Athir, Ibn al-Athiri chronicon quod Perfectissimum inscribitur, I, 1851-1853, II, 1867-1876); si tratta di un'edizione comunque incompleta. La traduzione più accessibile è quella pubblicata nei Recueil des Historiens des Croisades. Historiens Orientaux, II, Paris 1887. Per quanto riguarda il Caucaso mi sono basato sugli ampi brani tradotti ed editi da M. Defremery, Fragments de Geographes et d'Historiens arabes et persans inedit, in "Journal Asiatique», s. IV, 13 (1848) [d'ora in avanti Ibn al-Athir, I] e 14 (1849) [d'ora in avanti Ibn al-Athir, II]. Secondo Ibn al-Athir l'attacco dei Guzi intervenne in seguito alla chiamata da parte degli abitanti musulmani di Tblisi. Dice lo storico persiano: «Nell'anno 429 il re degli Abkhazi assediò la città di Tblisi. Gli abitanti opposero una fiera resistenza [...] allora gli assediati inviarono una deputazione in Azerbaijan, per implorare il soccorso dei musulmani. Allorquando i Ghozz furono entrati in Azerbaijan, e che gli Abkhazi ebbero conoscenza del loro avvicinamento e dei mali che avevano causato agli Armeni, smontarono gli accampamenti davanti a Tblisi velocemente per timore dei Ghozz»: Ibn al-Athir, I, p. 483. 
64 dell'era cristiana, vi fu annessa la Georgia occidentale. L'anno successivo fu inclusa la parte orientale del Paese caucasico, l'Iberia o Kart'li. Nel 72 la stessa sorte toccò all'Armenia. ${ }^{4}$

Gli sviluppi politici dell'impero hanno caratterizzato il procedere storico della Caucasia determinandone confini e influenze culturali. Dopo un lungo periodo durante il quale la regione oscillò fra l'influenza achemenide e quella di Roma, la crisi dell'imponente impalcatura costruita da quest'ultima lasciò l'eredità occidentale nelle mani di Bisanzio. Nel 629 l'imperatore Eraclio riuscì a sconfiggere l'Iran e gran parte dell'Armenia iranica passò sotto la diretta influenza greca. L'occupazione araba della seconda metà del VII secolo provocò una nuova definizione dei poteri e il territorio a sud del lago Sevan, passò sotto l'autorità islamica. La Georgia, dal canto suo, sin dall'Antichità, è stata divisa in due parti politicamente distinte: quella pontica o occidentale (Colchide per il mondo ellenico, Egrisi per i Georgiani ${ }^{5}$ ) e quella orientale (Iberia per i Greci e successivamente Kart'li in seguito alla supremazia della popolazione dei Kartveli). ${ }^{6}$ Essa subì la conquista dei principi Lazi da sud, acquistando nuova autonomia con la costituzione del regno di Lazica il quale era comunque vassallo dell'impero romano. Nello stesso periodo in cui l'Armenia passò sotto la sovranità bizantina, la dinastia lazica scomparve a vantaggio di piccoli principati la cui conseguenza sostanziale fu la consegna del regno nelle mani dei principi abkhazi.

L'Abkhazia (posta a nord-ovest dell'attuale Georgia e a sud dei monti del Caucaso), aveva resistito ai molti tentativi da parte degli arabi Ommayyadi di Murwan- $\mathrm{Kru}^{7}$ di invadere il territorio abkhazo, ma questi fallirono dopo l'occupazione araba dei passi caucasici di Darial e Derbend. Le truppe di Murwan-Kru riuscirono ad attaccare Sukhumi, ma non si spinsero oltre.

Durante il periodo cazaro 8 l'Abkhazia ottenne una forma di indipendenza piuttosto ampia grazie anche alle relazioni opportunamente costruite con il

${ }^{4}$ Cfr. D. Braund, Georgia in Antiquity: A History of Colchis and Transcaucasian Iberia, 550 $B C-A D$ 562, Oxford, 1995, in particolare il cap. quinto alle pp. 152-170.

5 Sul processo di colonizzazione della Colchide in epoca antica cfr. Ibid. pp. 87-117.

${ }^{6}$ C. Toumanoff, Caucasia and Byzantium, in «Traditio», 27 (1971), pp. 111-159, p. 119; Id., Medieval Georgian Historical Literature (VIIth-XVth Centuries), in "Traditio», 1 (1943), pp. 139-182, pp. 141-142, in particolare p. 142, nota 6.

7 Per una panoramica su questi eventi rimane utile l'antico saggio di M.F. Brosset, Histoire de la Géorgie cit., I, pp. 237-243; Encyclopaedia of Islam, 11 voll., London 1960, vol. I., pp. 100-101. Per un'indagine dettagliata della Georgia nel Medioevo resta insuperato W.E.D. Allen, A History of the Georgian People cit.; si veda anche G. Charachidzé, Introduction a l'étude de la féodalité géorgienne, Paris-Genève 1971.

${ }^{8}$ Sulla nascita e sugli sviluppi dell'impero dei Cazari si veda D.M. Dunlop, The History of the Jewish Khazars, Princeton (NJ) 1954; Id., The Khazars, in The World History of the Jewish People, a cura di W.H. Allen, London 1966, vol. II; assai utile è la voce Khazars in Encyclopaedia Britannica, 1973; per quanto riguarda la fonte documentaria per eccellenza nell'ambito degli studi sui Cazari, la corrispondenza cazara, la migliore edizione rimane quella di Kokovcov: P.K . 
potente impero giudaico. Il principe abkazo Leone II sposò la principessa cazara assumendo il titolo di re e istituendo la capitale a Kutaysi. ${ }^{9}$

Fra la metà del IX e la metà del secolo successivo i sovrani abkhazi regnavano non solo sull'Abkhazia, ma anche sulla Mingrelia, l'Imerezia e la Kart'lia. Il regno di Abkhazia/Abasgia che, nel 1008, unificò le due parti dando vita a un periodo di particolare fioritura economica e culturale, si costituì nel 790. L'epoca di transizione fra la dinastia lazica e quella abkhaza fu caratterizzata, per la Georgia, da una forte influenza bizantina. Per un lungo periodo, fra VIII e IX secolo l'impero bizantino aveva cercato di evitare che il regno ottenesse una sua autonomia, ma l'alleanza degli abkhazi con l'impero cazaro ne frenò le ambizioni di conquista; a tale fine aveva nominato un viceré in Iberia, inviso alla popolazione locale - a causa del palese squilibrio fra le imposizioni cui doveva procedere e il suo scarso legame col territorio - e i cui poteri erano piuttosto vasti. Era nominato da Bisanzio, pertanto agiva in forte dipendenza e fedeltà all'impero. Il viceré di Iberia regnava anche sull'Armenia settentrionale, sulla Lazica e sull'Albània. Secondo Toumanoff aveva compiti molto simili a quelli dell'esarca bizantino. ${ }^{10}$

Le guerre verificatesi fra i secoli VIII e IX in questa regione ne avevano profondamente mutati gli equilibri demografici. Molti nobili georgiani erano morti in battaglia o erano stati eliminati da Bisanzio al fine di poter meglio controllare il regno. Emersero in questi anni ceti dirigenti la cui posizione dominante non veniva minacciata dall'interno e la cui ascesa trovò minore concorrenza. La popolazione nutriva simpatia verso una dinastia indigena piuttosto che verso un governante esterno, magari inviato dallo stesso impero che vantava su un territorio lontano pretese autoritarie e che a quella stessa gente aveva, fino a poco tempo prima, fatto la guerra. I Bagratidi occuparono quindi i territori del Nord e dell'Ovest. ${ }^{11}$ Stessa sorte toccò alle altre regioni che, una dopo l'altra, caddero sotto l'influenza di poteri locali via via rafforzatisi. Gli Arsrunidi si impadronirono di parte dei territori di sud-ovest installandovi il regno di Vaspurakan. I Siunidi ottennero la parte orientale del principato di Siunia e i Mamikonidi riuscirono

Kokovcov, Evreisko-Khazarskaja Perepiska v X veke, Leningrad 1932; P.B. Golden, Khazaria and Judaism, in "Archivum Eurasiae Medii Aevi», III (1983), pp. 127-156, ripubblicato in Id., Nomads and their Neighbours in the Russian Steppe, Aldershot 2003. Qualche tempo fa è stato ripubblicato e tradotto anche in italiano il saggio, sempre piacevole, di Arthur Koestler La tredicesima tribù. Storia dei Cazari, dal Medioevo all'Olocausto ebraico, Torino 2003 (trad. it di The Thirteenth Tribe. The Khazar Empire and its Heritage, New York (NY) 1976).

${ }^{9}$ Encyclopaedia of Islam cit., vol. I, p. 101; Brosset, Histoire de la Géorgie cit. introduction, vol. 5, , pp. LXV-LXVIX.

${ }_{10}$ Toumanoff, Caucasia and Byzantium cit., p. 12-122.

${ }_{11}$ Toumanoff fornisce anche i nomi di questi distretti sui quali la dinastia Bagratide riuscì a estendere la propria autorità: Arsarunik, Siracene, Tayk Bagravendene, Taraun. Se li si immaginasse su una carta del Caucaso meridionale si apprezzerebbe la notevole estensione di dominio che questa famiglia riuscì a realizzare in tempi relativamente brevi. Si veda anche N. Adonz, Notes Arménobyzantines, in «Byzantion», X (1935), pp. 161-203 in particolare da p. 185. 
a imporre la propria autorità su altri territori a sud-ovest dell'Armenia attuale. I Guaramidi, un ramo dei Kosroidi, erano riusciti a monopolizzare la carica di viceré di Iberia. In Albània i principi di Garman erano la casa più eminente dal 628 e riuscirono a rendere l'ufficio di viceré quasi ereditario.

Fra tutti questi gruppi, la cui autorità era limitata sul territorio, emersero alla fine i Bagratidi riuscendo a rendere ereditario l'ufficio di viceré d'Armenia sin dall'806. Pochi anni dopo, nell'813, il ramo georgiano della medesima dinastia riuscì nella stessa operazione in Iberia. ${ }^{12}$ Fra l'884 e l'888 Armenia e Iberia ottennero una seppur informale autonomia da Bisanzio. Per un primo periodo l'impero accettò questa situazione de facto, ma ciò sarebbe durato poco. La sovranità Bagratide non era molto ben vista dagli altri principati. Tale situazione spinse i medesimi verso un irrigidimento delle proprie prerogative di dominio e verso un ripiegamento su una maggiore indipendenza dalla dinastia dominante. Nel 908 gli Arsrunidi, aiutati dagli Arabi, costituirono un regno separato nei loro territori a Vaspurakan; nel 970 i principi Sunia seguirono l'esempio. La lacerazione investì anche la dinastia Bagratide, la quale si scisse in più rami. Quello più giovane in particolare si divise in due branche distinte: nel 961 nacque il principato di Kars e nel 982 il principato di Lori. Mantennero sotto di sé i territori di Taraun, Syspiritis, Moxoene (cfr. cartina 1). Il regno d'Armenia era ridotto al principato di Ashot II. ${ }^{13}$

Il periodo che va dal IX al X secolo segna l'apice della potenza bizantina. L'esercito aveva a disposizione mezzi nettamente superiori a quelli dei piccoli nuclei politici caucasici. Questi, dal canto loro, costituivano per Bisanzio un imprescindibile argine per il mondo arabo oltre che la porta per accedere ai traffici commerciali d'Oriente. Tale ruolo si era notevolmente accresciuto in misura inversamente proporzionale alla decadenza dell'impero cazaro. ${ }^{14}$

12 C. Toumanoff, The Bagratids of Iberia from the Eighth to the Eleventh Century, in «Le Muséon», 74 (1961), pp. 5-42, pp. 11-12; Id., Caucasia and Byzantium cit., p. 122.

13 Per gli eventi si veda il quadro in Toumanoff, Caucasia and Byzantium cit., p. 125-127 e R. Bedrosian, The Turco-Mongol Invasions and the Lords of Armenia in the 13-14th Centuries, Ph. D. Dissertation, Columbia University (NY) 1979.

${ }^{14}$ L'impero cazaro fu determinante nel costituire un argine sulla linea del Caucaso agli attacchi arabi almeno per tutto l'VIII secolo. Nel 730 ad Ardabil essi inflissero loro una dura sconfitta e si spinsero fino quasi a Damasco, allora capitale del califfato. Gli studiosi che si sono occupati di storia cazara sono d'accordo nel ritenere fondamentale l'opera dei Cazari sia per le steppe del Volga sia per Bisanzio; si veda Golden, Khazar Studies, Budapest 1980; Koestler, The Thirteenth Tribe cit., pp. 17-21. Scrive inoltre l'archeologo russo Artamonov: «Fu solo grazie ai potenti attacchi cazari, che richiamarono il grosso degli eserciti arabi verso il Caucaso, che Bisanzio poté resistere» (M.I. Artamonov, Istorija Khazar, Leningrad 1962, p. 19). Dmitrij Obolensky era sulla stessa linea quando affermava che «il contributo principale dei Cazari alla storia del mondo fu l'aver difeso con successo la linea del Caucaso dai furiosi attacchi che gli arabi sferravano verso il nord», (D. Obolensky, Il Commonwealth bizantino: l'Europa Orientale dal 500 al 1453, RomaBari 1974, p. 172). 
La divisione che si era creata fra i poteri caucasici favorì l'azione dell'impero bizantino il quale ottenne parte dei domini bagratidi di Iberia. Nel 1021 e nel 1022 fra Bisanzio e il regno di Georgia - formatosi nel 1008 con l'unione dell'Abkhazia e dell'Iberia - si successero due guerre per i diritti sul ducato dell'alto Tao (Tayk'). In entrambi i casi i Greci ebbero la meglio e la Georgia ne uscì, se possibile, più debole di prima. ${ }^{15}$ In seguito Bisanzio acquisì il principato arsrunide di Vaspurakan. Stessa sorte toccò al regno bagratide d'Armenia dove Giovanni Smbat III dovette, dopo un'abile manovra condotta da Bisanzio, cedere all'autorità di questo. A Giovanni furono concesse terre in Cappadocia esattamente come al principe arsrunide spodestato poco prima da Vaspurakan.

Nei primi anni dell'XI secolo, come abbiamo visto, popolazioni di origine turca si erano già infiltrate a sud e a est della Caucasia passando dall'Azerbaijan. Il primo attacco di cui si abbia notizia è del $1016 .{ }^{16} \mathrm{Fu}$ in questa occasione che Bisanzio agì per occupare Vaspurakan. Il principe del regno arsrunide chiese aiuto a Bisanzio in cambio del riconoscimento formale dell'autorità greca. Questi accettò l'accordo, ma si limitò a occupare il principato e ad annetterlo (1021-1022).

Quanto abbiamo osservato è un esempio della politica condotta da Bisanzio sulla Caucasia fino alla seconda metà dell'XI secolo. Tale comportamento, molto ben organizzato e studiato attentamente, si rivelerà fatale non solo per le entità statali caucasiche, ma anche per l'impero stesso.

In questo periodo si verificò un esodo massiccio di Armeni verso la Cappadocia, la Cilicia e la Georgia. Un quinto della classe dirigente georgiana ai tempi della regina Tamara (a cavallo fra i secoli XII e XIII) era di origine armena. Toumanoff colloca in questo momento l'apice dell'immigrazione in Georgia. Dello stesso avviso Grousset e Charanis. ${ }^{17}$ Se prima del VI secolo gli Armeni erano, per Bisanzio, poco più che uno dei gruppi etnici minoritari arruolati nell'esercito, dal IX-X secolo essi divennero quantitativamente dominanti. Sia Charanis che Toumanoff concordano nella stima della percentuale di Armeni nell'esercito durante il periodo aureo di Bisanzio (IX-X secolo) affermando che

15 Toumanoff, Caucasia and Byzantium cit., p. 126-127.

${ }^{16}$ Ibidem, p. 128. P.B. Golden, The Turkic peoples and Caucasia, in Transcaucasia, Nationalism, and Social Change: Essays in the History of Armenia, Azerbaijan, and Georgia, a cura di R.G. Suny, Ann Arbor (MN) 1983, pp. 51-53 ripubblicato in Nomads and their Neighbours in the Russian Steppe. Turks, Khazars and Qipchaqs, Aldershot 2002; V.F. Minorsky, Studies in Caucasian History, Cambridge 1957, pp. 110 e segg.; C. Toumanoff, Studies in Christian Caucasian History, Georgetown (TE) 1963; Aristakès de Lastivert, Récit des Malheurs de la Nation armenienne in «Bibliothèque de Byzantion», 5 (1973); R.W. Thomson, The Crusaders through Armenian Eyes, in The Crusaders from the perspective of Byzantium and the Muslim World, a cura di A.E. Laiou e R. Parviz Mottahedeh, Washington (DC) 2001, pp. 71-82.

${ }_{17}$ R. Grousset, Histoire de l'Arménie, des origines à 1071, Paris 1947, pp. 586-587; P. Charanis, The Armenians in the Byzantine Empire, Lisboa 1963, pp. 50-53; Toumanoff, Caucasia and Byzantium cit., p. 129. 
essi costituivano almeno il $25 \%$ di tutte le forze armate. ${ }^{18}$ Tale massiccia presenza nelle fila dell'esercito bizantino era dovuta soprattutto alla forte migrazione di cui dicevamo; essa era il frutto di una politica in gran parte consapevole. Già durante il regno di Giustiniano venivano forzatamente trasferiti interi villaggi e annesse terre di confine alle quali era imposta regolarmente la leva militare.

Spesso il fenomeno migratorio era frutto di una situazione di guerra permanente nei territori dell'Armenia occidentale. Da una parte il flusso muoveva verso la Piccola Armenia, dall'altra verso la Siria settentrionale e la Cilicia. ${ }^{19}$ L'afflusso di elementi armeni influenzò profondamente la fisionomia sociale bizantina. La porta dell'esercito era un'entrata privilegiata per l'ascesa sociale. Non è un caso che nei secoli successivi, in particolare dal VII in poi, la classe dominante a corte avrà origini armene $o$ sarà in parte armenizzata. ${ }^{20}$

Molti furono inoltre i monasteri e le istituzioni ibere costituitesi entro i confini imperiali (Iviron sul Monte Athos ad esempio). Anche da un punto di vista culturale l'influenza caucasica fu forte su Bisanzio. ${ }^{21}$

Alle conquiste seguì spesso una condotta politica scellerata da parte di Bisanzio sulla Caucasia. L'imperatore Costantino IX abolì la leva obbligatoria in Iberia sostituendola con una maggiore tassazione. ${ }^{22}$ Tale iniziativa dette vita a un processo le cui conseguenze non tardarono a farsi sentire; i territori armeni e georgiani appena annessi erano non solo fortemente indeboliti dalle guerre, ostili in tutti gli strati della popolazione a Bisanzio, ma erano anche privi di difese sui confini. I regni di Kars, Lori e Siunia, il principato di Sasun e il principato arsrunide di Moxoene erano gli unici rimasti in Armenia con una sostanziale autonomia e in grado di difendersi militarmente da attacchi esterni. ${ }^{23}$ Non basta: le strade che portavano a Bisanzio attraverso la Caucasia non erano militarmente presidiate e difese in modo adeguato. L'opera di Bisanzio, portata avanti già dalla fine dell'XI secolo, aveva cioè provveduto a indebolire l'unità politica armena. L'emigrazione di cui abbiamo parlato aveva spostato non solo ampi strati della popolazione, ma anche molti personaggi eminenti (nobili, proprietari terrieri, dirigenti militari etc.) in zone periferiche. Tale fenomeno aveva generato un forte sfilacciamento non solo politico, ma anche sociale rendendo l'esercito debole e piegando le capacità

${ }^{18}$ Charanis, The Armenians cit., pp. 16-21 e 32-34; Toumanoff, Caucasia and Byzantium cit., p. 131.

${ }_{19}$ Charanis, The Armenians cit., pp. 13-14, 19-21; Toumanoff, Caucasia and Byzantium cit., pp. 132-133; Grousset, Histoire de l'Arménie cit., p. 522.

${ }^{20}$ In Toumanoff, Caucasia and Byzantium cit., si trovano in appendice le liste dei personaggi eminenti entrati stabilmente nelle fila del ceto dirigente bizantino. Si può così apprezzare che le percentuali di Armeni e Iberi, Abkhazi etc. sono, specialmente nei secoli X e XI, molto elevate.

${ }^{21}$ Toumanoff, Caucasia and Byzantium cit., p. 137.

22 Ibidem, Grousset, Histoire de l'Arménie cit., pp. 587-588.

23 Toumanoff, Caucasia and Byzantium cit., pp. 129-130; Grousset, Histoire de l'Arménie cit., pp. 607-608. 
economiche del regno. Quando le prime incursioni nomadi si affacciarono a sud dell'Armenia, questa non fu in grado di opporvi una vera resistenza. ${ }^{24}$

L'arrivo delle popolazioni turche accelerò un processo che era già in atto. Incrementò l'emigrazione verso quelle zone in cui vi era la possibilità di trovare sostentamento, le stesse entro le quali erano stati confinati molti appartenenti al ceto aristocratico armeno, causando un sensibile calo demografico all'interno del regno. Stessa sorte era toccata a una parte della Georgia.

Dal 1037-1038 i territori d'Armenia, Anatolia e Georgia furono sottoposti al fuoco di fila delle incursioni nomadi turche. Tale fenomeno non fu di breve durata. Esso ebbe inizio dalla prima metà del secolo XI e si concretizzò in un succedersi di incursioni di entità variabile che proseguì per decenni; le scorrerie si abbatterono sulla regione corrispondente grosso modo all'attuale Turchia orientale, l'Armenia e l'Azerbaijan. Questo fenomeno, che in seguito si ripeterà per oltre quattro secoli, ebbe conseguenze molto importanti sulla geografia politica del Caucaso.

Le ondate nomadi provocarono in primo luogo l'esplosione della Grande Armenia (in realtà le incursioni turche non riuscirono mai a sottomettere per intero il territorio armeno, ma ne distrussero definitivamente l'unità). Tale afflusso fu il risultato di massicci movimenti di genti, forse un tutt'uno etnico, divise, secondo gli studi di Cahen e Bedrosian, in due gruppi: $:^{25}$ un primo che si potrebbe definire l'esercito regolare selgiuchide, e un secondo costituito soprattutto da mercenari turkmeni difficilmente controllabili dai comandanti militari e autori spesso di devastazioni, rapine, stupri. ${ }^{26}$

Nel 1042, 15.000 Turkmeni provenienti dall'area dell'Urmiah attaccarono il principato di Vaspurakan nei pressi della costa nord-orientale del lago di Van sbaragliando le truppe bizantine che vi erano state poste a difesa. Quasi contemporaneamente un altro gruppo turco attaccò il distretto dell'Ayrarat.

Fra il 1045 e il 1063 truppe miste turche (composte sia da Selgiuchidi sia $\mathrm{da}$ altri gruppi nomadi più o meno direttamente controllati dai primi) entrarono in Armenia e attaccarono parecchie città fra cui Ani, Bayburt, Melitene, Sivas, Kars, Cesarea. ${ }^{27}$ Nel 1071 Romano IV fu sconfitto nella celebre battaglia di

${ }^{24}$ Golden, The Turkic peoples and Caucasia cit., pp. 53-57; Minorsky, Studies in Caucasian History cit., pp. 60-62. Sulle conseguenze nella Grande Armenia cfr. Bedrosian, The Turco-Mongol Invasions cit., pp. 68-69.

${ }^{25}$ C. Cahen, Pre-Ottoman Turkey, London 1968, pp. 27-32; Bedrosian, The Turco-Mongol Invasions cit., p. 69.

${ }^{26}$ Bedrosian, The Turco-Mongol Invasions cit., p. 70; S. Vyros, The Decline of Medieval Hellenism in Asia Minor and the Process of Islamization from the Eleventh through the Fifteenth Century, Los Angeles (CA) 1971, pp. 155-165.

${ }_{27}$ Bedrosian, The turco-Mongol Invasions cit., p. 65. Sulla composizione dei gruppi che attaccarono Vaspurakan non vi è accordo fra gli specialisti. Per un quadro del dibattito cfr. Golden, The Turkic peoples and Caucasia cit., p. 54, nota 27. 
Manzikert. Essa fu anche la naturale conseguenza della politica bizantina nel Caucaso; di un argine che non esisteva più. La battaglia di Manzikert determinò in modo decisivo gli equilibri politici, economici e culturali del Caucaso, ma non fu che la prima volta.

\subsection{Le cause della crisi}

Sino alla seconda metà del XII secolo l'impero bizantino era riuscito sostanzialmente a mantenere i suoi domini in una sorta di nucleo politico e amministrativo del quale esso stesso ne rappresentava l'unità. L'impero rimaneva un punto di riferimento politico per tutta la regione dai Balcani all'Anatolia e per le popolazioni che vi abitavano, fossero a esso sottomesse o ostili.

Tutto il bacino del Ponto rimaneva un'area irrinunciabile per Bisanzio soprattutto da un punto di vista economico. La costa settentrionale del Mar Nero, la Crimea e l'Azov in particolare, erano scali commerciali collegati con l'entroterra e con le maggiori direttrici di scambio verso nord, le quali conducevano nella Rus' e alla regione del Baltico (soprattutto la strada del sale o dei Varjagi ${ }^{28}$ ). La Crimea stessa, nella sua parte pianeggiante, era un granaio prezioso. Il noto trattato stipulato fra Manuele Comneno e Genova nel 1169, frutto di lunghe trattative e ripetute ambasciate, secondo cui la città ligure poteva commerciare nelle province dell'impero eccezion fatta per Rosia e Matrach ${ }^{29}$ e sul quale si è molto dibattuto, è una dimostrazione di quanto effettivamente fosse importante per l'impero mantenere il Mar Nero come un mare chiuso. Un trattato siglato nel 1192 fra Isacco Angelo e Genova riporta la stessa clausola. Analoga era la situazione per Venezia. Sia nel noto crisobullo del 1082 sia in quello stipulato nel 1198 con Alessio Angelo la Serenissima non poteva accedere al bacino del Ponto. E, nonostante i numerosi tentativi, non si è ancora riusciti a trovare mercanti italiani oltre il Bosforo prima della IV crociata. Vero è che tale iniziativa da parte di mercanti italiani non tardò a venire. Già nel maggio 1206 Zaccaria

${ }^{28}$ Per un quadro d'insieme, esaustivo e preciso sull'economia bizantina anche di questi anni, si veda G.G. Maniatis, The domain of private guilds in the Byhzantine economy, Tenth to Fifteenth Centuries, "Dumbarton Oaks Papers», 55 (2001), pp. 339-369 ma anche The Economic History of Byzantium: From the Seventh through the Fiftennth Century, a cura di A. Laiou, 3 voll., Washington (DC) 2002.

${ }^{29}$ Il documento in questione appartiene al crisobullo di Isacco II Angelo del 1192 ed è stato pubblicato negli Acta da F. Miklosich e J. Müller (Acta et diplomata greca medii aevi sacra et profana, 6 voll. Vienna 1860-1890, vol. III, p. 35, 1. 30-34) e da F. Dölger (Regesten der Kaiserurkunden des Oströmischen Reiches, 5 voll., Monaco 1924-1965, n. 1488). Sulle interpretazioni di questo passo vi sono opinioni divergenti per le quali sarà utile vedere M. Balard, La Romanie génoise (XIIe-début du XV'siècle), Roma-Paris 1978, p. 28, nota 44; Bratianu, La Mer Noire cit., p. 175; N.P. Sokolov, A proposito del significato di "he Rosia" in alcuni documenti bizantini dei secoli XII e XIII, in Storici sovietici del Levante genovese, a cura di A. Prefumo, Genova 1985, pp. 89-97. Si veda anche W. Heyd, Storia del commercio del Levante nel Medioevo, ed. It. Torino 1913, pp. 223 e sgg. 
Stagnario e Pietro di Ferraguto stipulano a Costantinopoli un contratto di accomandita per cui il primo concede al secondo 100 perperi d'oro per andare nel Mar Maggiore a commerciare sulla nave di Giorgio Barbaro, anconetano, usque in Soldadea, ${ }^{30}$ quindi in Crimea. Nel luglio del 1212 Angelo Abriano, che si definisce habitator in Costantinopoli, chiede a Giovanni Bianco di Cannaregio i soldi di una cauzione che avevano stipulato ad aprile dello stesso anno per darli a Zaccaria Stagnario (probabilmente un mercante di professione poiché pare essere il solito che compare nel 1206) che ne farà commercio a Smisso. In questo documento compare un altro particolare interessante: Zaccaria raggiungerà Smisso sulla nave de Provençalibus di Giovanni Longobardo. ${ }^{31}$

Non si è in grado di stabilire con esattezza quando la Crimea e l'Azov, la Gotia bizantina, caddero dalle mani dell'impero per finire sotto l'autorità latina. William Heyd nella sua antica, ma ancora per certi versi insuperata, storia del commercio levantino sosteneva che tale passaggio sarebbe avvenuto subito dopo e in conseguenza della crociata del $1204 .^{32}$

In effetti nella Partitio Romaniae ${ }^{33}$ la Crimea non compare, né si fa menzione della parte orientale dell'Anatolia. Un buon indizio in base al quale si può ritenere che tali possedimenti non fossero più pertinenza dell'impero.

La quarta crociata verificatisi dunque fra il 1203 e il 1204 ebbe un impatto devastante sugli equilibri politici di tutta la costa del Mar Nero. Le conseguenze della conquista latina di Costantinopoli e le atrocità che ne seguirono segnarono profondamente l'animo dei contemporanei e determinarono una profonda frattura fra due mondi che sino ad allora avevano vissuto lo scisma del 1054 come un fatto puramente dottrinale. I Latini erano, nonostante la divisione religiosa, percepiti come parte dell'ecumene cristiano. Dal 1204 in avanti per i bizantini muterà sostanzialmente questa prospettiva. Il cristiano occidentale sarà parte esterna a quella frontiera ideologica oltre la quale per Bisanzio esisteva il mondo barbaro, estraneo e avverso.

D'altra parte la crociata contro i cristiani ebbe anche pesanti conseguenze economiche. I rapporti frequenti che Bisanzio aveva col mondo circostante, i principati della Rus', la Georgia e la Crimea, verranno a mutare decisamente.

${ }^{30}$ Bratianu, La Mer Noire cit., p. 183; R. Morozzo della Rocca, A. Lombardo, Documenti del commercio veneziano nei secoli XI-XIII, 2 voll., Torino 1940, II, docc. 478-479, pp. 18-20. La Soldadea del documento è naturalmente Soldaïa.

${ }^{31}$ Morozzo della Rocca-Lombardo, Documenti del commercio veneziano cit., vol. II, doc. n. 541, pp. 84-85. Il nome della nave lascia pensare a un traffico piuttosto intenso sul Mar Nero solo pochi anni dopo la crociata. Purtroppo non si ha conoscenza di altri documenti così precoci che indichino altri mercanti sul Mar Nero per poter apprezzare il traffico pontico in questi anni.

32 Heyd, Storia del commercio cit., p. 777. Su questo credo abbia portato argomenti più che convincenti Michel Balard (La Romanie génoise cit., p. 28).

33 A. Carile, Partitio terrarum Imperii Romaniae, in «Studi Veneziani», VII (1965), pp. 125-305. 
Le potenze commerciali occidentali, in particolare le città marinare italiane, inizieranno una progressiva e inesorabile penetrazione che le porterà a istituire emporia commerciali su tutta la costa del Mar Maggiore e a formare una propria, seppur limitata sul territorio, sovranità nei punti strategici del Ponto.

$\mathrm{Ma}$ in che modo la crociata intervenne nel mutare tali equilibri? La morte di Manuele Comneno, l'imperatore che era riuscito a tenere a freno le velleità di conquista occidentali ${ }^{34}$ e intervenuta nel 1180 segnò l'inizio della decadenza bizantina. Come ha giustamente osservato Mario Gallina, la morte di Manuele I non generò il primo processo di disgregamento all'interno del territorio imperiale poiché esso si era già verificato altre volte in passato; tuttavia questa volta «nuovi e rovinosi ne erano gli immediati risvolti politici» poiché nessuno prima aveva mai messo in discussione «l'unità e il senso della basileia». ${ }^{35} \mathrm{Si}$ andava cioè delineando una nuova concezione dell'imperatore per cui egli non rappresentava più quella sorta di imperialismo escatologico che aveva contraddistinto l'impero sino dalla sua fondazione. In realtà il trono bizantino non era mai stato molto solido. Gli imperatori si succedevano con rapidità quasi doppia rispetto all'impero germanico occidentale. ${ }^{36}$ Con i Comneni però si verificò una sorta di consolidamento monarchico che, seppur effimero, dette nuova sicurezza allo stato rispetto al passato; tre imperatori regnarono per quasi un secolo. La morte di Manuele, il terzo di essi, rigettò la monarchia bizantina nell'instabilità e nella successione violenta. A ciò va aggiunta la grave situazione economica in cui versava la popolazione. I crescenti pericoli determinati dalle mire di conquista occidentali, germaniche soprattutto, avevano costretto le finanze verso un irrobustimento delle spese per la difesa le quali, come le altre, gravavano direttamente sulle tasche della gente. Inoltre già in questi anni la presenza dei mercanti italiani in primo luogo, ma non solo, nella capitale dell'impero stava diventando assai invadente. Alcuni di essi, come abbiamo visto, avevano già un quartiere nella capitale dell'impero e sarà solo l'inizio di una dominazione commerciale ben più estesa.

L'evento traumatico della IV crociata si inserisce quindi in un quadro di debolezza generale dell'impero il quale non poteva, in questo periodo, far fronte in alcun modo a una minaccia di queste proporzioni. E non era possibile fermare i crociati soprattutto se si pensa alla dinamica della spedizione e alle premesse

${ }^{34}$ In quegli anni vi era in Occidente un dibattito vivace fra i titolari del potere sulla necessità di promuovere una crociata contro il Saladino, il quale aveva occupato il Santo Sepolcro dopo la dura battaglia che ebbe luogo in Palestina nel luglio del 1187 in cui l'esercito latino venne sconfitto e il 2 ottobre Gerusalemme cadde. Due anni dopo si decise di agire: il Barbarossa, Filippo II Augusto di Francia e Riccardo d'Inghilterra presero la croce.

${ }_{35}$ M. Gallina, Potere e società a Bisanzio. Dalla fondazione di Costantinopoli al 1204, Torino 1995, pp. 313-314.

${ }_{36}$ Si pensi che dalla metà del IX secolo fino alla fine dell'XI salirono sul trono bizantino ventitre imperatori. A tale proposito si veda A.P. Kazhdan, Vizantijskaja kul'tura (X-XII vv.), Moskva 1968, tr. it. Bisanzio e la sua civiltà, Roma-Bari 1995, in particolare le pp. 27-66. 
diplomatiche che essa ebbe. Non è compito di questo lavoro indagare o riproporre l'evento del 1203-1204 in tutti i suoi particolari, ma giova stabilire un quadro generale per determinare con chiarezza quali conseguenze tale vicenda ebbe sulla regione in oggetto.

La IV crociata è stata definita, a ragione, la "crociata deviata" ${ }^{37} \mathrm{Vi}$ sono pochi dubbi sul fatto che l'intenzione originaria dei dirigenti crociati, esclusa Venezia, fosse quella di non muovere verso Costantinopoli; altrettanto acquisito è il fatto che la Serenissima abbia agito con forza al fine di spezzare definitivamente la resistenza bizantina alla sua penetrazione commerciale oltre il Bosforo (in questo periodo Genova era riuscita ad acquisire una posizione di privilegio rispetto alla rivale adriatica grazie ai buoni rapporti che aveva instaurato con Alessio III). Dopo alterne vicende Venezia riuscì a imprimere il proprio ritmo alla crociata e il 13 aprile 1204 l'esercito latino entrò nella capitale bizantina abbandonandosi a ogni genere di nefandezza. Lo scisma dell'XI secolo, come detto, ebbe in questo evento la sua realizzazione pratica. Le conseguenze politiche della crociata furono straordinariamente positive per la Serenissima che ottenne una vasta area dell'impero. Tutti i crociati ottennero benefici territoriali importanti, ma i vantaggi che andarono a Venezia superarono di gran lunga quelli di chiunque altro. Baldovino di Fiandra ottenne un quarto del territorio imperiale, degli altri tre quarti la metà spettava a Venezia e il resto era da distribuire fra i cavalieri che avevano partecipato alla crociata. Per la prima volta l'impero veniva frantumato da forze esterne. In seguito alla IV crociata nacque lo Stato Latino d'Oriente. Venezia si impadroniva di tutta la rotta marittima dalla madrepatria fino a Costantinopoli, controllava gli stretti e l'ingresso in città. I Franchi organizzarono l'impero frantumandone l'unità in un elevato numero di principati. Notevoli furono anche le conseguenze politiche, economiche e culturali nel bacino del Mar Nero caucasico e nell'Azov.

Lo studio delle regioni slavo-orientali non può prescindere dall'analisi delle vicende che legarono queste aree all'evoluzione politica, culturale ed economica dell'Impero Romano d'Oriente. Il contributo della storia bizantina alla formazione del mondo slavo e del Caucaso in particolare è un dato incontestabile e dai risvolti complessi. ${ }^{38}$ Le popolazioni slavo-meridionali (Serbi, Croati e Bulgari)

${ }^{37}$ La letteratura sulla IV crociata è copiosa. In questa sede basterà ricordare due fra le migliori opere di sintesi sulla storia di Bisanzio; Gallina, Potere e società cit., p. 318; Ostrogorsky parla di "diversione della crociata»: G. Ostrogorsky, Storia dell'impero bizantino, Torino 1993, p. 372. La IV crociata è stata definita anche «l'incompiuta» in un recente saggio (M. Meschini, 1204: l'incompiuta. La quarta crociata e le conquiste di Costantinopoli, Milano 2004). Si veda inoltre D.E. Queller, T.F. Madden, The Fourth Crusade: The Conquest of Costantinople, Philadelphia (PA) 1997.

${ }^{38}$ I. Duičev, Bisanzio e il mondo slavo, Medioevo Bizantino-slavo, vol. I, Roma 1965, pp. 3-22, p. 4; Id., Les Slaves et Byzance, in Études historiques à l'occasion du XIe Congrès international des sciences historiques, Sofja 1960, pp. 31-77; Ch. Diehl, Byzance, grandeur et décadence, Paris 1919, pp. 292 e sgg.; si veda anche Les centres proto-urbains russes entre Scandinavie, Byzance et Orient, a cura di M. Kazanski, A. Nercessian, C. Zuckerman, Paris 2000 in particolare la terza parte pp. 299-363. 
ebbero con Bisanzio rapporti stretti a livello politico, culturale ed economico. Meno continue e più indirette, ma altrettanto importanti, furono le relazioni fra Bisanzio e la Rus' e i popoli non slavi dell'area orientale (popolazioni turcomanne, Cazari, Polovcy/Cumani) ${ }^{39}$. La situazione del Vicino Oriente alla vigilia della IV Crociata era estremamente complessa e costituisce l'ambito entro il quale tale evento ebbe a generare uno scossone in grado di modificare in parte l'assetto politico della regione pontica.

Abbiamo detto che nella Partitio Romaniae, redatta all'indomani della disastrosa esperienza crociata, non si fa menzione della regione caucasica. ${ }^{40}$ D'altra parte in quest'area la situazione politica era ben diversa rispetto ai territori della costa anatolica o settentrionale del Mar Nero. Già la prima crociata era intervenuta in modo determinante sugli equilibri politici del Caucaso. I crociati non vi arrivarono mai, ma indebolirono fortemente il potere islamico. Tale evento inflisse un colpo durissimo ai Selgiuchidi e creò un temporaneo sfaldamento nelle maglie del potere turco. Proprio durante il XII secolo, mentre a Bisanzio era in corso la restaurazione dei Comneni, il Caucaso georgiano stava vivendo un periodo di forte sviluppo territoriale e politico. Non aveva del tutto torto in un suo celebre saggio sulla storia georgiana William Allen ad affermare che il regno di Georgia, alla vigilia dell'incoronazione di Tamara la Grande (1184), fosse in gran parte il frutto delle crociate. ${ }^{41}$ Approfittando della debolezza selgiuchide la dinastia bagratide aveva portato avanti una politica fortemente aggressiva ed era riuscita a respingere gli assalti del sultanato turco di Iconio.

In tale contesto emerse energicamente la rinnovata forza militare e culturale del regno di Georgia. Il re di Abkhazia e Georgia, David III, fu molto abile a introdursi entro tale sfaldatura e sconfisse a più riprese le forze musulmane. Egli fu in grado di riprendere parte dei territori caduti in mani turche durante le incursioni dell'XI secolo. Nel 1122 riprese Tblisi dopo ben quattro secoli, ${ }^{42}$

39 Per un quadro dei rapporti slavo-bizantini nel periodo pre-mongolo resta di riferimento G. Vernadsky, Relations bizantino-russes au XII siècles, in "Byzantion», 4 (1927-1929); cfr. anche. Per un aggiornamento del dibattito attorno al problema cfr. O. Pritsak, Origin of Rus', Harvard (MA) 1982 e Sverdlov, Domongol'skaja Rus', Sankt Peterburg 2003. Un quadro bibliografico esaustivo si trova in The Economic History of Byzantium cit., in particolare la parte IV, Exchange, Trade, and Markets.

40 Anche in Bratianu, La Mer Noire cit., p. 178.

${ }^{41}$ Allen, $A$ History of the Georgian People cit., p. 96; A.A. Vasiliev, The foundation of Trebizond, in «Speculum», 2 (1936), p. 13.

${ }^{42} \mathrm{Ibn}$ al-Athir, narrando della conquista georgiana di Tblisi traccia un ritratto molto positivo di David. Secondo lo storico persiano questi «si impegnò ad accontentare gli abitanti della città» accordandogli una serie di privilegi fra cui il divieto di uccidere maiali, mantenere la sacralità della preghiera del venerdì, la separazione nei bagni pubblici fra Georgiani e Musulmani; in pratica David «manifestò nei confronti dei musulmani più considerazione dei re musulmani stessi» (Ibn al-Athir, I, p. 486). 
occupò l'area di Shirvan, nel 1123 conquistò Ani (che fu più volte persa e ripresa negli anni successivi) ${ }^{43}$ e fu in grado di entrare nella Grande Armenia riprendendosi antichi possedimenti perduti. In questi anni molti Armeni combattevano nelle fila dell'esercito georgiano. ${ }^{44}$ Alla spinta in politica estera David dovette associare una forte attenzione verso l'interno dove l'aristocrazia di palazzo aveva molto potere e contrastava il progetto di uno stato monarchico centralizzato del sovrano. A questo scopo - e anche per rafforzare la propria forza militare verso l'esterno - David chiamò (1118) i Cumani a stabilirsi nelle terre attorno al regno georgiano; essi potevano andare a ripopolare le campagne che avevano subito un sensibile calo demografico in seguito alle incursioni dei Selgiuchidi. La creazione di un esercito composto esclusivamente da Cumani ${ }^{45}$ rappresentava un pericolo per la popolazione rurale con la quale i nomadi non erano in grado di interagire del tutto. Era di fatto un esercito salariato e che riceveva in cambio del proprio servizio terre buone per il pascolo. ${ }^{46}$

Ad alcuni anni di stagnazione, dopo la morte di re David, ${ }^{47}$ seguì la consacrazione al potere di Giorgio III il quale, sulla scia del predecessore, riprese le campagne contro i Selgiuchidi; questi portò avanti le conquiste in Armenia, soprattutto a Settentrione. Furono prese Gandzak, Dvin, Kars e Ani che divenne definitivamente georgiana nel 1199.48 A Giorgio III (1156-1185) successe sul trono di Georgia la figlia Tamara (1184-1213), figura mitica, leggendaria, ma certo una sovrana dotata di grande spessore culturale e molto abile politicamente. ${ }^{49} \mathrm{Fu}$ l'apogeo per il regno cristiano di Georgia che riuscì a sottomettere anche l'Azerbaijan. La regione che oggi corrisponde più o meno all'Azerbaijan

${ }^{43}$ La città cadde di nuovo sotto il controllo di Giorgio III nell'agosto del 1161 quando, al suo comando, i Georgiani «marciarono verso la città di Ani, nell'Arran. Si resero padroni, e uccisero molte persone» (Ibn al-Athir, In, p. 499), ma non fu la conquista definitiva.

${ }^{44}$ Allen, A History of the Georgian People cit., pp. 85-108.

455.000 uomini, secondo P. Golden; P.B. Golden, Cumanica I: The Qipčaqs in Georgia, "Archivum Eurasiae Medii Aevi», IV (1984), pp. 45-87, p. 61, ripubblicato in Id. Nomads and their Neighbours cit.

46 E probabile che in questa occasione siano giunti in Georgia almeno 200.000 individui dalle steppe e questo non solo per l'interesse che presso i nomadi poteva costituire il buon pascolo, ma anche perché all'inizio del XII secolo i Rus' operarono una massiccia offensiva contro i Cumani; essa culminò nell'avanzata del 1107/1109 sugli insediamenti cumani di Sugrov, Šarukan e Balin. Si veda su questo e sulle cifre Golden, The Qipčaqs in Georgia cit., pp. 70-71; O. Pritsak, The Polovcians and Rus', in "Archivum Eurasiae Medii Aevi» II (1982), pp. 303 e segg., p. 335 e 368.

47 Avvenuta nel 1124 secondo Ibn al-Athir (I, p. 488).

48 Per un quadro generale degli eventi si veda Storia degli Armeni, a cura di G. Dédéyan, Milano 2002, specialmente le pp. 235-268.

${ }^{49}$ Ancora oggi non esistono studi monografici dedicati alla regina georgiana in lingua italiana. Per un quadro generale, ben curato e per molti aspetti ancora oggi valido, si deve ricorrere all'opera antica di Brosset, Histoire de la Géorgie cit. soprattutto il vol. I, pp. 403-480 in cui, accanto alla traduzione francese della cronaca georgiana viene fatto un accurato studio sul paese caucasico nel Medioevo. 
fu per lungo tempo abitata da popolazioni iraniche fino all'assorbimento entro le fila dei nomadi turcofoni che, coi Guz prima (XII sec.) e poi brevemente sotto il sultano di Korazmia Jalal ad-Din - in fuga dai Mongoli - ne influenzarono profondamente lingua e cultura (su questo si veda più avanti, cap. quinto).$^{50}$

La Georgia perseguì quindi una politica estremamente oculata e organizzata acquisendo nuovi territori, stipulando alleanze matrimoniali con Bisanzio. L'equilibrio politico fra l'impero e il regno caucasico era mutato. Essi erano gli unici due regni cristiani del Medio Oriente e più volte si avvicinarono, principalmente nei periodi in cui la minaccia turca si intensificava. Fra il 1028 e il 1034, durante la reggenza di Romano III Macedone, la regina Miriam (regina di Iberia) visitò la capitale greca e nel 1032 suo figlio e la nipote dell'imperatore, Elena, si sposarono. ${ }^{51}$ Fra il 1065 e il 1071 si celebrò il matrimonio fra Marta, figlia di Bagrat IV di Georgia (1027ca-1072) e Michele VII Ducas. ${ }^{52}$ Durante il regno di Alessio I (1081-1118) Kata, una delle figlie di David II, sposò un nipote dell'imperatore. ${ }^{53}$

Non meno importante, per l'indirizzo delle vicende politiche nella regione, fu la terza crociata. Nel 1190, durante la spedizione capeggiata dal Barbarossa, i cavalieri della Croce entrarono nei territori dei Selgiuchidi in Anatolia. Il potere di questi ultimi e la loro effettiva autorità sul territorio, già indeboliti, continuavano in un processo di autoerosione a causa delle lotte fratricide che si verificavano frequentemente alla corte del sultano Kilij Arslan, i cui due figli non volevano cedere il potere uno all'altro. La debolezza selgiuchide aveva inoltre incoraggiato le incursioni lungo la diagonale che va della costa centro-orientale del Mar Nero a quella sud-occidentale del Caspio da parte dell'esercito georgiano. ${ }^{54} \mathrm{Il}$ Barbarossa e Kilij Arslan siglarono così un accordo in seguito al quale entrambi si impegnavano a combattere uniti contro un'eventuale alleanza fra Bisanzio e il Saladino. In questa occasione Kilij concesse la possibilità all'esercito crociato di passare in Anatolia, ma tale iniziativa si rivelò inutile poiché il Barbarossa come è noto, nella primavera del 1190, morì nelle acque dell'Oronte, in Cilicia.

${ }^{50}$ La conquista georgiana avvenne fra il 1202 e il 1203 quando i Georgiani si impadronirono di Dvin che, «come tutto il resto dell'Azerbaijan, appartiene all'emiro Abu-Bakr [...]» (Ibn al-Athir I, p. 501). Athir attribuisce all'emiro doti non proprio edificanti: «era sempre occupato a bere notte e giorno, senza sosta, e non cessava di essere immerso nella ubriachezza» (ibid.). Secondo il racconto dello storico persiano, quando gli abitanti di Dvin andarono a chiedergli soccorso egli non lo accordò rendendosi così corresponsabile della presa della città da parte georgiana e del conseguente massacro che la popolazione dovette subire. Sulla conquista dell'Azerbaijan si veda Ibn al-Athir I, pp. 501-509.

${ }^{51}$ HG, p. 314; Allen, A History of the Georgian People cit., p. 88-89; Vasiliev, The foundation cit., p. 4.

${ }_{52}$ HG, p. 330; Allen, A History of the Georgian People cit., p. 91. cit., p. 4.

53 HG, p. 360; Allen, A History of the Georgian People cit., p. 99; Vasiliev, The foundation

${ }^{54}$ Ibidem, p. 13; Allen, A History of the Georgian People cit., p. 106. 
Per Bisanzio la situazione divenne, se possibile, ancora più complessa. Ai problemi presenti nei Balcani (Bulgari e Serbi che, approfittando dell'impegno imperiale contro il Barbarossa, si erano allargati ulteriormente su Sofia e Skopie) si aggiunsero quelli col sultano di Iconio. Il 1190 fu un annus horribilis per Bisanzio. I Bulgari inflissero una dura sconfitta alle truppe imperiali proprio nei Balcani. Nel 1194 la situazione si ripeté e presso Arcadiopoli l'esercito di Isacco II fu sconfitto di nuovo. Nell'aprile del 1195 il fratello dell'imperatore, Alessio, si appropriò violentemente del trono. Fu il colpo di grazia.

Intanto in Anatolia al ritiro delle truppe crociate seguì la politica piuttosto aggressiva di Solimano, figlio del sultano di Iconio Kilij, il quale nel 1196 occupò Konya (Iconio) imponendo al nuovo imperatore Alessio III condizioni assai dure. L'imperatore bizantino doveva pagare un alto tributo al selgiuchide, ma nello stesso anno si verificò la pesante sconfitta inflitta dall'esercito cumano-georgiano ai danni di Solimano. Questi aveva deciso di attaccare l'Armenia di Leone II la quale aveva violato i confini del sultanato turco; nel frattempo l'esercito georgiano era avanzato fino a Erzrum. In questa occasione Solimano riuscì a sottomettere Salturid e ad avanzare verso la Georgia. Fu allora che l'esercito turco subì la pesante sconfitta che si rivelerà decisiva per le sorti del Caucaso e del regno georgiano in particolare. La cronaca georgiana ne racconta gli eventi con dovizia di particolari. Il sultano «camuffando la sua perfidia sotto forme amichevoli, inviava sovente ambasciatori per chiedere la pace, e dei bei e numerosi regali. Ma ben presto egli riunì ottanta migliaia di uomini, 800.000 soldati. L'esercito del sultano percorse tutti i suoi domini: la Mesopotamia, il Calonero, la Galatea, l'Ancouria, l'Isauro, la Cappadocia, la Grande Armenia, la Bitinia i confini della Paflagonia e obbligarono tutti a prendere le armi, non lasciando nei villaggi che le donne». ${ }^{55} \mathrm{Nel} 1199$, all'esordio della guerra contro i Selgiuchidi, «i Georgiani si mettono in battaglia. Il generalissimo Zakaria Mkhargrdzel, Chalwa e Iwane di Akhal-Tzikhé e gli altri Thoriani prendono l'avanguardia; da un lato piazzano gli Abhkazi, dall'altro gli Imerezi, da un altro i Kart'li, quelli di Cakheth e di Hereth». ${ }^{56}$ La guerra si rivelerà vittoriosa. È interessante osservare come il cronista chiami l'avversario barbaro. Tale denominazione emerge ogni volta che si vedono contrapposte le due parti. La percezione dell'altro come barbaro in quanto non cristiano denota una forte identità religiosa da parte dei Georgiani. ${ }^{57}$ Tale consapevolezza è uno dei motivi

55 HG, pp. 457-458.

${ }^{56}$ Ibidem, p. 460

57 Gli esempi sono molti nella cronaca georgiana. HG, p. 462: «Era uno spettacolo stupefacente quello di vedere questi barbari fuggire...»; Ibidem, p. 466: "questa regina brillante fra tutti quelli che hanno portato lo scettro, sempre occupata al servizio di Dio, alla costruzione ed all'abbellimento di Chiese e monasteri»; Ibidem, p. 467, in occasione della morte di David Soslan (1208) il cronista scrive: «era stato un principe pieno di tutte le virtù religiose». Di contro è interessante notare la serie di appellativi usati dalle fonti persiane nei confronti dei Georgiani. 
della forte influenza culturale che la Georgia esercitò sulla regione caucasica nei primi anni del XIII secolo. Una predominanza che avrebbe presto subito un colpo durissimo.

La vittoria sui Selgiuchidi favorì l'evolversi degli eventi successivi la quarta crociata i cui esiti si fecero sentire eccome in quest'area anche se, naturalmente, essa incise con più forza in Anatolia, sull'Egeo e nei Balcani, ovvero in quelle aree circostanti la capitale dell'impero. Sul Caucaso l'impatto della crociata fu meno diretto, ma ebbe conseguenze importanti.

Nel 1204 Solimano morì durante la sua seconda spedizione contro la Georgia e dopo aver conquistato Ankara. Gli successe il fratello che riuscì a riappropriarsi così del trono dal quale era stato estromesso da Solimano stesso. Kay Khusraw I, questo il suo nome, adottò una politica assai diversa da quella del fratello: molto meno aggressivo, con una minore tensione all'espansionismo. I progetti di una eventuale ulteriore marcia sulla Georgia vennero pertanto abbandonati.

\subsection{Le conseguenze della crociata. L'impero di Trebisonda}

Lo scossone causato dalla presa di Costantinopoli favorì la nascita di nuovi centri di potere più o meno direttamente legati al tragico evento. L'Impero Latino d'Oriente, estremamente frantumato già dalla nascita e il solido sistema "coloniale" 58 veneziano si affiancarono ai tre nuovi centri bizantini ben distribuiti sulla costa meridionale del Mar Nero. Il principato dell'Epiro, che dal 1222 diventerà impero di Tessalonica, scomparirà presto, in seguito alla dura sconfitta impostagli da Giovanni Asen nel 1230. L'impero di Nicea, che si rivelerà l'erede della tradizione imperiale, era geograficamente vicino alla capitale e ospitò il nuovo patriarca, ma un ruolo di primo piano nella politica della regione caucasica fu mantenuto dall'impero di Trebisonda. Lontano geograficamente dalla capitale, non potrà mai recuperarla, ma fu un centro importantissimo per i rapporti coi

Ad esempio Ibn al-Athir, I, pp. 509-512; e il funzionario dell'Ilkhan che scrive nel XIV secolo Ata Malik Juvaini (Juvaini, The History of the World Conqueror, ed. a cura di J. A. Boyle, 2 voll., Manchester 1958. Mi sono servito dell'edizione italiana Ata-Malik Juvaini, Gengis Khan, a cura di G. Scarcia, Milano 1962 rist. 1991, d'ora in avanti Juvaini), parte seconda (d'ora in avanti II), pp. 490-493.

${ }^{58}$ Il termine non è del tutto corretto poiché per Venezia, e ancor più per Genova, i caratteri della dominazione non furono quelli della colonizzazione. Le città nelle quali si insediarono i mercanti delle città italiane non vennero occupate interamente e anche quando ciò avvenne (il caso dell'isola di Chio è l'unico che abbia un simile carattere) mancò la dominazione territoriale e mancò lo sfruttamento delle risorse a vantaggio della madrepatria. La dominazione era di tipo economico e finanziario. Si può dunque parlare di colonie operando una semplificazione, ma sarebbe più corretto usare il termine emporia, stabilimenti ecc. Su questo punto è molto istruttivo il saggio di Michel Balard pubblicato come prefazione alla nuova edizione delle Colonie genovesi di Roberto Sabatino Lopez (R.S. Lopez, Storia delle colonie genovesi nel Mediterraneo, Genova 1997). 
poteri vicini, la Georgia in primo luogo. Lo fu anche economicamente in quanto diventò presto centro per i traffici commerciali di primissimo livello ed ebbe una profonda influenza culturale sulla regione dovuta in gran parte al peso dei sovrani che lo avevano fondato, i Comneni..$^{59}$

La dinastia cui apparteneva la regina Tamara di Georgia, i Bagratidi, aveva avuto rapporti con la corona di Bisanzio sin dalla fine dell'XI secolo, in particolare con la famiglia dei Ducas e dei Comeni.

Andronico nel 1170 si rifugiò sotto la protezione di Giorgio III (11551184) che lo accolse benevolmente. Secondo le parole del cronista autore della storia georgiana: «un giorno venne [da Giorgio III] Andronico Comneno, un cugino da parte di padre di Manuele il Grande, sovrano dell'intero Occidente e imperatore di Grecia; era accompagnato dalla moglie [...]. Giorgio concesse al principe accoglienza degna del suo alto casato, gli dette tutte le città e cittadelle di cui aveva bisogno e gli assegnò una residenza a lui vicina». ${ }^{60}$

È possibile, stando al passo sopra citato, che Andronico avesse legami di sangue con la casa regnante di Georgia. ${ }^{61}$ L'esperienza georgiana di Andronico potrebbe essere alla base della sostanziale accettazione popolare del governo dei Comneni nella città anatolica.

La rivolta anti-comnena che si verificò a Bisanzio nel 1185 costrinse i figli di Manuele, Alessio e David, a fuggire dalla furia di Isacco Angelo. I bambini si rifugiarono alla corte di Tamara e crebbero in un ambiente culturalmente vivace nel quale appresero la lingua georgiana. Tali vicende influenzarono la scelta di Trebisonda come centro dal quale ripartire per la ricostituzione di un territorio dopo la disfatta del 1204 . Tuttavia, in base alle date, sembra proprio

59 Sull'impero di Trebisonda e la sua formazione esiste una copiosa letteratura scientifica della quale non credo sia il caso rendere conto in questa sede in modo esaustivo. Per un approfon-

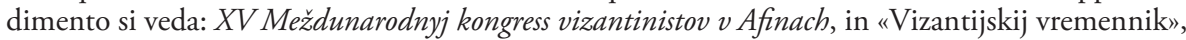
XXXIX (1978), pp. 17-45; F.I. Uspenskij, Vydelenie Trapezunta iz sostava Vizantijskoj imperii, in «Seminarium Kondakovianum», I (1927), pp. 21-34; S.P. Karpov, Empire of Great Comnenoi: Some Remarks on Particularities of its Foundation, in Actes du XVe Congrès International d'Études Byzantines, Atene 1980, pp. 153-159; Id., Trapezundskaja imperija i zapadnoevropejskie gosudarstva v XIII-XV vv., Moskva 1981, ed. it. L'impero di Trebisonda, Venezia, Genova e Roma: 1204-1461, Roma 1986; superato, ma sempre piacevole e istruttivo, rimane il saggio di N. Jorga, Une nouvelle théorie sur l'origine et le caractére de l'empire de Trébizonde, in "Revue historique du sud-est européen", XIII (1970-1971), pp. 107-116; M. Kuršanskis, L'Empire de Trébizonde et la Géorgie in "Revue des étude byzantines», XXXV (1977), pp. 237-256.

${ }^{60}$ Brosset, Histoire de la Géorgie cit., pp. 396-397; Vasiliev, The foundation cit., p. 6; il passo è riportato anche in Uspenskij, Vydelenie Trapezunta cit.

${ }^{61}$ Così in Vasiliev, The foundation cit., p. 6-8. Andronico è nonno del primo imperatore di Trebisonda. Vasiliev fa notare, opportunamente, che d'ora in avanti compare nella famiglia imperiale il nome David, sconosciuto prima di allora a Bisanzio. La cronaca georgiana reca un altro passo in cui il legame di parentela è espressamente indicato. Dopo che l'esercito georgiano aveva occupato «tutta la Paflagonia e il Ponto, [Tamara] li donerà al suo parente Alessio Comneno, figlio di Andronico, allora rifugiato presso di lei», HG, p. 465. 
che la fondazione del nuovo impero non sia stata una conseguenza diretta della seconda presa di Costantinopoli. In realtà i crociati violarono la capitale sul Bosforo per la prima volta il 18 luglio del 1203 . Nell'aprile dell'anno successivo Alessio Comneno entrò a Trebisonda; contemporaneamente, o quasi (13 aprile), Costantinopoli cadde per la seconda volta. A questo va aggiunto che la cronaca georgiana racconta un fatto, ${ }^{62}$ ormai accolto come attendibile, secondo cui agli inizi del 1203 Tamara accolse a corte un numero imprecisato di monaci. Alcuni venivano dal Monte Athos, altri da Cipro, altri ancora da Antiochia etc. Come era solita fare, la regina li congedò con ricche donazioni per gli enti religiosi cui appartenevano. Sulla strada del ritorno alcuni di essi fecero sosta a Costantinopoli. Essendo venuto a conoscenza del fatto Alessio III Angelo, ostile da sempre al regno georgiano, confiscò loro i beni che trasportavano. Tamara provvide a inviare nuove donazioni, ma non si accontentò di questo. Cercò di reagire al sopruso bizantino e lo fece marciando su Trebisonda. ${ }^{63}$ L'evento narrato sarebbe stato quindi un pretesto. È più probabile che la conquista di Trebisonda sia stata un atto programmato, da parte di Tamara, per espandere la propria influenza sull'Anatolia e avere una testa di ponte per il commercio sul Mar Nero. La politica espansionistica di Tamara è ben testimoniata dagli eventi che caratterizzarono il suo regno. Tamara riuscì non solo a consolidare la supremazia culturale del suo potere su tutta la Caucasia, ma riprese molti distretti e città ai Selgiuchidi. Emblematico è il caso della vittoria di Ani nel 1209. ${ }^{64}$ La conquista di Trebisonda fu, molto probabilmente, il tentativo di assicurarsi un punto di riferimento in Anatolia. Secondo la cronaca georgiana Tamara concesse ad Alessio le forze militari necessarie per portare a termine la spedizione $^{65}$ alla quale presero parte soldati georgiani ed imerezi in particolare. ${ }^{66}$ Quello che sembra certo è che non fu parte di un disegno più ambizioso che avrebbe previsto la riconquista di Costantinopoli.

Trebisonda, città bizantina, divenne la capitale di un piccolo impero, la cui importanza rimarrà a lungo vitale per la regione circostante e per la Transcaucasia

${ }^{62}$ Ibidem, pp. 464-465; Vasiliev, The foundation cit., pp. 18-19.

63 «La regina ne fu informata e per confondere il demone (evidentemente Isacco) rimanda a questi santi personaggi delle somme più considerevoli», HG, p. 465.

${ }^{64}$ La città cadde dopo che «12 mila uomini, senza contare quelli che morirono sul posto, furono massacrati, come quelli nelle chiese» (HG, p. 468). Quando la regina seppe la notizia scattò la rappresaglia georgiana. Le truppe, agli ordini del vecchio e geniale Zaccaria, ripresero Ani, «sconfissero i Saraceni (Ibid. cit., p. 469), e uccisero 12 mila persone». A parte le cifre, che pare siano del tutto approssimative e in gran parte simboliche, il cronista georgiano è spesso attendibile e in questo periodo non c'è da dubitare del potenziale militare di Tamara e delle ripetute vittorie di questa sui Turchi.

${ }_{65}$ Vasiliev, The foundation cit., p. 23.

66 «[Tamara] Fece partire un distaccamento di soldati imerezi che si impadronirono della Lazica, di Trebisonda, di Limon, di Samsun, Sinope, Cerasonte, Cythora, Amastri, Eraclea...»; HG, p. 465; Vasiliev, The foundation cit., p. 20. 
in particolare. Le mire di conquista georgiane sulla città anatolica sarebbero state giustificate dall'importanza che essa aveva da ben prima della presa da parte dei Comneni. Con la nuova reggenza Trebisonda crebbe, ma essa era già da tempo un florido centro per il commercio. La sua posizione geografica ne aveva favorito lo sviluppo. Già dall'VIII secolo appare come un porto di riferimento per la Georgia. ${ }^{67}$ Era frequentata da mercanti di varia provenienza, greci, armeni, circassi, ebrei, mercanti dalla Crimea, dall'Iberia. ${ }^{68}$ Da qui, sin dal XII secolo, partiva la via commerciale per la Persia e l'Asia centrale.$^{69}$ La crociata del 1204 ebbe l'effetto di rafforzarne decisamente il ruolo di referente commerciale nella regione. A una decadenza che nella capitale era in pieno sviluppo dalla seconda metà del XII secolo si contrapponeva, e forse ne era in parte causa, il rafforzamento delle città periferiche. ${ }^{70} \mathrm{~A}$ ciò si possono aggiungere la scomparsa del pesante prelievo fiscale operato da Bisanzio sulle città dell'impero in particolare dagli ultimi anni del XII secolo, la conquista tataro-mongola, la costituzione dell'Ilkhanato di Persia; la nascita di uno stato unitario significava soprattutto maggiore sicurezza delle vie di transito, quindi maggiore mobilità e aumento dei traffici commerciali. Se le vie di collegamento terrestri beneficiarono di questa situazione, lo stesso si può dire per i traffici marittimi. Trebisonda era collegata via mare con Costantinopoli e frequenti erano i contatti con la costa settentrionale del Mar Nero, la Crimea e il Mar d'Azov, dove l'insediamento latino della Tana rappresenterà uno dei centri di scambio più vivaci della regione per oltre un secolo. Tali relazioni commerciali si intensificarono ulteriormente nel momento in cui le città marinare italiane si stabilirono sul Mare Majus e dettero vita al nuovo sistema commerciale. I primi stabilimenti commerciali genovesi e veneziani sul Mar Nero furono istituiti in Crimea, con Caffa che divenne un centro di straordinaria importanza, ma Trebisonda rappresentava lo snodo attraverso il quale passavano i collegamenti terrestri con il Caucaso che dalla città anatolica proseguivano per Tabriz e l'Estremo Oriente. Questa sua posizione resisterà a lungo. Secondo M. Balard ci sono forti probabilità che l'installazione a Trebisonda di una comunità organizzata genovese sia più o meno contemporanea a quella di Caffa, quindi poco dopo la metà del XIII secolo. Nel 1291 un membro della famiglia Doria lavora per i Comneni e Galvano Negro è console genovese a Trebisonda. ${ }^{71}$

Giova infine ricordare che una delle voci più consistenti del giro d'affari cui i Latini avevano dato vita nei loro empori commerciali era costituito dagli

${ }^{67}$ Karpov, Limpero di Trebisonda cit., p. 35; A. Laiou, Exchange and Trade, Seventh-Twelfth Centuries, in The Economic History of Byzantium cit., pp. 689-754, alle pp. 726-728

${ }^{68}$ Karpov, Limpero di Trebisonda cit., pp. 29-30.

${ }^{69}$ Karpov, Limpero di Trebisonda cit., p. 30.

${ }^{70}$ Karpov, Limpero di Trebisonda cit., p. 31; F.I. Uspenskij, Istoria Vizantijskoj imperii, Moskva 1948 cit., p. 658.

${ }^{71}$ Balard, La Romanie Génoise cit., I, p. 134. La presenza di un console a questa data lascia pensare a un insediamento non troppo recente. 
schiavi e alla fine del XIII secolo la loro provenienza dimostra ancora una volta quanto fossero stretti i legami di questa città col Caucaso: Abkhazi, Lazi, Circassi, Zichi, Cumani. ${ }^{72}$

Dopo la morte di Tamara i rapporti fra l'impero trapesuntino e la Georgia mutarono. Con il successore della regina, Giorgio IV Lasha (1212-1223), le relazioni fra il regno cristiano e il giovane impero anatolico subirono un rallentamento. Fu una situazione temporanea in quanto gli eventi dell'Ovest imposero all'impero di Trebisonda una più intensa attività sul Caucaso e in Crimea.

Fra l'estate e l'autunno del 1214 David Comneno, fratello di Alessio, venne sconfitto duramente e ucciso dai Rum di Izz ad-Din; in quell'occasione fu catturato anche Alessio. L'impero di Trebisonda dovette accettare una sorta di protettorato da parte del sultano di Iconio al quale pagava un tributo oneroso. A questa data i confini occidentali dell'impero anatolico arrivavano ai fiumi Iris e Thermodon (Jeshil Yrmak e Terme), a sole 155 miglia dalla capitale. ${ }^{73}$

La presenza del sultanato di Iconio sulla costa centro-meridionale del Mar Nero, con tanto di sbocco sul medesimo, tagliò letteralmente in due l'Anatolia lasciando a ovest il regno di Lascaris e a est i Comneni di Trebisonda. ${ }^{74}$

Da Manzikert al 1204 si era determinato un quadro nuovo: il crollo di Bisanzio e la conseguente perdita di controllo sulla provincia ne aveva rafforzato il ruolo politico e aveva affrancato dalla pressione, soprattutto fiscale, ma anche militare, le città con elevate potenzialità autonome. Trebisonda aveva beneficiato di questa situazione rafforzando molto il suo ruolo sia politico sia commerciale. Dal punto di vista politico la città anatolica diventava il referente primo del regno cristiano di Georgia. Quando per quest'ultimo si avvicinerà la fine sarà già forte la dipendenza dei Comneni dal sultanato di Iconio il quale per altro

${ }^{72}$ Anche in questo caso è molto istruttivo il lavoro già citato di M. Balard ( La Romanie génoise cit., in particolare le pp. 289-309; C. Verlinden, Le recrutement des esclaves à Genes, du milieu du XII siècle jusque vers 1275, in Fatti e idee di storia economica nei secoli XII-XX. Studi dedicati a F. Borlandi, Bologna 1976, pp. 37-57; Id., Aspects de l'esclavage dans les colonies médiévaes italiennes, in Hommage à L. Febvre, Paris, 1953, t. II, pp. 91-103; Id. Esclaves du Sud-est et de l'Est européen en Espagne orientale à la fin du Moyen Age, in "Revue d'Histoire du Sud-Est européen», XIX/2 (1942); Id., Esclaves et ethnographie sur les bords de la mer Noire (XIIIe et XIVe siècles), in Miscellanea historica in honorem Leonis von der Essen, Bruxelles-Paris, 1947, pp. 287-298; Id., Esclaves alains en Italie et dans les colonies itliennes au XIVe siècle, in "Revue belge de Philologie et d'Histoire», 36 (1958), pp. 451-457.

${ }^{73}$ Vasiliev, The foundation cit., p. 29; W. Miller, Trebisond, the last greek Empire, London 1926.

${ }_{74}$ Sulle vicende politiche di questo periodo si veda R.M. Šukurov, Velikie Komniny i Vostok (1204-1461), Sankt Peterburg 2001; Id., D.A. Korobejnikov, Velikie Komniny, Sinop i Rym v 1223-1230 gg., in «Pričernomor'e v srednie veka», 3 (1998), pp. 178-200; R.M. Šukurov, Tjurki na pravoslavnom Ponte v XIII-XV vv.: načal'nyj étap tjurkizacii?, "Pričernomor'e v srednie veka», 2 (1995), pp. 68-103; Id., Velikie Komniny i "sinopskij vopros" v 1254-1277 gg., "Pričernomor'e v srednie veka», 4 (2000), pp. 177-208; S.P. Karpov, Latinskaja Romania, Sankt Peterburg 2000, in particolare il capitolo 3 (Ljudi i obstojatel'stva), pp. 128-214. 
non riuscirà mai a imporre un indirizzo proprio alla vita del giovane impero trapesuntino. Economicamente questo era ormai il trampolino da cui mercanti di provenienze diverse si lanciavano verso lucrose imprese sia per mare (verso Bisanzio e soprattutto verso il nord del Mar Nero, Crimea e Azov) sia per terra (Estremo Oriente e Persia, via Tabriz, la cui importanza commerciale stava lentamente crescendo verso uno straordinario processo di sviluppo che raggiungerà l'apice sotto il governo mongolo degli Ilkhan). A Trebisonda si installeranno le fiorenti comunità genovese e veneziana i cui rapporti commerciali col Caucaso andranno via via sempre più intensificandosi (si pensi, ad esempio, ai centri di Lo Vati/Faxium, Savastopoli e Batumi).

In seguito all'onda d'urto della crociata e alla conseguente nascita di nuovi poteri contrapposti la tradizione culturale bizantina dimostrò di non aver esaurito la propria energia positiva. Tale tensione si realizzò in parte nell'opera del giovane regno di Teodoro Lascaris sorto attorno a Nicea. Il risultato fu, grazie anche alla posizione geografica di questo centro, una lunga resistenza alle potenze vicine, l'Impero Latino d'Oriente in testa e la successiva presa di Costantinopoli. A est l'impero di Nicea era limitato dalla presenza e dalle velleità di conquista del sultanato di Iconio per il quale uno sbocco sul Mar Nero era irrinunciabile. Gli eventi dei primi due decenni del secolo XIII videro un susseguirsi di guerre fra Teodoro Lascaris e il nuovo imperatore latino Enrico, fratello di Baldovino di Fiandra. L'intera regione dai Balcani all'Anatolia attraversò un lungo periodo di instabilità e incertezza durante il quale il regno niceno riuscì a resistere ai Selgiuchidi da un lato (vittoria di Teodoro presso Antiochia nella primavera del 1211) e ai Latini d'Oriente dall'altro. Verso la fine del 1214 si giunse alla pace fra Enrico e Teodoro. L'evento ebbe effetti importanti sugli equilibri politici del Ponto poiché i due soggetti, fino ad allora in guerra, si riconoscevano reciprocamente rispettando uno i confini dell'altro. Proprio tale riconoscimento tenne fuori dagli scontri la parte orientale della costa anatolica del Mar Nero. Il sultanato di Iconio era una barriera che permetteva al piccolo impero di Trebisonda di vivere in sicurezza e ciò incrementò il suo ruolo di referente politico e commerciale per i centri del Caucaso e della Persia.

Il regno di Georgia da parte sua incontrò la fine dal 1220, quando un popolo venuto dall'Est si riversò sulle frontiere dell'Azerbaijan e distrusse la grande costruzione che la dinastia Bagratide aveva faticosamente creato. Quell'esercito, guidato da Gengis khan, cambierà le sorti di un intero continente dando vita a un nuovo soggetto politico, complesso e vastissimo del quale più avanti tenteremo di indagare parte della sua realizzazione in Caucasia e quella che si è soliti identificare col termine di pax mongolica.

Negli anni in cui si stava preparando la crociata verso il Mediterraneo partirono in sordina le operazioni militari al nord. Nel XIII secolo il mondo Baltico venne profondamente trasformato da una lunga serie di conflitti. Alcuni popoli vennero decimati dalle guerre, altri furono incorporati di fatto nell'orbita della 
cristianità occidentale, tutti dovettero accogliere il flusso commerciale da Ovest portato dai mercanti che seguivano, e talvolta partecipavano direttamente, alle operazioni militari. Sorsero in questa circostanza almeno quattro nuovi poteri: i domini di Livonia ${ }^{75}$ e Prussia e i ducati di Estonia e Finlandia. Nacquero una nuova arcidiocesi e otto nuovi vescovati, puntello religioso contro l'idolatria e l'eterodossia della cristianità orientale. Il controllo religioso divenne capillare con la dislocazione sul territorio delle diocesi e la presenza di religiosi che portavano avanti con zelo l'opera di conversione. ${ }^{76}$ La quarta crociata non fu dunque un evento circoscritto al Medio Oriente e all'impero bizantino, ma ebbe, come abbiamo visto in precedenza, un prologo determinante nelle prime incursioni nomadi in Anatolia e nel Caucaso. Essa fu, inoltre, anche il risultato di un profondo sconvolgimento che, politico soprattutto, divenne ben presto religioso. Fu un evento globale, che sconvolse non solo gli equilibri politici di tutto il bacino del Ponto, ma destabilizzò gran parte dei poteri acquisiti anche a nord, dove popolazioni diverse interagivano fra di loro da secoli e abbatté un'ulteriore barriera per l'avanzata mongola da est.

75 Sul concetto di Livonia è tornato recentemente Piero Bugiani nella sua edizione al Chronicon Livoniae di Enrico il Lettone. Cfr. Chronicon Livoniae, a cura di P. Bugiani, Livorno 2005, in particolare l'introduzione alle pp. XIII-XIV.

${ }^{76}$ Sulla crociata del Nord la bibliografia è copiosa. Basti in questa sede citare il lavori seguenti: E. Christiansen, The Northern Crusade. The baltic and the Catholic Frontier (1100-1525), London 1980, tradotto anche in italiano: Le crociate del nord, Bologna 1983; E.N. Johnson, The German Crusade on the Baltic, in A History of the Crusades cit., III, 1975, pp. 545-565; L. Leciejewicz, La nuova forma del mondo. La nascita della civiltà europea medievale, Bologna 2004. 



\section{LE STEPPE DEI POLOVCY COME ESITO TRANSITORIO}

La regione che si estende dal medio corso del Volga e giunge, a occidente, sino al basso Danubio abbracciando l'istmo naturale fra Mar Nero e Caspio è stata oggetto di conquista per i popoli nomadi delle steppe sin dal I secolo dell'era cristiana, sin da quando cioè Tacito rammenta il popolo dei Sarmati. È un ambito geografico vario la cui straordinaria eterogeneità etnica ne ha caratterizzati gli sviluppi politici, culturali ed economici sin dall'Antichità e di tali mutamenti ne è stata insieme una conseguenza. ${ }^{2}$

Tale area è oggi compresa fra l'Ukraina centro-orientale, la Russia meridionale e il Kazakistan centro-occidentale ed è caratterizzata da una prevalenza di pianura. Vi scorrono fiumi dalla portata imponente come il Dnepr, il Don, il Volga e l'Ural. Se tracciassimo una linea immaginaria seguendo il parallelo che da Kiev giunge fino ad Aktyubinsk (nel Kazakistan nord-occidentale) noi avremmo ottenuto due zone morfologicamente simili, ma due ambienti naturali piuttosto diversi. ${ }^{3}$ In effetti le pianure poste a nord della nostra linea sono dominate dalla foresta, mentre quelle meridionali consistono in zone prevalentemente erbose. A Nord si incontra una barriera naturale nella catena montuosa degli Urali, a Sud vi sono i mari. La zona settentrionale è ricca d'acqua. I piccoli ruscelli di oggi, nel periodo considerato, erano una risorsa idrica fondamentale per le popolazioni che abitavano la regione. Osservando una carta dell'Europa centro-orientale si noterebbe come a ovest del Caspio vi sia stata una forte urbanizzazione laddove a est la naturale vocazione del suolo non ha ancora subito le modificazioni imposte dall'uomo.

1 «Hiberi locorum potentes Caspia via Sarmatam in Armenios raptim effundunt[...]»e più avanti: «Enimvero apud Sarmatas non una vox ducis: se quisque stimulant, ne pugnam per sagittas sinerent: impetu et comminus praeveniendum» infine: «Sarmatae omissu arcu, quo brevius valent, contis gladisque ruerent», Tacito, Libri ab excessu divi Augusti, ed. a cura di B. Ceva, 3 voll., Milano 2000, vol. II, Libro VI-XXXIII, pp. 409-413; si veda a tale proposito anche A. Carile, L'ecumene romano-cristiana e i popoli della Rus', in "Rivista di Bizantinistica», 2 (1992), pp. 11-33; M. Bais, Albània Caucasica. Ethnos, storia, territorio attraverso le fonti greche, latine e armene, Milano 2001, pp. 43-44.

2 Per il periodo che riguarda il Caucaso e l'Albània in particolare si rimanda al sopra citato lavoro di Marco Bais. Per approfondire il problema etnico della regione, sin dall'Antichità, è necessario rivolgersi a una copiosa e solida tradizione storiografica per la quale rimandiamo alla bibliografia di questo volume.

${ }^{3}$ Si tratta di una suddivisione proposta da A. Bartha, The typology of nomadic empires, in I popoli delle steppe: Unni, Avari, Ungari (Atti della XXXV Settimana di Studi cisam), Spoleto 1987, pp. 151-174. 
La conoscenza dell'ambiente geografico in cui si svolse l'afflusso di popolazioni nomadi era una necessità anche nel Medioevo. Di tali descrizioni possediamo un esemplare unico per chiarezza compositiva e per il periodo preso in esame: il De administrando imperio dell'imperatore bizantino Costantino VII Porfirogenito. ${ }^{4}$ L'opera fu scritta attorno alla metà del X secolo e aveva lo scopo di istruire i successori del basileús sull'importanza delle zone che confinavano con Bisanzio e su come si dovesse condurre l'attività diplomatica con chi le abitava. ${ }^{5}$ Nei secoli successivi la situazione mutò politicamente e il De administrando non poté essere utilizzato come manuale di geografia storica, ma per lo studioso rimane un osservatorio privilegiato sulla regione. ${ }^{6}$

I popoli nomadi delle steppe hanno rappresentato per secoli un tópos profondamente radicato nella mentalità di chi si è dovuto confrontare con essi; autori romani, greci, bizantini tutti li hanno percepiti come popolo "altro". Anche una larga parte della storiografia sull'argomento se ne è spesso occupata sottolineandone le forme di vita primitive, i rozzi mezzi di scambio e soprattutto - errore questo assai più grave - ne ha sottovalutata l'importanza avuta nel processo formativo dell'identità europea. ${ }^{7}$

All'interno di questo complesso movimento umano e nel corso della sua evoluzione compare nelle fonti, dalla metà del secolo XI, un popolo, le cui caratteristiche sono assimilabili a quelle di altri gruppi etnici che avevano dimorato nelle steppe; un popolo destinato a soccombere sotto i colpi dell'esercito mongolo. I Cumani-Polovcy rappresentano quindi l'ultimo passaggio del nomadismo allargato prima di quella che potremmo definire "stabilizzazione" tataro-mongola, ovvero la costruzione di un sistema di potere organizzato con manifestazioni territoriali che, se in un primo momento ebbe un impatto devastante sulla regione conquistata, in seguito ne consolidò il carattere urbano pur senza mai entrare a farvi parte del tutto come elemento costitutivo. ${ }^{8}$ I Mongoli giunsero nei territori stepposi dell'Europa orientale come popolo sostanzialmente nomade, ${ }^{9}$ ma ben presto entrarono in una lunga fase di acculturazione i cui

${ }^{4}$ Costantinus VII Porfirgenitus, De administrando imperio, a cura di R. J. H. Jenkins, Washington (DC) 1967; Die Byzantiner und ihre Nachbarn: Die "De administrando imperio" genannte Lehrschrift des Kaisers Konstantinos Porphyrogennetos für seinen Sohn Romanos, a cura di K. Belke e P. Soustal, Wien 1995.

${ }^{5}$ Obolensky, Il Commonwealth cit., pp. 39-40.

${ }^{6}$ Costantino traccia un itinerario che, partito da Tessalonica, giunge sino a Sotirioupolis (oggi Pizunda). È un resoconto assai dettagliato di luoghi, popoli e geografia territoriale. Egli descrive con dovizia di particolari il Danubio, Belgrado, poi il Dnestr fino al Dnepr e ancora verso oriente fino a Cherson, in Crimea. Descrive il Bosporo e lo stretto di Kerč. Giunge a nord fino alla foce del Don, dando una descrizione del Lago di Meotia (il Mar d'Azov) e il territorio dei Cazari.

7 Tali posizioni sono mutate negli ultimi anni grazie soprattutto all'opera intelligente e molto solida di altomedievisti che hanno riconsiderato il problema con rigore.

${ }^{8}$ Questo fenomeno viene definito dagli antropologi anche come reidentificazione, per sottolineare i mutamenti della struttura nomade verso una maggiore sedentarietà.

${ }^{9}$ Su questo punto gioverebbe soffermarsi a lungo. Il processo evolutivo che ha visto prota- 
sviluppi ne modificarono le forme organizzative, anche se in modi assai diversi nelle singole aree in cui sorsero i diversi stati; quelli di nostro interesse, l'Orda d'Oro e l'Ilkhanato vissero processi di integrazione molto diversi fra loro.

In questa sede ci proponiamo di affrontare quindi gli aspetti e i problemi legati alla steppa nel periodo che va dalla prima metà del secolo XI fino alla conquista tataro-mongola, occorsa nei primi decenni del XIII. Lo scopo è quello di determinare le premesse a un evento la cui portata ebbe conseguenze che andarono ben oltre i confini della regione conquistata. In questa prima parte analizzeremo l'origine e le problematiche connesse con le popolazioni nomadi, e in particolare i Cumani o Polovcy, coloro cioè sui quali si abbatté l'avanzata tataro-mongola nella regione a nord dei monti caucasici, dal Volga fino alle steppe dell'Azov e della Crimea. Sono problemi aperti, domande alle quali ancora non è possibile dare risposte definitive, ma proprio per questo vale la pena operarvi una riflessione. L'analisi di tali questioni impone il parziale scollamento dall'andare cronologico a vantaggio di un'analisi oggettiva che evidenzi, se possibile, i caratteri e gli sviluppi di questi gruppi umani complessi la cui opera determinò per oltre un secolo la vita della regione considerata.

Alla vigilia dell'invasione tataro-mongola i Polovcy dominavano un'area vastissima compresa fra il medio-basso corso del Volga fino al Danubio. Nel XIV secolo il generale armeno Hethum, nella sua storia dei Tatari, descrive il territorio affermando che «il regno di Komania è estremamente vasto, ma a causa del suo clima ostile, è un luogo difficile per abitarvi [...] ad oriente, il Regno di Komania confina col Regno di Korazmia e un deserto; ad occidente, col Mare Grande [Mar Nero] e un mare piccolo (chiamato Mar di Reme [Azov]); a nord col Regno dei Kassi [Rus']; a sud, si distende sino al grande fiume chiamato Et'il [Volga]. La città principale del Regno di Komania si chiama Sarai. In passato era una città nobile e conosciuta, ma essa è stata attaccata e quasi completamente distrutta dai Tartari che la presero con la forza [...]». Qui Hethum commette un errore in quanto le due Saraj (Batu e Berke) furono entrambe costruite dai Mongoli, e portano i nomi di due khan. ${ }^{10}$ La loro presenza era massiccia anche nel territorio compreso fra Caspio e Mar Nero; i monti del Caucaso ne costitui-

gonista l'unione delle tribù mongole verso forme sociali più stabili, legate al territorio e a prime forme produttive di tipo agricolo ebbe inizio prima delle grandi conquiste dei gengiskhanidi. Ce ne occuperemo diffusamente in seguito.

${ }^{10}$ Hethum visse a cavallo fra XIII e XIV secolo. Militare di professione, egli fu testimone oculare di molti degli eventi di cui parla in quanto fece parte di numerose spedizioni militari come nobile locale nell'esercito mongolo che aveva da pochi decenni conquistato la Caucasia. Mi sono servito di due edizioni: quella in Recueil des Historiens des Croisades. Documents Arméniens, Paris 1869, I, pp. 469-490 e de La Storia dei Tartari di Hayton Armeno, in G.B. Ramusio, Navigazioni e Viaggi, vol. III, Torino 1980, pp. 299-355 (d'ora in avanti STHA), III, pp. 299-355, p. 352; per la traduzione in lingua inglese e un'aggiornata bibliografia si veda anche G. Burger, A Lytell Cronycle, Toronto 1988. Una moderna traduzione, anch'essa in lingua inglese, è data dall'armenista Robert Bedrosian, The Flower of Histories of the East (online all'indirizzo <http://rbedrosian.com/hetumint.htm>). 
vano il blando confine meridionale; erano una popolazione nomade di origine turca, la cui vicenda è ancora in gran parte da chiarire e rappresenta un punto fermo nell'indagine della regione compresa fra il Caucaso centro-settentrionale e l'Azov. Ma vi sono pochi dubbi sul fatto che in questo periodo essi fossero i "padroni assoluti di tutto il Dešt-i Kipciak». ${ }^{11}$

Il concetto di steppe dei Polovcy è alquanto ambiguo, poiché non consente una definizione geografica chiara; è un ambito spaziale rarefatto, anche se in parte determinato dalla struttura climatico-territoriale. Si può affermare con Tosi che sebbene il Caucaso sia un contesto naturale straordinariamente diversificato, non si può ricondurre al solo determinismo ambientale la complessità etnica che lo caratterizza; tuttavia essa rimane alla base del comportamento umano che contrassegnò i tratti salienti del nomadismo. ${ }^{12}$ In ogni caso le caratteristiche morfologiche del territorio compreso fra il Caucaso e l'Azov hanno giocato un ruolo fondamentale nello sviluppo delle vicende politiche, militari e sulla progressiva formazione del tessuto etnico delle popolazioni che abitarono questa zona. ${ }^{13}$ Tali condizioni non sono però sufficienti a spiegare gli sviluppi successivi che coinvolsero i Polovcy. Caratteri strutturali e dinamiche sociali hanno determinato un cambiamento niente affatto casuale, bensì strettamente legato alla dimensione intrinseca di quella società, ai suoi appartenenti tutti e alle loro caratteristiche. Lo studio della società nomade attraverso le fonti scritte rischia di deviare l'attenzione verso aspetti che l'estensore delle medesime considerava preminenti, ma che non lo erano affatto all'interno dei nuclei sociali considerati. Se per lo studioso contemporaneo l'indagine di questi modelli e del loro ruolo nel più vasto movimento storico del periodo considerato è un'operazione culturalmente difficile, essa non lo era di meno per chi guardava a questi popoli, spesso sulla difensiva, in relazione al proprio presente, al modello in seno al quale si era formato e nel quale era profondamente inserito. Non sorprende quindi che Niceta Coniate, Cinnamo, Anna Comnena, l'autore del Racconto dei Tempi Passati, Roberto di Clari o Ottone di Frisinga, per citare solo alcuni che ci hanno lasciato traccia dei nomadi della steppa e dei Cumani in particolare, li definiscano barbari e guardino a essi come ad un modello distante, da rifutare. ${ }^{14}$

11 B.D. Grekov, A.Ju. Jakubovskij, L'Orda d'Oro, Firenze 1959 (tr. it. di Zolotaja Orda i ee Padenie, Moskva 1958), p. 6. Dešt-i-Kipčak è la denominazione data dalle fonti arabo-persiane alle steppe dei Polovcy che essi chiamavano appunto Kipčaki.

${ }_{12}$ M. Tosi, Dalla tribù all'impero cit., I, pp. 246-270, alla p. 247.

13 Sulla geografia del Caucaso e circa il ruolo dell'ambiente sugli sviluppi della regione si vedano in particolare gli articoli di A. Carile, Il Caucaso e l'impero bizantino in Il Caucaso: cerniera fra culture cit., I, pp. 9-80; O. Pritsak, The Turcophone peoples in the Area of the Caucasus from the VI to the XI Century, in Il Caucaso: cerniera fra culture cit., I, pp. 223-245; N. Garsoïan, B. Martin Hisard, Unité et diversité de la Caucasie Médiévale (IVE-XI ${ }^{2}$ s.), in Il Caucaso: cerniera fra culture cit., I, pp. 275-347.

${ }^{14}$ La descrizione lasciataci da Roberto di Clari è particolarmente interessante perché è lo scritto di un uomo che, a differenza di Coniate, Frisinga etc., non era un intellettuale di profes- 
Per certi aspetti il movimento delle popolazioni nomadi che occuparono le steppe prima dell'esercito di Gengis Khan ha delle forti somiglianze con le migrazioni dei secoli V-VIII che giunsero in Europa occidentale provocando l'inizio di quello che siamo soliti chiamare, secondo una nostra consolidata categoria culturale, Medioevo occidentale. ${ }^{15}$

Nonostante gli sforzi posti in essere dalla storiografia russa, soprattutto del periodo sovietico, ${ }^{16}$ per tracciare un quadro evolutivo nitido della presenza dei Polovcy nelle steppe, non si è giunti a un risultato definitivo. Sebbene lo scopo di questa indagine ci allontani dal definire la storia della regione tutta nel periodo che precede il XIII secolo e tale compito, d'altra parte, ci sarebbe apparso proibitivo a patto di dedicare decenni di studio a un simile impegno, sarà utile tentare una definizione di ciò che si può legittimamente intendere per steppe dei Polovcy e quali sono stati i passaggi fondamentali che hanno visto questa popolazione appropriarsi di una regione strategicamente importante per gli equilibri politici, economici e culturali dell'Europa centro-orientale dominandola per poi crollare sotto i colpi dell'avanzata tataro-mongola.

Le fonti sulla vita dei Polovcy-Cumani sono relativamente scarse e si riducono sostanzialmente a quelle bizantine unite agli annali russi e alle fonti arabo-persiane.

sione. Roberto era un cavaliere che prese parte alla IV crociata. Inoltre essa è l'unico punto di vista occidentale per quel periodo. Le parole del cavaliere francese confermano alcuni caratteri di queste popolazioni e l'ingenua esagerazione con la quale egli sovrappone la propria percezione ai loro usi reali: «Sono un popolo selvaggio che non ara e non semina, che non ha casolari né case, posseggono soltanto tende di feltro, rifugio dove si nascondono, e vivono di latte, di formaggio e di carne [...]. Ciascuno di loro possiede almeno dieci o dodici cavalli; essi li hanno così addestrati che li seguono ovunque li vogliono portare e montano ora sull'uno ed ora sull'altro [...]. Non venerano null'altro che il primo animale che incontrano al mattino: colui che lo incontra lo venera per tutto il giorno, qualunque animale sia»; Roberto di Clari, La conquista di Costantinopoli, a cura di A. Nada Patrone, Genova 1972, LXV, pp. 196-197.

${ }^{15}$ D'altra parte uno dei popoli che più spaventarono l'Europa cristiana, gli Unni del leggendario Attila, provengono proprio dall'Asia centrale. Alla metà del V secolo il loro impero aveva dimensioni notevolissime e lo spostamento delle tribù unne a Ovest fu una delle cause della crisi definitiva che colpì l'impero romano.

${ }^{16}$ In particolare P. Golubovskij, Pečenegi, torki i polovcy do našestvija tatar, Kiev 1884; V.G. Ljaskoronskij, Russkie poxody v stepi v udelino-večevoe vremja, Sankt Peterburg 1907; P. Diaconu, Kvoprosu o glinjanych kotlach na territorii RNR., in «Dacia», n. s., 8 (1964), 249-264; S.A. Pletneva, Pečenegi, torki i polovcy v južnorusskich stepjach, in "Materialy i issledovanija po arxeologii SSSR», 62 (1958), 151-226; Id., Poloveckaja zemlja, Moskva 1975; D.A. Rasovskij, Polja poloveckogo in "Annaly Instituta im. N.P. Kondakova», 9 (1937), pp. 58-77; Id., Rol polovcev v vojnach Asenej s vizantijskoj i latinskoj imperijami v 1186-1207gg., in Spisanie na B'lgarskaja Akademija na naukite, Sofia 1939; Id., Voennaja istorija polovcev, in «Annaly Instituta im. N.P. Kondakova», 11 (1940), 108-114; Id., Pečeneghi, torki i berendei na Rusi i v Ugri, in «Seminarium Kondakovianum», VI (1933), pp. 9-18; M.V. Bibikov, Istočnikovedčeskie problemy izučenija istorii kočevnikov v Nižem Podunav'e v XII veke, in "Revue Roumaine d'Histoire», 19 (1980), 1, 47-52; S.A. Pletneva, Polovcy, Moskva 1990; P.P. Toločko, Kočevye narody stepej $i$ kievskaja Rus', Sankt Peterburg 2003. 
Premesso che si tratta di materiale riguardante soprattutto aspetti politico-militari, si può tuttavia tentare una riflessione su alcuni dei problemi che nascono dall'indagine di questa popolazione e del suo ruolo nel Dešt-i- Kipčak. ${ }^{17}$

La documentazione ha un carattere prevalentemente narrativo e presenta due impedimenti: di tipo linguistico e tipologico. Come detto sono fonti bizantine, annali e cronache antico-russe, qualche racconto di viaggio occidentale (anche se di epoca tarda rispetto alle altre: XII secolo), in particolare i racconti di Giovanni di Pian del Carpine, Guglielmo di Rubruck, Benedetto Polono; vi sono inoltre documenti letterari armeni, bulgari e romeni, storie scritte da storici arabi, persiani in epoche diverse ma anche fonti archeologiche, numismatiche $\mathrm{e}$ toponomastiche. Nel corso degli anni non sono mancati i lavori di bizantinisti e slavisti a riguardo. Purtroppo in Italia tale strada è rimasta poco battuta e bibliografia nella nostra lingua, sull'argomento, non se ne trova molta.

In Germania e nella Russia sovietica sono stati condotti molti studi e ben approfonditi (qua in particolare sta riprendendo vigore l'indagine etnologica sui nomadi delle steppe); di grande interesse è il lavoro che ormai da qualche anno stanno portando avanti a Vienna storici di primissimo piano, tra cui W. Pohl, i cui studi hanno contribuito in maniera determinante a liberare il campo da radicati pregiudizi sui popoli nomadi delle steppe e hanno dato un impulso nuovo a riconsiderare tutte le problematiche legate all' ambiente in cui vivevano, alle forme economiche su cui basavano la loro vita sociale etc. ${ }^{18}$ Forte è anche l'interesse statunitense e molto si sta facendo nei dipartimenti di bizantinistica e di slavistica a riguardo in quel Paese.

\subsection{Sull'origine dei Polovcy/Cumani}

Sull'etnogenesi dei Polovcy e sulle loro relazioni con i confinanti, principati russi e Bisanzio innanzitutto, ma anche Peceneghi sino alla fine del XII secolo, sono state scritte opere di grande pregio e spessore scientifico, ma una comune teoria sull'argomento è lontana a venire.

I Polovcy-Cumani compaiono nelle fonti con denominazioni diverse. I Russi li chiamavano Polovcy, i Latini e i Bizantini Cumani (o Komanoi), i Turchi li chiamavano Kipciaki, le fonti arabo-persiane parlano di Qoumani o Qipčaki e gli Ungheresi li chiamavano Kun. Tale confusione onomastica è dovuta a più

17 E.Č. Skržinskaja, Rus', Italija i Vizantija v Srednevekov'e, Sankt Peterburg 2000, pp. 3689; M.V. Bibikov, Vizantijskie istočniki po istorii drevnej Rusi i Kavkaza, Sankt Peterburg 2001, in particolare le pp. 83-98 e 199-293; Rasovskij, Polja Poloveckogo cit.

$18 \mathrm{Si}$ veda ad esempio il volume in cui sono raccolti alcuni saggi di W. Pohl, Le origini etniche dell'Europa. Barbari e Romani tra Antichità e Medioevo, Roma 2000; in particolare la parte seconda L'Europa Orientale e il mondo delle steppe, pp. 181-287, ma anche l'apparato bibliorafico delle opere citate, piuttosto dettagliato e ricco di fonti oltre che di saggi sull'argomento; W. Pohl, H. Reimitz, Strategies of distinction: the construction of ethnic communities, 300-800, Leiden 1998; W. Pohl, Kingdom of the Empire, the integration of Barbarians in Late Antiquity, New York (NY)-Leiden-Köln 1997. 
di un fattore. Innanzitutto i popoli di cui ci stiamo occupando erano realtà, come detto sopra, mobili, fluide, pertanto era difficile ottenere su di essi una conoscenza precisa per chi viveva all'esterno di tale contesto, e le nostre conoscenze riguardo i nomadi delle steppe, compresi i Polovcy, provengono quasi esclusivamente da fonti "altre". I Polovcy non ci hanno lasciato memoria scritta. In secondo luogo erano i popoli nomadi a utilizzare nomi diversi per autodefinirsi. Essi erano culturalmente impregnati di mito e leggenda. ${ }^{19}$ Infine non dobbiamo dimenticare quanto detto poco sopra: gli osservatori "esterni" (autori bizantini, slavi, persiani, etc.) anche se in misura diversa avevano nei confronti di queste popolazioni forti pregiudizi e tendevano a considerarli come identici ad altri gruppi coi quali potevano non avere alcun legame.

I Polovcy appartengono al gruppo turcomanno dei Ghuz (Ghuzi, Oguzi), ma sono da tenere ben distinti dall'altro ramo della medesima appartenenza, i Selgiuchi o Selgiuchidi, coloro che vinsero a Manzikert nel 1071 e occuparono gran parte della Persia iranica. ${ }^{20}$ Contrariamente a quanto accadde con i Ghuz Selgiuchidi che invasero il Vicino Oriente dopo aver aderito all'Islam, i Cumani rimasero sostanzialmente pagani ${ }^{21}$ fino alla conquista mongola.

I Polovcy farebbero parte del gruppo turco Kimäk di origine siberiana e proverrebbero dal medio Irtysh o dall'Oby. Sull'origine, anche geografica, dei Cumani/Polovcy non vi sono certezze. Ė però probabile che essi provenissero dalla zona in cui confluiscono l'Irtysh e l'Ob oltre gli Urali. Sono menzionati nelle fonti islamiche sin dal IX secolo. ${ }^{22}$ Secondo lo storico arabo Gardizi ${ }^{23}$

19 A tale proposito si veda Pohl, Le origini etniche cit., p. 188, ma anche alcuni saggi di Peter Golden recentemente raccolti e pubblicati insieme (Golden, Nomads and their Neighbours cit., in particolare il cap. I: Imperial Ideology and the Sources of Political Unity Amongst the Pre-Cinggisid Nomads of Western Eurasia, e il cap. IV: The Turkic Peoples and Caucasia).

${ }^{20}$ Golden, Imperial Ideology cit., pp. 49-50.

${ }^{21}$ Pritsak, The Turcophone peoples cit., p. 239; P.B. Golden, An introduction to the History of the Turkish People, Wiesbaden 1992; P. B. Golden, Wolves, Dogs and Qipćaq Religion, in «Acta Orientalia Academiae Scientiarum Hungaricae», L (1997), pp. 87-97, ripubblicato in Golden, Nomads and their Neighbours cit., cap. XIV.

${ }^{22}$ R. Grousset, L'empire des steppe: Attila, Gengis Khan, Tamerlan, Paris 1969, p. 241; V.V. Bartold, Turkestan v epokhu Mongolskago naščstviya, 2 voll., Sankt Peterburg 1900, ed. inglese Turkestan down to the Mongol invasion, London 1968 (solo il vol. 21986 Leiden ovvero il testo. Il vol. 1 contiene, nell'originale russo, l'edizione di parte delle fonti utilizzate dal Bartold per la sua monografia). Sulla localizzazione dei Kimäk c'è una profonda confusione nelle fonti, dovuta forse ai frequenti spostamenti di questa popolazione o forse alla sua dislocazione su un territorio piuttosto esteso. I Kimäk erano pastori nomadi e cacciatori di pellicce. L'unico insediamento stabile significativo dei Kimäk era la residenza estiva del Qan, Namakiyya. Si veda a tale proposito C.E. Bosworth, Kimäk, in Enc. Islamica, V, 1986 Leidden, pp. 107-108. Molto interessante è anche il capitolo dedicato ai Cumani da Jean Richard nel suo saggio sulle missioni degli ordini mendicanti in Oriente nel Medioevo: J. Richard, La papauté et les missions d'Orient au Moyen Age (XIIIe-XVe siècles), Roma 1998, pp. 20-33.

${ }^{23}$ Gardizi è uno storico persiano attivo dalla metà dell'XI secolo. Non si sa niente della sua vita, ma ha lasciato un lavoro di notevole utilità, Zayn al-akhbar, composto fra il 1049 e il 
i Polovcy si sarebbero separati dal resto dei Kimäk muovendosi verso Ovest attorno alla metà dell'XI secolo. Sicuramente attorno agli anni Quaranta del secolo successivo avevano occupato la vasta fascia di territorio compresa fra basso Volga e Dnepr, fino al medio-basso Danubio, una collocazione che ne avrebbe determinati i successivi insediamenti sparsi. Tra il Danubio e il Dnepr vi erano i Polovcy danubiani o di Lukomor, presso il Dnepr si erano stabiliti i Polovcy del Dnepr o di Zaporože. Tra il Dnepr e il corso inferiore del Don vi erano i Polovcy del litorale, mentre tra l'alto Donets e il Tor vi erano i Polovcy del Donets. Nel bacino del Don si erano stabiliti i Polovcy del Don. Infine tra l'Orel e la Samara si trovavano i Polovcy dell'oltre Orel (cfr. cartina 2). ${ }^{24}$ Erano popolazioni estranee a ogni forma di cultura urbana, ma non per questo "primitive"; anzi, le condizioni climatiche e la morfologia territoriale che dovevano affrontare rendeva necessarie forme di sopravvivenza piuttosto raffinate e modellate sull'ambiente nel quale vivevano. Si stanziarono progressivamente nella regione senza mai raggiungere una forma sociale saldamente basata sullo sfruttamento della terra. Il nomadismo rimase alla base del sistema produttivo, per cui l'allevamento era la principale fonte di sussistenza e il bestiame il possesso più prezioso. Resta da stabilire se, quando nei territori delle steppe giunsero i Tatari, l'organizzazione sociale comana stesse vivendo un momento di transizione verso forme insediative più stabili. ${ }^{25}$

Il significato etimologico dell'etnonimo ha subito numerose modificazioni. Come abbiamo visto le fonti sono discordanti nell'utilizzo di Cumani, Komanoi, Cumani, Kun, Qipčaki. Secondo l'ipotesi avanzata da Diaconu ${ }^{26}$ Kumani proviene dalla parola turca Kum (= steppa, pianura, sabbia) e gli abitanti delle steppe, quindi del kum sarebbero automaticamente Kumani. L'etnonimo usato dalle fonti russe dà

1052. Solo alcune parti del lavoro di Gardizi sono state tradotte in inglese (ed. E.G. Browne, Berlin-London 1928); l'opera migliore per conoscere il personaggio e la sua opera resta quella di Bartold, Turkestan cit.

${ }^{24}$ Questa è la suddivisione data da K.V. Kudrjašov, Poloveckaja step', Moskva 1948, p. 134; tale definizione è stata ripresa da Grekov, Jakubovskij, L'Orda d'Oro cit., p. 7-8. Indipendentemente dalla localizzazione e dai nomi che le varie componenti assunsero in seguito al loro stanziamento sul territorio è da notare come i Polovcy abbiano, piuttosto rapidamente, occupato capillarmente una regione molto vasta. Omelian Pritsak ne individuò dodici sottogruppi che corrispondevano all'incirca alla suddivisione suggerita da Kudrjašov: Pritsak, The Polovcians and Rus' cit., pp. 342343. Si veda anche Golden, Cumanica I: The Qipćaqs in Georgia cit., p. 54.

${ }^{25}$ Nella sua opera, ancora oggi riferimento imprescindibile per gli studi sull'Eurasia Bartold sostiene che nella regione del Syr-Darya (Kazakistan), fra Žend e Fārāb, sorgeva la città di Sighnāq, "dominio dei Qipčaki pagani”, contrapposti ai Selgiuchidi che già si erano islamizzati. Vi erano pertanto, stando al documentato lavoro di Bartold, centri importanti attorno ai quali ruotava questa civiltà prevalentemente nomade ben prima dell'invasione mongola. Non a caso Sighnāq rimarrà un centro di rilievo politico anche durante il dominio mongolo. Cfr. Bartold, Turkestan cit., pp. 178-179.

${ }_{26}$ P. Diaconu, Les Coumans au Bas-Danube aux XIe et XIIe siècle, Bucaresti 1978, p. 9. 
origine a interrogativi diversi. In primo luogo da dove viene Polovcy? E poi perché in russo esiste un nome diverso, diciamo "autonomo", mentre nelle altre fonti, bizantine, occidentali, arabo-persiane si usa l'etnonimo originario seppur nelle sue naturali variazioni $?^{27} \mathrm{E}$ poi, è veramente un nome autonomo quello russo di Polovcy o è piuttosto un calco? È un problema che solo inizialmente riguarda la linguistica poiché le sue implicazioni sono decisamente di tipo storico.

I Polovcy hanno fatto parte della storia della Rus' sin dagli anni Sessanta del XII secolo. Essi effettuavano incursioni rapide dagli effetti devastanti sulla campagna della Rus'. ${ }^{28}$ Le fonti parlano dei Polovcy come di una calamità, di un pericolo costante col quale gli abitanti dello stato kieviano, ma anche dei principati periferici, dovevano convivere. L'etimologia del termine va quindi ricercata nel complesso dei rapporti che i Polovcy avevano con la Rus'. ${ }^{29}$

Secondo una prima teoria avanzata da A. Kunik ${ }^{30}$ nel 1875 Polovcy deriverebbe dalla parola antico-russa polovyj (= giallastro, paglierino), quindi un'origine derivata dall'aspetto fisico - pelle e capelli - dei loro vicini. ${ }^{31}$ Secondo Miklosich $^{32}$ l'etnonimo potrebbe derivare non già dall'aspetto fisico dei Polovcy, quanto dal fatto che presso di loro esisteva un'orda, l'Orda Blu ed è da essa che deriverebbe tale nome. Sobolevskij ${ }^{33}$ concorda con questa teoria e aggiunge che anche in russo la parola polovyj talvolta è usata, in forma volgare, per indicare i buoi di colore bluastro. Di fatto l'Orda d'Oro nacque ben oltre e per opera dei Tataro-mongoli; non risulta ve ne fosse una anche presso i Polovcy. Nel primo dopoguerra ha avuto un certo successo la teoria ipotizzata da Gordljevskij ${ }^{34}$ secondo la quale Polovcy sarebbe la traduzione della parola kipčaki e non, come si pensava, di Kumani. Rashid ad Din, uno dei più attendibili storici della vicenda tataro-mongola vissuto a cavallo fra XIII e XIV secolo, nella sua Storia $^{35}$, sostiene che kipčak significherebbe albero vuoto e che sia da interpretare

27 Tutte le fonti russe utilizzano Polovcy, solo il Racconto dei Tempi Passati (ed. it. a cura di I.P. Sbrizolo, Torino 1971, alla p. 132) sostiene in un passo che "Ismaele generò dodici figli, dai quali discesero i Turcomanni e i Peceneghi, e i Turchi e i Cumani, cioè i Polovcy, che sono venuti dal deserto".

${ }^{28}$ Racconto dei Tempi Passati cit., p. 93: «Vennero, per la prima volta i Polovcy a combattere contro la terra russa [...] e dopo aver combattuto, si allontanarono».

${ }^{29}$ Skržinskaja, Rus', Italija i Vizantija cit., p. 38.

${ }^{30} \mathrm{La}$ teoria di Kunik è espressa nel libro di B.A. Dorn, Kaspij. O poxodax drevnix rusov v Tabaristan, Sankt Peterbury 1875, p. 387; Skržinskaja, Rus', Italija i Vizantija cit., p. 45.

31 Il russo polova (che in Slavo Ecclesiastico è plava) significa paglia.

${ }^{32}$ F. Miklosich, Etymologisches Wörterbuch der Slavischen Sprachen, Wien 1886, p. 154; Skržinskaja, Rus', Italija i Vizantija cit., p. 65.

${ }^{33}$ Skržinskaja, Rus, Italija i Vizantija cit., p.64; p. 175; D. A. Rasovskij, Polovcy, «Seminarium Kondakovianum», VII (1935), p. 255.

${ }^{34}$ Skržinskaja, Rus', Italija i Vizantija cit., p. 66.

35 Una prima parziale edizione dell'opera di Rashid ad-Din fu data alle stampe da E. Quatremère, Histoire des Mongols de la Perse, Paris, t. I, 1836; Ristampa: Amsterdam 1968 una 
come una combinazione fra prato e Polovcy (= il prato/la steppa dei Polovcy). Giustamente Elena Skržinskaja, nel suo saggio, fa notare come non vi siano tracce, nelle fonti russe, bizantine e occidentali, di questa parola; ${ }^{36}$ essa appare solo in Rashid ad-Din.

Elena Skržinskaja ha controllato anche le versioni fornite dai più autorevoli dizionari etimologici russi i quali danno spiegazioni diverse. ${ }^{37}$ Assai curioso è invece l'etnonimo usato per i Polovcy da Ottone di Frisinga ${ }^{38}$ il quale dice "quod ne cui incredibile videatur, audiat quod Pecenati et hii qui Falones dicuntur». A parte il fatto che anche Ottone, vescovo germanico, descrive i Polovcy piuttosto negativamente, resta da vedere perché usi questo etnonimo. L'aggetivo tedesco è falb (= giallo chiaro, giallo crema, o giallo rossiccio usato specialmente per designare il colore dei cavalli). L'Alto Tedesco Antico è fahl (= scialbo) la cui radice è palitá (è il greco poliós, pelitrós = grigio smorto, è il latino pallere, nell'inglese moderno è la parola fallow, oramai in disuso). Se notiamo bene la somiglianza fra il termine usato da Ottone e quello russo, almeno nel significato, possiamo ipotizzare che il vescovo germanico avesse appreso questo etnonimo dai Rus'. Ciò è assai probabile visto che questi frequentavano assiduamente, nella prima metà del XII secolo, la via di commercio fra Kiev e Regensburg sulla quale si trovavano alcuni fra i più importanti centri commerciali dell'Europa orientale. ${ }^{39}$

Non si è in grado di stabilire con certezza quando ebbe avvio la dominazione cumana sul Dešt-i Kipciak. La prima notizia che si ha sulla loro presenza in

versione dell'opera dello storico persiano si trova anche in C. D'Ohsson, Histoire des Mongols depuis Tchinguiz-qan jusqu'a Timour Bey ou Tamerlane, 4 voll., Amsterdam 1834-1835; un'altra buona traduzione, che a lungo ha influenzato gli storici, fu effettuata in russo da J. Berezin, Sbornik letopis'ej. Istoria Mongolov, sočinenie Rašid-Eddina, in Trudy Vostočnago otdelenija Imperatorskago Archeologičeskago Obščestra, Sankt Petersburg 1858-1883, voll. V, VII e XIII. Il testo persiano fu edito nel 1861. Si veda anche Bartold, Turkestan cit., e l'ottimo, seppur antico, saggio di E. Blochet, Introduction à l'histoire des Mongols, Leiden-London 1910. La traduzione più recente in lingua occidentale è quella del grande orientalista J. A. Boyle, The Successors of Genghis Khan, New York (NY) 1971. Si tratta di un'edizione ottima, ma parziale. L'opera completa di Rashid ad-Din è vastissima. Boyle ne selezionò alcune parti fra le più interessanti. Essa va da Ogödeï a Temur. La migliore edizione per un'indagine sistematica sull'opera dello storico persiano resta quella russa in tre volumi usciti in anni diversi e a cura di un gruppo di studiosi: Rašid ad-din, Jami' at-tawarikh in Sbornik Letopis'ej, vol. I, trad. e cura di L.A. Ketagurov e O.I. Smirnova, Moskva-Leningrad 1952; vol. II, trad. e cura di Ju.P. Verkovskij, Moskva-Leningrad 1960; vol. III trad. e cura di A.K. Arends, Baku (1946). Ė questa l'edizione che ho utilizzato (d'ora in avanti $\mathrm{RaD}$ ) in alternanza con quella di Boyle, The successors cit.

${ }^{36}$ Skržinskaja, Rus', Italija i Vizantija cit., p. 65.

37 Ibidem, pp. 66-68.

38 Ottone di Frisinga, Otto Frisingensis vescovo; 1114 ca.-1158. Ottonis episcopi Frisingensis Chronica, sive Historia de duabus civitatibus, a cura di A. Hofmeister, Hannover 1984, VI, 10.

${ }^{39}$ Skržinskaja, Rus', Italija i Vizantija cit., p. 60; Rybakov, Istorija Kul'tury Drevnej Rusi. Domongol'skij period, 2 voll. Moskva-Leningrad 1948, in particolare il cap. Torgovlja i torgovye puti, pp. 342-343. 
quest'area è degli anni Trenta dell'XI secolo, ${ }^{40}$ ma essi appaiono sporadicamente e non sembrano essere, in questi anni, una forza politica coesa e militarmente in grado di pericolose azioni offensive. Si ritrovano però già nella seconda metà dell'XI secolo (1087) nell'Alexiade di Anna Comnena come presenza stabile e con prerogative decisamente mutate.

\subsection{Le prime incursioni}

Le domande legate all'indagine delle steppe dei Polovcy prima che vi giungessero i Tataro-mongoli sono diverse. Come abbiamo visto non è semplice definire geograficamente i confini attribuibili alle steppe dell'Europa orientale, così come non lo è la messa a fuoco della tipologia di rapporti che queste popolazioni nomadi ebbero coi vicini e quali fossero le dinamiche a esse interne. Fra gli anni Trenta e Sessanta del Novecento si è sviluppato un vivace dibattito storiografico, soprattutto in URSS, fra coloro che attribuivano una vocazione aggressiva e distruttiva ai Polovcy e coloro che attribuivano alla Chiesa ortodossa la creazione di miti infondati sulla ferocia di questi popoli i cui rapporti con i principati russi sarebbero stati sostanzialmente pacifici. Altrettanto forte è stata la discussione scientifica (ma sarebbe meglio dire ideologica) fra gli storici russi marxisti sull'esistenza o meno di una struttura di tipo feudale all'interno di queste società che stavano progressivamente abbandonando il nomadismo a vantaggio di un più stabile e coerente rapporto col territorio e quindi con la produzione agricola a fronte di un progressivo abbandono dell'allevamento e della pastorizia. Ne parleremo diffusamente più avanti.

Per quanto attiene alla successione con cui queste popolazioni giunsero e si stanziarono nei territori considerati vi è oggi un sostanziale accordo fra gli studiosi. Un altro tema, oggetto di indagine da parte degli specialisti, ma ancora privo di univoche interpretazioni è quello delle conseguenze che tale successione ebbe sui gruppi sconfitti: l'arrivo dei Polovcy e la conseguente spinta ai danni dei Peceneghi verso Ovest generò una più o meno rapida scomparsa dell'elemento etnico, un loro assorbimento entro popolazioni la cui esistenza era tradizionalmente stanziale e i cui confini erano sottoposti a un potere organizzato (Bisanzio e il regno di Bulgaria in primo luogo) o la scomposizione in elementi che possiamo in seguito ritrovare, mutati, in realtà diverse? Sono domande alle quali

40 Secondo gli Annali di Lavrent'ev, (Lavrent'evskaja Letopis', Leningrad 1926-1927, rist. München 1977, p. 158), riportati da B.D. Grekov, A.Ju. Jakubovskij (L'Orda d'Oro cit., p. 6) e dalla Sovetskaja Istoričeskaja Enciklopedija, i Polovcy farebbero la loro prima comparsa nel 1054, ma Bartold (Turkestan cit., p. 296) nota una loro incursione contro Altūntāsh, viceré di Korazmia, già nella primavera del 1032. Nel suo lavoro il Bartold ha utilizzato copiosamente fonti arabe inedite. In questo caso egli ha appreso la notizia dal giurista e teologo ottomano Bahavi (G.L. Lewis, The Balance of Truth, London 1957). 
non è possibile dare una risposta chiara e definitiva; dubbi che rappresentano materia di riflessione e sui quali credo sia opportuno soffermarsi.

I Cumani popolarono per un secolo e mezzo il territorio compreso fra il fiume Dnepr e l'Ural e che sovrasta i due mari del Caucaso, il Mar Nero e il Caspio, fino all'avanzata tataro-mongola. Prima di tale evento traumatico la regione visse però una lunga stagione di mutamenti non solo politici, ma soprattutto etnico-culturali di rilevante entità. L'etnogenesi delle popolazioni che occuparono queste terre è un altro aspetto molto interessante e che merita di essere approfondito ${ }^{41}$ Le incursioni delle prime popolazioni nomadi, Peceneghi soprattutto, avevano destabilizzato la regione, ma il loro passaggio nelle steppe russo-meridionali era stato uno stadio, durato relativamente poco, verso la stabilizzazione nel bacino del basso Danubio da dove ebbero non pochi problemi con Bisanzio.

Il profondo mutamento politico che si verificò nei territori delle steppe russo-meridionali dovuto all'occupazione di Kiev da parte dei principi russi, alla cacciata violenta dei Magiari e all'inizio, di fatto, del declino cazaro agevolò l'insediamento di popolazioni nomadi nuove. I Cazari si erano convertiti al giudaismo nell'VIII secolo ${ }^{42}$ e avevano contribuito in maniera decisiva al mantenimento dei territori centro-meridionali delle steppe orientali; essi venivano a perdere ora la loro egemonia attorno alla città che diventerà la capitale della Rus'. Le guerre che opposero i Cazari ai Rus' si ripeterono nel corso del X secolo con sempre maggiore frequenza fino al 965 anno in cui cadde Sarkel (sul medio corso del Don), città fondamentale per l'impero giudaico in quanto situata all'inizio della cosiddetta strada Cazara che univa il Don al Volga.

I Rus' conquistarono quindi il territorio cazaro di Tmutorakan', davanti alla Crimea, ma non riuscirono a garantirsi uno sbocco sul Caspio, il mare dei Cazari. Il crollo definitivo dell'impero Cazaro può essere collocato nel 1016 anno in cui il suo esercito fu affrontato e sconfitto dalle armate russa e bizantina; nessuno dei due vincitori seppe però collocarsi rapidamente nei territori conquistati. La mancanza di un centro di potere che potesse definire confini e limiti di sovranità difendendoli favorì l'infiltrazione rapida e costante di popolazioni nomadi e semi-nomadi da Oriente e da Nord. Si è soliti collocare, in seguito al crollo dell'impero Cazaro, l'avanzata dei Peceneghi, ai quali sarebbero succeduti i Polovcy.

${ }^{41}$ Oggi si tende ad attribuiree all'opera dell'uomo un'importanza determinante negli sviluppi etnici (la costruzione delle appartenenze etniche), abbandonando la biologia come unico elemento alla base di tali trasformazioni. In questo senso il lavoro dello storico assume una responsabilità accresciuta, ma anche una maggiore gratificazione. Anche tali progressi si devono in gran parte agli studi di W. Pohl (Le origini etniche cit.)

${ }^{42}$ Sugli aspetti etnico-linguistici relativi ai Cazari, oltre alle opere citate sopra (cap. primo) si veda il saggio di Pritsak, The Turcophone peoples cit., p. 236. 
Il dominio dei Polovcy rappresenta un legame importante e coerente fra Bisanzio e i principati della Rus' kieviana e ha più volte opposto limiti precisi alle velleità espansionistiche di entrambi. Al tempo stesso ha mantenuto a lungo una vasta rete di rapporti, soprattutto commerciali, con entrambi i soggetti politici. ${ }^{43}$

All'inizio i movimenti sotto forma di rapide incursioni effettuati dalle popolazioni nomadi di origine turca, verificatisi nei primi anni del XII secolo, erano opera di gruppi disomogenei, un insieme di cellule distinte da un punto di vista etnico ${ }^{44}$. Bibikov parla espressamente di unione di forze pecenego-turche. ${ }^{45}$ A dire il vero la realtà della steppa era molto fluida. I clan dominanti cercavano, anzi promuovevano «'integrazione di tutti i popoli a cavallo nelle vicinanze» ${ }^{46}$ poiché era questa la loro forza. Il ciclo vitale dell'influenza esercitata da un clan sull'altro non durava più di due-tre generazioni. Tale situazione rendeva necessaria l'aggregazione in un contesto di generale tolleranza ideologica a vantaggio di una maggiore coesione oggettiva. D'altra parte le fonti bizantine parlano indistintamente di Sciti per indicare le popolazioni nomadi che vivevano ai confini dell'impero. E proprio le fonti bizantine che affrontano il problema dei rapporti fra l'impero e il nomadismo non sono poche. La denominazione di Sciti compare per designare gruppi etnici, la cui collocazione geografica pare più o meno chiara agli autori, che precedono l'arrivo dei Polovcy in quest'area. In particolare sono Sciti i Peceneghi/Patzinaki. ${ }^{47}$ Lo stesso etnonimo, per indicare genericamente le popolazioni turco-nomadi, è usato da Samuele di Ani in occasione della prima penetrazione crociata in Tracia ${ }^{48}$.

Dopo aver stabilizzato i rapporti con i Magiari (fondazione dello stato ungherese a opera della dinastia Arpád ${ }^{49}$ e loro definitiva collocazione a ovest del Danubio, fra i Carpazi e le Alpi transilvaniche) l'impero trovò nei Peceneghi un nuovo nemico da dover fronteggiare. La documentazione relativa ai rapporti fra Bisanzio e le popolazioni nomadi che si assestarono ai confini dell'impero rivela in modo piuttosto chiaro che tali relazioni vissero momenti diversi. Dopo

43 B.A. Rybakov, Istorija kul'tury Drevnej Rusi cit.; A. Ponomarev, Kumany-Polovcy, in «Vestnik Drevnej Istorii», 3-4 (1940), pp. 366-370; Skržinskaja, Rus', Italija i Vizantija cit., in particolare il capitolo Polovcy. Opyt istoričeskogo istolkovanija étnikona, pp. 36-89.

${ }_{44}$ Bibikov, Vizantijskie istočniki cit., pp. 231-32.

${ }^{45}$ Bibikov, Istočnikovedčeskie problemy cit., pp. 47-52.

46 Pohl, Le origini etniche cit., p. 186; si veda su questo anche Golden, Imperial Ideology cit.

${ }^{47}$ Bibikov, Vizantijskie istočniki cit., p. 232. Tali sono in Anna Comnena, Cinnamo, Niceta Coniate, Giovanni Tzetze e Michele Psello. Anna Comnena però usa anche l'etnonimo Cumani con precisione e li distingue dai Peceneghi/Sciti (cfr. nota 62).

48 «I Romani attraversarono la Tracia e penetrarono in Asia, per vendicare i cristiani delle tribolazioni che gli facevano subire gli Sciti, i Persiani e i Dadjigs (gli Arabi)», Samuel Anetsi, in Recueil des Historiens des Croisades. Documents Arméniens cit., pp. 445-468, p. 447.

49 Obolensky, Il Commonwealth bizantino cit., pp. 225-227. 
una prima fase sostanzialmente tranquilla in cui l'impero tentò di mantenere rapporti pacifici coi Peceneghi, la situazione mutò dal momento in cui questi aderirono alla campagna anti-bizantina del principe russo Svjatoslav ${ }^{50}$.

Dalla caduta dell'impero dei Cazari fino all'insediamento dei Peceneghi ai confini nord e nord-orientali dell'impero, nei confronti della Transcaucasia e della regione Danubiana, Bisanzio adottò una "coerente diplomazia della steppa ${ }^{51}$. Un atteggiamento usuale che serviva all'impero per mantenersi alleate quelle popolazioni che potevano costituire una barriera contro eventuali aggressioni da Est e da Nord. Bisanzio, come abbiamo visto, non poteva perdere il controllo sugli scali del Mar Nero, magazzino alimentare dell'impero. Più volte le autorità bizantine negheranno l'accesso oltre il Bosforo alle navi italiane che ne facevano insistente richiesta. ${ }^{52}$

In pratica l'impero aveva nei nomadi della steppa una sorta di scudo, dal Caucaso al Danubio, una barriera che poteva abbassarsi in qualsiasi momento e anzi rivoltarglisi contro. Erano queste le frontiere settentrionale e orientale di quello che Obolensky ha definito il "Commonwealth bizantino». ${ }^{53}$

Nel periodo di cui ci stiamo occupando il concetto di confine era spesso sovrapposto a quello di frontiera, entrambi categorie culturali, ideologiche più che determinati limiti fisici. ${ }^{54}$ Per Bisanzio la frontiera, ideologica, era la linea oltre la quale non vi era civiltà, bensì il mondo barbaro che necessariamente ruotava attorno all'impero e alla sua capitale. Tali limiti per Bisanzio erano segnati a est dal Caucaso e dall'Eufrate. ${ }^{55}$ All'interno di questo mondo civilizzato

${ }^{50}$ Ibidem, in particolare il capitolo VI, pp. 185-288.

${ }^{51}$ Secondo una felice definizione di Carile, Il Caucaso e l'impero bizantino cit., p. 37.

52 A tale proposito esiste una copiosa letteratura scientifica, qui collocata in bibliografia. In questa sede ci limitiamo alle opere di più ampio respiro e all'interno delle quali si trovano apparati bibliografici dettagliati. E.H. Byrne, Genoese Shipping in the Twelfth and Thirteenth Centuries, Cambridge (MA) 1930; M.E. Mallet, The Florentine Galleys in the XVth Century, Oxford 1967; G. Forcheri, Navi e navigazioni a Genova nel Trecento. Il 'Liber Gazarie', Genova 1974; R.S. Lopez, Su e giù per la storia di Genova, Genova 1975; G.G. Musso, Navigazione e commercio genovese con il Levante nei documenti dell'Archivio di Stato di Genova (secc. XIV-XV), Roma 1975; Balard, La Romanie Génoise cit.; M.E. Martin, The First Venetians in the Black Sea, in "Archeion Pontou", 35 (1979), pp. 111-122; A. Laiou, The Byzantine Economy in the Mediettanean Trade System: XIIIth-XVth Centuries, in "Dumbarton Oaks Papers», 34-35 (1980-1981), pp. 177-222; E. Ashtor, Levant Trade in the later Middle Ages, Princeton (NJ) 1983; Id. Storia economica e sociale del Vicino Oriente nel Medioevo, Torino 1982; G. Pistarino, I Gin dell'Oltremare, Genova 1988; S.P. Karpov, Ital'janskie morskie respubliki i Juznoe Pricernomor'e v XIII-XV vv.: problemy torgovli, Moskva 1990; Id. La navigazione veneziana nel Mar Nero, XIII-XV secolo, Ravenna 2000.

${ }^{53}$ Obolensky, Il commonwealth bizantino cit.; Carile, Il Caucaso e l'impero bizantino cit., p. 38 .

${ }^{54} \mathrm{H}$. Ahrwailer, La frontière et les frontières de Byzance en Orient, in Actes du XIVe Congrès International des Études Byzantines, Bucaresti 1971, pp. 209-230; A. Carile, L'Ecumene romanocristiana cit.; Id., I nomadi nelle fonti bizantine, in I popoli delle steppe cit., pp. 55-87.

55 Ahrwailer, La frontière cit., pp. 209-210. 
vi è l'ecumene cristiano, parte integrante dell'impero. Quindi quelle popolazioni cristiane geograficamente fuori dall'impero devono essere recuperate ${ }^{56}$. I nomadi che vivono ai confini con l'impero, ma che abbracciano il paganesimo non fanno quindi parte dell'ecumene recuperabile; sono omnium paganorum crudelissimi ${ }^{57}$ nemici dell'impero. Ciò non impedisce alla politica di Bisanzio operazioni diplomatiche frutto di un calcolo accurato, tese al controllo di tali confini e alla pacifica convivenza con queste popolazioni.

Le frontiere, fisicamente intese, sono sempre state rarefatte, tutt'altro che nettamente determinate. Di fatto la presenza di funzionari bizantini in certe aree ne sanciva il legame con l'impero. Di questi funzionari stabili non si hanno notizie presso le popolazioni nomadi turcomanne. ${ }^{58}$

L'irrigidimento di Bisanzio sulle frontiere orientali si verificò una prima volta in seguito alle incursioni arabe. L'impero non avvertì più come sicuri i confini orientali e cambiò il sistema di fortificazione delle città poste in tali regioni. Un'altra svolta nell'organizzazione dei limes si verificò proprio in occasione delle prime incursioni dei popoli nomadi. Secondo l'Ahrwailer è questo il periodo in cui le frontiere caucasiche a est e a nord del Ponto assumono il carattere fisicamente e culturalmente discontinuo che per Bisanzio non muterà più. ${ }^{59}$

La frontiera orientale è per Bisanzio la frontiera per eccellenza. Essa ha rappresentato la sottile separazione fra due mondi che si sono sentiti reciprocamente ostili e culturalmente diversi. L'arrivo, fra X e XII secolo, di popolazioni nomadi da Oriente pone in risalto tutti i limiti di tale linea di confine. Popolazioni mai assimilate del tutto che non saranno adeguato ostacolo all'avanzata dei Polovcy prima e dei Mongoli poi. In pratica si assiste, fra Manzikert (1071) e Myriokephalon (1176) a un processo di nomadizzazione delle frontiere. ${ }^{60}$

Nell'ambito di un processo piuttosto rapido come fu questo, Bisanzio ricorse spesso, come detto, alla diplomazia. La conformazione di questo sistema di relazioni, abilmente costruite dalla politica imperiale, ebbe a mutarsi nella seconda metà dell'XI secolo quando i Peceneghi vennero spazzati via dai Polovcy. Anch'essi rientrano nel processo di nomadizzazione di cui sopra, anzi ne sono la realizzazione ultima, prima del mutamento strutturale imposto dai Tataro-mongoli.

Si è soliti pensare a questi secoli come a un periodo caratterizzato da un succedersi confuso di gruppi etnici più o meno consistenti quantitativamente,

56 Ibidem, pp. 210-211; Carile, L'ecumene romano-cristiana cit.

57 Epistola Brunonis ad Henricum regem, in Monumenta Poloniae Historica, I, Lwów 1864, p. 224; Obolensky, Il commonwealth cit., p. 257-258.

58 Ahrwailer, La frontière cit., p. 215.

59 Ibidem, p. 219.

${ }^{60}$ Ibidem, p. 228. 
in cui il più forte spazzava via il più debole rapidamente, cancellandone ogni traccia. Le fonti che si hanno a disposizione dimostrano che la situazione fu assai più complessa. Il processo che, progressivamente, portò i popoli nomadi a stanziarsi nei territori delle steppe fu una successione di gruppi eterogenei che poggiavano su un substrato etnico costante, le cui modificazioni nel tempo erano minime. Vi era l'infiltrazione, talvolta massiccia, di elementi nuovi, ma essi si mischiavano ai precedenti dando vita a un processo piuttosto lento il cui risultato è la diversità etnica che in gran parte preesisteva a questo periodo, in parte è visibile ancora oggi nella regione compresa fra il Caucaso georgiano e la Russia meridionale.

\subsection{Alcune considerazioni sui rapporti coi vicini: Bisanzio e la Rus'}

Anna Comnena, nell'Alexiade, narra la guerra con gli Sciti che ebbe luogo negli anni $1087-1090 .{ }^{61}$ Gli Sciti cui si riferisce sono ancora i Peceneghi, autori di continue incursioni in territorio bizantino nella zona del Danubio inferiore. Poco oltre essa però afferma che Tatu (comandante degli Sciti/Peceneghi) "partì [dalla cittadina di Dristra] per guadagnare l'alleanza dei Cumani». ${ }^{62}$ In base a tale affermazione possiamo osservare che se gli eserciti di Alessio avevano preso Dristra (esclusa la zona fortificata) che si trova sul Danubio e se Tatu dovette partire per chiedere l'alleanza dei Cumani, allora questi ultimi non dovevano a loro volta essere troppo lontani dalla zona dello scontro. I Polovcy erano quindi già vicini al Danubio, probabilmente organizzati secondo il loro modello sociale originario, quello nomade, ma di sicuro non avevano ancora sconfitto e allontanato del tutto i Peceneghi. ${ }^{63}$

La successiva comparsa dei Polovcy nell'Alexiade si ha in occasione dell'epilogo della battaglia che vide opposto Bisanzio agli Sciti. L'esercito barbaro vinse la battaglia e i Cumani giunsero sul luogo, chiamati da Tatu, troppo tardi. Gli Sciti non avevano avuto alcun bisogno del loro aiuto. Secondo Anna ebbe luogo in questa occasione uno scontro durissimo fra i dirigenti cumani e

${ }^{61}$ Anna Comnena, Alexiade. Règne de l'empereur Alexis I Comnène (1081-1118), ed. a cura di B. Leib, II edizione, Paris 1967, II, VII, p. 87.

${ }^{62}$ Ibid.., II, VII, p. 95. È questo il primo caso nell'Alexiade in cui Anna Comnena usa il nome Cumani, dimostrando di distinguere bene chi fossero e di conoscerne l'identità rispetto a quella degli altri "Sciti".

${ }^{63}$ Ibidem, II, VII, p. 95. Il passo recita: «Paleologo e Gregorio Maurokatakalon, d'accordo per differenziare il combattimento coi Patzinaki, consigliarono di occupare militarmente la grande Pristlava» (Stamboul, Leib, p. 95, nota 3); J. Bromberg, Toponymical and historical Miscellanies, in «Byzantion», XII (1937), pp. 459-465. Ė la prima volta che Anna chiama questa popolazione turcomanna Peceneghi/Patzinaki. Sino a questo momento li ha chiamati Sciti. In Anna Comnena gli Sciti sono quindi da intendersi come i Peceneghi e non come i Polovcy che vengono chiamati Cumani. Siamo nel 1087 e questa popolazione turcomanna, che aveva dominato le steppe comprese fra il Don e il Danubio per due secoli circa, era ormai vicina alla disfatta. 
quelli peceneghi per la spartizione del bottino. Non sappiamo dalle fonti come andarono esattamente le cose e Anna Comnena non poteva essere informata con precisione sugli accordi intercorsi fra Peceneghi e Polovcy. Di fatto lo scontro che seguì l'arrivo delle truppe turcomanne nei pressi del Danubio contro i vincitori di Dristra fu un evento grandioso le cui conseguenze furono assai positive per Bisanzio. L'impero aveva perduto la battaglia decisiva, ma gli Sciti non furono in grado di infliggere il colpo di grazia sullo sconfitto in quanto dovettero fronteggiare un nuovo avversario. "Gli Sciti furono completamente battuti». ${ }^{64}$ Alcuni bizantinisti enfatizzarono l'importanza dell'intervento dei Cumani che, da alleati degli Sciti, si trasformarono nei loro carnefici salvando le sorti di Bisanzio. ${ }^{65}$

Per i due anni successivi (1088-1089) fra Bisanzio e i Peceneghi si protrasse una guerra di rapina fatta di rapide incursioni, attacchi repentini e saccheggi organizzati nelle zone di confine. Non si giunse più allo scontro diretto. In questi mesi la diplomazia bizantina si mise a lavoro per evitare la guerra. Alessio I non accettò di unirsi ai Cumani per annientare i Peceneghi. Non si fidava dei "nuovi Sciti”, voleva concludere un trattato di alleanza con i Peceneghi approfittando della durissima sconfitta che questi avevano subito per mano dei Polovcy. Ma l'opera della diplomazia di Bisanzio non poteva avere effetti definitivi poiché «i barbari sono infingardi». ${ }^{66}$

Le ostilità fra Bisanzio e gli Sciti ripresero nel 1091. È in questa occasione che venne offerta la possibilità ai Polovcy di sbarazzarsi una volta per tutte degli ingombranti nemici. Alessio I, consapevole dello squilibrio delle forze in campo a vantaggio dell'avversario, non aveva alcuna intenzione di affrontare l'esercito nemico da solo. Era ben conscio che avrebbe potuto sfruttare la spinta offertagli dai Cumani. Secondo Anna Comnena l'esercito di questi si presentò alla foce del Danubio, pronto alla battaglia, con ben 40.000 uomini, ${ }^{67}$ comandati da Tourgokan e Boniak. ${ }^{68}$ Questa volta l'imperatore «lascia [ai Cumani] piena libertà di attaccare gli Sciti». ${ }^{69}$ Questi, dal canto loro, cercarono l'alleanza dei Polovcy e del basileús contemporaneamente e in segreto. In realtà i Polovcy non avevano alcuna intenzione di allearsi coi Peceneghi. Il loro scopo era quello di impadronirsi dei territori della steppa da questi occupati. Ogni forma di alleanza

${ }^{64}$ Anna Comnena, Alexiade cit., II, VII, p. 105.

${ }^{65}$ Su questa linea F. Chalandon, Essai sur le règne d'Alexis I Comnène, Paris 1900; Id., Histoire de la Première Croisade, Paris 1925.

${ }^{66}$ Anna Comnena, Alexiade cit., II, VII, p. 107.

${ }^{67}$ Ibidem, II, VII, p. 136.

${ }^{68}$ I nomi dei due condottieri Cumani sono confermati dal Racconto dei Tempi Passati (ed. cit., p. 128 e 130): «Svjatopolk concluse la pace coi Polovcy e prese in moglie la figlia di Turgoka, principe dei Polovcy. E più avanti: Anno 6604 (1096) Intanto giungeva Bonjak a Kiev con i Polovcy, una domenica sera e combatté presso Kiev [...]».

${ }_{69}$ Anna Comnena, Alexiade cit., II, VII, p. 137. 
avrebbe semplicemente ostacolato tale piano. La battaglia divenne inevitabile ed ebbe luogo ai piedi della collina di Lebunian. Essa si rivelerà decisiva per le sorti dell'intera regione compresa fra basso Volga e Caucaso settentrionale, fino al Danubio. L'esito della battaglia fu scontato e «terribile fu il massacro degli Sciti». ${ }^{70}$

Il 1091 fu dunque la data della definitiva disfatta dei Peceneghi i quali persero una precisa identità e finirono in gran parte assimilati, in seguito alla sconfitta, ora dai Polovcy, ora da Bisanzio, ora da altri popoli vicini. Proprio l'impero li userà più volte nell'esercito. ${ }^{71}$ Gli eventi che seguono riguardano la guerra bizantino-cumana, e anche in questo caso l'impero impiega come truppe ausiliarie elementi nomadi a distanza di pochi anni dalla rotta dei Peceneghi. Dopo alterne vicende, sostanzialmente favorevoli a Bisanzio, si arrivò alla mobilitazione integrale dell'esercito cumano guidato dallo Pseudo Costantino Diogene. In tale occasione i generali bizantini cercarono di resistere all'urto nel modo migliore. Nicola Maurokatakalon, generale bizantino, «fece chiamare i capi delle truppe ausiliarie, Ouza, che era un Sarmato, lo Scita Karatzas, e anche il semi-barbaro Monastras». ${ }^{72}$ A distanza di soli tre anni dalla disfatta (siamo nel 1094) i Pecenghi erano in parte assimilati nelle fila dell'esercito, ma ancora sostanzialmente estranei alla struttura sociale dell'impero; come si è visto essi vennero utilizzati come truppe ausiliarie. Non erano più autonomi, e si può supporre che la perdita della loro autonomia abbia posto i resti della popolazione sotto la sovranità imperiale, magari confinati in zone di frontiera nell'area del basso Danubio.

La guerra coi Cumani ebbe un esito favorevole per Bisanzio in quanto dopo che i Greci ebbero catturato il sedicente figlio di Romano IV gli invasori

${ }^{70}$ Ibidem, II, VII, p. 142.

71 «Di quella gente che vive sui carri, ne cadono a migliaia [...] non pochi prigionieri furono arruolati nelle file degli alleati e la maggior parte, assegnata all'esercito, furono venduti come schiavi» (Niceta Coniate, Grandezza e Catastrofe di Bisanzio, ed. a cura di J. L. Van Dieten, A. Pontani, A. Kazhdan, 2 voll., Milano 2001, I, V. 6, p. 41); Coniate, Grandezza e Catastrofe cit., I, II, 4/5, pp. 69-70: «L'imperatore, dopo aver distinto le truppe, le divise per stirpe e tribù, affinché ogni tribù aiutasse l'altra: mise i Macedoni in un unico gruppo, in un altro i Celti, in un altro gli Sciti». «[L'imperatore Michele V] volle poi sostituire il suo seguito personale con dei giovinotti sciti che aveva comprati tempo addietro [...] e parte ne teneva come guardia del corpo, parte ad assisterlo in alcun altro dei suoi desideri» (Michele Psello, Imperatori di Bisanzio, Cronografia, ed. a cura di D. Del Corno, S. Impellizzeri, U. Criscuolo, S. Ronchey, 2 voll., Milano 1997, I, V.15, p. 203). Sempre Niceta Coniate, in occasione della spedizione di Isacco contro i Bulgari del 1187, dice che «i Mesi [...], arruolate truppe di mercenari Sciti, avevano fatto irruzione dalle parti di Agatopoli» (Coniate, Grandezza e Catastrofe cit., III, 1.1, p. 405). In Cinnamo: «Scelti alcuni degli Sciti che servivano nell'armata dei Romani, li inviava sotto il comando di Gifardos, un soldato assai esperto, sulle tracce del nemico", Giovanni Cinnamo, Epitome rerum ab Ioanne et Manuele Comnenis gestarum, in A. Tomei, Realtà e propaganda nell'opera di Giovanni Cinnamo, Tesi di Laurea, Università degli Studi di Firenze, Facoltà di Lettere e Filosofia, A. A. 2001/2002, III, 3.

${ }^{72}$ Ibidem, T. 2, lib. X, p. 204. 
subirono un duro colpo e non riuscirono a ricompattarsi subito. ${ }^{73}$ Nonostante la vittoria l'impero non pose fine alla minaccia cumana. Certo la sconfitta subita e la riappropriazione del bottino di guerra da parte di Bisanzio impose ai Polovcy di ripiegare indietro verso le steppe del Caucaso settentrionale, abbandonando velleità espansionistiche oltre il Danubio.

Dalla narrazione sulla guerra bizantino-cumana degli anni Novanta dell'XI secolo si avverte con molta forza lo sdegno della figlia di Alessio I contro «questi barbari, che erano avidi di saziarsi di sangue umano, di rimpinzarsi di carne umana e di prelevare un gran bottino dal nostro paese». ${ }^{74}$

Ancora nella campagna di Giovanni Comneno contro Sozopoli del 11201121 compaiono nuovamente i Peceneghi, ma questa volta è assai probabile che si tratti di un'unione di tribù, tra cui vi sono anche i Polovcy (ormai prossimi ad affermare la supremazia in questa regione) che, attraversato il Danubio, invadono «i territori dei Romani». ${ }^{75}$ Bisanzio riesce a sconfiggere gli invasori e anche in questo caso molti dei prigionieri andranno a ingrossare le fila dell'esercito. ${ }^{76}$

In Niceta Coniate si trova un passo molto interessante nel quale il funzionario bizantino descrive con accuratezza il modo di combattere el'equipaggiamento dei Cumani. Si tratta di un'incursione particolarmente violenta che il popolo nomade effettuò in territorio bizantino nel $1155^{77}$ e alla quale i Greci opposero le truppe di Kálmán/Coloman, pretendente al trono d'Ungheria sostenuto da Bisanzio. ${ }^{78}$

I Cumani sono descritti in questo caso come guerrieri abili con l'arco, dotati di armatura leggera. Usano talvolta giavellotti corti e vivono prevalentemente sui cavalli. ${ }^{79}$ Sono dotati di un sacco di pelle "pieno di strame" e impermeabilizzato del quale si servono per attraversare il fiume.

${ }^{73}$ Cfr. su questo anche Ostrogorsky, Storia dellimpero bizantino cit.; Rasovskij, Pečeneghi, torki i merendei na Rusi i v Ugri, in "Seminarium Kondakovianum", 1933, pp. 9-18; ne parla anche il Racconto dei Tempi Passati: «Anno 6603 (1095). Andarono i Polovcy contro i Greci, contro il figlio di Diogene, combatterono in terra greca; e l'imperatore fece prigioniero il figlio di Diogene, e ordinò di accecarlo».

${ }_{74}$ Anna Comnena, Alexiade cit., II, VIII, pp. 128-130; Cinnamo, T. 2, lib. X, p. 191. Il libro X narra gli eventi del 1094-1097, compresa la prima crociata.

75 Cinnamo, Epitome cit., I, 3.

${ }^{76}$ Ibidem, I, 3: «Per affetto nei confronti di quanti erano già stati fatti prigionieri, e dopo essere stati addestrati all'uso dei Romani, [gli Sciti/Cumani] servirono a lungo iscritti nei registri militari».

${ }_{77}$ Niceta Coniate, Grandezza cit., I, III, 11/12, pp. 215-216; per la datazione si veda Ottone di Frisinga cit., II, 53.

${ }_{78}$ Per la sequenza degli eventi si veda Bibikov, Vizantijskie istočniki cit., p. 244-246; per l'identificazione di Coloman si veda il commento a Niceta Coniate (Grandezza cit., vol. I, p. 582, n. 95).

${ }^{79}$ Questa immagine del nomade che vive sui cavalli ricorre spesso nelle fonti non solo bizantine, ma anche russe e occidentali. Niceta Coniate afferma poco più avanti che il cavallo "gli fornisce nutrimento [...] e montato dà sfogo alla bestiale sessualità del barbaro». Niceta Coniate, Grandezza cit., p. 215. 
I Cumani tentarono più volte di rompere la linea del Danubio. ${ }^{80}$ Bisanzio, dal canto suo, non riuscì più a estendere la propria sovranità oltre la linea del fiume. Le due forze rimasero speculari e contrapposte fino alla disfatta bizantina determinata dalla IV crociata.

I rapporti fra Rus' e Polovcy furono frequenti sin dai primi anni della comparsa di questi ultimi nelle steppe; potrebbe essere questo uno dei motivi per cui i Rus' utilizzano, soli, l'etnonimo Polovcy. ${ }^{81}$ I Polovcy si stanziarono al di là del Dnepr, il quale costituiva una barriera naturale, un confine fra le due popolazioni. E possibile che il fiume abbia influito sulla percezione della diversità etnica. Non va infine trascurato il tipo di relazioni che intercorreva fra la Rus' e i Polovcy. Un rapporto che troppo spesso è stato considerato solo entro i limiti di un continuo stato di guerra, di inimicizia. È probabile che si percepissero in genere come ostili, non solo fisicamente, ma anche e soprattutto mentalmente. Quasi sempre le fonti russe citano i Polovcy indicandoli come una sciagura. Per i Polovcy era indispensabile "versare sangue, e glorificarsene e nutrirsi di carogne e di ogni immondezza [...] accoppiarsi con la matrigna e con le loro nuore». ${ }^{82}$ Rappresentavano una "calamità derivata dai pagani»" ${ }^{83}$ erano gli «empi figli di Ismaele». ${ }^{84}$ Molti eventi del periodo kieviano mostrano però che le relazioni fra nomadi, Polovcy in particolare, e Rus' erano diverse.

La caduta dell'impero cazaro aveva fortemente favorito l'indipendenza dei Bulgari del Volga i quali intrapresero contatti commerciali in tutta la regione, soprattutto con la Rus'. Tali relazioni durarono con una buona intensità fino alla conquista mongola. Alla solidità dei legami economici faceva da contraltare la contemporanea situazione di scontro politico e militare che andò aggravandosi proprio fra la Rus' e i Bulgari, uno stato di conflitto in cui ebbe la sua parte anche la differenza confessionale fra i due stati. ${ }^{85}$

${ }^{80}$ Anna Comnena, Alexiade cit., t. 3, lib. XIV, p. 177: «i Comani avevano di nuovo oltrepassato il Danubio [...] all'inizio dell'ottava indizione, alla fine dell'autunno, nel mese di novembre»; Ibidem, t. 3, lib XIV, p. 182: i Cumani rompono di nuovo il limes danubiano, ma quando l'esercito imperiale si reca sul posto, questi sono già fuggiti. Inizia allora l'inseguimento, narrato con dovizia di particolari da Anna Comnena, secondo la quale «dopo un inseguimento di tre giorni e tre notti [i soldati imperiali] tornarono presso l'autocrator senza aver ottenuto il risultato».

${ }^{81}$ La letteratura della Rus' in questo periodo utilizza sovente il titolo di Khan (Kaqan) per indicare i principi kieviani, specialmente Vladimir e Jaroslav. Lo stesso sistema di successione in uso presso l'élite dirigente russa, il Lestvičnaja sistema, presenta forti somiglianze col modello turco dei Polovcy e dei Peceneghi. Si veda su questo Golden, Imperial Ideology cit., p. 39; V.O. Ključevskij, Sočinenija v devjati tomach, Kurs russkoj istorij, I, Moskva 1956; L.N. Gumilev, Drevnie Tjurki, Moskva 1967.

${ }^{82}$ Racconto dei Tempi Passati cit., p. 9.

${ }^{83}$ Ibidem, p. 93.

${ }^{84}$ Ibidem, p. 131

${ }^{85}$ I Bulgari del Volga avevano abbracciato l'Islam. Si veda su questo C. Halperin, Russia and the Golden Horde, The Mongol impact on Medieval Russian History, Bloomington (ID) 1985, specialmente il cap. I; B.D. Grekov, Voľ̌kia Bolgary v IX-X vv., in «Istoričeskij Zapiski», 14 
La compenetrazione di legami fra Rus' e popolazioni nomadi ebbe natura diversa nei diversi contesti cronologici. Abbiamo visto che già nell' 898 gli Ugri giunsero sulle rive del Dnepr poco a sud di Kiev; nel 915 i Peceneghi conclusero un trattato di pace con Igor' sul Danubio; di nuovo appaiono i Peceneghi in una alleanza coi Russi nel 979 e così accade altre volte durante il X secolo. Nel 1015 tocca a Vladimir I difendersi contro le incursioni dei Peceneghi.

Le incursioni delle prime popolazioni nomadi, soprattutto Peceneghi, avevano destabilizzato la regione, ma il loro passaggio nei territori delle steppe russo-meridionali era stato una fase, uno stadio, dalla durata relativamente breve, verso la stabilizzazione nel bacino del basso Danubio. Secondo Halperin i rapporti fra Rus' e Peceneghi furono esclusivamente militari, di scontro. ${ }^{86}$ Coi Polovcy le cose cambiano. Giustamente Elena Skržinskaja ha fatto notare come la presenza di questa popolazione turca stanziatasi fra basso Volga, Caucaso settentrionale e costa centro-nord del Mar Nero abbia fortemente influito sullo sviluppo dello stato variago-slavo. ${ }^{87} \mathrm{E}$ altresì vero che le relazioni fra Rus' e Polovcy vissero fasi molto diverse, talvolta sovrapposte. Un primo momento coincise con il periodo iniziale di vita della Rus', fino ai primi anni del XII secolo, e un secondo ebbe fine con l'invasione mongola.

La prima attribuzione dell'etnonimo Polovcy nelle fonti russe è quella riportata dal Racconto dei Tempi Passati datata all'anno $6406(898)^{88}$ in occasione della quale il cronista descrive lo stanziamento degli Ugri lungo le rive del Dnepr. Nel IX secolo non vi sono ancora i Polovcy, ma l'autore del Racconto li usa come termine di paragone. Egli sostiene che gli Ugri «giunti al Dnepr vi si accamparono, poiché essi erano nomadi come i Polovcy».

La prima incursione in terra russa da parte di nomadi, tra cui ci sono i Polovcy, è descritta come abbiamo visto dagli annali di Lavrent'ev e collocata nel 1054. Nel 1061 il Racconto dei Tempi Passati descrive una violenta incursione dei Polovcy, stavolta da soli, contro "la terra russa». ${ }^{89}$ Secondo il cronista questa fu «la prima calamità derivata dai pagani e dai nemici senza Dio». Le incursioni si succedono con sempre maggiore frequenza: 1068, 1071, 1078, 1082, sino ai fatidici 1092-1093, anni in cui i Polovcy riuscirono ad appropriarsi di ben tre insediamenti: Pesočev, Perevoloka e Priluk oltre a parecchi villaggi su entrambe le sponde del Dnepr. ${ }^{90}$ Tale evento riveste un'importanza a mio

(1945), pp. 3-37; Ju.A. Limonov, Iz Istorij vostočnogo torgovli Vladimiro-Suzdal'kogo Knjažestva: Meždunarodnye Svjazi Rossii do XVII v. in Sbornik Statei, Moskva 1961, pp. 55-63; T.S. Noonan, Suzdalia's Eastern Trade in the Century before the Mongol Conquest, in "Cahiers du monde Russe et Sovietique», XIX (1978), 4, pp. 371-384.

${ }^{86}$ Halperin, The Golden Horde cit., p. 13.

87 Skržinskaja, Rus', Italija i Vizantija cit., p. 78.

${ }^{88}$ Racconto dei Tempi Passati cit., p. 14.

89 Ibidem, p. 93.

${ }^{90}$ Ibidem, p. 121. 
avviso centrale nei rapporti russo-cumani di questa prima fase. La conquista delle città rappresentò l'istituzione di un punto di riferimento importante per i Polovcy, una linea di confine che li metteva di fronte ai principati della Rus' e coerentemente in contatto con un mondo diverso da quello cui essi provenivano. Un'economia sedentaria, basata sullo sfruttamento della terra estraneo alla cultura nomade dei Polovcy. Ma anche l'inizio di una serie di relazioni che non si possono considerare come univoche. Da questo momento i rapporti russo-cumani si concretizzano attraverso alleanze politiche ma anche relazioni commerciali. D'altra parte i regolari rapporti tra le due popolazioni sono confermati anche dalla perfetta conoscenza che l'autore dello Slovo o Polku Igoreve dimostra di avere della steppa. ${ }^{91}$

Nella seconda fase i rapporti russo-cumani si susseguono con un impressionante alternarsi di alleanze e guerre, incursioni e accordi portati avanti dai principi russi per affermare la loro sfera d'influenza su territori sempre più ampi e poter arrivare al trono del Gran Principato di Kiev. Se seguiamo l'andare degli eventi sulla base delle fonti si può osservare che alleanze e rotture si susseguono continuamente, in un contesto di forte precarietà. Le relazioni non furono né sempre amichevoli, né tanto meno sempre ostili. ${ }^{92}$

La decentralizzazione del potere che si verificò nella Rus' durante il XII secolo fu insieme causa ed effetto del mutamento nei rapporti coi Polovcy. Le inimicizie fra principi russi imponevano a questi la ricerca di un'alleanza per prevalere gli uni sugli altri. Ė assai probabile che i Polovcy avessero fatto volentieri a meno di spostarsi a Nord, verso la foresta. Era un ambiente estraneo al loro stile di vita. Ma quando erano chiamati dai principi per scopi meramente politico-militari essi ne approfittavano per effettuare delle incursioni. Bisogna considerare infatti che la Rus', a parte il primo periodo, non è mai stata uno stato unitario, ma un insieme di principati legati da rapporti parentali e di dipendenza quasi mai rispettata. Il termine Rus' non

${ }^{91}$ Halperin, Russia and the Golden Horde cit., p. 16. Lo Slovo è un poema epico russo che narra le gesta del principe Igor di Kiev impegnato nel recupero delle terre sottratte dall'avanzata cumana. Scritto attorno al 1185 ha suscitato un aspro dibattito (tuttora vitale) sulla sua autenticità. Il manoscritto originale andò perduto nell'incendio di Mosca del 1812. Recentemente, e grazie soprattutto agli studi del linguista russo Andrej Zaliznjak (cfr. A.A. Zaliznjak, "Slovo o polku Igoreve": Vzgljad lingvista, Moskva 2004) sembra si possa ritenere autentico e contemporaneo alle vicende in esso contenute. La bibliografia a riguardo è copiosa; per le traduzioni in italiano si può ricorrere alle seguenti: Il cantare della gesta di Igor, a cura di R. Poggioli, Torino 1954; Il Cantare di Igor, a cura di E.T. Saronne, Parma 1988; Il canto dell'impresa di Igor, a cura di E. Bazzarelli, Milano 1991.

${ }_{92}$ P.B. Golden, The peoples of the south Russian steppes, in The Cambridge History of Early Inner Asia, ed. Denis Sinor. New York (NY), 1990; Id., Imperial Ideology cit.; Halperin, Russia and the Golden Horde cit.; T. S. Noonan, Rus', Pechenegs, and Polovtsy: Economic Interaction along the Steppe Frontier in the Pre-Mongol Era, in "Russian History: Histoire Russe», 19 (ANNO?), 1-4, pp. 301-327. 
ha mai designato un'appartenenza etnica, bensì un insieme di popolazioni che si muovevano, almeno fino a tutto il secolo XI, lungo le principali vie commerciali del Volga e del Dnepr. ${ }^{93} \mathrm{Nel}$ momento in cui essa raggiunse il suo massimo splendore, alla metà dell'XI secolo, si estendeva su un territorio piuttosto vasto, di forma vagamente trapezoidale, compreso fra il Volga a est, la sorgente del Don e la regione di Kiev a sud, i territori prospicienti il Mar Baltico e il corso della Neva a nord, mentre a occidente era compresa fra il medio corso della Dvina occidentale, il Neman e l'Ungheria attuale. Tale estensione sarà uno degli elementi determinanti le relazioni coi popoli nomadi delle steppe (cfr. carta 3).

I momenti di accordo avevano natura militare, ma anche familiare. Non è raro incontrare nelle fonti riferimenti precisi a legami matrimoniali sanciti fra principi russi e classe dominante nomade. ${ }^{94}$ Le relazioni fra i principi della Rus' e i Polovcy erano estremamente precarie; nel giro di pochi mesi potevano mutare radicalmente. In un passo del Racconto dei Tempi Passati si legge che nel 1095 i Polovcy giunsero a Jur'ev e vi rimasero per circa un anno. Svjatopolk «se li riappacificò». ${ }^{95}$ Ma nel 1096 si verificò l'incursione dei nemici della Rus' nella regione di Kiev, dove essi riuscirono ad appiccare il fuoco al palazzo del principe a Berestovo. Il 19 luglio 1096 i Russi inflissero una dura sconfitta ai Polovcy sul Trubez. In quell'occasione uccisero Tugorkan. ${ }^{96}$

Nel quadro della labile impalcatura di rapporti messi in piedi dai Rus' coi nomadi delle steppe entra, dagli anni Quaranta del XII secolo, l'alleanza stipulata dal Gran Principe (il Principe di Kiev) con l'unione di tribù dei Cernye Klobuki (= cappelli/cappucci neri). Ne facevano parte Berendei, Torki, Peceneghi - quel che ne restava - e altri gruppi minori. Con essi una parte della classe dirigente russa cercò sempre di stabilire dei legami, spesso in funzione anti-cumana. Ė probabile che vi sia stata, da parte del sovrano di Kiev, la speranza di creare una milizia ben armata, consapevole degli usi militari nomadi e abituata a combattere in territori fisicamente ostili come la steppa, da poter utilizzare quando necessario contro una popolazione numerosa e ben organizzata quale era quella dei Polovcy alla metà del XII secolo. In un

$93 \mathrm{Si}$ veda a tale proposito P.B. Golden, The question of the Rus' Qaganate, in "Archivum Eurasiae Medii Aevi», 2 (1982), pp. 89-90 e soprattutto il recente e voluminoso lavoro di Sverdlov già citato: un lungo saggio molto ben documentato e frutto di un'accurata riflessione in cui viene ripresa l'eredità degli studi di Grekov e di Rybakov.

${ }^{4}$ Nel 1094 il principe russo Svjatopolk conclude la pace coi Polovcy e prende in moglie la figlia di Tugorkan, principe dei Polovcy (Racconto dei Tempi Passati cit., p. 128); nel 1118 il figlio di Vladimir Monomach, Andrej, sposa la nipote di Tugorkan (Cronaca di Nikonian: The Nikonian Chronicle, 2 voll, a cura di A. Serge, A. Zenkovskij, Princeton (NJ) 1984, I, p. 242); nel 1205, quindi in periodo piuttosto avanzato, Vsevolod Jurevič fa sposare il figlio Jaroslav con la nipote del Qagan cumano Končak (Nikonian Chronicle cit., II, 228).

95 Racconto dei Tempi Passati cit., p. 129.

${ }^{96}$ Ibidem, pp. 130-131. 
saggio uscito qualche anno fa Svetlana Pletneva ${ }^{97}$ affermava che il legame con quei nomadi era ormai frutto di un'alleanza, di un vincolo di vassallaggio con Izjaslav Mstislavič (principe di Kiev in quegli anni), come dimostrerebbe un passaggio degli Ipat'evskaja Letopis. ${ }^{98}$ I Černye Klobuki giocavano ormai un ruolo determinante nell'indirizzo politico della Rus'. Alcuni fatti: nel 1147 Izjaslav Mstislavič recluta soldati da Smolensk, Novgorod e fra i Berendei contro gli Olgoviči e Jurij Dolgorukij. ${ }^{99}$ L'anno successivo Jurij, figlio di Vladimir Monomach, vuole attaccare Izjaslav Mstislavič, ma esita davanti a forze composte da Russi e Berendei. ${ }^{100}$ Nel 1167 Vladimir Mstislavič, zio di Izjaslav, vuole occupare il trono di Kiev che appartiene al nipote, ma viene cacciato dalla città da truppe di Berendei. ${ }^{101}$ Nel 1185 Svjatoslav Vsevolodič invia Roman Nezdilovič come governatore di Poroz', roccaforte dei Černye Klobuki, per guidarli contro i Polovcy. ${ }^{102} \mathrm{Si}$ potrebbe continuare con gli eventi che mostrano l'utilizzo sistematico, dalla seconda metà del XII secolo da parte dei principi russi, di questa unione nomade che sta subendo una sorta di trasformazione verso una maggiore stabilità territoriale. ${ }^{103}$

Le conseguenze delle incursioni cumane in territorio russo furono decisamente negative, ma si differenziarono con ogni probabilità da regione a regione ed ebbero un impatto diverso sui distinti livelli della struttura sociale. Se i contadini ebbero a soffrire parecchio delle devastazioni provocate dalle scorrerie dei nomadi sui confini fra la steppa e la foresta, le relazioni commerciali fra le realtà che occupavano il territorio fra Don e Volga non si arrestarono mai del tutto; il ceto artigianale e mercantile molto probabilmente ne trasse benefici. In ogni caso le incursioni dei Polovcy in terra russa non si fermarono ché in seguito alla conquista mongola. ${ }^{104}$

97 S.A. Pletneva, Polovcy, Moskva 1990, p. 76.

98 Polnoe Sobranie Russkich Letopisej, Ipat'evskaja Letopis', a cura di A.A. Šachmatov, Moskva 1962, p. 323.

${ }_{99}$ Nikonian Chronicle cit., II, pp. 27-32.

${ }^{100}$ Ibidem, pp. 38-39.

101 Ibidem, pp. 134-35.

102 Polnoe Sobranie Russkich Letopisej cit., pp. 556-557.

103 Sotto l'etichetta di Črnyj Klobuk in realtà vi era un quadro etnico assai fluido. I gruppi dominanti erano, come detto, Torki, Berendei e Peceneghi, ma non è affatto escluso che vi fossero anche elementi Polovcy inglobati durante le numerose incursioni e assimilatisi nel corso del tempo attorno a Poroz'.

104 Secondo la Cronaca Nikoniana nel 1211 si vericò un'incursione particolarmente violenta nella regione di Kiev (Nikonian Chronicle cit., II, p. 245), pochi anni prima invece, 1205, vi era stata l'unione matrimoniale di Jaroslav Vsevolodič con la nipote di Končak. Nei primi anni del XIII secolo i Cumani erano ancora una forza militare temibile. Secondo il cronista della presa occidentale di Costantinopoli Roberto di Clari, "Giovanni di Valacchia [Kalojan] aveva questi Cumani in suo aiuto ed era solito ogni anno giungere a predare nelle terre dell'imperatore fin quasi alla stessa Costantinopoli, né l'imperatore aveva forze sufficienti per difendersene»; Roberto di Clari, La conquista di Costantinopoli cit., LXV, pp. 196-197. 
I rapporti della Rus' coi nomadi delle steppe quindi costituiscono materia di riflessione piuttosto ampia e confermano come lo studio della realtà nomade di origine turca stanziatasi nella steppa dell'Europa orientale richieda parecchie cautele. Tali relazioni, come abbiamo visto, erano costituite da incursioni rapide, fatte a scopo di rapina, per procacciarsi bottini sostanziosi, alternate a momenti di pace, durante i quali intercorrevano fra le popolazioni rapporti commerciali frequenti. ${ }^{105} \mathrm{Da}$ un punto di vista etnico la situazione non era meno fluida di quanto non lo fosse politicamente: non bisogna immaginare le popolazioni nomadi delle steppe come blocchi rigidi e contrapposti gli uni agli altri in una sorta di guerra fratricida. Lidentità collettiva come motivo di scontro era un elemento a mio avviso assente o quantomeno debole. I Berendei, o i Črnye Klobuki in generale, non attaccavano i Polovcy in quanto Polovcy, né in quanto percepiti come ostili. Difficile immaginare che vi fosse questa consapevolezza. Più probabile pensare a ragioni estremamente pratiche, legate alla sopravvivenza in un ambiente nel quale certe popolazioni vivevano da secoli e vi si erano adattate; in questo i nomadi delle steppe mostrano una complessità organizzativa non inferiore alla civiltà urbana occidentale. I principi della Rus' dal canto loro tentavano da un lato di difendere i confini del loro principato, dall'altro di espanderlo, utilizzando l'alleanza coi nomadi. Fra principi russi e popolazioni nomadi vi era spesso una mutua convergenza di interessi (vedremo che in occasione della prima incursione mongola una parte dell'aristocrazia russa stringerà alleanza coi Polovcy in funzione anti-mongola). Interessi commerciali, politici e militari; da questo punto di vista bisogna rifarsi a un'affermazione precedentemente illustrata: la Rus' non era, né fu mai uno stato unitario. I principi, intenti a mantenere il loro possedimento e ad allargarlo, se possibile basavano su questa necessità il carattere dei legami coi nomadi. Se serviva ci si alleavano, altrimenti li respingevano. I Polovcy, dal canto loro, necessitavano di beni di sussistenza e di uomini. Le città del Gaardariki (come veniva detta la Rus' dagli scandinavi per via della bellezza e della ricchezza delle sue città) rappresentavano un potenziale bottino cui attingere.

In ultima analisi si può osservare che tali relazioni non si arrestarono mai, fino alla conquista mongola, ma in seguito a essa tesero a subire uno sviluppo diverso; mutarono alcune vie di commercio e in generale cambiarono gli equilibri di forza. La conquista mongola ruppe la continuità insediativa dei Cumani e li privò di una autonomia militare ed economica indebolendoli anche a livello demografico, ma non riuscì mai a imporsi come elemento etnico dominante. La Rus' subì un colpo tremendo dalla conquista mongola, ma quando questa si verificò la crisi era già un processo ben avviato. Lo Stato slavo procedette verso un declino che fu anzitutto economico; il baricentro delle relazioni commer-

${ }^{105}$ Grekov - Jakubovskij, L'Orda d'Oro cit., pp. 14-15; Bartold, Turkestan cit., pp. 341-343 e 357-358. 
ciali si spostò verso Sud, verso la Crimea, dove la raggiunta unità territoriale determinata dalla conquista mongola e l'indebolimento di Bisanzio causato dalla quarta rovinosa crociata favorì l'arrivo dei mercanti occidentali. Contemporaneamente la condotta dei Khan dell'Orda d'Oro, non sempre favorevole ai mercanti musulmani, rafforzò i contatti commerciali fra questi e i nuovi emporia costituitisi in Crimea per opera delle città marinare italiane. La Rus' non scomparve come insieme di stati territoriali, ma venne posta, più o meno direttamente, sotto il controllo dell'amministrazione mongola. Un controllo che se dapprima fu stretto e diretto, divenne ben presto labile e distante. Fu sì un cambiamento politico, ma si affiancò a un disastroso declino economico che era già in corso e che le guerre aggravarono. D'altro canto la creazione di uno stato nuovo, con i suoi funzionari, la sua macchina amministrativa, ma poco radicato sul territorio come fu l'Orda d'Oro, consentì una maggiore libertà nei movimenti e una nuova rete di relazioni politiche, culturali ed economiche; ma di questo e delle conseguenze che l'invasione mongola ebbe sulla regione ci occuperemo dettagliatamente più avanti. 


\section{LA CAUCASIA FRA NOMADISMO E IMMIGRAZIONE}

\subsection{Il bosporo cimmerio fra Bisanzio e la seconda ondata nomade}

Lo stretto di Kerč è situato nell'odierna regione caucasica dell'Adigeia, fra le montagne e la vasta piana di Kuban. Sebbene la popolazione sia in maggioranza costituita da Russi e Adighé, ancora oggi esistono nell'area oltre ottanta nazionalità, specchio fedele della complessità etnica che caratterizza il Caucaso in ogni suo angolo. Gli Adighé sono noti alle fonti come Circassi sin dai tempi in cui nella regione vi erano le colonie greche. La cultura greca ha influenzato a lungo lo sviluppo di quest'area conferendole sin dal V secolo a. C. una fisionomia strettamente legata a quella civiltà, con la sua sviluppata cultura sedentaria, la struttura produttiva incentrata sulla lavorazione agricola e la presenza di centri urbani densamente popolati. Sulla riva destra del Kuban si sviluppò, sin dal VII-VI secolo a. C. la civiltà dei Meozi che ha dato il nome al Mar d'Azov così come lo conosciamo attraverso le fonti antiche. ${ }^{1}$ Sin dai primi secoli della nostra era fece la sua comparsa l'unione delle tribù semi-nomadi degli Zichi, anch'essi destinati a influenzare profondamente lo sviluppo della regione fino all'avvento dell'egemonia bizantina che si realizzò nella seconda metà del VI secolo. Quando nel $561 \mathrm{fu}$ stipulato il trattato di pace definitivo fra Bisanzio e la Persia Sassanide, oltre al controllo politico sull'Iberia - e quindi anche su Tbilisi - Giustiniano riuscì a mantenere i possedimenti sulla costa settentrionale del Mar Nero conservando l'unità "romana". ${ }^{2}$ In questi anni l'impero greco operò una conversione forzata presso le popolazioni del Caucaso nord-occidentale costituendovi una diocesi di Zichia. L'avanzata e il consolidamento della potenza Cazara, fra VIII e IX secolo giunse fino allo stretto di Kerč dove fu valorizzata la

${ }^{1}$ Lago di Meozia, Meotide etc. Secondo Strabone lo stretto di Kerč si inserisce fra il Bosporo Cimmerio dei Greci e la Sindike. Esso univa il Ponto Eusino (Mar Nero) con la Palude Meotide (Mar d'Azov). Sulla riva destra (il Bosporo Cimmerio) sorgevano Pantikapaion, il Villaggio delle formiche e Parthenion; la Sindike ospitava le città di Korokondame, Hermonassa, Apatouron e, più a sud, Gorgippia. A nord dello stretto si trova quella che i Greci chiamavano Korokondamitis, una piccola isola sulla quale erano sorti sei insediamenti importanti per la navigazione in quella regione: Satyros, Patrasys, Fanagoria, Giardini, Tyrambe e il Santuario di Achille. Il tutto costituiva la penisola di Taman: Strabone, Geografia, lib. XI, II, 2-11, ma anche Erodoto, Le storie, a cura di L. Annibaletto, Milano 1988, lib. IV, XI e segg.

${ }^{2}$ Bratianu, La Mer Noire cit. p. 120. In particolare furono manenuti la regione del Bosporo Cimmerio con Panticapea e Cherson; la stessa operazione politica fu approntata a ovest, in Dobrugia. 
città di Matrega (Matrica o Matracha) e da dove l'impero turco-giudaico poteva controllare l'ingresso nel Mar d'Azov e il passaggio delle derrate alimentari che avevano nella piana del Kuban un deposito granario di primaria importanza. ${ }^{3}$ I contatti commerciali di questa parte dell'impero Cazaro con l'Asia centrale e col Nord baltico sono sempre stati molto attivi. Il ritrovamento di monete arabe nell'area del Volga e nelle province baltiche ha confermato questo aspetto rafforzando la convinzione degli studiosi sul ruolo importante giocato dai Cazari come intermediari commerciali fra la Persia Sassanide e il Nord. Tutta l'area attorno a Kerč e la penisola di Taman in particolare era ricca di villaggi, stando a quanto dice al-Idrisi. ${ }^{4}$ La città di Matrega si trovava sulle rive del Kuban. ${ }^{5}$

La sconfitta dell'impero Cazaro per opera dei Rus' di Svjatoslav nel X secolo dette vita a una situazione di squilibrio politico per cui la Zichia finì sotto la sovranità di Bisanzio sul distretto di Tmutorakan attraverso un complesso gioco di alleanze che ebbe breve durata. Nel 971 fu concluso un trattato fra Svjatoslav e Vladimir che pacificò le violente lotte interne ai principi russi. Secondo il Racconto dei Tempi Passati Vladimir fu mandato a Tmutorakan nel 988. ${ }^{6} \mathrm{Nel}$ 1022 Mstislav "giunto a Tmutorakan, fondò la chiesa della Santa Madre di Dio, e la costruì. ${ }^{7}$ Vladimir si era da poco convertito al cristianesimo di Bisanzio e l'imperatore greco, Basilio II, colse l'occasione per infliggere il colpo di grazia ai Cazari della regione proprio attraverso il principe cristiano-slavo. La spedizione bizantina organizzata nel 1016 ebbe l'esito sperato e all'inizio dell'XI secolo era attiva una struttura amministrativa bizantina di Zichia della quale sono state scoperte tracce da Banescu e confermate dagli studi di Bratianu. ${ }^{8}$ Dal 1084 al 1094 si parla di un vescovo di Kerč che dipende dal patriarcato di Matrega, nella penisola di Taman. ${ }^{9}$

Nel X secolo le relazioni fra gli stati di questa regione erano frequenti e ben organizzate. I principi russi erano riusciti a imporsi come interlocutori politici credibili sia per Bisanzio sia per gli stati occidentali; le figlie di Jaroslav sposarono

${ }^{3}$ Nell'VIII secolo vi era a Matrega un governatore Cazaro, il Tudhun: G. I. Bratianu, Recherches sur le commerce génois dans la mer Noire au XIIIe siècle, Paris 1929, alle pp. 32-34.

${ }_{4}^{4}$ Tale indicazione è confermata da Rubruck (Guglielmo di Rubruc, Viaggio cit., I, 11, p. $10)$.

5 Per la verità su un braccio del Kuban, lo stesso che per Strabone si chiamava Hyspanis (Strabone, Geografia cit., lib. XI, II, 9-11) e per Idrisi Sakir. Si veda anche Heyd, Storia del commercio cit., p. 221.

${ }^{6}$ Racconto dei Tempi Passati cit., p. 70; è da questa data che si costituisce il principato russo indipendente di Tmutorakan (Bratianu, Recherches sur le commerce cit., p. 36).

7 Racconto dei Tempi Passati cit. p. 84.

${ }^{8}$ N. Banescŭ, La domination byzantine à Matracha (Tmutorokan) en Zichie, en Khazarie, et en "Russie» aù l'époque des Comnènes, in "Academie Roumaine, Bulletin de la Section Historique», XXII (1941), 2, pp. ??; Bratianu, La Mer Noire cit. p. 158.

9 G. Golubovich, Biblioteca bio-bibliografica della Terra Santa e dell'Oriente francescano, 5 voll., Quaracchi 1906-1927, II, pp. 558-560 e III, pp. 58 e 216. 
i sovrani di Francia, Ungheria e Norvegia. ${ }^{10}$ A ragione Bratianu affermava che Kiev e Novgorod' (Velikij) divennero delle piccole Costantinopoli. ${ }^{11}$ La complessa maglia di relazioni commerciali che si venne a creare vedeva nello stretto di Kerč e nel Bosporo un centro di fondamentale importanza per la raccolta e lo smistamento delle merci: mercanti russi, bizantini e Cazari acquistavano dalle popolazioni nomadi della zona, Peceneghi e Bulgari del Volga, per poi portarle a Nord, sul Baltico, o a Sud verso i mercati persiani. Lo stesso accadeva nella regione del Caucaso dove grande importanza aveva assunto la città di Trebisonda. Alla vigilia della seconda ondata nomade, alla metà dell'XI secolo, la striscia di territorio che dal Mar d'Azov giunge fino all'Armenia era inserita in un sistema politico ed economico funzionale e i cui rapporti con l'Asia centrale erano in ottima salute.

Quella seconda ondata nomade della quale ci siamo occupati nel primo capitolo di questo lavoro portò verso Occidente i Turchi Selgiuchidi e i Cumani. Proprio l'occupazione delle steppe da parte di questi ultimi cambiò nuovamente fisionomia alla regione e Tmutorakan venne presto invasa dall'elemento turcocumano. Durante tutto il secolo XI la regione fu oggetto della pressione esercitata dai Cumani da est e dalle frequenti lotte interne fra principi russi. ${ }^{12}$ Di fatto l'influenza di Bisanzio era ancora molto forte nel XII secolo se, alla stipula del trattato col quale Manuele Comneno concesse a Genova di stabilirsi nella capitale dell'impero, si vietava espressamente di commerciare in Rosia e Matracha. ${ }^{13}$ Durante gli anni in cui l'Impero Latino d'Oriente era al suo apogeo, dopo la IV crociata, molti mercanti latini si imbarcavano a Costantinopoli e giungevano fino a Matrega per poi andare alla foce del Don. Heyd afferma che essi non pensavano affatto ai prodotti asiatici; ${ }^{14}$ in realtà, come abbiamo avuto modo di dire sopra, la situazione politica del Turkestan e della Korazmia in particolare non permettevano questo tipo di contatti nel periodo di passaggio fra un prima e un dopo la seconda ondata nomade; fu proprio l'azione mongola a cambiare le cose. Dobbiamo ammettere però che, anche dopo la conquista e il mutato equilibrio continentale, lo stretto di Kerč e gli insediamenti di Matrega e La Copa conserveranno la loro importanza; si trattava di una zona ricca di pesce e già al tempo delle colonie greche esso costituiva il principale sostentamento per le popolazioni locali. A questo dobbiamo aggiungere la buona qualità e la

${ }^{10}$ Bratianu, Recherches sur le commerce cit. p. 37.

${ }^{11}$ Ibidem, pp. 37-38.

12 Racconto dei Tempi Passati cit. pp. 93-95, 113, 114, 116 e 128.

13 Abbiamo già sottolineato quanto sia controversa l'interpretazione di questo passaggio, ma in questa sede sembra utile vedere come Bisanzio non intendesse far passare dallo stretto di Kerč navi antagoniste, anche se alleate. Lo stesso divieto si ripeterà nel 1198; si veda Bratianu, $\mathrm{La}$ Mer Noire cit., p. 179. Per uno sguardo ampio e approfondito sulla regione in questo periodo cfr. Bortoli, Kazanski, Kherson and its region cit., pp. 660-665.

${ }^{14}$ Heyd, Storia del commercio cit., p. 312. 
notevole quantità di grano che le terre dello stretto erano in grado di fornire. ${ }^{15}$ Ritrovamenti archeologici recenti hanno infine dimostrato che anche in seguito alla distruzione di Kherson per mano mongola le manifatture e l'industria locale non persero dinamismo. ${ }^{16}$

\subsection{Le infiltrazioni nomadi in Georgia e le loro conseguenze}

Più avanti ci occuperemo dell'evoluzione politica nella Transcaucasia ponendo l'accento sull'elemento sedentario e sul ruolo che i centri di potere tradizionali ebbero nell'affrontare l'impatto col nomadismo. Le incursioni dei Selgiuchidi, la politica di Bisanzio, il regno di Georgia e l'invasione mongola, verranno analizzati nel contesto di un insieme di rapporti complessi e niente affatto univoci; essi ebbero un precedente importante nell'occupazione della zona da parte dei Cazari nell'VIII secolo, quando andava formandosi l'egemonia del popolo turco dal Volga al Caucaso. Non dobbiamo trascurare l'importanza che ebbe la presenza di altre realtà nomadi prima ancora che giungessero i Cazari - si pensi agli Unni - ma i loro contatti con la realtà sedentaria furono contrassegnati da rapporti occasionali, caratterizzati soprattutto da rapide scorrerie e incursioni dalle conseguenze pesanti sulla regione e sui villaggi in particolare. $\mathrm{Da}$ una prima fase che potremmo definire di contrasto, si passò a rapporti più articolati attraverso i quali le popolazioni del Caucaso georgiano vennero ad assumere una loro fisionomia peculiare. ${ }^{17}$

\subsubsection{Popoli di Georgia e organizzazione sociale}

Nel I secolo avanti l'era cristiana Strabone poteva osservare che «l'Iberia è ben popolata, soprattutto da città e insediamenti colonici; vi si trovano addirittura tetti in terracotta e le case sono costruite secondo i crismi dell'ar-

15 Si veda su questo il saggio, antico ma sempre molto utile, di G. I. Bratianu, La question de l'approvisionnement de Constantinople a l'époque byzantine et ottomane, in «Byzantion», 5, (1929-1930), pp. 83-107. Il divieto di oltrepassare gli stretti era legato, a mio avviso, alla politica fortemente protezionista e centralizzata che Bisanzio attuava in materia di politica economica. Fino al disastro del 1204 lo stato acquisiva grano in gran quantità registrando il tutto con estremo rigore; a ciò va aggiunta la tendenza al ripiegamento che l'impero operava su se stesso nel senso che si preoccupava molto dell'approvvigionamento delle derrate, ma curava assai poco l'aspetto commerciale. Questo favorirà l'ingresso delle repubbliche marinare italiane e dei mercanti catalani nel tessuto economico bizantino.

${ }_{16}$ Bortoli, Kazanski, Kherson and its region cit., p. 663.

17 Anche se bisogna ammettere che sul primo periodo i dati a nostra disposizione sono decisamente scarsi ed è forse questa la ragione per cui non siamo in grado di apprezzare appieno la complessità delle relazioni fra le due realtà antagoniste (ammesso che questo aspetto abbia predominato sui contatti di tipo pacifico). 
chitettura; ci sono piazze e gli altri edifici pubblici». ${ }^{18}$ Il geografo greco poteva apprezzare l'organizzazione sedentaria e la buona produttività del suolo anche se "la maggior parte [degli Iberi] è bellicosa e abita la montagna». ${ }^{19}$ Più avanti il Nostro aggiunge: "gli Albani, invece, sono più portati alla pastorizia e, se si esclude il fatto che non sono selvaggi, si avvicinano di più alle stirpi nomadi: pertanto sono moderatamente bellicosi». ${ }^{20}$ I termini usati da Strabone, selvaggi, bellicosi, nomadi, tradiscono la percezione di un uomo colto greco davanti a popolazioni che vivevano in modo diverso dal suo e che gli erano sostanzialmente sconosciute. Detto questo però bisogna riconoscere al geografo una notevole perspicacia poiché non sbagliava di molto quando affermava quello che abbiamo riportato. La divisione netta fra sedimenti nomadi e sedentari nel Caucaso e dell'area georgiana in particolare era un dato di fatto ai suoi tempi e lo era a maggior ragione la distribuzione geografica dei due modelli organizzativi. Il territorio georgiano, in cui predomina la montagna, imponeva un adattamento rigoroso da parte della popolazione e gli incontri che si verificarono nei secoli successivi col nomadismo eurasiatico non ebbero gli stessi effetti che produssero su popolazioni da secoli sedentarizzate. Per gli Iberi e gli Albani il nomadismo era un retaggio recente.

Il modello organizzativo più antico di cui si abbia notizia è quello del $s a$ gwareulo. ${ }^{21} \mathrm{Si}$ trattava di una sorta di clan, un nucleo molto simile all'oboq che vedremo quando ci occuperemo della società mongola; una comunità di famiglie con antenati in comune. Ogni sagwareulo, diviso a sua volta in gwari, era guidato dalla figura dell'anziano, in georgiano mamasakhlisi (padre della casa). L'elemento strutturale fondamentale non era la pastorizia, o non lo era necessariamente. Lo sfruttamento collettivo delle risorse era alla base del gwari, ma tali risorse potevano essere quelle del suolo. La redistribuzione della ricchezza era una delle principali forme di livellamento per cui ogni clan, o famiglia o singolo membro della medesima, doveva avere la propria parte. Di fatto possiamo immaginare che l'economia delle comunità georgiane di questo periodo fosse poco diversificata (non univoca, come spesso si pensa), una caratteristica comune al nomadismo tradizionale. La realtà sociale georgiana era composta da nuclei che potevano variare in dimensione e ricchezza. Questo modello o insieme di modelli sembra forte ancora al tempo di Strabone. Egli scrive: «gli uomini che popolano questa terra si classificano in quattro gruppi: il primo è quello da cui fanno discendere i re, in base alla parentela e alla maggiore anzianità (i cadetti, invece, si occupano della giustizia e dell'esercito). Il secondo è quello dei sacerdoti, che si occupano anche delle questioni con i popoli confinanti. Il terzo è quello dei soldati e dei

${ }_{18}$ Strabone, Geografia cit. XI, III, 1, p. 95.

${ }^{19}$ Ibidem, XI, III, 3, p. 96.

${ }^{20}$ Ibidem, XI, IV, 1, p. 99.

${ }^{21}$ Allen, A History of Georgian People cit., p. 221. 
contadini e il quarto è quello del "popolo", cioè degli schiavi reali, che si occupano di tutti i bisogni della vita quotidiana». ${ }^{22} \mathrm{Da}$ queste affermazioni si può evincere che la trasmissione del potere non era patrilineare, bensì seguiva il criterio, molto forte presso le popolazioni nomadi, della preminenza: poteva cioè essere il figlio, ma anche un fratello o un cugino. Il membro più anziano del sagwareulo più influente (in genere il più numeroso e quindi il più ricco) dominava l'intero sistema tribale dell'Iberia, amministrando la giustizia in tempo di pace e guidando l'esercito durante gli scontri. ${ }^{23}$ La quasi totalità della popolazione era libera. I beni immobili appartenevano al gwari tutto, ma spettava al membro anziano la gestione degli stessi. Come sottolineò a suo tempo Allen i nuclei familiari esistevano indipendentemente dal clan, ma era naturale farne parte e per ragioni di sicurezza. Le guerre combattute dall'Iberia contro Roma e il successivo trattato di pace incrementarono i contatti fra due modelli diversi e la società georgiana subì l'influenza dell'impero. Ciò si avvertì soprattutto dal II secolo, quando il sistema di successione si stabilizzò a vantaggio dell'avvicendamento patrilineare. ${ }^{24}$ Una profonda influenza ebbe anche il mutamento religioso; l'introduzione del cristianesimo in Iberia ebbe origini antiche, già in età apostolica si assiste ai primi contatti fra il mondo pagano delle popolazioni caucasiche e la religione monoteista. ${ }^{25} \mathrm{Il}$ processo che portò all'affermazione del cristianesimo fu lungo e complesso. Nel IV secolo esso divenne la religione ufficiale sia dell'Armenia sia dell'Albania e dell'Iberia. ${ }^{26} \mathrm{La}$ scelta religiosa degli stati caucasici fu di straordinaria importanza poiché, come ha ben scritto Bais, l'affermarsi del cristianesimo dette un impulso determinante «alla formazione della coscienza nazionale degli stati caucasici, terre di frontiera anche rispetto alla cristianità». ${ }^{27}$

Dal V secolo osserviamo una maggiore frammentazione sociale per cui all'organizzazione basata sul clan si sostituiscono progressivamente gruppi differenziati e omogenei con diritti e ricchezza diseguali. Questa disarticolazione crebbe con lo sviluppo del potere centrale e con il contemporaneo perfezionamento della macchina amministrativa. Lo squilibrio si accentuò particolarmente fra XI e XII secolo dando vita a condizioni libere, servili e portando una maggiore distanza all'interno dei ranghi stessi sulla base della ricchezza accumulata prestando servizi armati alle famiglie aristocratiche o al sovrano stesso. Il processo è assimilabile a quello che si era verificato nell'Europa Occidentale dall'epoca carolingia in avanti. I cosiddetti Aznaurni, ad esempio, compaiono nelle fonti

${ }^{22}$ Strabone, Geografia cit., XI, III, 6, p. 99.

23 Allen, $A$ History of Georgian People cit., p. 223.

${ }^{24}$ Ibidem, p. 224

25 «Allora mandarono un vescovo di nome Yovhannes, due preti, tre diaconi, una croce, e un'icona del Savio", HG, I, XI.

${ }^{26}$ Braund, Georgia in Antiquity; Outtier, La Christianisation du Caucase, in Il Caucaso: cerniera fra culture cit., I, pp. 553-568.

27 Bais, Albania caucasica cit., p. 103. 
georgiane proprio in questi secoli come nobiltà militare, ufficiali di prestigio a servizio del sovrano; in cambio ricevevano terre e diritti sui contadini che le lavoravano. All'alba del XII secolo alcuni di loro hanno accumulato vere fortune e possiedono aree fortificate. La mobilità sociale era scarsa, ma non sembra fosse impossibile l'ascesa, soprattutto se si prestava la propria opera nell'esercito. Anche il ceto mercantile esce dall'ombra documentaria in questi anni e acquisisce importanza crescente con lo sviluppo delle vie di transito internazionali che passavano per il Caucaso e per l'Armenia.

Sin dal VI secolo il territorio georgiano fu sottoposto a una ripartizione amministrativa che si normalizzò durante il regno bagratide. I sovrani della potente dinastia non poterono abbattere il sistema preesistente basato sulla presenza a livello locale degli Eristavni, governatori ai quali competeva l'amministrazione delle province sia da un punto di vista militare sia civile. Le notizie su questa carica e sulle sue funzioni sono scarse e frammentarie ${ }^{28}$ e con essa lo sono i dati sulla ripartizione amministrativa del regno prima dell'XI secolo. Col crescere del potere regio e dopo l'ascesa al trono di Bagrat III, la forza degli Eristavni diminuì poiché essi rappresentavano una seria minaccia per il sovrano e il suo potenziale di controllo sul territorio. Gli Eristavni avevano un seguito numeroso e ben organizzato; erano responsabili del reclutamento militare, amministravano la giustizia nei villaggi, beneficiavano di tributi annuali e potevano imporne di nuovi: un potere notevole che si scontrava coi progetti autocratici di Bagrat III. ${ }^{29}$ In effetti con l'avvento sul trono della regina Tamara il frazionamento territoriale aumentò e ciò fu l'effetto di molteplici fattori: il numero crescente di guerre e di vittorie, terre nuove da spartire, personaggi che le ricevevano in premio del loro apporto militare e ricchezza che finiva, sempre più copiosa, nelle mani di poche famiglie le quali erano di fatto in grado di amministrare il territorio in autonomia rispetto al potere centrale; cresceva infine il potere dell'esercito. All'aumento delle proprietà territoriali corrispose il rafforzamento dell' istituto della patronqmoba, ovvero un sistema in cui nelle campagne vi erano possessori di terre (Tsikhis o Midsis Patroni), o contadini che lavoravano quelle stesse terre (qmani) senza esserne i proprietari; contemporaneamente aumentò anche la protezione sulle campagne da parte del potere centrale, il quale sperava di poter esercitare così un maggior controllo sull'autorità dei proprietari locali. Durante il regno di Tamara si ebbe una svolta: Tamara legò la proprietà dei singoli distretti all'ufficio che vi si esercitava così, alla fine del mandato (che poteva essere revocato dal sovrano in qualsiasi momento), la terra e quel che vi stava sopra tornava alla corona. Non sempre il sistema funzionava e talvolta poteva capitare che il sovrano lasciasse la proprietà al funzionario destituito.

${ }^{28}$ Allen, A History of the Georgian People cit., p. 238.

${ }^{29}$ Ibidem, pp. 243-249; per un quadro più approfondito si veda anche Charachidzé, Introduction a l'étude cit. 
Da un punto di vista amministrativo le notizie sono più numerose e Allen nel suo lavoro ne illustrò a sufficienza le caratteristiche. Basterà in questa sede ricordare che gli ufficiali del regno erano numerosi e la macchina burocratica raggiunse nel XIII secolo dimensioni notevolissime. ${ }^{30}$ In questo contesto si abbatterono sulla Georgia le incursioni nomadi.

Dopo il periodo dell'apogeo politico, culturale ed economico, l'invasione mongola pose fine all'indipendenza del regno georgiano. È mia convinzione che l'impatto dell'esercito di Gengis khan ebbe effetti tremendi per la Georgia e per i piccoli centri di potere armeni in quanto essi erano deboli e fiaccati dalla politica bizantina da una parte e dalla prossimità geografica del sultanato selgiuchide dall'altra; a questi due elementi dobbiamo però aggiungerne un altro il cui peso è stato forse poco evidenziato dagli studi sull'argomento e cioè le infiltrazioni nomadi nel tessuto sociale georgiano sin dall'inizio del XII secolo e il loro ruolo sull'evoluzione del nucleo civile di quelle terre accanto agli eventi politici. I Cumani vennero invitati in Georgia da David in persona per ragioni di opportunità politica, ma vi rimasero a lungo e vissero un processo di assimilazione coerente nel tempo la cui influenza sui mutamenti in seno all'eterogenea società georgiana fu tutt'altro che trascurabile. Con questi elementi e con i risultati di questo processo dovettero fare i conti non solo i mercanti occidentali che si servirono, dalla seconda metà del XIII secolo, dei porti georgiani di Batumi e Savastopoli, ma anche gli ordini mendicanti che furono incaricati dalla Santa Sede di installarsi in questa zona stabilmente.

\subsubsection{Polovcy in Georgia}

Nel 1118 David III (1089-1125) decise di chiamare entro i confini del regno georgiano un gruppo di tribù cumane guidate dal khan Äträk. Il processo che vide questo contingente nomade installarsi stabilmente nel contesto strutturale di un regno sedentario come quello di Georgia fu lungo e complesso, ma di certo esso ebbe un impatto destabilizzante sugli equilibri sociali ed economici preesistenti nella regione. Le ragioni dell'iniziativa di David furono molteplici e su esse si è acceso un dibattito che ancora oggi rimane vivo. Gli elementi da considerare a tale riguardo sono, a mio parere, almeno quattro: le incursioni delle prime popolazioni turco-nomadi (Selgiuchidi soprattutto), unite alla politica che Bisanzio adottò verso l'Anatolia e la Caucasia, ridussero le potenzialità umane della Georgia; in pratica essa soffriva di una forte carenza di manodopera. ${ }^{31}$ Il secondo elemento lo abbiamo in parte già considerato (cfr. cap. 1) e riguarda la scarsa stabilità del potere centrale. Le dispute in seno all'aristocrazia per l'influenza sulle decisioni del sovrano inquietavano David che temeva la

30 Allen, $A$ History of the Georgian People cit., pp. 258-260.

31 Golden, Cumanica I cit., p. 59. 
crescente influenza a corte di alcune famiglie nobili. Crebbe quindi, da parte di quest'ultimo, l'esigenza di dotarsi di un esercito fedele, di uno strumento che fosse solo nelle sue mani. Non va inoltre trascurato il fatto che i Cumani erano una popolazione vicina e potenzialmente pericolosa per la Georgia. Essi costituivano un utile cuscinetto fra il Caucaso e la Rus. ${ }^{32}$ Metterli sotto la propria protezione poteva incrementare le capacità di controllo sull'elemento nomade. Il quarto e forse il più urgente dei motivi che spinsero David a concedere ospitalità ai Cumani fu l'emergenza in politica estera. In questo periodo la Georgia si stava riaffermando come nucleo politico rilevante e stava cercando di allargare la propria egemonia verso quelle terre che aveva perduto in seguito alle incursioni dei Selgiuchidi. Per porre in atto questo disegno David necessitava di un esercito forte, numeroso e ben addestrato, tutte cose che la provata popolazione georgiana non era, in questi anni, in grado di fornire.

Nel 1118 penetrarono così all'interno dei confini georgiani oltre 200.000 individui (40.000 furono quelli che vennero arruolati). ${ }^{33}$ Erano per lo più quelle cellule nomadi che abitavano la vasta regione delle steppe fra Donec, Don e Volga, la cosiddetta Cumania Nera. ${ }^{34}$ Essi furono distribuiti nelle campagne e gli furono concesse terre per il pascolo. A coloro i quali prestavano servizio militare fu corrisposto un salario.

Lo scopo di David III era quello di inglobare all'interno del tessuto umano indigeno questi elementi nomadi e per farlo cercò di concedere loro terre polverizzate: mai grandi appezzamenti, ma piccole porzioni fondiarie interne a zone abitate da Georgiani o Armeni. In questo modo sperava di indebolire l'elemento identitario turco e contemporaneamente accelerare il processo di assimilazione..$^{35} \mathrm{E}$ ovvio che tutto ciò avesse come scopo finale la cristianizzazione dei nuovi arrivati.

Se prendiamo per buone le cifre di cui disponiamo non possiamo esimerci dal porsi una domanda: che fine hanno fatto i 200.000 Cumani che sono entrati in Georgia se è vero che nelle fonti scompaiono già nella seconda metà del XII secolo e oggi nel paese caucasico i toponimi e i termini di origine turca sono assai scarsi? Questo interrogativo assume maggior interesse se confrontiamo l'esperienza georgiana con quella che i Cumani vissero in Ungheria. ${ }^{36}$ Difficile

${ }^{32} \mathrm{Su}$ questo aspetto si veda Š.A. Meschia, Ja. Cincadze, Iz istoria russo-gruzinskich vzaimootnošenij X-XVIII vv., Tbilisi 1958, pp. 7-9; Golden, Cumanica I cit., p. 60.

33 Sulle cifre c'è sostanziale accordo: Golden, Cumanica I cit., p. 62.

${ }^{34}$ Golden, Cumanica I cit., p. 66; Id., Polovcy Dikii cit.; Pletneva, Polovcy cit.

${ }^{35}$ Golden, Cumanica I cit., p. 63.

${ }^{36}$ Dove le tracce della presenza cumana sono ancora oggi visibili a livello lessicale e toponomastico. Su questo si vedano i lavori di N. Berend già citati. Sulla presenza cumana in Romania si veda invece L. Rásonyi, Contributions à l'histoire des premières cristallisations d'Etat des Roumanis, in «Archivum Europae Centro-Orientalis», I (1935), pp. 221-253; Diaconu, Les Coumans au Bas-Danube cit. 
pensare a un processo di assimilazione così completo. È più probabile che gran parte degli individui entrati nel 1118 se ne siano andati precocemente. In fondo sappiamo che lo stesso Äträk tornò nella steppa molto presto. ${ }^{37} \mathrm{Si}$ tratta di un problema centrale per comprendere il processo di interazione fra i due modelli che nel caso georgiano vissero un fase di confronto particolarmente interessante. Quella parte minoritaria che rimase in Georgia può essersi sedentarizzata in seguito a un processo imposto dal potere centrale e può aver avuto un confronto con la popolazione delle campagne. Non era raro che elementi nomadi entrassero a far parte della classe dirigente di regni sedentari in seguito ad alleanze che sfociavano in unioni matrimoniali. Da questo punto di vista la scarsa unità interna delle cellule nomadi e la loro conseguente disarticolazione politica erano un fattore che favoriva questo tipo di rapporti diversificati e talvolta contrapposti con le realtà sedentarie. In passato si è voluto vedere il caso della Georgia come emblematico non solo da questo punto di vista; ${ }^{38}$ la parziale, talvolta totale, integrazione dell'elemento nomade all'interno del ceto dirigente locale si sarebbe verificata spesso in epoche successive. Khazanov sosteneva che fra la fine dell'XI e la metà del XII secolo si sarebbero stabiliti nella Georgia orientale (la zona di Kartli, al confine con l'Azerbaijan) centinaia di migliaia di Cumani. Sono cifre poco verosimili poiché, come vedremo più avanti parlando del fattore demografico, la Georgia aveva una popolazione che superava appena i quattro milioni di abitanti. Piuttosto è assai probabile, e su questo concordo con Khazanov, che la corona abbia concesso ai Cumani entrati dal 1118 l'area pianeggiante al confine con l'Azerbaijan affinché vi potessero passare l'inverno senza che attraversase i monti del Caucaso per tornarsene nella steppa. Se questo nutrito gruppo nomade si sia del tutto sedentarizzato o abbia progressivamente abbandonato il modello tradizionale a vantaggio di una maggiore stabilità o di un'economia più diversificata è difficile dirlo con certezza. Certo si è che in condizioni ambientali favorevoli e in un contesto sociale sostanzialmente mutato il processo di sedentarizzazione potrebbe essere iniziato senza ostacoli insormontabili. D'altra parte quando le condizioni esterne lo permettono le società tradizionalmente nomadi non sono così avverse a modelli produttivi più diversificati come in genere si è soliti ritenere. Di fatto i pochi elementi che abbiamo a disposizione farebbero presumere a un crescente ricorso alla produzione agricola da parte di questi gruppi. Inoltre vediamo comparire Cumani nell'amministrazione di Stati vicini a quello georgiano nella seconda metà del XII secolo. L'atabeg azerbaijano Šams ad-Din Eldigüz era un mamelucco di origini cumane, e non è il solo esempio che si potrebbe fare. ${ }^{39}$

37 Golden, Cumanica I cit., p. 65.

38 A.M. Khazanov, Nomads and the outside World, Cambridge 1994², pp. 198-202.

39 Golden, Cumanica I cit., p. 79; Z.M. Bunijatov, Gosudarstvo atabekov Azerbaidžana (11361225 godu), Baku 1978, pp. 44-52; Minorsky, Studies in Caucasian History cit., pp. 93-96. 
In questo, come in tutti gli altri casi in cui troviamo personaggi eminenti di origine cumana inseriti nell'apparato amministrativo di un contesto sedentario, i Cumani non compaiono mai come sostenitori degli interessi cumani, bensì come politici o amministratori locali (Georgiani, Azerbaijani etc.) la cui matrice etnica è quella cumana,${ }^{40}$ segno evidente di un processo reidentificativo in avanzata fase di compimento.

\subsubsection{Il nomadismo in Georgia: contrasto e integrazione}

Nei primi decenni del XII secolo l'opera di Vladimir Monomach contro i Cumani - in particolare quelli che abitavano l'area compresa fra il Donec e il Volga - si era intensificata e la pressione esercitata sulla popolazione turconomade era divenuta poco sostenibile. Questo è forse un altro dei motivi che spinsero Äträk ad accettare la proposta di David III. Dopo la morte del sovrano slavo la politica dei Rus' verso i Cumani cambiò decisamente a favore di un minore antagonismo; la diminuita pressione può aver influito sul ritorno nella steppa da parte di alcuni gruppi di coloro che si erano spostati in Georgia (altri, come è noto, si insediarono nell'Anatolia orientale, fra i territori armeni e quelli selgiuchidi).

L'assenza di un modello coordinato del potere si scontrava con l'elemento sedentario indigeno. A fronte di nuclei che si stabilirono nelle campagne adattandosi - con tempi e modi assai diversi da zona a zona - al nuovo modello semi-nomade, c'erano gruppi staccati di Cumani che non potevano essere controllati in alcun modo e che perpetravano incursioni e razzie in quelle stesse campagne. ${ }^{41}$ Con molta probabilità si assistette a una frammentazione del gruppo che giunse in Georgia per cui se un nutrito numero venne con successo insediato nelle campagne sedentarizzandosi (almeno parzialmente), altri tornarono nella steppa appena ciò fu possibile. Dalla seconda metà del XII secolo e fino al regno di Tamara troviamo nomi turchi all'interno del ceto dirigente locale georgiano. La seconda o la terza generazione di quei Cumani che si erano stabiliti nelle pianure del regno caucasico erano riusciti ad affermarsi come governatori, atabeg, o ufficiali militari; si trova qualche nome cumano anche nei più alti ranghi dell'aristocrazia di corte. Dobbiamo però

${ }^{40}$ Golden, Cumanica I cit., p. 81; I. Vásáry, Cumans and Tatars. Oriental Military in the Pre-Ottoman Balkans, 1185-1365, Cambridge 2005, in particolare le pp. 22-25.

41 Non è argomento di questo lavoro capire se e come sia possibile pensare a una forma di Stato organizzato per la realtà nomade di questi anni e d'altra parte si tratta di una questione che ha per molto tempo impegnato gli studiosi. La letteratura a riguardo è copiosa. Fra i lavori di maggior rilievo ricordiamo L. Krader, Formation of the State, Englewood Cliffs (NJ) 1968; Id., Origins of the State Among the Nomads of Asia, Berlin 1981, p. 77 e segg.; Pletneva, Kočevniki cit.; O. Pritsak, The Origin of Rus', Cambridge (MS) 1981. Più avanti torneremo sull'argomento con particolare riguardo alla vicenda mongola. 
tenere sempre presente il carattere duale, fluido delle relazioni che intercorsero fra le due realtà. Negli ultimi trent'anni del XII secolo certe dinamiche interne ai diversi strati sociali emersero con evidenza. Durante i regni di Demetrio I e, soprattutto, di Giorgio III i contrasti fra la corona e l'aristocrazia si inasprirono in modo crescente. In Georgia il ceto aristocratico che operava prevalentemente a corte aveva anche notevoli proprietà nelle periferie rurali. Gran parte dei governatori locali provenivano dalle famiglie eminenti di Tbilisi, Kutais, Batumi; tale situazione di privilegio era stata indebolita dalla politica di Giorgio III che tendeva a concedere le terre in base ai servizi resi alla corona piuttosto che al lignaggio. Durante il passaggio dal regno di Giorgio III alla figlia, Tamara (1184), si assiste a un tentativo da parte della nobiltà di palazzo di ridimensionare il potere della nuova regina. In questa circostanza appare in modo evidente il carattere ambiguo e difficilmente inquadrabile delle dinamiche sociali che portavano all'ascesa elementi di origine nomade. Uno dei funzionari fedeli alla corona, Qubasar venne costretto a dimettersi dal suo incarico a causa delle pressioni esercitate dall'aristocrazia guidata da Qutlu Arslan. ${ }^{42}$ Ora, è piuttosto evidente che entrambi i nomi, Qubasar e Qutlu Arslan, siano di origine turco-cumanica. Ciò evidenzia come due personaggi, provenienti verosimilmente dall'emigrazione cumana del 1118, fossero schierati su sponde contrapposte dopo due generazioni e come fossero in grado, sul finire del XII secolo, di costituire un'alternativa politica a quella del legittimo sovrano. Vero si è che Tamara non cedette al ricatto aristocratico e la storia del suo regno dimostra come ella seppe reagire con forza alle pressioni esterne (da questo punto di vista furono molto importanti i successi in politica estera del regno georgiano).

La vicenda matrimoniale di Tamara è un altro evento che induce a riflettere sul dualismo fra elemento nomade e sedentarismo; la regina sposò Jurij, figlio del celebre principe rus' Andrej Bogoljubskij il quale era di origine cumana. ${ }^{43}$ Il nomadismo dunque appare come elemento solo parzialmente antagonista. Quanto abbiamo affermato circa i rapporti fra Polovcy e Rus' nel capitolo precedente andrebbe allargato alla Caucasia tutta in un quadro di rapporti complessi, differenziati e su vasta scala. Credo infatti che non vada trascurata l'affinità religiosa fra lo stato slavo e quello caucasico. Il principe kieviano Izjaslav

${ }^{42}$ Golden, Cumanica I cit., pp. 79-80; Allen, A History of the Georgian People cit., pp. 253254. In questa occasione, ad esempio, Tamara gli riprese Lori, cioè solo una parte del distretto di cui era governatore. La famiglia di Qubasar poteva così possedere terre nonostante la destituzione dell'alto ufficiale.

43 Il padre di Andrej, Jurij Dolgorukij, aveva sposato la figlia del qan cumano Ay-opa (Golden, Cumanica I cit., p. 82; Pritsak, Polovcians cit., p. 377; Allen, A History of Georgian People cit., pp. 104-106). Il matrimonio di Tamara naufragò assai precocemente (1187) e senza che fosse nato un erede per il trono di Georgia. Tamara sposò poco dopo (forse nel 1189) David Soslan un principe della dinastia bagratide. 
Mstislavovič sposò una georgiana, mentre il fratello di Andrej, Vsevolod, sposò una principessa di origini As. ${ }^{44}$

Infine durante la prima incursione mongola nel Caucaso (si veda oltre il cap. 5) si trovano Cumani sia nell'esercito del sultano di Korazmia sia in quello georgiano.

${ }^{44}$ Golden, Cumanica I cit., p. 83. 



\section{LA COSTRUZIONE DI UN POPOLO}

\subsection{I primi gruppi altaici. Acquisizioni e problematiche aperte}

L'origine delle tribù turco-mongole è strettamente legata al concetto di frammentarietà. Sebbene l'Occidente attorno alla metà del XIII secolo abbia percepito i Tataro-mongoli come un'entità politica e militare sostanzialmente omogenea, essi erano in quel periodo il frutto di un'evoluzione interna già avanzata. La storia di queste popolazioni è quella di realtà, più o meno vaste, ma spesso assai diverse fra loro per le quali l'unificazione fu la tappa di una evoluzione avviatasi decenni prima. Le fasi di tali mutamenti non sono facilmente individuabili per una serie di ragioni. Fra le altre si rilevano la scarsità documentaria innanzitutto, la tipologia delle fonti e la loro vasta distribuzione linguistica, i condizionamenti ideologici di parte della storiografia che si è occupata di mongolistica e i pregiudizi che si è soliti associare al nome "Mongoli". Per molto tempo si è studiato il nomadismo della steppa guardandovi come a un modello necessariamente alternativo; più spesso come antagonista rispetto all'economia $\mathrm{e}$ alla fisionomia sociale basate sulla sedentarietà di cui la produzione agricola era un aspetto imprescindibile. Gli studi pi recenti, giovandosi dell'apporto delle scienze sociali, hanno fortemente ridimensionato queste categorie. ${ }^{1} \mathrm{Da}$ un punto di vista storico, e scendendo nell'individualità etnica, potremmo citare a titolo d'esempio le parole di Edward G. Browne il quale, in una celebre antologia di letteratura persiana, scriveva a proposito delle differenze che caratterizzarono le invasioni araba e tataro-mongola che «in a word, the Tartars were cunning, ruthless and bloodthirsty marauders, while the Arabs were, as even their Spanish foes were fain to admit, Knights... and gentlemen, albeit Moors». ${ }^{2}$ L'opera citata è di straordinaria importanza, soprattutto se valutata insieme ai due volumi che Browne aveva già dato alle stampe fra il 1902 e il $1906,{ }^{3}$ ma in quelle parole è

1 In effetti la distanza fra gli studi storici e quelli antropologici oltre alla scarsa interazione fra le due discipline sono problemi ancora oggi molto presenti. Un fenomeno complesso e cronologicamente duraturo come il nomadismo dovrebbe essere studiato in un'ottica più ampia possibile, che si serva del ricorso alle fonti come della ricerca sul campo. Su questo si è espresso con chiarezza Anatoly Khazanov (Nomads and the Outside World).

2 E.G. Browne, A history of Persian Literature under Tartar dominion (AD 1265-1505), Cambridge 1920, p. 4.

${ }^{3}$ E.G. Browne, A Literary History of Persia from the earliest times until Firdawsi, London 1902 e Id., A Literary History of Persia from Firdawsi to Sa'di, London 1906, rist. in 4 voll., New Delhi 1997. 
contenuto un luogo comune che ancora oggi, a distanza di oltre ottanta anni, caratterizza gran parte della storiografia (non specialistica a dire il vero) sull'argomento. L'immagine dei Mongoli cattivi, assetati di sangue e portatori di distruzione e lacrime piuttosto che di un nuovo corso strutturale è ancora ben radicata nell'immaginario collettivo.

Non si può avere la pretesa di fare chiarezza su un argomento che, data la scarsità di riscontri documentari scritti, è destinato a rimanere per sempre nell'ombra, salvo ricevere un soccorso decisivo dall'indagine archeologica e antropologica. Certo, si può tentare, sulla base delle più recenti acquisizioni e analizzando le fonti che lo stesso Browne conosceva e tradusse, di rileggere una vicenda la cui portata storica fu straordinaria e coinvolse larga parte del mondo allora conosciuto. L'analisi delle dinamiche sociali interne ai primi gruppi altaici e il loro carattere nomade originario ritengo siano premesse irrinunciabili per capire la nascita dell'impero mongolo e in particolare la sua realizzazione più occidentale (Orda d'Oro e Ilkhanato), la stessa che coinvolse la Caucasia.

Quello che potremmo definire il "pregiudizio" tataro-mongolo è soprattutto il frutto di un punto di vista: le fonti attraverso cui li conosciamo sono tutte o quasi opera di chi subì la loro dominazione. Vi sono delle eccezioni - il caso della Storia Segreta è emblematico - ma tali restano. Eccezioni parziali sono invece costituite dall'opera di Rashid ad-Din ${ }^{4}$ e di Juvaini. ${ }^{5}$ In ogni caso entrambe sono caratterizzate dalla necessità da parte dell'autore di compiacere chi aveva commissionato l'opera (nella fattispecie l'Ilkhan in persona).

Credo che serva a poco ridiscutere il carattere più o meno violento delle invasioni mongole; esse rappresentarono certamente uno scossone improvviso, un fatto cruento, dalla Cina alla Persia, dal Turkestan alla Russia, in seguito al quale morirono centinaia di migliaia di uomini, furono distrutte intere comunità, cambiarono gli equilibri economici e in parte etnici di un intero continente. D'altra parte possediamo un numero di fonti scritte mongole, quindi di una prospettiva "interna", troppo esiguo per poter esaminare il fenomeno in un quadro di relativa imparzialità. E comunque varrebbe la pena osservare tali fenomeni in un'ottica comparativa per evitare di racchiudere questo popolo all'interno di un clichè estraneo alla realtà storica entro la quale esso agì e si sviluppò.

Sulla storia dei Mongoli vi è una buona produzione di resoconti di viaggio, cronache di storici coevi le vicende in questione, ma sono arabe, cinesi, persiane, slave o occidentali. Come dicevamo la memoria storica tataro-mongola si riduce alla dimensione collettiva solo nel caso della Storia Segreta, un documento di straordinaria importanza, molto dettagliato e ben scritto, ma è poco

\footnotetext{
${ }^{4}$ Per le edizioni di Rashid ad-Din utilizzate si veda il capitolo secondo, nota 35.

5 Per le edizioni di Juvaini utilizzate si veda il capitolo primo, nota 57.
} 
e comunque risale all'inizio degli anni Quaranta del XIII secolo, quindi a un periodo successivo l'etnogenesi dei primi gruppi altaici, sebbene se ne occupi con dovizia di particolari.

Con queste premesse l'unico tentativo, onesto, che si può fare è quello di porsi delle domande e cercare una riflessione globale attraverso le fonti accessibili. Un primo problema riguarda il periodo originario di sviluppo delle tribù tataro-mongole, la loro struttura sociale e la specificità del caso nel contesto nomade di questo periodo; in secondo luogo ci si dovrebbe chiedere perché questi popoli originari dell'estrema periferia orientale dell'Asia, fondamentalmente nomadi, si siano mossi in modo compatto verso occidente conquistando terre su terre fino a diventare, anche se per pochi anni, il più vasto impero che sia mai esistito. Il terzo quesito che ci si è posti, e sul quale ancora oggi si sta interrogando parte della storiografia specialistica, riguarda l'individuazione delle fasi attraverso cui gruppi nomadi siano entrati in una fase di sviluppo il cui risultato fu la creazione di imperi più o meno stabili; in pratica ci si chiede come si è giunti alla formazione di veri e propri organismi statali. ${ }^{6}$ Per quanto riguarda l'effetto esterno di tali fenomeni, ovvero le conquiste, possiamo dire che non sono mancati i tentativi per spiegarne l'origine e i caratteri fondamentali. Esamineremo nel dettaglio questi aspetti e proveremo a fornire qualche ipotesi su quesiti che scaturiscono da questo ulteriore problema. Ad esempio: quali furono le modalità della conquista nella regione che stiamo indagando? Davvero devastarono tutto? Quali caratteristiche aveva il loro modo di combattere che, nuovo per gli uomini occidentali, impressionava per la sua efficacia?? Quali furono le modalità del dominio nel Caucaso e attorno all'Azov? Anche questi sono gli interrogativi sui quali ho concentrato l'attenzione nelle pagine che seguono.

6 Si tratta di quella che negli Stati Uniti viene definita come "State formation" o "Early State". Su questo argomento è intervenuto, con un saggio pubblicato qualche anno fa, Nicola Di Cosmo le cui argomentazioni sono assai convincenti. Ne riparleremo poco oltre. Cfr. N. Di Cosmo, State Formation and Periodization in Inner Asian History, in "Journal of World History", 10 (1999), 1, pp. 1-40.

7 Qualche anno fa Aldo Settia ha pubblicato un libro nel quale analizza a fondo il fenomeno della guerra nel Medioevo. Dalla sua indagine rimane piuttosto estranea l'area in questione (d'altra parte pretendere un impegno geograficamente esaustivo sarebbe stato troppo e nessuno studioso da solo avrebbe potuto attendere a tale scopo), ma si tratta di un ottimo saggio, molto documentato e dal quale emerge con chiarezza il complesso sistema bellico medievale. Uno dei meriti che ritengo debbano attribuirsi al libro è quello di avere smontato, con il rigoroso ricorso alle fonti, il mito delle popolazioni naturalmente inclini alla distruzione e alla strage. Il modo di combattere nel Medioevo era profondamente diverso da quello cui la nostra memoria visiva è abituata. Su questo è emblematica una frase del saggio che mostra come tale sistema fosse generale, non esclusivo di un esercito piuttosto che di un altro: «per tutta l'età medievale infatti il modo di gran lunga più diffuso di guerreggiare consistette in scorrerie devastatrici generalmente limitate nel tempo e nello spazio»; esse costituivano "almeno l'80 per cento degli episodi militari attestati dalle fonti»: A.A. Settia, Rapine, assedi, battaglie. La guerra nel Medioevo, Roma-Bari 2002, p. 4. 
L'insieme di popolazioni che abitavano la regione altaica sin dall'VIII secolo era un universo composito, molto differenziato da un punto di vista etnico. Secondo Juvaini ${ }^{8}$

La patria dei Tartari ${ }^{9}[\ldots]$ è un'immensa vallata, la cui superficie si estende per sei o sette mesi di viaggio sia in lunghezza che in larghezza. A oriente confina con la terra del Khitai, ${ }^{10}$ a occidente con il paese degli Uighur, a settentrione con i Kirghisi ${ }^{11}$ e con il fiume Selenga, e a meridione con i Tangut e con i Tibetani.

L'etnonimo col quale si è soliti indicare questo popolo è sostanzialmente un'etichetta. È una classificazione semplicistica sotto la quale si trova un insieme di tribù distinte fra loro e la cui origine meriterebbe una singola trattazione per ciascuna. Volendo in questa sede indagare quale fu l'impatto dell'avanzata mongola nel Caucaso e in quelle che abbiamo definito come steppe dei Polovcy non possiamo tuttavia esimerci dal considerare i primi sviluppi di queste popolazioni e quali elementi agirono per dar vita all'unificazione di questo vasto e diversificato universo tribale.

Il contesto ambientale entro il quale ebbero luogo i primi fenomeni di unificazione è caratterizzato da estati temperate, talvolta piuttosto calde, e da inverni estremamente rigidi. I massicci montagnosi sono piuttosto numerosi. La vegetazione è povera e il suolo non dispensa risorse sufficienti per il sostentamento. Il nomadismo della steppa è un ottimo esempio di adattamento a queste condizioni estreme. L'adozione del nomadismo come modello predominante, e mai esclusivo, da parte di queste popolazioni fu il frutto di un lungo e complesso processo. Il possesso del bestiame divenne la fonte di sostentamento essenziale già quando le condizioni ambientali consentivano un modesto ricorso alla lavorazione della terra. Il concetto di gruppi nomadi dediti esclusivamente all'allevamento e alla raccolta (soprattutto caccia) e a cui l'agricoltura sarebbe stata del tutto estranea andrebbe ridimensionato. Vi erano nuclei più o meno popolosi che non erano in grado di lavorare la terra, alcune zone della regione in oggetto non lo permettevano, ma non dobbiamo associare i due concetti in modo univoco. Alcune aree della Mongolia attuale, al confine con la Cina e in particolare verso ovest, al confine con Kazakistan, presentavano una modesta fertilità per cui non

8 Juvaini, I, pp. 41-42.

9 Juvaini utilizza l'etnonimo generico di Tartari, ma intende i Mongoli tutti. Considerando che egli scrive negli anni Cinquanta del XIII secolo e che era un funzionario dell'Ilqan, penso si possa ben capire il motivo di questa scorretta denominazione.

${ }^{10}$ Si tratta della Cina Settentrionale, quella che nelle fonti occidentali appare come Catai, Cattai, Gattaio etc.

${ }^{11}$ Gli Uighur erano, come i Kirghisi, una popolazione turca. I primi erano originari della Mongolia attuale, ma da essa erano stati cacciati dai Kirghisi i quali, prima che ciò avvenisse, abitavano la regione dell'alto Yenissei. 
sarebbe stato impossibile trarne un seppur minimo mantenimento. Gran parte dei prodotti del suolo entravano nelle società nomadi per appropriazione (scambio e razzia) ed erano uno degli elementi chiave nel regolare i rapporti con le società sedentarizzate. I caratteri dei sistemi produttivi di questa regione erano cioè più sfumati, meno netti di quanto non si sia spesso ritenuto. ${ }^{12}$

Alla fine del XII secolo le popolazioni nomadi dalle quali ebbe origine il gruppo dei Mongoli erano dedite principalmente all'allevamento e risultavano pressoché sconosciute alla totalità dell'Occidente. Esse abitavano l'immensa regione posta a ovest e a nord della Grande Muraglia cinese; la Cina dal canto suo non ne aveva un'idea precisa e le considerava più che altro un pericolo da cui difendersi. ${ }^{13}$ L'area entro la quale presero le mosse i primi gruppi altaici è molto vasta: a nord si trova la regione siberiana del lago Baijkal, a sud l'India, a est la Cina e a ovest l'attuale Kazakistan. Giovanni di Pian del Carpine, che si recò in questa zona alla metà del XIII secolo dice che «il clima è incredibilmente variabile. Infatti in piena estate, quando il caldo è forte in altri luoghi, qui esplodono violenti temporali con fulmini a causa dei quali restano uccise molte persone; si hanno anche, nella stessa stagione, abbondanti nevicate e violente tempeste di venti freddissimi tanto che, talvolta, gli uomini riescono a cavalcare con fatica». ${ }^{14}$ Nella sua

${ }_{12}$ Per questi aspetti, soprattutto per i rapporti fra nomadi dell'Asia Centrale e la Cina, si veda N. Di Cosmo, Ancient Asia Nomads; Their Economic Basis and its Significance in Chinese History, in "The Journal of Asian Studies», 53 (1994), 4, pp. 1092-1126; B.O. Bold, Mongolian Nomadic Society, a Recontruction of Medieval History of Mongolia, New York (NY) 2001, pp. 25-32.

${ }^{13} \mathrm{La}$ Cina interveniva spesso per fomentare conflitti fra le popolazioni nomadi dell'Asia centroorientale con lo scopo primario di favorire la disgregazione interna alle tribù che per dimensioni $e$ preparazione militare costituivano un potenziale pericolo. A tale proposito è emblematico il caso dei Tatari; si veda per questo M. Hoàng, Gengis Khan, Milano 1992, pp. 45-65; Grousset, L'empire des steppe cit., pp. 243-245; V.V. Bartold, Turkestan cit., pp. 381-382. Ora la monografia più completa e aggiornata sui rapporti fra la Cina e il nomadismo della steppa è N. Di Cosmo, Ancient China and its Enemies: The Rise of Nomadic Power in East Asian History, Cambridge 2004; sul fattore ambientale cfr. la parte I, alle pp. 13-91; cfr. anche già citato Di Cosmo, Ancient Asian Nomads.

${ }_{14}$ Giovanni di Pian del Carpine, Storia dei Mongoli, a cura di P. Daffinà, C. Leonardi, M.C. Lungarotti, L. Petech, Spoleto 1989, I, 5, p. 339. Questa ottima edizione contiene sia il testo in latino (d'ora in avanti Historia Mongalorum) sia la traduzione in italiano (d'ora in avanti Storia dei Mongoli). L'Historia di frate Giovanni è un'opera molto nota ai medievisti anche se non specialisti di mongolistica. Essa fu scritta in due diverse redazioni, la prima alla fine del viaggio sulla scia emotiva della sua straordinaria esperienza, la seconda più tardi, al fine di rendere credibile il suo viaggio in terre che per gli uomini del suo tempo erano poco più che un'immagine mentale. Nella seconda redazione Giovanni aggiunse un capitolo, il IX, come ha ben descritto M. Cristiana Lungarotti nel suo saggio contenuto nella Storia di cui sopra (pp. 79-92). L'Historia ha avuto varie edizioni, con traduzioni talvolta lacunose, ma in genere il livello scientifico è molto alto. Rammentiamo in questa sede le principali edizioni. Accanto alla fondamentale versione data da padre A. Van den Wyngaert, Fr. Johannes de Plano Carpini, Ystoria Mongalorum, in Sinica Francescana I: Itinera et relationes Fratrum Minorum saeculi XIII et XIV, a cura di A. Van De Wyngaert, Quaracchi-Firenze 1929; possiamo ricordare il primo vero tentativo di tradurre e commentare l'opera già nel 1838: M. D’Avezac, 
Storia della Nazione degli Arcieri (ca. 1273), il monaco armeno Gregorio di Akner afferma che

come abbiamo appreso da alcuni di loro, queste genti [i Mongoli] vennero dalle loro terre del Turkestan e si spostarono da qualche parte verso Est, vivendo là in estrema povertà per molto tempo come razziatori e selvaggi. ${ }^{15}$

Il generale armeno Hethum, all'inizio del XIV secolo, afferma che

il territorio nel quale primieramente abitarono i Tartari è di là dal gran monte Belgian, del quale è fatta menzione nell' 'istorie di Alessandro. Vivevano i detti in quella regione a guisa di bestie, non avendo né lettere né fede, pascolando i loro armenti di luogo in luogo, dove trovavano i pascoli migliori, né esperienza alcuna aveano nell'arte delle armi $[\ldots]$ come gente rozza da tutti erano stimati e angariziati. Furono anticamente più nazioni di Tartari, i quali comunemente si chiamavano Moglí. ${ }^{16}$

I Mongoli Gengiskhanidi sarebbero i discendenti di un'antica tribù composta da elementi mongoli e tungusi: i She-wei della quale parlano per primi gli Annali cinesi Liao (X secolo). Fra i sottogruppi mongoli che facevano parte della tribù She-wei vi erano i Meng-wu, dai quali deriverebbe l'etnonimo di Mongoli.

Al tramonto del XII secolo l'Europa orientale e l'Asia erano dominate da una straordinaria frammentazione politica e culturale. L'Anatolia era soggetta al sultanato selgiuchide. Nella parte orientale del Ponto si era alla vigilia della costituzione dell'Impero di Trebisonda; il Caucaso era, come abbiamo visto, dominato dalla forte presenza del regno di Georgia, potenza cristiana in una regione marcatamente caratterizzata dall'Islam. Oltre la catena montuosa,

Relation des Mongols ou Tartares par le frère Jean du Plan de Carpine de l'Ordre de Frères Mineurs, Paris 1838. Ricordiamo inoltre l'edizione di Giorgio Pullè, Historia Mongalorum. Viaggio di F. Giovanni da Pian del Carpine ai Tartari, in Firenze 1913, rist. Milano 1929 e 1956², quella di Becquet - Hambis, Jean de Plan Carpin, Histoire des Mongols, traduit et annoté par Dom Jean Becquet et par Louis Hambis, Paris 1965. L'edizione del Wyngaert e quella, citata all'inizio, del Centro italiano di studi sull'alto medioevo di Spoleto con l'edizione in latino a cura di E. Menestò, sono le migliori. Gioverà quindi basarsi su queste ogni qual volta si attingeranno dati dall'opera del francescano di Magione.

${ }^{15} \mathrm{Si}$ è a lungo dibattuto sulla paternità di questa Storia. Sembra oggi appurato che debba ascriversi a Gregorio (Grigor) monaco originario di Akner, in Cilicia. Per le edizioni e il dibattito attorno al testo cfr. R. Blake, R. Frye, History of the Nation of Archers, in "Harvard Journal of Asiatic Studies", 3-4 (1949), pp. 400-444; Id., History of the Nation of the Archers (the Mongols) by Grigor of Akants, Cambridge (MA) 1954. Questa è l'edizione su cui mi sono basato, con l'aggiunta delle correzioni apportate alla medesima dall'armenista Robert Bedrosian, il quale ha fornito a sua volta un'edizione del testo ora disponibile online: Gregor of Akner, History of the Nation of Archers, $<$ http://rbedrosian.com/ga1.htm>.

${ }^{16}$ Hayton l'Armeno, La Storia dei Tartari cit., p. 311. 
frontiera naturale, si trovavano i Polovcy, elemento maggioritario all'interno di un universo etnico complesso. La Transoxiana e parte dell'Iran erano sotto il potere del sultanato della Korazmia, di origine turca, religione musulmana e culturalmente influenzato dalla solida tradizione arabo-persiana. Il califfato di Baghdad, abbaside, costituiva un puntello per l'Islam a sud del Caucaso. Nell'Asia orientale, regione d'origine dei primi gruppi altaici, la situazione politica era più complessa. Il Qara-Kitai, posto fra la regione dell'Issiq-Kul, il Tchu e la Kagaria, era abitato da una popolazione di origine mongola, ma culturalmente cinese. La Cina era sostanzialmente divisa in tre aree: a nord vi era il regno tunguso dei Kin (Chin), con capitale Pechino. A sud il forte impero Song, la cui capitale era Hang-Tche Ou, mentre a nord-ovest, fra l'Ordos e il Kan-Sou, si trovava il regno tangut dello Si-Hia. ${ }^{17}$ Oltre i monti Altaj, nelle zone comprese fra le steppe siberiane e il deserto dei Gobi vi era un autentico crogiuolo etnico costituito da tribù nomadi di origine altaica, ovvero mongola, turca e tungusa. Fra di esse i più numerosi erano i Naiman ${ }^{18}$ una popolazione di Turchi mongolizzati che abitava la regione posta fra l'Irtysh, e le sorgenti dello Yenisey, nell'attuale Mongolia nord-occidentale. L'influenza culturale dei Naiman sull'unione mongola sarà importante poiché, a parte il fattore quantitativo, essi conoscevano la scrittura; lo stesso Bartold affermava che le prime transazioni commerciali mongole di cui abbiamo attestazione scritta sono in lingua uigurica e compaiono dopo il $1206,{ }^{19}$ cioè dopo che i Naiman erano stati sconfitti dai Mongoli di Gengis khan. È infatti noto che i Mongoli non avessero alcuna scrittura prima dell'unificazione. ${ }^{20}$ Inoltre i Naiman erano entrati in contatto

${ }_{17}$ Si veda Grousset, L'empire des steppes cit., pp. 243-244; Bartold, Turkestan cit., p. 381; Di Cosmo, Ancient China cit., pp. 206-253.

18 "I Naiman si vantano fidando sul loro ulus grande e popoloso»; Storia Segreta dei Mongoli, ed. a cura di S. Kozin, M. Osulfieva, Milano 2000, VII, 190, p. 160 (d'ora in avanti SSM); si veda anche Bold, Mongolian Nomadic Society cit., pp. 56 e 82. Il nome Naiman è di origine mongola (naiman = otto); si veda su questo P. Pelliot, La Haute Asie, Paris 1937, p. 28; Grousset, L'Empire des steppes cit., p. 244. Nella Storia Segreta compare il nome del qan dei Naiman, Tayang (da ta-wang, in cinese "Gran Re": Grousset, L'empire des steppes cit., p. 244): SSM, VII, 189, p. 157.

19 Bartold, Turkestan cit., p. 387.

${ }^{20}$ Juvaini, I, p. 44: «[...] poiché i popoli tartari non avevano scrittura propria, ordinò che i bambini dei Mongoli imparassero a scrivere dagli Uighur; e che questi yasa e queste ordinanze fossero scritte su rotoli». Anche Bar Ebreo afferma che: "poiché i Mongoli non hanno una loro scrittura, Gengis Khan aveva istruito i bambini Tatari con la scrittura uigurica. Pertanto il Mongolo è scritto in lettere uiguriche come fanno gli Egiziani coi Greci e i Persiani con gli Arabi». Bar Ebreo era nato da famiglia ebrea a Melitene, sull'Eufrate, attorno al 1225. I suoi studi gli avevano garantito un'ampia conoscenza linguistica. Divenne cristiano monofisita all'età di 17 anni e fu ordinato vescovo della sua città natale nel 1246 circa. Fu poi vescovo di Aleppo e di Sis, in Cilicia. Tale formazione ne fa uno degli osservatori contemporanei più credibili e la sua opera è ricca di particolari interessanti. Vi sono diverse edizioni dell’̀̀opera di Bar Ebreo. Si veda Grigorius Bar Hebraus, Chronicon Syriacum, a cura di Paul Bedjan, Paris 1890, p. 411; cfr. anche B. Spuler, History of the Mongols. Based on Eastern and Western Accounts of the Thirteenth and Fourteenth Centuries, 
col nestorianesimo e ne avevano assimilata in parte l'identità; ciò nonostante non avevano perduto la tradizione sciamanista legata alla loro origine nomade. Ad esempio quando, dopo aver ucciso il Van-khan, leader della tribù dei Kerait da poco sconfitta dai Gengiskhanidi, i Naiman praticarono sul suo corpo uno speciale rito propiziatorio; la madre del khan dei Naiman ordinò che le venisse portata la sua testa:

venne steso per terra un grande pezzo di feltro bianco e dopo avervi posto la testa si compirono sacrifici, si tennero le mani giunte in preghiera mentre le nuore, secondo il rito, cantavano al suono di liuti $[\ldots]$

Tale rito si concluderà col sinistro presagio della sconfitta che di lì a poco $\mathrm{i}$ Naiman avrebbero patito per mano dei Gengiskhanidi del giovane Timujin. ${ }^{21}$ Sembra quindi che la loro identità religiosa fosse blanda e che la tradizione dominasse ancora nei rituali più frequenti. ${ }^{22}$

Quando il sultano di Korazmia iniziò la marcia che lo avrebbe portato fino al Caucaso attirandosi la reazione mongola, compaiono anche i Naiman; ${ }^{23}$ in particolare in occasione dell'occupazione da parte del sultano di Bukhara da dove avanzò fino a Samarcanda. La città cadde nelle mani di Muhammed e di lì a poco si venne allo scontro. Nell'anno dell'egira 607, fra la fine di agosto e l'inizio di settembre, l'esercito del sultano e quello del Qara-Kitai si fronteggiarono nella piana dell'Ilamish. ${ }^{24}$

Fra le altre popolazioni nomadi citate nella Storia Segreta ed entrate a far parte dell'unione Gengiskhanide vi erano i Merkit, abitanti della regione a sud del lago Baijkal. ${ }^{25}$ Nelle fonti vengono spesso distinti in diversi gruppi, ma agiscono sempre come una sola tribù. I Kerait erano, insieme ai Naiman, la tribù numericamente più consistente e militarmente più temibile della regione alla vigilia dell'unifica-

Berkeley (CA) $1972^{2}$, p. 39-40; Bar Hebraeus, The Chronography of Abu'l Faraj (Bar Hebraeus), trad. e cura di A. E. Wallis Budge, London rist. 1976 (ristampato recentemente, Piscataway (NJ) 2003); L. Conrad, On the Arabic Chronicle of Bar Hebraeus: His aims and audience, in "Parole de l'Orient», XIX (1994), pp. 320-378; crf. anche A Syriac Encyclopaedia Of Aristotelian Philosophy: Barhebraeus (13th C.), Butyrum Sapientiae, Books Of Ethics, Economy, And Politics, a cura di N.P. Joosse, Leiden 2004.

${ }^{21}$ SSM, VII, 189, pp. 157-158.

22 Su questo aspetto si veda C. Humphrey, A. Hürelbaatar, Regret as a Political Intervention: An Essay in the Historical Anthropology of the Early Mongols, in "Past and Present», 186 (2005), pp. 3-45.

${ }^{23}$ Per gli eventi si veda Juvaini, II, pp. 387-390 e 401-407; sull'evolversi della situazione politica del Turkestan orientale rimane per certi versi insuperata la monografia di Bartold citata sopra.

${ }^{24}$ Juvaini, II, pp. 387-390, in particolare la p. 389: «I due eserciti si affrontarono e si incontrarono un venerdì del Rabi I 607» (agosto-settembre 1210). Si veda anche il cap. X, II parte, pp. 401-407.

${ }^{25}$ Grousset, L'empire des steppes cit., p. 246; P. Pelliot, A propos des Comans, in "Journal Asiatique», I (1920), p. 145; SSM, II, 102, p. 85. 
zione Mongola. Vivevano lungo il corso del Selenga. ${ }^{26}$ Anch'essi avevano subito l'influenza nestoriana e, rispetto ai Naiman, si sarebbero convertiti già dall'inizio dell'XI secolo. ${ }^{27}$ Furono sconfitti nel 1203 dai Mongoli di Gengis khan. ${ }^{28}$ La popolazione contro la quale si concentrarono gli sforzi maggiori del khan mongolo al fine di sottometterla fu proprio quella dei Tatari. Essi vivevano fra il basso corso del Kerulen e il deserto dei Gobi, nell'odierna Mongolia orientale.

$\mathrm{Si}$ è ritenuto a lungo che i Tatari fossero di origine tungusa e si fossero in seguito mongolizzati. Grazie agli studi di Renè Grousset e Paul Pelliot, per citare i più eminenti specialisti, si è stabilito che i Tatari fossero mongoli o «apparentemente di lingua mongola». ${ }^{29}$ Tribù tatare compaiono già nelle iscrizioni turche del Kocho Tsaïdam dell'VIII secolo, «epoca durante la quale forse abitano già la regione del Basso Kerulen». ${ }^{30}$ In questo periodo i confini fra le terre da pascolo utilizzate dalle diverse popolazioni erano labili e indefiniti; ciò determinava anche la natura dei rapporti fra le medesime tribù. Alla fine del XII secolo l'autorità imposta dai Mongoli sugli altri gruppi ebbe l'effetto di creare aree di influenza più marcate. I Tatari rappresentarono per decenni uno dei principali ostacoli all'ascesa mongola. ${ }^{31}$ Le fonti ne parlano attribuendo loro qualità negative e considerandoli come i nemici principali. ${ }^{32}$

Il mosaico etnico costituito da queste tribù fu unificato da Gengis khan, ma è difficile ancora oggi stabilire se, alla vigilia dell'unificazione, fossero gruppi ben distinti o avessero dato vita a fenomeni di risocializzazione di qualche tipo costituendo nuovi e più grandi insiemi. Alcuni aspetti dei rapporti fra le varie popolazioni ci sono noti grazie alla Storia Segreta, ma sono molte di più le zone d'ombra di questa fase che potremmo definire come protomongola.

${ }^{26}$ Anche su questo si è molto dibattuto, ma non credo sia un problema centrale. Si veda a riguardo Grousset, L'empire des steppes cit., pp. 245-246; Pelliot, L'Haute Asie cit., p. 25.

27 È quanto afferma Bar Ebreo, Spuler, History of the Mongols cit., pp-39-40.

${ }^{28}$ Bartold, Turkestan cit., p. 382. La Storia Segreta narra con dovizia di particolari i fatti inerenti la guerra fra Mongoli e Kerait: SSM, VI, pp. 137-154.

29 Su questi aspetti rimane fondamentale il lavoro di René Grousset (L'empire des steppes cit., p. 246); per un'analisi lucida e più recente si veda il già citato N. Di Cosmo, Ancient China, in particolare il cap. VII, In search of grass and water: Etnography and History of the North in the Historian's Records, pp. 255-293; Id., Warfare in Inner Asian History 500-1800: 500-1800, Leiden 2002.

${ }^{30}$ Grousset, L'empire des steppes cit., pp. 246-247.

${ }^{31}$ Lo scontro dal quale i Tatari usciranno definitivamente sconfitti è narrato in SSM, V, 148-169, pp. 119-136.

32 SSM, IV, 133, pp. 106-107: «Činggis-qan disse: I Tatari sono nostri vecchi nemici. Uccidevano i nostri padri e avi. Quindi dobbiamo attaccarli [...] poi, chiedendo l'aiuto della tribù Č̈rkin, l'ormai Gengis Qan dice: vi invitiamo insieme a noi per annientare i Tatari che da tempi immemorabili furono gli assassini dei nostri avi e padri». Ancora in SSM, V, 153, p. 123: «Uccidemmo i capi Tatari delle stirpi Čaga, Alči, Dutaud, Aluqai». E più avanti dovendo decidere cosa fare dei dirigenti Tatari catturati i generali di Gengis-Qan dissero: «i Tatari furono da tempi immemorabili assassini dei nostri avi e padri. Distruggiamoli fino all'ultimo, e i bambini piccoli rimasti, quelli più bassi dell'assale di un carro, e le donne, facciamo schiavi e disperdiamoli in luoghi diversi». 
La situazione che siamo in grado di studiare meglio, quella determinatasi in seguito alle conquiste, è quindi il frutto anche dell'elemento originario di queste popolazioni. Dell'insieme di queste realtà frammentate e della loro unificazione, unitamente all'interazione con gli elementi assoggettati. Sono aspetti che meritano di essere esaminati con ordine.

\subsection{Etnogenesi e nomadismo: i primi mutamenti}

La totalità delle popolazioni turco-mongole prima dell'unificazione era strutturata sulla base di un'economia prevalentemente nomade, sebbene non sia dato sapere con esattezza quale fase di sviluppo stesse attraversando ognuna di esse singolarmente. Fra la seconda metà del XII secolo e la prima metà del successivo esse vissero un momento di sviluppo che mutò la loro struttura sociale a vantaggio di un'accresciuta stabilità sul territorio e un'economia caratterizzata da un maggiore ricorso allo sfruttamento individuale delle risorse. ${ }^{33}$ Questo avvenne soprattutto nel periodo a cavallo delle grandi conquiste che, iniziate con Gengis khan, proseguirono ininterrotte fino agli ultimi decenni del XIII secolo. Tali popolazioni si stanziarono in aree vicine fra di loro e vaste dando vita a un lungo e complesso processo di unificazione le cui conseguenze si fecero sentire in tutto il mondo allora conosciuto. Partiti dall'Asia orientale i Tataro-mongoli giunsero fino alle porte dell'Europa occidentale fondando quello che le fonti orientali chiamano Ulus Giuči o Orda Azzurra e i Russi Orda d'Oro (Zolotaja Orda).

L'indagine sugli aspetti sociali, politici, economici del processo evolutivo che portò alla formazione dell'impero rimangono ancora oggi patrimonio di una corrente storiografica di nicchia alla quale l'Italia è spesso rimasta impermeabile. ${ }^{34}$ In Russia si è cominciato a studiare il fenomeno con rigore e metodo già nell'Ottocento, ma gli studi prodotti in questo secolo sono spesso rimasti ostaggio delle vicende che hanno caratterizzato ideologicamente il grande Paese slavo fino al

33 Di fatto il nomadismo non è mai stato un modello economico rigidamente autarchico. Anatoly Khazanov ha ampiamente dimostrato che se l'economia nomade presenta caratteri di una forte monovalenza e di un ricorso modesto alla tecnologia più avanzata, essa ha sempre dialogato con modelli sedentari in cui le risorse erano più differenziate e quindi lo erano anche i settori della produzione. Cfr. A.M. Khazanov, Nomads and the outside World cit; N. Di Cosmo, Ancient Asian Nomads cit., pp. 1092-1126.

${ }^{34}$ A dire il vero gli studiosi che si sono occupati di Tataro-mongoli e di Orda d'Oro non sono mancati da noi, ma se negli Stati Uniti vi sono stati e vi sono studi di pregio (in coerenza con la cosiddetta world history e alimentati dall'emigrazione russa dei primi anni Novanta) e in Francia o nel Regno Unito la mongolistica è una disciplina a tutti gli effetti, in Italia le cose stanno diversamente. Si studiano i Mongoli, ma lo fanno soprattutto gli slavisti, i sinologi, i filologi e i linguisti. Pochi gli storici che se ne sono occupati, quasi nessun medievista. Non esistono centri di studio dedicati a questa disciplina. Il fatto è che non sempre si è riusciti a cogliere appieno la portata storica della vicenda tataro-mongola. A questo va aggiunta l'oggettiva difficoltà dovuta alla lingua nella quale certi studi sono scritti e l'egemonia culturale esercitata dal Medioevo occidentale e italiano in particolare. 
definitivo compimento della Rivoluzione del 1917. Anche in Mongolia gli storici si sono dedicati allo studio della società mongola tradizionale. Fortunatamente alcuni fra i migliori contributi sono stati tradotti in russo o, più recentemente, in inglese. ${ }^{35}$ Gli sforzi rivolti alla conoscenza della società tataro-mongola giunsero a un buon livello scientifico già nella prima metà del Novecento e culminarono nel 1934 quando venne pubblicato il libro dell'accademico Boris J. Vladimircov, frutto di un lungo lavoro e sin troppo dotto. ${ }^{36}$ Le teorie su cui Vladimircov basava le sue conclusioni uscivano in parte dalla rigida concezione materialista, pur rimanendo fortemente ancorata alla linea interpretativa marxista. Proprio per questo motivo l'opera ha un valore e suscita un interesse maggiore oggi di quanto non ne riconobbero gli studiosi sovietici fino agli anni ' 80 del secolo scorso (a tale proposito è emblematico il giudizio che ne danno Grekov e Jakubovskij nella loro opera sull'Orda d'Oro). ${ }^{37}$ In ogni caso l'Obščestvennyj Stroj Mongolov è un lavoro in gran parte insuperato e comunque imprescindibile per qualsiasi indagine sulla società tataro-mongola precedente le conquiste di Gengis khan. La storiografia russa sui Mongoli ha inoltre risentito del movimento eurasista, protagonista di un vivace dibattito sin dalla seconda metà dell'Ottocento sull'eterno dilemma della Russia/Europa o Russia/Asia. Tale movimento, che ha avuto fra i suoi più illustri esponenti G. Vernadsky, ${ }^{38}$ ha insistito sul carattere sostanzialmente positivo dell'intervento mongolo sulla storia del mondo slavo cercando di ridimensionare il concetto di Tatarskoe jarmo (giogo tataro). I cosiddetti eurasisti hanno pertanto guardato ai popoli della steppa con occhio critico senza attribuire loro una connotazione distruttiva a tutti i costi. ${ }^{39}$ Non a caso il movimento fu duramente perseguitato dal bolscevismo.

Per comprendere a fondo le modalità delle conquiste mongole fra Asia ed Europa e per poter meglio analizzare l'organizzazione dei territori che essi

${ }_{35}$ N. Ishjamc, Istorija Mongol'skoj Narodnoj Respubliki, Moskva 1954 e n. ed. 1964; S. Nacagdorj, Iz istorii aratskogo dvizenija vo vnešnej mongolij, Moskva 1958; S. Jagchid, P. Hyer, Mongolia's culture and society, Boulder (CO) 1979; N. Jagvaral, Aratstvo i aratskoe chozjaistvo, Ulambaatar 1974; C. Dalai, Mongolija XIII-XIV vv., Moskva 1983.

36 V.Ja. Vladimircov, Obščestvennyj stroj mongolov, Moskva-Leningrad 1934. Il libro fu poi tradotto in francese col titolo di Le régime social des Mongols. Le féodalisme nomade, Paris 1948. L'opera risente della formazione di Vladimircov il quale era soprattutto un linguista, ma tale impostazione non danneggia affatto la qualità dell'indagine.

${ }^{37}$ Grekov- Jakubovskij, L'Orda d'Oro cit. pp. 22-23.

38 Si veda a tal proposito G. Vernadsky, The Mongols and Russia, New Heaven-London 1953; G. Vernadsky, Kievan Russia, New Heaven-London 1948; Id., The scape and content of Chingis Qan's Yasa, «Harvard Journal of Asiatic Studies», 3 (1938), pp. 337-360.

39 Il dibattito sull'Eurasismo sarebbe un capitolo da approfondire in uno spazio apposito. Rimandiamo per questo al saggio di A. Ferrari, La foresta e la steppa. Il mito dell'Eurasia nella cultura russa, Milano 2003. Di fondamentale importanza rimane l'intervento di R. Risaliti a riguardo: Sull'Eurasia e dintorni. A proposito del dibattito russo sui modelli di civiltà, in "Religioni e società", XXXIV (1999), pp. 98-104. 
assoggettarono dal Caucaso al Mar d'Azov penso sia necessario entrare dettagliatamente all'interno della struttura sociale delle popolazioni turco-mongole alla vigilia dell'unificazione operata da Gengis khan e valutarne i caratteri intrinseci in relazione ai loro successivi mutamenti.

Il nomadismo della steppa ha introdotto alcune novità sostanziali nel rideterminare la fisionomia di quella che gli antropologi chiamano società egualitaria..$^{40} \mathrm{La}$ trasformazione dei fondamenti economici collettivi, avvenuta attorno al II millennio a. C., è alla base della configurazione che si realizzò durante il predominio di Gengis khan e dei Mongoli.

Il contesto sociale delle tribù nomadi alla vigilia dell'unificazione era basato sul clan di tipo agnatizio patrilineare, l'oboq o obob; ${ }^{41}$ in esso si praticava l'esogamia ${ }^{42}$ la quale era anche il veicolo principale attraverso cui venivano a crearsi alleanze fra clan. La poligamia era regolare, ma vi era una rigida gerarchia delle mogli. ${ }^{43}$ Dice Juvaini che «secondo le usanze dei Mongoli, il grado dei figli dello stesso padre dipende da quello delle rispettive madri, cosicché il figlio di una moglie più anziana gode della preferenza e del diritto di precedenza». ${ }^{44}$ Juvaini aggiunge che "se sono in corso opere alle quali un uomo deve contribuire col proprio lavoro forzato, e questi è personalmente assente, si presenta la moglie a eseguire il compito in vece sua». ${ }^{45}$ Guglielmo di Rubruck, che fu inviato da Luigi IX di Francia presso i Mongoli nel 1253 per capire, indagare e apprendere il maggior numero di notizie possibile su questo popolo misterioso e inquietante per le potenze occidentali, afferma che, nell'organizzare l'accampamento (orda), la prima moglie aveva la precedenza sulle altre nella disposizione della propria ger, la tenda posta sui carri. ${ }^{46}$ L'oboq viveva su un $u l u s,{ }^{47}$ un territorio astrattamente delimitato che costituiva

${ }^{40}$ Tosi, Dalla tribù all'impero cit., p. 269; Khazanov, Nomads and the outside world, in particolare il capitolo 3, pp. 119-196.

${ }^{41}$ Bold, Mongolian nomadic Society cit., pp. 79-80; Vladimircov, Le régime social cit., pp. 56-73; SSM, I, 9 cit., p. 52 e 28, p. 57; RaD, I, p. 148.

${ }^{42}$ SSM, I, 53, p. 62: «Una volta Ambaqai-qagan andò personalmente ad accompagnare la figlia, che dava in sposa a un Tataro della tribù Airiud Buiruud...»; SSM, I, 61, p. 65: "quando Temučin ebbe nove anni Yesügai-Bagatur decise di trovargli una fidanzata presso gli zii materni...della stirpe di Olqonogud».

${ }^{43}$ Durante la narrazione dell'origine di Timujin la Storia Segreta sottolinea il ruolo preminente della prima moglie: Timujin era figlio di Yesügai-Bagatur e la sua prima moglie (SSM, I, 59, p. 65).

44 Juvaini, I, p. 61.

45 Ibidem, I, p. 50.

${ }^{46}$ Guillelmus de Rubruc, Itinerarium cit., pp. 145-332, pp. 173-174: «Et quando deponunt domus, prima uxor deponit suam curiam in capite occidentali, et postea alie secundum ordinem suum ita quod ultima uxor erit in capite orientali...»; recentemente ripubblicato tradotto Guglielmo di Rubruc, Viaggio nell'impero dei Mongoli, a cura di L. Dalledonne e G. L. Podestà, Genova 2002, II, 4, pp. 15-16.

47 Si veda a tale scopo SSM, II, 73, pp. 70, 82-82 e 96; III, 139, pp. 90, 110 e 106; V, 151, pp. 123, 133-134 e 166. 
l'ambito spaziale entro il quale esso esercitava i propri tradizionali diritti di pascolo, caccia, pesca etc. ${ }^{48} \mathrm{Il}$ carattere fondamentalmente nomade dell'economia mongola portava a concepire l'ulus non tanto come entità spaziale quanto soprattutto demografica; l'ulus era rappresentato, per i Mongoli, soprattutto dagli individui, i nuclei familiari che si trovavano su un determinato territorio piuttosto che dal territorio stesso. ${ }^{49}$

Il prestigio dell'oboq era determinato in gran parte dalla sua origine. Le fonti fanno spesso riferimento agli avi dei Mongoli. Ecco allora che Qabulqagan era sovrano di tutti i Mongoli e dopo la sua morte "governò tutti i Mongoli il figlio del cugino di lui». ${ }^{50} \mathrm{Si}$ è a lungo dibattuto sull'esistenza o meno del concetto di proprietà privata presso le tribù turco-mongole in questo periodo. A mio avviso la proprietà delle terre per il pascolo era un concetto estraneo alla cultura nomade tradizionale, mentre era ben presente il concetto di proprietà per quanto riguarda il bestiame. Per una società organizzata sul nomadismo era assai più importante la mobilità, ovvero la possibilità di andarsi a cercare pascoli migliori quando le condizioni ambientali non consentivano più l'utilizzo di un determinato territorio. Senza questa possibilità era arduo sopravvivere; il possesso della terra era il più delle volte un impedimento. ${ }^{51}$ L'ineguaglianza sociale all'interno di una comunità nomade esiste e può derivare sia dalla proprietà degli animali, ovvero i mezzi della produzione, sia dall'acquisizione di una posizione egemone all'interno di un clan o di una tribù, cioè la gestione della produzione..$^{52}$ Tuttavia le società nomadi tendono all'uguaglianza e la differenziazione delle risorse, o della loro gestione, può essere motivo di scollamento di uno o più individui dalla comunità; uno dei bisogni fondamentali di un'organizzazione mobile è l'integrità della comunità medesima. L'eccessiva povertà di un individuo o di un clan può destabilizzare questa situazione spingendo verso l'abbandono chi non ha più possibilità di sopravvivenza. La redistribuzione della ricchezza era dunque una delle forme principali di livellamento sociale per cui ogni elemento poteva beneficiare del frutto delle razzie o delle altre fasi del processo produttivo. Naturalmente anche la redistribuzione della ricchezza poteva essere, e spesso lo era, diseguale. Le

48 Vernadsky ha tradotto ulus come "stato" (Vernadsky, The Mongols and Russia cit., p. 123). Tale concetto rende più o meno l'idea, ma non bisogna immaginare l'ulus come un territorio ben definito e sul quale un oboq agiva imponendovi la propria autorità normatizzata. Era un "possesso" sui generis.

49 Per uno sguardo d'insieme si veda l'ottimo e già citato studio di Bet Ochir-Bold Mongolian Nomadic Society, in particolare i capp. 2-4, pp. 25-129.

${ }_{50}$ SSM, I, 52, p. 62; anche Rashid ad-Din dice che gli antenati di Timujin sono quelli di tutti i Mongoli (RaD, II, p. 69); Vladimircov, Le régime social cit., p. 59.

${ }^{51}$ Su questa opinione si veda anche Ochir-Bold, Mongolian nomadic Society cit., p. 49; F. Scholz, Nomadismus. Teorie und Wandel einer sozio-okologischen Kulturweise, Stuttgart 1995, pp. 25-27.

52 Cfr. Khazanov, Nomads and the Outside World cit., p. 152. 
differenze sociali erano il più delle volte temporanee, ma c'erano. Potremmo dire che la società nomade di questi anni tendeva all'eguaglianza senza tuttavia raggiungerla. Detto questo dobbiamo anche aggiungere che la stratificazione si stabilizza difficilmente col ricorso esclusivo a elementi interni al nomadismo. Più spesso è proprio l'interazione con l'esterno, col mondo sedentarizzato, che consolida le differenze. È quello che accadrà con la formazione dell'impero mongolo. Ad esempio la rigida gerarchia con la quale i beni paterni venivano distribuiti fra i figli è un esempio di come le cose tendano al cambiamento già dopo l'unificazione. Come abbiamo detto quelli nati dalla prima moglie godevano di una posizione di privilegio rispetto agli altri. Fra quelli nati dalla stessa moglie il figlio primogenito ereditava i beni principali del padre. Generalmente veniva mandato a controllare i pascoli più lontani, i figli minori rimanevano sui territori più vicini, mentre il più giovane diveniva guardiano del focolare. ${ }^{53}$ Secondo Vladimircov il figlio cadetto riceveva lo yurt del padre e delle sue mogli diventando äžän cioè maître, propriétaire.$^{54}$ Su questa figura è più chiaro Rashid ad-Din: «l'usanza dei Mongoli vuole che il cadetto dei figli si chiami Ažän; poiché egli resta in casa, i beni, gli affari e l'economia domestica spettano a lui». ${ }^{55}$ Lo yurt (parola turca che ha il suo corrispondente mongolo in nutunq/nutuq) era il territorio sul quale un oboq, o parti di esso, aveva deciso di stabilirsi per periodi più o meno lunghi. ${ }^{56}$ Rashid ad-Din ne parla in più di un'occasione come del territorio sul quale si accampano i clan e lo descrive come un ambito spaziale impermeabile alle infiltrazioni esterne. ${ }^{57}$ Il concetto di nutug assume grande importanza per l'organizzazione produttiva degli oboq. Esso è, oltre alla residenza dei nuclei familiari, il pascolo e costituisce «il prerequisito naturale per l'economia nomade». ${ }^{58}$

Di grande importanza era la figura dell'anda. Un anda o "fratello giurato" era un elemento molto importante per la coesione sociale fra appartenenti a oboq diversi. Timujin e Žamuka divennero anda quando avevano appena undici anni. ${ }^{59}$ E probabile che tale legame avesse radici profonde nella tradizione delle tribù turco-mongole. ${ }^{60}$ Lo status di anda era trasmissibile in modo volontario.

53 SSM, I, 54, p. 63: Yesügai Bagatur, il padre mitico di Timujin «tornò in fretta a casa e riportò con sé due fratelli, il maggiore Nekün e il minore Maritai, guardiano del focolare». Anche in $\mathrm{RaD}$, II, p. 30: «Ažän significa figlio cadetto, colui che resta in casa e nella yurt; cioè il maestro del fuoco della yurt».

54 Vladimircov, Le régime social cit., p. 67.

${ }_{55} \mathrm{RaD}, \mathrm{II}$, p. 60.

56 SSM, II, 85, p. 77; II, 93, p. 81; III, 215, p. 89; Bold, Mongolian nomadic Society cit., pp. 36-37 e 41-42; Vladimircov, Le régime social cit., p. 69.

${ }_{57} \mathrm{RaD}, \mathrm{I}, \mathrm{pp} .49,79,87,93$; si veda anche Vladimircov, Le régime social cit., p. 71.

58 Bold, Mongolian nomadic Society cit., p. 41.

59 SSM, III, 116, p. 95.

60 «Avevano sentito dire agli anziani che la legge del gemellaggio impone agli anda, fratelli giurati, di essere come un'anima sola» SSM, III, 117, p. 95. 
«Una volta il Van-khan si fece anda, fratello giurato, di mio padre Yesügei. Chi è anda di mio padre è come se fosse padre mio». ${ }^{61}$ Secondo Rashid ad-Din il Van-khan divenne padre adottivo di Timujin poiché era stato anda di suo padre ${ }^{62}$ e il Van-khan chiamava Timujin figlio per la stessa ragione. ${ }^{63}$ Era un legame molto forte che rendeva l'amicizia duratura, ma non era raro che tale vincolo si spezzasse. La forza che caratterizzava il legame fra due anda è testimoniata anche da un altro passo della Storia Segreta nel quale Žamuka, stimolato dal Van-khan dei Kerait che vuole attaccare i Mongoli di Timujin dice: «Io non posso combattere contro un anda [in questo caso si riferisce a Timujin] e lui [il Van-khan] mi comanda di dirigere l'esercito». ${ }^{64}$

Fra le diverse tribù turco-mongole alla vigilia dell'unificazione una pratica assai diffusa era la vendetta. ${ }^{65} \mathrm{Si}$ trattava di un dovere trasmissibile. Ambaqaiqagan, mongolo, dopo aver subito violenza dai Tatari «mandò a dire al mediano dei sette figli $[\ldots]$ affinché lui a sua volta lo trasmettesse a [...] uno dei propri dieci figli, quanto segue: vendicate me [...] come sovrano di tutti i Mongoli. Vendicatemi e continuate a far vendetta fino a che non avrete perduto le unghie [...]». ${ }^{66}$ Ancora: "Qutula-qagan e Qadagan-Tayži [...] andarono a vendicarsi del popolo tataro. Essi si batterono tredici volte senza riuscire a vendicarsi e riparare l'offesa». ${ }^{67}$ Il padre di Timujin Yesügai Bagatur incontra dei Tatari. Questi tentano di avvelenarlo, allora Yesügai dice: «mi hanno avvelenato i Tatari di nascosto» ${ }^{68}$ non a caso, come abbiamo visto sopra, i rapporti fra Tatari e Mongoli saranno caratterizzati da inimicizia e conflitti. ${ }^{69}$ Ancora, in occasione della guerra contro i Merkit, Žamuka dice: «Nel fare vendetta distruggeremo i Merkit Uduid e Uwas». Così anche i capi Merkit: volendo vendicare il rapimento di una donna (Höelün, la madre di Timujin) da parte di Yesügai-Bagatur, intrapresero l'incursione che era culminata nel rapimento della sposa promessa

${ }^{61}$ SSM, II, 96, pp. 82-83. Tale affermazione è confermata in SSM, III, 104, p. 87.

${ }_{62} \mathrm{RaD}$, II, p. 106.

${ }^{63} \mathrm{RaD}$, II, pp. 135-140.

64 SSM, VI, 170, p. 138; e più avanti l'ormai Gengis khan dice a Žamuka: «Per quanto divergessero le nostre vie, tu sei sempre stato il mio sacro fedele amico» (SSM, 200, p. 175). Alla fine però il rapporto fra i due si spezzerà e Žamuka verrà ucciso dai Mongoli per aver tradito il suo anda Timujin.

65 Secondo Vladimircov (Le Régime social cit., pp. 65-66) tale prassi era ormai in disuso nei primi anni del XIII secolo, ma nella Storia Segreta si trovano molti esempi di come ancora vi si facesse ampiamente ricorso.

${ }^{66}$ SSM, I, 53, p. 62.

${ }^{67}$ Ibidem, I, 58, p. 65.

68 Ibidem, I, 67, p. 67.

${ }^{69}$ In Ibidem, III, 133, p. 106. Timujin, oramai eletto Gengis Khan, dice: «i Tatari sono nostri vecchi nemici. Uccidevano i nostri padri e avi. Quindi dobbiamo attaccarli». E più avanti, Ibidem, III, 133, p. 107: i Tatari [...] da tempi immemorabili furono gli assassini dei nostri avi e padri». 
del futuro Gengis khan, Börte. ${ }^{70}$ Altri esempi si trovano nella Storia Segreta e in Rashid ad-Din. ${ }^{71}$

L'oboq era un'unità in continuo movimento. Le migrazioni individuali diventeranno frequenti solo in un periodo successivo. ${ }^{72} \mathrm{La}$ transumanza imponeva continui spostamenti, ma la mobilità non era solo quella dei carri; vi era una sensibile tendenza all'interscambio fra clan. I gruppi familiari vivevano su terreni spesso contigui fra di loro e le relazioni fra oboq erano assai flessibili. Non era raro il caso che in uno di questi potessero entrare a far parte elementi esterni. Talvolta a un oboq numeroso e in grado di assicurare protezione contro gli attacchi esterni si aggiungevano interi gruppi di oboq o tribù (irgän). Dopo aver sconfitto i Merkit (probabilmente nell'ultimo decennio del XII secolo), Timujin decide di spostare il suo ayil. L'ayil viene definito dalle fonti come un modello organizzativo che in molti hanno visto come l'evoluzione individualistica del nomadismo mongolo. Sulle caratteristiche dell'ayil e sulle sue dinamiche interne mi soffermerò oltre. Si trattava, in ogni caso, di un piccolo gruppo di carri, una famiglia nomade, un accampamento di dimensioni ridotte contrapposto alle notevoli dimensioni del kuren. La Storia Segreta elenca una serie di nomi di individui che, pur appartenendo a tribù diverse, si uniscono ai Mongoli. In totale entrano a far parte dei Mongoli di Timujin individui da 19 diverse tribù e un intero kuren. ${ }^{73}$ Un simile flusso di persone si verifica ancora poco dopo: «Passarono dalla parte di Timujin altri ancora». ${ }^{74}$ L'afflusso di questi elementi "esterni" è emblematico del movimento di genti che si verificava fra oboq e, spesso, fra intere irgän. Quando parliamo di impermeabilità del clan, a mio avviso, ci si deve riferire soprattutto alla scarsa apertura della famiglia; il vincolo di sangue era fondamentale. Tant'è vero che i consanguinei o coloro che avevano comunque antenati in comune vengono chiamati dalle fonti uruq mentre esiste un termine diverso per coloro i quali sono entrati a far parte dell'oboq pur non avendo con questo alcun legame di sangue: questi sono gli $\check{z} a t .{ }^{75} \mathrm{Va}$ aggiunta, a questo riguardo, un'altra figura; uno žat poteva diventare parente per alleanza; era questo il caso di un rapporto alla base del quale non vi era alcun legame di sangue, ma che si saldava tramite un patto stipulato fra due o più individui sulla parola; era in genere la conseguenza di unioni matrimoniali. In questo caso la sfumatura è resa dalle fonti con un altro termine, quda. ${ }^{76}$

70 SSM, III, 111, p. 92.

71 Ibidem, II, 76, pp. 71-72, 73; 102, p. 85 e 136, p. 108; RaD, I, p. 52 e 53.

72 Ochir-Bold, Mongolian nomadic Society cit., pp. 61-63; Khazanov, Nomads and the outside world cit., pp. 37 e segg.

73 SSM, III, 120, p. 97-98. Il kuren era il sistema organizzativo che coinvolgeva più famiglie. Ci tornerò sopra più avanti.

74 Ibidem, III, 121, p. 99. È in questo caso che Timujin viene nominato qan dei Mongoli. Un insieme già eterogeneo di elementi appartenenti a tribù diverse.

75 Vladimircov, Le régime social cit., pp. 73-74.

76 Ibidem, p. 58 e 74; RaD, I, p. 87, 157, 175. 
Una prassi consolidata presso le popolazioni nomadi delle steppe eurasiatiche consisteva nel trasformare i prigionieri di guerra, risparmiati all'esecuzione seduta stante, in servi o schiavi. ${ }^{77}$

Il clan era dunque un soggetto preciso: «Ciascuno dei loro rami è ben definito, designato da un nome o un nomignolo, e diviene un clan». ${ }^{78}$ Esso era tuttavia soggetto a processi di frantumazione, disgregazione e ricomposizione molto frequenti. Come abbiamo osservato la società mongola sul finire del XII secolo era un universo dinamico, in continuo movimento, un magma elastico i cui sviluppi non sono sempre facilmente individuabili. Di fatto la lotta per l'indipendenza dei clan più piccoli contro quelli più forti e numerosi, la corsa all'accaparramento dei pascoli migliori, le continue guerre fra tribù, la ricerca di clan più forti per potersi meglio difendere, e non ultime le annessioni coercitive da parte di clan particolarmente aggressivi e ricchi, erano sviluppi esterni che costituivano alcune delle cause che generavano una scarsa stabilità sociale. La disuguaglianza sociale all'interno degli oboq e soprattutto fra oboq diversi era causa e motivo del rafforzamento di personaggi (di solito pastori) che acquisivano, incrementandole, nuove ricchezze a svantaggio di altri che, già deboli, piombavano inesorabilmente in una condizione di ulteriore precarietà. La nascita di un ceto dominante all'interno della società mongola affonda le sue radici ben prima che le nostre fonti inizino a parlare. Quando noi possiamo osservare le dinamiche interne a tale organismo vi sono già elementi eminenti e gruppi impoveriti che ne subiscono l'autorità. I personaggi che ben presto allargheranno il loro potere su interi ulus vengono chiamati dalle fonti noyon.$^{79}$ Essi sono anche il risultato della lotta vittoriosa sul terreno della produzione nomade in un contesto economico di generale debolezza, ${ }^{80}$ ma anche - nota giustamente Vladimircov - il risultato di un' usurpazione, dell'acquisizione di un'autorità nuova con la forza a svantaggio dell'ormai in disuso modello di kuren secondo il quale era l'anzianità a determinare l'autorità e il potere sulla collettività. ${ }^{81}$ Il tema della lotta di classe, caro alla storiografia marxista, rimane a mio avviso centrale nell'indagine delle dinamiche sociali interne che portarono alla stratificazione delle comunità e all'emergenza di ceti dominanti. Detto questo dobbiamo tuttavia sottolineare ancora come l'economia produttivo-alimentare

77 Vedremo più avanti se e in quale misura si può parlare di schiavitù per i Mongoli.

${ }^{78} \mathrm{RaD}, \mathrm{I}, \mathrm{p} .136$.

79 Ad esempio in SSM, I, 51, 62: «Qulan Bagatu ebbe per figlio Yeke Čere. Fu lui il noyon di Badai $[\ldots] »$ 26-27.

${ }^{80}$ Vladimircov, Le régime social cit., pp. 93-94 ; Grekov, Jakubovskij, L’Orda d'Oro cit., pp.

${ }^{81}$ Vladimircov (Le régime social cit., p. 94) traduce il termine noyon con 'maitre', mentre Pelliot (P. Pelliot, Les Mongols et la Papauté, in "Revue de l'Orient Chrétien», XXIV (1924), pp. 225-335, p. 306) con quello di "signeurs". Il principio tradizionale della leadership basata sull'anzianità risulta decisamente indebolito nel processo di costruzione dell'unità voluto da Gengis Khan. Cfr. C. Humphrey, A. Hürelbaatar, Regret as a Political Intervention cit., pp. 22-23. 
nomade di questi anni era modesta e strumentale alla sussistenza delle comunità $\mathrm{e}$ a ciò il più delle volte insufficiente. Un fenomeno sostanzialmente privato $\mathrm{e}$ non destinato allo scambio che, pur non assente, aveva dimensioni e caratteri del tutto diversi da quello che siamo soliti intendere per le società sedentarizzate dell'Occidente europeo. ${ }^{82}$

\subsection{Alla base dell'unificazione}

Quando Timujin dette inizio all'assoggettamento delle tribù turco-mongole la struttura sociale delle stesse stava vivendo probabilmente una fase di cambiamento. Rashid ad-Din e la Storia Segreta mostrano come la vecchia forma organizzativa del Kuren fosse entrata in crisi a vantaggio di una maggiore tendenza allo sfruttamento individuale delle risorse tradizionali (caccia, pesca, allevamento e prodotti da esso derivati) contemporaneamente all'emergere del ceto dirigente costituito da quella che alcuni studiosi hanno chiamato "aristocrazia nomade" (la qual cosa presuppone l'utilizzo di categorie interpretative di tipo occidentale e sulla quale gioverà soffermarsi) ovvero i noyon di cui sopra.

La base dell'organizzazione sociale delle popolazioni considerate, alla metà del XII secolo, era quindi il Kurigen o Kuren. Tale modello organizzativo tradizionale era tipico delle tribù nomadi che abitavano l'Asia centro-orientale e in base a esso le famiglie che erano a capo della comunità disponevano le loro tende in cerchio (Kuren significa letteralmente "anello"). ${ }^{83}$ Le famiglie del Kuren si stabilivano per periodi più o meno lunghi su un determinato territorio e la loro economia si fondava sulle risorse tipiche di una produzione poco diversificata: pastorizia, caccia, produzione di latte e di formaggio. La transumanza e la migrazione di una parte della società erano aspetti centrali dell'attività di queste popolazioni.$^{84} \mathrm{Al}$ centro dell'anello formato dalle tende stava l'abitazione del più anziano membro della comunità.

Dopo essersi dato all'inseguimento di briganti che gli avevano rubato otto cavalli giallo-fulvi Timujin e un compagno che aveva trovato per strada si avvicinarono «alle tende di una certa tribù e là videro gli otto cavalli fulvi che pascolavano al margine di quel gran kuren». ${ }^{85}$ Più avanti la Storia Segreta mostra che, quando Žamuka, anda di Timujin, gli dichiara guerra egli aveva riunito attorno a sé tredici tribù con le quali aveva formato tre tümen $;^{86}$ subito dopo

82 Ochir-Bold, Mongolian nomadic Society cit., pp. 22-24.

${ }^{83}$ La parola mongola Chureen (russo Курень, Kuren') acquisisce il significato di "cerchio".

${ }^{84}$ Da notare come, in occasione della vittoria dei Mongoli sui Merkit e la conseguente elezione di Timujin a qan, nei primi anni del XIII secolo (1203), questi avverta l'esigenza di nominare un suo fedele come custode dei carri da transumanza (SSM, III, 124, p. 100).

${ }^{85}$ SSM, I, 90, pp. 79-80.

${ }^{86}$ Il tümen era l'unità militare tipica delle popolazioni nomadi della steppa. Esso era costituito $\mathrm{da}$ diecimila uomini. Anche su questo concetto e le sue caratteristiche torneremo con più precisione in seguito, quando ci occuperemo dell'organizzazione militare dei Mongoli. 
aggiunge che «anche Činggis-qan disponeva di tredici kuren e con queste formò tre tümen...». ${ }^{87}$ Dall' uso da parte del cronista di "anche" sembrerebbe che tribù e kuren in questo momento (all'inizio del XIII secolo) si equivalessero, che avessero lo stesso significato. Per parte mia ritengo che non fosse così. Il kuren non è mai indicato esplicitamente col significato di tribù. Come abbiamo già sottolineato la tribù, irgän, era formata da un gruppo di individui fra loro associati, mentre il kuren era un modello associativo, un modo di accamparsi con le tende e coi carri, un sistema produttivo che prevedeva il concorso di tutti gli appartenenti al nucleo sociale. Giustamente Bet-Ochir Bold ${ }^{88}$ afferma che il kuren fosse non solo un modello organizzativo di tipo socio-economico, ma anche un sistema difensivo-militare contro le aggressioni esterne. Si trattava in sostanza di una necessità, vista la straordinaria precarietà cui il nomadismo centro-asiatico doveva far fronte in questi anni. È molto probabile che il kuren, un accampamento di grandi dimensioni, venisse identificato con la tribù per consuetudine. I Mongoli credo sapessero bene quale fosse la differenza fra i due concetti, ma tendevano a identificarli per distinguerli dal modello più in uso al tempo in cui la Storia Segreta fu scritta: l'ayil. Tant'è vero che, oltre all'esempio citato sopra, la fonte cita un altro passo incerto; dice che «I vecchi e i bambini di un kuren di Činggis-qan, detto Aurug [...] di quella gente lasciata ad Aurug [...]», ovvero al kuren si attribuiva un nome. Sembra che esso acquisisse la funzione di villaggio, di insediamento provvisorio comunque ben distinto dalla tribù che vi abitava. ${ }^{89}$

Non possiamo ancora oggi collocare esattamente il momento in cui il modello del kuren entrò in crisi a vantaggio dell'ayil. Di sicuro la transizione fu lenta e non riguardò tutte le tribù della regione in modo uniforme. Quando l'esercito di Timujin mosse verso occidente, il kuren era praticamente del tutto scomparso. I riferimenti al kuren infatti si ripetono nella Storia Segreta $a^{90}$, ma già in occasione dello scontro dei Mongoli coi Merkit, la cronaca cita l'ayil. ${ }^{91}$

${ }^{87}$ SSM, III, 129, p. 104

${ }^{88}$ Bold, Mongolian nomadic Society cit., pp. 61-62.

89 Ancora nella Storia Segreta (SSM, V, 151, p. 123) è scritto che Činggis-qan, vedendo il Van-qan (ovvero il sovrano dei Kerait) «del tutto esausto dalla fame [...] lo introdusse nel proprio kuren e lo mantenne a sue spese». Insomma, gli esempi in cui irgän e kuren sono ben distinti, nelle fonti, non mancano e indicano che tale differenza fosse ben marcata per i Mongoli stessi. Sulla liceità di utilizzare il termine di tribù per contesti estranei a quelli africani si veda quanto afferma Nicola di Cosmo; State formation and Periodization cit., p. 18, nota 60.

90 Dopo la sconfitta dei Merkit molte tribù si unirono ai Mongoli. La Storia Segreta, III, 119120, ne cita i nomi. Ancora in SSM, III, 120, p. 98: «Inoltre vennero anche con un kuren quelli di Baarin...». E più avanti, SSM, III, 122, p. 99: "passarono dalla parte di Timujin altri ancora: un kuren di Geniges, Qunan ed altri, con un kuren Daritai-Očigin, un kuren di Unčin-Saqaid...con unkuren Sača-Beki». Indipendentemente dai nomi, ai quali è difficile stare dietro, si nota come, nel momento delle prime unificazioni, quasi tutte le tribù turco-mongole vivevano su kuren. Ancora in SSM, IV, 136, p. 108: «I vecchi e i bambini di un kuren di Činggis-qan, detto Aurug...»

${ }_{91}$ SSM, III, 112, p. 93: "Qatai-Darmala [uno dei capi Merkit] fu catturato...gli misero un collare di legno, e lo portarono sul monte Burqan. Indicarono a Belgütai l'ayil in cui si trovava sua madre ed egli andò a cercarla». 
Il nodo che mi pare sia ancora ben stretto, circa i mutamenti sociali in seno alla struttura delle tribù di questo periodo, resta la modalità con la quale si verifica la successione dei due modelli. Da questo punto di vista ha contribuito senza dubbio il mutamento delle basi economiche collettive e la loro organizzazione. La discendenza diventa un elemento preminente e insieme ad essa la capacità da parte di singoli individui di acquisire autorità attraverso due vie: una astratta (la sfera sacra, lo sciamanesimo) ed una ben più concreta (il controllo sulle risorse, pascoli e bestiame soprattutto).$^{92}$ Questi individui sono coloro che organizzano la produzione, le migrazioni e sono quelli che ridistribuiscono il bottino delle razzie. Nell'ambito delle tribù di cui ci stiamo occupando, fra la metà del XII secolo e il successivo l'insieme delle popolazioni turco-mongole, dedite al nomadismo e organizzate sul kuren cominciano a possedere beni, a riunirsi attorno a figure carismatiche e a sfruttare le risorse disponibili attraverso gruppi più esigui spostandosi lentamente verso un maggiore sfruttamento individuale delle risorse, quello che le fonti chiamano ayil $; ;^{33}$ nuclei familiari più piccoli in cui la produzione perde in parte la sua dimensione collettiva a vantaggio di un maggiore individualismo. ${ }^{94}$ Tale fenomeno fu conseguenza delle conquiste di Gengis khan, quindi dei vasti territori che si avevano ora a disposizione o piuttosto tale mutamento era già in atto fra i gruppi etnici dominanti e in generale entro il vasto magma umano che componeva il complesso di queste popolazioni fra XII e XIII secolo? In realtà l'opera di Gengis khan rappresentò un forte scossone sia interno sia esterno. L'unificazione avviò un lento processo che portò a delle modificazioni in seno alla struttura sociale e di conseguenza dell'esercito. Va inoltre considerato il fatto che le conquiste creavano bottino, ricchezza da distribuire e, contemporaneamente, un incremento dell'autorità di chi era in grado di gestire questo surplus. La stratificazione sociale diviene ancora più marcata quando queste popolazioni si troveranno coinvolte in un sistema a loro sostanzialmente estraneo, quello dello scambio, del commercio e quindi della ricchezza mobile..$^{95}$ Pur con qualche riserva ci sentiamo di sottoscrivere quello che affermava, ormai qualche anno fa, Owen Lattimore, cioè che il modello nomade entra in crisi, paradossalmente, quando comincia a vincere. Ovvero la sedentarizzazione è fonte di debolezza per una società tradizionalmente nomade. ${ }^{96}$

${ }_{92}$ Tosi, Dalla tribù allimpero cit., p. 262.

${ }^{93}$ L'aiyl era la base militare ed economica della società tataro-mongola all'indomani delle conquiste di Gengis qan. Si trattava di una ripartizione territoriale che potremmo definire, con qualche riserva, feudale. Naturalmente il termine va inteso in senso generale, senza assimilazioni rigide ai modelli europei.

${ }_{94}$ Proprio questo elemento, lo sfruttamento collettivo delle risorse, è una delle caratteristiche fondamentali del kuren. Si veda in proposito anche la voce della Sovetskaja Istoričeskaja Enciclopedija, Moskva 1965, vol. VIII, col. 311.

${ }_{95}$ Tosi, Dalla tribù allimpero cit., p. 270.

${ }_{96}$ O. Lattimore, The Geographical Factor in Mongol History, in «The Geographical Journal», LXXXXI (1938), 1, pp. 1-16. 
Ritengo che il passaggio dal kuren all'ayil si sia verificato non già quando tribù importanti, per numero di individui e retroterra culturale, vennero sconfitte e assoggettate dai Mongoli di Timujin (si pensi ai Naiman, Merkit, Kerait, Tatari etc.), ma prima. Stando agli esempi riportati nella Storia Segreta, in Rashid ad-Din e in Juvaini e grazie molti studi, il kuren era un modello generico, svuotato del suo significato sociale, alla fine del XII secolo. Esso esisteva, ma era entrato in una fase di crisi definitiva, per cui sempre più elementi (in particolare i noyon, ma anche personaggi ricchi appartenenti ai bogo $P^{7}$ favoriti da noyon eminenti) si staccavano dal grande anello, per costituire nuclei mobili, più scarsi quantitativamente, con una maggiore propensione allo sfruttamento individuale delle risorse. Questo dette vita a una rapida disgregazione per cui gran parte degli equilibri sociali vennero a mutare. L'antica struttura della tribù nomade andava scomponendosi in parti più piccole. I noyon vennero a staccarsi dalla base comunitaria cui appartenevano. L’oboq veniva così a inglobare altri oboq. I vincitori assoggettavano i vinti dando origine a due fenomeni solo apparentemente diversi: il forte aumento dei vincoli di dipendenza (bogol) e il rafforzamento del noyonato, ovvero di quei personaggi eminenti il cui prestigio verrà definitivamente e formalmente riconosciuto da Gengis khan. I piccoli possessori di bestiame ( $q a$ araču$_{\text {) }}$ da parte loro mantennero la propria condizione di piccoli proprietari strettamente legati al possesso del bestiame e dei prodotti che potevano trarre da esso, ma in un contesto di crescente precarietà $\mathrm{o}$, potremmo dire, mobilità. Cambiavano in sostanza referente con rapidità e non riuscivano a raggiungere una stabilità economica tale da potersi permettere di rimanere da soli. La costante situazione di conflitto in cui vivevano le popolazioni turco-mongole generava insicurezza e spingeva le classi più deboli verso i noyon più influenti e meglio organizzati militarmente, quindi anche i più ricchi. L'affermazione di quella che Vladimircov ha definito "aristocrazia della steppa" e il parallelo assoggettamento a essa dei ceti più deboli, era già un risultato quando, nel 1206, Timujin poté completare l'unificazione delle tribù turco-mongole e avviare le conquiste, non già una fase transitoria; con le conquiste aumenta però in modo sostanziale la differenziazione sociale e quella che possiamo a buon diritto definire come società egualitaria diventa, di fatto, un modello dell'ineguaglianza. Tale dinamica ha suggerito un accostamento della struttura sociale mongola di questo periodo a certe manifestazioni feudali dell'Europa occidentale.

\subsection{I mutamenti successivi: è lecito parlare di "feudalesimo nomade"?}

È lecito definire la società mongola di questi anni come "feudale" o si tratta di una forzatura? Se ciò è possibile, quali sono le caratteristiche di questo modello sociale che rendono attuabile un simile paragone? Per coerenza concettuale

${ }^{97}$ Una categoria che in questa sede definiamo, per semplificare, come schiavi. Vedremo più avanti che in realtà la definizione non è del tutto appropriata. 
avremmo dovuto seguire un percorso espositivo che ignorasse categorie teoriche proprie di ambiti sociali, politici ed economici distanti da quello in oggetto, ma poiché si è a lungo - e inevitabilmente verrebbe da dire - tirato in ballo il concetto di feudalesimo nomade allora penso valga la pena soffermarsi. Le definizioni attorno a questo termine per quanto riguarda l'Europa occidentale sono state moltissime e notevole è la bibliografia a riguardo. Basterà citare le opere che hanno posto le basi per la riflessione attuale. Marc Bloch intitolò il suo libro La società feudale, ponendo l'accento sull'aspetto sociale e sull'errata concezione del termine come esclusivamente giuridico. D'altra parte François Louis Ganshoff scrisse più o meno il contrario. ${ }^{98}$ Quel che appare dalla lettura delle due opere è che entrambe abbiano punti di forza e fondano la loro analisi su basi solide. Se esiste un diritto feudale sarà difficile che esso si esprima in una società regolata da dinamiche di tipo diverso. Il diritto è espressione anche degli sviluppi sociali, pertanto i due punti sembrano strettamente legati. È casomai difficile stabilire se l'uno è il frutto dell'altro e casomai in quale ordine. Riproporre in questa sede il lungo dibattito sull'esistenza di uno o più feudalesimi (della cosiddetta mutazione feudale, quindi di periodi distinti in cui il fenomeno si sarebbe manifestato in forme diverse) sarebbe difficile e ci porterebbe lontano dal tema che abbiamo scelto di trattare. ${ }^{9}$ Per una semplificazione che ben si attaglia alle esigenze della nostra esposizione possiamo tuttavia rifarci a quanto hanno scritto in un recente e agile saggio Luigi Provero e Giuseppe Albertoni i quali hanno fornito una definizione che, propria della realtà italiana (ovviamente si parla dell'Italia centro-settentrionale), può costituire un buon mezzo comparativo per il contesto in esame. Nelle pagine introduttive si legge:

Parlare di feudalesimo nell'Italia medievale, quindi non significa affermare che la società medievale italiana fosse feudale. Significa piuttosto analizzare una delle molte componenti della società e dell'organizzazione dei poteri medievali [...]. Questa componente era costituita essenzialmente dalle clientele armate, ovvero dal seguito dei guerrieri che giuravano fedeltà a un signore». Più avanti gli autori aggiungono che caratteri dominanti di questa componente erano «la dominazione dell'aristocrazia militare sulla società; il fondamento militare del potere regio; il consolidamento di queste basi militari tramite un sistema di raccordi di fedeltà e di lealtà; [...] l'uso della terra come strumento di ricompensa [...]. Per definire questo sistema non vediamo nome migliore di "feudalesimo". ${ }^{100}$

98 F.L. Ganshoff, Che cos’è il feudalesimo?, Torino 1982-2003³.

99 Per un'analisi comparativa, ben documentata e con una ricca bibliografia, si rimanda all'opera di E. Bournazel, J.P. Poly, Les féodalités. Histoire générale des sysèmes politiques, Paris 1998, in particolare il libro secondo: Féodalité: universalité du concept, pp. 513-750.

100 G. Albertoni, L. Provero, Il feudalesimo in Italia, Roma 2003, p. 9. 
Da questo si notano almeno due cose: feudalesimo è un' etichetta alla quale si possono attribuire diversi significati e su questo la storiografia del Medioevo ha versato fiumi di inchiostro. Per certe aree dell'Europa occidentale quanto affermato dagli autori citati è corretto. Ma, ed è il secondo aspetto, provando a svuotare il termine dei significati che siamo soliti attribuirgli in base alla nostra esperienza, che cosa possiamo osservare per la realtà mongola di fine XII secolo? Sin dall'Ottocento molti studiosi si sono occupati di questo aspetto e del concetto di feudalesimo nomade. Negli anni Venti, in Unione Sovietica, si sono moltiplicati i tentativi di far entrare la società mongola di fine XII secolo dentro alla definizione di feudale, ponendo l'accento sull'aspetto produttivo. ${ }^{101}$ La Rivoluzione d'Ottobre era cosa recente e gli studi di Weber sull'ordine sociale pure; l'influenza di questi due fattori sul pensiero storico russo fu notevole. Le fasi dello sviluppo storico in senso socialista furono applicate a contesti diversi provocando spesso interpretazioni inesatte, ma fornendo un contributo la cui importanza per l'evoluzione della conoscenza storica non può essere sottovalutata. L'impatto dello stalinismo negli anni precedenti il secondo conflitto mondiale sull'opera degli storici sovietici fu castrante e impose una forte limitazione alla genuina interpretazione marxista e all'uso degli strumenti tipici del materialismo per comprendere i processi storici; la linea sulla quale ci si doveva muovere era quella delle cinque fasi: società primitiva, schiavismo, feudalesimo, capitalismo e infine la realizzazione socialista della società. ${ }^{102}$ Tutto questo ha inibito la possibilità di indagini non ideologizzate del fenomeno, ma ha anche contribuito a incrementare l'uso delle scienze sociali ausiliarie alla storia; categorie interpretative di tipo antropologico e sociologico sono state ampiamente utilizzate dai massimi storici sovietici e i risultati sulla ricerca ne hanno positivamente risentito.

Dopo questa breve premessa proviamo a tornare sulla liceità dell'uso del termine feudalesimo per una società nomade come quella mongola dei secoli XII e XIII.

Se volessimo adottare il termine feudalesimo nel suo significato strettamente economico allora potremmo facilmente scartarne l'efficacia per la realtà mongola tradizionale. Sarebbe una forzatura. L'aspetto più interessante mi pare sia piuttosto l'insieme delle relazioni umane e la formazione di solidarietà costruite e ramificazioni parentali; in quest'ultimo caso qualcosa di simile, a mio avviso, è riscontrabile presso la società mongola tradizionale. Riguardo al primo aspetto: l'economia europea di età feudale presenta alcuni caratteri che nell'economia

101 In realtà se per Vladimircov all'alba del XIII secolo tali relazioni, che egli definisce come feudali, erano già pienamente compiute, per Grekov e Jakubovskij esse si sarebbero realizzate appieno solo in seguito alla formazione dell'impero mongolo allorquando esso ebbe conquistato e governato per alcuni decenni regioni in cui il modello feudale era ben radicato.

102 Ochir-Bold, Mongolian nomadic Society cit., p. 16. 
nomade erano del tutto assenti ed essi riguardano soprattutto lo sfruttamento della terra e la distribuzione della medesima come elemento per consolidare le relazioni fra i poteri.

Per quanto concerne invece le relazioni umane e la crescente disuguaglianza fra ceti si nota che la nascita di un gruppo emergente, fenomeno già in pieno sviluppo nella seconda metà del XII secolo, uscito vittorioso dalla lotta interna ai clan e frutto di un'affermazione coercitiva della propria supremazia sugli altri individui appartenenti al clan medesimo, segna il passaggio da una concezione antica del potere, a una nuova; la prima basata sul diritto, tipico dell'organizzazione tribale, dell'anzianità, la seconda strettamente legata allo sfruttamento individuale delle risorse disponibili e quindi alla forza oggettiva, potremmo dire economica. Timujin rappresenta il livello più alto di questo ceto emergente, è l'esponente di quella classe egemone, chiamiamola pure aristocrazia nomade, che nella seconda metà del XII secolo stava estendendo la sua autorità sul proprio clan/oboq e, attraverso lo sfruttamento delle risorse umane dello stesso, su clan esterni ed economicamente più deboli. La prima parte della Storia Segreta, presentando la genealogia e l'infanzia del condottiero, fa spesso riferimento agli avi del khan e ai suoi amici delineando chiaramente la fisionomia degli oboq cui essi appartenevano; clan aristocratici il cui carattere dominante su altri e più deboli clan non è meno evidente. Le relazioni di alleanza che troviamo fra il clan di Timujin e altri eminenti sono ben salde prima che egli venga nominato khan. La figura del khan è quella del capo clan o, all'interno della tribù/irgän, quella del personaggio più influente fra i clan che vi appartengono. La propria vena carismatica si esplicitava soprattutto in occasione di razzie, incursioni, rapine o guerre. ${ }^{103}$

Prendiamo in esame uno degli aspetti che caratterizzano il fenomeno feudale europeo, ovvero le clientele armate. Presso le società mongole precedenti l'unificazione rivestiva una grande importanza la figura del nökör/nököd. I nököd erano persone di condizione libera che, spontaneamente, si mettevano a servizio di un capo clan, di un noyon col quale il più delle volte non avevano legami di sangue. Anzi, era assai frequente il caso in cui il nökör prestava servizio in un oboq diverso da quello di provenienza. ${ }^{104}$ Erano fideles ai quali non poteva essere imposto di prestare servizio. Erano legati ai loro noyon da un vincolo di fedeltà; ${ }^{105}$ il loro dovere principale era quello di prestare servizio militare permanente. Erano la

103 Vladimircov, Le régime social cit., pp. 100-110.

104 Ochir-Bold, Mongolian nomadic Society cit., p. 110; Vladimircov, Le régime social cit., p. 111 e 113. Era frequente anche il caso in cui un nököd abbandonasse il proprio noyon per entrare a servizio da un altro, magari rivale. In questo caso il noyon "tradito" non reagiva violentemente; il passaggio da un clan all'altro era una pratica diffusa e non ritenuta oltraggiosa dai noyon e dai qan. Din.

${ }^{105}$ In questo senso gli esempi non mancano nella Storia Segreta, in Juvaini e in Rashid ad- 
guardia armata dei capi clan, la parte direttamente controllata e controllabile dell'esercito, un'élite. Vivevano costantemente col loro capo e lo servivano durante le battute di caccia. In tempo di pace erano servitori domestici, ma dovevano essere pronti in qualsiasi momento a prendere le armi e seguire il loro signore. Non possono essere assimilati ai bogol in quanto, se questi ultimi erano inscindibilmente e unilateralmente legati al loro padrone, i nököd non lo erano affatto. Potevano scindere il loro vincolo in qualsiasi momento, inoltre il compito dei bogol non era di tipo militare. A ciò va aggiunto che le clientele armate dei noyon ricevevano quello che i Mongoli chiamavano qubi, una retribuzione in natura (assimilabile grossolanamente a quelle che era il beneficium in Occidente). ${ }^{106}$ L'economia mongola di questo periodo era ancora prevalentemente nomade. I mutamenti che si erano verificati e che erano in atto negli anni a cavallo fra XII e XIII secolo non avevano ancora prodotto uno scollamento della società dalla sua forma produttiva tradizionale. Il nomadismo comportava una concezione della terra, del territorio assai mobile. Come abbiamo visto parlando dell'ulus il territorio/patrimonio non era un bene apprezzabile in quanto entità spaziale, ma soprattutto per la sua consistenza demografica. Cioè l'ulus era un popolo, in particolare la gente che viveva su un determinato territorio. Il territorio in sé interessava in modo relativo ai pastori nomadi; si spostavano continuamente per cui una terra non era migliore di un'altra a meno che non avesse determinate caratteristiche adatte al pascolo. Il qubi si traduceva pertanto il più delle volte in una retribuzione in ayil. Il nököd meritorio riceveva determinati ayil dei quali poteva disporre liberamente, nei limiti della tutela fisica dei suoi appartenenti. I diritti del nököd sui suoi ayil e sul territorio all'interno del quale essi vivevano si concretizzavano nell'esercizio della giustizia e nella gestione delle clientele verso il basso e verso l'alto. ${ }^{107}$ Oltre a questo compenso in natura, il nököd otteneva anche la totale protezione da parte del suo noyon.

In questo modo i nököd potevano utilizzare i propri uomini, ottenuti tramite qubi, anche militarmente nel comune obiettivo di servire il noyon di riferimento. Tale sistema verrà utilizzato e riorganizzato da Gengis khan allorquando, nel 1206, opererà l'integrale ristrutturazione dell'esercito. Ma se da un lato questo sistema consentiva un maggiore controllo sul territorio attraverso le solidarietà e le clientele oltre che una buona organizzazione dell'esercito, dall'altro esso produceva un processo di disintegrazione sociale, per cui il potere andava disperdendosi in una serie di figure più o meno forti politicamente, ma anche assai mobili da un punto di vista del servizio. In altri termini, prima della creazione dell'impero, si vennero a costituire ceti sempre più forti, ege-

106 Bet Ochir Bold ha proposto un parallelo a mio avviso accettabile fra i nökör mongoli e quello che rappresentavano gli hus-carl nel mondo anglo-sassone e i Druženniki nella Rus' (OchirBold, Mongolian nomadic Society cit., p. 110), una sorta di fideles.

107 Vladimircov, Le régime social cit., p. 151. 
moni su una crescente massa di individui, le cui rivendicazioni porteranno a una concezione personale del potere. Pertanto Gengis riuscirà a tenere sotto di sé questa impalcatura ben salda, ma alla sua morte le divisioni emergeranno drammaticamente dando luogo a un impero sí vastissimo, ma la cui unità sarà solo un fatto formale.

Quando Gengis khan sconfisse i Kerait e i Naiman proclamando l'impero nell'anno della Pantera, ${ }^{108}$ procedette alla distribuzione dei benefici ai propri fedeli, coloro che lo avevano sostenuto e aiutato nell'opera ormai giunta a compimento. Tutte le tribù assoggettate divennero in questa circostanza unagan bogol dell'unico grande ulus di Gengis khan. ${ }^{109}$ Questo ulus, le cui proporzioni erano notevolissime, fu prerogativa di colui che uscì eletto dal Quriltai, dall'assemblea del clan egemone. I discendenti maschi di Gengis divennero automaticamente köbägün, ovvero "principi imperiali". ${ }^{110}$

Sebbene vi fossero, a mio parere, delle somiglianze fra la struttura sociale delle popolazioni nel periodo precedente l'unificazione e quello che intendiamo per feudalesimo europeo, erano assai evidenti anche le differenze. Nell'Occidente europeo, nonostante la notevole disorganicità delle fasi di sviluppo, la città non perse mai, sin dall'impero di Augusto, il suo ruolo di fulcro del sistema di potere territoriale. Nella società mongola la città non ebbe mai questo ruolo. Essa rappresentò un centro di potere comune solo nel XIV secolo, dopo che le conquiste ebbero il loro definitivo compimento dando vita a fenomeni di acculturazione le cui dinamiche non sono oggetto del nostro studio. Va in secondo luogo sottolineata la profonda differenza che l'Europa, e l'Italia in particolare conobbero, riguardo i centri di potere politico dalle prime grandi migrazioni del $\mathrm{V}$ secolo all'affermazione della supremazia comunale. L'esistenza di signorie rurali, territoriali e fondiarie, il fenomeno dell'incastellamento. Tutti aspetti di una lunga fase, ben studiata per l'Italia e parti dell'Occidente europeo, ma che non si possono ragionevolmente applicare alla comunità mongola, né prima, né dopo la sua parziale stabilizzazione sul territorio. Il modello nomade da cui essa ebbe origine influenzò gli sviluppi successivi della società rimanendo come preminente anche quando $\mathrm{i}$ Mongoli entrarono in contatto con modelli urbani e strutture sociali di tipo stratificato, né il popolo della steppa seppe adattarsi alla disponibilità di un territorio che per estensione avrebbe permesso loro un maggiore avvicinamento alla cultura urbana.

${ }^{108}$ SSM, VIII, 202, p. 178; cioè a dire il 1206.

109 Ibidem, VIII, 203, pp. 180-181. Rashid ad Din dice: «Poiché Gengis qan divenne qan, maestro della congiunzione dei pianeti, autocrate della terra e del tempo, tutti i clan e le tribù mongole, apparentate o estranee, divennero suoi schiavi e servitori», RaD, II, p. 12; Vladimircov, Le régime social cit., p. 129.

110 Secondo una definizione di Vladimircov (Le régime social cit., p. 127), il quale sostiene che letteralmente la parola significa figlio; SSM, VIII, 202, p. 178 e VIII, 207, p. 183. 


\subsection{Lo strumento dell'esercito e la realizzazione delle solidarietà}

In un passo dell' Historia Mongalorum di Giovanni di Pian del Carpine si legge: «Dicto de imperio, dicendum est hoc modo de bello: primo de ordinatione acierum $[\ldots] »{ }^{111}$ L'aspetto militare è uno dei più interessanti per noi che leggiamo con occhio critico l'opera del francescano e lo era anche per lui in quanto la raccolta delle informazioni sul modo di combattere, sugli armamenti, le tecniche e le tecnologie utilizzate dai Tataro-mongoli, era uno degli obiettivi primari del suo viaggio. L'Historia Mongalorum resta quindi un osservatorio documentario privilegiato per approfondire l'elemento militare che tanto incuriosiva e terrorizzava le genti dell'Europa occidentale. Esso costituisce però, per sua natura, un punto di vista parziale; l'impressione di un "agente" in missione esplorativa il cui scopo era quello di rendere conto al papa, Innocenzo IV, su come si potesse far fronte a una eventuale minaccia da est.

Una delle conseguenze dell'unificazione portata a termine da Timujin fu proprio la riorganizzazione dell'esercito. Una massa compatta che combatteva quasi esclusivamente a cavallo e che seguiva disciplinatamente gli ordini dei comandanti. Questo imponente sistema militare era il frutto della complessa struttura sociale che stava alla base del reclutamento. In pratica, come scrisse George Bratianu: «l'armèe représente toute cette féodalité nomade en marche». ${ }^{112}$

Alla fine del XII secolo Timujin viene eletto khan ${ }^{113}$ dopo che tutto «l'ulus dei Merkit era fuggito preso dal panico, lungo il corso dello Slengge e le nostre truppe li inseguivano uccidendo e catturando i fuggiaschi». ${ }^{114}$ Appena eletto, il nuovo khan riorganizzò il proprio seguito. Furono nominati tre kortšin ovvero incaricati di portare la faretra; due addetti alla mensa (čerbi), ${ }^{115}$ un suo fedele, Degai, fu investito del ruolo, importantissimo per l'organizzazione nomade dell'ulus, di controllore delle greggi. Il fratello di Degai, Güčügür fu invece nominato custode dei carri da transumanza. Un altro fedele, Dodai-čerbi, fu incaricato di dirigere i servi e gli schiavi dell'ulus, mentre altri tre uomini divennero portatori di spada al comando di Qasar, un altro partigiano di Timujin. Tre uomini furono nominati custodi della mandria di cavalli, altri quattro divennero

111 Historia Mongalorum cit., p. 275.

112 Bratianu, La Mer Noire cit., p. 187.

113 Tale esito è ancora oggi poco chiaro in quanto Timujin, al momento della sua elezione, era a capo di un'unione di tribù relativamente esigua, non aveva discendenze particolarmente eminenti e la sua carriera militare non era certamente d'eccellenza. Purtroppo le fonti, e la Storia Segreta in particolare, su questo aspetto tacciono.

114 SSM, III, 110, p. 91.

115 Il termine Čerbi viene utilizzato, nella Storia Segreta, per indicare coloro i quali in tempo di guerra servivano i capi militari e in tempo di pace si occupavano del bestiame e della mensa. Il termine è stato utilizzato in Mongolia sino a tempi recenti per indicare funzionari militari e amministrativi. Si veda anche Bold, Mongolian nomadic Society cit., pp. 110-111. 
esploratori (un compito molto importante all'interno dell'organizzazione militare mongola, poiché erano quelli che compivano le perlustrazioni preliminari in seguito alle quali si decideva se e in quale misura agire con l'esercito); infine fu scelto un consigliere del khan nella persona di Sübeetai-Bagatur. La Storia Segreta dice che

quanto a Sübeetai-Bagatur [Timujin] disse: Mi farò topo per raccogliere e mettere da parte per te. Mi farò corvo nero per ripulire insieme a te quanto sta fuori. Mi farò feltro per coprirmi insieme con te; mi farò feltro da jurta e cercherò di avvolgermi insieme con te. ${ }^{116}$

Dopo questa prima generale organizzazione dei suoi più stretti collaboratori Timujin provvide all'esercito. La prima volta vi mise mano nel 1203, dopo la vittoria sui Kerait. ${ }^{117}$ Fu selezionata la guardia del corpo del khan, i kešigten, divisi in due unità: 70 turgewuts per la guardia diurna e 80 kebtewuts per quella notturna. Del corpo delle guardie «facevano parte i figli e i fratelli minori dei noyon fra i più valorosi e prestanti, come pure i figli di persone di stato libero». ${ }^{118}$ Altri mille uomini andarono sotto il comando di Arqai-Qasar per le battaglie, mentre in tempo di pace egli sarebbe dovuto essere il comandante dei kešigten. I čerbi furono portati da due a sei. Distribuì ai noyon il comando delle formazioni di mille, cento e dieci. Organizzò i turni e i tempi del cambio di guardia fra notte e giorno e iniziò la campagna che lo avrebbe messo a capo di tutte le tribù nomadi della regione, quella contro i Naiman. ${ }^{119}$

La guardia del khan era sottoposta a una rigida disciplina e operava sotto il carico di una pesante responsabilità. Chi ritardava nel prendere servizio veniva punito con trenta frustate la prima volta, settanta la seconda e se ciò accadeva per la terza volta egli veniva frustato trentasette volte e quindi espulso dal corpo con conseguente crollo sociale. Infatti se da un lato vi erano pesanti responsabilità dall'altro la guardia del khan godeva di privilegi esclusivi. Un appartenente alla guardia era un personaggio socialmente eminente; «un qualsiasi soldato del mio keshig sia al di sopra di qualsiasi minggan dell'esercito». ${ }^{120}$ La guardia del khan non partecipava alle guerre di conquista; essa interveniva soprattutto verso l'interno e in casi eccezionali quando era direttamente guidata dal khan in persona. Gengis khan sceglieva i propri generali quasi esclusivamente dalla sua guardia. Questo sistema era concepito molto attentamente. Nessun generale e nessun noyon in genere poteva punire un soldato di sua iniziativa. Ogni decisione in questo senso doveva passare dal khan il quale, se si agiva in assenza di una sua

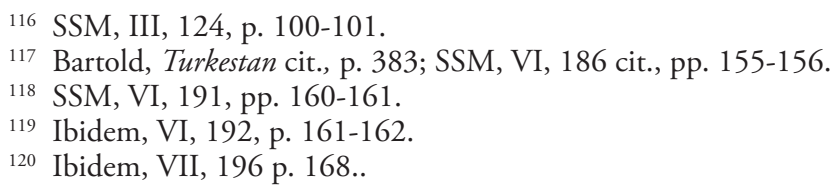


approvazione, puniva il colpevole nello stesso modo con cui questi intendeva punire il soldato. ${ }^{121}$ Inoltre, essendo i generali parte della guardia del khan, questi li conosceva personalmente e aveva esperienza diretta delle loro capacità.

Secondo Juvaini i dirigenti militari godevano di privilegi particolari: erano esenti da ogni forma di tributo; tutti coloro i quali cadevano prigionieri in guerra erano automaticamente loro proprietà e ne potevano disporre a piacimento, potevano presentarsi al khan senza particolare permesso; se condannati a morte si poteva procedere contro di loro solo al nono crimine commesso; infine durante le feste occupavano un posto d'onore e ricevevano vino. ${ }^{122}$

Nel 1206 Gengis khan aveva proceduto a una seconda e ben più radicale organizzazione dell'esercito:

Hoc modo Chingiscan ordinavit, ut decem hominibus preponeretur unus, et ille secundum nos appellatur decanus; decem autem decanis preponeretur unus, qui centenarius nominatur; decem vero centenariis preponeretur unus, qui millenarius appellatur; decem millenarii preponeretur unus, et ille numerus vocatur tenebre apud eos. ${ }^{123}$

Quindi la riforma del khan portò, secondo frate Giovanni, a una precisa divisione gerarchica per cui la base dell'esercito erano le chiliarchie, composte da mille uomini ciascuna; secondo la Storia Segreta le chiliarchie create dalla riforma del 1206 erano $95 .{ }^{124}$ Juvaini dice:

Essi [i Mongoli] hanno diviso tutto il popolo in compagnie di dieci, e nominato uno dei dieci comandante degli altri nove; e di ogni dieci comandanti, uno ha ricevuto il titolo di comandante dei cento, ché tutti i cento sono stati sottoposti al suo comando. E così via ogni mille uomini, e così pure ogni diecimila, al di sopra dei quali essi hanno posto un comandante che chiamano "comandante del tümen". ${ }^{125}$

Il tümen era composto da 10.000 uomini. ${ }^{126}$ A ogni tümen era a capo un comandante del tümen, tale entità comprendeva dieci chiliarchie, composte

121 Bartold, Turkestan cit., p. 383.

122 Juvaini, I, p. 27; Bartold, Turkestan cit., p. 385-386. Il vino era un bene raro e molto apprezzato presso i Mongoli.

123 Historia Mongalorum, p. 266.

124 «[Gengis Qan] nominò chiliarchi e mise al comando di mille guerrieri i seguenti novantacinque noyon» e ancora «in tutto Činggisqagan promosse così novantacinque chiliarchi del popolo mongolo», SSM, VIII, pp. 178-179; Historia Mongalorum, p. 462.

125 Juvaini, I, p. 52.

126 Petech scrive opportunamente che Giovanni ha tradotto tümen con tenebre forse perché il turco antico tuman significa nebbia (Historia Mongalorum, p. 462, nota 2); anche in russo moderno tuman significa nebbia. 
ciascuna da mille uomini e guidata da un chiliarca, in ogni chiliarchia vi erano dieci centurie, composte ciascuna da cento uomini e comandate da un centurione. L'unità più piccola era composta da dieci soldati di cui uno era il referente per i superiori e gli altri nove gli dovevano obbedire. Quando vi era necessità di uomini ci si rivolgeva al comandante del tümen che si rivolgeva al chiliarca e così via. ${ }^{127}$ Naturalmente il capo supremo dell'esercito era il Gran khan che nominava direttamente i suoi generali.

L'organizzazione in tümen, migliaia, centinaia e decine non fu una novità introdotta dai Mongoli; già da tempo le popolazioni nomadi adottavano questa ripartizione quantitativa. Con Gengis khan si assiste per la prima volta alla nomina di tre generali per ogni tümen. ${ }^{128}$ Uno di essi guidava l'ala sinistra (al tempo della riforma era Muquli), un altro, Bughurij, comandava l'ala destra e Naya stava a capo della parte centrale. ${ }^{129}$ Tale ordine era insindacabile. Chiunque osasse cambiare ala d'operazione veniva giustiziato immediatamente davanti all'esercito. ${ }^{130}$

Le fonti arabo-persiane e la Storia Segreta ci forniscono i termini mongoli per le singole ripartizioni militari: come detto il tümen era la ripartizione principale, la più grande. Ogni tümen comprendeva dieci mingghan (le chiliarchie di frate Giovanni), ogni mingghan era composto a sua volta da dieci jaghun e ogni jaghun comprendeva dieci arban. Il sistema che portava un individuo alla guida delle singole ripartizioni era molto semplice. Abbiamo visto che gli arban erano guidati da comandanti eletti dai componenti degli arban stessi. Il meccanismo era uguale per gli jaghun, mentre cambiava per il mingghan e il tümen. In questi casi era il Gran khan in persona che sceglieva i comandanti. Questi avevano una certa autonomia tattica e potevano prendere decisioni autonomamente sul campo di battaglia. Era un aspetto fondamentale in quanto rendeva ogni singolo soldato fondamentale non solo come forza fisica, ma anche in quanto organizzatore attivo della strategia bellica. I comandanti delle unità maggiori, jaghun e mingghan provenivano soprattutto dai noyon più influenti. Era una gerarchia molto precisa, ma altrettanto fluida. I piccoli esponenti delle tribù minori, tarkhat, potevano, se eccellenti in battaglia, raggiungere anche il comando di una mingghan.

La rigorosa disciplina cui era sottoposto l'esercito mongolo era un altro degli elementi che colpiva i contemporanei. Juvaini non esita a decantarne le lodi ${ }^{131}$ e afferma:

127 Juvaini, I, p. 52.

128 «Cuncto vero exsercitui preponuntur due duces vel tres, ita tamen quod habeant respectum ad unum», Historia Mongalorum, p. 276. «E [Gengis Qan] comandò [che] Qorči sia a capo [di un] tümen, condividendo però il comando con Taqai e Ašig», SSM, VIII, 205, p. 183.

129 Bartold, Turkestan cit., p. 386; SSM, VIII, 202, pp. 178-179.

${ }^{130}$ Ibidem, pp. 391-392. In un passo della sua storia Juvaini sostiene che durante le battute di caccia, le quali per i Mongoli erano una cosa molto seria, non si potevano infrangere le rigide disposizioni del Khan. Anche in questo caso si rischiava la morte.

131 Juvaini, I, p. 50. 
Se sorge un'improvvisa necessità di armati, si dà ordine che tante migliaia di uomini si presentino al tal posto alla tal ora di un certo giorno o di una certa notte [...] ed essi arrivano, non un batter d'occhio prima o dopo l'ora assegnata. ${ }^{132}$

L’obbedienza e la disciplina sono tali che

se c'è un comandante di centomila a una distanza dal Khan pari all'intervallo tra alba e tramonto [...] il Khan manda un solo cavaliere a punirlo.

A nessuno era concesso trasgredire l'ordine secondo il quale ogni soldato doveva prendere posto nei singoli reparti. ${ }^{133}$ Nell'esercito venivano spesso inseriti prigionieri catturati durante le spedizioni di conquista; essi venivano utilizzati come primo contingente da opporre al nemico frontalmente. ${ }^{134}$ La cavalleria mongola interveniva successivamente e con tattiche molto ben studiate. Un sistema di combattimento che apparteneva al regolare stile di vita nomade. Il prigioniero utilizzato in battaglia aveva spesso il compito di andare contro al nemico come forza d'urto iniziale, destabilizzante. Talvolta veniva loro assegnato il compito di andare in missione esplorativa presso popoli dei quali i Mongoli non si fidavano e che temevano potessero uccidere gli ambasciatori anziché rimandarli indietro sani e salvi. ${ }^{135}$

I cavalieri mongoli erano arcieri formidabili e la loro tattica in battaglia assolutamente nuova per chi si trovava ad affrontarli. Essi univano i sistemi tipici dei cavalieri delle steppe a una nuova e migliore organizzazione. Attaccavano a cavallo per poi sparire e ricomparire sull'avversario disorientato. Ma, appunto, l'esercito tataro-mongolo era anche molto ben strutturato.

Più si approfondiscono gli studi sull'organizzazione militare tataro-mongola e più ci si rende conto di come la dimensione quantitativa non fosse determinante nelle loro vittorie. A dire il vero questo è un aspetto sul quale ci si è interrogati a lungo. ${ }^{136}$ Mason Smith affermava che fra la Russia e la Caucasia vi fossero 600.000 mongoli impegnati militarmente. ${ }^{137} \mathrm{E}$ un numero eccessivo se consideriamo che solo nel 1206 Gengis, riformando la guardia, costituì 95 migliaia (quindi compresa la guardia circa 96.000 uomini). De Hartog e Dunn confermano sostanzial-

132 Ibidem, I, pp. 51-52.

133 Ibidem, I, p 54: «Nessun soldato può arruolarsi se non nel centinaio, migliaio o decina che gli è stato assegnato, e non può rifugiarsi altrove. [Chi trasgredisce questo ordine] viene messo a morte sotto gli occhi delle truppe».

${ }_{134} \mathrm{Si}$ veda a riguardo quanto dice Giovanni di Pian del Carpine, Historia Mongalorum, VI, 14, pp. 281-282.

135 Juvaini, I, p. 73.

136 Si veda ad esempio J. Mason Smith, Mongol manpower and Persian population, "Journal of the economic and social History of the Orient», 18 (1975), pp. 271-299.

137 Ibidem, p. 272. 
mente questi dati fornendo altre cifre: stimano che fino alla fine del XIII secolo vi fossero nell'esercito mongolo circa 70.000 uomini che diventavano 250.000 comprese le truppe ausiliarie di altre aree soggiogate. ${ }^{138}$ Di Cosmo afferma che nel 1206 l'esercito mongolo non superasse di molto le 100.000 unità. ${ }^{139}$ Ora, le cifre - considerando le fonti che abbiamo a disposizione per questo periodo - non sono molto attendibili; sarebbero in ogni caso stime approssimative. Se volessimo rendere l'idea della consistenza di cui poteva disporre l'esercito di Gengis dovremmo considerare il metodo utilizzato in battaglia dai Mongoli. È noto che essi utilizzavano la popolazione maschile delle città conquistate (quella che non serviva ad altri scopi) come avanguardia, in un tragico gioco al massacro in cui i disgraziati scampati all'esecuzione in loco venivano mandati contro gli eserciti nemici prima che la cavalleria mongola intervenisse. Dunque la percezione che molti autori hanno avuto dell'esercito mongolo può essere stata filtrata da questo. L'efficacia dell'apparato militare di Gengis era il frutto di varie componenti fra le quali la quantità degli uomini era la meno importante. La sistematica militarizzazione della società, il metodo di combattere, la preparazione e la tattica impiegata in battaglia ebbero un peso assai maggiore. L'esercito tataro-mongolo era costituito quasi esclusivamente dalla cavalleria (in maggioranza pesante e in piccola parte leggera). La fanteria diventerà un corpo militare a tutti gli effetti solo in seguito alla costituzione dell'impero; in particolare dopo la parziale riorganizzazione militare operata da Tamerlano dopo il 1372. Oltre all'uso frequente della fanteria questi limitò il ricorso alla leva obbligatoria, arruolando solo un segmento della popolazione delle campagne e vincolando alla decisione dei governanti locali il numero di soldati da corrispondere all'esercito centrale.

È difficile analizzare tutti i fattori che determinarono i successi travolgenti delle armate di Gengis khan sin dalle sue prime apparizioni a Ovest. Esclusa la particolare malvagità che avrebbe animato questi demoni a cavallo si rende necessario indagare quali elementi nuovi essi apportarono al modo di combattere medievale. L'equipaggiamento e l'uso delle armi fu sicuramente uno di questi fattori. Gengis khan aveva proceduto a rendere l'esercito compatto, ben organizzato e aveva trasferito in ambito militare le caratteristiche della società nomade. Il cavallo era quindi utilizzato non solo come un mezzo pesante in ambito bellico, ma anche come strumento di trasporto veloce col quale effettuare rapide e improvvise incursioni e con altrettanta rapidità allontanarsi prima che il nemico potesse reagire; movimenti armonici e collettivi di migliaia di guerrieri

138 L. De Hartog, The Army of Chinghis Qan, in «Army Quarterly and Defence Journal», 109 (1979), 476-485, p. 484; J.L. Dunn, The Ageless Chinese: a History, New York (NY) 1978, pp. 246-247.

139 Di Cosmo, State formation cit., p. 17. Su posizioni analoghe V.P. Alekseev, Some Aspects of the Study of Productive Forces in the Empire of Chengiz Khan, in Rulers from the Steppe: State Formation on the Inner Eura-sian Periphery, a cura di G. Seaman, D. Marks, Los Angeles (CA) 1991, p. 191. 
durante i quali si portava l'attacco con gli archi (un'arma che i cavalieri mongoli sapevano utilizzare benissimo anche in movimento) etc.

Le informazioni sulle tecniche militari e sull'equipaggiamento da battaglia dei Mongoli si ricavano soprattutto da fonti successive le loro grandi conquiste. L'Historia Mongalorum e l'Historia Tartarorum per prime, ma anche l'Itinerum di Guglielmo di Rubruck, insieme ad altre fonti sparse (si pensi ad esempio all'Encyclica contra Tartaros e alla lettera de adventu Tartarorum, entrambe di Federico II e scritte nel 1241) $)^{140}$ costituiscono un bacino informativo di straordinaria importanza. Sull'armamento che ogni cavaliere mongolo doveva avere Giovanni di Pian del Carpine è molto chiaro:

Arma autem ista ad minus omnes debent habere: duos arcus vel tres, vel unum bonum ad minus, et tres pharetras magna plenas sagittis, et unam securim, et funes ad machinas trahendas. ${ }^{141}$

I più ricchi, sostiene il francescano, posseggono anche spade appuntite e bilama. Sono ben corazzati e bardano accuratamente anche il cavallo. Non è del tutto chiaro l'uso delle diverse armature e la loro composizione. Va detto che non si hanno a riguardo e per questo periodo descrizioni precise come quella di Pian del Carpine. ${ }^{142}$ Secondo il francescano essa poteva essere di due tipi: di cuoio o metallica. La prima consisteva in strisce di cuoio legate fra di loro «corrigioli sive corduli ${ }^{143}$ e sovrapposte una all'altra per una piccola porzione lungo il bordo inferiore e superiore. Era quindi un'armatura molto mobile che permetteva movimenti bruschi, più delle pesanti armature di maglia o di quelle metalliche utilizzate dagli eserciti europei. L'armatura metallica era costruita in modo simile a quella di cuoio. Era fatta con strisce di metallo molto sottili, munite di «otto forellini» ${ }^{144}$ dai quali passava una cordicella che teneva le strisce assieme. Alcuni cavalieri erano muniti di una lancia all'estremità della quale vi era un gancio che serviva per disarcionare l'avversario. I Mongoli erano soliti costruire anche scudi piuttosto piccoli, ma essi non erano usati nelle battaglie, perché troppo ingombranti per un arciere. Secondo frate Giovanni li utilizzavano soprattutto durante la guardia notturna. ${ }^{145}$

140 La prima in Monumenta Germaniae Historica. Costitutiones et Acta Publica Imperatorum et Regnum, II, Hannover, 1896, pp. 322-325; la seconda in Matteo Paris, Cronica Majora, a cura di H. R. Luard, IV, London 1877, pp. 112-119; si veda anche Historia Mongalorum, Note, p. 453.

141 Ibidem, VI, 4, p. 276.

142 Quella di Giovanni è la prima descrizione che si ha circa l'armatura mongola ed è anche, come ha fatto giustamente notare P. Daffinà, la più completa se si considera che il nostro «tratta una materia non ancora trattata, così ampiamente, da altri» (Ibidem, Note, p. 463).

143 Ibidem, VI, 4, p. 277.

144 Ibidem, VI, 8, p. 278.

145 Ibidem, VI, 10, p. 279. 
Secondo quanto afferma Giovanni di Pian del Carpine in un passo che abbiamo già citato ${ }^{146} \mathrm{i}$ Mongoli usavano delle «funes ad machinas trahendas». $\mathrm{E}$ verosimile che l'esercito tataro disponesse di macchine da guerra ben costruite e tecnicamente evolute. D'altra parte l'accaparramento di sapere umano da parte dei Mongoli era uno degli obiettivi primari durante le loro conquiste. Seguendo l'itinerario delle campagne militari successive la conquista della Cina (1211), grazie alla precisa descrizione che ci ha lasciato Juvaini, si nota come in ogni città che cadeva nelle loro mani i Mongoli giustiziavano senza esitazione chi aveva osato combatterli, ma risparmiavano regolarmente gli artigiani. ${ }^{147}$

Prima di ogni battaglia i generali mongoli mandavano avanti degli uomini in avanscoperta, i precursores di Giovanni di Pian del Carpine, ${ }^{148}$ i quali avevano il compito di osservare e riportare le condizioni in cui si sarebbe potuta svolgere la battaglia, di quale potenziale potevano disporre i nemici e talvolta avevano il compito (soprattutto se si trattava di prepararsi all'assedio di una città) di dissuadere l'avversario dal resistere. ${ }^{149}$

Nel paragrafo successivo Giovanni di Pian del Carpine offre un particolare curioso. Dice che i Mongoli guadavano i fiumi utilizzando un pezzo di cuoio

146 Ibidem, VI, 4 (crf. anche la nota 40).

147 Juvaini, I, p 107, dice che in seguito al primo attacco contro Bukhara «la cittadella e le mura vennero rase al suolo e i Mongoli se ne andarono. E portarono seco la gente comune e gli artigiani che erano sfuggiti alla spada, o perché prestassero servizio come coscritti, o perché esercitassero il loro mestiere». Quindi quella che Juvaini chiama gente comune entrava nell'esercito, mentre gli artigiani venivano utilizzati in quanto potessero mettere la loro arte a disposizione dei Mongoli. Gengis Khan mostrerà più volte ammirazione per l'arte manuale e intellettuale. Passi simili si trovano in Juvaini, I, pp. 117, 120 e 122. Emblematico è il caso della presa di Samarcanda. Durante il cammino che portava alla ricca città del territorio selgiuchide l'esercito mongolo attaccò molti villaggi, ma non vi furono stragi. Ai Mongoli servivano gli uomini per l'esercito e ne arruolarono molti. Quando, fra il maggio e il giugno 1220, Samarcanda cadde essi arruolarono nell'esercito la gente comune, asservirono donne e bambini e portarono con sé gli artigiani (Juvaini, I, p. 142). Ancora un buon esempio si trova dopo la conquista di Urgench, la capitale dellla Korazmia: «Allora sospinsero la popolazione in aperta campagna, e separarono dagli altri gli artigiani e gli operai, che assommavano a più di centomila; le donne giovani ed i bambini furono ridotti in schiavitù e condotti in cattività; e gli uomini che restavano vennero ripartiti in modo che ad ogni combattente dell'esercito toccasse di mettere a morte ventiquattro persone [...]. Poi l'esercito si dedicò al saccheggio e alla rapina, e distrusse quel che restava delle case e dei quartieri». E più avanti: «quando i Mongoli terminarono la battaglia ed ebbero finito di far prigionieri, di saccheggiare, uccidere e sterminare, quegli abitanti che erano artigiani vennero suddivisi e mandati nei paesi dell'Oriente», Juvaini, I, pp. 154-155. Ancora, dopo essere entrati a Merv, nel febbraio del 1221: «i Mongoli ordinarono di uccidere l'intera popolazione, donne e bambini compresi, e di non risparmiare nessuno [...] ad eccezione di quattrocento artigiani, espressamente scelti tra gli uomini, e di alcuni giovani, ragazzi e ragazze, che condussero seco in cattività», Juvaini, I, p. 184.

148 Historia Mongalorum, VI, 11, p. 279.

149 Così fu per la presa di Bukhara, Žend, Merv, Urgench etc. Si veda a tal proposito la prima parte di Juvaini. 
rotondo e leggero legato ben stretto, all'interno del quale mettevano i loro pochi oggetti, e che usavano come zattera trainata dal cavallo al quale era legata. ${ }^{150}$

Anche sulla disposizione dell'esercito durante le battaglie Giovanni di Pian del Carpine è preciso. I Mongoli attaccavano prima, more nomadum, con schermaglie portate da arcieri a cavallo che con estrema rapidità attaccavano e retrocedevano sperando di essere seguiti fino a un punto in cui era disposto il grosso delle armate. Se le dimensioni dell'esercito avversario lo consentivano, attaccavano in massa, altrimenti fuggivano fino a far perdere le loro tracce per poi tornarvi sopra accerchiandolo di sorpresa. Giovanni di Pian del Carpine osserva che spesso, per sembrare più numerosi, i Mongoli utilizzavano fantocci posti sopra i cavalli. ${ }^{151}$

L'esercito di Gengis khan non amava molto il combattimento corpo a corpo. La straordinaria abilità dei cavalieri mongoli con l'arco faceva preferire loro quest'arma e il combattimento a distanza. Quando il contatto diveniva inevitabile essi usavano tecniche precise. Ad esempio, se durante una battaglia gli avversari riuscivano a impegnarli e a metterli in difficoltà i Mongoli aprivano i ranghi, lasciando uscire l'avversario, quindi lo inseguivano attaccandolo a cavallo.

\begin{tabular}{|l|l|l|}
\hline & \multicolumn{1}{|c|}{ secoli XII-XIII } & \multicolumn{1}{c|}{ secoli XIV-XVII } \\
\hline $\begin{array}{l}\text { Decine } \\
\text { Arban }\end{array}$ & $\begin{array}{l}\text { Unità militare composta da } \\
\text { dieci uomini }\end{array}$ & In disuso \\
\hline $\begin{array}{l}\text { Centinaia } \\
\text { Jaghun }\end{array}$ & $\begin{array}{l}\text { Unità militare composta da 100 } \\
\text { uomini }\end{array}$ & In disuso \\
\hline $\begin{array}{l}\text { Migliaia } \\
\text { Mingghan }\end{array}$ & $\begin{array}{l}\text { Unità militare composta da } \\
1000 \text { uomini }\end{array}$ & Termine scomparso \\
\hline Tümen & $\begin{array}{l}\text { Unità militare composta da } \\
\text { più } \text { oboq }\end{array}$ & $\begin{array}{l}\text { Unità amministrativa civile e } \\
\text { militare composta da } 100.000 \\
\text { uomini }\end{array}$ \\
\hline
\end{tabular}

Tabella 1. Struttura militare e amministrativa monogola (fine XII e secoli XIV-XVII) (riadattata da B.-O. BolD, Mongolian Nomadic Society cit., p. 98)

Una simile impalcatura militare era possibile solo come conseguenza di una serie di relazioni, clientele e solidarietà ben costruite e radicate nei diversi clan, o meglio nei diversi ulus. L'esame dell'organizzazione militare mostra

150 Tale riferimento si trova anche in Federico II, Encyclic,: «Ipsi autem Tartari, sagittarii incomparabiles, utres ferunt artificialitar factos, quibus flumina transmeant indempnes rapacissima et paludes»; Historia Mongalorum, Note, p. 463. Questo sistema era tutt'altro che improvvisato. Se ne ha dimostrazione nel fatto che, ad esempio, Erodoto e Senofonte parlano di un metodo simile per i loro tempi (si veda Niceta Coniate, Grandezza e catastrofe cit., vol. I, commento, p. 582, nota 98).

151 Historia Mongalorum, VI, 14, p. 281; ma tale metodo veniva usato anche dalle tribù prima dell'unificazione portata a termine da Gengis khan. La Storia Segreta (VII, 193, p. 163) parla di uno stratagemma simile durante la guerra fra Gengis Khan e i Naiman. 
come l'unagan bogol, prima una fascia della popolazione mongola ben visibile e individuabile, adesso sia parte di queste relazioni e scompaia nelle maglie di un sistema più complesso. Non è raro trovare comandanti delle centinaia $o$ delle migliaia discendenti di seconda generazione degli unagan bogol. Questi individui possono arrivare fino al titolo di noyon. Aumenta la mobilità sociale, ma diminuisce drasticamente la libertà dei nököd e di tutti gli esponenti dell'aristocrazia militare; nessuno poteva più cambiare referente senza la nomina di Gengis khan, capo supremo delle forze armate, colui che nomina personalmente i comandanti dei tümen. ${ }^{152}$

\subsection{Nomadismo e schiavitù}

Indagare il ruolo degli schiavi e la concezione della pratica stessa presso un modello sociale come quello nomade è un'operazione piena di limiti a causa delle scarse evidenze documentarie relative al nomadismo, ma credo valga la pena fare un tentativo poiché nella regione compresa fra la Caucasia e il Mar d'Azov si verificò, in condizioni uniche, l'incontro dei due modelli, società domestica e ceto mercantile urbano. I rapporti che intercorsero fra questi due mondi furono vari e imposero l'adattamento degli uni verso gli altri; talvolta ne accentuarono le chiusure.

In epoca romana e sino all'epoca dell'impero gran parte della struttura produttiva si basava sulla manodopera di schiavi. Ciò era possibile soprattutto in un contesto nel quale la produzione agricola era dominante. Giustamente Marc Bloch sottolineò a suo tempo quanto la trasformazione che intervenne nelle produzioni, conseguenza del generale declino demografico, economico e politico nell'ultimo periodo imperiale, interessò e in parte mutò il sistema di reclutamento degli schiavi. "Lo schiavo è un cattivo lavoratore»" ${ }^{153}$ e l'utilizzo della manodopera di schiavi presenta notevoli inconvenienti, ma fino a quando la situazione politica permise un agevole reclutamento questi problemi non si presentarono. Il cattivo lavoratore o il lavoratore ribelle veniva facilmente sostituito. Il complesso sistema organizzativo romano si serviva abbondantemente degli schiavi poiché le conquiste procedevano rapide e i successi si ripetevano incessantemente, generando un costante afflusso di persone laddove ce n'era bisogno. In questo periodo non vi erano problemi di prezzo né di quantità. In pratica la forza lavoro disponibile era superiore alle necessità del contesto sociale in cui essa veniva richiesta. Sul finire dell'era romana ad un brusco rallentamento

152 Vladimircov, Le régime social cit., p. 135.

${ }_{153}$ M. Bloch, Comment et pourquoi finit l'esclavage antique in "Annales», V/II (1947), pp. 30-44, 161-170; ripubblicato Come e perché fini la schiavitù antica in Lavoro e tecnica nel Medioevo, Roma- Bari 1990, pp. 221-263. Noi abbiamo utilizzato la versione riproposta in La servitù nella società medievale, a cura di G. Cherubini, Firenze 1993, pp. 3-40, p. 9. 
delle conquiste si accompagnò la straordinaria diffusione del Cristianesimo il cui sistema dottrinale imponeva una nuova riflessione sulla liceità di possedere schiavi. Opportunamente Bloch scriveva che «per quanto [il Cristianesimo] favorisse gli affrancamenti, esso non aveva condannato la schiavitù». ${ }^{154}$ Certo esso impose limiti e costrinse alla generale rielaborazione del trattamento che si poteva riservare ad uno schiavo, ma di fatto non si giunse mai ad una netta e definitiva condanna della pratica.

La tratta degli schiavi subì una notevole impennata in epoca carolingia, ${ }^{155}$ ma in questo periodo non possiamo più parlare con disinvoltura di schiavi, si tratta soprattutto di servi. Gioverà soffermarsi su questo aspetto per poterlo poi affiancare ad alcune figure tipiche del nomadismo. In Occidente la differenza si accentua soprattutto in epoca alto-medievale. Uno scostamento che coincide con la «dissoluzione delle vecchie gerarchie sociali». ${ }^{156}$ Intorno al X secolo la documentazione comincia ad usare vocaboli diversi per designare le figure servili. Lo schiavo/oggetto di epoca romana ${ }^{157}$ diminuisce progressivamente e lascia il posto al servo, a un individuo di condizione non libera, ma che non è legato ad un padrone da un vincolo di proprietà. Lo schiavo in quanto oggetto rimarrà quasi esclusivamente una condizione riservata allo straniero. ${ }^{158}$ In alcune aree dell'Europa Occidentale al generale impoverimento determinato dal declino della bassa Antichità si accompagnò l'esigenza, da parte dei ceti più deboli, di trovare protezione e mezzi di sussistenza adeguati per far fronte alla nuova situazione e ciò fu possibile solo accettando di finire sotto proprietari ricchi e signori che avevano un forte radicamento locale. Il numero degli schiavi è in questo periodo esiguo e ciò frena il ricorso indiscriminato ai loro servizi i quali non solo vanno mantenuti, ma vanno anche controllati; lo schiavo diventa un bene di lusso che non tutti possono permettersi. Quello di servo è un concetto legato all'aspetto demografico più che a quello commerciale e militare; i servi non si acquistavano sul mercato, non erano estranei, nati altrove e "importati"

${ }_{154}$ Bloch, Come e perché cit., p. 31.

155 P. A. Milani, La schiavitù nel pensiero politico. Dai greci al basso Medioevo, Milano 1972, p. 341.

156 Bloch, Come e perché cit., p. 31.

157 In realtà la denominazione di schiavo-oggetto meriterebbe un approfondimento. Si è soliti utilizzare questa categoria semantico-culturale applicandola a quel contesto sociale in cui l'individuo veniva considerato merce scambiabile e della quale si poteva arbitrariamente disporre. Dobbiamo però sottolineare che spesso, se non sempre, il diritto risulta distante dalla realtà oggettiva cui si riferisce; pertanto in una società come quella romana, dove il diritto aveva raggiunto livelli di raffinatezza straordinari, lo schiavo-oggetto era tale solo a livello giuridico poiché ad esso venivano richieste cose che ad un oggetto non ci si sarebbe mai sognati di chiedere. La considerazione dello schiavo come individuo in quanto biologicamente simile era presente anche in quei contesti sociali in cui lo schiavo era trattato, a livello estrinseco, come oggetto.

158 Bloch, Come e perché cit., p. 32. In questa sua affermazione Bloch utilizza una categoria antropologica molto utilizzata ancora oggi dagli specialisti. 
coercitivamente in una realtà diversa da quella di origine. Il servo si riproduceva in un contesto sociale che era il medesimo del suo padrone; da un punto di vista economico un servo deve essere in grado di produrre più possibile in quanto non solo deve generare un sovraprodotto, ma deve poterne garantire anche al padrone. Il servizio cui era obbligato aveva spesso carattere costante, autonomo dalla produttività che era in grado di provvedere (sia che la terra rendesse tanto, sia che rendesse poco, il servo doveva al padrone la medesima quantità di prodotto). Raramente quindi un servo era in grado di accumulare riserve, il più delle volte viveva in una costante condizione di debito nei confronti del padrone. Dunque doveva operare una sorta di controllo sulla crescita della prole per evitare di impoverirsi ulteriormente. Tutto questo rendeva il servo un elemento demografico attivo ma sfavorevole alla crescita quantitativa. ${ }^{159}$ Infine possiamo osservare che se in una società che si basa sull'attività degli schiavi la produzione aumenta indipendentemente dalla produttività del lavoro in virtù dell'afflusso costante di manodopera attraverso il reclutamento (guerra e commercio), con la servitù è il fattore demografico a determinare la produttività; essa ne è a sua volta influenzata poiché lo schiavo è sostanzialmente desocializzato ${ }^{160}$ mentre il servo è parte integrante del contesto sociale che ne utilizza il lavoro.

Come detto nella concezione occidentale della schiavitù ha giocato un ruolo determinante l'intervento del Cristianesimo. Le prese di posizione, più o meno rigide, dei religiosi hanno influenzato il pensiero politico e indirizzato l'atteggiamento degli individui nei confronti di questa pratica; pur tuttavia non si è mai giunti ad una condanna definitiva della stessa. L'entrata in gioco dell'elemento religioso come identificante costituisce uno dei fattori di distinzione fra l'atteggiamento delle società sedentarie e quelle nomadi verso la pratica servile. Non credo vi siano dubbi sul fatto che la diffusione del Cristianesimo in Europa abbia accentuato il carattere etno-confessionale dell'assoggettamento. Il rafforzarsi dell'identità collettiva basata sul religioso poneva dei freni all'asservimento di similes; diminuirono effettivamente i fenomeni in cui cristiani riducevano in schiavitù altri cristiani. ${ }^{161}$ La chiesa dovette riadattare la propria struttura dottrinale e talvolta ripensarne alcuni aspetti in base alla liceità davanti alla presenza di individui che possedevano altri individui. Schiavitù e libertà furono posti sul piano di valori positivi o meno, buoni o cattivi, che Dio di-

159 Si veda su questo la lucida analisi offerta da Meillassoux: Antropologia della schiavitù, il parto del guerriero e del mercante, Milano 1992, in particolare le pp. 90-133.

160 Ho qui preso in prestito il concetto di desocializzazione dall'antropologia in quanto mi sembrava pertinente. Semplificando un aspetto assai complesso potremmo dire che si ha desocializzazione di uno schiavo quando esso viene sottratto al suo ambiente sociale per essere inserito in uno nuovo e nel quale rimane marginalizzato socialmente (Meillassoux, Antropologia cit., pp. 104-105).

161 Milani, La schiavitù nel pensiero politico cit., pp. 344-345; Verlinden, L'esclavage cit., I, pp. 122 e 137-138. 
stribuisce in base a principi che l'uomo non può cogliere o può semplicemente ricondurre a meriti e colpe delle quali non è in grado di stabilire l'origine. Come ha giustamente sottolineato Piero Milani la differenza apportata dal Cristianesimo e dalla scolastica in particolare fu la speranza; speranza di riscatto, visione escatologica del percorso umano per cui è la Giustizia divina cui spetta lo scioglimento della condizione attuale. Sin dall'antichità (lo stoicismo, Seneca, i primi giuristi dell'era cristiana e i padri della Chiesa) l'idea di libertà naturale è stata sottoposta ad attente e complesse riflessioni. Tuttavia, come abbiamo sottolineato, nessuno mise in discussione la legittimità del principio; non vi furono intellettuali, religiosi o laici, che giunsero a posizioni di condanna nei confronti della schiavitù e di chi la praticava.

La chiesa romana non fu l'unica ad intervenire sull'argomento. Nella Caucasia la chiesa georgiana condannò ripetutamente e con durezza il commercio degli schiavi imponendo pene severe per chi lo praticava. Nel codice di leggi promulgato da Vakhang si arrivò a minacciare la scomunica per chi vendeva schiavi cristiani ai mercanti musulmani, ${ }^{162}$ ma questo è un discorso che ci porterebbe troppo lontano. Era utile a mio avviso richiamare alcuni atteggiamenti e le principali correnti di pensiero che hanno influenzato, più $o$ meno direttamente, l'atteggiamento degli uomini nei confronti della schiavitù in epoca medievale per poi affrontare l'altro tema, la condizione servile presso le popolazioni nomadi, la percezione che esse ebbero di tale pratica e quella che nutrirono per gli individui ridotti in schiavitù.

Dicevamo sopra che prima dell'unificazione operata da Gengis khan lo sfruttamento collettivo delle risorse naturali e quindi il raggruppamento di più clan in aree vicine erano il modello più diffuso. Ma all'inizio del XIII secolo la crescente stratificazione sociale rendeva ancor più dinamico il sistema di relazioni interne al clan. L'oboq era una realtà in continuo movimento e la sua fisionomia mutava in modo rapido. La comunità familiare era percepita come "aperta", ma in realtà non lo era poi tanto. In un modello patrilineare la discriminante fra individuo produttivo e individuo non produttivo ha a che fare soprattutto con le caratteristiche intrinseche della persona piuttosto che con il suo status sociale. Per tanto era necessaria un'apertura verso l'esterno al fine di incrementare l'entità demografica del clan e, più in generale, garantirsi una qualche continuità. ${ }^{163} \mathrm{Il}$ legame di sangue era importante, ma aveva un peso notevole anche l'acquisizione volontaria di alleanze esterne (la figura del quda di cui ci siamo occupati poco

162 Allen, A History of Georgian People cit., p. 286. Di fatto gran parte di questi provvedimenti rimasero sulla carta e raramente vennero applicati.

163 Nelle piccole comunità, come erano i nuclei familiari protomongoli, era sempre più difficile mantenere un rapporto accettabile fra elementi produttivi ed elementi improduttivi; è questo uno dei motivi che stanno alla base della naturale propensione, da parte del nomadismo, alla guerra. 
sopra ne è un esempio). Ai legami di sangue si aggiungevano quelli di amicizia giurata (la figura dell'anda). La nascita di queste figure e il rafforzamento dei legami extra-familiari progredì nei decenni successivi. La Storia Segreta è ricca di episodi del genere e mostra il numero sempre crescente di adozioni - a cavallo fra XII e XIII secolo - di giovani appartenenti ai clan sconfitti da parte di quelli che prevalevano nelle guerre. ${ }^{164}$ I fenomeni di adozione non consentivano tuttavia ai nuovi componenti dell'oboq di trarre beneficio da tutti i diritti materiali di cui godevano gli appartenenti originari (in primo luogo i pascoli ereditati dal clan stesso); ciò che invece ottenevano appieno era la protezione da parte del clan: un privilegio importante nel contesto di durevole conflitto che vigeva nei territori occupati dal nomadismo turco-mongolo nella seconda metà del XII secolo e che dobbiamo considerare all'interno di una situazione estremamente fluida (non era raro che gli appartenenti a uno stesso clan passassero in campi avversi e si combattessero a vicenda).

L'insicurezza non era solo una condizione esterna; conflitti feroci potevano esplodere anche all'interno degli stessi clan portando alla rottura dei rapporti di sangue e a maggior ragione di fratellanza giurata. ${ }^{165}$ Per lo stesso motivo il numero di persone potenzialmente assoggettabili aumentava costantemente e molti elementi dell'esercito Gengiskhanide, quando il khan lo riorganizzò, appartenevano a membri di clan vinti ed asserviti.

Dobbiamo tuttavia operare delle distinzioni per capire quale concetto avessero i Mongoli e in generale le società nomadi della servitù; un concetto assai differenziato e certamente diverso rispetto a quello che si aveva in Occidente. Esistevano vari gradi di asservimento e non tutti generavano condizioni definitive. Anche in questo caso la conquista mongola e l'espansione commerciale delle città italiane in Oriente mise in contatto due modi diversi di concepire l'utilità dell'individuo assoggettato.

Nella società mongola tradizionale il concetto di libero era strettamente legato a quello di oboq, assumeva cioè grande importanza la parentela. Gli uomini liberi erano quelli nati e formatisi all'interno della comunità, mentre era straniero colui che si era formato in un ambiente sociale diverso, estraneo. ${ }^{166}$

164 SSM, I, 14, p. 52: «strada facendo [Dobun, un antenato di Gengis] incontrò un poveraccio che conduceva con sé il figlioletto». L'uomo scambiò il figlio con della selvaggina; la Storia Segreta in questo punto dice: "[Dobun] si portò a casa il ragazzo facendone il proprio servo»: SSM, I, 16, p. 54. In SSM, III, 97, p. 83 un vecchio dice al giovane Timujin «volevo darvi allora anche questo mio figlio [...] perché tu, Temüjin, gli comandi di sellare il cavallo, perché tu gli comandi di aprirti la porta». Ancora «nell'accampamento abbandonato dei Tayčiud e dei Besüd i nostri raccolsero un bambino di nome Kököčü e lo portarono alla madre Höelün, e lei lo prese per allevarlo», SSM, III, 120, p. 97; gli esempi potrebbero continuare.

165 I casi che potremmo citare dalla Storia Segreta e dalla Summa di Rashid ad-Din sono numerosi. Basterà, a titolo esemplificativo, RaD, I, p. 52; SSM, III, 104, p. 87 e 105, p. 88; III, 116, p. 95; V, 150, p. 122.

166 Meillassoux, Antropologia cit., p. 29. 
La sovrapposizione di categorie valide per la società occidentale con fenomeni endogeni alla realtà nomade nel Medioevo non è dunque del tutto praticabile. Come dicevamo sopra, il contesto sociale delle comunità protomongole era privo di una stratificazione rigida in quanto le relazioni che regolavano le dinamiche sociali profonde si basavano sulla redistribuzione interna della ricchezza prodotta e il sovrapprodotto era assente o minimo. Pertanto, sebbene vi siano forme di asservimento rintracciabili, non possiamo parlare di schiavi, né di servi come talvolta è stato fatto e tanto meno possiamo parlare di società schiavista.

Nella società tradizionale mongola coloro i quali erano caduti in una condizione di dipendenza costituivano in generale i qaraču. Fra di essi la mobilità sociale era una realtà consolidata già prima che Timujin unificasse le genti turco-mongole. Nel periodo in cui lo sfruttamento collettivo delle risorse naturali era il fattore dominante gli oboq vivevano in comune condividendo molteplici aspetti del quotidiano. L'ulteriore frammentazione dei clan poteva essere volontaria, ma anche imposta. Quando era quest'ultima eventualità a verificarsi nascevano legami alterati e determinati dalla prevaricazione per cui un $o b o q$ vincitore poteva decidere quale sorte riservare al vinto. Generalmente si tendeva a utilizzare il nucleo vinto come nuova linfa per il proprio clan incorporandolo a questo. Allora gli individui inglobati diventavano stranieri, ma non vi era desocializzazione.

Nelle fonti la parola con la quale vengono designati gli individui o interi gruppi asserviti è bogol. La storiografia sovietica ha voluto spesso vedere nei bogol degli schiavi sottomessi e obbligati a partecipare al processo produttivo sotto il vincolo di servitù, ma l'analisi delle fonti mi pare non permetta questa equazione. La traduzione fatta da Berezin a suo tempo della parola bogol con il russo rab (cioè schiavo) ha generato un insieme di fraintendimenti dai quali si è spesso verificato il tentativo di piegare i fatti ad una concezione meccanicistica per cui nella società nomade tradizionale dovesse per forza esistere la figura dello schiavo o del servo nel senso che questa parola acquista per l'Europa del Pieno e Basso Medioevo. Il bogol non compare mai come assoggettato ad un padrone, non risulta che sia mai proprietà di un altro individuo. Le dinamiche che portavano al processo di assoggettamento erano di tipo diverso; spesso predominava la necessità di ricevere protezione, in cambio si forniva un servizio. Un passaggio della Storia Segreta recita: «Allora i fratelli [della stirpe di Gengis], in cinque, fecero prigionieri tutti quegli uomini [della tribù Jarčut] che divennero loro servi e schiavi, e lavorarono con le mandrie e in cucina». ${ }^{167}$ Gli individui incorporati nei clan dominanti entravano a far parte del processo produttivo andando ad occupare mansioni che non si differenziavano da quelle svolte dagli appartenenti originari di quel medesimo clan. Nascevano vincoli che non si concretizzavano attraverso la disponibilità arbitraria di un individuo. Carattere dominante di

167 SSM, I, 39, p. 59. 
questi vincoli era la fedeltà spesso volontaria e la necessità di rimanere presso clan forti, in grado di assicurare protezione agli elementi deboli e alla loro unica ricchezza, il bestiame. Il vincolo era raramente sancito in modo ufficiale e si poteva rompere con facilità. Tant'è vero che poter disporre di bogol era un privilegio riservato a pochi singoli individui.

Se nel XIII secolo l'uso di servi domestici era relativamente diffuso nell'Occidente europeo e in generale presso le società sedentarie, i Mongoli continuavano a possedere servi "collettivi". Una simile caratteristica è rintracciabile presso le prime comunità georgiane. Ancora nei primi secoli dell'era cristiana la stratificazione sociale era un fenomeno in lenta progressione. Solo gli appartenenti alla classe dominante, quella dei gwari, erano autorizzati a tenere dei servi. In ogni caso anche nel sistema del sagwareulo georgiano gli schiavi erano proprietà del gwari e ne facevano parte a tutti gli effetti prestando servizi assimilabili a quelli dei bogol mongoli. ${ }^{168}$

Accanto alla figura del bogol si è spesso fatto riferimento, nella letteratura scientifica sull'argomento, a quella degli unagan bogol (ütegu bogol, ongu bogol, ütele bogol) che Vladimircov tradusse come "vassalli servi". ${ }^{169}$ L'unagan bogol non era una figura molto diversa da quella del bogol. Il termine non compare nella Storia Segreta, ma Berezin lo utilizzò nella sua traduzione russa della Summa di Rashid ad-Din; ${ }^{170}$ era molto probabilmente una sorta di vassallo, un fedele costretto all'obbedienza in quanto libero, ma discendente di bogol, ${ }^{171}$ una condizione successiva e forse caratterizzata da un legame più labile con il clan dominante. Rashid ad-Din ne parla probabilmente perché quando egli scrive vi erano personaggi che, partiti da una condizione umile, erano riusciti a migliorarla e ad occupare un ruolo importante nella società, ma solo in un contesto che era quello sedentario e non più tradizionale, cioè il suo (di un funzionario persiano del XIV secolo). È nostra convinzione che le due parole designassero una condizione simile in seno alla società mongola tradizionale, con la differenza che l'unagan bogolgiunse ad avere un vincolo più stretto verso un solo individuo piuttosto che appartenere ad un intero oboq. In cambio del loro servizio i bogol ricevevano il necessario per la propria sussistenza. Non potevano scindere di loro iniziativa tale legame. Anche gli unagan bogol - se accettiamo che fossero qualcosa di diverso dai bogol in quanto rilasciati, cioè affrancati - erano qualcosa di diverso da quello che si è soliti associare al concetto di servo medievale o

168 Si veda su questo il già citato studio Allen, A History of the Georgian People cit., pp. 221-225.

169 Vladimircov, Le régime social cit., p. 78 e segg.

170 È una variante del termine mongolo ütegu, cioè separato, rilasciato. L'edizione del monumentale lavoro di Berezin uscì nell'arco di trent'anni a Sankt Peterburg: I.N. Berezin, Trudy Vostočnogo Otdelenija Imperatorskago Archeologičeskago Ob̌̌čestva, Sankt Peterburg 1858-1888, nel nostro caso: Sbornik letopisej. Istoria Mongolov sočinenie Rašid-Eddina, voll. V, VII, XIII.

171 Bold, Mongolian nomadic Society cit., p. 115. 
di schiavo classico. Le fonti ne parlano in generale come appartenenti a tribù vinte e obbligati a servire presso quelle prevalenti. Essi subivano l'imposizione di un vincolo di dipendenza, ma con delle differenze sostanziali. Innanzitutto in questo periodo non erano soggetti ad un solo padrone. Il bogol era a disposizione dell'intero oboq o di una parte di esso ${ }^{172}$ (una famiglia) anche se non era considerato una proprietà a tutti gli effetti: conservava quasi sempre i suoi beni, ${ }^{173}$ non doveva la totalità del proprio lavoro al clan cui era assoggettato; il suo servizio consisteva essenzialmente nei compiti tipici della produzione nomade: portare gli animali al pascolo, seguire la transumanza, la cucina e soprattutto servire il proprio oboq in tempo di guerra fornendo prestazioni militari; manteneva una relativa libertà personale. ${ }^{174}$ Proprio grazie alle prestazioni militari poteva innalzare la sua condizione sociale. La mobilità non è un fatto ricorrente nelle fonti, ma i noyon avevano possibilità che altri non avevano, prerogative esclusive frutto del ruolo che essi occupavano in quanto clientela armata del khan e spesso potevano affidare compiti di alta responsabilità anche ai bogol. Di fatto l'economia nomade era troppo poco diversificata perché vi fossero strati sociali sistematicamente impegnati nel processo produttivo.

Gengis Khan rivolto ad un anziano del suo ulus, Usun, dice: «esiste da noi Mongoli la tradizione di elevare alla dignità di noyon, di beki, coloro che lo meritano». Il khan poteva innalzare alla dignità di noyon anche i bogol che si erano distinti per la loro fedeltà e il loro coraggio durante le guerre contro le tribù antagoniste. ${ }^{175} \mathrm{La}$ posizione occupata dai bogol all'interno della società mongola tra XII e XIII secolo dipendeva verosimilmente dalla condizione dell'oboq cui questo era soggetto. Era piuttosto frequente il caso in cui a cadere sotto il vincolo di assoggettamento fossero interi oboq. ${ }^{176} \mathrm{La}$ condizione degli unagan bogol era ereditaria. ${ }^{177} \mathrm{~A}$ tale proposito è emblematico un passo della Storia Segreta; dopo la sconfitta dei Kerait e la successiva spartizione del bottino da parte dei Mongoli vincitori, un membro della tribù dei Žirgin di

172 Ad esempio quando i Mongoli sconfissero i Merkit «presero prigioniere tutte le donne fino all'ultima e tutti i bambini. Distrussero i sacri ripari catturando tutto quanto l'ulus», SSM, III, 109, p. 91.

173 Secondo RaD (III, p. 126) una parte del popolo Žalair era da tempo unagan bogol del clan di Borižigin. Un membro di questo clan viveva indipendentemente dal clan medesimo e con cavalli di sua proprietà; si veda a tal proposito anche Vladimircov, Le régime social cit., p. 84.

174 Ibidem, p. 80.

${ }^{175}$ Ad esempio in SSM, IX, 212, pp. 187-188; e in particolare Ibidem, p. 191. Quest'ultimo episodio è riportato in Ibidem, IX, 219, p. 193. Il coraggio e la fedeltà erano doti fondamentali per ottenere il favore del Khan. Oltre ad una rapida ascesa sociale tali meriti determinavano concessioni di vario tipo. Poteva essere l'attribuzione di una bella moglie o di un gruppo di bogol (Ibidem, VIII, 208, p. 185), ma anche un territorio per il pascolo (Ibidem, IX, 219, p. 192).

176 Ibidem, III, 137-139, pp. 109-110.

177 Si veda su questo punto Vladimircov, Le régime social cit., pp. 82-83; SSM, III, 137, p. 109; Ibidem, III, 139, p. 110; Ibidem, V, 154, p. 126; Ibidem,VII, 186, p. 155. 
nome Qadag-Bagatur riuscì a far fuggire il suo signore, il Van khan dei Kerait. Ammirato dal coraggio dell'uomo, che a rischio della vita, era riuscito a salvare quella del suo signore, Timujin lo graziò e stabilì che "Qadag Bagatur e cento Žirgin servano la sua famiglia» (di Quyildar, un mongolo deceduto nella battaglia contro i Kerait). «E i figli loro siano servi dei figli e dei nipoti di Quyildar. E i genitori Žirgin non potranno maritare le loro figlie a chi vogliono. Siano essi servi dei membri della famiglia di Quyildar, precedendoli e accompagnandoli». ${ }^{178}$ Spesso fra clan dominanti e clan asserviti si verificavano unioni matrimoniali che potevano mutare lo stato sociale dei secondi elevandolo. Non è infatti raro trovare nelle fonti accenni a clan di bogol le cui condizioni economiche erano relativamente buone. ${ }^{179}$ Gli unagan bogol erano una massa la cui composizione era complessa e potevano essere parte della classe dominante del clan ma anche di gruppi socialmente più deboli. Nel primo caso la loro condizione era migliore, nel secondo svolgevano mansioni più umili, ma sempre rimanendo proprietari di quei beni strettamente legati alla loro attività (bestiame e attrezzi per il pascolo e per la fabbricazione del kumys). Come abbiamo detto la sconfitta di intere tribù poteva coincidere col loro asservimento; ad esempio dopo aver soggiogato i Kerait Gengis si rivolse ai propri partigiani e «distribuì fra tutti i prigionieri Kerat in quantità. Il tümen dei Tübegen fu distribuito fino all'ultimo uomo». ${ }^{180}$

Dopo la conquista le cose però cambiano in parte. Con l'intensificarsi delle conquiste e quindi degli scontri fra tribù, si assiste, sul finire del XII secolo, ad un mutamento della condizione dei bogol; la transizione da un'organizzazione collettiva della società ad una più individuale si accompagnò anche ad un'accresciuta individualità nella pratica servile. I bogol diminuiscono nelle fonti a vantaggio dei servi di un solo padrone. Accanto a essi compare, nella Storia Segreta e nella Summa di Rashid ad Din, anche un'altra figura: quella degli inžä, ovvero servitori di casa; individui che prestavano la loro opera a singole persone e nella loro abitazione. Potevano entrare nella dote delle giovani donne. ${ }^{181}$ Adesso i bogol potevano essere affrancati divenendo darqan. ${ }^{182}$ L'emergere di queste nuove figure è il frutto di più fattori tra i quali giova ricordare l'incremento demografico generato dalle conquiste di Gengis khan, l'accrescimento dei Mongoli mediante assorbimento di gruppi rivali e, come dicevamo sopra, l'inglobamento di una realtà sedentaria il cui effetto fu soprattutto il consolidamento della stratificazione sociale.

178 Ibidem, VI, 185, p. 154.

179 Vladimircov, Le régime social cit., p. 84; RaD, I, pp. 169-170 e III, p. 126.

180 SSM, VII, 187, p. 156.

181 Vladimircov, Le régime social cit., p. 85; RaD, I, p. 194.

182 «Qulan-Bagatur ebbe per figlio Yeke-Čeren. Fu lui il noyon di Badai e Kišlig, darqan, cioè servi emancipati», SSM, I, 51, p. 62. 
La società mongola tradizionale non può dunque essere considerata come schiavista nel senso che ricorre sistematicamente alla manodopera di schiavi, né di servi, per attendere alle proprie necessità produttive. In essa non vi era un insieme di funzioni assolte istituzionalmente da una classe di individui e in modo permanente tale da permettere ad un ceto sfruttatore di aver garantite certe mansioni ed esserne quindi definitivamente liberato.

L'incontro fra due concezioni dell'asservimento così diverse - da una parte la schiavitù "assente" della società tradizionale mongola e dall'altra l'ideologia commerciale del ceto mercantile urbano - ha prodotto risultati, per lo studioso, molto interessanti. Il commercio degli schiavi ha investito direttamente la Caucasia ed ha avuto conseguenze sulla popolazione niente affatto trascurabili. Nell'ambito spaziale del vasto impero mongolo la funzione commerciale esercitata dagli operatori musulmani del Turkestan e delle regioni sotto il dominio Ilkhanide fu determinante nell'incremento della compravendita di schiavi. L'Islam aveva in sé, e prima che venisse a confronto coi Mongoli, creato le condizioni sociali necessarie allo sviluppo di un'economia individualistica finalizzata alla realizzazione del profitto. ${ }^{183}$ La società di classe, in cui lo schiavo è un individuo totalmente desocializzato e talvolta desessualizzato, era una realtà consolidata alla vigilia dell'invasione mongola. Con questo aspetto i nuovi dominatori dovettero confrontarsi.

Gli eventi politici, dai primi decenni del XIII secolo in poi, favorirono il reclutamento di persone in questa regione e l'efficienza con la quale gli operatori occidentali e mediorientali si occuparono del traffico fu notevolissima. Nel XIII secolo l'esplosione demografica di gran parte delle città europee e la crescita economica generalizzata fu un fenomeno quasi contemporaneo alle invasioni mongole in Oriente. $\mathrm{Al}$ commercio degli schiavi già fiorente nei Balcani, ${ }^{184}$ cui partecipava soprattutto Venezia, si aggiunse quello orientale tanto che il Mar Nero divenne il bacino di reclutamento più fruttuoso dell'intero mondo di allora. ${ }^{185} \mathrm{Il}$ commercio degli schiavi crea sempre l'offerta nelle aree circostanti a quelle della cattura (o del reclutamento). D'altra parte la schiavitù ha origine in un contesto commerciale poiché gli schiavi vengono scambiati dove si scambiano anche gli oggetti del loro lavoro. Tuttavia essa ha sempre avuto, anche in Occidente, uno stretto legame con vicende di tipo politico e militare. Quando, ad esempio, giunse in Europa l'onda lunga delle crociate e della dominazione

${ }^{183}$ Meillassoux, Antopologia cit., p. 236.

184 Balard, La Romanie génoise cit., pp. 797-798.

185 Verlinden, Aspects de l'esclavage cit.; Id. Le recrutement des esclaves à Genes già citato.; Id. Esclaves et ethnographie già citato.; Id. La colonie vénitienne de Tana centre de la traite des esclaves au XIVe et au début du XVe siècle, in Studi in onore di G. Luzzatto, Milano 1950, vol. 2, pp. 1-25; id., L'esclavage cit.; D. Gioffrè, Il mercato degli schiavi a Genova nel secolo XV, Genova 1971; G. Balbi, La schiavitù a Genova tra i secoli XII e XIII, in Mélanges offerts à René Crozet, Poitiers 1966; Malowist, La schiavitu cit. 
latina nell'Oriente il numero degli schiavi saraceni era predominante sui mercati italiani e non solo. ${ }^{186}$ La penetrazione veneziana e soprattutto genovese nel Mar Nero, dalla fine del XIII, mutò questo stato di cose, tanto che gli schiavi tartari divennero i più numerosi. ${ }^{187}$ Inoltre le città occidentali in crescita necessitavano di nuova manodopera, i mercanti avevano disponibilità maggiori e a ciò si aggiunga la singolare situazione dell'Egitto, dove i Mamelucchi non erano altro che degli intrusi i quali fornivano protezione armata alla popolazione in cambio di un vero e proprio mantenimento. ${ }^{188}$ Erano turchi in un paese arabo che avevano estremo bisogno di manodopera, sia per l'esercito, sia per la produzione. I Mamelucchi controllavano gran parte dei mercati mediorientali dell'epoca: Beirut, Damasco, Alessandria e naturalmente il Cairo. Si veniva pertanto a creare un reticolo commerciale per cui i mercanti occidentali, Veneziani e Genovesi, utilizzavano i loro emporia sul Mar Nero per acquistare schiavi e rivenderli proprio ai Mamelucchi. In questo modo essi potevano garantirsi la benevolenza dei sultani turchi, continuare a frequentare i porti sud-orientali e mantenere elevato il volume dei loro traffici. Tuttavia le istituzioni ecclesiastiche non potevano trascurare che l'Egitto era un paese musulmano, di infedeli, e l'afflusso costante di persone asservite - sovente circassi, russi o caucasici di rito cristiano - era condannato con durezza dalla Santa Sede. Si è spesso tentati di osservare questo fenomeno dal punto di vista occidentale, ma dobbiamo tener conto della rigidità dottrinale islamica che ebbe sulla schiavitù effetti simili nelle società musulmane rispetto a quelli avuti dal Cristianesimo in Europa. L'infedele è straniero, è assoggettabile, ma non lo è il musulmano. Non è quindi casuale che i principali referenti commerciali dei mercanti occidentali nel traffico di schiavi fossero proprio i Mamelucchi egiziani.

186 D. Gioffrè, Il mercato degli schiavi cit., p. 13.

187 Lintervento occidentale nel commercio di schiavi sui mercati di Levante è da far risalire a ben prima del XIII secolo, ma fino a questo periodo esso non assume dimensioni rilevanti; Balard, La Romanie génoise cit., p. 289; G. Balbi, La schiavitù a Genova cit.; C. Verlinden, Le recrutement des esclaves à Genes cit.

188 Sui rapporti fra i Mamelucchi e i Mongoli cfr. R. Amitai-Preiss: Mongols and Mamluks: The Mamluk-Ilkhãnid War 1260-1281. Cambridge, 1995, Cambridge 1995. 


\section{L'OPERA DI TIMUČIN \\ E L'ESPANSIONE FRA CAUCASO E AZOV}

La figura di Gengis khan ha da sempre suscitato sentimenti forti. I contemporanei ne ebbero una percezione contraddittoria, determinata dal mito delle grandi conquiste compiute con una rapidità e un'efficacia che lasciavano sbalorditi unita alla ferocia che seguiva, e spesso precedeva, le sue azioni militari. Anche gli storici si sono spesso soffermati sull'aspetto leggendario del khan, talvolta esaltandone le doti di condottiero, talaltra raschiando appena la superficie sulla quale appariva soprattutto l'efferatezza del soldato. Recentemente l'approccio alla figura dell'imperatore dei Mongoli è stato più rigoroso, attento alla realtà presentata dalle fonti. Ne emerge un quadro assai complesso il cui carattere dominante sembra essere quello di un abile uomo politico, che seppe utilizzare gli strumenti a sua disposizione per raggiungere obiettivi spesso pianificati con maniacale attenzione. La sua impresa più complessa, l'unificazione delle tribù turco-mongole, fu però la conseguenza di una situazione preesistente. L'opera di Gengis non mutò nella sostanza alcun equilibrio sociale, almeno nei venti anni successivi le conquiste, semmai gettò le basi per gli sviluppi che si verificarono dopo la sua morte e accelerò processi già in atto. Egli si limitò a tenere in piedi l'impalcatura basata sull'antica fisionomia tribale del nomadismo in cui l'oboq era comunque il nucleo organizzato sostanziale. La sua azione, nei primi anni dell'impero, si concretizzò nell'imposizione di tale modello organizzativo a un numero notevolmente allargato di tribù, esito delle conquiste, le quali erano adesso parte dell'unico grande ulus imperiale, quindi legate da un vincolo di appartenenza al khan; i personaggi eminenti di ogni singolo clan/oboq, i noyon, erano d'altra parte legati al Gran khan da un vincolo di fedeltà che, come abbiamo visto, prevedeva il servizio reso sotto forma di clientela armata. In questo senso Gengis introdusse davvero un elemento di novità: la fedeltà dell'aristocrazia nomade fu rafforzata e indirizzata verso la figura dell'imperatore. In pratica il quadro sociale divenne più stabile, segno di una gerarchizzazione la cui rigidità poteva essere allentata solo dai provvedimenti del khan. Contemporaneamente rimasero intatti tutti i legami che univano gli strati inferiori della struttura sociale. Gengis, ed è l'altra grande novità prodotta dall'unificazione, procedette a una generale riorganizzazione che interessò ogni componente del nuovo impianto politico; l'indiscutibile genio di cui era dotato gli permise di capire rapidamente che, solo utilizzando questa nuova e accresciuta forza come un unico grande popolo, solidale alla vita dell'impero e alla 
composizione dell'esercito, poteva sfruttarne al meglio le potenzialità. Riuscì a porre fine alle continue lotte fra le tribù turco-mongole garantendo una nuova, benché relativa, sicurezza nella steppa.

Il vasto organismo che egli creò resse coi suoi successori immediati, i figli, ma entrò in crisi già alla terza generazione a causa di una serie di circostanze che non rientrano integralmente nello scopo della nostra indagine e d'altra parte meriterebbero una monografia a sé. In estrema sintesi, e per riprendere quanto accennato nel capitolo precedente, una delle ragioni sta proprio nell'origine stessa dei Mongoli Gengiskhanidi: il fatto che fossero un'entità unitaria imposta, frutto di necessità impellenti molto più che di un'integrazione ideologica solida. Inoltre non va dimenticato che la successione all'interno del clan seguiva logiche distributive che tendevano alla frantumazione. Limpero mongolo cioè nacque già diviso. Ogni figlio del khan (ma poteva essere un fratello, un cugino, uno zio, chiunque in quel momento godesse della stima del khan medesimo) ereditava una porzione del territorio paterno in base alla successione anagrafica, ma con criteri diversi da quelli in uso presso la maggior parte dei regni occidentali. ${ }^{1}$ Fatto si è che la conseguenza più evidente della frantumazione verificatasi pochi anni dopo la morte del khan fu la divisione dell'impero in parti diverse, tutte direttamente dipendenti dalla sede del Gran khan (che alla morte di Gengis fu Ogödeï, il terzo figlio e non il primogenito Giuči). Tale dipendenza dalla "sede centrale" si rivelò ben presto un fatto solo nominale tanto che gli ulus periferici ottennero unampia autonomia andando a costituire stati indipendenti il cui sviluppo si staccò nettamente dal progetto originario e unitario di Gengis khan. Tutto questo non modificò la sostanza delle conquiste. Quella che si è soliti definire come pax mongolica fu una situazione oggettiva in seguito alla quale l'Oriente divenne un mondo finalmente aperto e raggiungibile. Ecco, credo che se non si apprezza nella sostanza questo esito delle conquiste di Gengis e dei suoi successori riesce difficile capire anche perché dalla seconda metà del XIII secolo viaggiatori occidentali siano potuti andare fino in Cina e la poderosa macchina commerciale delle città marinare italiane (e non solo la loro) abbia potuto dirigersi sino al Caspio, alla Russia, alla Persia, alla Cina traendone profitti eccezionali e stabilendo in quelle regioni sedi stabili, emporia solidi e ben organizzati. Se la quarta crociata aveva, da un lato, annullato "l'ostacolo" bizantino, le conquiste mongole avevano letteralmente spianato la strada dell'estremo Oriente. Coi primi trent'anni del XIII secolo si venne cioè a creare una situazione politica nuova.

Per capirne le conseguenze proviamo a percorrere le tappe fondamentali della conquista a Ovest dei Mongoli con particolare riguardo alla Caucasia;

${ }^{1}$ Si ricordi quanto abbiamo detto nel capitolo precedente; il figlio maggiore ereditava la porzione di ulus più lontana dal campo in cui viveva l'oboq di origine; ques'ultimo spettava al figlio minore, il guardiano del focolare. Gli altri figli non avevano diritti predeterminati, dipendeva dalla volontà del padre. 
quali furono le ragioni che spinsero l'esercito mongolo in questa direzione non è ancora oggi chiaro. Le ipotesi si sono succedute; la crescente desertificazione e la necessità di trovare nuovi territori, più fertili e meglio sfruttabili sono fra le cause di questo fenomeno. ${ }^{2}$ Per parte mia ritengo più interessante analizzare le conseguenze di questo evento, i passaggi che dettero luogo a un'ampia riorganizzazione territoriale e politica, un processo che coinvolse ampi spazi in modi diversi. Il nuovo assetto dei territori conquistati dai Mongoli, dal Caucaso al Mar d'Azov, fu il risultato di un processo apparentemente e cronologicamente rapido, ma nella sostanza piuttosto lungo e complesso. L'aristocrazia degli stati conquistati, dal potere centrale alla feudalità periferica, dovette accettare la nuova dominazione - e in molti casi vi si adattò - ma se quest'ultima ebbe conseguenze immediate da un punto di vista politico, non si verificò mai, d'altra parte, una vera assimilazione culturale. Sono aspetti complessi, che meritano di essere trattati con ordine.

\subsection{Tempi e modi dellinvasione ad Ovest}

La prima spedizione promossa ed effettuata dall'ulus di Gengis khan fuori dai territori della steppa si verificò contro la Cina. All'inizio del XIII secolo questa era divisa in tre stati diversi. A nord-ovest l'impero del Minyak, a nord l'impero Chin degli Jurčen e a sud l'impero Sung. ${ }^{3}$ In un tempo relativamente breve i Mongoli riuscirono a conquistare l'intero territorio cinese a nord e a sud della Grande Muraglia. Nell'aprile del 1214 ebbe inizio l'assedio a Pechino, capitale dell'impero Chin che l'anno successivo, giugno 1215, cadde. I fatti seguenti la conquista a Est videro l'esercito mongolo impegnato contro il sultano della Korazmia, Muhammed prima e il figlio del medesimo, Jalal ad-Din, poi; entrambi, dopo un lungo inseguimento, vennero travolti dall'urto mongolo. Per molto tempo si è insistito sul ruolo della campagna contro il sultano e sul fatto che esso avrebbe costituito il pretesto principale per l'avanzata a Ovest. I Mongoli erano attratti dalla civiltà urbana non in quanto tale (era un modello estraneo alla loro tradizione e verso alcuni aspetti di essa nutrivano una certa diffidenza), ma perché in grado di produrre ricchezza. In effetti, come notò a suo tempo Bartold nella sua monografia sul Turkestan, la conquista della Cina valse a Gengis khan una notevole fama anche oltre i confini del suo ulus. ${ }^{4} \mathrm{La}$ Cina era un paese in cui il diffuso benessere costituiva una forte attrattiva per i sovrani musulmani della Korazmia e la notizia che i Mongoli erano riusciti a

2 Tenderei ad escludere un eventuale incremento demografico poiché i Mongoli Gengiskhanidi erano, alla fine del XII, decisamente pochi.

${ }^{3}$ Di Cosmo, Ancient China cit. Cfr. anche Hoàng, Gengis Khan cit. p. 177; Grousset, L'empire des steppes cit. pp. 243-273; Bartold, Turkestan cit. p. 381-384.

${ }^{4}$ Ibidem, p. 393. 
conquistarla dovette suscitare in loro una profonda impressione. Si ha indicazione di un'ambasciata inviata dal sultano della Korazmia alla fine del 1215.5 Gengis ricevette i messi con molte aspettative in quanto era convinto che il sultano gli riconoscesse un'autorità frutto delle sue imprese militari. In pratica voleva che, come padrone dell'Oriente, il sultano accettasse di giurargli fedeltà e governasse l'Occidente sotto la sua protezione. ${ }^{6} \mathrm{Da}$ abile politico quale era Gengis si rendeva perfettamente conto di quanto fosse importante assicurare unità a un numero di territori più vasto possibile. Egli, appartenente a una cultura nomade e a essa fortemente legato, comprendeva quanto potesse essere produttivo uno stretto legame con economie sedentarie e quanto le produzioni di queste fossero utili per il suo popolo. ${ }^{7}$ Panni, ma anche, grano erano prodotti dei quali i Mongoli apprezzavano l'utilità e che non erano in grado di produrre; per non parlare poi del vino del quale erano ghiotti. È questa una delle ragioni dell'attacco alla Cina, a mio avviso la principale. Nelle terre del Turkestan, dove l'economia sedentaria era una realtà consolidata e prosperosa, i mercanti traevano enormi profitti dal commercio con i paesi vicini, Rus' e Anatolia in particolare. Ma le forme di pagamento prevedevano spesso dilazioni per cui ogni conflitto o impedimento rischiava di provocare danni enormi ai mercanti stessi. Tant'è che, ad esempio, la spedizione selgiuchida contro l'impero di Trebisonda, dei primi anni del XIII secolo, causò la rovina di molti mercanti; ${ }^{8}$ lo stesso avvenne durante la prima incursione mongola nel Caucaso e nella successiva battaglia contro Mstislavl di Černigov. ${ }^{9}$

Il commercio terrestre dei mercanti musulmani del Turkestan con la Cina era molto intenso all'inizio del XIII secolo e la conquista mongola del 1214-1215 aveva allarmato il sultano il quale probabilmente temeva un irrigidimento dei rapporti col Paese orientale. E dunque possibile che Gengis non avesse alcuna intenzione di muovere guerra contro il sultano e che anzi preferisse di gran lunga mantenere sicure le strade di collegamento fra i suoi territori e la Korazmia. Si trattava a questo punto di verificare quali margini di trattativa esistessero perché il sultano accettasse una pacifica sottomissione finalizzata alla messa in sicurezza dei territori che, a questo punto, avrebbero abbracciato un'estensione

5 Ibidem, pp. 393-394. È in questa occasione che lo storico Juzjani, citato da Bartold, riporta la descrizione delle scene di devastazione che erano "ovunque visibili» alle porte di Pechino dalle mura della quale «le giovani donne si gettavano l'una l'altra per scampare la furia dei Mongoli».

${ }^{6}$ Era parte del sistema ideologico tradizionale mongolo la convinzione che il khan, investito dal Cielo (Tenngri), dovesse governare il mondo e porre sotto la propria protezione i sovrani antagonisti. Si veda su questo il saggio già citato di Golden, Imperial Ideology cit.

7 «Negli ultimi anni del suo regno egli [il sultano] aveva procurato pace e calma assoluta, e sicurezza e tranquillità, e aveva conseguito il massimo della prosperità e del benessere; le strade erano sicure e le sommosse sedate: di guisa che ovunque vi fosse guadagno o profitto, nell'estremo Occidente come nel più lontano Oriente, ivi si dirigevano mercanti» (Juvaini, I, p. 98).

${ }^{8}$ Juvaini, II, p. 395.

9 Ibn al-Athir, II, pp. 457-460. 
notevolissima: dalla Cina al Caspio. Naturalmente la pace cui Gengis aspirava doveva concludersi alle sue condizioni, ma in Juvaini si ha il racconto degli eventi, forse un po' sbilanciato a sfavore del sultano, ritenuto responsabile da tutti gli storici a lui contemporanei di aver attratto l'ira dei Mongoli e aver così, sebbene indirettamente, promosso la distruzione dei Paesi che essi conquistarono. Juvaini dice che tre mercanti musulmani (dei quali fornisce il nome e le merci che avevano con loro) ${ }^{10}$ si recarono nei territori dei Mongoli per commerciare. Quando giunsero alla frontiera, le guardie che avevano il compito di controllare le merci in entrata si resero conto dell'elevata qualità di quanto portavano i mercanti e li inviarono direttamente dal khan, come questi aveva ordinato. In quegli anni Gengis aveva promulgato uno yasa (una legge) con il quale garantiva la piena immunità ai mercanti che avessero avuto intenzione di recarsi nei territori a lui sottomessi. ${ }^{11}$ Gengis apprezzò molto la mercanzia che portavano i mercanti, la pagò adeguatamente e li rimandò indietro insieme a mercanti dei suoi territori per portare un messaggio di pacificazione al sultano. Questa iniziativa - non sappiamo quanto reale - ebbe un esito tragico a Utrar, città posta al confine orientale del territorio di Korazmia. Stando ai racconti di Ibn al-Athir e Juvaini, ${ }^{12}$ Gengis fu comunque soddisfatto del risultato diplomatico. Fatto si è che nello stesso anno 1218 la carovana dei mercanti in provenienza dalla Mongolia giunse a Utrar. Il governatore della città sospettò i mercanti di essere spie. Non vi sono dati certi sulla dinamica degli eventi, è dubbio cioè se fu il governatore o il sultano in persona a prendere una decisione sulla sorte dei mercanti. Il fatto sicuro è che questi furono massacrati. Secondo Juvaini tutta la carovana morì in questa occasion $\mathrm{e}^{13}$ tranne uno che riportò la notizia a Gengis. La reazione del khan fu piuttosto composta se è vero che si limitò a inviare tre persone presso il sultano per chiedere spiegazioni e farsi consegnare

${ }^{10}$ Juvaini, I, p. 98: «[... e dopo aver radunato una quantità smisurata di mercanzia - stoffe arabescate d'oro, cotoni, zandanichi [le stoffe di Zandana, un villaggio nelle vicinanze di Bukhara] e quant'altro ritennero adatto - si misero in cammino».

${ }_{11}$ Su alcuni degli yasa promulgati da Gengis Khan si vedano Bar Ebreo, Chronicon cit., p. 412; Spuler, History of the Mongols cit., pp. 40-41. Di notevole interesse è la risposta che Güyük fa pervenire a Innocenzo IV dopo l'ambasciata di Giovanni di Pian del Carpine. In un passo il khan mongolo afferma «se tu agisci in accordo con le tue parole, allora vieni; tu, il gran papa e i re, tutti di persona a rendere omaggio a noi. Allora potremmo far conoscere gli yasa che già esistono" riferendosi alle leggi promulgate entro i confini dell'impero e in vigore nei territori del medesimo, cfr. Ibidem, p. 68.

12 Bartold, Turkestan cit. p. 398; Juvaini, I, pp. 100-101.

13 «Prima che giungesse quest'ordine [l'uccisione di tutti i mercanti], uno dei mercanti escogitò uno stratagemma e sfuggì alle strettezze della prigione» (Juvaini, I, p. 101); Bartold, Turkestan cit. p. 399. Data la dinamica narrata dallo storico persiano è probabile che egli non conoscesse i fatti nel dettaglio e abbia costruito la storia dell'unico sopravvissuto in grado di riportare personalmente la notizia a Gengis khan. Indipendentemente da questo, il massacro dei mercanti a Utrar è confermato da altre fonti attendibili (da questo punto di vista lo è senza dubbio anche Juvaini) per le quali si veda Bartold, ed. russa, vol. I. 
il governatore di Utrar ritenuto il responsabile degli eventi. ${ }^{14}$ La risposta del sultano fu alquanto esplicita: i tre ambasciatori furono uccisi. ${ }^{15}$ A questo punto il casus belli era creato e l'invasione della Korazmia ampiamente giustificabile (ammesso che a Gengis interessasse avere una giustificazione per muovere guerra contro qualcuno). Bartold sostiene ${ }^{16}$ che in un primo momento Gengis aveva un'alta considerazione delle capacità militari del sultano e dell'efficacia del suo esercito; non voleva pertanto scatenare una guerra della quale non avrebbe potuto prevedere l'esito. Da questo punto di vista i Mongoli erano molto accorti, non intraprendevano mai campagne militari alla cieca. Si informavano sempre con accuratezza facendo perlustrare minuziosamente i territori sui quali avrebbero dovuto combattere e non è escluso che le ambasciate inviate nel 1218 avessero anche questo scopo. Resta il fatto che l'uccisione dei mercanti e la successiva eliminazione dei messi legittimava anche giuridicamente l'intervento militare contro il sultano. Juvaini e prima di lui Ibn al-Athir e Nasawi, sono molto precisi nell'indicare i tempi e i modi dell'invasione a Ovest da parte dell'esercito mongolo. Senza fare la cronistoria delle conquiste ritengo utile fissare alcuni punti che possono chiarire i successivi eventi che investirono la regione caucasica.

\subsection{La prima campagna contro il sultano di Korazmia e l'invasione del Turkestan}

Nell'estate del 1219 l'esercito mongolo si riunì sull'Irtysh pronto a scatenarsi contro il sultano della Korazmia. ${ }^{17}$ Nell'autunno dello stesso anno giunse nella regione anche Gengis, che si fermò a Qayaligh. ${ }^{18}$ Stando ai calcoli dei turcologi che si sono occupati di queste vicende, ${ }^{19}$ l'esercito mongolo disponeva in questo momento di circa 150-200.000 uomini cioè un numero molto inferiore a quello di cui poteva disporre il sultano. Uno dei fattori di debolezza degli eserciti che si trovarono ad affrontare i Mongoli era costituito dalla scarsa unità dei primi rispetto alla formidabile compattezza dell'esercito invasore. Di questo aspetto sono stati spesso indicati come responsabili nelle fonti i dirigenti dei centri attaccati. Va però sottolineato che in questo periodo l'esercito del sultano non poteva che essere diviso e frammentato. Esso era il risultato di una situazione d'emergenza in cui

${ }^{14}$ Juvaini, I, p. 102.

15 Mohammed en-Nasawi, Histoire du sultan Djelal ed-Din Mankobirti, prince du Kharezm, trad. e a cura di O. Houdas, Paris 1895 (d'ora in poi Nasawi), pp. 60-61.

${ }^{16}$ Bartold, Turkestan cit. pp. 396-397.

17 Juvaini, I, p. 107; Grousset, L'empire des steppes cit. pp. 298-299.

18 Qayaligh, a sud-est del lago Balkhash, oggi al confine fra il Khazakistan orientale e la Cina, era nelle mani dei Turchi Qarluc. Vi regnava Arslan il quale chiese il riconoscimento in quanto vassallo a Gengis khan e lo ottenne (Juvaini, I, pp. 95-97); egualmente si unirono all'esercito mongolo, accettando il vincolo di sottomissione, il re degli Uighuri Bartchuq e il governatore di Almaligh, «la città più importante di quella regione» (Juvaini, I, p. 95).

19 Bartold, Turkestan cit. 404; Grousset, L'empire des steppes cit. p. 299. 
l'aristocrazia locale, atabeg, vizir ed emiri, aveva accettato la guida di Mohammed solo perché seriamente preoccupata dell'avanzata mongola. La milizia del sultano era guidata da esponenti di quei ceti eminenti che governavano in totale autonomia nelle città del Turkestan e della Transoxiana. Ora questa autonomia veniva congelata senza garanzie su un suo futuro ripristino. Non potevano riconoscere alcuna autorità consolidata al sultano; oltre a questo si hanno notizie, soprattutto in Juvaini, di una scarsa stima, da parte dei generali musulmani, nei confronti delle capacità militari del sultano medesimo. ${ }^{20}$ Infine, quando Mohammed ebbe imposto la propria sovranità sugli stati della Transoxania perseguì una politica che si sarebbe rivelata disastrosa per le sorti dei sultanati. Imponeva tributi sempre più alti alla popolazione senza riuscire mai a ottenere vittorie importanti che potessero giustificare tali prelievi. Nasawi, la cui attendibilità è fuori discussione, sostiene che dopo aver fatto assassinare gli ambasciatori dei Mongoli il sultano corse ai ripari in modo del tutto scoordinato:

La prima misura che [il sultano] pensava di prendere in queste circostanze critiche [...] fu di circondare, malgrado la sua immensità, la città di Samarcanda di mura il cui sviluppo, per ciò che se ne sappia, sarebbe dovuto essere 12 farsakh, ${ }^{21} \mathrm{e} \mathrm{di}$ installarvi una guarnigione stabile. ${ }^{22}$

Ancora, la seconda misura presa dal sultano fu l'invio di nuovi funzionari col compito di prelevare imposte (la terza volta nello stesso anno) e uomini da addestrare come arcieri; ${ }^{23}$ la situazione divenne tesa al punto che i notabili di Baghdad, guidati dal principe della città an-Nasir li-Din-Allah, gli schierarono contro l'esercito senza però alcun esito. ${ }^{24}$

Lo scarso coordinamento risultò nella distribuzione eccessiva delle risorse sul territorio. Anziché opporsi ai Mongoli concentrando le forze nelle città strategiche, i generali del sultano si stabilirono col grosso dell'esercito lungo la linea del Sir Darya presidiando città molto distanti fra di loro (come Žend, Sighnaq, Utrar). Nasawi dice che

un'altra misura funesta fu quella che prese il sultano di disseminare le sue truppe nelle città della Transoxiana e del Turkestan. Così lasciò Inal-Khan (il governatore oggetto della discordia) a Utrar con ventimila uomini, Qotlo-

20 È un aspetto questo sul quale ha molto insistito Bartold (Bartold, Turkestan cit. pp. 404406) e che viene sottolineato con forza da Nasawi (Nasawi, p. 61). Anche Juvaini ne fa menzione (Juvaini, I, p. 142).

${ }^{21}$ Farsakhlfarsang è termine persiano e proviene dall'antica unità di misura, parasang, che un cavallo era in grado di percorrere in un'ora; corrispondeva a circa 4,8 chilometri. Nel XIX secolo la farsakh persiana erano $6,23 \mathrm{~km}$, mentre quella araba erano $5,76 \mathrm{~km}$.

${ }^{22}$ Nasawi, p. 61

${ }^{23}$ Ibidem, p. 62.

${ }^{24}$ Juvaini, II, pp. $472-477$. 
gh-Khan con un gruppo di diecimila cavalieri a Chehrkent, l'emiro Ikhtijar ed-Din Kechki, [...] con trentamila uomini a Bukhara. ${ }^{25}$

I Mongoli agirono come loro solito dividendo le ali dei loro tümen e avanzando sulla base di una buona conoscenza del territorio e delle città che avevano ben studiato in precedenza. Una prima divisione, al comando del khan in persona, avanzò su Utrar, un'altra al comando di Čagataï e Ogödeï rimase a presidiare le retrovie, mentre una terza divisione, comandata da Giuči (il primogenito di Gengis) scese lungo il fiume e si diresse contro Sighnaq e Žend (al confine fra l'Uzbekistan e il Kazakistan).

Dopo un lunghissimo assedio Utrar cadde:

La cittadella e le mura vennero rase al suolo e i Mongoli se ne andarono. E portarono seco la gente comune e gli artigiani che erano sfuggiti alla spada, o perché prestassero servizio come coscritti, o perché esercitassero il loro mestiere. $^{26}$

La presa di Utrar fu fondamentale per gli sviluppi militari successivi perché aprì la breccia sulla linea del Syr Darya consentendo all'esercito mongolo di penetrare a Ovest. Tuttavia la campagna di Gengis prevedeva l'attacco totale alla regione e l'assoggettamento di tutte le città più ricche della Korazmia. Dopo aver preso Utrar, il Gran khan si diresse con il figlio Tuli su Bukhara. ${ }^{27}$ "Essa è la cupola dell'Islam ed è, in quelle regioni, pari a Baghdad, la città della Pace»; ${ }^{28}$ Bukhara cadde nel febbraio del $1220 .^{29}$ La battaglia per la presa della città fu violentissima; fra i soldati di un gruppo che era uscito dalle mura per tentare di sorprendere i Mongoli «non fu risparmiato nessuno [...] che fosse più alto di un manico di frusta e si contarono più di trentamila morti». ${ }^{30}$ L'esercito del khan entrò in città fra il 10 e il 16 febbraio $1220 .{ }^{31}$ La cittadella resistette per dodici giorni. Entrati in città i Mongoli vollero che gli venisse consegnato ogni bene che gli abitanti avevano preparato per rifornire l'esercito del sultano. Gli

${ }^{25}$ Nasawi, pp. 62-63.

${ }^{26}$ Juvaini, I, p. 107. Secondo Bartold l'assedio ebbe inizio nel settembre del 1219 e durò complessivamente sei mesi: Bartold, Turkestan cit. p. 406. Quanto al governatore della città, che secondo i Mongoli era il responsabile della morte degli ambasciatori, Gengis ordinò che gli venisse colato argento fuso negli occhi e nelle orecchie e «questa morte crudele fu il giusto castigo di InalKhan al quale la sua condotta ignobile, i suoi atti barbari e i suoi provvedimenti crudeli avevano avuto la riprovazione di tutti» (Nasawi, p. 63).

27 Sulla quale si veda anche Nasawi, pp. 73-76.

${ }^{28}$ Juvaini, I, p. 121.

29 Secondo Juvaini l'assedio ebbe fine nel marzo (Juvaini, I, p. 126); Bartold, Turkestan cit. pp. 406-407; Grousset, L'empire des steppes cit. p. 299.

${ }^{30}$ Juvaini, I, p. 130; Nasawi, p. 73.

31 Sono le due versioni date da Ibn al-Athir e da Juzjani (Bartold, Turkestan cit. p. 410). 
uomini che avevano difeso Bukhara vennero uccisi durante la battaglia invece «i bambini piccoli, i figli dei nobili e le loro donne, snelle come cipressi, furono ridotti in schiavitù» $\mathrm{e}$ ancora fra la popolazione superstite il khan non procedette a una strage «ma i giovani e gli uomini fatti idonei a tal uopo furono arruolati in una leva per l'offensiva contro Samarcanda e Dabusiya». ${ }^{32}$ Infatti l'obiettivo successivo era Samarcanda. La città, una delle più ricche della regione, era ben difesa stando a quel che dice Juvaini. ${ }^{33}$ Samarcanda

era per ampiezza di territorio il più grande dei paesi dell'impero del Sultano, la più piacevole delle sue terre per fertilità di terreno, e per unanime consenso il più delizioso dei paradisi di questo mondo, tra i quattro Eden. ${ }^{34}$

Gli abitanti di Samarcanda si erano preparati a respingere l'assedio dei Mongoli; avevano rinforzato la cittadella e avevano costruito

diverse file di fortificazioni esterne, le mura erano state innalzate fino alle Pleiadi, e il fossato affondato attraverso la terra asciutta fino all'acqua sottostante..$^{35}$

Come erano soliti fare i Mongoli mandarono avanti nell'assalto alla città le leve prese durante assedi precedenti. In questo caso furono, secondo Juvaini, prigionieri presi a Bukhara e

quando i villaggi che [Gengis khan] incontrava sulla sua strada gli si sottomettevano egli non li molestava affatto; ma quando opponevano resistenza, come avvenne a Sar-i-Pul e a Dabusiya, lasciava distaccamenti di truppe ad assediarli. Personalmente egli non si fermò prima di arrivare a Samarcanda. ${ }^{36}$

Quando Čagataï e Ogödeï ebbero preso Utrar si ricongiunsero con l'esercito del padre davanti alle mura di Samarcanda. Prima di decidere per l'attacco furono ispezionate le mura e i sistemi difensivi che gli abitanti della città avevano preparato per resistere ai Mongoli. Nello stesso periodo (marzo 1220) Gengis inviò i suoi due generali più esperti e noyon anziani Žebe e Subeteï all'inseguimento del sultano. ${ }^{37}$

Nella presa di Samarcanda fu utilizzata una delle tattiche ossidionali tipiche degli eserciti nomadi in genere e molto apprezzata dagli strateghi mongoli

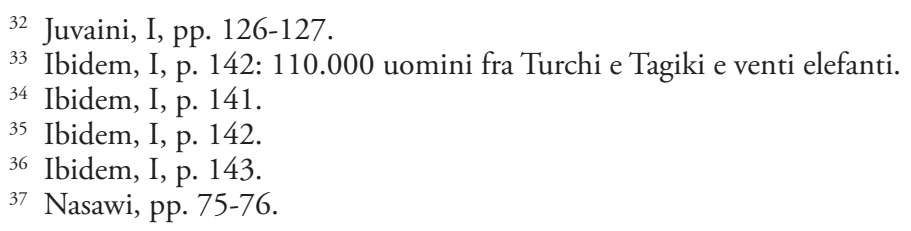


in particolare: Gengis in persona si mise alla testa dell'esercito. Ordinò ai suoi noyon di disporre le truppe in cerchio attorno alla città. Attesero che reparti di fanteria uscissero all'esterno e li massacrarono. Poi scagliarono una fitta pioggia di frecce sulla città e si ritirarono. La scena si ripeteva generalmente più volte, sino a che gli abitanti della città assediata non cedevano per le privazioni. Anche gli abitanti di Samarcanda non resistettero a lungo e le autorità cittadine decisero rapidamente per la resa. ${ }^{38}$ Non avendo alcun interesse a uccidere gli abitanti, Gengis ordinò che essi accettassero la protezione dei Mongoli, «si contarono più di cinquantamila persone che rimasero sotto tale protezione». ${ }^{39}$ I Mongoli e Gengis in particolare apprezzavano molto il coraggio e l'abilità dei soldati. Era un buon motivo per avere salva la vita. In effetti coloro i quali avevano resistito, nonostante la situazione disperata, vennero distribuiti nelle fila dell'esercito. Juvaini dice che furono 30.000 i giovani che per la loro abilità furono distribuiti ai figli del Gran khan e ai loro congiunti. E che altrettanti furono scelti per formare una leva ulteriore. ${ }^{40}$ Dopo la conquista della città $\mathrm{i}$ Mongoli vi lasciarono, come erano soliti fare, un governatore, un funzionario che esercitasse il potere per loro attraverso il quale potevano esigere il pagamento dei tributi, la leva obbligatoria e controllare l'ordine pubblico, nonché amministrare la giustizia. ${ }^{41}$

Dopo Samarcanda fu la volta di Urgench, la capitale della Korazmia. Juvaini dimostra di conoscere bene la città e la regione tutta: «era la sede del trono dei sultani del mondo e il domicilio delle celebrità dell'umanità». ${ }^{42}$ La città fu presa nell'aprile del 1221 dopo un lungo assedio durante il quale la popolazione resistette eroicamente. I generali del sultano si opposero con ogni mezzo all'assalto dell'esercito mongolo che, forte anche dei rinforzi giunti dalle città da poco conquistate, sbaragliò la resistenza locale e varcò le mura.

I Mongoli conquistarono la città quartiere per quartiere, casa per casa, distruggendo gli edifici e facendo strage degli abitanti, finché alla fine la ebbero tutta nelle loro mani. Allora sospinsero la popolazione in aperta campagna, e separarono dagli altri gli artigiani e gli operai, che assommavano a più di 100.000; le donne giovani ed i bambini furono ridotti in schiavitù e condotti

${ }^{38}$ Ibidem, I, pp. 145-156.

39 Ibidem, I, p. 146.

${ }^{40}$ Ibidem, I, p. 147; Grousset, L'empire des steppes cit. pp. 300-301; Bartold, Turkestan cit. p. 409-410. Stando alle versioni di Ibn al-Athir e di Juzjani, la presa di Samarcanda causò la morte di un numero spaventoso di persone, da 50 a 70.000. Ibn al-Athir sostiene che l'esercito del sultano non prese parte alla battaglia, che fu combattuta dai soli abitanti della città; Juvaini invece afferma che un reparto dell'esercito formato esclusivamente da Turchi e guidato da un gruppo di generali, uscì dalla città facendo qualche prigioniero fra i Mongoli prima di arrendersi assieme alla popolazione stremata dall'assedio (Juvaini, I, pp. 143-144).

${ }^{41} \mathrm{Su}$ questo aspetto, estremamente complesso e interessante, mi soffermerò in seguito.

42 Juvaini, I, p. 149. 
in cattività; e gli uomini che restavano vennero ripartiti in modo che ad ogni combattente dell'esercito toccasse di mettere a morte ventiquattro persone [...]. Poi l'esercito si dedicò al saccheggio e alla rapina, e distrusse quel che restava delle case e dei quartieri.

E più avanti:

Quando i Mongoli terminarono la battaglia ed ebbero finito di far prigionieri, di saccheggiare, uccidere e sterminare, quegli abitanti che erano artigiani vennero suddivisi e mandati nei paesi dell'Oriente. ${ }^{43}$

La conquista di Samarcanda e Urgench destò una forte impressione presso i contemporanei e il sultano stesso dovette riceverne una lezione durissima. Privo oramai di punti di appoggio strategicamente validi, responsabile più o meno diretto della distruzione delle città più ricche della Korazmia nonché della morte di decine di migliaia di abitanti e braccato dall'esercito di Žebe e Subeteï, il sultano cercò rifugio prima a Balkh e poi a Nishapur, il 18 aprile 1220 secondo Juvaini:

Egli entrò in città, e, per lo smodato terrore che lo pervadeva, continuò a spaventare la popolazione con lo spauracchio dell'esercito tartaro, lamentando la distruzione delle fortezze ordinata nei giorni della prosperità, allorché credeva che il semplice orgoglio sarebbe bastato a trarlo d'impaccio in caso di bisogno. ${ }^{44}$

Entrambe le città si trovavano nella Transoxiana meridionale; Balkh a sud di Samarcanda e Nishapur fra Turkmenistan e Iran settentrionale, a est del Caspio. Già in occasione dell'assedio a Samarcanda Gengis era venuto a sapere che «il sultano aveva passato il fiume a Tirmiz». ${ }^{45}$ Decise di mandargli incontro l'esercito al comando dei suoi migliori generali. Gli eserciti di Žebe e Subeteï lo inseguirono fino a entrare in entrambe le città. A Balkh «i notabili della città mandarono loro incontro una delegazione». ${ }^{46}$ In questa occasione i due generali mongoli non operarono alcuna ritorsione nei confronti degli abitanti della città che si erano immediatamente arresi. Il percorso dell'esercito mongolo sulle tracce del sultano condusse il primo davanti a Nishapur (24 maggio 1220). Fu

43 Ibidem, I, pp. 154-155.

${ }_{44}$ Ibidem, I, p. 198. Secondo il nostro cronista il sultano vi rimase per quasi un mese, fino al 12 maggio. Nasawi conferma tale informazione dicendo: «Durante un mese che passò a Nisabour [il sultano] inviò messaggi su messaggi in tutte le direzioni per domandare che si riunissero le truppe e che si ammassassero approvvigionamenti, ma i Tatari ebbero notizia di questi preparativi e lo obbligarono ad abbandonare precipitosamente i suoi piani» (Nasawi, I, pp. 107-108).

45 Juvaini, I, p. 170. Tirmiz è oggi Karshi, in Uzbekistan.

46 Ibidem, I, pp. 170-171; Grousset, L'empire des steppes cit. p. 301. 
in questa occasione che l'esercito mongolo si divise in due parti. Žebe diretto a Juvain (l'attuale Jaghatai a nord-ovest di Nishapur) e Subeteï a Tus (a pochi chilometri da Mashhad, vicino alla frontiera irano-afghana). Attraversando l'Iraq persiano i due generali mongoli attaccarono senza esitazione i villaggi che gli si opponevano e si limitavano ad accogliere sotto la loro protezione quelli che si arrendevano rapidamente. ${ }^{47}$ Tale fu la sorte di Tus, Damghan, Semnan. «Misero a ferro e fuoco la maggior parte dell'Iraq persiano» quindi si recarono ad Ardabil, che presero dopo un assedio in seguito al quale massacrarono gli abitanti e saccheggiarono i loro beni. ${ }^{48}$

Il sultano si rifugiò sul Caspio dove trovò la morte nel dicembre $1220 .{ }^{49} \mathrm{La}$ scomparsa del sultano di Korazmia Muhammad Khorazm-Shah non pose fine alle conquiste mongole. Il figlio del sultano Muhammad, Jalal ad-Din, avrebbe di lì a poco dato luogo a una sorta di restaurazione nel territorio persiano con conseguente ripresa delle ostilità da parte dei Mongoli.

Dopo essersi sbarazzato del sultano, ${ }^{50}$ Gengis intraprese la conquista dell'Afghanistan e del Khorassan. La prima città a cadere fu Balkh che accettò immediatamente la sottomissione, ma fu egualmente distrutta e la popolazione sterminata. $^{51}$

A Tuli fu affidato il compito di portare a termine la conquista del Korasan. ${ }^{52}$ L'impresa del figlio di Gengis si concluse con la conquista di Merv e la strage dei suoi abitanti (febbraio 1221). Nel frattempo a Nishapur erano scoppiati disordini e la popolazione, nel novembre 1220, aveva ucciso lo shahna (governatore) lasciato dai Mongoli in città al tempo del loro primo passaggio pochi mesi addietro. ${ }^{53}$ Intervenne in questa occasione Toghachar (o Toquchar), uno dei generi di Gengis ${ }^{54}$ il quale assediò la città. La difesa della popolazione sfociò in una violenta battaglia nella quale Toghachar trovò la morte. L'esercito mongolo si ritirò, ma la ritorsione sarebbe stata tremenda. «Venuta la primavera del 618 [1221-1222], avuta ragione di Merv, Tuli si diresse su Nishapur, senza che

${ }^{47}$ Juvaini, I, pp. 170-175.

48 Ibidem, I, p. 174.

49 Juvaini dice letteralmente: «Il sultano si riposava nelle isole di Abaskun» (è il Caspio con un gioco di parole fra sukun, cioè riposo e Abaskun, v. Ibidem, p. 181, nota 8). È probabile che Mohammed sia morto sull'isola di Ashur-Ade, vicino alla foce del Gurgan. Anche sulla data non si è sicuri. Quella più probabile è il dicembre 1220; anche Nasawi sembra confermarlo: «Il sultano fu sotterrato sull'isola nell'anno 617» (8 marzo 1220 - 25 febbraio 1221), Nasawi, p. 81; si veda anche Bartold, Turkestan cit. p. 426.

${ }^{50}$ L'episodio è narrato in Juvaini, I, pp. 162-165.

51 Ibidem, I, pp. 158-161; Grousset, L'empire des steppes cit. p. 301.

52 Juvaini, I, pp. 176-178.

53 «Frattanto le leve di Tus guidate dal loro capo, un certo Siraj-ad-Din [...] uccisero lo Shahna e ne mandarono la testa a Nishapur, senza rendersi conto che con quell'unica testa avevano mozzato il capo a una grande moltitudine» (Juvaini, I, p. 201).

${ }^{54}$ Ibidem, I, p. 201. 
nessuno sapesse del suo arrivo». La popolazione difese strenuamente la città, utilizzando ogni arma per respingere l'assalto dei Mongoli, ma non servì. Il 7 aprile $1221 \mathrm{fu}$ portato l'assalto definitivo. Tre giorni dopo esso si concluse. Nishapur fu presa il 10 aprile 1221 e rasa al suolo. ${ }^{55}$ La vedova di Toghachar assistette al massacro. ${ }^{56}$

Dopo la presa di Nishapur, Tuli col suo esercito raggiunse il padre presso Talekhan. Čagataï e Ogodeï, che avevano preso Urgench, raggiunsero egualmente l'esercito presso la medesima località.

Come abbiamo detto la morte del sultano Muhammed non pose fine alle ostilità coi Mongoli. Il figlio di Muhammed, Jalal ad-Din, si era rifugiato nel cuore delle montagne afghane, a Ghazna, ed era riuscito a ricostituire un esercito. ${ }^{57}$ Nei pressi di Perwan il figlio del sultano era riuscito a sconfiggere un distaccamento mongolo guidato dal luogotenente di Gengis, Chig-qutuqu e questo evento deve aver ancor più convinto il Gran khan della necessità di eliminare Jalal ad-Din. Gengis giunse a Ghazna dove "venne informato che Jalal ad-Din se ne era allontanato quindici giorni prima». ${ }^{58} \mathrm{Il} 24$ novembre del 1221 l'esercito mongolo al comando di Gengis in persona dopo aver superato Ghazna - nella quale lasciò un suo noyon come basqaq ${ }^{59}$ - proseguì sulle tracce del sultano fino alla battaglia che ebbe luogo sulle rive dell'Indo. "Gengis Khan era arrivato sulla costa dell'Indo [...] nella mattina dell'anno 618 [1221-1222] le armate si misero in linea ${ }^{60}{ }^{6}$ Lesercito mongolo riuscì ad accerchiare quello del sultano e mise in atto una strategia assai frequente in questi casi: si dispose in schiere ognuna delle quali a forma di arco, una dietro l'altra con il fiume che «fungeva da corda» dice Juvaini. ${ }^{61}$ Avanzando verso l'esercito nemico i Mongoli riducevano sempre più lo spazio di manovra di quest'ultimo. L'esercito mongolo travolse gli uomini di Jalal ad-Din, il quale però riuscì a porsi in salvo. ${ }^{62}$ Dopo questa vittoria che avrebbe avuto conseguenze importanti per la

55 Ibidem, I, p. 204.

56 Ibidem, I, p. 203: «Allora entrò in città con la sua scorta una figlia di Gengis-Khan, che era la moglie favorita di Toghachar, e mise a morte tutti i sopravvissuti, tranne quattrocento persone scelte per la loro abilità nell'artigianato, che vennero condotte nel Turkestan, dove vivono tuttora i discendenti di alcuni di loro»; Grousset, L'empire des steppes cit. p. 302.

57 Ibidem, pp. 445-461; Nasawi, p. 93.

58 Juvaini, I, p. 162.

59 I basqaq erano, insieme ai darugal darugačin, funzionari locali che operavano alle strette dipendenze dei governatori nominati dal khan nelle singole città e nei villaggi periferici più grandi. Il loro compito era sostanzialmente fiscale: dovevano controllare che i tributi imposti alla popolazione venissero da questa regolarmente versati. Per farlo potevano ricorrere alla forza in quanto disponevano di un manipolo di militari che comandavano.

${ }^{60}$ Nasawi, p. 139. Nasawi parla in realtà del 25 novembre anziché del 24.

${ }^{61}$ Juvaini, I, pp. 163-165.

62 "Come il fulmine piombò in acqua/ e come il vento ripartì (Ibidem, p. 163); l'episodio è narrato anche in Nasawi, p. 140. 
Transoxiaia, Gengis rimandò Ogödeï a Ghazna dove la popolazione non aveva opposto resistenza all'operato del basqaq insediato dal khan. Non è chiaro per quale motivo dunque Gengis «ordinò di far uscire tutti all'aperto, e gli artigiani vennero radunati da una parte, mentre gli altri furono messi a morte e la città fu distrutta». ${ }^{63}$

Dopo aver sottomesso quasi tutta la Transoxiana, Gengis khan decise che avrebbe potuto far ritorno in Mongolia e delegare ai figli e ai generali il proseguimento delle ricerche del sultano. L'inverno del 1222 lo passò a Samarcanda. All'inizio del 1223 risalì il corso del Sir Darya per dirigersi verso le steppe del Talas e del Tchu. Nell'estate del 1224 era sull'Irtysh. All'inizio del 1225 ritornò nella sua patria d'origine come un trionfatore e decisamente rafforzato nel suo ruolo di Gran khan e padrone dell'ulus mongolo; un ulus le cui dimensioni adesso erano aumentate nettamente.

\subsection{La prima incursione fra Caucaso e Azov (1220-1221)}

Mentre il khan faceva ritorno in Mongolia, il suo esercito proseguiva l'inseguimento alla ricerca di Jalal ad-Din. L'intervento mongolo nel Caucaso infatti ebbe un prologo drammatico nel 1221. I Tatari guidati da Žebe e Subeteï, attesero l'inverno presso Hamadan e il Dkébel e «soffrirono un freddo violento ed una neve spessa». ${ }^{64}$ Marciarono poi verso l'Azerbaijan e «commisero sul loro cammino, nelle borgate e nei piccoli villaggi, uccisioni e saccheggi». ${ }^{65}$ I due generali d'eccellenza del Gran khan giunsero, passando quindi da sud, nella Caucasia e penetrarono entro i confini della Georgia. In quegli anni il regno di Giorgio III Lacha, successore di Tamara, stava vivendo un momento di straordinaria fioritura artistica e culturale nonché una buona fase economica. A questo si aggiunga che la cavalleria georgiana era fra le più preparate e meglio equipaggiate della regione. «Un copioso distaccamento di truppe georgiane (circa 10.000 combattenti) gli andarono incontro». ${ }^{66}$ Giorgio III pensò in quella circostanza di chiedere aiuto all'atabeg dell'Azerbaijan, Uzbek. Fu inviata un'ambasciata in due direzioni. Da una parte si cercò di allacciare relazioni diplomatiche con l'Azerbaijan, dall'altra si chiese l'aiuto di Melik al-Ashraf, governatore di Khilat e Djezireh, per creare un fronte comune contro i Mongoli. "Alla fine questi barbari giunsero nelle vicinanze

${ }^{63}$ Juvaini, I, pp. 164-165.

${ }^{64}$ Ibn al-Athir, II, p. 448.

${ }^{65}$ Ibidem, II, p. 450. In questa circostanza Athir aggiunge che i Mongoli si diressero verso il Caspio poiché «il clima da quelle parti è migliore e vi sono pascoli in abbondanza per le loro bestie da soma»; secondo Juvaini, I, p. 172 «il verde dei prati e la gran copia di sorgenti gli piacquero tanto che non torse un capello agli abitanti [di Radkan, nell'area a est del Caspio] e lasciò sul posto uno shahna».

${ }^{66}$ Ibn al-Athir, II, p. 448. 
di Tblisi». ${ }^{67}$ Nel febbraio $1221^{68}$ l'esercito cristiano, insieme con elementi musulmani inviati dall'Azerbaijan e da Khilat, affrontò quello mongolo presso Tblisi e venne annientato. ${ }^{69}$ Dopo la vittoria sui Georgiani l'esercito mongolo mosse verso Est, sull'Azerbaijan e pose l'assedio a Maraga, a est di Mosul (marzo 1221). Dopo aver conquistato anche la città sul Tigri, Žebe e Subeteï si diressero verso il califfato abbaside di Baghdad. ${ }^{70}$ In questa occasione l'unica conquista mongola in territorio musulmano fu la città di Hamadan e, dopo averla rasa al suolo e data alle fiamme, ripresero la via del Nord. Passando da Derbend, sulla costa occidentale del Mar Caspio ed evitando Tblisi, giunsero sui territori delle steppe del Caucaso settentrionale dove si scontrarono con le popolazioni che vi abitavano, Lazi, Circassi, Alani e soprattutto Cumani. Ibn al-Athir descrive con attenzione gli eventi:

Quando i Tatari ebbero attraversato la linea di Derbend e dello Shirvan, avanzarono in quelle contrade, dove abita un gran numero di nazioni, fra le quali ricordiamo gli Alani, i Lazi, e dei popoli turchi. Li saccheggiarono, uccisero molti Lazi, popolo che si componeva di musulmani e di infedeli e si scagliarono contro quegli abitanti che gli si dimostrarono ostili. ${ }^{71}$

A questo punto Athir racconta di un'ambasciata che i Mongoli avrebbero inviato presso i Cumani cercando di convincerli che, appartenendo a «una stessa razza» non aveva senso che si combattessero fra di loro e che sarebbe stato molto meglio se si fossero uniti contro il comune nemico, che in quel caso erano gli Alani.

Convennero coi Qipciaki di pagargli una somma di argento e di dargli stoffe ed altri oggetti, e di rendergli ciò che gli avevano preso. I Qipciaki abbandonarono gli Alani. ${ }^{72}$

I Mongoli sterminarono gli Alani e poi, naturalmente, si diressero verso i Cumani; li attaccarono, li vinsero e "presero loro il doppio di quanto gli aveva-

${ }^{67}$ Ibidem, II, p. 449. È assai curioso notare come Athir chiami in questa circostanza i Mongoli. Raramente usa questa espressione e stranamente lo fa parlando dei Mongoli in contrapposizione ai Georgiani, un popolo nei confronti del quale gli storici musulmani non avevano alcuna simpatia.

${ }^{68}$ Secondo Athir in gennaio.

${ }_{69}$ Grousset, L'empire des steppes cit. pp. 306-307.

${ }^{70}$ Se tale progetto si fosse realizzato le conseguenze per l'Islam mediorientale sarebbero state tragiche. Nello stesso periodo dell'anno 1221 infatti i crociati avevano preso Damietta costringendo alla retrocessione i musulmani dell'Egitto. La conquista di Baghdad avrebbe isolato l'Islam sia a Occidente che a Oriente vibrando un colpo durissimo allo sviluppo dell'intera regione.

${ }^{71}$ Ibn al-Athir, II, p. 455.

72 Ibidem, II, p. 456. 
no dato». ${ }^{73}$ Dopo questo primo scontro vittorioso contro i Cumani i Mongoli «arrivarono nella città di Sudak», capitale del Kipciak. ${ }^{74}$

Dal secondo scontro coi Cumani scaturì anche la prima battaglia fra Mongoli e Russi. All'alba del terzo decennio del Duecento i Cumani stavano vivendo un lento processo di acculturazione coi Russi la cui conseguenza più evidente fu l'adozione, ancora timida allora, del cristianesimo. I Cumani chiesero aiuto a Mstislav di Galič (che aveva sposato la figlia del khan Kutan) per fronteggiare l'esercito mongolo. I principi russi accolsero la richiesta, ma agirono divisi. Decisero che sarebbe stato meglio non attendere i Mongoli nei propri territori, ma affrontarli nelle steppe (si trattava del territorio posto nell'imbuto che oggi forma la Russia meridionale, fra Rostov e Astrakhan). I Mongoli, come loro solito, inviarono prima un messo che consentisse alle loro vittime designate di evitare lo scontro accettando la sottomissione. $\mathrm{Ma}$ la risposta russa si concretizzò nell'assassinio degli ambasciatori mongoli, fornendo così all'esercito di Žebe e Subeteï il pretesto per l'inizio delle ostilità. Lo scontro fra le truppe mongole e quelle russe si verificò sul fiume Kalka (forse il Kalets, un fiume che scorre fra il Dnepr e il Don e si getta nelle acque del Mar d'Azov) nel $1223 .{ }^{75}$ Ma non tutti i principi russi erano d'accordo nell'affrontare i Mongoli. Dunque il più deciso per lo scontro, Mstislav, affrontò gli invasori insieme ai Cumani uscendone duramente sconfitto. ${ }^{76}$ Dopo una battaglia che Athir definisce molto lunga e incerta, i Mongoli sconfissero l'unione cumano-russa e "fecero una carneficina». ${ }^{77}$ In seguito i Mongoli si diressero verso il bacino del Sir-Darya dove avrebbero dovuto ricongiungersi con il resto dell'esercito. Il colpo definitivo alla Russia e alla Caucasia fu pertanto rimandato, ma non ancora per molto.

In quella che fu in sostanza solo una rapida scorreria nel Caucaso i Mongoli avevano fortemente destabilizzato la sovranità musulmana della regione e

73 Ibidem.

74 Ibidem, II, p. 457. Athir dice che Sudak «è situata sul mare dei Khazari [...]. I Qipciaki vi vendono e acquistano delle ragazze, schiavi, borthasi, volpi nere, castori, zibellini, ed altre produzioni del loro paese». Quando i Mongoli arrivarono in città gli abitanti la abbandonarono rifugiandosi sulle montagne con le loro famiglie e le loro ricchezze, altri si imbarcarono e fuggirono in Asia Minore.

75 «Nell'anno 6732 [1224] a causa dei nostri peccati, giunsero dei pagani sconosciuti. Nessuno sa bene chi siano [...] né di dove siano usciti, né che lingua parlino, né di che stirpe siano, né quale sia la loro fede» e ancora "Dio permise che accadesse per l'orgoglio e per la presunzione dei principi russi» (Cronaca di Tver' in Polnoe Sobranie Russkich Letopisej, t. XXX, Vladimirskij Letopisec, Moskva 1965, alcuni brani sono tradotti in Pianto sulla distruzione di Rjazan', a cura di E.T. Saronne, Parma 1992, pp. 171-224 alle pp. 171-172).

76 Secondo la Cronaca di Tver' il khan cumano Kotjan, suocero di Mstislav di Galič, nel chiedere aiuto a questi disse: "Oggi hanno preso la nostra terra: domani sarà presa la vostra», Pianto, p. 173.

77 Ibn al-Athir, II, p. 459. 
avevano indebolito le potenzialità militari del regno di Georgia. Tale situazione impressionò parecchio i principi islamici che trovarono nel sultano di Korazmia un valido esponente in grado di avviare una manovra di recupero dei territori perduti. Jalal ad-Din, figlio di quel Mohammed in fuga dai Mongoli già da alcuni anni, riuscì a trovare asilo e protezione presso Iltoutmich, sultano turco di Delhi, il quale gli mise a disposizione un piccolo esercito che utilizzò per dirigersi verso il Caucaso e tentare il recupero dei territori recentemente devastati dalla scorreria mongola. Nel 1224 fece il suo ritorno in Iran dove, grazie all'intervento degli atabeg, i governatori ereditari locali, poté figurare come l'unico, in quanto musulmano, in grado di guidare legittimamente quel principato e insieme con esso la riscossa dell'Islam ferito dai Mongoli.

Jalal ad-Din giunse nelle terre dell'Azerbaijan nell'agosto del $1225 .^{78}$ L'Azerbaijan era uno stato molto forte, dotato di un esercito ben armato e adeguatamente preparato. In seguito alla prima incursione mongola nelle steppe dei Polovcy questi si erano in gran parte mossi migrando nella direzione opposta a quella cui andarono poi gli invasori, cioè verso sud. ${ }^{79}$ Questo esercito improvvisato, composto soprattutto da profughi disperati in fuga dalla guerra, si riversò confusamente entro i confini dell'Azerbaijan. Ibn al-Athir dice che i Cumani avrebbero inviato al principe del Paese un messaggio col quale lo pregavano di accoglierli in quanto $\mathrm{i}$ "Tatari si sono impadroniti del nostro paese, ed hanno saccheggiato le nostre ricchezze». ${ }^{80}$ Dopo alcune resistenze il governatore di Derbend, che dipendeva dal principe dell'Azerbaijan, accettò che i Cumani si stanziassero ai confini. La presenza dei nomadi alla frontiera determinò la reazione dei Georgiani; le fonti parlano di frequenti scorrerie in questi mesi da parte dei Cumani nei territori georgiani; tali iniziative si concretizzavano sempre in incursioni nei villaggi, razzie, vessazioni ai danni della popolazione locale. Si giunse presto allo scontro. Verso la fine del 1223 l'esercito georgiano affrontò quello musulmano e ne uscì sconfitto. Uzbek, principe dell'Azerbaijan, inflisse dunque un altro duro colpo alla Georgia. Quando Jalal ad-Din iniziò la sua opera di restaurazione dei distretti musulmani dell'Iraq e della Persia iranica l'unico distretto musulmano della regione che non ne riconobbe subito l'autorità fu proprio l'Azerbaijan. Uzbek dovette piegarsi a Jalal ad-Din dopo che questi lo ebbe sconfitto militarmente nel 1225. Lo scopo dell'Islam, rappresentato in questa circostanza dal sultano, era quello di restaurare a ovest l'impero della Korazmia. Il territorio azerbaijano era strategico poiché costituiva la testa di ponte per il commercio

${ }_{78}$ Ibidem, II, p. 482.

79 Athir dice che una parte dei Cumani si diresse verso il «Paese dei Russi», un'altra si rifugiò nelle montagne e una terza "si riunì e marciò verso il Derbend e Shirvan», (Ibidem, II, pp. 463464). Vedi anche Grekov - Jakubovskij, L’Orda d'Oro cit. pp. 34-35.

${ }^{80}$ Ibidem, p. 464. 
con l'Oriente e la navigazione sul Caspio. Una risorsa che era nelle mani dei Turchi sin dal 1136 (cfr. cap. 1). ${ }^{81}$ Il sultano si rendeva perfettamente conto che le conseguenze del passaggio mongolo in Caucasia avevano seriamente indebolito l'esercito georgiano. Si trattava di un'occasione da sfruttare; la mossa successiva del sultano fu proprio l'attacco ai Georgiani ${ }^{82}$ Questi ultimi si prepararono alla battaglia con oltre 70.000 uomini secondo Athir ${ }^{83}$ ma non bastò. I due schieramenti si affrontarono nella valle del Garni ${ }^{84}$ nell'agosto del 1225 e quattro anni dopo la sconfitta subita a opera dei Mongoli, l'esercito georgiano capitolava per la seconda volta. Athir dice che in questa occasione furono uccisi 20.000 Gorgiani. ${ }^{85} \mathrm{Nel}$ marzo dell'anno successivo si verificò il secondo terribile attacco del sultano in Georgia. Il sultano procedette su Dvin. ${ }^{86}$ Passando per i monti del Panbak (nella regione del lago Sevan, in Armenia) giunse sino a Lori, capoluogo del distretto armeno di Tashir. Sbaragliata la resistenza dell'esercito georgiano gli uomini del sultano si diressero a sud del Kur. Fra gennaio e febbraio del 1226 l'esercito georgiano tentò il contrattacco, ma senza esiti. Anzi, l'avanzata di Jalal ad-Din a questo punto fu ancora più violenta e divenne inarrestabile. Marciando su Tblisi il sultano attaccò tutti i villaggi che si trovarono sulla sua strada, demolì chiese facendo costruire al loro posto luoghi di culto per l'Islam. ${ }^{87}$ Conquistò Ani e Kars; poi si diresse verso il paese degli Abkazi, dove c'erano piccole guarnigioni georgiane rimaste a presidio dei territori, e lo conquistò con relativa facilità. ${ }^{88}$

Un contrattempo fermò l'avanzata del sultano sulla Georgia; un suo generale «si era sottratto al collare della fedeltà, ed era partito dal Kerman col proposito

${ }^{81}$ A tale proposito si veda anche quanto dice Grousset, L'empire des steppes cit. pp. 324325.

82 Juvaini, II, p. 478 dove lo storico persiano afferma che i Georgiani «fidando nella prodezza dei loro uomini e nell'acutezza delle loro lance e sciabole, radunarono le loro forze; e raccolti più di trentamila uomini, balzarono avanti».

${ }^{83}$ Ibn al-Athir, , II, p. 482. Juvaini parla di 30.000 uomini (Juvaini, II, p. 478).

${ }^{84}$ Juvaini, II, p. 479; il primo scontro fra i Georgiani e il sultano è narrato anche da Ibn alAthir, II, pp. 483-484, che colloca però l'evento nell'agosto anziché nel marzo del 1225. Il Garni, già noto come Azat, è un affluente dell'Arasse che scorre nell'odierna Armenia a est di Erevan; si veda a tal proposito Minorsky, Studies in Caucasian History cit. p. 124.

${ }^{85}$ Juvaini, II, pp. 482-483.

86 Secondo Juvaini quando il sultano apprese che la Georgia era retta da una donna (la regina Rasudan) avrebbe pronunciato queste parole: «E meschino chi affida i suoi affari ad una donna!» (Juvaini, II, p. 480). Tale reazione ingiuriosa, anche se con parole diverse è riportata anche nella cronaca georgiana (Brosset, Histoire de la Géorgie cit. p. 469).

87 Le nostre fonti, la cui attendibilità è in genere fuori discussione, spesso esagerano in negativo con la Georgia. Si tenga presente che essa era l'unico regno cristiano nella regione. La forte identità religiosa di Juvaini, così come quella di Rashid ad-Din, Ibn al-Athir o di Nasawi (tutti musulmani), è certamente un freno all'obiettività. Ė quindi necessario il confronto con una voce opposta che in questo caso è sicuramente la Cronaca Georgiana.

${ }^{88}$ Ibn al-Athir, II, p. 495. 
di conquistare l'Iraq». ${ }^{89}$ Lallarme rientrò nel momento in cui il sultano giunse alle porte di Kerman, città nella quale il generale ribelle governava; questi non esitò a presentare le sue scuse al sultano chiedendo il suo perdono. Le fonti tacciono sul seguito della vicenda. ${ }^{90}$

Quel che è certo è che fra la fine del 1227 e l'inizio del 1228 Jalal ad-Din si rimise in marcia contro la Georgia.

Ora i sultani di Rum, Siria, Armenia e di tutta quella regione [...] avevano formato una confederazione e si erano uniti per respingerlo; e fu adunato un esercito consistente di Georgiani, Alani, Armeni, Sarir, Lakz, Qufchaq, Svan, Abkhaz, Chanet, Siriani e gente di Rum. ${ }^{91}$

In questa occasione venne attaccata, saccheggiata e data alle fiamme Tblisi. L'ultimo durissimo colpo al regno cristiano fu inferto dal sultano nel 1228 quando l'esercito guidato dal vecchio generale Ivane venne inesorabilmente sconfitto.

La manovra operata da Jalal ad-Din ebbe quindi successo. Egli era riuscito a riportare sotto la bandiera dell'Islam parte dei territori perduti durante la prima incursione mongola. Ma a quale prezzo? Le guerre che ebbero luogo nella regione della Korazmia, della Transoxiana e del Caucaso ebbero effetti devastanti sia a livello demografico sia (ma forse le due cose sono strettamente legate) economico. Non è detto che le cifre fornite dalle nostre fonti siano del tutto attendibili, ma si possono prendere per buone come linea generale. Non è detto cioè che siano morti 20.000 Georgiani in seguito alle battaglie con Jalal ad-Din, ma quel che è certo è che fu un massacro. L'impressione percepita dallo storico che ci racconta gli eventi è lo specchio del carattere straordinario di quegli eventi stessi. Quindi, se è vero che la storia di questi anni è quella di una guerra perpetua più o meno dappertutto, è altrettanto vero che queste guerre non erano la norma; non in questi termini, con queste conseguenze. Le ripetute sconfitte georgiane, pagate a prezzo di migliaia di morti, portarono a un crollo verticale della popolazione maschile giovane, della forza lavoro. Contemporaneamente gli assalti ai centri abitati, piccoli o grandi che fossero, si concretizzavano nell'uccisione indiscriminata di ampi strati della popolazione, comprese donne e bambini. Gli effetti di queste perdite furono disastrosi. Non solo la produzione si bloccò, ma anche gli scambi subirono una battuta d'arresto drammatica. Con lo stato di guerra permanente, le strade insicure, i mercanti non si muovevano. Da un punto di vista militare

89 Juvaini, II, pp. 484-485.

90 Si veda comunque Ibn al-Athir, II, pp. 498-500.

${ }^{91}$ Juvaini, II, p. 490. I Sarir erano gli Avari del Daghestan, gli Svan erano una popolazione dell'Alto Ingur, in Georgia, i Chanet erano i Lazi, una popolazione che viveva a sud-est del Mar Nero. 
poi la situazione non era migliore: la Georgia aveva rappresentato un punto di equilibrio nel Caucaso musulmano. Unico stato cristiano, ricco, militarmente forte, scoraggiava i piccoli stati circostanti dal muovere guerra, tant'è vero che fino all'intervento del sultano questa regione era rimasta relativamente tranquilla. Infine va considerato il fattore debolezza. Cioè l'indebolimento generalizzato, quindi anche militare, cui era stata ridotta la Georgia rappresentò un vantaggio di non poco conto per i Mongoli i quali, quando giunsero nella regione, trovarono una resistenza scarsa ad attenderli.

Per quanto riguarda i governatorati e gli stati musulmani circostanti la situazione non era molto diversa. Le lacerazioni che aveva prodotto la costante campagna militare del sultano emersero in tutta la loro gravità all'arrivo delle truppe mongole. I prelievi fiscali, la leva obbligatoria e le guerre sul territorio avevano prodotto una situazione di profonda incertezza il cui esito sarà, anche in questo caso, una debolezza che non fu conseguenza delle conquiste mongole; essa era già un fatto concreto prima che questi si affacciassero oltre il Caspio per la seconda volta e facilitò non poco il loro compito.

Dal 1224 Jalal ad-Din si era scagliato contro il califfato di Baghdad. ${ }^{92}$ Nella sua marcia era riuscito a conquistare la città di Khilat, punto strategico fondamentale poiché posta sulle alture nel nord-ovest del lago di Van (oggi nella Turchia orientale), nell'aprile del 1230 , causando la durissima reazione dei sultani selgiuchidi che si videro minacciati da vicino. ${ }^{93}$ Nell'agosto del 1230 il sultano selgiuchide Ala ad-Din Kaï-Qubad I del sultanato di Iconio e il sultano ayyubide al-Achraf inflissero una dura sconfitta all'esercito di Jalal ad-Din nei pressi di Erzindjan ${ }^{94}$ a sud di Trebisonda in Turchia. Per l'esercito del sultano di Korazmia fu una disfatta. D'altra parte la conquista della regione meridionale posta fra Mar Caspio e Mar Nero era un elemento che poneva i sultanati selgiuchidi in una condizione pericolosa non solo per la perdita del territorio, ma anche e soprattutto in prospettiva occidentale. La presenza dell'impero latino a Costantinopoli, la crescente potenza del ricostituito impero bizantino a Nicea, l'impero di Trebisonda sulla costa anatolica del Mar Nero, la Georgia nella Caucasia, costituivano tutti un accerchiamento che creava allarme presso i Selgiuchidi i quali non potevano permettersi di perdere anche Baghdad e il suo straordinario potenziale culturale e commerciale. Lasciare campo libero a Jalal ad-Din significava in questo momento chiudere le porte dell'Oriente e

${ }_{92}$ Il principe di Khilat, Al-Malik al-Ashraf della dinastia ayyubide e quindi discendente del Saladino, aveva concluso un trattato di pace con la Georgia nel 1223, Ibn al-Athir, II, p. 474; Nasawi, pp. 303-329.

${ }^{93}$ Ibn al-Athir, II, pp. 497-500; Juvaini, II, p. 504: «La conquista di Akhlat (Khilat) fu aggiunta a queste conquiste [...]. La paura del sultano si sparse per tutte quelle regioni, e la fama della sua severità e del suo rigore si diffuse vasta e lontana».

${ }^{94}$ Grousset, L'empire des steppes cit. p. 325; Nasawi, pp. 342-346; Juvaini, II, pp. 504505. 
interrompere i traffici commerciali con le regioni del Turkestan, oltre che con la Persia iranica.

\subsection{La seconda incursione mongola a Ovest e la conquista definitiva della Caucasia (1230-1236)}

Nell'estate del $1227,{ }^{95}$ durante l'assalto finale portato alla tenace resistenza dello Si-Hia (in particolare nell'assedio all'attuale città di Neijiang, a ovest di Chong Qing in Cina), il khan mori ${ }^{96} \mathrm{e}$ due anni dopo fu eletto nel gran quriltai il suo successore. Prima di morire Gengis aveva designato a tale scopo Ogödeï, suo terzo figlio; è con lui che la capitale dell'impero viene stabilita a Qaraqorum. Grousset sottolinea l'importanza di questa scelta poiché Qaraqorum era la città nella quale avevano avuto la loro capitale la maggior parte degli antichi imperi turco-mongoli; ${ }^{97}$ Juvaini sostiene che

secondo gli Uighur, la loro generazione e il loro incremento ebbero inizio sulle rive del fiume Orkhon, la cui sorgente scaturisce da una montagna che essi chiamano Roccia Nera (Quara-Qorum)..$^{98}$

Il più anziano dei figli di Gengis khan, Giuči, era morto sei mesi prima del padre, nel febbraio $1227,{ }^{99}$ pertanto l'ulus che spettava a questo finì per essere assegnato al figlio di Giuči e nipote di Gengis, Batu (al quale toccò la regione più a Ovest; sarà lui l'artefice della nascita dell'Orda d'Oro). Il secondo dei figli di Gengis, Čagataï ricevette la zona delle steppe dell'antico impero del Kara-Qitai, cioè la zona fra il fiume Itil e l'Issiq-kul, comprese le città di Samarcanda, Bukhara, Merv. Il figlio più giovane, Tuli, come da tradizione era designato guardiano del focolare, quindi ottenne la patria antica del padre, fra l'Onon e il Kerulen.

Il nuovo khan Ogödeï decise di portare a termine la conquista della Korazmia e armò un esercito che, nell'inverno fra il 1230 e l'anno successivo, giunse nel Khorassan. Jalal ad-Din, appresa la notizia, si dette alla fuga. Questa è la versione delle fonti; per parte mia sono più propenso a ritenere che stesse perseguendo una strategia di difesa, ben consapevole dell'efficacia dell'avversario. La fuga verso luoghi impervi poteva costituire un vantaggio per le sue truppe in quanto costituite da indigeni, mentre forse sperava che i Mongoli non avessero 309

95 Forse il 18 agosto, ma non è una data certa; si veda Grousset, L'Empire des steppes cit. p.

${ }^{6}$ SSM, pp. 236-237; Bartold, Turkestan cit. p. 459. Se è vero che Gengis nacque nel 1155, allora sarebbe morto all'età di 72 anni.

${ }_{97}$ Grousset, L'empire des steppes cit. p. 320.

98 Juvaini I, p. 74.

99 Bartold, Turkestan cit. pp. 458-459; Grousset, L'empire des steppes cit. p. 317. 
queste conoscenze. Dopo aver corso per un territorio piuttosto esteso giunse nei pressi di Dyarbekir (Diyar-Bekr) ${ }^{100}$ dove fu assassinato in circostanze poco chiare nell'agosto del $1231 .{ }^{101}$

Il generale mongolo Čormaghan rimase per dieci anni nel nord-est della Persia. Stabilì la sua residenza presso la pianura fra il corso inferiore del Kura e dell'Arasse. ${ }^{102} \mathrm{Da}$ questa posizione l'esercito mongolo si diresse più volte verso i confini degli stati vicini per fare razzia; prima verso l'Armenia, poi in Azerbaijan, poi ancora nei villaggi attorno a Mosul, nella regione dell'alto corso del Tigri. Nel 1236 i Mongoli decisero di salire verso il Caucaso e per la Georgia fu la fine. La regina Rasudan fu costretta ad abbandonare Tblisi e a rifugiarsi a Kutaisi, nella piana che guarda al Mar Nero. L'aristocrazia georgiana fu posta sotto la protezione mongola e obbligata a prestare il servizio militare in cambio della vita. Nel 1239 Cormaghan conquistò Ani e Kars nella Grande Armenia. Gli stati situati fra Caucaso e Anatolia dovettero a questo punto fare una scelta. Potevano resistere davanti ai Mongoli, cercando di mantenere la loro sovranità indipendente nella regione, col rischio di essere sconfitti a prezzo di orribili massacri; potevano però accettare da subito la sottomissione al Gran khan e avere salva la vita della popolazione evitando distruzioni e saccheggi. Gli atteggiamenti del sultanato selgiuchide di Anatolia e del sovrano armeno di Cilicia Hethum I furono diversi fra di loro. Il primo cercò la reazione all'avanzata delle armate di Čormaghan. Kaï-Khosrau II, questo il nome del sultano di Iconio, aveva riportato il sultanato selgiuchide a un livello di ricchezza eccellente. L'esercito era forte e lo stato versava in buone condizioni economiche. Probabilmente ritenne di poter affrontare i nuovi conquistatori con efficacia. Nel 1242 i Mongoli presero e saccheggiarono Erzurum, a nord del lago di Van, oggi in Turchia orientale. Il 26 giugno 1243, presso Erzindjan, ebbe luogo la battaglia fra l'esercito turco e quello mongolo. Le armate del generale mongolo Baïju (che aveva sostituito Čormaghan) sbaragliarono l'esercito avversario. Dopo il saccheggio di Sivas, nel cuore dello stato selgiuchida il sultano accettò la tutela mongola diventando vassallo del khan, evitando un inutile massacro alla sua gente. Tale scelta fu fatta, con molta astuzia, anche da Hethum I che non aspettò l'arrivo dei Mongoli. Si dichiarò da subito loro suddito e vassallo dei noyon di Ogodeï. Entrò sotto la protezione mongola riuscendo comunque a esercitare la propria sovranità esattamente come aveva fatto fino ad allora e pagando ai Mongoli un tributo. Una politica analoga fu seguita dall'atabeg di Mosul, il quale accettò di buon grado la protezione mongola e salvò la vita alla popolazione della città. L'esercito di Baïju riuscì ad affermare la presenza mongola nel Kurdistan e nella Georgia sud-occiden-

100 Juvaini, II, p. 509.

101 Grousset, L'empire des steppes cit. p. 326; Juvaini, II, p. 515.

102 Ibidem, II, pp. 508-509. 
tale. In pratica i Mongoli erano riusciti a conquistare la Caucasia e la Persia determinando un impero che, dalla Cina al sultanato selgiuchide di Iconio, in Anatolia, non aveva soluzione di continuità. Restava da portare avanti l'opera di conquista verso Nord. Le steppe dei Polovcy sarebbero rimaste nelle mani di questi ultimi ancora per poco.

Nel quriltai convocato da Ogödeï nel 1235 (632 dell'Egira) ${ }^{103}$ i Mongoli avevano pianificato le operazioni militari future; ne erano state decise quattro.

Egli [il khan] si dedicò agli affari di stato e all'organizzazione degli eserciti [...]. Assegnò a ciascun figlio o parente una spedizione diversa e decise ancora una volta di partecipare di persona alla guerra e di volgere le redini verso l'azione. ${ }^{104}$

Di fatto il khan fu convinto a non partecipare personalmente alle operazioni e

poiché non erano state ancora schiacciate completamente le tribù dei Qifchaq e dei Keler, in primo luogo ci si occupò delle conquista e della sottomissione di quei popoli. Fra i principi per dirigere questa campagna vennero prescelti Batu, Mengü Qa’an e Güyük. ${ }^{105}$

Oltre alla spedizione ai danni dei Cumani e degli Ungari (i Keler di Juvaini) si decise di muovere guerra alla Cina dei Sung i quali, già sotto la protezione mongola, si erano arrogati il diritto di occupare territori che erano stati loro interdetti. La terza spedizione doveva essere diretta in Corea, la quarta in Asia Minore.

Le incursioni operate dai popoli nomadi della steppa ai danni delle città vicine erano quasi sempre operazioni fulminee, rapidi attacchi il cui scopo principale era la razzia. Quando erano riusciti ad accumulare un bottino soddisfacente essi si dileguavano con la stessa rapidità con la quale erano giunti. Le prime invasioni mongole a Ovest dovettero apparire ai contemporanei occidentali come rapide scorrerie. In effetti la prima incursione nei territori del Caucaso e della Rus' (quella degli anni Venti del Duecento) aveva avuto quelle caratteristiche. Venti anni dopo giunsero in Occidente notizie simili. Città distrutte, massacri ripetuti ai danni delle popolazioni conquistate, ovunque devastazione; in Occidente non venne subito compresa la gravità della situazione. La prima incursione mongola, che aveva attraversato il Caucaso ed era giunta fino ai confini meridionali della Rus', era stata un'operazione, potremmo dire, di "spionaggio". I Mongoli avevano semplicemente perlustrato le terre contro

$\begin{array}{ll}103 & \text { Ibidem, I, p. } 224 . \\ 104 & \text { Ibidem, I, p. } 224 . \\ 105 & \text { Ibidem, I, p. } 225 .\end{array}$ 
le quali avrebbero scatenato guerra al momento opportuno. Oramai l'esercito di Baiju aveva consolidato la presenza mongola nel Caucaso, in Anatolia e in Persia. Bisognava salire verso Nord. Il khan dei Polovcy Kutan (che abbiamo visto in precedenza come alleato dei Rus') fu l'unico a comprendere la gravità della situazione e, immaginando quale disastro si sarebbe verificato di lì a poco sulle steppe russo-meridionali, non attese che i Mongoli gli arrivassero addosso. Quando apprese la notizia che si erano stabiliti sulla linea al di là del Caucaso riunì le proprie genti e cercò rifugio a Occidente. Dopo aver attraversato il Dnepr e il Dnestr si fermò ai Carpazi. Inviò un'ambasciata al re Bela di Ungheria offrendo la sua sottomissione in cambio di protezione. Non vi sono cifre sicure sull'entità dei Polovcy di Kutan, ma è probabile che fossero almeno 200.000 persone. Naturalmente l'offerta del khan era molto allettante per il re cristiano. Se essi avessero accettato la sottomissione diretta al sovrano, aggirando quella dell'aristocrazia, avrebbero rafforzato l'autorità del primo ai danni della seconda. A ciò si aggiunga che il clero ungherese avrebbe accettato di buon grado di proteggere questi pagani in cambio di una loro conversione di massa. L'Ungheria accolse dunque i Polovcy in fuga dai Mongoli. ${ }^{106}$ La decisione ungherese di accogliere i nomadi cumani entro i propri confini creò non pochi problemi. La loro tradizionale struttura sociale basata sul nomadismo si conciliava male con la radicata civiltà sedentaria ungherese; convertirli al cristianesimo poteva funzionare, anche se continuarono a mantenere le loro tradizioni pagane a lungo, ma imporgli sistemi economici a loro estranei si rivelò un disastro. Tant'è vero che scoppiarono presto scontri con la popolazione locale, contadini soprattutto, ai quali il bestiame cumano in pascolo devastava i campi. I conflitti si successero con crescente intensità e crearono forti tensioni fra la popolazione locale e i nuovi ospiti; Bela dovette far ricorso a più riprese a provvedimenti che stabilivano nettamente l'entità delle terre adibite al pascolo e quelle che non potevano essere destinate altro che allo sfruttamento agricolo. Dopo essere stati espulsi nel 1241 furono richiamati nel 1246. La comunità cumana in Ungheria costituì una minoranza poco significativa. Secondo i recenti studi di Nora Berend essi non superavano il $7-8 \%$ sul totale della popolazione. ${ }^{107}$

106 Su questi eventi si veda D.G. Chrustalev, Rus': ot našestvija do "iga”. 30-40gg. XIII veka, Sankt Peterburg 2004; J.J. Sounders, The History of the Mongol Conquests, University of Pennsylvania (PA) 2001, specialmente i capp. 5 e 6; D.O. Morgan, The Mongols, Malden (MA) 1990, il cap. 6 Expansion to the West: the Mongols in Russia and Persia; The Mongol Empire and its Legacy, a cura di R. Amitai-Preiss Preim e D.O. Morgan, Leiden-Boston-Köln (MA) 1998; J.P. Roux, Genghis Khan et l'empire Mongol, Paris 2002.

107 N. Berend, Cuman Integration in Hungary in Nomads in the Sedentary world, a cura di A.M. Khazanov, Richmond (VA) 2001, pp. 103-127 alla p. 105; Vasary, Cumans and Tatars cit. pp. 138139. Sulla emigrazione cumana in Georgia si veda Golden, Cumanica I cit. dove l'autore fornisce una cifra che è la stessa data generalmente per l'emigrazione in Ungheria, cioè 40.000 persone. 


\subsection{La conquista del Dešt i-Qipčak (1236-1242)}

Alla fine del 1236 l'esercito inviato dopo il quriltai dell'anno precedente verso Ovest e al comando di Batu giunse sui ghiacci del Volga. ${ }^{108}$ Batu era il comandante supremo dell'esercito, ma lo stratega delle operazioni era il vecchio, geniale Subeteï, artefice di molte vittorie mongole. Durante il quriltai la guerra a Ovest era stata pianificata nei minimi dettagli e sarebbe dovuta durare diciotto anni. Ne bastarono molti di meno. Nei primi giorni del 1237 l'esercito mongolo entrò nelle steppe dei Polovcy. Coloro i quali non erano fuggiti con Kutan si sottomisero agli invasori. Ma una parte della popolazione cumana non si rassegnò alla resa. Agli ordini del khan Batčman si riorganizzò una autentica lotta di resistenza combattuta non solo da Cumani, ma anche dai Bulgari del Volga e dagli Alani. ${ }^{109}$

Passata la linea del grande fiume che si getta nel Mar Caspio, fra 1236 e 1237, gli eserciti mongoli dilagarono nelle steppe senza incontrare ostacoli e procedettero con le campagne in Europa e a Nord, contro i principati russi. I nuclei di resistenza guidati da Batčman continuavano però a effettuare rapide incursioni e rientrare altrettanto velocemente nelle foreste sul Volga nascondendosi all'esercito mongolo. ${ }^{110}$ Per stroncare i ribelli furono richiamati Subeteï e Möngke, figlio di Tuli (i quali si trovavano in Europa con Batu). In seguito a una lunga e faticosa ricerca i generali mongoli riuscirono a individuare l'accampamento cumano e a catturarlo. Batčman fu eliminato e con lui gran parte dei ribelli. ${ }^{111}$

Fra la fine degli anni Trenta del XIII secolo e il 1242 i Mongoli guidati da Batu salirono verso la Rus', dilagarono nell'Europa Orientale fino a giungere in Ungheria. Nel febbraio del 1238 fu devastato il piccolo villaggio di Mosca; poi in rapida successione fu la volta di Suzdal' e Vladimir'. Il 4 marzo 1238 Jurij II, granduca di Suzdal', fu sconfitto dall'esercito mongolo sul Sit affluente

${ }^{108}$ La cronologia degli eventi non è chiarissima. Alcune delle fonti persiane, Juvaini e Rashid ad-Din (che si è comunque largamente basato sul primo), parlano appunto della fine del 1236 . I Russi hanno sempre sostenuto che si trattasse dell'anno seguente basandosi sugli annali e il Racconto dei Tempi Passati. Si veda Grousset, L'empire des steppes cit. p. 329; P.P. Toločko, Kočevye narody stepej cit.; G.A. Fedorov - Davydov, Zolotoordynskie goroda povolžja, Moskva 1994, pp. 5-20; Grekov - Jakubovskij, L'Orda d'Oro cit. pp. 40-41.

109 Spuler, Die Goldene Horde cit., p. 16; Grekov - Jakubovskij, L'Orda d'Oro cit. pp. 42-43. A tale proposito Juvaini dice: «uno degli sconfitti, capotribù qifchaq, un tale chiamato Bachman, era sfuggito all'inseguimento insieme a una banda di guerrieri qifchaq, ed era stato raggiunto da altri fuggitivi» (Juvaini, III, p. 626).

${ }_{110}$ Ibidem, III, p. 626: «La maggior parte dei suoi rifugi e nascondigli era sulle rive dell'Etil [il Volga]. Qui si teneva celato nelle foreste, da cui saltava fuori come uno sciacallo, si impadroniva di qualcosa e si nascondeva di nuovo".

111 Grousset, L'empire des steppes cit. p. 329; Pelliot, A propos des Comans cit. pp. 166-167; Juvaini, III, p. 627, secondo cui Möngke ordinò che venisse tagliato in due. 
della Mologa. Ancora, distaccamenti mongoli si diressero verso Jarovslavl' e Tver'; entrambe le città furono conquistate. Le operazioni si protrassero fino al 6 dicembre 1240 allorquando le armate di Batu piombarono su Kiev e la distrussero. ${ }^{112}$ I Mongoli portarono a termine la campagna della Rus' con la conquista di Galič.

Durante le operazioni nella regione delle foreste una parte dell'esercito si diresse verso Ovest. Se è vero che l'Europa occidentale sapeva poco dei Mongoli, non è altrettanto vero il contrario; i Mongoli conoscevano perfettamente l'Europa centro-orientale. Conoscevano i rapporti familiari delle più importanti casate regnanti, conoscevano il territorio, le potenzialità militari dei singoli regni. Non si muovevano senza aver ottenuto le informazioni che ritenevano sufficienti per intraprendere una campagna militare. Per questa ragione e temendo che un attacco unificato alla Polonia potesse suscitare la reazione di Enrico di Slesia e di Venceslao di Boemia, il vecchio Subeteï pensò di dividere l'esercito in tre parti. La prima, al comando di Kaidu, doveva muovere verso la Polonia, la seconda, al comando di Kadan, doveva invadere da sud l'Ungheria e Subeteï, con Batu, avrebbero portato il grosso delle truppe, la terza parte, su Pest. Nell'inverno fra il 1240 e l'anno successivo i Mongoli passarono la Vistola. Il 18 marzo vi fu il primo scontro con i Polacchi che vennero battuti.

Il 24 marzo 1241 le truppe di Kaidu dilagarono in Polonia, gli eserciti assediati cercarono di contenere l'attacco, ma non vi riuscirono. Cracovia fu distrutta. Nei primi giorni di aprile stessa sorte toccò alle campagne di Breslavia e l'8 aprile Kaidu riportò le truppe a Liegniz. Il 9 l'esercito formato da Polacchi, crociati tedeschi e cavalieri teutonici fu sbaragliato nella città. Nel frattempo le truppe al comando di Batu e Subeteï erano penetrate in Ungheria. Gli eventi che ebbero luogo in Europa centrale sono narrati nei dettagli soprattutto dalle fonti orientali, Juvaini e Rashid ad-Din, le quali non mostrano di avere chiara la geografia europea. È pertanto probabile che date e luoghi non siano sempre corretti. Quel che è certo è che all'inizio di aprile del 1241 il vecchio generale mongolo Subeteï sconfisse inesorabilmente le armate di re Bela IV di Ungheria alla confluenza fra il Sajo e la Theiss, davanti a Pest. ${ }^{113}$

Dopo la vittoria i Mongoli incendiarono la città e si dettero alla caccia di Bela, fuggito sull'Adriatico, esattamente come avevano fatto col sultano venti anni prima. Il 25 dicembre 1241 Batu passò il Danubio all'altezza di Gran (in Strigonia). Kadan nel frattempo proseguiva l'inseguimento fino a Spalato dove fu

112 Sulla presa di Kiev da parte dei Mongoli giova vedere gli estratti degli annali russi pubblicati in appendice del Povest' o Nikole Zaraskom, Povest'o razorenii Rjazani Btyem, Pochvala rodu Rjazanskich knjazej, nella trad. it.: Pianto cit.

${ }^{113}$ Grousset, L'Empire des steppes cit., p. 331. Secondo lo storico francese la battaglia di Pest si sarebbe svolta l'11 aprile; Vasary, Cumans and Tatars cit. pp. 138 e segg.; Spuler, Die Goldene Horde cit. pp. 10 e segg. 
raggiunto dall'ordine di Batu che gli intimava di tornare indietro. L'11 dicembre era morto Ogödeï e nessuno poteva mancare al quriltai che si sarebbe tenuto per l'elezione del nuovo khan. Anche Batu dovette rientrare e in molti hanno attribuito a questo evento la salvezza dell'Europa dai Mongoli.

Dopo venti anni di incursioni, guerre, conquiste in successione e vittorie eclatanti, i Mongoli avevano allargato l'ulus di Timučin fino a farne l'impero più vasto che fosse mai esistito nella storia dell'uomo. È difficile dire se davvero la morte di Ogödeï sia stata la causa della rinuncia mongola a proseguire nella loro avanzata. Il quriltai col quale il nuovo khan aveva ordinato le campagne in Europa non prevedeva confini massimi, né limiti minimi ai quali i comandanti militari avrebbero dovuto attenersi con rigore. Le guerre dalle quali i Mongoli uscivano vittoriosi erano, come abbiamo visto, molto ben programmate e tarate sulle reali possibilità di riuscita. L'immagine che i contemporanei ebbero del loro impatto a Ovest ha per molto tempo influenzato il punto di vista della storiografia critica. L'organizzazione con la quale combattevano e le strategie militari certamente innovative che attuavano in battaglia impressionavano in tal modo che a un certo punto la loro fama superò di sicuro l'effettiva minaccia che avrebbero potuto costituire per l'Europa. La stessa denominazione, Tartari, utilizzata costantemente nelle fonti occidentali è la prova dell'associazione demoniaca, soprannaturale, operata in Occidente all'immagine di questo popolo del quale si utilizzò una componente etnica, quella dei Tatari appunto, per deformarla in Tartari, provenienti dal Tartaro. Le operazioni militari di cui furono protagonisti mutarono completamente la geografia politica di un intero continente e di tutta la regione che abbraccia il Mar Nero lasciando esclusa solo la parte occidentale, la Dobrugia e Bisanzio. Gli effetti della dominazione mongola si fecero sentire non solo a livello politico, con il parziale e disomogeneo rinnovamento istituzionale degli stati conquistati, ma anche dal punto di vista economico e culturale. Il Caucaso e l'Azov rappresentarono la porta d'accesso a questa nuova entità politica e alle sue potenzialità economiche. Divennero centro di una complessa rete commerciale che da qui si ramificava in tutte le maggiori piazze dell'Oriente unificato dai Mongoli. È difficile stabilire fino a che punto l'opera dell'esercito di Gengis abbia trasformato una pura immagine mentale in realtà concreta e accessibile. Fatto si è che nei primi quaranta anni del XIII secolo cambiò tutto. Dalla IV crociata alla conquista di Kiev l'Oriente si spalanca all'intraprendenza e alla formidabile organizzazione dei mercanti occidentali. La devastazione di cui parlano i viaggiatori occidentali, riferendosi alle città che furono teatro delle battaglie combattute dai Mongoli lasciò il posto alla ricostruzione e alla riorganizzazione istituzionale. Batu khan, erede del territorio più lontano dalla sede dell'impero mongolo, fondò l'Orda d'Oro e si dimostrò particolarmente sensibile alle necessità di una ricostruzione. Non è un caso che di lui gli osservatori del tempo ebbero ad ammirare l'apertura a Ovest e l'apprezzamento per l'attività di scambio. Batu si rese perfettamente 
conto che dopo la conquista bisognava riavviare un processo virtuoso col quale riportare ricchezza nelle regioni devastate dalla guerra.

Era un uomo assai giusto e amico dei musulmani; sotto la sua protezione i musulmani vivevano liberamente [...]. All'ombra della sua protezione i musulmani del Turkestan godevano di gran tranquillità e di straordinaria sicurezza. ${ }^{114}$

Anche Juvaini rileva la particolare attenzione di Batu per i mercanti e la loro attività:

Da ogni parte i mercanti gli portavano merci; qualunque cosa fosse, la prendeva, e la pagava a un prezzo di gran lunga superiore al suo valore. Ai sultani di Rum, e della Siria e di altri paesi largiva privilegi e jarlyk (un lasciapassare) e chiunque veniva al suo servizio non tornava senza un utile. ${ }^{115}$

L'invasione mongola si era abbattuta sulla Caucasia violentemente, scompaginando una serie di equilibri in parte acquisiti e in parte già indeboliti. Di fatto la generale devastazione prodotta dalle guerre imponeva una rapida ricostruzione e su molti fronti. Di questo i Mongoli si dimostrarono consapevoli.

${ }^{114}$ Grekov - Jakubovskij, L'Orda d'Oro cit. p. 45. Sono parole dello storico persiano AlJuzjani, autore della Tabakat-i-Nasiri (Le tavole di Nasir) il quale non nutriva per i Mongoli sentimenti particolarmente positivi; anche per questo la sua testimonianza sulla condotta di Batu è interessante.

115 Juvaini, I, pp. 300-301. La percezione delle doti di Batu come buon amministratore e protettore dei mercanti si riscontra in tutte le fonti. 


\section{GLI EFFETTI POLITICI DELLE CONQUISTE E LA SEPARAZIONE CAUCASICA FRA ORDA D'ORO E ILKHANATO}

\subsection{Dal nomadismo alla formazione dello stato}

Nel suo celebre saggio sul nomadismo Khazanov affermava che la conquista e la sottomissione di una società sedentaria da parte di un organismo nomade è solo uno dei modi di formazione di quello che egli chiama «nomadic state», Stato nomade appunto. ${ }^{1} \mathrm{Al}$ contrario è altrettanto vero che non tutte le conquiste da parte di formazioni nomadi hanno generato organismi che possiamo lecitamente definire come stati. L'espansione per una realtà nomade era un'esigenza spesso irrinunciabile, ma non sempre ha condotto alla formazione di stati nomadi. ${ }^{2}$ Come abbiamo già affermato le società nomadi non sono in grado di provvedere totalmente ai propri bisogni esenziali; si rende pertanto necessaria una complessa interazione con quei contesti stabili ed economicamente diversificati basati su una buona organizzazione produttiva. Come ha posto in rilievo $\mathrm{Di}_{\mathrm{C}} \mathrm{Cosmo}^{3} \mathrm{per}$ accettare la teoria secondo la quale i rapporti fra nomadismo e vicini sedentari si baserebbero su una sorta di costante bisogno del primo nei confronti delle risorse dei secondi bisognerebbe dare per scontato che nelle steppe dell'Asia Centrale fosse impossibile produrre alcunché dal suolo; ovvero che l'agricoltura sarebbe stata del tutto assente.

\subsubsection{La razzia come prima risposta}

L'ambiente ostile entro il quale sorsero le prime unioni nomadi fu determinante nell'influenzare gran parte dei comportamenti ritualizzati e coerenti; da

1 Potremmo dire, semplificando, che per Stato nomade si può intendere quell'organismo sociale che, in seguito ad un processo evolutivo costante, ha raggiunto una solida organizzazione politica e all'interno del quale la maggior parte della popolazione vive in una condizione di nomadismo. Cfr. Khazanov, Nomads and the outside world cit., p. 228.

${ }^{2}$ Per un inquadramento del problema oltre al già citato saggio di Khazanov si veda N.N. Kradin, Nomadism, Evolution and World-Systems: Pastoral Societies in Theories of Historical Developement, in «Journal of world-systems research», VIII (2002), 3, pp. 368-388; Di Cosmo, State formation cit. Molto interessanti anche se in gran parte discutibli sono le conclusioni cui giunse L. Krader, The origin of the State Among the Nomads of Asia, in The Early State a cura di H. Claessen, Mouton 1978, pp. 93.107. A tale proposito si veda N.C. Munkuev, Zametki o drevnich mongolack, in Tataro.mongoly v Azii i Evrope. Sbornik statei, Moskva, 1977, pp. 409-446 e Khazanov, Nomads and the outside world cit., p. 234.

3 Di Cosmo, Ancient Asian Nomads cit., p. 1094. 
questo punto di vista una delle risposte più urgenti alle necessità impellenti delle cellule associative fu la razzia o l'accaparramento istituzionalizzato di beni altrui. È questo un aspetto peculiare del nomadismo, sebbene gran parte delle guerre di epoca medievale avessero caratteristiche simili anche in contesti sedentari.

Sin dalle prime fasi del processo di unificazione i Mongoli avevano una forte propensione all'espansione cui faceva seguito la sottomissione delle tribù circostanti. Nel contesto di un'economia nomade la razzia era l'elemento fondamentale per appropriarsi di quei beni che la tribù non era in grado di produrre e di cui aveva bisogno (e riemerge l'influenza dell'ambiente, la sua scarsa generosità e l'esigenza, adattandovisi, di provvedere laddove esso manca). $\mathrm{Da}$ questo punto di vista il contatto con le società sedentarie era necessario e frequente. La razzia non era un fenomeno estemporaneo e improvvisato. Al contrario, si trattava di iniziative molto ben organizzate e parte di un sistema di approvvigionamento complesso. A titolo di esempio si pensi al passo della Storia Segreta in cui, sconfitti i Merkit, Gengis Khan «catturò l'intero suo ulus [si riferisce a Togtoga, il beki dei Merkit] con tutti i suoi sudditi e prese le loro abitazioni». ${ }^{4}$ Lo stesso Juvaini sostiene che durante le operazioni militari nel Turkestan i Mongoli prendevano regolarmente «bestiame e tessuti». ${ }^{5}$ Ibn alAthir afferma che in Georgia i Mongoli chiedevano alla popolazione della città di Ganja (Gandzak, oggi nell'Armenia settentrionale, cfr. cap. I) tessuti dorati e denaro. ${ }^{6}$ Durante la presa di Gandzak, nella seconda campagna di Gengis Khan nel Caucaso,

i Tartari giravano per i distretti della città in cerca di beni e ricchezza. Essi scoprirono molti oggetti fatti d'oro, argento, bronzo ferro così come abiti nascosti nelle cantine e nelle camere sotterranees.?

Anche Kiriakos Gandiate descrive la presa di Gandzak come una sciagura nella quale la razzia fu perpetrata più volte ai danni della popolazione civile:

Allora presero la grotta nella quale stava il gran Vardapet Vanakan, piena di ricchezze e portarono via il glorioso vardapet in persona, coi suoi studenti

${ }^{4}$ SSM, VII, 197, p. 168.

5 Juvaini, I, p. 119; Allsen, Commodity and exchange cit., p. 27.

${ }^{6}$ Ibn al-Athir, in Allsen, Commodity and exchange, pp. 27-28; Brosset, Histoire de la Géorgie cit., p. 71.

7 Kirakos Gandzakets, History of the Armenians, ed. a cura di R. Bedrosian, New York (NY) 1986 (d'ora in avanti KG), cap. 21, p. 199; Kirakos Gandzaketsi, Istorija Armenii, ed. a cura di A. Khanlarian, Moskva 1976. Si veda anche Z. Arzoumanian, Studies in Armenian Historiography: Bishop Sebeos, Lewond the Priest, Kirakos of Gandzak, Wynnewood (PA) 1981. Il monaco armeno Kirakos nacque probabilmente nei primi anni del XIII secolo. Operò nei monasteri di Getik e di Gandzak fino all'invasione mongola della Caucasia allorquando fu catturato. Cfr. J.A. Boyle, Kiriakos Gandzak on the Mongols, in "Central Asiatic Journal», (1963), pp. 199-214. 
[...]. Tutto il paese, unito nella disgrazia, dette loro un gran tesoro e oro per riscattare il vardapet e i suoi studenti ${ }^{8}$.

Gli episodi di razzia durante le conquiste sono frequenti e le fonti ne parlano con grande impressione e sdegno. Naturalmente i cronisti armeni e georgiani sottolineano il furto di sacre scritture, libri religiosi e il saccheggio delle chiese con maggiore indignazione e dovizia di particolari. Nel corso delle razzie i pezzi migliori toccavano ai personaggi eminenti, mentre il resto dell'esercito si doveva accontentare di quel che rimaneva. Questo sistema dette avvio a una prima forma di circolazione delle merci all'interno del vasto e neonato impero.

Un'altra forma di razzia fu senza dubbio la tassazione imposta agli stati conquistati nella prima fase di dominio che non era il frutto di una politica strutturale volta al mantenimento dell'amministrazione pubblica. Questo aspetto emergerà col tempo, quando il ceto dirigente inizierà ad acquisire piena consapevolezza delle esigenze amministrative nelle realtà locali e dopo che l'aristocrazia mongola si sarà anche solo parzialmente assimilata al contesto locale. Naturalmente questo fenomeno poté verificarsi solo in quelle parti dell'impero dove la classe dirigente mongola si affiancò o si sostituì a quella locale, mentre nei territori dove ciò non avvenne (come nella Rus') l'aspetto fiscale rimase in sostanza una forma di razzia arbitraria e irregolare. Ad esempio quando Čormaghan ebbe portato a termine la campagna del Caucaso, negli anni Trenta del XIII secolo, Ogödeï gli ordinò di esigere un tributo fisso dal califfo di Baghdad; tale onere consisteva in bestiame, oro e tessuti (soprattutto i nasij persiani e le stoffe di Damasco). . Si trattava di un efficace sistema di controllo sull'aristocrazia locale, ma anche di una pratica alla quale i Mongoli non sapevano ancora rinunciare. Il processo di reidentificazione fu lento e molto complesso, ma si realizzò solo in alcune parti dell'impero generando come conseguenza, fra le altre, la cessazione del prelievo indiscriminato e arbitrario. Abbiamo più volte posto l'accento sulla buona predisposizione da parte dei Mongoli verso il commercio e i suoi attori, ma non è mai abbastanza se consideriamo quali effetti ebbe questo atteggiamento della classe dirigente nomade verso la pratica mercantile. ${ }^{10}$

\subsubsection{I processi organizzativi}

Non v'è dubbio che l'impero mongolo fosse una forma di stato nata in seguito alle straordinarie conquiste conseguite da Gengis Khan e dai suoi successori. Proprio la politica posta in essere dai successori portò a un sensibile incremento del flusso di merci attraverso l'Eurasia, in particolare di tessuti, per

${ }^{8}$ GA, cap. 3, p. 4.

9 Allsen, Commodity and exchange cit., p. 29.

${ }^{10} \mathrm{Su}$ questo si veda anche T.T. Allsen, Mongolian Princes and Their Merchant Partners, in "Asia Major», s. III, 2 (1989), 2, pp. 83-126. 
i quali i Mongoli avevano una particolare predilezione. Dopo pochi decenni di dominio il ceto dirigente mongolo comprese appieno l'importanza di una pratica con la quale non aveva familiarità, la produzione finalizzata allo scambio. Alla metà del XIII secolo molte zone dell'impero, dalla Cina alla Persia, erano in grado di produrre gran parte dei prodotti di cui la popolazione aveva bisogno e potevano creare quel surplus in virtù del quale presero vigore gli scambi su scala internazionale. I governatori della Cina Yüan ad esempio si rendevano perfettamente conto che sostenere la produzione in loco piuttosto che sprecare energie e mezzi per acquisire quegli stessi beni altrove era una pratica poco conveniente. D'altra parte l'origine nomade dei Mongoli creava loro alcuni problemi pratici che poterono risolvere solo col tempo e mai in modo definitivo. Uno dei principali ostacoli coi quali si trovarono a confronto dopo le conquiste fu la gestione di un sistema che conoscevano solo "teoricamente", ma che non erano in grado di realizzare in concreto. Come abbiamo visto parlando dell'organizzazione sociale presso le tribù mongole tradizionali il nomade aveva un'identità sociale frutto di un livellamento che non era integrale, ma piuttosto diffuso. In virtù di questo paradigma associativo e delle particolari condizioni ambientali con le quali dovevano confrontarsi ogni giorno, i nomadi erano in grado di svolgere gran parte delle mansioni richieste dalla vita itinerante nella steppa. Quando questi stessi individui vennero in contatto con una realtà nuova, in cui vi erano esigenze completamente diverse (o per lo meno erano diversi i modi per soddisfarle) si trovarono in difficoltà e dovettero ricorrere allo sfruttamento di risorse umane di cui dovevano potersi fidare. Fu esattamente ciò che accadde nella Cina degli Yüan e, anche se in misura minore, nell'Ilkhanato. Gli Yüan in particolare furono gli unici dirigenti mongoli di tutto l'impero a non convertirsi mai all'Islam; né si affidarono alla dirigenza locale. L'aristocrazia territoriale soffrì molto durante il governo mongolo e la vicenda dei Polo è, da questo punto di vista, emblematica. Stranieri, musulmani del Turkestan soprattutto, venivano chiamati e talvolta inseriti nei ranghi più alti dell'amministrazione pubblica. Se in Cina i burocrati locali di professione vennero spesso sostituiti da funzionari stranieri, nell'Ilkhanato ai primi furono affiancati mercanti e religiosi nell'amministrare le istituzioni. Due aspetti, questi, nevralgici per la vita mediorientale e il controllo su di essi significava spesso poter controllare la popolazione con maggiore efficacia. ${ }^{11}$ Nell'Orda d'Oro la situazione si stabilizzò in modo ancora diverso. Vedremo poco più avanti che la classe dirigente mongola non esercitò un controllo diretto sul territorio tranne che in alcune zone e nei centri che considerava fondamentali per la gestione degli affari più redditizi. Anche in questo caso l'ambiente giocò un ruolo importante poiché la Rus' era immersa quasi per la sua totalità in una regione ricca di foresta; i Mongoli non conoscevano la foresta, né sapevano come potervi sopravvivere sfruttandone le risorse. Anche per questo motivo, oltre che per le molteplici ragioni

${ }^{11}$ Allsen, Commodity and exchange cit., p. 31. 
politiche sulle quali ci soffermeremo diffusamente, non vi si stabilirono mai in modo definitivo, preferendo le fertili pianure sul basso corso del Volga e lasciando solo quando necessario un governatore ad amministrare i distretti più lontani. Possiamo citare, a titolo di esempio, il caso dell'immigrazione occidentale: la città di Caffa raggiunse un'autonomia totale, sia dalla madrepatria sia dal governatore mongolo di Solgat col quale finì anche per fare la guerra; lo stesso si può dire per Soldaïa e per la Tana ${ }^{12}$. È vero che i mercanti occidentali dovettero spesso far fronte ai cambiamenti politici repentini che intervenivano nella regione e furono sottoposti sempre a una rigida tassazione, ma è altrettanto vero che questo non ne frenò la spinta migratoria, né ridimensionò i guadagni di gran parte di loro. Nell'Ilkhanato molti personaggi scalarono posizioni sociali importanti grazie ai Mongoli (si pensi a Juvaini e a Rashid ad-Din). Ma la classe lavoratrice su cui più si concentrarono gli sforzi di "reclutamento" fu quella degli artigiani. Essi venivano utilizzati nelle province dell'impero secondo una generale suddivisione che prevedeva tre grandi categorie: quelli che lavoravano a corte, quelli che venivano impiegati nell'esercito e quelli che producevano "privatamente" ed erano tassati dal governo. La condizione artigianale era un marchio sociale in Cina, mentre non abbiamo notizie altrettanto chiare sull'Ilkhanato e sull'Orda d'Oro. Di fatto dalla seconda metà del XIII secolo la produzione (soprattutto di tessuti) subì un forte incremento in gran parte dell'impero. Quando parliamo di effetti della pace mongola bisogna porre l'accento anche su questo aspetto: l'apertura dei mercati orientali garantì una circolazione su lunghe distanze di merci che venivano prodotte anche prima del XIII secolo, ma dalla metà del XIII secolo certe lavorazioni vennero organizzate sistematicamente per far fronte $\mathrm{a}$ un mercato più esigente $\mathrm{e}$ nel segno di una precisa volontà che era quella del ceto dirigente mongolo.

I Mongoli rimasero dunque sostanzialmente nomadi, ma furono molto rapidi nel comprendere l'importanza della produzione finalizzata allo scambio e nel creare le migliori condizioni affinché essa potesse prosperare. Se proviamo poi a osservare cosa accadeva all'interno dell'Asia ci accorgiamo che anche i rapporti commerciali fra la Cina degli Yüan e l'Ilkhanato furono intensi sin dai primi anni di vita dello stato mongolo mediorientale e andarono via via inglobandosi in un più ampio contesto animato da mercanti di varia provenienza e fra i quali spiccavano quelli egiziani. ${ }^{13}$ I Mongoli partecipavano attivamente a questo commercio. Ibn Muham-

${ }^{12} \mathrm{Su}$ Caffa si veda il già citato lavoro di Michel Balard (La Romanie génoise). Per la Tana si veda E. Skržinskaja, Storia della Tana, in «Studi Veneziani», X (1968), pp. 3-47; M. Berindei, G. Veinstein, La Tana-Azaq de la présence italienne à l'emprise ottomane (fin XIIIe-milieu XVIe siècle), in «Turcica», VIII (1976), pp. 110-201; S. P. Karpov, On the origin of medieval Tana, in «Bizantino-Slavica», LVI (1995), pp. 225-235; Id., Srednevekovyj Pont, New York (NY) 2001, alle pp. 343-362; L. Pubblici, Venezia e il mar d'Azov. Alcune considerazioni sulla Tana nel XIV secolo, in "Archivio Storico Italiano", CLXIII (2005), pp. 435-84.

13 T. Allsen, Culture and Conquest in Mongol Eurasia, Cambridge 2001, p. 42; Amitai Preiss, Mongols and Mamluks cit. 
med al-Tibi, il soprintendente per i Mongoli deputato alla riscossione dei tributi nell'Ilkhanato meridionale, aveva messo in piedi un sistema commerciale estremamente vivace ed efficiente fra i territori sotto la sua amministrazione e la Cina degli Yüan. Wassaf sostiene che quello che veniva prodotto nella Cina più remota si consumava in Occidente. ${ }^{14}$ Ibn Battuta, che giunge in Cina nel 1327, sostiene che molte navi cinesi prendevano il largo in direzione del Golfo Persico. ${ }^{15}$ Dalla seconda metà del Duecento la Cina visse un momento di straordinaria crescita economica veicolato sia dall'aumento della produzione sia dallo sfruttamento delle nuove opportunità commerciali. La dinastia dei Sung incoraggiava il commercio con il Turkestan musulmano e, quando gli Yüan mongoli prevalsero, il grande paese orientale ricevette un impulso nuovo e positivo. Cambiò sostanzialmente la situazione politica. Il mercato cinese era del tutto nuovo e aveva potenzialità enormi. Sorsero associazioni di mercanti musulmani (ortakh) che ottenevano prestiti dal governo per il sostegno delle loro attività; sorsero moschee, ospedali e bazar e ottennero una sostanziale autonomia amministrativa. Le vie di commercio che collegavano il Turkestan al suo interno con l'Estremo Oriente erano ben sviluppate già prima della conquista mongola, ma anch'esse beneficiarono della maggiore sicurezza garantita dal nuovo organismo politico-amministrativo. I Mongoli conoscevano bene le aree entro le quali passavano le principali strade carovaniere ed erano profondi conoscitori delle potenzialità dei fiumi; amavano assai meno la montagna e le difficoltà che essa presentava per l'approvvigionamento delle risorse indispensabili alla sussistenza. Marco Polo, allorquando passa per la strada del Tibet, attraverso le città di Wakhān e Shughnān per recarsi nella regione di Kāshghar, afferma che dopo venti giorni di viaggio per luoghi deserti «si truova castella e case assai»; ${ }^{16}$ e più avanti aggiunge che

è si grande, che v'ha bene otto reami grandi e grandissima quantità di cittade e di castella. Egli v'ha in molti luoghi fiumi e laghi, e havvi montagne, ove si truova l'oro di pagliuola in grande quantità». ${ }^{17}$

${ }_{14}$ Wassaf (J.von Hammer-Purgstall, Geschichte Wassafs, Wien 1856), pp. 37-39; Spuler, History of the Mongols cit., pp. 165-168; si veda anche Allsen, Culture and conquest cit., p. 42; J. Aubin, Les princes d'Ormuz du XIII eau XVe siècle, in «Journal Asiatique», 241 (1953), pp. 89-99.

${ }^{15}$ Le edizioni dei viaggi di Ibn Battuta sono molte, dalla fondamentale traduzione di C. Defremery - B. R. Sanguinetti, Voyages, Paris 1854, più volte ristampata e tradotta (ho utilizzato prevalentemente l'edizione Cambridge: The Travels of Ibn Battuta, AD 1325-1354, 1962, in 2 voll.), all'ottima edizione italiana di F. Gabrieli, I viaggi di Ibn Battuta, Firenze 1961. Si veda anche il recente studio di R. E. Dunn, Gli straordinari viaggi di Ibn Battuta, Milano 1998. Il passo in questione è in Ibn Battuta, The Travels cit., vol. II, p. 320

${ }^{16}$ M. Polo, Il Milione, a cura di M. Ciccuto, Milano 1998, XCIX, p. 275; Delle numerose edizioni dell'opera di Marco Polo ve ne sono molte che meriterebbero una citazione. In questa sede, e per ragioni pratiche, ci siamo serviti dell'edizione citate insieme a altre tra: Il Milione, a cura di D. Olivieri, Bari 1928² Il Milione, a cura di D. Ponchiroli, Torino 1982; Le divisament dou Monde, a cura di G. Ronchi, Milano $2006^{3}$.

${ }_{17}$ Milione, ed. Ronchi, p. 468. L’oro di pagliuola è la polvere d'oro (pagliuola = pagliuzze). 
Molti studiosi hanno fatto ricorso alla narrazione di Marco Polo per dimostrare la straordinaria mobilità della quale poteva beneficiare un mercante, fosse esso occidentale o meno, entro l'impero mongolo. L'opera del veneziano è sostanzialmente attendibile e i suoi racconti, sebbene non siano sempre di prima mano, sono precisi e molto istruttivi. Una lettura del Milione dà l'idea di quello che ha rappresentato l'amministrazione mongola di un territorio così vasto negli anni successivi alle conquiste.

La formazione dell'impero mongolo è un esempio di come un organismo politico possa formarsi in tempi relativamente brevi e altrettanto rapidamente collassare. Certo si è che la dominazione ebbe caratteristiche assai diverse nei molti territori sui quali si estese. Giova pertanto considerare il caso della Caucasia nel dettaglio distinguendo l'area meridionale dall'Azov, rimanendo fra l'influenza degli "Stati" più occidentali di tutto il sistema politico creato dai Mongoli: l'Orda d'oro e l'Ilkhanato.

\subsection{La riorganizzazione del territorio e la nuova geografia fra Caucaso e Azov}

Gli effetti delle campagne mongole dettero vita a una profonda riorganizzazione del potere. Dal 1236 Tblisi era stata posta sotto l'autorità mongola. La città manteneva sostanzialmente le sue istituzioni, ma queste erano direttamente responsabili del loro operato nei confronti degli esponenti mongoli nella regione (subito dopo la conquista tale compito spettava direttamente al generale Čormaghan). Nel 1239 era caduta la Grande Armenia con la conquista di Kars e Ani; nel 1242 il generale mongolo aveva portato l'attacco al cuore del sultanato selgiuchide; cadute Erzrum e Erzindjan si determinò il passaggio del territorio di Iconio nelle mani dei Mongoli (26 giungo 1243). Fra la conquista dei territori di Armenia e Georgia e quella del sultanato selgiuchide di Iconio, si era verificata la capitolazione dei principati russi. Tutta l'area compresa fra Mar Nero e Mar Caspio, oltre alle steppe dei Qipciaki, era, più o meno direttamente, sotto l'autorità mongola. Alla morte del gran Khan Ogödeï (1241) la guida dell'impero fu assunta dal figlio Güyük (1246-1248). La spinta espansionistica dei Mongoli si affievolì temporaneamente a causa dei contrasti interni alla famiglia imperiale e alle dispute sorte in merito alla successione e alla morte prematura del Khan (nel 1248 aveva appena 43 anni).

I contrasti a corte erano già fortissimi fra il partito di Čagataï/Ogödeï da una parte e quello di Tuli dall'altra. Batu, figlio di Giuči prese posizione in favore dei Tulidi, rappresentati da Möngke. Questi era un fermo sostenitore della necessità di muovere gli eserciti soprattutto a Est e consolidare il proprio potere sulla Cina. È probabile che Batu vedesse di buon occhio l'elezione di Möngke proprio per questo: concentrando i suoi sforzi a Est il nuovo Khan lo avrebbe lasciato tranquillo a ovest e questi avrebbe potuto accrescere la propria autonomia dall'impero. Probabile che Batu desiderasse scendere verso sud fino 
alla Transcaucasia ed estendere la propria sovranità sulle regioni del Califfato abbaside di Baghdad.

Güyük, salito al potere grazie alla ferma volontà della madre, Törägän, è colui presso il quale si recò Giovanni di Pian del Carpine che ce lo descrive come un uomo energico, severo, assai attaccato al suo potere. ${ }^{18}$ Di certo il Khan era convinto della necessità di consolidare e, se possibile, estendere il potere mongolo a Ovest. Nella Caucasia Güyük impose la separazione della Georgia in due parti separate: la Kartlia e l'Imerezia. La Georgia, come detto, non era direttamente controllata dai Mongoli. Essi avevano obbligato al vincolo di fedeltà l'aristocrazia locale che governava in modo sostanzialmente autonomo, ma in questo periodo lo stato maggiore mongolo (che aveva il suo quartier generale a ridosso della Georgia, sulla piana di Mughan nell'Azerbaijan) era ancora in grado di mutarne gli equilibri politici a suo piacimento. La Kartlia fu assegnata a David Lacha, mentre il figlio della deposta regina Rasudan, David Narin, conservò il territorio dell'Imerezia. ${ }^{19}$ Nella Caucasia il sistema di controllo esercitato dai nuovi dominatori si concretizzava spesso nella deposizione improvvisa di un sovrano e nella nomina immediata di un successore estraneo alla linea dinastica locale. ${ }^{20}$ In Anatolia i nuovi padroni concessero il trono di Iconio al sultano Kili Arslan IV spodestando il legittimo erede, il fratello anziano Kai-Kavus II. Anche in quest'area sono molti gli esempi di manipolazioni istituzionali operate dai governatori mongoli nelle città o nei distretti conquistati. ${ }^{21}$

Alla morte di Güyük i contrasti dinastici in seno al clan imperiale si acuirono e sfociarono in un autentico colpo di Stato dal quale uscì vittorioso il partito di Möngke (1251-1259), ${ }^{22}$ nipote di Gengis in quanto figlio di Tuli. Batu aveva partecipato attivamente a questa operazione. L'elezione del nuovo Khan determinò la successiva politica estera dei Mongoli, ma essa non attese esattamente le speranze di Batu. In effetti rispetto a Güyük il nuovo Khan produsse il suo massimo sforzo sulle regioni orientali e sulla Cina in particolare, ma si preoccupò ben presto anche di consolidare il suo potere in Occidente. Sotto di lui il vasto

18 Rashid ad-Din sostiene che l'elezione di Güyük rappresentò una trasgressione degli ordini di Ogodeï, il quale voleva porre al comando dell'impero il nipote Shiremün (Boyle, The successors cit., p. 19). Più avanti lo storico persiano spiega come Güyük ottenne il potere (Boyle cit., pp. 180-184). Si veda anche quanto dice Hethum (STHA, pp. 319-322).

19 Grousset, L'empire des steppes cit., p. 337; KG, pp. 264-266.

${ }^{20}$ Cfr. Bedrosian, The Turco-Mongol invasions cit., in particolare Mongol Control Techniques in the 13th Century, pp. 180-201).

21 Bedrosian, The Turco-Mongol invasions, specialmente il terzo capitolo Armenia's Lords and Their Reactions to the Turco-Mongol Invasions and Domination of the 13-14th Centuries cit., pp.156-179.

22 Su Möngke cfr. Rashid ad-Din (Boyle, The successors cit., pp. 187-237) ed Hethum (STHA, pp.319-328). Si veda anche l'ottimo articolo di Thomas Allsen (Guard and Governament in the Reign of the Grand Qan Mongke, 1251-59, in Harvard Journal of Asiatic Studies 46 (1986), 2, pp. $495-521$. 
territorio sottomesso alla dominazione mongola riprese una certa stabilità e fu possibile avviare nuove campagne di conquista. Nel 1253 venne pianificata la spedizione a Ovest che contemplava l'attacco alla potenza più antica e stabile del Medio Oriente, il califfato di Baghdad. Non potendo intervenire in alcun modo sui territori di Batu (in quanto quest'ultimo lo aveva concretamente aiutato a prendere il trono e soprattutto perché l'ulus di Batu era un beneficio concesso da Gengis in persona ed era quindi intoccabile), progettò l'allargamento delle conquiste in Medio Oriente. Le operazioni, iniziate nel 1253, ebbero il loro epilogo nel 1258 con la conquista della Mesopotamia e di Baghdad. Nel 1260 l'esercito di Hülegü, fratello di Möngke e responsabile della campagna in Medio Oriente, venne sconfitto dalle armate dell'Egitto Memelucco a 'Ain Jālūt, in Siria. ${ }^{23}$ Ma tale evento non mutò nella sostanza le cose per i Mongoli, mentre si rivelò determinante per l'Egitto.

La conquista di Baghdad da parte di Hülegü dette vita a quello che conosciamo come Ilkhanato di Persia. ${ }^{24}$ La nascita di un nuovo regno sostanzialmente autonomo - non si sa quanto voluto da Möngke, ma certamente molto apprezzato da Hülegü - fu un ulteriore motivo di irrigidimento nei rapporti con i dirigenti dell'ulus di Giuči i quali vedevano nella creazione dell'Ilkhanato un pericoloso antagonista, uno spostamento delle dispute familiari a Ovest. Il titolo di Ilkhan (Khan minore, sottomesso) fu forse adottato proprio per sottolineare, da parte di Hülegü, la propria formale subordinazione al Khan del Nord, a Batu e ai suoi successori ${ }^{25}$ per non avere problemi con questo. Di fatto, dopo l'arrivo di Hülegü in Iran e l'instaurazione della sua sovranità fino alla Caucasia, i rapporti fra i due si irrigidirono notevolmente. È probabile, come ha ipotizzato Thomas Allsen, che il fratello del Khan fosse stato inviato in Iran temporaneamente, senza che a Karakorum vi fosse l'intenzione di creare in quell'area un regno unico sotto il controllo diretto di Hülegü. ${ }^{26}$ Ritengo che, alla luce degli sviluppi militari legati alla conquista del territorio in Persia, il Khanato centrale avesse intenzione di porre sotto la formale autorità di Batu quello che diventerà poi l'Ilkhanato.

Möngke è stato definito da Grousset l'unico erede degno di Gengis. ${ }^{27}$ La sua fu una figura molto presente nelle vicende politiche dell'impero. Politico duro,

${ }^{23}$ Amitai-Preiss, Mongols and Mamluks cit., pp. 26-48; J. Masson Smith, Ayn Jalut: Mamluk sucess or Mongol failure?, in Harvard Journal of Asiatic Studies, 44 (1984), 2, pp. 307-345.

${ }^{24}$ La conquista mongola della Persia meriterebbe una trattazione a sé. Si veda a tale riguardo il lavoro di T.T. Allsen, Culture and Conquest cit., in particolare i capp. 3-5, pp. 15-40, dove si può trovare un'esauriente e aggiornata bibliografia.

${ }^{25}$ Allsen, Culture and conquest cit., pp. 22-23; P. Pelliot-L. Hambis, Histoire des campagnes de Gengis Khan, Leiden 1951, pp. 363-364; J.A. Boyle, The Death of the Last 'Abbasid Caliph': A Contemporary Muslim Account, "Journal of Semitic Studies», 6 (1961), pp. 151-152.

${ }^{26}$ Allsen, Culture and Conquest cit., p. 20.

27 Grousset, L'empire des steppes cit., p. 341. 
uomo energico ma intelligente, convinto sostenitore della necessità di tenere unito l'impero e di non conferire eccessive autonomie ai territori periferici, egli riuscì a stabilizzare il vasto territorio in tutte le sue regioni. Dovette lottare non poco a corte e fuori, negli ulus periferici, per affermare la propria volontà ma alla fine vi riuscì. Il vero ostacolo alla politica unitarista di Möngke fu sempre e solo Batu; altro uomo forte e di grande carisma. I rapporti fra i due erano buoni, ma essi avevano una diversa concezione dell'impero. Batu, discendente dell'oboq imperiale, tendeva a rendersi il più possibile indipendente da Karakorum; Möngke dal canto suo era convinto che l'impero dovesse avere una sua formale unità e lasciare agli ulus periferici una limitata autonomia di gestione, condizione inapplicabile all'interno di una realtà così vasta.

Quando Batu dette avvio all'organizzazione del vasto territorio ereditato e da lui notevolmente ampliato doveva rendere conto delle sue operazioni al Gran Khan. Gli ulus periferici erano ancora saldamente legati alla sede dell'impero, all'ulus di Gengis. Ma ben presto l'Orda d'Oro acquisì una sostanziale indipendenza dal resto dell'impero e visse una lunga fase di sviluppo che ne rafforzò progressivamente l'autonomia. La politica interna del nuovo stato influenzò direttamente anche quella delle zone a essa periferiche. La Subcaucasia, pur formalmente nelle mani dell'aristocrazia locale, subì le conseguenze dell'apertura a Ovest voluta e posta in essere da Batu. Stessa sorte toccò alla Transcaucasia contemporaneamente al consolidamento della presenza mongola - di Hülegü - da Sud. Lo scontro fra i due stati mongoli sarebbe divenuto presto inevitabile e avrebbe coinvolto da vicino tutta la linea del Caucaso provocando un nuovo stravolgimento politico.

\subsection{La Caucasia e l'Ilkhanato}

Sin dall'invasione mongola del 1236 la Caucasia entrò irrimediabilmente nell'orbita dei Mongoli. Il periodo che va da questa data fino al 1261, al primo scontro cioè fra il nuovo soggetto politico (l'Ilkhanato) e l'Orda d'Oro di Batu, vide profondi mutamenti politici e sociali, soprattutto nella classe dirigente. I rapporti fra i Mongoli e gli stati della Caucasia attraversarono momenti diversi nel corso degli anni; una situazione a fasi alterne che andò via via irrigidendosi fino a raggiungere i connotati della tirannia per poi mutare in un sistema più equo con i suoi abitanti e favorire una condizione di relativo sviluppo generalizzato.

Sia l'Armenia storica che la Georgia avevano pesantemente risentito delle guerre avvenute fra 1220 e 1242. L'Armenia fu in pratica attaccata quattro volte dalle ondate nomadi. Una prima volta durante la spedizione lampo mongola del 1220-1221, una seconda volta dai Cumani in fuga nel 1222 (come abbiamo visto in precedenza). La terza invasione fu la conseguenza della strategia (o della fuga) del sultano Jalal ad-Din che, fra 1225 e 1230, si riversò nel Caucaso con un esercito numerosissimo e attaccò la regione conquistandone città e campagne; 
una guerra intrapresa dal sultano contro i Mongoli che costò perdite ingenti in vite umane e danni enormi alle infrastrutture in regioni terze. A nulla valse lo sforzo militare armeno-georgiano per contrastare l'incedere dell'esercito messo insieme da Jalal ad-Din (composto in gran parte da Cumani e Turkmeni). La quarta invasione fu però diversa. Essa ebbe luogo dal 1236 e durò per quasi dieci anni. Stavolta i Mongoli non erano di passaggio. Avevano programmato attentamente la conquista della Caucasia. Nel 1236 il nomadismo della steppa si mise in marcia fino a stanziarsi nella piana di Mughan, in Azerbaijan, dove fu collocato il campo centrale. Della Georgia abbiamo detto in precedenza. Dopo la guerra vittoriosa coi Selgiuchidi i Mongoli avevano completato l'opera nella regione.

In Armenia, nel primo periodo della dominazione mongola non sembra vi sia stato un diretto controllo sulla politica. Hethum accolse di buon grado e precocemente la protezione dei Mongoli. Questi dovettero lasciare le istituzioni esistenti per un lungo periodo. R. Bedrosian, nel suo lavoro sulle relazioni fra Armenia e invasioni mongole, afferma che «fino al 1243 non fu imposta alcuna tassa permanente in Armenia, i conquistatori si accontentarono del ricco bottino ottenuto dalle molte aree prese con la forza militare». ${ }^{28}$ In estrema sintesi si potrebbe dividere la dominazione mongola nella Caucasia in quattro grandi fasi distinte. Una prima, che potremmo collocare cronologicamente dal $1236 \mathrm{al}$ 1243 , corrispose a una dominazione piuttosto "morbida". Si era in piena guerra e la regione era letteralmente in ginocchio. Un sensibile calo demografico, la stagnazione economica, intere comunità quantitativamente ridimensionate, insieme all'emergenza militare alla quale i Mongoli stavano ancora attendendo, erano elementi che rendevano necessaria una presenza costante nelle città conquistate, al fine di sostituire provvisoriamente la recente destrutturazione del potere, ma non permettevano una troppo severa imposizione fiscale. ${ }^{29} \mathrm{I}$ nuovi dominatori si accontentavano spesso del bottino racimolato durante le frequenti incursioni.

La seconda fase ebbe inizio con i provvedimenti adottati dal nuovo Khan, Güyük, nel 1243. Furono inviati in ogni parte dell'impero funzionari addetti all'imposizione e al prelievo fiscale. Tali funzionari avevano il compito di costituire un'imposta obbligatoria di un decimo di tutte le proprietà. Secondo Kiriakos i collettori

erano severi e rapaci [...]. Questo Bugha [uno dei due funzionari menzionati da Kiriakos] venne con l'esercito Tataro, entrava nelle case dei nobili, e senza

${ }^{28}$ Bedrosian, Armenia cit., p. 117; KG, in particolare il cap. 32 cit., pp. 233-238.

${ }^{29}$ Molto probabilmente, sempre a causa della situazione di guerra costante che la regione stava vivendo, i Mongoli non avevano nemmeno una sufficiente quantità di uomini da lasciare a presidio nelle città conquistate. 
pietà prendeva ciò che gli piaceva. Nessuno osava dirgli niente, poiché aveva riunito briganti fra gli Iraniani e i Tachidi che [...] erano soprattutto nemici verso i Cristiani. ${ }^{30}$

L'altro funzionario di cui Kiriakos fa il nome è Arghun; un fedelissimo di Güyük che viaggiò in lungo e in largo per l'impero a organizzare la macchina fiscale dei Mongoli. Lo troviamo in Azerbaijan già nel 1243-1244. Poi a Tabriz, dove ricevette ambasciate dal sultano selgiuchide e dagli Ayyubidi di Damasco e Aleppo. ${ }^{31}$ Con l'elezione di Güyük, Arghun venne riconfermato nel suo incarico e fu deputato a organizzare il sistema fiscale della Caucasia. Tornò nel Khorasan nel 1247, in primavera. Nell'aprile del 1248, quando morì il Khan, Arghun si trovava probabilmente a Talas (l'odierna Jambul nel Kazakistan). In questa seconda fase le fonti locali, armene e georgiane, riportano il carattere rapace, talvolta arbitrario delle esazioni e la dura reazione mongola a ogni rifiuto di pagare. Contemporaneamente viene istituito l'obbligo del servizio militare che ogni appartenente all'aristocrazia locale doveva prestare nell'esercito dei Mongoli in cambio della conferma nel suo status sociale, poiché era considerato come vassallo del Khan. ${ }^{32}$ Gli stessi esponenti dell'aristocrazia locale cui era stato concesso un determinato distretto da amministrare avevano il diritto/dovere di recarsi dal Khan (il Gran Khan, a Karakorum) senza filtri intermedi per ogni richiesta avessero da fare. ${ }^{33}$ Era una situazione estremamente

30 KG, pp. 260-261.

31 J. A. Boyle, Dynastic and political History of the il-Khāns, in The Cambridge History of Iran, vol. 5, pp. 303-421, p. 338.

32 [I principi georgiani e aghuaniani] per la loro forza e abilità accompagnarono [I Mongoli] nelle incursioni con la cavalleria e presero le città e le fortezze che non erano state sottomesse, distruggendo e traendo in schiavitù (GA, pp. 5-6); cfr. anche Bedrosian, The Turco-Mongol invasions cit., p. 120.

${ }^{33}$ Così alla regina georgiana Rasudan furono inviati ambasciatori mongoli che le portarono l'ordine di recarsi da Batu «che era nelle regioni del nord, un parente stretto del Khan [...e da] l'altro generale di nome Baiju che era nella regione armena» (KG, pp. 262-263). Ella preferì inviare il figlio David per paura di essere «violata» dice Kiriakos. In seguito alle insistenze del Khan di volere presso di sé la regina in persona, questa decise di suicidarsi piuttosto che adempiere all'ordine. David Lasha, figlio di Giorgio, e David Narin, figlio di Rasudan, si recarono in questa occasione presso il Khan Güyük in persona il quale «deliberò che essi avrebbero governato il regno a turno, prima David figlio di Giorgio Lascha, il maggiore dei due» (KG, p. 264). Sappiamo, come già detto sopra, che in realtà il regno di Georgia fu da Güyük diviso in due parti e concessa una per ognuno dei due. Ancora Kiriakos narra del viaggio che Smbat, fratello di Hethum re di Cilicia, effettuò presso il Khan con «ricchi doni». Non ebbe problemi per tutta la durata del suo viaggio e fu ricevuto con tutti gli onori da Güyük il quale gli concesse numerosi distretti e fortezze che erano già state armene, ma erano poi cadute sotto il dominio Selgiuchide (KG, pp. 265-266). Infine Kiriakos riporta il viaggio effettuato da Hethum di Cilicia in persona presso il Khan, che in quegli anni era già Möngke (il viaggio si svolse fra il 1254 e il 1255). È un racconto estremamente interessante e dettagliato nel quale Kiriakos sottolinea che il Khan "gli concesse un editto ricchissimo per cui nessuno osasse molestare lui o il suo paese. Gli dette anche un documento 
onerosa per i nobili, ma tutto sommato vantaggiosa. Essi potevano mantenere un diretto controllo sul territorio, potevano accrescere le loro ricchezze con le iniziative militari cui erano costretti a partecipare e avevano un contatto diretto col Khan (anche se, per ovvie ragioni, vi si potevano recare una, massimo due volte nella vita), del quale erano vassalli e dal quale ricevevano il riconoscimento formale della loro autorità sul territorio. Ma durante questo periodo si verificò un evento che in parte mutò l'atteggiamento dei Mongoli verso la popolazione locale. Fra il 1248 e il 1249 il re georgiano di Kartlia, David, organizzò insieme a una parte dell'aristocrazia armena e georgiana una rivolta anti-mongola. L'iniziativa non poté concretizzarsi poiché i Mongoli scoprirono la cospirazione quando solo pochi villaggi nella Georgia meridionale avevano aderito all'azione. ${ }^{34}$

Dopo questo tentativo di sovversione si entra a mio avviso in una terza fase; potremmo collocarla fra il 1251 e il 1259 , periodo in cui il Gran Khan dei Mongoli è Möngke. La sua visione unitaria dell'impero si realizzò in un progetto preciso di tassazione allargata per cui fu incaricato ancora Arghun di recarsi nelle terre occidentali affinché organizzasse il nuovo sistema impositivo. ${ }^{35}$ Kiriakos parla della tassazione di questi anni con dovizia di particolari nella sua storia. Lo storico armeno conferma che «nell'anno 703 [1254] Möngke Khan e il gran generale Batu inviarono un ostikan chiamato Arghun, che era già stato nominato da Güyük Khan di supervisionare le tasse di corte esatte dai paesi soggiogati». ${ }^{36}$ In questa occasione i funzionari mongoli si recarono nella Grande Armenia, in Cilicia, in Georgia e nell'Azerbaijan, oltre che nei distretti periferici, dove registrarono tutti dall'età di undici anni in avanti eccetto le donne. E imposero tasse gravosissime, più di quanto un uomo fosse in grado

che concedeva alla Chiesa [cristiana] libertà ovunque» (KG, pp. 301-309); del viaggio di Hethum parla anche Hethum l'Armeno affermando che «nell'anno del signore 1253 il signore Hayton, re d'Armenia [...] secondo il consiglio de' suoi savi, deliberò d'andare in persona dall'imperatore de' Tartari, acciò più facilmente potesse acquistare la sua benevolenza e [...] fare con quello sempiterna pace» (STHA, pp. 322-324).

${ }^{34}$ KG, p. 267-270; GA, pp. 9-10. La reazione mongola, secondo Kiriakos, fu rabbiosa «gettandosi su molti distretti, quelli che si erano ribellati e quelli che non lo avevano fatto» (KG, p. 369).

${ }^{35}$ Arghun, partigiano anti-Tulidi, fu comunque incaricato di gestire il sistema fiscale nella Caucasia (in Rashid ad-Din, Boyle, The Successors cit., p 218: «All'emiro Arghun Aqa [...] fu concessa l'autorità sui paesi di Persia, come il Khurāsān, Māzandarān, Irāq, Fārs, Kirmān, Adhrāijan, Georgia, Lūr, Arrān, Armenia, Rūm, Diyār Bakr, Mosul, e Aleppo». Si veda anche Allsen, Culture and Conquest cit., p. 19; Bedrosian, The Turco-Mongol invasions cit., p. 195. Secondo quest' ultimo, che usa opportunamente molta cautela con le stime, la popolazione armena della Grande Armenia, escludendo quindi la Cilicia, doveva aggirarsi attorno ai 4 milioni di persone. Per qualche mese Arghun fu accompagnato, verosimilmente durante il 1251, nei suoi viaggi da Juvaini in persona. Sugli eventi relativi ad Arghun e al suo operato come funzionario mongolo in Iran si veda anche Boyle, History of the Il-Khanns cit., pp. 338-339.

${ }^{36}$ KG, pp. 298-299. L'ostikan era un funzionario fiscale (si veda più avanti). 
di pagare». ${ }^{37}$ In effetti Arghun si era reso perfettamente conto della necessità di una riforma fiscale che potesse sostenere e in parte stimolare la ricostruzione. Tornato per la via di Derbent, sul Caspio, provvide a istituire il qubchur, una nuova forma contributiva che consisteva in un'imposizione diretta sul pascolo. ${ }^{38}$ Fu una tassazione durissima e rigorosa, anche perché il sistema era realizzato male; i funzionari mongoli che dovevano far fronte alla riscossione di rado se ne occupavano direttamente. Essi appaltavano questo servizio a personaggi locali, spesso membri dei ceti aristocratici minori che svolgevano con molto zelo il loro compito. Chi non poteva pagare veniva privato dei figli e chi si nascondeva veniva ucciso. Ancora Kiriakos svela il ruolo tenuto dall'aristocrazia dei distretti in questo tipo di operazioni:

I principi, signori dei distretti, divennero loro aiutanti nell'imporre ed esigere tasse, per il loro profitto personale. E non erano soddisfatti di questo. Tutti gli artigiani, nelle città come nei villaggi furono tassati. Poi anche i pescatori dei mari e dei laghi, minatori e maniscalchi e pittori [...]. Allo stesso modo fecero grandi profitti coi mercanti e prelevarono una gran quantità di oro, argento e pietre preziose. Così che tutto divenne costoso e il paese divenne colmo di lamentazioni e dolore. Poi lasciarono nel paese rapaci ostikan che chiedevano lo stesso valore ogni anno in base alla lista scritta. ${ }^{39}$

Come nel caso dell'Orda d'Oro anche presso i Mongoli in Caucasia i mercanti erano rispettati più degli altri. Il clero, se crediamo al racconto di Kiriakos (non smentito da altre fonti e anzi confermato dalle fonti russe circa la situazione nell'Orda d'Oro), era esentato dal pagamento di qualsiasi tributo. Prima della creazione dell'Ilkhanato la Caucasia dovette dunque subire una durissima pressione da parte dei Mongoli, sia per quanto riguarda la fiscalità sia per la massiccia presenza militare sul territorio.

La quarta e ultima fase di questo periodo può essere collocata fra il 1256 e il 1261 e corrisponde grosso modo alla creazione dell'Ilkhanato (anche se di stato unificato si potrà parlare solo in seguito alle riforme di Ghazan alla fine del XIII secolo) fino al primo scontro di questo con l'ulus di Giuči.

37 KG, p. 299.

${ }^{38}$ Il sistema fiscale mongolo, sia quello applicato nelle regioni conquistate a Ovest che quello in vigore a Est, non è stato chiarito del tutto, né è semplice farlo visto lo stato attuale della documentazione. Si veda ad esempio Juvaini, I, p. 54: «[I Mongoli hanno] richiesto [a tutti i popoli e tutti i paesi che sono caduti sotto il loro dominio] servizio militare ed equipaggiamento di yam, nonché le spese relative all'approvvigionamento di foraggio, e tutto ciò a prescindere dalle tasse ordinarie; ed oltre questo hanno imposto anche il qupchur». Lo storico persiano conosceva bene la situazione del suo tempo. Sui dubbi che hanno animato gran parte degli studiosi sull'imposizione mongola di questi anni e sul qubčur in particolare si veda P. Pelliot, Qubčiri-qubčir et qubčiurqubcur, in "T’oung pao», 37 (1944), pp. 153-164, in particolare le pp. 154-155.

39 KG, pp. 299-300. 
Prima dell' istituzione dell'Ilkhanato i Mongoli avevano raccolto la Caucasia in un'unità amministrativa divisa in cinque distretti (Vilayet): ${ }^{40}$ la Georgia e la Grande Armenia, composte in maggioranza da popolazione armena, erano i primi due. La Georgia a sua volta era stata suddivisa in otto tümen (otto distretti capaci di fornire all'esercito 10.000 uomini atti alla leva ciascuno). La Grande Armenia era invece suddivisa nei tümen di Mamikonean di Sasun e Vaspurakan con centro a Erzrum. La Cilicia era sostanzialmente una singola unità affidata al suo re, vassallo del Khan. Quando Hülegü, con la benedizione di Möngke, mosse a Ovest (contemporaneamente il fratello Qubilaj - lo stesso che diventerà khan e protettore dei Polo - attaccò la Cina a Est), lo fece portandosi dietro una massa di genti consistente, forse più di quanto non aveva fatto anni prima Gengis khan. Questo esercito comprendeva non solo gli armati, ma anche ampie parti del clan di Hülegü. Scelse come sede per sé e per la sua gente l'ambita piana di Mughan, in Azerbaijan con conseguenze disastrose per tutta la regione. Come abbiamo visto questa zona era stata scelta già da Čormaghan e dal suo successore Baiju per pianificare le operazioni militari nella regione sin dal 1236 . Durante i quasi venti anni intercorsi fra quella data e l'arrivo di Hülegü vi si erano stabilite le genti al seguito di Čormaghan prima e di Baiju poi; si erano accumulate famiglie non solo mongole, ma di tutti quei soldati che avevano servito nell'esercito durante le battaglie e che si erano aggregati in un modo o nell'altro alla compagine dei vincitori. Hülegü ordinò a Baiju di sgombrare la piana. Baiju era un eminente noyon mongolo, ma Hülegü era un esponente dell'oboq di Gengis, inviato in Occidente dal Khan in persona, pertanto i suoi ordini non potevano essere messi in discussione. Baiju noyon non aveva scelta. Un fiume di genti si riversò nella Caucasia e, dopo essersi mosso verso ovest, dove sperava di trovare terre simili a quelle abbandonate, si dette al saccheggio in aree già duramente provate dalla guerra. Sivas, Erzindjan, Erzrum, Cesarea, Iconio (Konya) furono tutte attraversate dalle genti turco-mongole in movimento. ${ }^{41}$

Oltre a ciò la creazione di un centro istituzionale vicino determinò un cambiamento drastico della condizione giuridica dei ceti eminenti armeni e georgiani, i quali divennero una sorta di sottovassalli del Khan, andando a costituire dei vassalli diretti dell'Ilkhan al quale dovevano obbedienza (naturalmente erano dal medesimo meglio controllabili). L'aristocrazia locale, armena e georgiana, doveva adesso il servizio militare direttamente all'Ilkhan e doveva fornire al suo esercito una quantità di soldati relativa alle necessità militari. Le

${ }^{40}$ KG, p. 125. I Vilayat erano ripartizioni amministrative in uso nella Persia iranica ben prima dell'arrivo dei Mongoli. In tutto i Vilayat dell'Ilkhanato erano 17 (I.P. Petruševskij, The Socio-economic condition of Iran under the Il-Khans, in The Cambridge History of Iran, Cambridge 1968, vol. 5, pp. 483-537, p. 497).

${ }^{41}$ Bedrosian, The Turco-Mongol cit., p. 126; anche Kiriakos ne parla fornendo dettagli sulla dinamica degli eventi e sulle loro conseguenze (KG, cap. 59, p. 310). 
conseguenze di questa situazione non tardarono a farsi sentire. Il reclutamento forzato fra la popolazione aveva un duplice effetto: da un lato spopolava le campagne, con immaginabili danni economici; dall'altro privava il territorio, nelle sue manifestazioni politiche e militari periferiche, di un efficace sistema difensivo. Indeboliva la regione in tutti i sensi e ciò andava a sovrapporsi a una situazione già abbastanza critica. Non a caso fra il 1259 e il 1261 un nutrito gruppo di nobili armeno-georgiani dette avvio a una seconda ribellione; stavolta l'iniziativa fu concepita e organizzata meglio. Di nuovo alla testa dei rivoltosi c'era il re georgiano David Lasha. Dopo alterne vicende anche questa rivolta fu sedata nel sangue. David trovò la morte in questa circostanza. ${ }^{42}$

Il compito affidato dal Khan a Hülegü prevedeva l'attacco a Baghdad e la conquista delle terre del califfo abbaside, oltre alla definitiva soppressione del principato degli Ismaeliti del Mazenderan (gli Assassini che vivevano nella striscia di territorio che segue la curva della costa meridionale del Caspio). ${ }^{43} \mathrm{Le}$ roccaforti degli Assassini caddero nel novembre del 1256; prima Meimoundiz e poi Alamut. ${ }^{44}$ Rukn ad-Din, capo della setta, fu inviato da Hülegü al Gran Khan Möngke, ma non vi arrivò mai; fu ucciso durante il viaggio. Nessuno era mai riuscito prima a violare i rifugi di questo gruppo armato che per decenni aveva terrorizzato mercanti e diplomatici e aveva lasciato la via di transito sudcaspica praticamente in disuso. I Mongoli li cancellarono.

Due anni dopo e in seguito a uno scambio epistolare fra Hülegü e il califfo di Baghdad al-Musacim, ${ }^{45}$ l'esercito mongolo affrontò quello abbaside nel gennaio

${ }^{42}$ Gli eventi sono narrati da Kiriakos (KG, pp. 326-330). La vicenda in questione pone un ulteriore problema interpretativo. Kiriakos sostiene chiaramente che «il gran ostikan Arghun reclutò un ingente esercito e andò dietro al fuggitivo re David per prenderlo» (KG, p. 326). Sembra cioè che Arghun abbia partecipato in prima persona alla soppressione della rivolta. Se è così allora Arghun, che compare sempre nelle fonti come amministratore civile, non militare, avesse compiti militari eccome. Si è sempre ritenuto, sulla base delle fonti disponibili, che i Mongoli avessero istituito nei territori conquistati una sorta di potere duale: civile e militare, strettamente connessi e sullo stesso piano gerarchico, ma con compiti leggermente diversi, entrambi direttamente responsabili del loro operato verso il Khan e la cui importanza gerarchica mutava a vantaggio dell'uno o dell'altro in base alla situazione politica del distretto in cui operavano: in emergenza il funzionario militare aveva più potere. Nel caso di Arghun i due poteri non sembrano separati. Egli è un militare la cui opera è prevalentemente amministrativa. Sulla dualità dell'amministrazione mongola nelle terre soggiogate - civile/militare - si veda anche D. Ostrowsky, Muscovy and the Mongols: Cross-Cultural Influences on the Steppe Frontier, 1304-1589, Cambridge 2002, in particolare il cap. 2 della prima parte, pp. 36-65 e la tabella a p. 40.

${ }^{43}$ In realtà è molto probabile che la spedizione prevedesse sin da subito la conquista della Siria. Grousset, L'empire des steppes cit., p. 427; Rashid ad-Din, trad. Quatremère, p. 145; trad. D’Ohsson, III, p. 139. Anche Amitai-Preiss, Mongols and Mamluks cit., pp. 23-25

${ }_{44}$ Rashid ad-Din, trad. Quatremère cit., p. 145; trad. D’Ohsson, III cit., p. 197.

${ }^{45}$ Con il quale il Khan mongolo intimava al suo avversario, come al solito, di sottomettersi. Ce ne ha lasciato un resoconto completo Rashid ad Din; ne parla anche Nasir ad-Din Tusi (1200-1274), storico persiano che ha lasciato un particolareggiato resoconto degli eventi relativi alla presa di Baghdad in appendice all'opera di Juvaini (G.M. Wickens, Nasir ad-Din Tusi on the 
1258. ${ }^{46}$ Le truppe mongole, guidate dal generale Baiju, raggiunsero la periferia di Baghdad il 22 gennaio. Negli stessi giorni un altro distaccamento armato al comando di Hülegü si avvicinava alle mura occidentali della città, mentre un terzo contingente guidato dal noyon Ket-Buqa, passando da sud, penetrava nel distretto di Karkh. ${ }^{47}$ Lassedio ebbe inizio il 29 gennaio e si concluse nei primi giorni di febbraio, quando (esattamente il 13) Baghdad fu spaventosamente saccheggiata per sette giorni consecutivi. ${ }^{48} \mathrm{La}$ conquista della città irachena rappresentò un colpo durissimo per l'Islam. Si è molto insistito nel passato sulle conseguenze disastrose che la caduta di Baghdad e la contemporanea devastazione portata dai Mongoli nella regione avrebbero avuto per l'economia mediorientale. Le conseguenze di questo evento furono indubbiamente negative, la guerra non produce mai ricchezza nelle regioni in cui ha luogo; specie se dura costantemente per oltre trent'anni (e non vi sono dubbi che il modo in cui Baghdad fu presa ebbe un impatto tremendo sull'equilibrio demografico, sulle infrastrutture di tutto il territorio circostante e sulla percezione dell'evento da parte dei contemporanei), ma se leggiamo i resoconti di viaggio arabi o persiani del periodo precedente la conquista mongola, ci rendiamo conto che la regione stava vivendo un momento di crisi già nella seconda metà del XII secolo dovuto, credo, in misura notevole agli effetti delle prime incursioni turche in Medio Oriente. ${ }^{49}$

Fall of Baghdad: a Further Study, in «Journal of Semitic Studies», 7 (1962), pp. 23-35; ma anche Boyle, The death cit., pp. 145-161, in particolare le pp. 150-161); cfr. anche Amitai-Preiss, Mongols and Mamluks cit., pp. 22-24.

${ }^{46}$ Boyle, Dynastic and political Historys cit., pp. 346-347.

47 Secondo il racconto di Tusi i reparti impiegati nell'assedio di Baghdad furono due, ma è l'unica fonte a offrire questa versione: Wickens, Nasir ad-Din Tusi cit., p. 30.

${ }^{48}$ Secondo il racconto di Bar Ebreo (The Cronography cit., pp. 471-475; Wickens, Nasir ad-Din Tusi cit., pp. 33-35; Boyle, The death, 150-161; KG, pp. 313-320; STHA, pp. 325-326). La morte del Califfo fu un trauma e viene raccontata in modo diverso dalle fonti musulmane e da quelle cristiane. Queste ultime parlano di un'esecuzione con la spada (da parte di Hülegü in persona piuttosto che da un suo noyon); le fonti musulmane raccontano di una pratica che veniva usata dai Mongoli in quanto strettamente legata alle loro convinzioni religiose: il califfo venne avvolto in un tappeto e ucciso a calci: Juzjani, (Tabaqat-e Naseri, trad. e cura H. G. Raverty, London 1881, pp. 1252-1253); Wassaf, Geschichte cit., I, 79; Boyle, The death cit., p. 150. Per i Mongoli spargere sul terreno il sangue di un nobile, fosse anche straniero, costituiva un affronto alla terra la quale si sarebbe ribellata producendo calamità e lutti contro il popolo trasgressore (si veda anche Grousset, L'empire des steppes cit., p. 360). M. Polo (Il Milione cit., XIX, pp. 113-114) fornisce una versione errata, leggendaria - dell'aurum sitisti, aurum bibe - per cui il califfo sarebbe stato rinchiuso in una torre e lasciato morire di fame insieme al suo tesoro. Versione analoga è data da Hethum (STHA, p. 326) secondo il quale «[Hülegü comandò ch'ei fosse serrato in una camera e avanti gettate le perle e l'oro, acciocché di quelle si cibasse a sua satisfazione, né gli fosse portato cosa di sorte alcuna [...]»

${ }^{49}$ E delle crociate. Si veda ad esempio Ibn Jubayr, Viaggio in Ispagna, Sicilia, Siria e Palestina, Mesopotamia, Arabia, Egitto, trad. C. Schiaparelli, Palermo 1995, pp. 149-160. Già Ashtor riconosceva, nel suo celebre saggio (Storia economica e sociale cit., cap. VII, pp. 262-295, in particolare p. 265) che un processo di decadenza era in atto ben prima che i Mongoli iniziassero le campagne occidentali. 
Poco dopo ebbe luogo l'altro grande evento che caratterizzò la spedizione di Hülegü in Occidente: il tentativo di conquistare la Siria e l'Egitto mamelucco. Un'iniziativa questa che non ha ancora trovato una spiegazione convincente. In effetti, dopo aver raggiunto il vero obiettivo della missione militare, cioè il califfato di Baghdad e l'annientamento degli Assassini, Hülegü non aveva ragioni evidenti per spingersi fino all'Egitto col rischio di impattare contro eserciti forti e ben organizzati, di entrare nell'orbita dell'impero latino d'Occidente e di allontanare troppo il baricentro del Khanato di Persia. Si è parlato di una forte acrimonia personale fra Hülegü e l'ayyubide di Siria Nasir Yusuf, ${ }^{50}$ ma non può bastare per giustificare una così onerosa campagna militare. Grousset ha scritto, sulla base di un passo in Rashid ad-Din, che Gengis Khan aveva da subito pensato la conquista di tutti i popoli «dai confini dell'Amu-Darya fino all'estremità dei paesi d'Egitto", ${ }^{51}$ ma di questa affermazione non si fa parola nella Storia Segreta. E probabile, come ha ipotizzato John A. Boyle, che la ragione di questa iniziativa sia da ricercarsi nell'opera persuasiva condotta dal sovrano armeno Hethum I nei confronti di Hülegü. ${ }^{52} \mathrm{E}$ chiaro che il cristiano Hethum temeva parecchio la presenza così vicina dei regni musulmani che vedevano in esso un'appendice pericolosa sul Mediterraneo; forse l'armeno sperava di ottenere, in una simile iniziativa, l'appoggio del Regno Latino d'Oriente che in Siria era costituito dalla zona lungo la costa del principato di Antiochia, la contea di Tripoli e il regno di Gerusalemme (senza la città). Aggiungiamo che, se a sud il regno di Gerusalemme era nelle mani dell'aristocrazia locale, la parte settentrionale, quella di Antiochia e Tripoli, era di Boemondo VI, genero di Hethum. Una eventuale sconfitta dell'Egitto avrebbe portato importanti vantaggi economici alla Cilicia e alla Caucasia tutta; si consideri che l'obiettivo dichiarato di questa spedizione mongola fu da subito Aleppo. Fatto si è che l'esercito mongolo, composto da numerosi elementi armeni e georgiani ${ }^{33}$ provenienti dai distretti in cui era obbligatoria la leva, penetrò nella città di Aleppo il 24 gennaio 1260; l'assedio durò circa un mese dopo il quale la città cadde provocando il panico nella regione fra i musulmani. Boemondo, che aveva attivamente partecipato all'iniziativa, ricevette

${ }^{50}$ Grousset, L'empire des steppes cit., p. 434. Questa ipotesi è riportata, con qualche scetticismo, anche da Boyle (The History cit., p. 350).

51 Grousset, L'empire des steppes cit., p. 427.

52 Amitai-Preiss, Mongols and Mamluks cit. pp. 11-14. Aggiungerei, fra le ipotesi avanzate a riguardo, il fatto che Hülegü è sempre stato considerato filo-cristiano. Con ogni probabilità lui, da buon mongolo, non aveva una solida autocoscienza religiosa, al contrario della prima moglie. Doquz-qatun era una donna molto stimata dal Gran Khan Möngke, ma era soprattutto la nipote dell'ultimo van-Khan dei Kerait i quali avevano da tempo abbracciato il cristianesimo di Nestorio. Gran parte degli atti a favore dei cristiani che indubbiamente Hülegü fece credo si debbano a lei. Non so fino a che punto la principessa potesse influenzare la politica militare del marito, ma è un aspetto da considerare con attenzione.

53 Un contingente georgiano pare abbia partecipato attivamente anche alla presa di Baghdad (KG, pp. 315-316). Si veda anche Amitai-Preiss, Mongols and Mamluks cit. p. 26. 
come premio i territori che aveva perduto a causa della campagna di Saladino. Il 1 marzo cadde Damasco il cui governo fu messo nelle mani di un governatore mongolo. Nell'estate del 1260 le truppe mongole, sempre con l'ampia partecipazione dell'aristocrazia armena e georgiana, penetrarono fino a Gaza. In questa circostanza si ripeté quanto era già accaduto per Batu in Ungheria. Möngke morì l'11 agosto 1259 e Hülegü dovette far ritorno in Persia. Lasciò il comando delle operazioni militari al suo generale di fiducia, il noyon Ket-Buqa ${ }^{54}$ Indebolito dal prelievo di truppe che dovettero seguire Hülegü, l'esercito mongolo si trovò ad affrontare la controffensiva egiziana (sostenuta da reparti latini, giunti da Acri e costituita in gran parte da mercenari) a 'Ain Jālūt e per la prima volta venne sconfitto; era il 3 settembre $1260 .{ }^{55}$ La sconfitta subita in questa circostanza ebbe un duplice effetto politico: da un lato essa rafforzò la presenza dei Mamelucchi in Palestina e in Siria andando a costituire un valido argine per le potenziali mire mongole in quella regione e su ciò che essa rappresentava (si pensi soprattutto alla posizione strategica della regione che si affaccia tutta sul Mediterraneo), dall'altro costrinse Hülegü a guardarsi da nord, dove i suoi parenti, fondatori dell'Orda d'Oro, ritenevano che l'Ilkhanato si stesse allargando troppo nella Caucasia. Oltre a questo, si tenga presente che il successore di Batu, Berke, simpatizzava per l'Islam e non aveva tollerato il massacro perpetrato a Baghdad nel 1258 dalle truppe di Hülegü; questi era un mongolo ancorato alle tradizioni culturali del suo popolo, uno sciamanista. ${ }^{56} \mathrm{Si}$ stava profilando uno scontro fratricida fra due stati mongoli che ritengo, pur non sottovalutando l'aspetto religioso e ideologico, sia stato determinato soprattutto dalla volontà politica di ottenere il controllo sulla Transcaucasia e sulle ricchezze del suo territorio. ${ }^{57}$

\subsection{Nell'Orda d'Oro}

[Batu] era un re che non aveva inclinazioni particolari verso qualsiasi credo o religione: riconosceva soltanto la fede in Dio e non seguiva ciecamente alcuna setta. La sua generosità era al di là di qualsiasi calcolo e la sua liberalità era smisurata $[\ldots]$ e i mercanti gli portavano da ogni parte ogni genere di mercanzia, ed egli prendeva tutto, e ne raddoppiava il prezzo a più riprese. Ed emetteva tratte sui Sultani di Rum e Siria, e concedeva loro jarligh; e nessuno di coloro che venivano a trovarlo ripartiva senza aver raggiunto il proprio scopo. ${ }^{58}$

${ }^{54}$ KG, p. 325; STHA, pp. 327-328; Bar Ebreo, The Cronography cit., p. 436.

55 Boyle, The death cit., p. 351; Amitai-Preiss, Mongols and Mamluks cit., pp. 39-45.

${ }^{56}$ A Hülegü si rimproverava l'aver preso una decisione senza essersi consultato con gli altri nobili mongoli prima della distruzione di Baghdad e dell'esecuzione del califfo. Rashid ad-Din, trad. Quatremère cit., p. 393; Grousset, L'empire des steppes cit., p. 475.

57 Amitai-Preiss, Mongols and Mamluks cit., pp. 78-91.

58 Juvaini, I, p. 300. Su Batu si veda anche la descrizione data da Rashid ad-Din in più di un'occasione nella sua Storia (Boyle, The Successors cit., pp. 56-57, 59-60, 107-110, 119-122, 200-203). 
Questa descrizione di Batu, fatta da Juvaini, rispecchia piuttosto bene il carattere del sovrano per quanto se ne sappia anche da altre fonti, lontane da questa che, comunque, non è del tutto imparziale. Batu pare essere stato un uomo estremamente attento alle esigenze dei mercanti e della propria gente in genere. Di certo era ben consapevole che dopo le devastazioni prodotte dal costante stato di guerra in cui aveva contribuito a gettare la Rus' e le steppe del Qipciak, si rendeva necessario un lungo processo di ricostruzione che potesse rendere il territorio e le città di nuovo produttivi.

Come abbiamo visto nel capitolo precedente, il territorio sul quale sarebbe in seguito sorta l'Orda d'Oro era quello che Gengis khan aveva concesso al figlio maggiore, Giuči. ${ }^{59}$ Con la morte di questi, avvenuta nel 1227, pochi mesi prima del padre, il possesso dell'ulus di Giuči passò a Batu, suo secondo figlio e nipote di Gengis. La gente di Batu, il suo oboq, vi regnò fino al 1359. Purtroppo sulla storia economica e sociale dell'Orda d'Oro non si hanno le stesse conoscenze che si hanno per l'Ilkhanato; su quest'ultimo e sulle sue condizioni socio-economiche - oltre che sulle istituzioni che vi governavano - abbiamo le storie di Rashid ad-Din e di Juvaini, che in quanto funzionari di primissimo piano del sistema amministrativo nell'Ilkhanato, costituiscono una testimonianza straordinaria per completezza e attendibilità. Rashid fu al servizio dell'Ilkhan Ghazan e si occupò in prima persona delle riforme promosse dal sovrano mongolo e della loro applicazione. Juvaini fu governatore di Baghdad durante il regno di Hülegü. Ė proprio sul periodo delle innovazioni introdotte da Ghazan che siamo meglio documentati. ${ }^{60}$ È probabile che alcune istituzioni adottate dai Mongoli a sud del Caucaso fossero altresì utilizzate a nord, ma è necessario muoversi con cautela a questo proposito. ${ }^{61}$

Sin dalla prima conquista delle steppe dei Qipčiaki i Mongoli introdussero un sistema di controllo decentrato e diversificato. La vastità del territorio da amministrare e le forti differenze istituzionali che vi trovarono imposero ai nuovi dominatori l'adozione di metodi di governo che cambiavano da regione a regione. Spesso le forme organizzative e i modi di gestione locali venivano lasciati intatti, talvolta venivano sostituiti o modificati dando luogo a nuove

${ }^{99}$ E in effetti sarebbe corretto chiamarlo ulus Giuči piuttosto che Orda d'Oro. Questa denominazione è un'invenzione russa del XVII secolo (probabilmente il primo a utilizzarla fu il mercante Kotov che nel 1624 viaggiò fino in Persia e nel suo racconto utilizza il termine Золотая Орда, ma non ne sappiamo la ragione). Nelle fonti contemporanee, anche quelle russe, il termine Orda è utilizzato per designare il campo, la città, talvolta la capitale. A riguardo si veda G.A. Fedorov Davydov, Obščstvennyj Stroj Zolotoj Ordy, Moskva 1973; V.L. Egorov, Gosudarsvennoe i Administrativnoe ustrojstvo Zolotoj Ordy, in "Voprosiy Istorij», 2 (1972), pp. 32-42; Id. Istoričeskaja Geografija Zolotoj Ordy, Moskva 1985.

60 Il periodo delle riforme di Ghazan è molto ben illustrato da Petruščevskij, The socio economic conditions cit.

${ }^{61}$ Nel loro lavoro sull'Orda d'Oro Grekov e Jakubovskij sovrappongono spesso e consapevolmente i due modelli. Talvolta pare una forzatura. 
istituzioni frutto di una fusione fra l'elemento indigeno e quello esterno. Ad esempio nelle steppe abitate dai Cumani essi soppiantarono del tutto il sistema che trovarono. Ogni singola unità amministrativa, fosse essa una comunità di villaggio piuttosto che un'assemblea cittadina, venne cancellata a vantaggio dell'istituzione di governatori locali, mongoli, che avevano il compito di gestire la comunità cui erano stati addetti e provvedere alla riscossione dei tributi. ${ }^{62} \mathrm{Ma}$ nella regione corrispondente, grosso modo, all'estensione della Rus' kieviana essi agirono diversamente. Non soppressero le forme di potere che vi trovarono. Lasciarono che i principi russi continuassero a governare i loro stati, ma imposero loro la presenza nelle città di esattori mongoli (baskak e daruga) ai quali i sovrani russi, nei primi anni della dominazione, dovevano totale adesione nell'amministrazione fiscale. Novgorod' ebbe una vicenda ancora diversa. I Mongoli non vi entrarono mai, non la attaccarono e adottarono nei confronti del principato settentrionale un atteggiamento del tutto particolare. Novgorod' rimase nelle mani del principe russo, senza che i conquistatori gli imponessero la presenza di alcun funzionario fiscale, ${ }^{63}$ ma dovette riconoscere la sovranità di Batu. L'aspetto fiscale e il prelievo militare erano totalmente a carico del principe, il quale costituiva a tutti gli effetti un vassallo del Khan dell'Orda d'Oro e a questi doveva obbedienza.

La regione settentrionale, nella quale i Mongoli avevano trovato la Rus' con le sue città, le sue istituzioni, non venne alterata. I funzionari locali, dopo un primo periodo, divennero automaticamente i principi russi. Come abbiamo detto sopra, questi erano obbligati ad accettare la presenza di un funzionario mongolo che controllasse l'esazione fiscale.

Nel resto dell'Orda d'Oro la classe dirigente dello stato era tutta scelta fra i noyon di Batu, fra coloro cioè che appartenevano al suo oboq. Essi erano strettamente legati al Khan; gli dovevano fedeltà e servizio e ottenevano in cambio un beneficio, una terra o meglio un territorio comprese le genti che vivevano. Ma non avevano con essa alcun legame. Il più delle volte non vi risiedevano nemmeno in modo stabile; era pertanto una prassi consolidata il trasferimento del controllo territoriale nelle mani di funzionari terzi che avevano un forte radicamento territoriale successivo. Essi cioè erano il più delle volte mongoli, incaricati di stabilirsi su un determinato territorio (che avevano ricevuto per meriti militari durante il quriltai più recente) e potevano vivere costantemente sull'area affidatagli. L'ex possedimento cumano veniva a costituire uno spazio

${ }^{62} \grave{E}$ un aspetto questo sul quale non sappiamo molto. Si veda a tal proposito G. Vernadsky, Political and Diplomatic History of Russia, London 1937; Ostrowsky, Muscovy and the Mongols cit., 36-63; Spuler, Die Goldene Horde cit., 300-360.

${ }_{63}$ "In quell'anno [1257] giunse dalla Russia la cattiva notizia, che i Tatari pretendono da Novgorod il tamga e i dazi, e la gente fu molto agitata per tutto l'anno» (Polnoe Sobranie Russkich Letopisej, t. I, Lavrent'evskaja Letopis', Moskva 1997, pp. 278-280; The Chronicle of Novgorod, trad. di R. Michell, N. Forbes, London 1914, pp. 95-96). 
unitario, un puzzle composto da tante piccole entità territoriali ognuna delle quali era strettamente legata alla sede del Khan dell'Orda (Saraj, alla foce del Volga) e costituiva da sé una autonoma unità militare. Il noyon che governava un distretto era tenuto a fornire al Khan un certo numero di soldati (generalmente una mingghan, un migliaio, più raramente un tümen). Alla classe dirigente militare apparteneva anche la figura del bukaul. Si trattava di un alto funzionario che provvedeva alla distribuzione delle truppe, alla loro destinazione e al movimento dei soldati verso i luoghi delle operazioni; controllava che non vi fossero deroghe al tradizionale sistema di ripartizione del bottino e a loro faceva capo ogni singolo tümen. I noyon che comandavano un distretto capace di fornire un migliaio di soldati erano direttamente responsabili verso i bukaul del loro operato. ${ }^{64}$

Anche nell'Orda d'Oro l'amministrazione era bipartita fra militare e civile; quest'ultima veniva impiegata soprattutto per la riscossione fisica dei tributi. Nelle fonti russe compare il termine baskak per indicare l'ufficiale militare e quello di daruga (darugačin) che designava l'amministratore civile.

Nei principati della Rus' il decentramento fiscale e amministrativo fu un fenomeno piuttosto precoce. Jaroslavl', fratello di Jurij Vsevolodovič e principe di Vladimir', fu insignito da Batu in persona del titolo di «primo principe di lingua russa» già nel 1243, quindi all'indomani della conquista mongola. Jaroslavl' ebbe così piena podestà sui territori attorno $\mathrm{a}$ Vladimir Suzdal' oltre che $\mathrm{a}$ Kiev. ${ }^{65}$ Poco dopo, nel 1250, il principe di Galizia Danijl, seguì l'esempio di Jarovslavl'. Poi, come abbiamo detto sopra, dovette fare la stessa cosa, con molti problemi in più, ${ }^{66}$ Aleksandr di Novgorod' (il celebre Nevskij). Il principe russo era direttamente investito di questa autorità dal Khan dell'Orda o, indirettamente, da un suo funzionario (talvolta poteva essere il metropolita). Come abbiamo visto il principe era affiancato nella conduzione del territorio da un funzionario mongolo; nei primi anni della dominazione si trattava quasi sempre di un baskak. Progressivamente le fonti menzionano sempre meno questa figura che, se in altre aree dell'ulus diventa effettivamente rara, nei territori russi essa scompare del tutto. ${ }^{67}$ I Mongoli fecero

${ }^{64}$ Grekov - Jakubovskij, L'Orda d'Oro cit., p. 99. Di questa figura parla lo yarlyk di Mengli Hirei del 1453 con riferimento alla Crimea. Si tratta di un documento stampato da Veselovskij nel numero XXIV del «Zapiskij Vizantjnskij Očerki» ma, non essendo riuscito a reperire il volume, cito di seconda mano.

${ }_{65}$ Grekov - Jakubovskij, L'Orda d'Oro cit., p. 182; The Chronicle of Novgorod cit., pp. 87-88.

${ }^{66}$ Novgorod' era una città ricca; era stata per anni il centro di un vivace traffico commerciale con le città baltiche e con Bisanzio; aveva istituzioni cittadine molto forti (il veče, l'assemblea cittadina, poteva porre il veto alle decisioni del principe) per cui la gente non accettava l'imposizione di un tributo dall'esterno; era intollerabile che un'autorità straniera si permettesse di governare la città. Nevskij dovette più volte ricorrere alla forza per evitare che focolai di rivolta sfociassero in aperta guerriglia cittadina.

67 Questo punto ha suscitato un vivace dibattito storiografico per cui alcuni storici hanno voluto considerare la scomparsa del termine dalle fonti come la fine dell'uso della pratica stessa. Personalmente concordo con chi afferma che fra la fine del XIII secolo e i primi venti anni del 
largo uso delle cariche militari per affiancare i governanti locali nella conduzione della cosa pubblica nei primi anni successivi le guerre di conquista. C'erano dei problemi oggettivi cui bisognava far fronte. Era necessario puntare a una rapida pacificazione delle zone conquistate e a una loro ripresa economica e culturale. La presenza del funzionario militare, che potesse cioè intervenire per conto del Khan quando necessario e mantenere l'ordine pubblico, era strumentale a situazioni d'emergenza. Calato il livello della tensione nelle aree sottoposte al dominio, più o meno diretto, dei Mongoli i funzionari militari venivano definitivamente sostituiti o con i soli membri dell'aristocrazia locale, o con altri funzionari di formazione e con compiti civili. ${ }^{68}$

Il principe regnava sulle terre russe solo se in possesso dello jarlych del Khan. Lo jarlych era un documento col quale il Khan concedeva a un suo vassallo l'autorità su una parte del suo ulus.

L'elemento mongolo nelle terre dell'ulus di Giuči era assolutamente minoritario. Dai brani del testamento di Gengis khan si evince che a Giuči furono assegnati non più di 4.000 Mongoli per la colonizzazione del suo ulus. ${ }^{69}$ L'esercito col quale Batu portò a compimento le sue conquiste era composto in gran parte da Mongoli, ma essi furono presto sovrastati dalla netta predominanza demografica dei Cumani. Inoltre una componente minoritaria dell'esercito, ma molto forte, era costituita da uomini caduti schiavi durante le campagne precedenti. Dopo l'acquisizione dei territori dell'Orda l'esercito verrà agevolmente integrato con l'afflusso delle genti dai distretti, sia del Qipciak che dei principati russi. Non è un caso che il territorio delle steppe si sia, in tempi relativamente brevi, turchizzato. ${ }^{70}$

successivo il baskak sia stato tolto nella conduzione dello stato (su questa linea Grekov, Jakubovskij, L'Orda d'Oro cit., pp. 181-182, ma anche Ostrowsky, Muscovy cit., pp. 42-43).

${ }^{68} \mathrm{Su}$ questa posizione Donald Ostrowsky (Muscovy cit.) il quale ha sostenuto che se la situazione politica lo permetteva era prassi, da parte dei Khan, concedere una certa autonomia ai vassalli locali - cioè ai principi russi - in modo che essi potessero meglio adempiere ai loro compiti e far affluire senza problemi il gettito fiscale nelle casse dell'impero. Questo sistema permetteva da una parte di stimolare alla pacificazione l'aristocrazia locale in cambio di una larga autonomia amministrativa, dall'altra e in conseguenza della prima, di consentire ai Khan di non tenere costantemente gli eserciti in allarme per sedare rivolte. Naturalmente in questo periodo i Mongoli non si auto-esclusero dalla vita dei principati russi, né dai distretti in cui avevano diviso il vasto territorio delle steppe. Alcuni punti erano per loro imprescindibili. I governanti locali dovevano provvedere all'imposizione e alla raccolta dei tributi nella misura in cui ciò veniva stabilito dal Khanato dell'Orda d'Oro; dovevano fornire soldati quando il Khan ne faceva richiesta (emblematico è il caso della guerra fra l'esercito dell'Orda d'Oro e quello dell'Ilkhanato negli anni '60 del XIII secolo); dovevano accettare i funzionari mongoli inviati periodicamente nelle terre russe garantendo loro protezione mentre svolgevano i loro compiti di controllo fiscale etc. Gli obblighi quindi non mancavano, ma l'assenza durevole di un funzionario mongolo che controllasse l'operato dei principi era significativa ed ebbe effetti benefici sulla ricostruzione.

${ }^{69}$ Grousset, L'empire des steppes cit., p. 469.

${ }^{70}$ Halperin, Russia and the Golden Horde cit., in particolare I cap. III e VII, pp. 21-32 e 75-86. 
Il rapporto fra i Rus', principati della Rus', e l'Orda d'Oro è in gran parte difficile da interpretare. Ė un problema che ha per molto tempo impegnato gli specialisti senza che ancora oggi si sia giunti a una sua univoca interpretazione. Ci si chiede se la Russia moderna sia erede, e in quale misura, della Rus' e soprattutto quale ruolo ebbero le invasioni mongole in questo processo. Non è compito del presente lavoro occuparsi di questo problema. Mi limiterò a osservare che dopo un periodo relativamente breve, diciamo fra il 1240 e i primi anni del Trecento, principati russi e Orda d'oro seguirono destini diversi. Mentre l'Orda d'Oro andrà incontro a una lenta ma inesorabile disgregazione, la Rus', o quel che ne resta, verrà coinvolta nello sviluppo che, dapprima timido, si concretizzerà attorno al nuovo centro di Mosca. La divisione che maturò nell'Orda d'Oro ebbe conseguenze profonde anche nella regione dell'Azov. Il distretto sul Don era parte integrante dell'Orda, ma beneficiò fortemente della sua divisione, confermando il ruolo di referente commerciale degli operatori che vi confluivano da tutta Europa. Lo sviluppo e la ricchezza che raggiungeranno gli insediamenti veneziano e genovese della Tana (il più lontano di tutto il sistema commerciale italiano dalla madrepatria) è uno degli aspetti più interessanti da indagare nella sua origine e nelle sue manifestazioni successive. Alla foce del Don stabilì la propria residenza Nogaï, fedele esponente della casata di Giuči che fu il vero autore della politica nell'Orda d'Oro durante il Khanato di Tulabuqa (1287-1290). La regione dell'Azov vivrà il suo momento peggiore durante il governo di Toqtai che fece prima arrestare i residenti genovesi di Saraj (1307) e poi attaccare Caffa nel 1308 con conseguenze disastrose per la cittadina di Crimea. Alla morte di Toqtai i latini verranno ammessi a ritornare nella regione.

Alla fine del XIII secolo l'unificazione mongola era conclusa. La geografia di un intero continente era sostanzialmente mutata. Gran parte dell'Asia era nelle mani dei Mongoli. L'Europa Orientale aveva subito lo stesso destino. L'ulus di Batu si estendeva fino ai confini orientali dell'Ungheria e aveva il controllo, seppur indiretto, di tutti i principati russi a nord. La Crimea e tutta la costa settentrionale del Mar Nero erano parte dell'Orda d'Oro fino alla linea del Danubio, in Dobrugia. I Balcani costituivano, insieme al ricostituito impero greco una rottura nella continuità geografica che i Mongoli avevano acquisito attorno al Mare Maggiore. L'Anatolia era sotto il controllo mongolo, col sultanato selgiuchide costretto alla fedeltà verso l'Ilkhanato. La Cilicia era riuscita a mantenere una sostanziale indipendenza dalla rigida autorità tatara, ma dei nuovi dominatori era uno stato vassallo. La Caucasia era completamente soggiogata. Tutta la costa orientale del Mar Nero era mongola, ma divisa fra due potenze contrapposte la cui influenza era divisa dai monti del Caucaso. A nord l'Orda d'Oro e l'ulus di Giuči, a sud l'Ilkhanato. Era una configurazione geografico-politica completamente nuova. Un territorio immenso sotto l'autorità formale di una sola potenza. I precoci dissidi sorti in seno ai diversi Khan per il 
dominio dei territori confinanti (non si dimentichi che nella seconda metà del XIII secolo il successore di Batu, Berke, rivendicò il proprio diritto su parte della Korazmia e non esitò a scatenare un conflitto contro l'ulus che legittimamente possedeva quella regione, quello di Čagataï), e soprattutto la debolezza del sistema politico instaurato porterà l'impero mongolo a rappresentare un'unità che oggettivamente non c'era. Ma l'opera di Gengis khan aveva comunque rotto il velo sotto il quale si trovava l'Oriente, in particolare quello centro-asiatico e la sua vitalità commerciale. Non è casuale il fatto che la penetrazione occidentale in queste regioni d'ora in avanti diventerà costantemente crescente, anche se, in seguito, dovrà fare i conti con l'instabilità politica dei vari distretti. La Caucasia dovette subire, oltre alle devastazioni che seguirono alle guerre, il trauma del precoce crollo Ilkhanide (con la morte dell'Ilkhan Abu Said, nel 1335, l'impero mongolo di Persia inizierà una inesorabile fase di declino dalla quale non si riprenderà mai completamente fino a crollare definitivamente sotto i colpi dell'esercito di Tamerlano).

Nell'Orda d'Oro la situazione fu diversa. I principati russi già dalla metà del XIV secolo non andavano più a rendere omaggio o a ricevere lo jarlych del Khan per governare sui loro territori. Il declino dell'ulus di Giuči fu più lento rispetto a quello dell'Ilkhanato; ciò è dovuto a mio avviso a tre fattori diversi. In primo luogo l'Orda d'Oro, a differenza dell'Ilkhanato, nasce già divisa. Batu non aveva alcun interesse a occuparsi direttamente dei principati russi. I Mongoli trovarono nella steppa un territorio per loro ideale, mentre le foreste russe costituivano una barriera, mentale molto più che fisica, ma pur sempre un ostacolo all'espansione verso nord. Non erano attratti da quel mondo che percepivano come distante. Inoltre il territorio che si trovavano ad amministrare era vastissimo e credo che a un certo punto abbiano preferito accertarsi che i principi russi non maturassero velleità di rivolta piuttosto che stabilirsi a stretto contatto con loro; da qui la forte autonomia di cui la Rus' beneficiò sin dai primi anni della dominazione mongola. Poi bisogna tenere presente che, sempre a causa delle distanze, anche le regioni poste direttamente sotto il controllo del Khan beneficiarono più delle altre dell'immigrazione commerciale. Il governatore di Solgat, che sovrintendeva all'amministrazione in Crimea per conto del Khan, si arricchiva parecchio coi traffici che passavano dalla Tana, da Caffa, Soldaïa, etc. E queste ricchezze contribuivano a uno sviluppo rapido e più duraturo, causa e frutto di una generale sicurezza che caratterizzò, quasi costantemente, certe regioni; non solo la Crimea, ma anche il distretto di Saraj, sul Volga fu il centro di un florido traffico commerciale che, prendendo le merci dal bacino del Caspio, le portava a Nord e viceversa; di notevole importanza era inoltre il legame commerciale fra la capitale dell'Orda e il Turkestan. Infine credo non vada sottovalutata la genesi politica dei due stati. L'Orda d'Oro fu il frutto di una campagna voluta a tutti i costi dalla sede centrale dell'impero. A Batu furono messe a disposizione le migliori risorse per portare a termine il 
compito. E soprattutto, quando Batu si rese conto, grazie all'intelligenza dei suoi generali, che non era il caso distendere la dominazione fino all'Ungheria, fece retromarcia. L'Ilkhanato trovò invece nell'Egitto dei Mamelucchi un ostacolo durissimo da superare e dovette guardarsi sempre da questo avversario (che lo batterà ancora). A ciò si aggiunga che il fattore religioso nella Caucasia e sul Mediterraneo era fondamentale. L'attacco a Baghdad, a Damasco, Aleppo etc. aveva umiliato i Musulmani della regione compattandoli; alla prima occasione si sarebbero vendicati e da questo punto di vista la vittoria dell'Egitto sulle truppe Hülegü fu uno stimolo per tutti i fedeli dell'Islam a non arrendersi all'idea di un esercito, quello mongolo, estraneo e invincibile. Nell'Orda d'Oro questo era un problema minore. Il fattore religioso poteva essere importante per i Russi, e lo era, ma nelle steppe dove Batu fondò la capitale era un aspetto assolutamente secondario. I Cumani non avevano una identità collettiva basata sul religioso tale da poter chiamare alle armi migliaia di individui sotto una bandiera ideologica. I Russi, dal canto loro, pur umiliati anche religiosamente, furono in parte risarciti; per un verso con la rapida esenzione fiscale riservata al clero e per un altro con il blando controllo religioso cui i Khan sottoponevano l'area russa.

A parte queste considerazioni si può senz'altro affermare che l'Orda d'Oro è ancora oggi e per molti versi un mistero storico e per quante spiegazioni accettabili si possano trovare, credo che non se ne possa prendere una come definitiva, soprattutto alla luce di un fenomeno così complesso. In questo caso gioverà sottolineare che gli equilibri di forza verificatisi nell'immenso territorio dell'ulus di Giuči produssero una rapida frammentazione del potere dando avvio a una serie di zone di influenza indipendenti. L'Azov fu insieme alla Crimea la regione che più di ogni altra beneficiò di questo stato di cose. Per la Caucasia fu diverso. I traffici si svilupparono ed ebbero un ruolo importante anche per la rinascita di quella zona, ma la maggiore pressione esercitata dal centro sulla periferia costituì un freno allo sviluppo commerciale. Dovunque però il crescente volume d'affari che si venne a creare attorno al Mare Maggiore attrasse i governatori mongoli che a lungo ne favorirono lo sviluppo consapevoli del profitto che avrebbero potuto trarne.

Il frenetico succedersi di momenti di pace e conflitti feroci, sia all'interno dei due soggetti politici sia fra di loro, non scoraggiò mai definitivamente $\mathrm{i}$ mercanti occidentali. Il popolo dei nomadi aveva favorito la rinascita di una vivace struttura urbana che poteva, per la prima volta dopo secoli, confrontarsi con un mondo fino ad allora sconosciuto. La pace mongola, come questo periodo è stato definito, ebbe effetti benefici sul commercio internazionale, che solo in seguito all'apertura dell'Oriente poté affacciarsi su mercati fino ad allora sconosciuti. Questa condizione di sviluppo, successiva alle grandi devastazioni provocate dalla guerra, ebbe conseguenze molto positive, ma di queste poterono beneficiare soprattutto gli operatori esterni: mercanti occidentali che si trovarono nella possibilità di avvicinare mercati fino ad allora solo immaginati ed ebbero 
accesso a prodotti rarissimi sulle piazze occidentali dando vita ad un commercio internazionale su vasta scala. La popolazione locale, e i mercanti in particolare, riuscivano a realizzare buoni guadagni, ma la situazione di guerra che aveva preceduto questo periodo costituiva un freno per la ripresa in loco. 



\section{IL FATTORE RELIGIOSO E IL PROBLEMA DELL'INTEGRAZIONE DOPO LA CONQUISTA}

\subsection{Le premesse culturali}

Quando si affronta il problema religioso nelle aree conquistate dai Mongoli si è tentati di seguire una tradizione storiografica, dominante, che ci ha presentato questo popolo guerriero come piuttosto tollerante nei confronti delle confessioni con le quali si dovette confrontare. Oggi fortunatamente questo aspetto si è ridimensionato. La tanto decantata tolleranza religiosa dei Mongoli è un mito storiografico estrapolato da alcuni brani riportati dalle fonti attraverso le quali studiamo la storia di questo popolo e cristallizzatosi nella comune percezione in base a una convinzione preconcetta piuttosto che a un'oggettiva disamina della documentazione. Alcuni passaggi dell' Historia Mongalorum o dell'Itinerarium, ma anche della cronaca di Rashid ad-Din, di Juvaini e di quelle di Kiriakos e di Gregorio di Akner, parlano di un sostanziale disinteresse della classe dominante mongola per le faccende religiose. Così in Juvaini si legge che

[Gengis] non essendo partigiano di alcuna religione o seguace di alcun credo, evitò il bigottismo, e il preferire una fede ad un'altra, e l'esaltarne una al di sopra delle altre ${ }^{1}$

o in Giovanni di Pian del Carpine che "per quel che ne sappiamo non hanno mai costretto nessuno fino ad ora a rinnegare la propria fede o la propria legge, eccettuato il caso del già esposto Michele». ${ }^{2}$ Per Gregorio di Akner

1 Juvaini, I, p. 46.

2 Historia Mongalorum, III, 5, p. 343. Il "già esposto Michele" è Michajl Vsevolodovič, principe di Kiev ed eletto nel 1245, da Batu, principe anche di Černigov. Quando fu chiamato davanti a Batu fu costretto a inchinarsi davanti all'idolo che rappresentava Gengis. Egli si rifiutò e venne decapitato insieme con un suo soldato. Come ha giustamente rilevato Paolo Daffinà nelle sue note all'Historia di frate Giovanni, «la tanto asserita tolleranza religiosa dei Mongoli va intesa in senso molto relativo" (Historia Mongalorum, p. 415, nota 5 al cap. III). Se volessimo citare un altro esempio della scarsa tolleranza mostrata dai Mongoli nei confronti di ciò che essi ritenevano intollerabile potremmo ricorrere ancora a Giovanni di Pian del Carpine il quale racconta di quel che accadde alla vedova e al fratello di Andrej di Černigov. Questi, principe della città omonima e figlio di Mstislavl III Romanovič, fu accusato di furto di bestiame e fatto uccidere da Batu. Il fratello, poco più che un adolescente e la vedova si recarono dal khan mongolo per chiedere giustizia; in tutta risposta gli uomini di Batu «mettendo il giovane su di lei che urlava e piangeva e con un ordine perentorio e senza condizioni li costrinsero ad unirsi» (Historia Mongalorum, III, 6, p. 344). 
[I Mongoli] non hanno alcuna religione, eccetto che per le immagini che portano sempre con loro per stregoneria. Avevano timore del sole, poiché pensavano fosse un potere divino. ${ }^{3}$

Hülegü, primo Ilkhan di Persia, non fu affatto elastico nei confronti dei musulmani della regione. Sartach, successore di Batu nell'Orda d'Oro seguì la stessa linea, essendo nestoriano. Arghun, figlio primogenito di Hülegü, fu favorevole ai cristiani, suo fratello Tagudar, invece, fu loro particolarmente ostile dopo che ebbe abbracciato l'Islam. Quando Ghazan fu eletto Ilkhan, nel 1295, egli si era già convertito all'Islam da circa quattro mesi. La sua linea in materia di politica religiosa non fu esattamente tollerante; perseguitò sistematicamente il cristianesimo. Si tratta insomma di un problema assai complesso. Si può tuttavia tentare di tracciare un quadro generale dei rapporti fra le nuove istituzioni e le vecchie, fra la classe dirigente nelle città e nei distretti e la popolazione che dovette subire le scelte di questa.

I Mongoli irruppero a Occidente con violenza sconvolgendo in pochi decenni il complesso sistema amministrativo e infrastrutturale preesistente. Tale caratteristica rende le incursioni di questo popolo diverse da altri fenomeni che si sono verificati nel nostro Medioevo solo apparentemente simili; si pensi ad esempio alle cosiddette invasioni barbariche le quali, come oggi insiste ad affermare la storiografia specialistica, non costituirono uno stravolgimento del vecchio sistema, bensì un processo graduale e molto complesso che pose uno davanti all'altro sistemi politici e culturali profondamente diversi fra di loro. Da questo punto di vista la vicenda mongola ebbe un impatto diverso nella regione che stiamo considerando. Essa dovette confrontarsi con equilibri fortemente basati sul religioso. Islam e cristianesimo costituivano un pilastro ideologico per le popolazioni assoggettate e i Mongoli ebbero un atteggiamento diverso nei confronti delle due confessioni, ondivago e caratterizzato più dall'orientamento individuale dei singoli khan piuttosto che da una programmata politica religiosa o appunto, come spesso si è detto, da una generale tolleranza. Il problema legato all'atteggiamento dei khan mongoli verso le confessioni è stato sovente semplificato presentando sostanzialmente questo quadro generale: Gengis e Ogödeï erano sciamanisti convinti, Güyük aveva forti simpatie filo-cristiane, Möngke era estraneo a ogni faccenda legata al culto, mentre Qubilaj e Hülegü sarebbero stati buddisti, il primo convinto e precoce sostenitore della religione orientale, il secondo uno scopritore tardo e un fedele più pigro. ${ }^{4}$ Per avere un

${ }^{3}$ GA, p. 2.

${ }^{4}$ È questa un'opinione piuttosto diffusa fra gli storici e in particolare fra gli islamisti; si veda ad esempio quanto afferma nel suo, comunque fondamentale, saggio A. Bausani, Religion under the Mongols, in The Cambridge History of Iran cit., vol. 5, pp. 538-549, p. 540. Hülegü condusse una politica comunque filo-cristiana; in questo il contributo della moglie, nestoriana di formazione, fu probabilmente decisivo. 
quadro esaustivo dell'atteggiamento che i khan mongoli ebbero in concreto verso le varie confessioni con le quali si dovettero confrontare bisognerebbe indagare ogni singola area e ogni singolo periodo della dominazione mongola: nel Caucaso come nel Nord, nell'Asia centrale come in Cina. Fra Caucasia e Azov l'atteggiamento del ceto dirigente forestiero verso i gruppi confessionali indigeni fu molto diverso nel corso degli anni.

In realtà una delle conseguenze più vistose della conquista mongola fu il progressivo, ma inesorabile, processo di acculturazione che si verificò fra i conquistatori e le popolazioni delle aree entro le quali essi si stanziarono. Tale processo fu tutto a svantaggio dei Mongoli. La vastità del territorio che i Tatari riuscirono ad assoggettare presenta unità etno-linguistiche alle quali non sempre corrispondono simili identità culturali. La Caucasia, ad esempio, è una regione sulla quale, in spazi relativamente ristretti, si trovano tre gruppi confessionali assai diversi fra di loro e profondamente radicati nel tessuto sociale del contesto cui appartengono: cristiani, musulmani, il paganesimo turco-nomade dei Cumani e aree in cui erano fortemente radicate pratiche sciamaniche. Larrivo dei Mongoli ebbe, da questo punto di vista, un impatto a effetto "ritardato". L'irruzione violenta dell'esercito tataro e la creazione di nuove strutture istituzionali modificarono da una parte l'organizzazione del potere, dall'altra lasciarono pressoché intatta la fisionomia culturale delle popolazioni assoggettate. Anzi, talvolta ne rafforzarono il sentimento identitario esclusivo proprio a causa del terrore trasmesso dai sistemi di conquista e dalle devastazioni che seguivano le operazioni militari.

Durante gli oltre due secoli di governo i Mongoli non riuscirono a imporre un andamento culturale egemone; il modello che resistette fu quello dei gruppi, maggioritari, estranei alla gestione del potere. I ceti dirigenti, quando erano costituiti da funzionari mongoli, subirono il più delle volte l'influenza culturale delle regioni nelle quali operarono e lo stesso accadde per i khan. Il culto praticato dai Mongoli, lo sciamanesimo, non costituiva un elemento di auto-riconoscimento collettivo; al contrario questa era una caratteristica molto presente presso i popoli assoggettati. La pratica sciamanica è strettamente legata all'ambiente naturale nel quale essa nasce e prende forza. Il culto monoteista, quali erano cristianesimo e Islam, tendeva invece a isolare la credenza dalle influenze impreviste e imprevedibili del contesto naturale. Nelle due grandi religioni monoteiste c'è - in misura assai diversa fra loro - normatizzazione del culto, creazione della neutralità, laddove questo elemento era del tutto assente presso i popoli nomadi della steppa. A questo va aggiunto che l'Islam dell'Ilkhanato e il cristianesimo della Rus' erano un collante sociale fortissimo estraneo alla cultura mongola e a quella nomade in generale. Ma nel caso dei Mongoli la supremazia culturale del sedentarismo non corrispose a una supremazia politica, almeno nei primi decenni della dominazione. ${ }^{5}$

5 Si veda su questo la lucida analisi di Golden, Imperial Ideology cit., pp. 75-76. 


\subsubsection{Prima delle conquiste}

Con l'amichevole aiuto di mio padre-qan e dell'anda Žamuka, con forze moltiplicate dal Cielo e dalla Terra, chiamati dal possente Tenggeri, e con l'ausilio della Madre Terra... ${ }^{6}$

queste parole, pronunciate da Timučin in ringraziamento agli elementi naturali, oltre che agli amici, per aver sconfitto i Merkit, sono un esempio del carattere delle credenze religiose dei Mongoli alla vigilia dell'unificazione (quindi ancora alla fine del XII secolo). I Mongoli erano pagani e presso di loro le pratiche sciamaniste erano molto radicate. ${ }^{7}$ Gengis khan era fortemente legato al culto originario mongolo, ma mai i Mongoli ebbero nelle credenze religiose un veicolo sociale; da questo punto di vista ebbe un ruolo determinante l'etnicità "costruita" dei Mongoli (ovvero di quell'ideologia collettiva imposta di cui ci siamo occupati nel cap. terzo). Kiriakos Gandiate, nel XIII secolo, sostiene che

non c'è religione o venerazione fra di loro, ma chiamano spesso il nome di Dio per ogni cosa. Non sappiamo, e nemmeno loro, se ciò sia per ringraziare Dio dell'essere o qualcosa che essi chiamano dio. Tuttavia sostengono che il loro re sia parente di Dio. Dio si è preso il cielo ed ha lasciato la terra al Khan. ${ }^{8}$

Secondo Giovanni di Pian del Carpine i Mongoli, quando li visitò lui, cioè nel 1245, credevano in un solo Dio (il Cielo) che «pensano sia il creatore di tutte le cose visibili e invisibili e credono che sia il dispensatore, in questo mondo, tanto del bene quanto del male». ${ }^{9}$ Ma i Mongoli presso cui si recò Giovanni di Pian del Carpine veneravano anche il sole, la luna, il fuoco, l'acqua e la terra e "offrono loro primizie di cibi e bevande». ${ }^{10}$ Facevano largo uso di idoli di stoffa, seta e feltro fabbricati in genere dalle donne. ${ }^{11} \mathrm{Nel}$ carro che disponevano davanti al campo utilizzavano un idolo che rappresentava il "primo Imperatore». ${ }^{12}$ Juvaini è molto chiaro nell'affermare che Gengis non era

partigiano di alcuna religione o seguace di alcun credo[...] al contrario, onorava e rispettava i dotti e i venerabili di qualsiasi setta, poiché riconosceva in tale comportamento la via per la Corte di Dio. ${ }^{13}$

${ }^{6}$ SSM, III, 113, p. 94

7 Historia Mongalorum, in particolare il cap. III; Guglielmo di Rubruck, Viaggio cit., pp. 104-108.

${ }^{8}$ KG, pp. 234-235.

9 Historia Mongalorum, III, 2, p. 342.

${ }^{10}$ Ibidem, III, 5, p. 343.

${ }_{11}$ Ibidem, III, 3, p. 342; Guillelmus de Rubruc, Itinerarium cit., II, 7, p. 17.

12 Historia Mongalorum, III, 3, p. 343, laddove il primo imperatore è ovviamente Gengis.

13 Juvaini, I, p. 46. Ė probabile che qui Juvaini esageri poiché se è vero che Gengis non aveva un credo che rispettava con devozione è altrettanto vero che i riti pagani mongoli gli erano familiari 
È però evidente che il forte radicamento col territorio e la natura nomade della società mongola tradizionale comprendessero una presenza capillare delle credenze e delle pratiche sciamaniste sin negli strati più bassi del complesso sociale. ${ }^{14}$ Ad esempio uno dei termini che ricorrono spesso nella Storia Segreta è quello di beki (biki), il quale, sia secondo Bartold ${ }^{15}$ che Vladimircov ${ }^{16}$ designerebbe una sorta di stregone, un "sacerdote capo". Questa figura godeva di una alta considerazione presso le tribù che Rashid ad- Din chiama straniere, $\mathrm{i}$ Merkit e gli Ö̈rat. Il beki era un personaggio eminente nella gerarchia sociale mongola: secondo Vladimircov, che attinge abbondantemente dalla storia di Rashid ad-Din, il beki era posto, nell'esercito, alla destra del khan, come i principi di sangue. ${ }^{17}$ Nella Storia Segreta c'è un passo molto chiaro sulla funzione di questo personaggio:

Esiste da noi Mongoli la tradizione di elevare alla dignità di noyon, di beki, coloro che lo meritano...la dignità di beki è ereditata dal maggiore di una famiglia [il beki] ci indichi e ci illumini sul corso degli anni e dei mesi beki:

la Storia Segreta prosegue definendo la simbologia cui doveva sottostare il

Quando sarà insignito della dignità di beki, gli si faccia indossare una pelliccia bianca, sia fatto salire sopra un cavallo bianco e poi fatto sedere su di un trono»..$^{18}$

Questa figura sembra dunque preesistere l'opera di Gengis e appartenere a una lunga e radicata tradizione mongola. Spesso era il noyon più anziano in una famiglia in cui ve ne erano più di uno.

Già nel XIII secolo verrebbe da pensare che l'aspetto religioso fosse ormai confuso con pratiche quotidiane individuali piuttosto che costituire un elemento identitario collettivo; in Juvaini, ad esempio, la figura del beki è del tutto assente. Si rileva tuttavia che, sulla base delle notizie fornite da Giovanni di Pian del Carpine, la figura dello stregone che assiste gli individui nei casi in cui essi disattendono alcune pratiche, è ancora presente alla metà del XIII secolo. Secondo il Nostro se qualcuno infrange volontariamente una delle regole rituali (come battere il cavallo con gli speroni, uccidere o catturare giovani uccelli etc.) viene ucciso,

e li frequentava spesso. Credo che il nostro storico persiano qui sovrapponga la sua immagine dei Mongoli, come essi si comportavano nell'Ilkhanato ai suoi tempi alla reale situazione che vigeva presso i Mongoli gengiskanidi oltre un secolo prima.

${ }^{14}$ Cfr. Humphrey, Hürelbaatar, Regret as a Political Intervention cit., pp. 9 e segg.

15 Bartold, Turkestan cit., pp. 391-392.

16 Vladimircov, Le Régime social cit., p. 61.

17 Ibidem, p. 62; Bartold, Turkestan cit., p. 391.

18 SSM, IX, 216, p. 191. 
si autem aliter, oportet quod pecuniam multam solvat incantatori qui purificet eos, et faciat et stationem et ea que in ipsa sunt, inter duos ignes transire, sed antequam sic purificetur nullus audet intrare nec de ipsa aliquid exportare. ${ }^{19}$

Sul ruolo delle donne entro i rituali magico-religiosi mongoli abbiamo il racconto di Kiriakos in parte confermato da Giovanni di Pian del Carpine per cui esse erano una sorta di streghe che "divinano ogni cosa». ${ }^{20}$ Fra le credenze più radicate i Mongoli avevano una concezione del post mortem piuttosto concreta; per loro esisteva un mondo simile a quello terreno nel quale «greges multiplicent, comedant, bibant et alia faciant que in hoc seculo a viventibus hominibus fiunt». ${ }^{21}$

La mancanza di una cultura scritta presso i Mongoli e l'importazione di questa dagli Uiguri (attraverso i Naiman) aveva probabilmente influenzato anche il carattere devozionale dei Mongoli in generale e dei Gengiskanidi in particolare. Gli Uiguri erano per la maggior parte nestoriani (anche i Naiman, che vissero per molto tempo a contatto con loro, adottarono il nestorianesimo) riuscirono a far penetrare il cristianesimo di Nestorio presso i Mongoli. La regione abitata dagli Uiguri rimase a maggioranza nestoriana anche dopo la conquista. Su di essa conviveva un coacervo di confessioni tra le quali solo l'ebraismo era assente. ${ }^{22}$ Anche secondo Giovanni di Pian del Carpine essi erano prevalentemente nestoriani, ${ }^{23}$ mentre secondo l'autore persiano Awfi veneravano in parte il sole, in parte erano cristiani. ${ }^{24} \mathrm{Il}$ buddismo era molto presente. Le fonti non evidenziano motivi di contrasto fra le diverse confessioni all'interno del complesso mondo uigurico. Si potrebbe pensare che buddisti, cristiani e pagani convivessero in modo sostanzialmente pacifico. D'altra parte parlare di unicità religiosa, così come di una qualche omogeneità etnica, per i popoli nomadi della steppa è, a mio avviso, fuorviante. Come abbiamo visto parlando dell'origine di quello che a Occidente veniva percepito come popolo

19 Historia Mongalorum, III, 7, p. 239-240.

${ }^{20} \mathrm{KG}$, pp. 236-237.

${ }^{21}$ Historia Mongalorum, III, 9, p. 240; Ibidem, Note, p. 415, nota 8; sulla concezione della vita dopo la morte presso i Mongoli anche J.P. Roux, La mort chez les peuples altaiques anciens et médiévaux, Paris 1963; Id., Les astres chez les Turcs et les Mongols, in "Révue de l'histoire des religions», CXCV (1979), pp. 153-192 ; M. Waida, Central Asian Mythology of the Origins of Death, in «Antropos», LXXVII (1982), pp. 663-702; Id. Myths of the Origin of Death: The Central Asian Type, in «Temenos», XVIII (1982), pp. 87-98; le pratiche descritte da Giovanni di Pian del Carpine sono confermate dal racconto di Kiriakos Gandiate (cit. pp. 234-238).

${ }_{22}$ Bartold, Turkestan cit., p. 388.

${ }^{23}$ Historia Mongalorum, V, 8, pp. 255-256: «Chingis [...] contra terram Huyrorum processit ad bellum. Isti homines sunt christiani et de secta Nestorianorum erant».

${ }^{24}$ Bartold, Turkestan cit., p. 388. 
dei Mongoli, questi erano un'esigua parte di un universo etnico molto elastico, fluido, all'interno del quale l'auto-identità era un concetto in gran parte estraneo. Così i Mongoli di Gengis odiarono a lungo i Tatari, fino a quando li ebbero completamente sconfitti, ma dieci anni dopo li troviamo insieme ad attaccare la Korazmia; oltre a questo le due componenti avviarono un lungo processo di assimilazione tanto che gli osservatori occidentali (e non solo) chiamavano Tartari i Mongoli tutti. ${ }^{25}$ I Kerait, un tempo alleati dei Mongoli, divennero presto loro nemici mortali e furono per questo attaccati e sconfitti. I Naiman ci vengono spesso presentati come avversari nella Storia Segreta, ma il debito culturale dei Mongoli nei confronti di questa grande tribù è notevolissimo e riconosciuto dalle fonti mongole o filo-mongole (e se ciò è vero, come è dimostrato dalla Storia Segreta e non solo, pare difficile pensare che dopo gli eventi militari che li videro contrapposti, i due popoli non abbiano vissuto un processo di assimilazione). Cioè, il mondo della steppa, è un ambito entro il quale certe categorie culturali a noi familiari non stanno in piedi in alcun modo. Non solo l'identità etnico-religiosa era debole, ma contemporaneamente era molto forte il complesso delle pratiche spicciole, quotidiane che caratterizzavano la vita della popolazione di questa regione indistintamente. I rituali presentati dalle nostre fonti, siano esse Giovanni di Pian del Carpine o Juvaini, Rubruck piuttosto che Kiriakos, risultano, oltre che incomprensibili ai nostri narratori, disomogenee, lontane da una qualsiasi codificazione normativa. Avevano una dimensione individuale e non costituivano legante collettivo. Il culto era al tempo stesso oggetto e veicolo orale.

Dicevamo del ruolo che rivestirono gli Uiguri su molti aspetti della formazione culturale mongola quando Gengis non aveva ancora completato l'unificazione. Sulle pratiche buddiste degli Uiguri ha lasciato molti dettagli Rubruck: «Dietro ad una cassa, che essi adoperavano come altare e sopra la quale mettono lampade e offerte, vedevo, infatti, la rappresentazione di una figura con le ali...». ${ }^{26}$ Secondo il frate fiammingo i buddisti pregavano rivolti verso Nord, con le mani giunte, inginocchiandosi a terra e appoggiando la fronte sulle mani, «per questo motivo i nestoriani che vivono in quelle zone non pregano mai con le mani unite, bensì

${ }^{25}$ Questo è un punto sul quale non c'è accordo fra gli specialisti. Non si è stabilito cioè per quale motivo i Mongoli vennero percepiti e metabolizzati presso i popoli terzi come Tatari/Tartari. Fermo restando che l'assimilazione occidentale della parola Tataro con Tartaro, cioè venuto dal Tartaro (dall'inferno), è più che verosimile, resta da capire se, prestando fede a Rubruck, ciò sia dovuto all'uso da parte dei Mongoli di Tatari come avanguardie nelle operazioni militari (Guillelmus de Rubruc, Itinerarium cit., XVII, 6, p. 72) o se, come io credo, ciò fosse dovuto piuttosto al fatto che i Tatari fossero una componente maggioritaria negli eserciti che combatterono in Europa o in Cina, senza essere stati necessariamente le avanguardie o comunque non solo esse. Si veda su questo anche quanto dice Daffinà, Historia Mongalorum, Note, pp. 426-427, nota 5.

${ }^{26}$ Guglielmo di Rubruck, Viaggio cit., p. 102 e nota 3; si veda anche l'edizione di padre Van De Wyngaert, (Guillelmus de Rubruc, Itinerarium cit., p. 227, nota 2). 
tenendole distese davanti al petto». ${ }^{27}$ Le notizie fornite da Guglielmo confermano la presenza di pratiche fortemente legate alla cosmografia. I templi buddisti «sono rivolti da oriente verso occidente ed esternamente sul lato settentrionale, costruiscono una stanza che esce a forma di coro».$^{28}$ Nell' Itinerarium si trovano molti esempi di questo tipo. ${ }^{29}$ Il viaggio di Guglielmo di Rubruck si colloca all'inizio degli anni Cinquanta del XIII secolo, pertanto le informazioni in esso contenute sono il ritratto di una situazione immediatamente successiva alle conquiste. Un osservatorio, da questo punto di vista, privilegiato.

\subsection{Mongoli, l'Islam e il Cristianesimo armeno-georgiano fino alla scelta di Ghazan (1220-1295)}

Nei primi anni del XIII secolo la Caucasia era una regione fortemente differenziata da un punto di vista religioso. La Georgia era un regno piuttosto vasto la cui religione ufficiale era il cristianesimo greco, lo stesso si può dire per l'Armenia (monofisita), o meglio per quelle aree conquistate dalla Georgia, ma la cui popolazione era in maggioranza armena (come i distretti di Kars, Ani, la zona di Erzrum e le aree attorno al lago Sevan). L'Anatolia era divisa fra il ricostituito impero greco di Nicea, cristiano, il sultanato musulmano-sunnita dei Turchi Selgiuchidi di Iconio, la Piccola Armenia o Cilicia, anch'essa ufficialmente cristiana e il piccolo impero di Trebisonda, cristiano. Il resto della regione, Azerbaijan, penisola Arabica e Persia iranica, era musulmana (Ayyubidi, il Califfato di Baghdad, con una forte predominanza del sunnismo). Oltre i monti del Caucaso vivevano le popolazioni nomadi, pagane, della steppa: Alani e Cumani. A Nord si trovavano i principati russi, cristiani. Dopo la conquista della Caucasia e delle steppe dei Cumani, i Mongoli si trovarono nel mezzo di un autentico accerchiamento monoteista.

Il ceto dirigente mongolo si limitò a utilizzare la devozione popolare in modo strumentale al controllo dell'andamento economico, dell'ordine pubblico, del riassetto istituzionale dopo le devastazioni prodotte dalle campagne militari. L'aspetto religioso, per come appare dalle fonti, sembra essere stato l'ultimo dei loro problemi, almeno in apparenza. Il territorio sul quale sarebbe sorto l'Ilkhanato era caratterizzato da una predominanza dell'Islam, nelle sue diverse correnti dottrinali. La Georgia e l'Armenia cristiane, l'Azerbaijan e il califfato musulmani e il sultanato selgiuchide anatolico hanno convissuto in uno stato di conflitto costante ben prima che arrivassero i Mongoli. Una lunga guerra dovuta a ragioni di tipo strategico piuttosto che a dispute dottrinali. ${ }^{30}$ Ciò nonostante la solida identità

27 Ibidem, p. 103.

${ }^{28}$ Ibidem, pp. 102-103.

${ }^{29}$ Ibidem, pp. 102-107.

30 Anche se la percezione popolare dei conflitti veniva sovente convogliata sull'aspetto religioso. 
religiosa dei Georgiani, così come quella di tutti gli stati musulmani contribuirono ad acuire il clima di inimicizia nella regione. I Mongoli portarono a termine le operazioni militari in una regione in cui il sentimento religioso era strettamente connesso con l'esercizio del potere, pratica questa assolutamente estranea alla loro cultura. Se è vero che il khan aveva una missione predeterminata in quanto volontà del Cielo (Tenggri), è altrettanto vero che il potere conferito al khan passava comunque attraverso il popolo e le sue esigenze. Era un culto del sovrano ibrido, a metà fra soprannaturale e terreno, fra spirituale e temporale (laddove, come abbiamo visto, il potere spirituale, era prerogativa dello sciamano). E comunque il volere del Cielo, come imperativo per il khan, e al tempo stesso sua suprema legittimazione, verrà elaborato solo a partire dal khanato di Güyük.

Durante gli anni della dominazione, le realtà indigene, in Caucasia come nell'Azov, hanno conosciuto i conquistatori attraverso il filtro della paura che ha impedito uno scambio culturale paritario. E ciò fu vero per l'Islam come per il cristianesimo monofisita armeno, per il nestorianesimo come per le pratiche pagane.

I rapporti fra i Mongoli e l'Islam furono caratterizzati, come gli altri, da un succedersi di atteggiamenti molto diversi nel corso degli anni. La quasi totalità della regione mediorientale al tempo dell'invasione mongola era ufficialmente sunnita. ${ }^{31}$ Gli eventi del 1258, con la presa e il saccheggio di Baghdad e l'uccisione del Califfo, gettarono l'Islam nel terrore; era una situazione senza precedenti poiché il dominio di una forza non musulmana, anzi all'inizio decisamente ostile all'Islam, rappresentava una novità assoluta per il sunnismo. ${ }^{32}$

La morte del califfo di Baghdad, ucciso dai Mongoli di Hülegü, ebbe un duplice effetto pratico: se da un lato i sunniti si trovarono per la prima volta senza il loro primo referente spirituale e politico, ${ }^{33}$ dall'altro questa decapitazione favorì il rafforzamento della corrente sciita in aree che erano state da sempre a forte maggioranza sunnita; ${ }^{34} \mathrm{a}$ ciò va aggiunta l'eliminazione degli

31 Lo era soprattutto presso la classe dirigente in quanto fra i ceti popolari predominava lo scitismo.

32 Bausani, Religion under the Mongols cit., p. 538.

33 Sulla confusione delle prerogative califfali vi sono varie testimonianze. Nel suo diario il diplomatico siriano Jamal ad-Din, che fu in Italia alla corte di Manfredi nel 1260, chiama il papa «il Califfo dei Franchi» (Bausani, Religion under the Mongols cit., p. 540). Lo storico tunisino Ibn Khaldûn (1332-1406) parlando del ruolo del Califfo dice che egli «agisce come vicario del Legislatore [Maometto] [...] il Legislatore è investito di un doppio compito: spirituale, poiché deve trasmettere al popolo gli obblighi religiosi e farlo agire nella concordia; e temporale, in qualità di responsabile degli interessi della civiltà umana (al-umrân al-basharî)» (Ibn Khaldûn, Discours sur l'Histoire universelle Al-Muqaddima, trad. e cura di V. Monteil, Arles 1997, pp. 338-339). Dobbiamo però rilevare che in questo periodo (seconda metà del XIII secolo) l'autorità temporale del Califfo si era ridotta a una quasi totale formalità, mentre era ancora molto forte l'influenza spirituale.

${ }^{34}$ Per il ruolo dell'Islam nella Persia selgiuchida si veda il saggio del grande orientalista A. Bausani, Religion in the Saljuq Period, in The Cambridge History of Iran cit., vol. 5, pp. 283-302. 
"eretici" Assassini di Alamut, che liberò l'ortodossia da un ostacolo di non poco conto.

Dal 24 al 30 gennaio del 1260 i Mongoli si scatenarono contro Aleppo, retta da un governatore ayyubida; secondo il racconto di Abu'l Feda fu un massacro. ${ }^{35}$ La presa di Baghdad aveva avuto un seguito immediato in Siria e in Palestina. La partecipazione di Hethum I di Armenia e di Boemondo VI alle operazioni militari determinò un clamoroso squilibrio fra l'accanimento portato sui musulmani della città rispetto alla sistematica salvezza concessa ai cristiani giacobiti di rito siriano. Anche su Damasco i Mongoli di Hülegü si abbatterono con violenza. Sebbene la città si fosse arresa senza combattere, il generale mongolo Ketbuqa volle egualmente uccidere il governatore della città. Sempre secondo Abu'l Feda rileviamo che ai cristiani fu concesso di consacrare a chiesa di Cristo una moschea che già i sunniti di Damasco avevano ricavato da una chiesa greco-ortodossa. ${ }^{36}$ Le fonti armene su questo tacciono. ${ }^{37}$ In ogni caso dalla conquista di Baghdad ai massacri perpetrati in Siria fra i musulmani della regione si creò certamente un clima di panico; una tensione che forse è fra le cause principali della spedizione mamelucca verso queste terre e la vittoriosa battaglia che le truppe egiziane combatterono contro i Mongoli di Ketbuqa.

Dopo la controffensiva egiziana in Siria i musulmani della regione poterono ottenere una notevole rivincita sui cristiani che si erano appoggiati ai Mongoli.

In Caucasia, e più in generale nell'Ilkhanato, la politica religiosa fu influenzata in minima parte dalle vicende mediterranee. Come detto l'atteggiamento dei funzionari mongoli in materia religiosa era determinato soprattutto dalle mutevoli convinzioni dei khan, i quali oscillarono sempre fra tradizionalismo sciamanista, nestorianesimo e Islam. Non v'è dubbio sul fatto che Hülegü, primo Ilkhan, fu filo-cristiano e abbiamo già esposto le ragioni che ci spingono a ritenere questo; questa sua tendenza anti-islamica gli costò cara quando Berke, alleatosi col sultano mamelucco, gli dichiarò guerra e lo fece utilizzando a pretesto la vicinanza di Hülegü col cristianesimo contrapposta alla sua (di Berke) recente conversione all'Islam. Le scelte politiche operate dall'Ilkhan però non vanno prese, a mio avviso, come dettate da un fervente sentimento religioso. Il politico e il religioso erano molto distinti nella mente dei sovrani mongoli in questo periodo. L'attacco alle città simbolo dell'Islam era dettato più da ragioni politiche, da esigenze di conquista, che dalla volontà di eliminare i musulmani della regione. Resta il fatto che alla morte di Hülegü le cose per la cristianità

35 Résumé de l'Histoire des Croisades tiré des Annales d'Abou'l-Feda, in Recueil des Historiens des Croisades. Historiens Orientaux,II, 1887; Géographie d'Aboulféda, a cura di J.T. Reinaud, 2 voll., Paris 1848 (d'ora in avanti Abaul-Feda). Aboul-Feda, I, pp. 140-141 : «Il massacro e la razzia durò fino al venerdi 14 safer, quando Hulagu fece arrestare la carneficina e proclamare un'amnistia generale»; Grousset, L'empire des steppes cit., p. 436.

${ }^{36}$ Abaul-Feda, I, p. 143.

37 Kiriakos liquida rapidamente la presa di Aleppo e di Damasco (KG, pp. 324-325). 
caucasica cambiarono sensibilmente e in modo brusco. Prima dell'avvento al potere nell'Ilkhanato da parte di Ghazan, vi fu un periodo sostanzialmente positivo per i cristiani. Abaqa, figlio di Hülegü, era buddista come il padre, ma mostrò una sostanziale tolleranza verso Armeni, Georgiani e giacobiti siriani. Fu protettore del patriarca nestoriano Mar Denha ${ }^{38}$ e sposò la figlia dell'imperatore bizantino Michele Paleologo. Fu un tenace avversario dell'Egitto mamelucco e dovette combatterlo mentre a Oriente stava avanzando l'esercito mongolo dei Čagataidi. ${ }^{39}$ Abaqa fu forse il primo mongolo a cercare l'alleanza con i poteri occidentali. La situazione nella quale versava richiedeva una stabilizzazione diplomatica almeno con l'Europa. Fra maggio e giugno 1274 Abaqa inviò due ambasciatori presso il pontefice romano, Gregorio X. Nel 1277 ne inviò altri due presso la corte di Eduardo I d'Inghilterra per concludere un trattato di alleanza contro i Mamelucchi. ${ }^{40}$ Non ottenne risposta. Morì poco dopo la disfatta dell'esercito mongolo-armeno che ebbe luogo il 30 ottobre 1281 contro i musulmani d'Egitto. ${ }^{41}$ Ad Abaqa successe Tagudar, cristiano di formazione, ma convertitosi precocemente all'Islam. I due anni durante i quali egli governò furono il preludio allo scossone che dal 1295 avrebbe apportato alla struttura politica, fiscale e anche culturale dell'Ilkhanato Ghazan. Secondo la testimonianza di Hethum, Tagudar fu nemico dei cristiani e ordinò che «tutte le chiese cristiane fossero distrutte»; perseguitò i cristiani al punto che dovette intervenire il Gran khan Kubilai a ordinargli di mitigare le sue posizioni. ${ }^{42}$ Fra la morte di Tagudar (1284) e l'avvento al trono di Ghazan, regnarono nell'Ilkhanato Arghun, filo-cristiano, e Geikhatu, un debole inviso al clan regnante, tanto che fu ucciso durante una rivolta di palazzo nel 1295. Durante il regno di Arghun, il monaco cinese Rabban Sauma, insieme a Rabban Marco, viaggiò in Occidente fino all'Italia e alla Francia. Il viaggio di Rabban Sauma è narrato in un documento straordinario conservatosi fino a noi e rinvenuto nel XIX secolo nel Tyari. È un manoscritto in siriaco che ha avuto molte edizioni ${ }^{43}$ e attraverso il quale possiamo ricostruire non solo la vicenda dei due monaci nestoriani, ma anche della Chiesa Orientale negli ultimi anni del XIII secolo, specialmente in Persia.

${ }^{38}$ Grousset, L'empire des steppes cit., p. 442.

39 Amitai-Preiss, Mongols and Mamluks cit., pp. 129-133 e 183-201.

${ }^{40}$ Grousset, L'empire des steppes cit., pp. 445-446 ; Amitai-Preiss, Mongols and Mamluks cit., pp. 95-105.

41 STHA, pp. 335-336.

42 Ibidem, p. 336.

${ }^{43}$ La prima edizione della Storia è stata pubblicata nel 1888 (P. Bedjan, Histoire de Mar Jab-alaha, patriarchi, et de Raban Sauma, Paris 1888); l'opera ebbe successo e fu ripubblicata varie volte, oltre alla seconda edizione di Bedjan del 1895. Io ho utilizzato l'edizione italiana a cura di P.G. Borbone, Storia di Mar Yabballaha e di Rabban Sauma, Torino 2000 alla quale rimando per la bibliografia e l'ottimo commento. 
Con l'avvento al potere di Ghazan si verificò un netto e durevole cambiamento di rotta nella politica dell'Ilkhanato nei confronti dei rapporti confessionali. Era il 16 giugno 1295. Ghazan fu una figura di grande rilievo nella vita politica del regno mongolo; le sue riforme, in materia fiscale, mutarono in meglio lo stile di vita di molte regioni sottoposte all'imperio ilkhanide e da un punto di vista religioso il sovrano non lasciò impronta più leggera. Divenne un musulmano devoto e la sua scelta influenzò profondamente la politica governativa in materia religiosa. Molti dirigenti dello Stato, governatori, emiri, ministri, si convertirono e questa loro conversione fu alla base di un sostanziale irrigidimento della classe dirigente nei confronti della diversità: ebbe inizio una sistematica persecuzione verso cristiani e buddisti, ma anche verso quelle cellule minoritarie di culto (come ad esempio coloro i quali rimanevano legati a pratiche sciamaniste e gli zoroastristi). ${ }^{44}$ Rashid ad-Din dice chiaramente che quando «il Signore dell'Islam, Ghazan» divenne musulmano fece distruggere tutti gli idoli e i templi e che i non musulmani dovevano essere convertiti anche con la forza. ${ }^{45}$ Più avanti, aggiunge lo storico persiano, data l'impossibilità di convertire alla vera fede con la forza, Ghazan lasciò che ognuno professasse il proprio credo: «Lasciamoli credere in ciò che essi hanno nei loro cuori...e se giungerà alle mie orecchie che essi stanno costruendo templi o idoli io, senza esitazione, li passerò a fil di spada». ${ }^{46}$ Appare chiaro quindi che il khan cercasse di mantenere un rigido controllo su coloro che erano controllabili, sugli strati superiori della società, quelli che vivevano attorno al sovrano. Per quanto riguarda la diffusione dell'Islam nei villaggi e, più in generale, presso i ceti più bassi, tale controllo era inapplicabile. Non si poteva andare in ogni singolo villaggio a controllare quante volte la gente andasse in moschea o farsi guarire da uno sciamano.

Sostenuto dal suo emiro fidato Nawruz, anch'egli musulmano devoto, si dimostrò particolarmente efficiente nel distruggere chiese, sinagoghe e templi buddisti. ${ }^{47}$ Anche la moneta fu coniata con immagini del Profeta e dal maggio del 1299 fu proibita l'usura, particolarmente avversata dalla dottrina islamica. Secondo lo storico persiano Khwandamir, autore dell'Habigu's-Siyar, alla fine del 1297 Ghazan fece uccidere non meno di cinque principi e trentasette emiri a causa della loro riluttanza verso l'Islam. ${ }^{48} \mathrm{Fu}$ sciita da subito, come il figlio e successore Oljeitu. Nonostante le scelte operate dagli Ilkhan, la frammentazione presente

${ }^{44}$ Lo Zoroastrismo era molto radicato nella Korazmia dove l'Avesta colloca la dinastia dei Paradhâta, dinastia mitica della religione iranica. La stessa famiglia del Profeta è tradizionalmente associata alla regione iranico-orientale per la sua origine. Si veda a tale proposito P. du Breuil, Lo zoroastrismo, Genova 1993, in particolare le pp. 16-17; id., Zarathustra, Genova 1990; G. Widengren, Les Religions de l'Iran, Paris 1968.

45 Bausani, Religion under the Mongols cit., p. 542.

46 Ibidem, p. 543.

47 Browne, A History of Persian Literature cit., vol. I, p. 40.

48 Ibidem, vol. I, p. 41. 
all'interno dell'Islam in generale e di quello sunnita in particolare, disorientava i Mongoli. Tale situazione non impedì alla popolazione di fede musulmana una rigida coerenza dottrinale. La maggior parte del ceto dirigente persiano rimase sunnita anche durante il regno di Ghazan. I centri storici del sunnismo, Isfahan e Shiraz, oltre a Baghdad, resistettero orgogliosamente alle pressioni aristocratiche (di tutti quei funzionari operanti sul territorio che si erano, per convenienza, convertiti allo scitismo). Da questo punto di vista la conversione di Ghazan rappresentò una novità: se i Selgiuchidi erano sempre stati filo-sunniti, la tendenza dell'Ilkhanato fu quella opposta e ciò mitigò in parte il contraccolpo derivato dall'abbattimento del califfato. Lo scitismo divenne presso i ceti dirigenti, da corrente di resistenza, l'Islam ufficiale dell'Ilkhanato. Bisogna inoltre aggiungere che proprio durante il periodo mongolo la teologia sciita raggiunse una sua definitiva canonizzazione. Nei confronti della popolazione, a maggioranza sunnita, non si verificarono in generale soprusi, a parte i primi anni del regno di Ghazan. Vi fu un sostanziale equilibrio fra la corrente maggioritaria a livello popolare e quella ufficiale adottata a corte. Il sunnismo era troppo forte e radicato in queste regioni per poter essere soppiantato dalla corrente antagonista solo perché ufficialmente adottata dalla casa regnante, che tra l'altro non era nemmeno persiana.

La Storia di Gregorio di Akner contiene numerose notizie circa gli eventi che caratterizzarono gli anni centrali delle conquiste mongole in Caucasia. Attraverso la sua narrazione è possibile vedere quale percezione avesse di loro un uomo dotato di una buona cultura e quanto scrive non conforta:

I primi che vennero contro il nostro paese non somigliavano a persone. Erano orribili alla vista, impossibili da descrivere. Avevano teste grosse come un bufalo e occhi piccoli come un pollo [...] la morte non compare fra di loro così che possono vivere fino a trecento anni. ${ }^{49}$

La descrizione fatta da Giovanni di Pian del Carpine non è molto diversa:

Inter oculos enim et inter genas plus quam alii homines sunt lati. Gene etiam satis prominent a maxillis; nasum habent planum et modicum, oculos habent parvos et palpebras usque ad supercilia elevatas. Graciles sunt generaliter in cingulo, exceptis quibusdam paucis; pene omnes mediocris sunt stature. Barba fere omnibus minime crescit: aliqui tamen in superiori labio et in barba modicos habent crines, quos minime tondent. ${ }^{50}$

Anche Kiriakos Gandiate ci descrive i Mongoli affermando che «non hanno barba, sebbene alcuni di loro abbiano pochi peli sopra le labbra o sulle guance. Hanno occhi rapidi e stretti, voci alte e grosse; erano robusti e longevi». ${ }^{51}$

\footnotetext{
${ }^{49}$ GA, p. 3.

50 Historia Mongalorum, III, p. 232.

${ }^{51} \mathrm{KG}$, p. 233.
} 
La cristianità caucasica accolse l'arrivo dei Mongoli in una situazione di profondo disagio economico e politico, ma con una coesione ideologica senza precedenti. Dai racconti di Gregorio di Akner, di Kirakos, ma anche di Hethum, traspare un forte senso di identificazione collettiva, di unione contro i "barbari invasori", veicolato e reso concreto dalla fede cristiana; le due diverse confessioni armena e georgiana sembrano in questo momento perdere la loro crisi polemica davanti a un pericolo maggiore costituito dall'arrivo del sultano prima e dei Mongoli poi.

Il periodo precedente alle incursioni di Jalal ad-Din e dell'esercito tataro fu particolarmente positivo per la Caucasia cristiana la quale, sotto la spinta georgiana, visse un vigoroso sviluppo economico. La regina Tamara, fervente cristiana, finanziò la costruzione di chiese, monasteri e conferì a essi terre, interi villaggi. Così alla fine del XII secolo (attorno al 1191) il

Vardapet e i suoi monaci iniziarono a lavorare sulla costruzione di un monastero e una chiesa nella già citata valle del Tandzut per ordine del gran principe Ivane. Costruirono una splendida chiesa di legno consacrata al nome di S. Gregorio. ${ }^{52}$

Ancora, nello stesso anno, venne costruita una chiesa a Nor, consacrata a S. Giovanni Battista, ${ }^{53}$ alla quale furono concessi "terre estese segnate ai confini con fiumi che si estendevano da monte a monte, come quelli di Abasadzor, e Zoradzor nel distretto di Bjini, e Ashawan sul monastero». ${ }^{54}$

L'incursione del sultano rafforzò il sentimento anti islamico della cristianità nella regione, unificando in sostanza il legame fra le città armene e il regno di Georgia. Kiriakos, armeno e religioso di professione, non esita a prendere le parti della Georgia quando questa viene attaccata e sconfitta dalle truppe di Jalal ad-Din. D'altra parte l'equilibrio, già fortemente sbilanciato verso il conflitto, fra Islam e cristianesimo si rompe definitivamente. Durante la presa di Tblisi da parte del sultano molti Georgiani preferirono la morte alla conversione forzata. ${ }^{55}$ Dopo aver conquistato Gandzak le guarnigioni militari lasciate a presidio della città si abbandonarono a ogni sorta di vessazione nei confronti della popolazione civile. ${ }^{56}$ La città era popolata soprattutto da musulmani, i cristiani erano una minoranza, nemmeno troppo tollerata, se crediamo a Kiriakos. ${ }^{57}$

52 Ibidem, p. 174; la chiesa fu posta sotto la diretta protezione del re Giorgio, padre di Tamara, il quale le concesse due villaggi: Abasadzor e Tandzut, oltre a una vigna a Mijnashen (KG, p. 175).

53 Ibidem, p. 176.

54 Ibidem, p. 180.

55 Ibidem, pp. 188-189.

56 Ibidem, pp. 189-190; tale atteggiamento è confermato anche da Juvaini (II, pp. 480-481).

57 KG, p. 198: «[La città] era fortemente nemica di Cristo e della sua venerazione, insultava e malediceva la Croce, deridendo e disprezzando i preti e gli aiutanti». 
Sempre attraverso il racconto del monaco armeno possiamo osservare quale fosse la situazione per le aree a maggioranza cristiana subito dopo la prima conquista mongola: «La fede fu bloccata e le cerimonie cessarono di esistere, non si udivano più canti. Il paese sprofondò nell'oscurità e la gente preferì la notte al giorno». ${ }^{58}$ Un altro duro colpo alla cristianità caucasica fu inferto dai Mongoli quando presero la città di Ani; ricca, ben fortificata e popolata da una netta maggioranza di cristiani in gran parte Armeni e qualche piccola comunità di Georgiani. Dopo che gli assalitori ebbero forzato la cinta difensiva «entrarono in città, presero tutti i beni e possedimenti, depredarono tutte le chiese, rovinarono e distrussero l'intera città e corruppero la gloria della sua bellezza». ${ }^{59}$ La mancanza di fonti documentarie riguardo queste regioni durante gli anni delle invasioni non ci permette di seguire adeguatamente lo sviluppo della politica mongola relativa al credo della popolazione caucasica. Si può tuttavia osservare che la distruzione delle chiese generasse nei confronti degli oppressori un odio fortissimo, la sensazione di una violazione totale e intima alla quale doversi ribellare. In questa prima fase degli eventi - che potremmo far iniziare con la prima incursione del 1220 - le distruzioni subite dalle comunità cristiane non poterono che determinare una profonda spaccatura fra la popolazione e il ceto dirigente straniero; è presumibile cioè che i governi provvisori mongoli abbiano avuto parecchi problemi a tenere sotto controllo l'ordine pubblico nelle città e nei villaggi periferici. L'indignazione con la quale Kiriakos descrive le violenze alle quali la popolazione di Ani, ma anche di altre città, venne sottoposta è fortissima. ${ }^{60}$

Vi furono casi in cui principi georgiani o armeni accettarono la sottomissione ai Mongoli ottenendo in cambio da una parte di scampare alla loro devastazione, dall'altra il rispetto del culto. Fu il caso di un nipote di Ivane e Zakaria di nome Jalal (poi principe di Kachezia), descritto come cristiano devoto da Kiriakos. ${ }^{61}$ Avendo egli accettato il vincolo di fedeltà con Čormaghan fu autorizzato a costruire una chiesa nel monastero «chiamato Gandzasar», alla cui consacrazione parteciparono «il catholicos di Aghbania, Nerses con molti vescovi, il gran Vardapet ${ }^{62}$ Vanakan con molti maestri, il santo Vardapet di Kachetia Gregorio e Eghia, entrambi». ${ }^{63}$

58 Ibidem, p. 202.

59 Ibidem, p. 221.

${ }^{60}$ Ibidem, pp. 222-224: «Era una scena pietosa. Genitori morti e i loro figli accatastati uno sopra all'altro, come una pila di pietre, preti e diaconi e prelati della Chiesa, vecchi, giovani, bambini, adolescenti e molte vergini [...] la terra bevve il sangue dei colpiti [...] donne prudenti e morigerate deflorate da uomini volgari e libidinosi; vergini benedette che si erano votate a Dio per mantenere le loro anime e i loro corpi puri furono insozzate da prostituzioni e corrotte senza dignità».

${ }^{61}$ Ibidem, pp. 230-232.

${ }^{62}$ Il Vardapet era il dottore della chiesa armena, la guida spirituale dei monasteri.

${ }^{63} \mathrm{KG}$, p. 232. Jalal poté completare la chiesa nel 1240. 
Tutto sommato Čormaghan non fu ostile al cristianesimo e durante la sua permanenza a governatore della Caucasia si adoperò affinché non vi fossero vessazioni nei confronti della popolazione. Fu lui che, probabilmente nel 1233, chiamò Rabban Ata a curare gli affari religiosi nella regione. "Quando arrivò Rabban molte cose divennero propizie per i Cristiani, e le uccisioni e le riduzioni in schiavitù cessarono». ${ }^{64}$ Furono erette nuove chiese e restaurate alcune fra quelle distrutte durante le guerre. I cristiani, sempre secondo Kiriakos, potevano adesso professare apertamente la loro fede anche a Tabriz e Nxchawan, cioè in quelle regioni dell'Iran e dell'Azerbaijan a forte maggioranza musulmana. Poterono ricomparire le sepolture con la croce ben visibile (non si ha notizia nelle fonti che tale pratica fosse stata interdetta dai Mongoli, ma evidentemente ciò era accaduto); i mercanti poterono ottenere il tamgha, ovvero una sorta di salvacondotto che i funzionari locali erano autorizzati a fornire affinché si potesse circolare senza essere molestati. Rabban Ata volle vedere tutti i capi della cristianità nella regione. ${ }^{65}$

Nei primi anni Quaranta, dopo che i Mongoli ebbero conquistato gran parte della Caucasia, Georgia e territori armeni compresi, il problema religioso per loro divenne certamente secondario. L'aristocrazia locale, come abbiamo visto nel capitolo precedente, dovette garantire all'esercito mongolo truppe, mezzi e gli stessi nobili dovettero partecipare in prima persona alle campagne militari mongole nella regione. Quando Baiju, sostituto di Čormaghan, iniziò le operazioni contro il sultanato selgiuchide di Iconio i maggiori dignitari armeni e georgiani presero parte alle spedizioni contro Karin, i distretti di Erzindjan, Cesarea, Sebastia, Tiwrike e altri ancora. In cambio del servizio militare i nobili ottenevano una certa libertà d'azione nei distretti che erano loro appartenuti prima dell'arrivo dei Mongoli e che adesso potevano amministrare solo in quanto vassalli di questi. La popolazione cristiana, in questo periodo, non ebbe pertanto a subire ritorsioni per via della propria confessione. Gli anni in cui Rabban Ata aveva sovrinteso gli affari religiosi della Caucasia erano serviti a stabilizzare la situazione, anche se con un equilibrio assai precario. Nelle pagine in cui parla della guerra fra Mongoli e Iconio Kiriakos dice che le truppe guidate dai nobili armeni e georgiani e composte in gran parte da leve prelevate da quelle aree, cercarono sempre di salvare la popolazione cristiana dei distretti che attaccavano. Così vediamo che dopo

${ }^{64}$ Ibidem, p. 238.

65 Narsete, Catholicos di Aghbania, fu richiamato più volte da Rabban Ata perché non era andato di sua volontà a visitare il funzionario mongolo. Narsete fu invitato anche dal principe armeno Awag il quale insistette affinché il prelato andasse a rendere omaggio a Rabban e alla moglie di Čormaghan. Fu ciò che fece. Si recò nella piana di Mughan dove rese omaggio a Elt'ina Khatun e fu ricompensato con un tamgha che lo rendeva praticamente intoccabile entro il territorio conquistato dai Mongoli e con l'autorizzazione a praticare il suo credo nel suo monastero a Xamshi (Ibidem, pp. 252-254). 
avere con successo assediato Karin i soldati cristiani acquistarono Vangeli dai Mongoli che li avevano rubati per distribuirli poi alla popolazione che non ne aveva. ${ }^{66}$

Dalla prima incursione mongola nella Caucasia fino alla morte di Hülegü, il generale atteggiamento nei confronti dei cristiani fu favorevole. Il successore di Čormaghan, Baiju, procedette a una parziale restaurazione della situazione precedente la guerra concedendo terre e distretti ai nobili locali, in gran parte religiosi. Così nel 1245 il generale mongolo concesse Khilat a T’amt'a (armeno) e ordinò la ricostruzione della città di Karin, nella quale autorizzò l'ingresso di un vescovo cristiano. ${ }^{67}$ In questi anni i catholicos armeni potevano circolare liberamente e quasi tutti i vescovati armeni furono ricostruiti: Karin, Ani, Kars, Bjni, Anberd, Haghbat; così come molti monasteri che erano rimasti chiusi a lungo per via della guerra. Tra questi Sanahin, Getik, Haghartsin, Kerch'arhun, Hawuts', T'arh, Ayrivank'. ${ }^{68}$

Nel 1246 fu eletto khan Güyük, incline per formazione al nestorianesimo. I ministri che scelse, il suo cancelliere, il suo precettore, erano tutti nestoriani. ${ }^{69}$ Come ministro per gli affari religiosi scelse proprio Rabban Ata. Su questo aspetto è interessante quel che dice Juvaini:

Poiché [Qadaq, ovvero il servo che allevò Güyük sin da quando era bambino] era di religione cristiana, anche Güyük fu allevato in quella fede [...] egli pertanto non lesinò onori ai Cristiani e ai loro preti, e quando questo si seppe in giro per il mondo, diversi preti si diressero alla sua Corte da Damasco, da Rum e da Baghdad, e dalle terre degli As [gli Alani] e dei Russi; e al suo servizio c'erano per lo più medici crisitani [...]. La causa dei cristiani trionfo, quindi, durante il suo regno, e nessun Musulmano osava alzare la voce contro di loro. ${ }^{70}$

Nel 1247 il catholicos armeno Kostantin si adoperò per la costruzione di una chiesa «nelle regioni orientali»; il governatore militare della regione di Kayean, Anagurak noyon, autorizzò la costruzione della chiesa e, secondo Kiriakos, «allargò le strade su tutti i lati in modo che i pellegrini potessero passare fra le truppe senza paura $[\ldots] »{ }^{71}$

${ }^{66} \mathrm{KG}$, p. 241; in occasione della presa di Tiwrike, nel 1243, «i Cristiani nelle truppe [assedianti] liberarono molta gente dalla schiavitù, sia apertamente che in segreto, preti e chierici. Ciò fu vero soprattutto per i grandi principi Awag, Shahnshah, Vahram e suo figlio Aghbugha [...] e altri principi e le loro truppe [che liberarono tanta gente] quanta potevano», (KG, p. 246).

67 Ibidem, p. 255.

${ }^{68}$ Ibidem, pp. 256-259.

${ }^{69}$ Grousset, L'empire des steppes cit., p. 336; P. Pelliot, Chrétiens d'Asie Centrale et d'ExtremeOrient, «T'oung pao», XV (1914), pp. 623-644, p. 628; Id., Les Mongols et la Papauté cit., p. 247.

70 Juvaini, I, p. 292.

71 KG, pp. 260-261. 
Nei distretti in cui operavano i funzionari fiscali mongoli, dopo che Güyük ebbe mandato Arghun in Caucasia, la situazione divenne più tesa anche a causa dell'uso frequente fatto dai Mongoli stessi di elementi locali che potessero muoversi senza difficoltà entro i difficili territori armeno-georgiani. Così uno dei funzionari di Arghun, un certo Bugha (di cui abbiamo già fatto menzione), si servì ampiamente di «briganti fra gli iraniani e i Tachiki » i quali perpetrarono «violenze e crudeltà ed erano nemici soprattutto dei Cristiani». ${ }^{72}$ Nel periodo che coincise con la riorganizzazione del sistema fiscale furono in molti i nobili che cercarono di piegare la situazione a loro vantaggio. I Mongoli non avevano possibilità di operare un controllo capillare sul territorio pertanto vi furono spesso episodi di ribellione e resistenza da parte della popolazione dei villaggi che non accettava le vessazioni dei «barbari» e degli «empi». ${ }^{73}$

In questi anni sembra che i funzionari locali mongoli tollerassero le manifestazioni di culto. Quando, nel marzo 1249, morì il vardapet Vanakan il lutto nella comunità armena di Xoranashat fu profondo. Alle esequie parteciparono tutti i più alti prelati della zona: Sargis, il vescovo della diocesi dove sorgeva il monastero di Xoranashat, Narses, catholicos di Aghbania, Giovanni, vescovo di Garni e molti altri fra «vardapet e preti». ${ }^{74}$ Sembra cioè che in questo periodo i Mongoli non si intromettessero nel dualismo istituzionale fra temporale e spirituale che caratterizzava l'organizzazione del potere in Armenia (nell'Armenia storica come in Cilicia).

Anche a Nord la situazione per i cristiani fu favorevole in questa fase. Batu era sostanzialmente un mongolo fortemente legato al culto tradizionale, ma il figlio Sartach era stato allevato e cresciuto da tutori cristiani siriani, era quindi un nestoriano e piuttosto devoto. Ebbe un atteggiamento favorevole verso il cristianesimo, ma secondo Kiriakos protesse anche i musulmani nell'area in cui governava. ${ }^{75} \mathrm{Nel} 1249$ Hasan, principe di Kachezia, andò a rendere omaggio a Sartach il quale lo condusse da Batu. Questi gli riservò una buona accoglienza e lo congedò con ingenti concessioni (i distretti di Ch'araberd, Akanay e Karkarhn, che «i Turchi e i Georgiani gli avevano precedentemente strappato»). ${ }^{76}$ In questa occasione egli ottenne anche l'esenzione di Narses dalle tasse e la concessione al catholicos della piena autorità nella sua diocesi.

Durante le operazioni di censo portate avanti da Möngke per mezzo dell'ostikan Arghun il clero rimase escluso da ogni forma di tassazione. ${ }^{77}$ Anche quando il Gran khan ricevette il re di Cilicia Hethum gli fece importanti con-

72 Ibidem, p. 261.

73 Di particolare rilievo le ribellioni avvenute nelle aree di Ded e Tsiranak'ar.

${ }^{74} \mathrm{KG}$, pp. 280-285.

75 Ibidem, pp. 294-296.

76 Ibidem, p. 295.

77 Tale ordine fu confermato anche al principe di Kachezia Jalal, quando questi si recò da Batu. 
cessioni, fra le quali «un documento che proclamava la libertà per la Chiesa [cristiana] dovunque». ${ }^{78}$ Tale ordine fu ratificato da Batu, che ancora nel 1255 era evidentemente la massima autorità mongola a Ovest.

Come abbiamo visto in precedenza le scelte operate da Ghazan provocarono un drastico mutamento sia politico sia culturale in tutto l'Ilkhanato. Tali conseguenze si fecero sentire anche nella Caucasia. La conversione all'Islam da parte del nuovo sovrano impose a quest'ultimo di rivolgersi a funzionari in maggioranza musulmani, e ciò fu necessario per almeno due ragioni: $\mathrm{i}$ funzionari mongoli dislocati sul territorio erano talvolta influenzati culturalmente dalle comunità alle quali sovrintendevano; più spesso erano mongoli tradizionali, legati allo sciamanesimo e fortemente avversi a ogni forma di culto imposto, specialmente all'Islam e al Cristianesimo. Ghazan dovette limitare il loro potere. In secondo luogo il nuovo khan doveva rimettere in ordine le finanze che nei decenni intercorsi fra la conquista della regione e la sua elezione erano precipitate verso il disastro più totale. Dunque era necessario affidarsi a personaggi colti, preparati e buoni conoscitori della realtà indigena. Non è casuale che gran parte delle riforme di Ghazan siano state portate avanti da Rashid ad-Din, fonte storica preziosissima e abile uomo di potere (si arricchì moltissimo in questi anni). ${ }^{79}$

Durante la dominazione mongola in Medio Oriente e nella Caucasia la geografia religiosa dell'Islam subì una serie di mutamenti negli equilibri fra confessioni, ma non veri e propri stravolgimenti. In primo luogo si verificò un forte aumento della presenza buddista, di provenienza indiana e grazie anche all'inclinazione che l'Ilkhan Arghun aveva verso questo culto. Un secondo aspetto della questione è costituito dalla buona diffusione del cristianesimo nestoriano - come ben compare dalle pagine di Rubruck e Giovanni di Pian del Carpine - e soprattutto fra le donne dei ceti eminenti, spinte in questa direzione dalla scelta operata da quelle della famiglia reale: la madre di Möngke, parecchie delle sue mogli e la moglie di Hülegü divennero nestoriane. ${ }^{80}$ In ultima analisi credo vada posto in luce il ruolo dell'ebraismo nell'Ilkhanato; la religione giudaica non era particolarmente avversata dai Mongoli. Anzi, essa si rafforzò spesso nella misura in cui si verificavano successi individuali di personaggi eminenti a corte. Sa'd al-Dula, ministro di Arghun, era ebreo e fu sempre un nemico dell'Islam; durante gli anni in cui questi ebbe potere gli ebrei ricevettero un trattamento di favore. La componente nettamente maggioritaria della popolazione, quella musulmana, non resistette a lungo alle malversazioni dell'emiro di Arghun,

78 KG, p. 304.

79 Rashid possedeva, all'inizio del XIV secolo, un intero quartiere a Tabriz che gli fu confiscato quando fu giustiziato nel 1318. Naiman.

80 Ibidem, p. 541. La moglie di Hülegü lo era sin da bambina in quanto della tribù dei 
tanto che nel 1291 quest' ultimo fu linciato dalla folla. Lo stesso Rashid ad-Din era ebreo di nascita.

\subsection{L'Orda d'Oro, l'Azov e una riconsiderazione delle influenze}

Una delle differenze di maggior rilievo fra la dominazione mongola nella Caucasia e quella che si verificò nel Qipčak fu l'impossibilità, per quest'ultimo, di vedere una reale integrazione fra dominatori e dominati. A differenza di quanto accadde nell'Ilkhanato i Mongoli dell'Orda d'Oro non si fusero mai con la popolazione locale; non vi fu mai vera assimilazione, anche se da questo punto di vista credo vadano ancora sottolineati lo squilibrio demografico fra Mongoli e popolazione locale a tutto vantaggio della seconda e soprattutto il carattere dei rapporti che si crearono fra potere centrale e istituzioni preesistenti. Queste, come abbiamo visto, riuscirono a mantenere una certa autonomia rispetto al khan dell'Orda. Quando, sul finire del XIII secolo, l'Orda d'Oro scelse l'Islam esso fu un fatto puramente formale e non influenzò la politica. A questo va aggiunto che la presenza dei Cumani - la maggioranza da un punto di vista quantitativo nelle steppe conquistate dai Mongoli e pagani - non favoriva un processo di acculturazione coi popoli russi la cui identità religiosa era molto forte.

Le fonti russe hanno dipinto Batu come nemico della cristianità $;{ }^{81}$ mentre l'impressione che ne trassero i religiosi occidentali è più sfumata. ${ }^{82}$ In effetti la conquista a Ovest coincise con l'attacco e la distruzione di Paesi dove il cristianesimo era religione di stato: Rus', Polonia, Ungheria, Moravia. Batu era un mongolo legato alle proprie tradizioni, non era cristiano né musulmano. Come ha rilevato a suo tempo René Grousset i rapporti religiosi all'interno del vasto territorio del Qipčak avrebbero potuto prendere una diversa direzione se a Batu fosse successo il figlio Sartach. Questi era nestoriano, ed evidentemente era anche un convinto sostenitore della fede cristiana. ${ }^{83}$ Rubruck dice che alla

81 «[Nell'anno 1237] sul fiume Voronež fu ucciso dall'imperatore senzadio Batu il pio principe Fedor Jur'evič di Rjazan»: Pianto sulla distruzione di Rjazan cit., p. 69; «[Nell'anno vigesimo secondo della traslazione da Cherson della miracolosa immagine di Nicola] giunse in terra russa l'empio Batu imperatore»: Povest' o razorenii Rjazani Batyem, in Pianto sulla distruzione di Rjazan' cit., p. 75. Nelle cantiche sulla distruzione di Rjazan' Batu è sempre dipinto come «l'empio» o «'indegno»: pp. 77, 81, 83, 85, 91, 109, 135; «Batu imperatore il maledetto», Povest' cit., p. 103 o a p. 111: «Batu infedele imperatore»; «Dio con la sua potente mano aveva salvato dall'infedele imperatore Batu [i cristiani]»: Pochvala rody Rjazanskich Knjazej, in Pianto sulla Distruzione di Rjazan' cit., p. 159; «svernarono i tatari maledetti», Tverskij Letopis', in Pianto sulla distruzione di Rjazan' cit., p. 184; «Batu, da vera bestia feroce, non risparmiò la sua giovinezza [di Vsevolod, figlio di Jurij di Rjazan'], Galicko-Volynskaja Letopis', in Pianto sulla distruzione di Rjazan' cit., p. 197.

${ }^{82}$ Per ovvie ragioni, sebbene anche Giovanni di Pian del Carpine, Rubruck o Benedetto Polono abbiano percepito Batu come avverso alla loro fede.

${ }^{83}$ Grousset, L'empire des steppes cit., p. 474 ; Guillelmus de Rubruc, Itinerarium cit., XV, pp. 59-64; KG, pp. 292-297. 
corte di Sartach c'erano «molti Tartari, sia cristiani, sia saraceni a cavallo»; il sovrano non esigette dal francescano la tripla genuflessione che era obbligo presso i Mongoli. ${ }^{84}$ Secondo Kirakos egli «credeva in Cristo [...] garantì molte libertà alla Chiesa e ai Cristiani». ${ }^{85}$ Sartach decretò che nessuno doveva richiedere tasse dalla chiesa "e dai suoi servitori», indipendentemente dalla nazionalità; e lo stesso fece coi musulmani.

In ogni caso alla morte di Batu (1255) salì sul trono dell'Orda d'Oro Berke; fu una scelta obbligata in quanto Sartach era morto poco prima durante il viaggio di ritorno dalla sede del Gran khanato dove si era recato per rendere omaggio a Möngke. Berke simpatizzò decisamente per l'Islam. Il suo regno fu contrassegnato da buoni successi in politica estera e militare, ma anche da durissime sconfitte. Dovette difendersi dall'attacco di Alghan, khan dei Čagataidi che conquistò Utrar. ${ }^{86}$ Riuscì a respingere l'avanzata del rivale mongolo, ma la perdita di Utrar fu un colpo assai duro al commercio dell'Orda d'Oro con l'Oriente. La maggior parte dei mercanti che si recavano in Cina passava da questo centro della Transoxiana. ${ }^{87}$

I successi maggiori Berke li ottenne contro l'Ilkhanato. Pur di prevalere su Hülegü, il khan dell'Orda si alleò con l'Egitto mamelucco, agitando anche il vessillo dell'Islam contro il responsabile della distruzione di Baghdad ${ }^{88}$ Lo scontro fra Hülegü e Berke si inasprì al punto che entrambi si rivalsero sui mercanti dell'uno sul territorio dell'altro; così Hülegü fece uccidere tutti i mercanti di provenienza qipčaka che si trovavano in territorio ilkhanide e lo stesso fece Berke coi mercanti caucasici e persiani. ${ }^{89}$ Fino al 1266 le due potenze si fronteggiarono a forza di incursioni, rotture del confine nei pressi di Derbend, senza che nessuna riuscisse a prevalere sull'altra. ${ }^{90}$ Gli scontri che si protrassero per anni fra Orda d'Oro e Ilkhanato ebbero il duplice effetto di indebolire militarmente

${ }^{84}$ Guillelmus de Rubruc, Itinerarium cit., XV, pp. 61-62.

${ }^{85}$ KG, p. 295.

${ }^{86}$ Grousset, L'empire des steppes cit., pp. 474-475; L.V. Čerepnin, Obrazovanie Russkogo Centralizovannogo Gosudarstva v XIV-XV vekach, Moskva, 1960, cap. V, pp. 675-683 .

${ }_{87}$ Grousset, L'empire des steppes cit., p. 475; T.T. Allsen, Commodity and exchange in the Mongol Empire: a cultural history of islamic textiles, Cambridge 1997, in particolare il cap. I.

${ }_{88} \mathrm{Su}$ questi eventi si veda soprattutto G. Lane, Early mongol Rule in 13th centry Iran: a Persian Renaissance, New York (NY) 2003, in particolare il cap. 5, pp. 96-176. Interessante la lettura fornita a riguardo da Golden, Imperial Ideology cit., p. 71 secondo il quale l'alleanza fra Orda d'Oro e Ilkhanato sarebbe stata agevolata dal comune sostrato etnico delle due realtà politiche. È vero infatti che nell'Egitto mamelucco vi era una numerosa comunità di Qipciaki che mantenne a lungo la propria identità etnica. Cfr. anche S. Zakirov, Diplomatičeskie otnošenie Zolotoj Ordy s Egiptom, Moskva 1966, in particolare le pp. 35 e segg.; Amitai-Preiss, Mongols and Mamluks cit., pp. 78-79.

89 Wassaf, Histoire cit., III, p. 381.

${ }_{90}$ Bratianu, Recherches sur le commerce cit., p. 259; Amitai-Preiss, Mongols and Mamluks cit., pp. 78-89. 
entrambi e di rafforzare contemporaneamente il mondo islamico tradizionale, con l'Egitto dei Mamelucchi in primo luogo. Da questa situazione il debole legame col territorio che caratterizzava la dominazione mongola nell'Orda d'Oro contribuì a indirizzarne la politica. Si pensi ad esempio al soccorso prestato prima ai Bulgari balcanici contro Michele Paleologo (che fuggì su una nave genovese nei pressi della foce del Danubio). ${ }^{91} \mathrm{Nel} 1281$ Tuda, successore di Berke, firmò un trattato di alleanza con Bisanzio e i Selgiuchidi contro Venezia, l'Ilkhanato e Carlo d'Angiò. ${ }^{92}$

Dopo la reggenza di Berke le condizioni dei cristiani nei territori dell'Orda d'Oro migliorarono decisamente. Timur, il successore, rafforzò i privilegi concessi ai religiosi cristiani (Greci ortodossi soprattutto). A Saraj, la capitale dell'Orda sul Volga, c'era un vescovo ortodosso prima del $1280 . .^{93}$

Durante la reggenza di Nogaï - vero artefice della politica mongola nell'Orda d'Oro durante l'ultimo decennio del XIII secolo - si mantennero sostanzialmente intatti i privilegi ottenuti dai cristiani. La tolleranza mostrata dal khan fu probabilmente dettata dalla consapevolezza dell'importanza economica rappresentata dai cristiani. Ė emblematico infatti che, nonostante l'islamizzazione, l'Orda d'Oro abbia mantenuto un atteggiamento generalmente favorevole nei confronti della religione cristiana proprio negli anni in cui si stavano consolidando le colonie commerciali genovesi e veneziane sul Mar Nero.

Nonostante la dominazione mongola si fosse realizzata nell'Orda d'Oro con la conquista violenta dei principati russi questi - lo abbiamo visto nel capitolo precedente - ebbero coi nuovi dominatori dei rapporti indiretti sotto tutti i punti vista. Dopo la partenza dei governatori locali, basqaq e daruga, l'aristocrazia locale poté regnare con una certa autonomia. Ciò avvenne perché ai Mongoli non interessava affatto dirigere la politica di un'area che ritenevano assolutamente marginale. Il cuore dell'Orda era nella steppa e quella era la regione che a loro premeva dominare. ${ }^{94}$

Se l'aspetto religioso ebbe importanti conseguenze sulla condotta politica dell'Ilkhanato, ciò non accadde nell'Orda d'Oro. In Cina, ad esempio, la reli-

${ }_{91}$ Bratianu, Recherches sur le commerce cit., pp. 206-208.

92 Il trattato ci è pervenuto in una copia lasciataci da al-Qalqašandi. Si veda M. Canard, Le traité de 1281 entre Michel Paléologue et le sultan Qalâun, in "Byzantion», X (1935), pp. 669680; F. Thiriet, La Romanie venitienne au moyen age: le developpement et l'exploitation du domaine colonial venitien (12.- 15. siecles), Paris 1959, p. 151; Balard, La Romanie génoise cit., pp. 53-55; Bratianu, Recherches sur le commerce cit., pp. 141-142; Grousset, L'empire des steppes cit., p. 477; R. Grousset, Storia delle crociate, 2 voll. Casale Monferrato 1998, pp. 613-625.

${ }_{93}$ Grousset, L'empire des steppes cit., p. 479; Bratianu, Recherches sur le commerce cit., p. 259, nota 1. Secondo George Vernardsky, in «Seminarium Kondakovianum», I (1927), pp. 78-80, gli annali russi parlano del vescovo ortodosso di Saraj il quale nel 1279 sarebbe giunto da Bisanzio per la terza volta nella capitale sul Volga.

${ }^{4}$ C. Halperin, Russia in the Mongol Empire in Comparative Perspective, in "Harvard Journal of Asiatic Studies», 43 (1983), pp. 239-261, p. 250. 
gione fu un fattore di divisione. La dinastia mongola degli Yüan, propendeva decisamente verso il confucianesimo, in parte verso buddismo e taoismo. ${ }^{95}$ Essi si scontrarono però con il retroterra culturale solidissimo di cui la Cina era il risultato.

Nell'Orda tale situazione non si verificò mai. I Cumani vennero letteralmente assimilati entro la struttura sociale mongola che in questo caso non dovette adattarsi alle steppe dei Qipčaki; fu semmai il contrario. ${ }^{96}$ Per quanto riguarda i principati russi, la loro identità religiosa non costituì mai una minaccia per l'integrità dell'autorità mongola sul territorio. I Russi erano distanti geograficamente e culturalmente dall'Orda d'Oro e questo ne favorì la sostanziale autonomia anche per quanto riguarda l'aspetto confessionale. Le altre confessioni presenti sul territorio costituivano una minaccia ancora minore. I cristiani occidentali rappresentati dai mercanti avevano altre esigenze, il loro collante sociale era di tipo economico e questo i khan lo sapevano bene. Anche l'Islam, tradizionalmente estraneo a questa regione, non era forte al punto da dover essere controllato dall'autorità mongola. In conclusione credo si possa affermare, con Charles Halperin, che durante la dominazione mongola nell'Orda d'Oro le istituzioni religiose aumentarono di numero e influenza sulla popolazione. Ciò fu il risultato di una effettiva tolleranza che in questa regione si verificò assai più che nell'Ilkhanato, in Cina o nella Transoxiana, ${ }^{97}$ almeno sull'area russa. Tolleranza che fu il frutto di almeno due fattori: il primo è quella caratteristica di indiretta dominazione che i Mongoli applicarono sui principati russi ${ }^{98}$ il secondo è rappresentato dalla solida barriera religiosa costituita dal cristianesimo russo, molto radicato ed elemento identitario collettivo fondamentale. I Mongoli furono influenzati molto più dall'elemento turco cumano che non da quello slavo russo.

${ }_{95}$ Halperin, Russia in the Mongol Empire cit., p. 254; I. de Rachewiltz, Personnel and Pesonalities in North China in the Early Mongol Period, in "Journal of the Economic and Social History of the Orient», 9 (1966), pp. 88-144.

${ }^{96}$ G.A. Fedorov-Davydov, Kočevniki Vostočniki Evropy pod vlastju zolotoordynskich chanov: Archeologičeskie pamiatniki, Moskva 1966; Id., Obščstvennyj stroj cit.

${ }_{97}$ Halperin, Russia in the Mongol Empire cit., pp. 255-256; M.D. Priselkov, Chanskie jarliki russkim mitropolitam, Petrograd 1916. Su questo è molto interessante l'itinerario di Afanasij Nikitin, mercante russo di Tver' che nel 1466 si recò in India visitando popolazioni turche, persiane, arabe riportato nel suo Chozenie za tri morja, ed. it. a cura di E.T. Saronne, Roma 2003.

${ }_{98}$ Gli storici che hanno parlato di dominazione ininfluente, da parte dei Mongoli sulla Russia, tranne che per le distruzioni da essi causate hanno spesso trascurato l'ottica comparativa con le altre dominazioni mongole in Caucasia e in Cina. Credo abbia ragione Charles Halperin quando afferma che sarebbe più corretto parlare non tanto di influenza mongola sulla Russia, sull'Iran o sulla Cina quanto del contrario. L'Orda d'Oro poi presenta questa ambivalenza del tutto peculiare. Le steppe, predilette dai Mongoli rispetto alla foresta russa, hanno avuto una forte influenza sui Mongoli e la loro turchizzazione è un fatto piuttosto evidente; altrettanto acquisito è il fatto che tale processo fu lento e complesso e si protrasse per tutto il periodo in cui i Mongoli dominarono nella steppa. 
La dominazione mongola non ebbe grande influenza sulla cultura russa. In realtà non ne ebbe molta su nessuna delle culture dominate. Questo non deve indurre nell'errore portato avanti per decenni da parte di certa storiografia critica per cui l'inferiorità culturale dei Mongoli avrebbe impedito loro di influire in qualche modo sulle civiltà conquistate. Da un punto di vista politico e istituzionale l'eredità mongola lasciò tracce durature sia nell'Ilkhanato sia, soprattutto, nella Rus'. Nell'Ilkhanato poi i khan promossero l'attività culturale grazie a una sensibilità che era in gran parte il frutto della sintonia confessionale. Tale aspetto rimase marginale in Russia e nelle steppe dove, pur non ostacolando mai l'arte e la cultura slave, i Mongoli non riuscirono nemmeno a stimolarle. La fioritura artistica che si verificò fra XIV e XV secolo nella zona nord-orientale della Russia fu un fenomeno endogeno, promosso e portato avanti da elementi slavi locali ai quali i "barbari invasori" non posero alcun ostacolo, né potevano porlo. 


\section{POPOLAZIONE E CONVIVENZA. L'ASPETTO DEMOGRAFICO FRA CONQUISTA E RICOSTRUZIONE}

\subsection{Alcune considerazioni sulle conseguenze demografiche delle conquiste}

L'analisi dell'andamento demografico sulla popolazione della fascia territoriale che comprende la Caucasia fino al Mar d'Azov presenta molteplici aspetti d'interesse, ma anche elementi problematici che cercheremo di chiarire per quanto possibile. Ci siamo già in parte soffermati sull'evoluzione politica della regione e sui risultati scaturiti dall'incontro di realtà diverse, nomadismo e sedentarismo, cultura urbana e costante movimento di genti, guerra e ricostruzione. Tutti questi eventi hanno generato - e sono stati in parte il risultato - di una straordinaria complessità etnica alla quale abbiamo più volte, nel corso di queste pagine, accennato. Adesso si tratta di vedere nel dettaglio quali conseguenze il processo storico in oggetto ha portato sulla popolazione della Caucasia e dell'Azov, quali erano le proporzioni etniche distribuite su un territorio molto vasto, ma che egualmente ha visto una singolare differenziazione. Si tratta di capire quali equilibri si ruppero in seguito ai molteplici traumi sofferti da questa regione e quali invece furono le alleanze, le solidarietà fra le diverse etnie.

Alcuni aspetti peculiari dell'andamento demografico europeo di epoca preindustriale - e in particolare dei secoli altomedievali - caratterizzarono anche il territorio di nostro interesse. L'elevata mortalità (soprattutto infantile) veniva aggravata dalle crisi periodiche causate da eventi traumatici tra i quali guerre, carestie e le molte epidemie che di questi ultimi erano una diretta conseguenza. A ciò possiamo aggiungere le condizioni ambientali particolarmente ostili le quali erano insieme causa ed effetto del calo demografico. L'abbandono di terreni, specialmente nelle aree a forte vocazione agricola, ne causava il depauperamento. Era in parte diversa la condizione delle terre del Qipčiak dove l'allevamento era il fattore produttivo fondamentale. La transumanza e il nomadismo tempravano la popolazione aumentandone la resistenza alle difficoltà ambientali.

Nella Caucasia l'intensa successione delle incursioni nomadi iniziate già sul finire dell'XI secolo fu alla base di una lentezza del processo di recupero demico a fronte di un rapido decremento delle risorse umane. Se nel Deśt i-Kipčiak il crollo avvenne soprattutto in seguito alla seconda incursione mongola (12361240), nella Caucasia questo fenomeno era già in una fase di compimento dalla metà del XII secolo.

Il rapporto fra popolazione urbana e aree rurali era diverso fra le due zone di cui ci stiamo occupando. Nelle steppe del Qipčiak vi era una scarsa quantità 
di poli d'attrazione urbana, concentrati prevalentemente sulle grandi arterie fluviali. Nella Rus' la situazione era diversa. Le città erano numerose, ma anche in questa regione il reticolo urbano aveva maglie piuttosto larghe. A loro volta il Caucaso e la Korazmia rappresentavano realtà con caratteristiche diverse. Le città, più o meno grandi, soffrirono l'invasione mongola; in particolare nella Korazmia città come Urghench, Samarcanda e Bukara vennero letteralmente distrutte e furono teatro di eccidi spaventosi. Per la Caucasia fu importante anche la frequente ridefinizione dei confini che ebbe a verificarsi in questo periodo. La destabilizzazione di cui soffrivano i centri di potere in seguito alle incursioni nomadi (si pensi soprattutto alla Georgia o ai califfati) si accompagnava a una mancanza di centri egemoni che potessero affermare la loro supremazia sulle aree rurali nel lungo periodo, che avrebbero portato cioè a una maggiore stabilità politica e a una maggiore sicurezza. Questa condizione verrà raggiunta solo qualche decennio dopo l'insediamento dei nuovi conquistatori e in seguito alla riorganizzazione del potere in tutte le aree soggiogate.

In questo contesto va però segnalata la maggiore mobilità garantita a uomini e merci e favorita dalla costituzione di un nuovo impero unificato. La forte attrattiva rappresentata dalla diminuita incertezza delle vie di transito terrestri e dal conseguente incremento degli scambi originò un fenomeno immigratorio il cui effetto più evidente fu l'insediamento, e talvolta la costruzione ex novo, di comunità straniere molto attive e precocemente organizzate (genovese, veneziana, catalana). La presenza del mare per la Caucasia costituì un formidabile mezzo per la ripresa economica; la promozione degli scambi sostenuta dai rappresentanti del potere mongolo in loco trovò nell' acqua uno strumento efficace. Nell'Orda d'Oro ciò fu possibile sia col Mar Caspio nel quale sfocia l'arteria di comunicazione tradizionalmente più sviluppata della regione: il Volga, sia col Mar Nero (Don e Dnepr).

Non vi sono fonti specifiche attraverso le quali poter inquadrare con completezza il problema da un punto di vista demografico. Per farlo dovremo ricorrere a indizi indiretti. Da questo punto di vista la produzione narrativa risulta più accessibile in quanto permette di ottenere uno sguardo generale su un territorio di maggiori dimensioni, laddove la fonte documentaria ci presenta la realtà di cui essa fu espressione peculiare (il caso del notaio è emblematico). Si è dovuta pertanto operare una distinzione almeno geografica procedendo a partire da nord verso la Caucasia, specificamente alla foce del Don. Il periodo considerato è più lungo dei limiti imposti alla nostra ricerca per poter apprezzare al meglio fenomeni complessi e scarsamente sostenuti dalla documentazione.

\subsection{Nell'Orda d'Oro e nella regione dell'Azov (1237-1395)}

La prima incursione mongola nella Caucasia si verificò mentre gli stati di quest'area attraversavano una fase di declino. La scarsa resistenza opposta 
all'avanzata dell'esercito gengiskanide fu anche il frutto di questa situazione de facto. Ciò premesso credo sia fuorviante negare, o cercare di ridimensionare, l'evento e le disastrose conseguenze che esso produsse a livello demografico. La Rus' non aveva conosciuto niente di simile in precedenza; lo stesso si può dire per gran parte della Korazmia; in Caucasia la situazione era, come vedremo, in parte diversa. La storiografia russa del secolo scorso ha insistito molto sul caos politico che regnava nello stato slavo alla fine del XII secolo individuando in esso la causa principale della debole resistenza opposta all'avanzata mongola. ${ }^{1}$ Non si può negare che tale disarticolazione organizzativa fosse un dato di fatto, ma credo che essa vada collocata in una dimensione più adeguata e, magari, comparativa. Tutti i regni dell'epoca, quando furono attaccati, opposero una resistenza insufficiente ai Mongoli. Nessuno, durante le campagne del 1236-1240 riuscì a impedirne l'avanzata e ciò fu il risultato di un complesso di fattori dei quali ci siamo già ampiamente occupati. Insistere troppo sulla disorganizzazione dei principati russi, sulle frequenti lotte fratricide è, a mio avviso, limitante. Alla fine del XII secolo era in corso, soprattutto nelle zone settentrionali e sudoccidentali della Rus' un processo di generale ripresa economica, spinto dai successi commerciali di città come Novgorod' Velikij. Vero si è che gran parte della Crimea fu conquistata, proprio in questi anni, dai Polovcy. A ciò vanno aggiunte le minacce portate dall'Ungheria, dalla Polonia, dagli ordini di Livonia e Teutonico e dalla Lituania sulla Rus'. In estrema sintesi possiamo concordare con chi afferma che alla vigilia dell'invasione mongola la Rus' era fortemente indebolita da un punto di vista politico e militare, ma gioverà ripetere che una grande compattezza fra i principi russi non c'era mai stata e che l'organizzazione di uno stato feudale come la Rus' è ancora oggi oggetto di dibattito. Meglio limitarsi a osservare che la resistenza opposta dai Russi ai nuovi invasori non differì granché da quella opposta, pochi mesi dopo, dalla Polonia e dall'Ungheria. Di fatto la discrasia fra crescita economica, iniziata sul finire del XII secolo, e debolezza politica nella Rus' alla vigilia dell'invasione fu da questa interrotta e i massacri perpetrati ai danni dei villaggi nelle zone rurali e della popolazione assediata ebbero effetti pesanti a tutti i livelli. Nelle città della Korazmia non fu diverso. La produzione, gli scambi e le potenzialità umane della regione subirono un colpo durissimo. ${ }^{2}$ Gran parte delle attività artigianali furono ridimensionate

${ }^{1}$ Grekov, Jakubovskij, L'Orda d'Oro cit. in particolare il cap. I della II parte, pp. 145-163; V.V. Kargalov, Vnešnepolitičeskie faktory razvitija feodal'noj Rusi. Feodal'naja Rus' i kočevniki, Moskva 1967, soprattutto le pp. 219-255; N.S. Borisov, Otečestvennaja istoriografija o vlijanii tataro-mongol'skogo našestvija na russkuju kul'turu, in «Problemy istorii SSSR» V (1976), pp. 129146; Halperin, Russia and the Golden Horde cit. introduzione; Sverdlov, Domongol'skaja Rus' cit., in particolare il cap. I.

2 Sulla Rus' si possono vedere i lavori di Kargalov, Vnešnepolitičeskie faktory cit. pp. 173217, ma anche Halperin, Russia and the Golden Horede cit. pp. 75-76. Il tentativo più recente di tracciare un quadro completo della Rus' pre-mongolica è quello effettuato da Sverdlov nel suo saggio già citato (Domongol'skaja Rus'). 
drasticamente a causa non solo delle distruzioni generate dalla guerra, ma anche dalle sistematiche deportazioni che i Mongoli effettuavano per assicurarsi i servizi dei lavoratori specializzati nelle loro città in Oriente. ${ }^{3}$ La circolazione di forze produttive fu un fenomeno che assunse dimensioni notevoli nei primi anni di governo mongolo. Esso non fu una novità assoluta per l'Eurasia poiché rappresentava, in parte, la continuazione di una seppur modesta mobilità già attiva da secoli fra Vicino Oriente e Asia Orientale; tali rapporti erano di tipo prevalentemente commerciale e avevano come oggetto non solo tessuti (in primo luogo seta), ma anche bronzo e vetro. ${ }^{4}$

Non fu minore il danno causato nelle campagne. Gran parte dei villaggi russi erano situati nelle foreste settentrionali, dove la cavalleria mongola non penetrava, ma le zone di confine con le steppe furono duramente danneggiate dalle scorrerie dell'esercito di Batu. La produzione agricola ne uscì fortemente ridimensionata e in alcuni casi risulta difficile dar torto agli studiosi russi di epoca sovietica quando affermavano che l'invasione mongola non aveva semplicemente danneggiato l'economia produttiva della regione, ma l'aveva portata a un processo regressivo che ebbe lunga durata, ben oltre il crollo dell'Orda d'Oro. ${ }^{5}$ Questa considerazione non può tuttavia assumere un carattere generale. Gli effetti delle conquiste furono diversi per le diverse aree in cui esse si realizzarono. Se è vero che le città meridionali della Rus' furono le più colpite, ciò non è altrettanto vero per il Nord. Dobbiamo inoltre tener presente il complesso gioco di alleanze che si svolse fra Mongoli e principi russi durante gli anni della dominazione.

Il territorio dell'Orda d'Oro fu sfruttato dai Mongoli in modo consapevole e in base alle sue reali potenzialità. Le aree a forte vocazione commerciale furono sostenute da una politica organizzata e da accordi internazionali in un contesto di generale apertura verso l'esterno. Le aree in cui la produzione agricola era dominante, come Bolgar e parti della Crimea, vennero lasciate a questo modello produttivo che prevedeva anche un massiccio ricorso alla manodopera artigiana (lavorazione del cuoio, delle pellicce, ma anche una sviluppata coltivazione del grano).

Tale atteggiamento dette i suoi frutti duraturi solo a partire dall'inizio del XIV secolo. Il 1300 non fu un anno favorevole per l'Orda d'Oro: una forte siccità, seguita da una violenta epidemia che colpì equini e ovini, falcidiarono la popolazione dei distretti ed ebbero effetti pesanti anche nelle città. Scrive lo storico egiziano al-Makrizi (1364-1442) che questa epidemia fu «talmente

\footnotetext{
${ }^{3}$ Allsen, Exchange and commodity, cit; Id., Culture and conquest cit.

${ }^{4}$ Li Chih-Chang, Travels of an Alchemist, a cura di A. Waley, London 1963, pp. 93 e 124; Allsen, Commodity and exchange cit. p. 35.

5 Per un quadro bibliografico di riferimento si veda C. Halperin, Soviet Historiography on Russia and the Mongols, in "Russian Review», 41 (1982), 3, pp. 306-322.
} 
grave che essi [gli abitanti delle campagne] più nulla avevano da mangiare, e vendevano i figli e i congiunti ai mercanti, che portavano in Egitto e in altri luoghi». ${ }^{6}$ La ripresa ebbe inizio dalla fine del primo decennio del secolo. In questi anni si verificarono una evidente crescita delle forze produttive e il recupero di alcuni dei maggiori centri urbani. Anche la pressione esercitata dal potere centrale dell'Orda d'Oro sui principi russi andava diminuendo progressivamente.

Nel primo periodo (fino grosso modo alla prima metà del XIV secolo) lo stato maggiore mongolo trovò in Mosca un alleato fedele; conseguenza di questi accordi furono le frequenti incursioni punitive nei confronti delle città nemiche della futura capitale russa. Quando però questi accordi mutarono fu proprio Mosca a subire ripetuti attacchi da parte dei Mongoli.

Da un punto di vista demografico questo stato di cose ebbe effetti contraddittori. Premesso e ripetuto che le fonti a nostra disposizione sono comunque scarse per un'analisi completa della questione, possiamo osservare due fenomeni diversi. I danni prodotti alle campagne del territorio compreso fra Dnepr e Volga misero in ginocchio la capacità di approvvigionamento dei villaggi rurali della zona e ciò fu vero soprattutto per i primi due decenni della dominazione. Tutto questo ebbe luogo immediatamente dopo la tragedia bellica; il risultato fu una migrazione forzata di ampi strati della popolazione verso Nord dove le zone di Beloozero, Viatka, ma anche il bacino del Don e Jaroslavl', furono colpite in misura minore rispetto alle altre (anche Mosca e Tver' uscirono senza danni consistenti dagli effetti delle guerre). ${ }^{7}$ L'altro aspetto della questione è rappresentato dagli esiti economici che la politica mongola ebbe nello stesso contesto geografico. La condotta favorevole al commercio e l'incoraggiamento che i Mongoli fornivano ai mercanti attirò operatori commerciali dall'estero. L'insediamento in centri che fino a poco prima erano stati inaccessibili rivitalizzò l'economia delle steppe dove i sovrani mongoli furono entusiasti sostenitori della pratica mercantile. Senza negare in alcun modo l'effetto catastrofico che l'invasione mongola ebbe sulla Rus' dobbiamo però interrogarci sulla durata di tali conseguenze. A mio avviso l'impatto subito dalla struttura demografica e dall'organizzazione economica della regione fu assorbito nei decenni successivi quando, pur non raggiungendo i livelli demici precedenti, si assistette a una crescita progressiva che rallentò con la crisi di metà Trecento: un destino comune del resto con tutto il mondo allora conosciuto, per poi subire il colpo di grazia dall'invasione timuride degli anni Novanta.

${ }^{6}$ Grekov-Jakubovskij, L'Orda d'Oro cit., pp. 124-125. Questa situazione è confermata dagli atti notarili veneziani del XIV secolo rogati alla Tana e per i quali cfr. nota 12 cap. sesto.

${ }^{7}$ Mosca in particolare beneficerà dell'afflusso massiccio di genti. Il principe della città fu abile a sfruttare una situazione politica complessa, assecondando le richieste mongole quando non avrebbe potuto opporsi alla loro volontà per poi ribellarvisi quando ciò fu possibile. 


\subsection{Nella Caucasia}

Diversa [dalla Transoxiana] è la situazione del Khorasan e dell'Iraq, paesi che sono afflitti da una febbre etica e da una malattia cronica: ogni città ed ogni villaggio è stato ripetutamente sottoposto al saccheggio e al massacro, ed ha sopportato per anni una tale confusione, che [...] la popolazione non raggiungerà la decima parte di quella che era prima ${ }^{8}$

con queste parole Juvaini, negli anni Sessanta del XIII secolo, descrive la desolazione che seguì la conquista mongola di quello che divenne l'Ilkhanato. Esso, a differenza dell'Orda d'Oro, coincise politicamente con l'organizzazione istituzionale che si trovò a sostituire, laddove i principi della Rus' rimasero al loro posto come intermediari fra il governo mongolo e la popolazione locale. La politica volta alla ricostruzione e allo stimolo verso i commerci fu simile a quella adottata nell'Ulus Giuči, ma qua i Mongoli si trovarono di fronte a un'economia strutturale saldamente organizzata sul commercio, sui mercati stabili e sugli scambi internazionali.

A cavallo fra XII e XIII secolo la popolazione della Caucasia, spinta dai successi del regno di Georgia guidato dalla regina Tamara, era considerevole per numero e con ogni probabilità la densità era elevata nelle zone pianeggianti. I primi anni della dominazione mongola furono contrassegnati da una profonda crisi economica e demografica, conseguenze dirette della conquista. ${ }^{9}$ La lunga durata degli eventi bellici e l'estensione del territorio sul quale si verificarono resero questa fase di decadenza molto lunga (fino almeno agli anni Novanta del XIII secolo). Il mutamento istituzionale che si verificò con l'avvento degli Ilqani (1256) non ebbe effetti immediati. Di fatto in questi decenni la popolazione si ridusse notevolmente, le condizioni delle campagne peggiorarono in modo sensibile e la produzione agricola diminuì a vantaggio degli spazi aperti destinati al pascolo che tanto piacevano alle tribù turco-mongole giunte con gli eserciti (e che di quegli eserciti erano parte integrante). Il declino, che Petruševskij ha definito colossale, ${ }^{10}$ investì anche le città, dove il crollo demografico - soprattutto fra la popolazione maschile - e le crescenti difficoltà di approvvigionamento rendevano la vita durissima. A ciò bisogna aggiungere ancora la forte tassazione imposta dai Mongoli nei primi decenni della dominazione i cui effetti si fecero sentire soprattutto sulle zone rurali che si vedevano depredare delle poche risorse rimaste.

8 Juvaini, I, p. 119.

9 Le fonti descrivono gran parte delle sofferenze cui furono sottoposte le città della Caucasia. Ad esempio durante la presa di Erzrum da parte dei Mongoli, avvenuta nel 1242, gran parte della popolazione venne uccisa e molti dei superstiti ridotti in schiavitù o deportati. Si veda su questo Bar Hebraeus, The Cronography cit. p. 406; KG, pp. 279-280; GA, pp. 7-8; Bedrosian, The Turco Mongol cit. pp. 113-114.

${ }_{10}$ Petruševskij, The socio-economic condition cit. p. 483. 
L'organizzazione fiscale promossa da Möngke in tutto l'impero e realizzata dall'emiro Argun in Caucasia nel 1254 non ha lasciato traccia diretta nella documentazione. Alcune stime avanzate da Bedrosian e da altri armenisti porterebbero a credere che la popolazione armena della Caucasia (esclusa quindi la Cilicia) non superasse in quegli anni i 4.000.000 di unità. ${ }^{11}$ Non abbiamo dati precisi su questo, ma se consideriamo ciò che il Caucaso e tutta la regione circostante rappresentarono nel Medioevo, allora possiamo ammettere che non solo i principali centri urbani, ma anche i villaggi rurali avessero un elevato numero di abitanti. Città come Barda, Baylakan, Derbend, Shamakha, Ganja, Dabil, rappresentavano mercati importanti per tutto l'Islam. Senza operare forzature possiamo stimare la popolazione della sola Georgia in circa $4.500 .000 .^{12}$

Prima dell'ulteriore mutamento intervenuto con la nascita dell'Ilqanato la Caucasia costituiva un'unità amministrativa autonoma, posta sotto la diretta autorità del noyon che aveva condotto le operazioni militari nella regione (Čormaghan e Baiju). Essa era composta da cinque distretti (vilayet). Di questi cinque, due erano composti da popolazione in maggioranza armena, gli altri erano popolati da Georgiani, Azerbaijani ed etnie diverse prevalentemente di rito cristiano. Nel vilayet di Georgia, o Guristan, predominava l'elemento armeno. Ogni vilayet doveva essere in grado di fornire un determinato numero di tümen. Ad esempio il vilayet della Grande Armenia era composto da otto tümen; esso era quindi in grado di fornire almeno 80.000 uomini per l'esercito qualora ve ne fosse stato bisogno.

L'istituzione dell'Ilqanato e l'obbligo da parte dell'aristocrazia locale di prestare servizio militare nelle fila dell'esercito mongolo portò a un ulteriore spopolamento dei distretti caucasici e al conseguente indebolimento della regione. La popolazione maschile veniva reclutata per le campagne militari mongole che in quegli anni furono frequenti sia contro l'Egitto mamelucco che contro l'Orda d'Oro sul confine caucasico.

Un lento, seppur evidente, miglioramento delle condizioni generali nella regione si rileva già a partire dai primi anni del XIV secolo quando le riforme di Ghazan ebbero effetti positivi soprattutto sulla produzione agricola. Un terzo momento di questo processo è rintracciabile dalla morte di Abu Said (1335) al disastro provocato dalla conquista timuride. La disintegrazione dell'Ilqanato

11 Bedrosian, The Turco-mongol cit. pp. 120-121; Dedeyan, Storia degli Ameni cit. p. 242, le cui stime coincidono con quelle fornite da Bedrosian.

12 Allen, A History of Georgian People cit. p. 285. Altrove si è forse esagerato stimando la popolazione del Caucaso tutto in circa 16.000.000 di individui. Purtroppo, ripetiamo, le fonti utilizzabili per questo tipo di operazione non consentono acquisizioni certe. È dunque opportuno utilizzare le cifre date solo come indicative di una situazione che, nonostante le forti difficoltà cui gli abitanti della Caucasia dovettero far fronte, non appare come disastrosa da un punto di vista demografico alla metà del XIII secolo. 
generò una profonda crisi politica e le lotte per il potere si scatenarono. Le autorità locali, fino a poco tempo prima rappresentanti del potere centrale nelle diverse zone dell'Ilqanato, entrarono in guerra per sostenere un pretendente al trono ilqanide piuttosto che un altro. Tutto ciò proseguì almeno fino alla morte violenta dell'ultimo ilqan: Togha-Timur avvenuta nel 1353.

Secondo i dati forniti da Rashid ad-Din, vizir dell'ilqan Ghazan e attuatore delle riforme poste in essere da quest'ultimo, possiamo apprezzare quali mutamenti intervennero fra X secolo e fine del XV nei distretti dell'ilqanato. Nella regione di Hamadan all'inizio del XIII secolo vi erano circa 660 villaggi; nel 1340 non erano più di 220. In quella di Herat nel X secolo figurano 400 villaggi mentre all'inizio del XV secolo erano appena $167 .{ }^{13}$ Uno degli elementi caratteristici della riorganizzazione mongola in Caucasia fu l'impossibilità, da parte dei nuovi conquistatori, di instaurare un regime di pace duratura. Le guerre con l'Orda d'Oro e i problemi politici con l'Egitto mamelucco rendevano difficile l'avvio di una ripresa economica che poggiasse su basi solide.

\begin{tabular}{|l|c|c|c|}
\hline Distretto (vilayat) & $\begin{array}{c}\text { primi anni } \\
\text { del XIII sec. }\end{array}$ & $1340 \mathrm{ca}$ & $\begin{array}{c}\text { primi anni } \\
\text { del XV sec. }\end{array}$ \\
\hline Hamadan & 660 & 212 & - \\
\hline Rudhravar & 93 & 73 & 30 \\
\hline Khwaf & 200 & - & 26 \\
\hline Isfara'in & 451 & 50 & 84 \\
\hline Baihaq & 321 & 40 & 29 \\
\hline Juvain & 189 & - & 20 \\
\hline Turshiz & 226 & - & - \\
\hline
\end{tabular}

Tabella 2. Numero di villaggi prima e dopo la conquista mongola nell'Ilqanato.

Dalla tabella si può evincere che la regione di Hamadan, nell'arco di tempo di oltre un secolo che separa le due notizie, ha subito una diminuzione di villaggi pari al $67,88 \%$. A Rudhravar l'emorragia è minore, ma si attesta comunque su un $21,51 \%$. Nelle zone di Isfara'in e Baihaq lo spopolamento fu particolarmente drammatico: rispettivamente $88,92 \%$ e $87,54 \%$. I dati riportati nella terza colonna ci aiutano a valutare quali effetti ebbero altri eventi traumatici che intervennero durante il XIV secolo: l'epidemia di peste del 1348 e la catastrofica invasione dell'esercito di Tamerlano sul finire del secolo. Possiamo apprezzare come nel distretto di Isfara'in la popolazione si attesta, all'inizio del XV secolo sul 5,76\% di quella dei primi anni del secolo XIII, con un'ulteriore diminuzione, rispetto a sessant' anni prima, di quasi il 6\%. Una sensibile ripresa si avverte nella zona di Baihaq dove la crescita si attesta su oltre il $50 \%$ in più

13 Petruševskij, The socio-economic condition cit. pp. 496-497. 
rispetto al 1340 con una percentuale che non supera comunque il 26,16\% del numero di villaggi presenti all'inizio del XIII secolo.

Il prelievo fiscale è un altro degli indici attraverso cui è possibile valutare i movimenti demografici del periodo. In questo sono estremamente utili i dati forniti da Hamd Allah Qazvini ${ }^{14}$ il quale, come funzionario del dipartimento finanziario dell'Ilqanato, è piuttosto attendibile.

\begin{tabular}{|l|c|c|c|}
\hline Regioni & $\begin{array}{c}\text { Tassazione (primi } \\
\text { anni del XIII sec.) }\end{array}$ & $\begin{array}{c}\text { Tassazione durante } \\
\text { il periodo ilqanide }\end{array}$ & $\begin{array}{c}\text { Tassazione (primi } \\
\text { anni del XV secolo) }\end{array}$ \\
\hline Azerbaijan & 20.000 .000 & 2.160 .000 & - \\
\hline Mughan e Arran & 3.000 .000 & 303.000 & - \\
\hline Shirvan & 1.000 .000 & 113.000 & 820.000 \\
\hline $\begin{array}{l}\text { Gushtasfi (al } \\
\text { delta del Kur e } \\
\text { dell'Arasse) }\end{array}$ & 1.000 .000 & 118.500 & - \\
\hline $\begin{array}{l}\text { Georgia (Guristan } \\
\text { e Abkhazia) }\end{array}$ & 1.000 .000 & 1.202 .000 & 400.000 \\
\hline Grande Armenia & 2.000 .000 & 390.000 & - \\
\hline
\end{tabular}

Tabella 3. Totale dell'esazione fiscale fra periodo pre-mongolico e Ilqanato (in dinar).

Se consideriamo che la tassazione imposta alle terre conquistate fu piuttosto pesante, soprattutto nel primo periodo della dominazione, allora le cifre riportate sopra impressionano non poco per la loro pochezza rispetto alla capacità contributiva che le regioni caucasiche avevano fino ai primi decenni del XIII secolo. L'Azerbaijan, regione sottoposta al più duro regime fiscale in seguito all'assenza di intermediari fra Ilqani e popolazione da una parte e dalla ricchezza delle sue terre dall'altra, paga nel XIV secolo il 10,8\% di quello che pagava poco più di un secolo prima. L'Armenia il 19,5\%, mentre la Georgia paga circa il $20 \%$ in più e questo è il risultato sia della situazione politica che vedeva l'Armenia come alleata degli Ilqani, sia della oggettiva difficoltà che essa aveva a ricuperare le perdite di popolazione seguite agli eventi del XIII secolo e aggravate dalla crisi di metà Trecento. La Georgia, da parte sua, costituiva uno stato vassallo, sottoposto indirettamente all'autorità degli Ilqani e per questo fortemente controllato nel flusso fiscale. È difficile pensare che avesse subito danni minori rispetto ad altri stati della stessa regione.

Il reticolo urbano fra Caucasia e Transoxiana era tradizionalmente ben sviluppato e le città, più o meno grandi, avevano un rapporto organizzato con le aree rurali. L'invasione mongola ebbe effetti devastanti sulle città più grandi (Merv, Herat, Nishapur, Tbilisi, Kars e Ani) e alcune di esse non raggiunsero mai

${ }^{14}$ Ibidem, p. 497. 
le dimensioni precedenti la conquista. Altre videro un'autentica rinascita durante il periodo mongolo. È il caso di Tabriz e di tutte quelle città che si trovavano più o meno direttamente coinvolte nel complesso degli scambi che si svilupparono rapidamente dalla seconda metà del XIII secolo. Tabriz crebbe notevolmente: dai 6.000 passi di circonferenza che aveva a metà del XIII giunse ad averne oltre 25.000 con Hassan qan (1295-1304); stessa cosa si può dire per le due Saraj, ma anche Urgench e Samarcanda ripresero vigore e tornarono a rappresentare centri per gli scambi di vitale importanza. Le città emporio, che basavano la loro prosperità economica sul commercio, allacciarono contatti con altri centri dell'Europa centro-occidentale allargando la rete di interessi internazionali verso est. Il ceto mercantile urbano europeo incontrerà la società nomade dell'Asia centro-orientale proprio in Caucasia, laddove i Mongoli gestivano, più o meno direttamente, zone la cui importanza strategica ed economica era straordinaria. Questa esperienza, nel cuore della regione che ha visto l'incontro di molteplici espressioni etniche, sociali e culturali, merita, oggi più che mai, l'attenzione degli studiosi e una riconsiderazione delle problematiche storiche. 


\section{CONCLUSIONI. RISULTATI E PROSPETTIVE}

Scriveva Tosi che il Caucaso è il complesso orografico più vicino al mare di tutto il pianeta e questo ha fortemente contribuito a renderlo una frontiera naturale fra spinte integrative contrapposte e sempre incomplete. Non si può non convenire sul fatto che

la frantumazione culturale e il disequilibrio politico del Caucaso sia derivato dall'interdipendenza storica della regione con il mondo delle steppe, innestatasi su un meccanismo interattivo che ha determinato un'influenza reciproca. ${ }^{1}$

Tosi, da archeologo e studioso dell'età antica, si riferiva alla preistoria della regione, ma ritengo si possano applicare con efficacia le sue convinzioni anche al periodo che abbiamo qui preso in esame. La Caucasia ha rappresentato per secoli il punto di contatto fra modelli diversi, spesso contrapposti, che non l'hanno mai conquistata del tutto, dando all'etnicità quel valore collettivo generalizzante che hanno avuto altre aree del pianeta (l'Europa cristiana, ad esempio). Le spinte all'unificazione politica e quindi anche ideologica non sono mai state sufficienti affinché tale processo si realizzasse del tutto poiché ogni conquista è stata imperfetta $\mathrm{o}$ «interrotta». Il nomadismo è dovuto scendere a patti col modello sedentario e quest'ultimo non è mai riuscito ad affermarsi come preminente su tutta la Caucasia. La vicenda mongola è un laboratorio etnico, culturale, economico e politico di straordinaria complessità, ma costituisce altresì un momento in cui le fonti parlano e ci permettono di cogliere aspetti che per il periodo precedente sono meno facilmente rintracciabili. E in effetti l'impatto della conquista mongola sulla Caucasia ha suscitato il vivo interesse degli specialisti. In particolare si è molto insistito sul ruolo distruttivo che questo evento ebbe e sul carattere «epocale» delle sue conseguenze; spero di aver in qualche modo esposto i dati disponibili o per lo meno quelli cui sono potuto accedere al fine di ridimensionare la questione che è stata spesso oggetto di esagerazioni, in un senso o nell'altro. In primo luogo non credo sia lecito parlare di migrazioni di massa poiché a muoversi furono alcune parti di quella composita società nomade che abbiamo analizzato. A portare a termine

1 Tosi, Dalla tribù all'impero cit. p. 250. 
le campagne militari fu un esercito che era l'espressione diretta di quella società. A stabilirsi nelle terre conquistate fu una classe dirigente esigua per numero e riluttante all'insediarsi in un contesto ambientale che non riconosceva come proprio. Il caso della Rus', con la creazione dell'Orda d'Oro, ne è l'esempio più chiaro. Non vi fu una sostituzione di genti, né una rivoluzione etnica; l'elemento originario predominò durante e anche dopo l'invasione e questo avvenne, pur se in termini diversi, in tutte le aree conquistate dalla Persia alla Cina, dalla Russia all'Asia Centrale. Basti pensare che l'Ilkhanato, dove l'assimilazione e la cooperazione fra ceto dirigente mongolo e le élites locali furono maggiori rispetto alle altre aree dell'impero, fu il primo a disintegrarsi.

Ci siamo inoltre interrogati sull'eredità lasciata dai Mongoli nelle regioni che subirono il loro dominio. Anche questo è un aspetto che spero di aver trattato con misura e con l'equilibrio che merita. Mi limiterò in questo caso a tirare le fila del lungo discorso fatto nelle pagine che precedono.

Concordo con chi ha guardato alle conquiste mongole del XIII secolo come ad un evento di straordinaria portata storica; ciò non solo, come in molti hanno giustamente e da tempo riconosciuto, perché grazie alla pax mongolica si aprirono nuove vie di transito, le strade divennero più sicure, i commerci internazionali ripresero vigore in aree fino ad allora quasi sconosciute; questo è tutto vero, ma non basta: l'unificazione di un territorio che copriva, nel momento del suo massimo sviluppo, due terzi dell'Asia e un terzo dell'Europa, fu soprattutto una rivoluzione culturale. Non credo che la definizione sia troppo audace. Prima della conquista mongola l'Oriente era, per l'uomo occidentale, una pura immagine mentale, una categoria culturale astratta, in proiezione verticale. Il XIII secolo segna un mutamento per cui fu possibile realizzare viaggi come quello dei Polo; le città italiane più attrezzate abbandonarono Bisanzio come punto di arrivo per i loro investimenti di Levante e si gettarono in un'avventura nuova che le portò fino in Cina. Certo, affinché si realizzasse la grandiosa stagione «coloniale» di Genova e Venezia furono necessarie l'audacia, la preparazione mentale e le infrastrutture del ceto mercantile il quale non aveva problemi ad imbarcarsi su una galea e farsi sei mesi di viaggio per andare fino alla foce del Don o in Crimea o a Trebisonda in Anatolia. Ma la concomitanza di questi fattori generò una stagione nuova e aprì ad un mondo nuovo già tre secoli prima della traversata colombiana.

L'altro aspetto che mi ha spinto a realizzare questo studio è il carattere nuovo della dominazione mongola. I Mongoli furono i primi a controllare non solo le steppe eurasiatiche, ma anche popoli sedentari, organizzati e culturalmente distanti da loro. Anche su questo le posizioni si sono spesso trovate come contrapposte e talvolta eccessive. L'accresciuta mobilità dei mercanti fu reale e coinvolse non solo l'Europa, ma anche gran parte del continente asiatico: viaggiavano i mercanti musulmani verso Settentrione e verso Oriente (i mercati della Transoxiana, dell'Orda d'oro e della Cina), quelli occidentali verso Oriente. Aumentarono le distanze di percorrenza perché si potevano raggiungere paesi 
lontani; aumentava la sicurezza delle strade (meno quella dei mari). È però altrettanto vero che mutarono forzosamente - non sempre e non dappertutto allo stesso modo - le capacità produttive in quanto gran parte della manodopera specializzata fu allontanata con coercizione dai propri paesi d'origine per finire nelle botteghe controllate direttamente dal ceto dirigente centrale del nuovo impero. Questo fenomeno ebbe una conseguenza di tipo culturale niente affatto trascurabile. La pace mongola rappresentò una condizione oggettiva in seguito alla quale poté realizzarsi l'incontro di due mondi assai distanti fra di loro. Essa non fu però una condizione permanente ed eguale in tutte le sue manifestazioni locali. Né fu con la conquista mongola che si prese a viaggiare su lunghe distanze; lo si faceva anche prima. Gli spostamenti di beni e di persone da Est ad Ovest e viceversa erano attivi sin dai primi secoli dell'era cristiana, ma circoscritti all'Asia ed alla Transoxiana. In Estremo Oriente, ad esempio, le merci (soprattutto stoffe) viaggiavano dalla Cina verso l'Asia Centrale ben prima dell'avvento della dinastia mongola degli Yuan, ma fu solo con questa che vennero istituiti centri di produzione organizzati e sottoposti a regole più rigide, magistrature addette all'industria tessile e a quella aurea; vi furono sistematici innesti di manodopera specializzata che arrivava con un flusso continuo sia durante le conquiste, sia (almeno) nei tre decenni successivi. Con l'avvento del ceto dirigente mongolo cambiarono quindi due cose da questo punto di vista: l'organizzazione della produzione e le dimensioni del mercato internazionale su cui venivano collocati i prodotti grazie all'estensione del dominio. Il processo di reidentificazione che vide i Mongoli sedentarizzarsi, fu lento e raramente si verificò davvero, ma ad esso fece da contraltare una pianificazione dei modi attraverso cui potevano essere meglio sfruttate le risorse di cui i Mongoli sapevano aver bisogno. Come ha osservato Allsen essi acquisivano le necessarie risorse economiche e i vari elementi culturali dal mondo sedentario ogni qual volta li ritenevano vantaggiosi. ${ }^{2}$ Il loro guardare al mondo sedentario come ad un contesto estraneo, ma da usare a proprio favore, era il frutto di una preesistente tensione verso quelle strutture del tutto intrinseca alla loro cultura (o a quegli aspetti del loro bagaglio culturale che erano parte integrante della società mongola tradizionale). Anche in questo caso credo si possa concordare con Allsen quando dice che il significato cosmologico dell'oro e i messaggi metafisici derivanti dalla manipolazione delle stoffe hanno favorito la forte tolleranza e la promozione verso i mercanti occidentali che di quei materiali facevano commercio arricchendosi. ${ }^{3} \mathrm{E}$ anche

2 Allsen, Commodity and exchange cit. p. 101; si veda anche l'articolo di M. Chazanov, Ecological Limitations of Nomadism in the Eurasian Steppes and Their Social and Cultural Implications, in "Asian and African Studies. Journal of the Israel Oriental Society», 24 (1990), pp. 1-15; H.G. Barnett, Culture Processes, in "American Anthropology», 42 (1940), pp. 31-37.

${ }^{3}$ Allsen, Commodity and exchange cit. p. 102. Ė questo un aspetto poco indagato, ma centrale nell'ambito dell'analisi sull'apprezzamento e la sostanziale propensione che i Mongoli avevano nei confronti delle pratiche commerciali. 
in questo caso torna con forza la convinzione di un nomadismo non autarchico, ma teso costantemente all'interazione con contesti produttivi diversi.

L'altro aspetto che fu nuovo e determinato direttamente dalle conquiste sorprendenti di questo popolo, dicevamo è costituito dall'estensione del dominio. Anche se l'impero si frantumò ben presto in quattro entità distinte esse erano di per sé molto estese e crearono una sorta di continuità in regioni generalmente ostili fra di loro. Vennero abbattute barriere ideologiche che, se resistevano senza sforzo a livello locale, garantivano comunque lo spostamento e l'insediamento più o meno stabile di genti in luoghi nei quali altrimenti non si sarebbero in alcun modo avventurati. Non abbiamo notizie di viaggi in Cina da parte di mercanti o viaggiatori occidentali prima delle conquiste mongole. Centri come quello della Tana o la foce del Volga sarebbero state forse irraggiungibili nel XII secolo. D’altra parte aree come lo stretto di Kerč subirono un crollo repentino in seguito alla conquista di Costantinopoli da parte dei crociati; a beneficiarne, in questo caso, fu Trebisonda. La conquista mongola, favorendo l'immigrazione commerciale dall'Occidente, dette stimoli nuovi a quell'area che acquisì di nuovo importanza sia per la sua ricchezza cerealicola sia per la buona pescosità del suo mare. ${ }^{4}$

Il nomadismo della steppa ha contribuito in modo attivo allo sviluppo culturale dell'Occidente aprendogli le porte di un mondo fino ad allora sconosciuto. A viaggiare su lunghe e nuove distanze non erano solo le merci o le persone, ma anche la conoscenza. La caduta delle frontiere mentali e il rinnovamento - lento e discontinuo - di alcune consolidate categorie culturali fu fra gli elementi più evidenti di quell'eredità. Da questo punto di vista e con le dovute cautele l'opera di Timučin e dei suoi immediati successori rappresentò un cambiamento sostanziale che rese reale e concreto quell'Oriente Aperto di cui iniziano a darci conto i protagonisti di una stagione nuova. L'emigrazione occidentale, dal canto suo, ha colto subito questi stimoli. Il mercante è uomo di pace, è quel fattore di contatto fra due contesti che permette l'apprendimento e la conoscenza dell'altro attraverso una pratica che non è quella militare; egli garantisce un rapporto completo e pacifico con l'esterno e anche in virtù di queste sue caratteristiche i Mongoli ne sostennero l'attività. I Mongoli non erano in grado di gestire un impero come quello che avevano costruito nella seconda metà del XIII secolo. Questa incapacità era conseguenza di molti fattori; le dimensioni delle loro conquiste sarebbero state eccessive per qualunque organismo organizzato (infatti l'esperienza mongola non avrà eguali nella storia dell'uomo), ma più di ogni altra ragione credo che si debba rilevare la loro incapacità peculiare di amministrare un universo così eterogeneo. Questa difficoltà era un retaggio del loro adattamento ambientale originario, tanto che il ricorso all'opera di stranieri

${ }^{4}$ Si veda su questo il saggio di M.G. Kramarovskij, Džučidy i Krym: XIII-XVvv., in Materjaly po Archeologii, Istorii, i Étnografii Tavrii, Simferopol 2003, X, pp. 506-531. 
fu massiccio e socialmente trasversale. Il caso della Cina è emblematico perché non furono chiamati soltanto gli artigiani a lavorare per gli Yüan, ma anche amministratori, funzionari militari e manovalanza di ogni tipo.

Su queste tematiche c'è ancora molto da fare. Gli spunti sorti durante la preparazione di questo lavoro superano certamente le acquisizioni; i dubbi sono di gran lunga superiori alle convinzioni. Si è a lungo parlato degli effetti demografici delle conquiste; abbiamo cercato di darne conto, ma restano ancora molte questioni aperte sull'entità dei rifugiati che si mossero dalle città conquistate in altri centri più o meno lontani dalla rotta dell'esercito mongolo. Queste migrazioni di massa furono frequenti soprattutto in Asia Centrale dove molte città vennero distrutte e le campagne devastate. Lo stesso lavoro andrebbe fatto per la Persia ilkhanide, dove lo spopolamento, soprattutto rurale, fu notevole.

Il rapporto fra l'uomo e l'ambiente, studiato con grande preparazione e buoni risultati in Italia negli anni Ottanta del secolo scorso, è un aspetto poco indagato per il contesto nomade e sarebbe estremamente interessante una ricerca che fosse in grado di mettere a confronto l'utilizzo fatto dalle società nomadi delle risorse ambientali con il rapporto che con esse avevano le società sedentarie con le quali il nomadismo si trovò a confronto dal XII secolo in avanti (Cumani e Mongoli soprattutto). Sarebbe oltremodo interessante studiare quale percezione si ebbe nell'Italia comunale delle conquiste mongole; quali echi giunsero al di qua del Mediterraneo e quali conseguenze vi furono sulla politica europea. Ma più di ogni altro si avverte il bisogno di gerarchizzare le problematiche storiche che riguardano la vicenda mongola e il suo impatto non solo sulla Caucasia, ma sull'Eurasia tutta. Tale necessità è il frutto di una tradizione che ha visto impegnati su questo fronte linguisti e filologi assai più che gli storici e questo è causato in gran parte dalle difficoltà linguistiche che le fonti per lo studio della storia caucasica presentano. Come si potrà notare gran parte della letteratura scientifica utilizzata per questo lavoro è in lingua inglese, russa e francese. L'Italia ha un Medioevo straordinariamente ricco che ha impegnato e impegna gli storici, assorbendo gran parte delle loro energie; tuttavia non esaurisce la loro curiosità. Lo storico interpreta il passato e non può predire il futuro e se è vero che la comprensione del passato aiuta a capire il presente uno sguardo ad Est, oggi più che mai, si rende necessario. 

CARTINE 



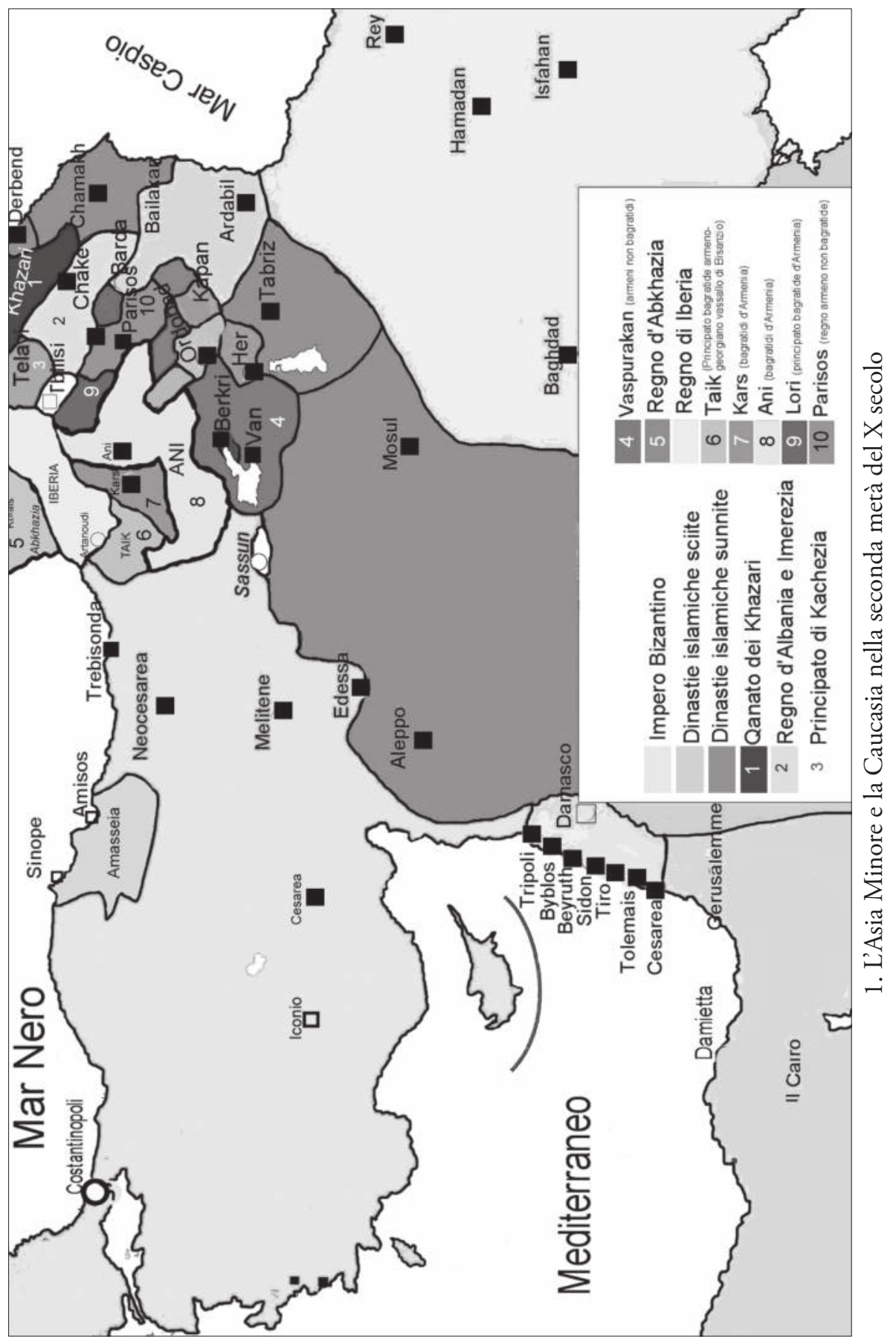

Lorenzo Pubblici, Dal Caucaso al Mar d'Azov : l'impatto dell'invasione mongola in Caucasia fra nomadismo e società sedentaria (1204-1295), ISBN 978-88-8453-436-1 (online), ISBN 978-88-8453-437-8 (print) @ Firenze University Press, 2007 


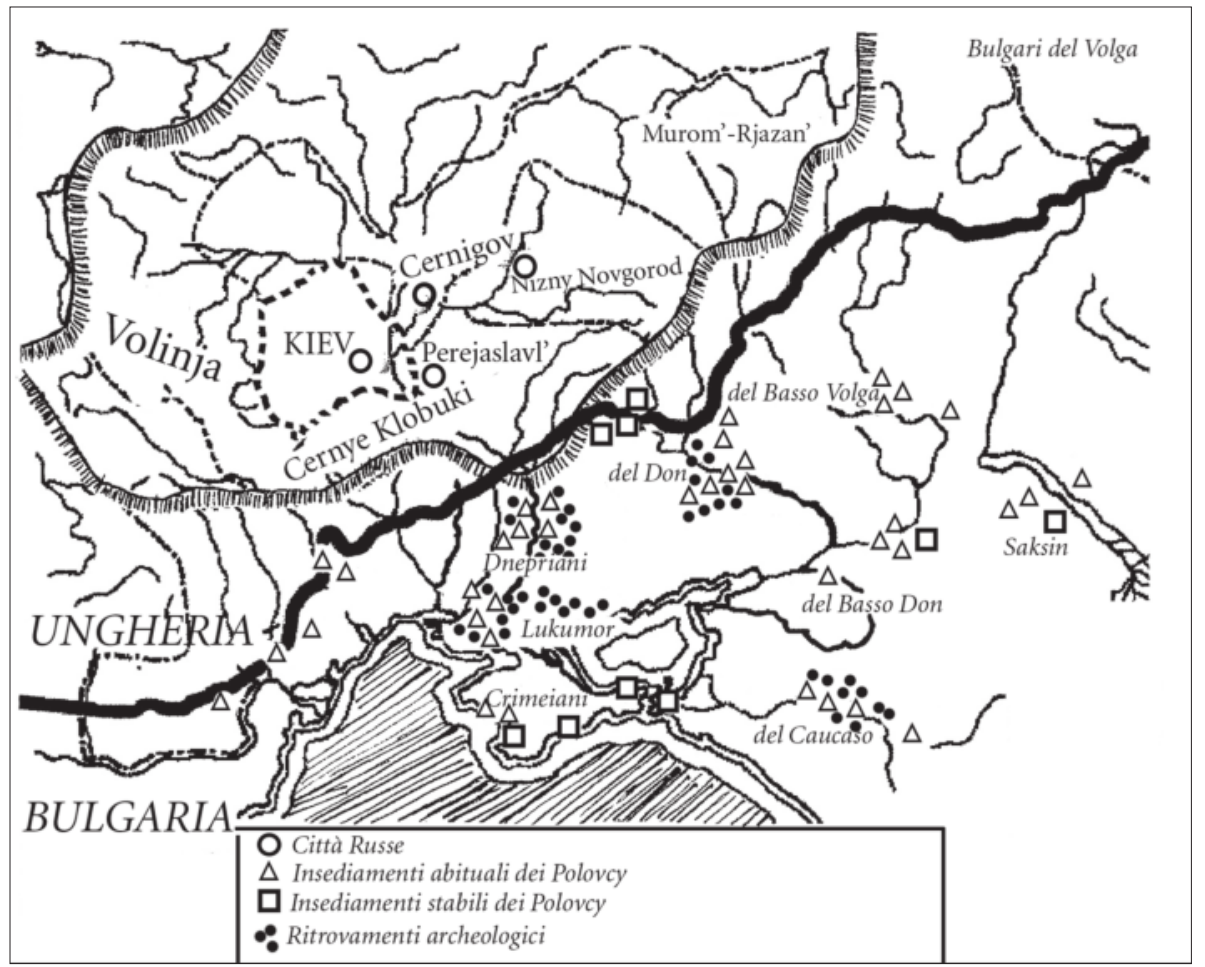

2. La Rus' e i Polovcy

(rielaborazione da Tolocko, Kocevye narody cit., p. 94) 


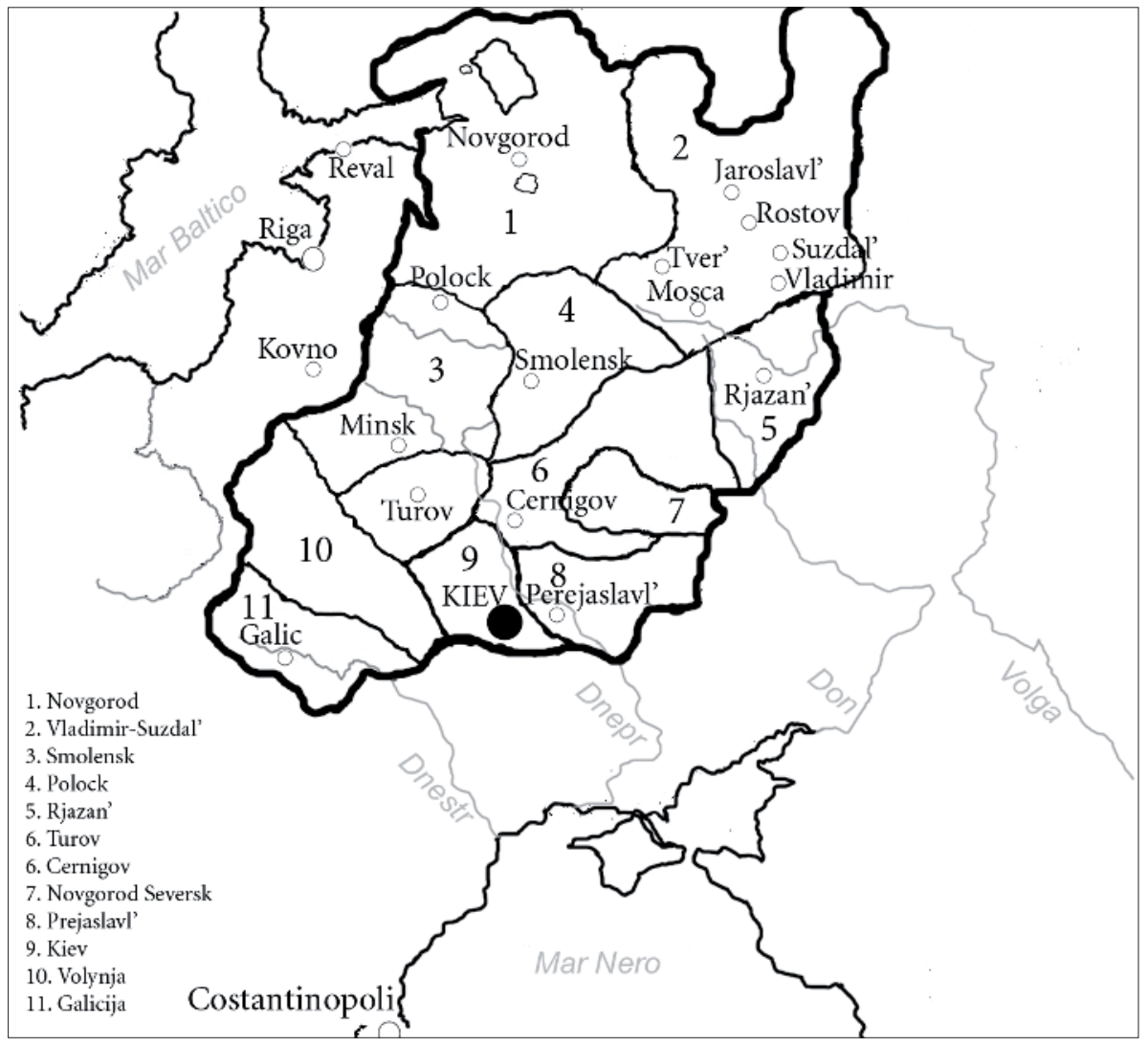

3. La Rus' di Kiev e i singoli principati alla metà del XII secolo (rielaborazione da Tolocko, Kocevye narody cit., p. 94) 


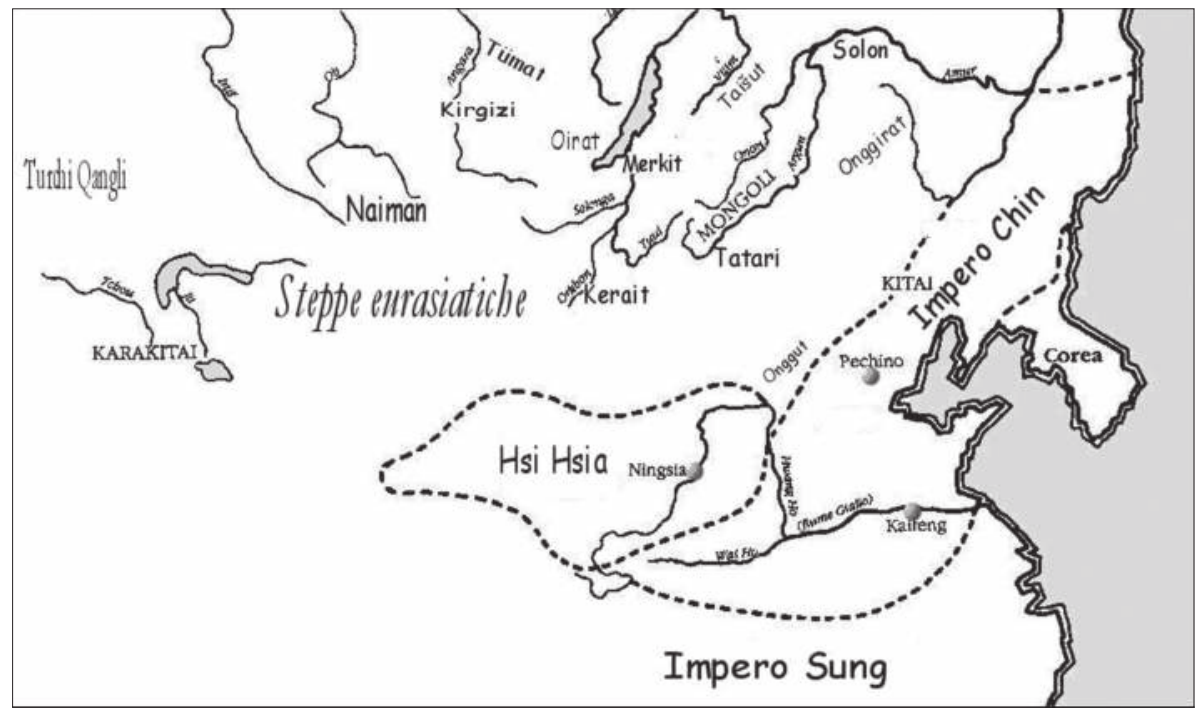

4. Il nomadismo centroasiatico alla vigilia dell'unificazione operata da Gensis khan (rielaborazione da Hoang, Gengis Khan cit., p. 81)

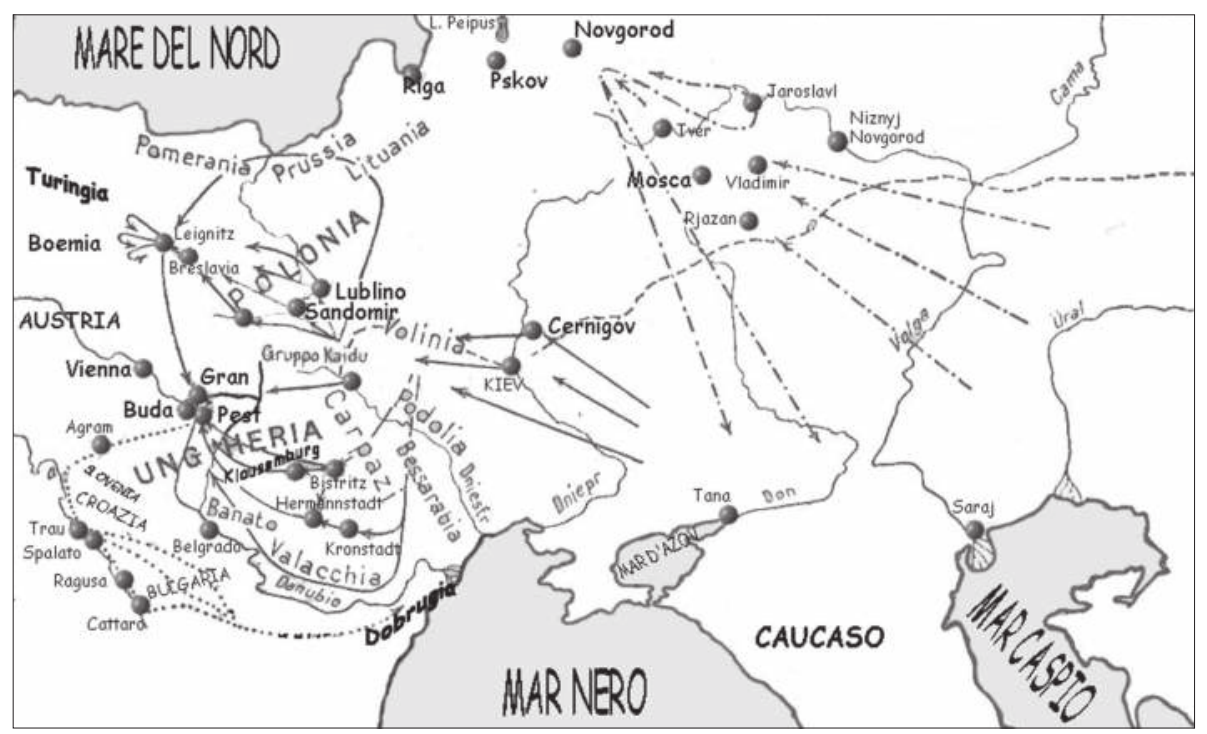

5. L'invasione mongola dell'Europa (rielaborazione da Prawdin, Il retaggio di Gengis Khan cit., p. 25) 
BIBLIOGRAFIA 



\section{A. Fonti EDITE}

$A$ history of Persian Literature under Tartar dominion (AD 1265-1505), a cura di E.G. Browne, Cambridge 1920

A Literary History of Persia, 4 voll., a cura di E.G. Browne, London 1902-1924

A lytell chronycle, a cura di G. Burger, Toronto 1988

A Syriac Encyclopaedia Of Aristotelian Philosophy: Barhebraeus (13th C.), Butyrum Sapientiae, Books Of Ethics, Economy, And Politics, a cura di N.P. Joosse, Leiden 2004

A. Comnena, Alexiade. Règne de l'empereur Alexis I Comnène (1081-1118), ed. a cura di B. Leib, II edizione, Paris 1967

Aвоu'L Feda, Géographie d'Aboulféda, a cura di J. T. Reinaud, 2 voll, Paris 1848

Aвоu'l Feda, Résumé de l'Histoire des Croisades tiré des Annales d'Abou'l-Fedâ, in Recueil des Historiens des Croisades. Historiens Orientaux, II, 1887

Acta et diplomata greca medii aevi sacra et profana, a cura di F. Miklosich e J. Müller, 6 voll., Vienna $1860-1890$

Afanasij Nikitin, Viaggio in tre mari, ed. it. a cura di E. T. Saronne, Roma 2003

Al UMARI, Das mongolische Weltreich, tr. e cura di K. Lech, Wiesbaden 1968

Ata Malik Juvaini, Gengis Khan, il conquistatore del mondo, a cura di G. Scarcia, Milano 1962, rist. 1991

Ata Malik Juvaini, The History of the World Conqueror, a cura di J. A. Boyle, 2 voll., Manchester 1958

Banescu, N., La domination byzantine à Matracha (Tmutorokan) en Zichie, en Khazarie, et en "Russie» aù l'époque des Comnènes, in "Academie Roumaine, Bulletin de la Section Historique», XXII (1941), 2, pp. 57-77

Bar Ebreo, Bar Hebraeus, The Chronography of Abu'l Faraj (Bar Hebraeus), trad. e a cura di A.E. Wallis Budge, London 1976, rist. Piscataway (NJ), 2003

Berezin, I.N., Trudy Vostočnogo Otdelenija Imperatorskago Archeologičeskago Obščestva, Sankt Peterburg 1858-1888 
Berezin, J., Sbornik letopis'ej. Istorija Mongolov sočinenie Rašid-Eddina, in Trudy Vostočnago otdelenija Imperatorskago Archeologičeskago Obščestva, voll. V, VII e XIII, Skt. Peterburg 1858, 1861, 1868 e 1888

BLochet, E., Introduction à l'histoire des Mongols, Leiden-London 1910

Brosset, M.F., Additions et éclaircissements a l'histoire de la Géorgie : depuis l'antiquité jusquien 1469, Sankt Peterburg 1851

Brosset, M.F., Histoire de la Géorgie depuis l'antiquité jusqu'au XIX siècle, 7 voll., Sankt Peterburg $1849-1858$

Carile, A., Partitio terrarum Imperii Romaniae, «Studi Veneziani», VII (1965), pp. 125-305

Chronicon Livoniae, a cura di P. Bugiani, Livorno 2005

Costantinus VII Porfirogenitus, De administrando imperio, a cura di R. J. H. Jenkins, Washington (DC), 1967

Cronaca di Tver' in Polnoe Sobranie Russkich Letopisej, t. XXX, Vladimirskij Letopisec, Moskva 1965, in parte trad. in Pianto sulla distruzione di Rjazan', a cura di E. T. Saronne, Parma 1992, pp. 171-224

Dölger, F., Regesten der Kaiserurkunden des Oströmischen Reiches, 5 voll., München 1924-1965

Dunn, R.E., Gli straordinari viaggi di Ibn Battuta, Milano 1998

Encyclopaedia of Islam, 12 voll., London 1960-2002

Epistola Brunonis ad Henricum regem, a cura di Hedvigis Karwasińska, in Monumenta Poloniae Historica, N.S. T.4/3, Warszawa, 1973, pp. 77-106

Epistola de adventu Tartarorum, Matteo Paris, Cronica Majora, a cura di H.R. Luard, IV, London 1877, pp. 112-119

Erodoto, Le storie, a cura di L. Annibaletto, Milano 1988

F. Balducci Pegolotti, La pratica della mercatura, a cura di A. Evans, Cambridge (MS) 1936

Federico II, Encyclica contra Tartaros, in Monumenta Germaniae Historica: Costitutiones et Acta Publica Imperatorum et Regnum, II, Hannover, 1896. pp. 322-325

Fr. Johannes de Plano Carpini, Ystoria Mongalorum, a cura di A. Van den Wyngaert, «Sinica Franciscana», I: Itinera et relationes Fratrum Minorum saeculi XIII et XIV, Quaracchi-Firenze 1929

Fragments de Géographes et d'historiens arabe et persane inédits, relatifs aux anciens peuples du Caucase et de la Russie Méridionale, a cura di M. Defremery, in "Journal Asiatique», s. IV, 13 (1848), pp. 457-551

Fragments des géographes et historiens arabo-persans inédits relatifs aux ancients peuples du Caucase, a cura di M. Defremery, Paris 1849 
G. Cinnamo, Epitome rerum ab Ioanne et Manuele Comnenis gestarum, a cura di A. TomeI, Tesi di Laurea, Facoltà di Lettere e Filosofia, Università degli Studi di Firenze, A. A. 2000/2001

Die Byzantiner und ihre Nachbarn: Die "De administrando imperio" genannte Lehrschrift des Kaisers Konstantinos Porphyrogennetos für seinen Sohn Romanos, a cura di K. Belke e P. Soustal, Wien 1995

G. De Villehardouin, La conquista di Costantinopoli, a cura di F. Garavini, Milano 1988

Giovanni di Pian del Carpine, Storia dei Mongoli, a cura di P. Daffinà, C. Leonardi, M. C. Lungarotti, E. Menestò, L. Petech, Spoleto 1989

Golubovich, G., Biblioteca bio-bibliografica della Terra Santa e dell'Oriente francescano, 5 voll., Quaracchi 1906-1927

Gregor di Akner, History of the Nation of Archers, tr. e cura di R. Bedrosian, Long Branch (NJ) 2003. Online [12/06]: < http://rbedrosian.com.gal.htm >

Grigor of AKants, History of the Nation of the Archers (the Mongols) by Grigor of Akants, a cura di R. Blake e R. Frye, Cambridge (MS), 1954

Guglielmo di Rubruck, Viaggio nell'impero dei Mongoli, a cura di L. Dalledonne e G. L. Podestà, Genova 2002

Guillelmus de Rubruc, Itinerarium, in Sinica Francescana, Vol. I, Itinera et relations fratrum minorum, saeculi XIII et XIV, a cura di A. Van De Wyngaert, Firenze 1929

Hayton Armeno, La Storia dei Tartari di Hayton l'Armeno, G. B. Ramusio, Navigazioni e Viaggi, vol. III, Torino 1980, pp. 299-355

Héthoum l'Historien, in Recueil des Historiens des Croisades. Documents Arméniens, Paris 1869, I, pp. 467-687

Hethum, The Flower of Histories of the East, Long Branch (NJ), 2004, trad. e cura di R. Bedrosian. Online [12/06]: < http://rbedrosian.com/hetumint.htm >

Histoire de Mar Jab-alaha, patriarchi, et de Raban Sauma, a cura di P. Bedjan, Paris 1888

Historia Mongalorum. Viaggio di F. Giovanni da Pian del Carpine ai Tartari nel 1245-47, a cura di G. Pullè, Firenze 1913

History of the Mongols. Based on Eastern and Western Accounts of the Thirteenth and Fourteenth Centuries, a cura di B. Spuler, Berkeley (CA) $1972^{2}$

History of the Nation of Archers, trad. e cura di R. Blake e R. Frye, «Harvard Journal of Asiatic Studies", 3-4 (1949), pp. 400-444

I viaggi di Ibn Battuta, a cura di F. Gabrieli, Firenze 1961

Ibn al-Athiri chronicon quod Perfectissimum inscribitur, a cura di C. I. Tornberg, I (1851-1853), II (1867-1876) Leiden 
Ibn aRABshah, Tamerlano or Timur the great Amir, trad. e cura di J. H. Saunders, rist. Lahore 1976.

Ibn Jubayr, Viaggio in Ispagna, Sicilia, Siria e Palestina, Mesopotamia, Arabia, Egitto, trad. C. Schiaparelli, Palermo 1995

IBn Khaldûn, Discours sur l'Histoire universelle Al-Muqaddima, trad. e cura di V. Monteil, Arles 1997

Il cantare della gesta di Igor, a cura di R. Poggioli, Torino 1954

Il Cantare di Igor, a cura di E.T. Saronne, Parma 1988

Il canto dell'impresa di Igor, a cura di E. Bazzarelli, Milano 1991

J.A. Boyle, The Successors of Genghis Khan, New York (NY) 1971

Jami at-tawarikh, «Sbornik Letopis'ej», vol. I trad. e cura di L.A. Ketagurov, Moskva-Leningrad 1952; vol. II trad. e cura di Ju.P. Verkovskij, Moskva Leningrad 1960; vol. III trad. e cura di A.K. Arends, Baku 1957

Jean de Plan Carpin, Histoire des Mongols, trad. e cura di D. J. Becquet, L. Hambis, Paris 1965

JuZJAni, Tabaqat-e Naseri, trad. e cura H. G. Raverty, London 1881

Kiriakos Ganjakets'is History of the Armenians, tr. e cura di R. Bedrosian, New York (NY) 1986

Kокоvсоv, P.K., Evreisko-Khazarskaja Perepiska v X veke (la corrispondenza ebraico-cazara nel X secolo), Leningrad 1932

Lavrent'evskaja Letopis', Leningrad 1926-1927, rist. München 1977

Li Chin-Chang, Travels of an Alchemist, a cura di A. Waley, London 1963

M. Polo, Il Milione, a cura di M. Ciccuto, Milano 1998

M. Polo, Il Milione, a cura di D. Olivieri, Bari $1928^{2}$

M. Polo, Il Milione, a cura di D. Ponchiroli, Torino, 1982

Le divisament dou Monde, a cura di G. Ronchi, Milano $2006^{3}$

M. Psello, Imperatori di Bisanzio, Cronografia, a cura di D. Del Corno, S. Impellizzeri, U. Criscuolo, S. Ronchey, 2 voll. Milano 1997

Mohammed en-Nasawi, Histoire du sultan Djelal ed-Din Mankobirti, prince du Kharezm, trad. a cura di O. Houdas, Paris 1895

Morozzo della Rocca R.-Lombardo A., Documenti del commercio veneziano nei secoli XI-XIII, 2 voll., Torino 1940 
N. Coniate, Grandezza e Catastrofe di Bisanzio, a cura di J. L. Van Dieten, A. Pontani, A. Kazhdan, 2 voll. Milano 2001

Nestore l'Analista, Cronaca degli anni passati (XI-XII secolo), trad. e cura di A. Giambelluca Kossova, Milano 2005

Notai genovesi in oltremare, atti rogati a Caffa e a Licostomo (sec. XIV), a cura di G. Balbi e S. Reiteri, Bordighera 1974

Otto Frisingensis vescovo; 1114 ca.-1158. Ottonis episcopi Frisingensis Chronica, sive Historia de duabus civitatibus, a cura di A. Hofmeister, Hannover 1984

Pianto sulla distruzione di Rjazan', a cura di E.T. Saronne, Parma 1992

Polnoe Sobranie Russkich Letopisej, Ipat'evskaja Letopis', a cura di A. A. Šachmatov, Moskva 1962

Racconto dei tempi Passati, Cronaca russa del secolo XII, a cura di I.P. Sbrizolo, Torino 1971

S. Rapp, Studies in Medieval Georgian Historiography: Early texts and Eurasian Contexts, Lovanii 2003

Rashid AD-Din, Histoire des Mongols de la Perse, a cura di E. Quatremere, Paris, 1836. Ristampa anastatica Amsterdam 1968

Rashid aD-DIn, Histoire des Mongols depuis Tchinguiz-khan jusqu'a Timour Bey ou Tamerlane 4 voll., a cura di C. D’Ohsson, Amsterdam 1834-1835

Istoria Mongolov, sočinenie Rašid-Eddina, a cura di J. Berezin, in Trudy Vostočnago otdelenija Imperatorskago Archeologičeskago ObŠčestra, Sankt Petersburg 1858-1883, voll. V, VII e XIII

Relation des Mongols ou Tartares par le frère Jean du Plan de Carpine de l'Ordre de Frères Mineurs, trad. e cura di M. D’Avezac, Paris 1838

Roberto di Clari, La conquista di Costantinopoli, a cura di A. Nada Patrone, Genova 1972

Ruj González de Clavijo, Dal Mare di Alboran a Samarcanda. Diario dell'ambasciata castigliana alla corte di Tamerlano (1403-1406), a cura di Anna Spinelli, Ravenna 2004

Ruj González de Clavijo, Viaggo a Samarcanda (1403-1406). Un ambasciatore spagnolo alla corte di Tamerlano, Roma 1999

Storia degli Armeni, a cura di G. Dédéyan, Milano 2002

Storia di Mar Yabballaha e di Rabban Sauma, a cura di P.G. Borbone, Torino 2000

Storia segreta dei Mongoli, ed. a cura di S. Kozin, M. Osulfieva, Milano 2000

Strabone, Geografia. Il Caucaso e l'Asia Minore, trad. e cura di R. Nicolai e G. Traina, Milano 2000

T'ovma Metsobets'i, The History of Tamerlan and His Successors, a cura di R. Bedrosian, New York 1987 
TAсіто, Libri ab excessu divi Augusti, a cura di B. Ceva, 3 voll., Milano 2000

The Chronicle of Novgorod, trad. di R. Michell, N. Forbes, London 1914

The Nikonian Chronicle, 2 voll, a cura di A. Serge, A. Zenkovskij, Princeton (NJ) 1984

The Secret History of the Mongols: A Mongolian Epic Chronicle of the Thirteenth Century, a cura di I. De Rachewiltz, Leiden 2004

The Secret History of the Mongols: for the First Time done into English out in the Original Tongue and Provided with an Exsegetical Commentary, a cura di F.W. Cleaves, Cambridge (MS) 1982

The Travels of Ibn Battuta, AD 1325-1354, a cura di C. Defrémery, B. R. Sanguinetti, 2 voll., Nendeln 1956 - rist.Cambridge 1962

The Wellspring of Georgian historiography: the early medieval historical Chronicle, a cura di K. B. Lerner, London 2004

von Hammer-Purgstall, J., Geschichte Wassafs, Wien 1856

\section{B. SAgGi e Contributi}

Adonz, N., Notes Arméno-Byzantines, in «Byzantion», X (1935), pp.161-203

Agadžanov, S.G., Ocerki istorii Oguzov i Turkmen Srednej Azii IX-XIII vv., Asxabad 1969

Agbunov, V.M., Antičnaja lozja Černogo morja, Moskva 1987

Ahrwaller, H., Byzance et la Mer. La marine de guerre, la politique et les institutions maritime de Byzance aux VIIe-Xve siècles, Paris 1966

Ahrwailer, H., La frontière et les frontières de Byzance en Orient, in Actes du XIVe Congrès International des Études Byzantines, Bucaresti 1971

Airaldi, G., Colonie genovesi nel Mar Nero, Studi Storici in Romania, Polonia e Bulgaria, in «Liguria», t. XXXVII, 5 (1970), pp. 9-12

Airaldi, G., Marinai, etnie e società nel Mediterraneo medioevale. Il caso di Genova, in Le genti del Mare Mediterraneo, a cura di R. Ragosta, Napoli 1981, pp. 59-85

Airaldi, G., Studi e documenti su Genova e l'Oltremare, Genova 1984

Ajbabina E. A.-BočArov C. G., Novye materialy po istorii srednevekovoj armjanskoj kolonii Kaffy, in «Visantijskij Vremennik», T. 57 (1997), pp. 210-233

Alberini, R., L'alimentazione degli equipaggi nelle antiche marine italiane, in «Rivista Marittima», LXIV, 7 (1931), pp. 71-85

Albertoni, G.-Provero, L., Il feudalesimo in Italia, Roma 2003 
Alekseev, L.V., Polockaja zemlja, Moskva 1966

Alekseev, L.V., Smolenskaja zemlja v IX-XIII vv., Moskva 1980

Alekseev, V.P., Some Aspects of the Study of Productive Forces in the Empire of Chengiz Khan, in Rulers from the Steppe: State Formation on the Inner Eura-sian Periphery, a cura di G. Seaman, D. Marks, Los Angeles (CA) 1991

Allen, W.E.D., A History of Georgian People: from the beginning down to the Russian conquest in the nineteenth Century, London 1932

Allsen, T.T., (Guard and Governament in the Reign of the Grand Qan Mongke, 1251-59, in "Harvard Journal of Asiatic Studies ", 46 (1986), 2, pp. 495-521

Allsen, T.T., Culture and Conquest in Mongol Eurasia, Cambridge 2001

The Mongol Empire and its Legacy, a cura di Amitai Preiss R.- Morgan, Leiden-Boston-Köln 1998

Artamonov, M.I., Istoria Khazar, Leningrad 1962

Ashtor, E., Levant Trade in the later Middle Ages, Princeton (NJ) 1983

Ashtor, E., Storia economica e sociale del Vicino Oriente nel Medioevo, Torino 1982

Ashtor, E., Tecnology, industry and trade: the Levant versus Europe, 1250-1500, Aldershot 1992

Aubin, J., Les princes d'Ormuz du XIII eau XVe siècle, in "Journal Asiatique», 241 (1953), pp. 89-99.

BaIs, M., Albania Caucasica. Ethnos, storia, territorio attraverso le fonti greche, latine e armene, Milano 2001

Balard M., Byzance et les régions septentrionales de la mer Noire (XIIIe-Xve siècles), in "Révue Historique», 288 (1993), pp. 19-38

Balard M., La Mer Noire et la Romanie Génoise (XIIIe-Xve siècles), London 1989

Balard M., Les Génois en Crimée aux XIIIe-XIVe siècles, in «Apxeion Ponton», 35 (1979), pp. $2-217$

BALARD, M., Costantinopoli e le città pontiche all'apogeo del Medioevo, in Le città del Mediterraneo all'apogeo dello sviluppo medievale: aspetti economici e sociali, a cura di G. Cherubini, Pistoia 2003, pp. $1-18$

Balard, M., Gênes et l'Outre-Mer, les actes de Caffa du notaire Lamberto di Sambuceto (1289-1290), Paris-Le Haye 1973

Balard, M., La Romanie génoise (XII - début du XVE siècle), Roma-Paris 1978 
Balard, M., Les Génois en Romanie entre 1204 et 1261 - Recherches dans les minutiers notariaux génois, in «Mélanges d'Archéologie et d'Histoire publiés par l'École Française de Rome», 78 (1966), pp. 467-502

BALBI, G., La schiavitù a Genova tra i secoli XII e XIII, in Mélanges offerts à René Crozet, a cura di P. Gallais e J.Y. Riou, Poitiers 1966

Barnett, H.G., Culture Processes, in «American Anthropology», 42 (1940), pp. 31-37

BARTha, A., The typology of nomadic empires, in I popoli delle steppe: Unni, Avari, Ungari (Atti della XXXV Settimana di Studi CiSAM), Spoleto 1987, pp. 151-174

Bartold, V.V., Turkestan down to the Mongol invasion, London, London $1977^{4}$

Bartold, V.V., Turkestan v epokhu Mongolskago naščestviya, 2 voll., Sankt Peterburg 1900

Bausani, A., Religion in the Saljuq Period, in The Cambridge History of Iran cit., V, pp. 283-302

Bausani, A., Religion under the Mongols, in The Cambridge History of Iran cit., V, pp. 538-549

Bautdinov, G., Gli italiani in Russia, Milano 1986

BAUTIER, R.H., Les relations économiques des occidentaux avec les Pays d'Orient, au Moyen Âge. Points de vue et documents, in Sociétés et compagnies de commerce en Oriente et dans l'Ocean Indien, Actes du huitième colloque International d'Histoire Marittime (Beyrut 5-10 settmbre 1966),

Paris 1970, pp. 263-332

Bedrosian, R., The Turco-Mongol Invasions and the Lords of Armenia in the 13-14th Centuries, Ph. D. Dissertation, Columbia University (NY) 1979

Belin F.A., Histoire de la latinité de Costantinople, Paris 1894

Benet, S., Abkhasians: the long-living people of the Caucasus, rist. New York 1974

Beradze T.N., Moreplavanije i morskaja torgovlia v srednievekovoj Gruzii, Tiblisi 1989

Berend, N., Cuman Integration in Hungary, in Nomads in the Sedentary world, a cura di A. M. Khazanov, Richmond 2001, pp. 103-127

Berindei M.-O'Riordan G., Venise et l'Horde d'Or, fin XIIIe-début XIVe siècle, in "Cahiers du monde Russe et Sovietique», XXIX (1988), 2, pp. 243-256

Berindei, D.-Veinstein, G., La Tana-Azaq de la présence italienne à l'emprise ottomane (fin XIIIemilieu XVIe siècle), in «Turcica», VIII (1976), pp. 110-201

Bıвıкоv, M.V., Istočnikovedčeskie problemy izučenija istorii kočevnikov v Nižem Podunav'e v XII veke, in «Revue Roumaine d'Histoire», 19 (1980), 1, pp. 47-52

Bıвıкоv, M.V., Vizantijskie istočniki po istorii drevnej Rusi i Kavkaza, Sankt Peterburg 2001 
Bliznjuk S.V., Gorodskoj Pejzaz Famagusty v XIII-XIV vekax, Gorod v srednewekovoj civilizacii Zapadnoj Evreopy, t. 1., Moskva 1999, pp. 363-369

BLOch, M., Comment et pourquoi finit l'esclavage antique, «Annales», V/II (1947), pp. 30-44, 161170; poi in Come e perché fini la schiavitù antica in ID., Lavoro e tecnica nel Medioevo, Roma- Bari 1990, pp. 221-263

BLOCH, M., La servitù nella società medievale, a cura di G. Cherubini, Firenze 1993

Bočarov, S.G., Fortifikacionnye sooruženija Kaffy (konec XIII-vtoraja polovina XV vv.), in Pričernomor'e v srednie veka, a cura di S. P. Karpov, S. Peterburg 1998, III, pp. 82-116

Bold, B.O., Mongolian Nomadic Society: A Reconstruction of the 'Medieval' History of Mongolia, New York (NY) 2001

Borisov, N.S., Otečestvennaja istoriografija o vlijanii tataro-mongol'skogo našestvija na russkuju kul'turu, in «Problemy istorii SSSR» V (1976), pp. 129-146

BorsarI, S., Studi sulle colonie veneziane in Romania nel XIII secolo, Napoli 1966

Bosworth, C.E., The Ghaznavids, Edimburgh 1963

Bournazel, E., Poly, J.P., Les féodalités. Histoire générale des sysèmes politiques, Paris 1998

Boyle, J.A., Dynastic and political History of the il-Khanns, in The Cambridge History of Iran, V, 1968, pp. 303-421

BoyLe, J.A., Kiriakos Gandzak on the Mongols, in "Central Asiatic Journal», 8 (1963), pp. 199214

Boyle, J.A., The Death of the Last 'Abbasid Caliph': A Contemporary Muslim Account, in "Journal of Semitic Studies», 6 (1961), pp. 145-161

Bratianu, G.I., La Mer Noire, des origines a la conquête Ottomane, München 1969

Bratianu, G.I., La question de l'approvisionnement de Constantinople a l'époque byzantine et ottomane, in «Byzantion», 5 (1929-1930), pp. 83-107

Bratianu, G.I., Recherches sur le commerce génois dans la mer Noire au XIIIe siècle, Paris 1929

Braund, D., Georgia in Antiquity. A History of Colchis and Transcaucasian Iberia 550BC-AD 562, Oxford 1994

Bromberg, J., Toponymical and historical Miscellanies, in «Byzantion», XII (1937), pp. 459-465

Bubenok, O. B., Otnositel'no mongol'skogo prisutstwija na Bospore v 1222-1223 i 1238-1239 gg., in «Bosporskie Issledovanija», V (2004), pp. 388-395

Bunjatov, Z. M., Gosudarstvo atabekov Azerbaidžana (1136-1225 godu), Baku 1978 
Byrne, E. H., Genoese Shipping in the Twelfth and Thirteenth Centuries, Cambridge (MS) 1930

Cahen, C., Pre-Ottoman Turkey, London 1968

Cahen, C., The Turkish Invasion: The Selchükids, in A History of the Crusades, a cura di K.M. Setton, Madison (WI)-London 1969, I, pp. 135-176

Canard, M., Le traité de 1281 entre Michel Paléologue et le sultan Qalâun, in «Byzantion», X (1935), pp. 669-680

Cardini F., I costi della crociata. L'aspetto economico del progetto di Marin Sanuto il Vecchio (13121321), in Studi in memoria di F. Melis, Napoli 1978, t. 2, pp. 170-210

Carile, A., I nomadi nelle fonti bizantine, , in I popoli delle steppe: Unni, Avari, Ungari (Atti della XXXV Settimana di Studi CISAM), Spoleto 1987, pp. 55-87

CARILE, A., Il Caucaso e l'impero bizantino, in Il Caucaso: cerniera fra culture dal Mediterraneo alla Persia (secoli IV-XI) (Atti della XLIII Settimana di Studi ciSAM), Spoleto 1996, pp. 9-80

CARILE, A., Impero romano e Romania, "La nozione di Romano tra cittadinanza e università», Napoli 1984, pp. 247-261

CARILE, A., L'ecumene romano-cristiana e i popoli della Rus', in «Rivista di Bizantinistica», 2 (1992), pp. 11-33

Carile, A., La cancelleria sovrana dell'Impero Latino di Costantinopoli (1204-1261), in «Studi Veneziani», n.s. 2(1978), pp. 37-73

Carile, A., Partitio terrarum Imperii Romanie, in «Studi Veneziani», VII (1965), pp. 125-305

Čerepnin, L.V., Monolo-tatary na Rusi (XIII v.), in Tataro-mongoly v Azii i Evrope, Moskva 1977, pp. 186-209

Čerepnin, L.V., Obrazovanie Russkogo Centralizovannogo Gosudarstva v XIV-XV vekach, Moskva, 1960

Chalandon, F., Essai sur le règne d'Alexis I Comnène 1081-118, Paris 1900

Chalandon, F., Histoire de la Première Croisade, Paris 1925

Charachidzé, G., Introduction a l'étude de la féodalité géorgienne, Paris-Genève 1971

Charachidzé, G., La mémoire indo-européenne du Caucase, Paris, 1987

Chazanov, M., Ecological Limitations of Nomadism in the Eurasian Steppes and Their Social and Cultural Implications, in "Asian and African Studies. Journal of the Israel Oriental Society», 24 (1990), pp. 1-15

Chozenie za tri morja, ed. it. a cura di E.T. Saronne, Roma 2003 
Christiansen, E., The Northern Crusade. The baltic and the Catholic Frontier (1100-1525), London 1980, trad. it. Le crociate del nord, Bologna 1983

Conrad, L., On the Arabic Chronicle of Bar Hebraeus: His aims and audience, in "Parole de l'Orient", XIX (1994), pp. 320-378

Constable, O.R., The Problem of jettison in medieval Mediterranean maritime law, in "Journal of Medieval History», XX (1994), pp. 207-20

Čukaeva, V.A., Russkie knjažestva i Zolotaja Orda. 1243-1350gg, Dnepropetrovsk 1998.

CurTa F., Qagan, Khan, or King? Power in Early Medieval Bulgaria (Seventh to Ninth Century), «Viator» 37 (2006) pp. 1-31.

CURTA F., Southeastern Europe in the Middle Ages, 500-1250, Cambridge 2006.

CurTa F., East Central and Eastern Europe in the Early Middle Ages, Ann Arbor 2005.

DalaI, C., Mongolija XIII-XIVvv., Moskva 1983

Danceva-Vasileva, A., B'lgarija i Latinskata Imperija (1204-1261), Sofjia 1985

Danilova L.V., Stanovlenie sistemy gosudarstennogo feodalizma v Rossii: pričiny, sledstvija, in Sistema gosudartvennogo feodalizma $v$ Rossii, I, Moskva 1993

De Clary. R., La Conquete de Constantinople, Paris 1924

De Hartog, L., The Army of Chinghis Khan, in «Army Quarterly and Defence Journal», 109 (1979), pp. 476-485

De Rachewiltz, I., Personnel and Pesonalities in North China in the Early Mongol Period, in "Journal of the Economic and Social History of the Orient», 9 (1966), pp. 88-144

Delacroix-Besnier, C., Les dominicains et la chrétienté grecque aux XIVe et Xve siècles, Roma 1997

Di Cosmo, N., Ancient Asia Nomads; Their Economic Basis and its Significance in Chinese History, in «The Journal of Asian Studies», 53 (1994), 4, pp. 1092-1126

Di Cosmo, N., Ancient China and its Enemies. The rise of nomadic power in East Asian History, Cambridge 2002

Di Cosmo, N., State Formation and Periodization in Inner Asian History, in "Journal of World History», 10 (1999), 1, pp. 1-40

Di Cosmo, N., Warfare in Inner Asian History 500-1800: 500-1800, Leiden 2002

Diaconu, P., K voprosu o glinjanych kotlach na territorii RNR., in «Dacia», n.s. 8 (1964), pp. 249-64

Diaconu, P., Les Coumans au Bas-Danube aux XIe et XIIe siècle, Bucaresti 1978 
DieHL, CH., Byzance, grandeur et décadence, Paris 1919

Dorn, B.A., Kaspij. O poxodax drevnix rusov v Tabaristan, in «Zapiski Imp. Akademii Nauk», 26 (1875), pp. 451-459

Du Breuil, P., Lo zoroastrismo, Genova 1993

Du Breuil, P., Zarathustra, Genova 1990

DuIČEv, I., Bisanzio e il mondo slavo, in Medioevo Bizantino-slavo, Roma 1965, vol. I, pp. 3-22

DuičEv, I., Les Slaves et Byzance, in Études historiques à l'occasion du XIe Congrès international des sciences historiques, Sofia 1960, pp. 31-77

Dulaurier, E., Le Royame de la petite Arménie, Paris 1908

Dunlop, D.M., The History of the Jewish Khazars, Princeton (NJ) 1954

Dunlop, D.M., The Khazars in The World History of the Jewish People, a cura di W.H. Allen, London 1966, vol. II, London 1966

Dunn, J.L., The Ageless Chinese: a History, New York (NY) 1978

Egorov, V.L., Gosudarsvennoe i Administrativnoe ustrojstvo Zolotoj Ordy, in «Voprosiy Istorij», 2 (1972), pp. 32-42

Egorov, V.L., Istoričeskaja geografija Zolotoj Ordy v XIII-XIV vv., Moskva 1985

Encyclopedia of Islam, seconda edizione, 12 voll. Leiden 1965-2005

Fedalto, G., La chiesa latina d'Oriente, 2 voll. Verona 1973-1976

Fedorov-Davydov, G.A., Iskusstovo kočevnikov i Zolotoj Ordy, Moskva 1976

Fedorov-Davydov, G.A., Kočevniki Vostocnniki Evropy pod vlastju zolotoordynskich chanov: Archeologičeskie pamiatniki, Moskva 1966

Fedorov-Davydov, G.A., Kočevniki Vostočniki Evropy pod vlastju zolotoordynskich chanov: Archeologičeskie pamiatniki, Moskva 1966

Fedorov-Davydov, G.A., Obščestvennyj Stroj Zolotoj Ordy, Moskva 1973

Fedorov-Davydov, G.A., The Culture of the golden Horde Cities, Oxford 1984

Fedorov-Davydov, G.A., The Silk Road and the Cities of the Golden Horde, Berkley (Ca) 1991

Fedorov-Davydov, G.A., Zolotoordynskie goroda povolžja, Moskva 1994

Ferrard, C.G., The Amount of Constantinopolitan Booty in 1204, «Studi Veneziani», 13 (1971), pp. $95-104$ 
FERrari, A., La foresta e la steppa. Il mito dell'Eurasia nella cultura russa, Milano 2003

Field, H., Contributions to the anthropology of the Caucasus, Harvard (MA), 1953

FolKer, E.R., Incontri con la Cina. La scoperta dell'Asia Orientale nel Medioevo, Milano 1997

Forcheri, G., Navi e navigazioni a Genova nel Trecento. Il 'Liber Gazarie', Genova 1974

Frazee, A., The Catholic Church in Constantinople, 1204-1453, in "Balkan Studies», XIX (1978), pp. 33-49

Frejdenberg, M.M., Dubrovnik i Osmanskaja imperija, Moskva 1989

Fumagalli, V., L'uomo e l'ambiente nel Medioevo, Roma-Bari 1992, $2003^{2}$

Gallina, M., Potere e società a Bisanzio. Dalla fondazione di Costantinopoli al 1204, Torino 1995

Ganshoff, F.L., Che cos’è il feudalesimo?, Torino 1982-2003³

Gardizi, Zayn al-akhbar, a cura di E.G. Browne, Berlin-London 1928

Garsoïan, N.-Martin Hisard, B., Unité et diversité de la Caucasie Médiévale (IV-XI ${ }^{e}$ s.), in Il Caucaso: cerniera fra culture, pp. 275-347

Geiger, B., Peoples and languages of the Caucasus; a synopsis, S. Gravenhage 1959

GiLl, J., Innocent III and the Greeks: Aggressor or Apostle?, in «Relations between East and West in the Middle Ages», Edimburgo 1973, pp. 95-108

Giofrrè, D., Il mercato degli schiavi a Genova nel secolo XV, Genova 1971

Golden, P.B., An introduction to the History of the Turkish People, Wiesbaden 1992

Golden, P.B., Cumanica I: The Qipčaqs in Georgia, Cumanica I: The Qipčaqs in Georgia, in «Archivum Eurasiae Medii Aevi», IV (1984), pp. 45-87

Golden, P.B., Khazaria and Judaism, in «Archivum Eurasiae Medii Aevi», III (1983), pp. 127156

Golden, P.B., Nomads and their Neighbours in the Russian Steppe, Aldershot 2003

Golden, P.B., The peoples of the south Russian steppes, in The Cambridge History of Early Inner Asia, a cura di Denis Sinor. New York (NY), 1990

Golden, P.B., The question of the Rus' Qaganate, in «Archivum Eurasiae Medii Aevi», II (1982), pp. $89-90$

Golden, P.B., The Turkic peoples and Caucasia, in Transcaucasia, Nationalism, and Social Ch'ange: Essays in the History of Armenia, Azerbaijan, and Georgia, a cura di R.G. Suny, Ann Arbor (MN) 1983, pp. 45-67 
Golden, P.B., Wolves, Dogs and Qipčaq Religion, in «Acta Orientalia Academiae Scientiarum Hungaricae», L (1997), pp. 87-97

Golovкo, A.B., Knjaz’ Roman Mstislavič, in «Voprosy Istorij», 12 (2002), pp. 52-70

Golubovsкij, P., Pečenegi, torki i polovcy do našestvija tatar, Kiev 1884

Gorelik, M.V.-Fomičev, N.M. "Rizarskie dospechi XIV veka iz Azova" Severnoe Pričernomor'e I Povolžye vo vzaimootnašeniyach Vostoka I Zapada v XII-XIV vekach, Rostov na Donu 1989.

GorskiJ, A.A., Rus'v konce X-načale XII veka: territorial'no-političeskaja struktura, in «Otečesvennaja Istorija», 4 (1992), pp. 154-161.

Gorskij, A.A., Rus’v konce X-načale XII vekach: Territorial’no-političeskaja struktura ("zeml»i $i$ "volosti»), in "Otečestvennaja Istorija», 4 (1992), pp. 154-161

Gorskij, A.A., Russkie zemli v XII-XV vekach: Puti političeskogo razvitija, Moskva 1996

Goutalier, R., Privateering and Piracy, in «Journal of European Economic History», 6 (1977), pp. 199-213

Grekov, B.D., Volžkia Bolgary v IX-Xvv., in «Istoričeskij Zapiski», 14 (1945), pp. 3-37

Grekov, B.D., Jakubovskij, A.Ju., Zolotaja Orda i ee Padenie, Moskva 1958, trad. it. L'Orda d'Oro, Firenze 1959

Grousset, R., Histoire de l'Arménie, des origines à 1071, Paris 1947

Grousset, R., L'Empire des Steppes : Attila, Gengis Khan, Tamerlan, Paris 1969

Grousset, R., Storia delle crociate, 2 voll. Casale Monferrato 1998

Gumilev, L.N., Drevnie Tjurki, Moskva 1967, $1993^{2}$

Gumilev, L.N., Drevnjaja Rus' i Velikaja step’', Moskva 1992

Halperin, C., Russia and the Golden Horde, The Mongol impact on Medieval Russian History, Bloomington (ID) 1985

Halperin, C., Russia in the Mongol Empire in Comparative Perspective, in "Harvard Journal of Asiatic Studies», 43 (1983), pp. 239-261

Halperin, C., Soviet Historiography on Russia and the Mongols, in «Russian Review», 41 (1982), 3, pp. 306-322

Halperin, C., The Russian Land and the Russian Tsar: The Emergence of Muscovite Ideology, 13801408, in «Forschungen zur osteuropaischen Geschichte», XXIII (1976), pp. 7-103

Hamm, M., The Black Death in Russia: its Effect Upon Urban Labor, in «Russian History», II (1975), pp. 53-77 
Hamm, M., The city in Russian History, Lexington 1976

Heers, J., Esclaves et domestiques au Moyen Age dans le monde méditerranéen, Paris 1981, rist. 1996

HeYD, W., Storia del commercio italiano col Levante nel Medioevo, ed. it., Torino 1913

HoÀng, M., Gengis Khan, Milano 1992

Hrochovà , V., Aspects des Balcans Médiévaux, Praha 1989

Humphrey, C., Hürelbaatar, A., Regret as a Political Intervention: An Essay in the Historical Anthropology of the Early Mongols, in «Past and Present», 186 (2005), , pp. 3-45

Il Codice cumanico e il suo mondo, Atti del Colloquio internazionale (Venezia, 6-7 dicembre 2002), a cura di F. Schmieder e P. Schreiner, Roma 2005

IORGA, N., Une nouvelle théorie sur l'origine et le caractére de l'empire de Trébizonde, in "Revue historique du sud-est européen», XIII (1970-1971), pp. 107-116

Isнuamc, N., Istorija Mongol'skoj Narodnoj Respubliki, Moskva 1954, $1964^{2}$

Jaсову, D., La population de Constantinople ò l'époque byzantine; un problème de démographie urbaine, in "Byzantion", 31 (1961), pp. 101-102

JАсову, D., Les quartiers juifs de Constantinople à l'époque bizantine, in «Byzantion», 37 (1967), pp. 167-226

JACOBY, D., Recherches sur la Médirranée orientale du XIIe-au Xve siècle, London 1979

JaCoBy, D., Social Evolution in the Latin Greece, in A History of the Crusades, a cura di K.M. Setton, vol. VI, Madison 1989, pp. 175-221

JaCOBY, D., Société et démographie à Byzance et en Romanie latine, London 1975

JACOBY, D., The Jewish Community of Constantinople from the Komnenan to the Palaiologan Period, in «Vizantijskij vremennik», 55 (1998), pp. 31-40

JaGchid, S.-Hyer, P., Mongolia's culture and society, Boulder (CO) 1979

Jagvaral, N., Aratstvo i aratskoe chozjaistvo, Ulambaatar 1974

Jakobson, A.L., Krym v srednie veka, Moskva 1973

Janin, V.L., Ja Poslal Tebe Berestu, Moskva 1998

Johnson, E.N., The German Crusade on the Baltic, in A History of the Crusades cit., III, 1975, pp. 545-565

Kargalov, V.V., Mongolo-tatarskoe našestvie na Rus'. XIII vek, Moskva 1966 
Kargalov, V.V., Vnešnepolitičeskie faktory razvitija feodal'noj Rusi. Feodal'naja Rus' i kočevniki, Moskva 1967

Karpov, S.P., Black Sea and the crisis of the mid XIVth Century: an underestimated turning point,

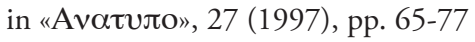

Karpov, S.P., Dokumenty po istorii venecianskoj faktorii Tana vo vtoroj polovine XIV v., in Pričernomor'e v srednie veka, a cura di S.P. Karpov, I (1991), pp. 198-199

Karpov, S.P., Empire of Great Comnenoi: Some Remarks on Particularities of its Foundation, in Actes du XVe Congrès International d'Études Byzantines, Atene 1980, pp. 153-159

Karpov, S.P., Ital'janskie morskie respubliki i Juznoe Pricernomor'e v XIII-XV vv.: problemy torgovli, Moskva 1990

Karpov, S.P., Latinskaja Romania, S. Pietroburgo 2000

Karpov, S.P., Novye dannye o pravoslavnom prixode v Azove, Moskva 2000

Karpov, S.P., On the origin of medieval Tana, in «Bizantino-Slavica», LVI (1995), pp. 225-235

Karpov, S.P., Putjami srednevekovych morechodov, Černomorskaja navigacija Venecianskoj Respubliki v XIII-XV vv., Moskva 1944, ed. It. La navigazione veneziana nel Mar Nero, XIII-XV secolo, Ravenna 2000

Karpov, S.P., Srednevekovyj Pont, New York (NY) 2001

KaRpov, S.P., The empire of Trebizond and Venice in 1374-76 (a chrisobull redated), in Centre for Byzantine Studies. XIIth Spring Symposium, Birmingham 1978

Karpov, S.P., Trapezundskaja imperija i zapadnoevropejskie gosudarstva v XIII-XVvv., Moskva 1981, ed. it. L'impero di Trebisonda, Venezia, Genova e Roma: 1204-1461, Roma 1986

Karpov, S.P., Venecianskaja Tana po aktam kanclera Benedetto B'janko (1359-60gg), in Pričernomor'e $v$ srednie veka, a cura di S.P. Karpov, V (2002), pp. 9-26

KašTanov, S.M., Finansy srednevekovoj Rusi, Moskva 1988

Kazhdan, A.P., Vizantijskaja kul'tura (X-XII vv.), Moskva 1968, tr. it. Bisanzio e la sua civiltà, Roma-Bari 1995

Kedar, B.Z., The Franks in the Levant, 11th to 14th Centuries, London 1993

Kehren, L., Tamerlan, l'Empire du Seigneur de fer, Neuchatel 1978

Khazanov, A.M., Nomads and the outside World, Cmbridge $1994^{2}$

KLjučEvskij, V.O., Sočinenija v devjati tomach, Kurs russkoj istorij, I, Moskva 1956

Koestler, A., The Thirteenth Tribe. The Khazar Empire and its Heritage, New York (NY) 1976, trad. it. La tredicesima tribù, Storia dei Cazari, dal Medioevo all'Olocausto ebraico, Torino 2003 
Kокоvсоv, P.K., Evreisko-Khazarskaja Perepiska v X veke, Leningrad 1932

Koprulu, M.F.-Leiser, G., The Seljuks of Anatolia: Their History and Culture Accordino to Local Muslim Sources, Salt-Lake City (UT) 1992

Korinny, N.N., Prejaslavskaja zemlja X-pervaja polovina XIII veka, Kiev 1992

Korobejnikov, D.A., Velikie Komniny, Sinop i Rym v 1223-1230 gg., in Pričernomor'e v srednie veka, a cura di S.P. Karpov, III (1998), pp. 178-200

Krader, L., Formation of the State, Englewood Cliffs (NJ) 1968

Krader, L., Origins of the State Among the Nomads of Asia, Berlin, in The Early State, a cura di H.J.M. Claessen e P. Skalnik, The Hague, 1978, pp. 93-109

Krader, L., Principles and structures in the organization of the Asiatic Steppe-pastoralists, in «Southwestern Journal of Anthropology», 11 (1955), pp. 65-92

Krader, L., Social organization of the Mongol Turkic pastoral nomads, The Hague 1963

Kradin, N.N., Nomadism, Evolution and World-Systems: Pastoral Societies in Theories of Historical Developement, in «Journal of world-systems research», VIII (2002), 3, pp. 368-388

Kramarovskij, M.G., Džučidy i Krym: XIII-XVvv., in Materjaly po Archeologii, Istorii, i Étnografii Tavrii, Simferopol 2003, X, pp. 506-531

Kramarovskij, M.G., The Golden Horde and Levant in the epoch of Fr. Petrarca: trade, culture, handcrafts, in "Rivista di Bizantinistica», 3 (1993), pp. 249-280

Kretschmer, K., Die italienischen Portolane des Mittelalters, Berlino 1909

KrivošEev, Ju.V., Rus' i Mongoly, Sankt Peterburg $2003^{2}$

KuČKIn, V.A., Rus pod igom: kak eto bylo?, Moskva 1991

Kudrjašov, K.V., Poloveckaja step', Moskva 1948

KuRšAnskis, M., L'Empire de Trébizonde et la Géorgie in "Revue des étude byzantines», XXXV (1977), pp. 237-256

KuZa, A.V., Drevnerusskie knjažestva X-XIII vv., Moskva 1975

Laiou, A., The Byzantine Economy in the Mediterranean Trade System: XIIIth-XVth Centuries, in «Dumbarton Oaks Papers», 34-35 (1980-1881), pp. 177-222

Lane, G., Early mongol Rule in 13th centry Iran: a Persian Renaissance, New York (NY) 2003

LatTimore, O., The Geographical Factor in Mongol History, in «The Geographical Journal», LXXXXI (1938), 1, pp. 1-16 
LeCIEjEwicz, L., La nuova forma del mondo. La nascita della civiltà europea medievale, Bologna 2004

Les centres proto-urbains russes entre Scandinavie, Byzance et Orient, a cura di M. Kazanski, A. Nercessian, C. Zuckerman, Paris 2000

Lewis, A.R.-Runyan, T.J., European naval and maritime history, 300-1500, Bloomington 1985

LewIs, G.L., The Balance of Truth, London 1957

Limonov, Ju.A., Iz Istorij vostočnogo torgovli Vladimiro-Suzdal'kogo Knjažestva: Meždunarodnye Svjazi Rossii do XVII v., in Sbornik statei, Moskva 1961, pp. 55-63

Limonov, Ju.A., Vladimiro-Suzdaliskaja Rus'. Očerki social'no-političeskoj istorii, Leningrad 1987

Ljaskoronskij, V.G., Russkie poxody v stepi v udelino-večevoe vremja, Sankt Peterburg 1907

Loenertz, R.J., Les etablissement dominicains de Péra-Constantinople, in «Echos d'Orient», T. XXXIV, n. 179 (1935), pp. 332-349.

Lopez, R.S., Genova marinara nel Duecento. Benedetto Zaccaria, ammiraglio e mercante, Milano 1933

Lopez, R.S., Nelle terre dell'Orda d'Oro : tre documenti genovesi inediti, in Studia Slavica Mediaevalia et Humanistica Riccardo Picchio dicata, 2 voll. Roma 1986, pp. 463-474

Lopez, R.S., Su e giù per la storia di Genova, Genova 1975

Mallet, M.E., The Florentine Galleys in the XVth Century, Oxford 1967

Malowist, M., La schiavitù nel Medioevo e nell'età Moderna, Napoli 1987

Malowist, M., Les routes du commerce et les marchandises du Levant dans la vie de la Pologne au Bas Moyen Âge et au début de l'époque moderne, in Mediterraneo e Oceano Indiano, atti del VI Colloquio Internazionale di Storia Marittima (Venezia 20-29 settembre 1962), Firenze 1970

Maniatis, G.G., The domain of private guilds in the Byhzantine economy, Tenth to Fifteenth Centuries, in «Dumbarton Oaks Papers», 55 (2001), pp. 339-369

Martin, M.E., The First Venetians in the Black Sea, in «Archeion Pontou», 35 (1979), pp. 111 122

Mason Smith, J., Mongol manpower and Persian population, in "Journal of the Economic and Social History of the Orient», 18 (1975), pp. 271-299

Masson Smith, J., Ayn Jalut: Mamluk sucess or Mongol failure?, in «Harvard Journal of Asiatic Studies», 44 (1984), 2, pp. 307-345

Mavrodin, V.V., Russkoe morechodstvo na juznych moriach (Cernom, Azovskom i Kaspiskom s drevnejsich vremen do XVI v. vkljucitel'no), Simferopol' 1955 
Meillassoux, C., Antropologia della schiavitù, il parto del guerriero e del mercante, Milano 1992

Menade, L., Centres of ancient Georgian culture, Tblisi 1968

Merren, L., La Vie quotidienne des marins du moyen age. Des Vikings aux galèleres, Paris 1969

Meschia, Š.A.-CincadZe, Ja., Iz istoria russo-gruzinskich vzaimootnošenij X-XVIII vv., Tbilisi 1958

Meschini, M., 1204: l'incompiuta. La quarta crociata e le conquiste di Costantinopoli, Milano 2004

Miklosich, F., Etymologisches Wörterbuch der Slavischen Sprachen, Wien 1886

Miklosich, F., Müller, J., Acta et diplomata greca medii aevi sacra et profana, 6 voll., Wien $1860-1890$

Milani, P., La schiavitù nel pensiero politico. Dai greci al basso Medioevo, Milano 1972

MilLER, W., Trebisond, the last greek Empire, London 1926

Minorsky, V., Studies in Caucasian History, Cambridge 1957

Minorsky, V., The Turks, Iran and the Caucasus in the Middle Ages, London 1978

Morgan, D.O., The Mongols, Malden (MA) 1990

Morozzo della Rocca, R.-Lombardo, A., Documenti del commercio veneziano nei secoli XI-XIII, 2 voll., Torino 1940.

Munkuev, N.C., Zametki o drevnich mongolack, in Tataro.mongoly v Azii i Evrope. Sbornik statei, Moskva, 1977

Musso, G.G., Navigazione e commercio genovese con il Levante nei documenti dell'Archivio di Stato di Genova (secc. XIV-XV), Roma 1975

NACAGDORJ, S., Iz istorii aratskogo dvizenija vo vnešnej mongolij, Moskva 1958

Namiток, A., Origines des circassiens, Paris, 1939

Nasonov, A.N., Russkaja Zemlja i obrazovanie territorii drevnerusskogo gosudarstva: istorikogeograficeskoe issledovanie. Mongoly i Rus', Sankt Peterburg 2002

NaZArova, E.L., Krestovyj pochod na Rus' 1240g., in Vostočnaja Evropa v istoričeskoj retrospektive: k 80-letiju, cur. V.T. Pašuto Moskva, pp. 190-201

Nicol, D.M., The Byzantine View of Western Europe, in «Greek, Roman and Byzantine Studies», T. VIII, (1967), pp. 315-339

Nicol, D.M., The last centuries of Byzantium, 1261-1459, Cambridge 1993 
Noonan, T.S., Rus', Pechenegs, and Polovtsy: Economic Interaction along the Steppe Frontier in the Pre-Mongol Era, «Russian History: Histoire Russe», 19 (1992), 1-4, pp. 301-327

Noonan, T.S., Russian's Eastern Trade, 1150-1530: the Archeological Evidence, in «Archivum Eurasiae Medii Aevi», III (1983), pp. 201-264

Noonan, T.S., Suzdalia's Eastern Trade in the Century before the Mongol Conquest, in "Cahiers du monde Russe et Sovietique», XIX (1978), pp. 371-384

ОвоLеnsкy, D., Byzantines frontier zones and cultural exchanges, in Actes du XIVe Congrès International des Études Byzantines, Bucarest 1971, pp. 303-314

Овоlensкy, D., Il Commonwealth bizantino: l'Europa orientale dal 500 al 1453, Roma-Bari 1974

Occidente e Oriente tra Medioevo ed Età Moderna, a cura di L. Balletto, 2 voll., Genova 1997

Origone, S., Bisanzio e Genova, Genova 1992, cit. pp. 121-123

Ostrogorsky G., Storia dell'impero bizantino, Torino 1993

Ostrowsky, D., Muscovy and the Mongols: Cross-Cultural Influences on the Steppe Frontier, 13041589, Cambridge 2002

Panitescu, P.P., La route commerciale de Pologne à la mer Noir eau Moyen Âge, Bucarest 1934

Papacostea, S., La pénétration du commerce génois en Europe centrale: Maurocastrum (Moncastro) et la route moldave, in «Il Mar Nero» III (1997-1998), pp. 149-158.

Papacostea, S., Quod non iretur ad Tanam. Un aspect fondamental de la politique Génoise dans la Mer Noir au XIVe siècle, in «Revue des études sud-est Européenne» XVII (1979), pp. 201-18

PAšuto, V.T., Mongol'skij pochod v glub' Evropy, in Tataro-mongoly v v Azii i Evrope, Moskva 1977, pp. $210-227$

Pelliot, P. - Hambis, L., Histoire des campagnes de Gengis Khan, Leiden 1951

Pelliot, P., A propos des Comans, «Journal Asiatique», I (1920), pp. 125-186

Pelliot, P., Chrétiens d'Asie Centrale et d'Extreme-Orient, in «T’oung pao», XV (1914), pp. 623644

Pelliot, P., La Haute Asie, Paris 1937

Pelliot, P., Les Mongols et la Papauté, in «Revue de l'Orient Chrétien», XXIII (1922-23), pp. 3-30, XXIV (1924), pp. 225-335, XXVIII (1931), pp. 3-84

Pelliot, P., Qubčiri-qubčir et qubčiur-qubčur, in «T’oung pao», 37 (1944), pp. 153-164

Pertusi, A., Fine di Bisanzio e fine del mondo. Significato e ruolo storico delle profezie sulla caduta di Costantinopoli in Oriente e Occidente, a cura di E. Morini, Roma 1988 
Petruševskij, I.P., The Socio-economic condition of Iran under the Il-Khans, in The Cambridge History of Iran, Cambridge 1968, vol. 5, pp. 483-537

Pistarino, G., I Gin dell'Oltremare, Genova 1988

Pletneva, S.A., Pečenegi, torki i polovcy v južnorusskich stepjach, in «Materialy i issledovanija po arxeologii SSSR», 62 (1958) , pp.151-226

Pletneva, S.A., Polovcy, Moskva 1990

Pletneva, S.A., Poloveckaja zemlja, Moskva 1975

Pohl, W. - Reimitz, H., Strategies of distinction: the construction of ethnic communities, 300-800, Leiden 1998

PoHL, W., Kingdom of the Empire, the integration of Barbarians in Late Antiquity, New York (NY)Leiden-Köln 1997

PoHL, W., Le origini etniche dell'Europa. Barbari e Romani tra Antichità e Medioevo, Roma 2000

Polubojarinova, M.D., Russkie ljudi v Zolotj Orde, Moskva 1978

Ponomarev, A., Kumany-Polovcy, in «Vestnik Drevnej Istorii», 3-4 (1940), pp. 366-370

Ponomarev, A.L., Naselenie i territorija Kaffy po dannym massarii-buchgalterskoj knigi kaznačejstva sa 1381-1382 gg., in Pričernomor'e v srednie veka, a cura di S.P. Karpov, IV, S. Pietroburgo 1998, pp. $317-442$

Prawdin, M., Il retaggio di Genghiz-Khan, Firenze 1940

PraWdin, M., L'Empire mongol et Tamerlan, Paris 1937

Priselkov, M.D., Chanskie jarliki russkim mitropolitam, Petrograd 1916

Pritsak, O., The Origin of Rus, Cambridge (MS) 1981

Pritsak, O., The Polovcians and Rus', in «Archivum Eurasiae Medii Aevi» 2 (1982), pp. 321-380

Pritsak, O., The Turcophone peoples in the Area of the Caucasus from the VI to the XI Century, in Il Caucaso: cerniera fra culture cit., pp. 223-245

Prochorov, G.M., Etničeskaja integracija v Vostočnoj Evrope v XIV veke (ot isichastskich sporov do Kulikovskoj bitvy), in Rus' i Vizantija v epoche Kulikovskoj Bitvy, S. Pietroburgo 2000, pp. 5-43.

Pubblici, L., La costruzione di un popolo. Timujin e l'unificazione turco-mongola, in "Quaderni del MAES», VII (2004), pp. 3-33

Pubblici, L., Venezia e il mar d'Azov. Alcune considerazioni sulla Tana nel XIV secolo, in «Archivio Storico Italiano», CLXIII (2005), pp. 435-484 
Queller, D.E., Madden, T.E., The Fourth Crusade. The Conquest of Costantinople, Philadelphia (PA) 1997

Rásonyı, L., Contributions à l'histoire des premières cristallisations d'Etat des Roumanis, in «Archivum Europae Centro-Orientalis», I (1935), pp. 221-253

Rasovskij, D.A., Pečeneghi, torki i berendei na Rusi i v Ugri, in «Seminarium Kondakovianum», VI (1933), pp. 9-18

Rasovskij, D.A., Polja poloveckogo, «Annaly Instituta im. N.P. Kondakova», 9 (1937), pp. 5877

Rasovskij, D.A., Polovcy. I: Proischoždenie Polovcy, «Seminarium Kondakovianum», VII (1935), pp. $254-262$

Rasovskij, D.A., Rol'polovcev v vojnax Asenej s vizantijskoj i latinskoj imperijami v 1186-1207gg., «Spisanie na B'lgarskaja Akademija na naukite», LVIII, (1939), pp. 203-211

Rasovskij, D.A., Voennaja istorija polovcev, in «Annaly Instituta im. N.P. Kondakova», 11 (1940), pp. 108-114

Richard, J., La Papauté et les missions d'Orient au Moyen Âge (XIIIe-XVe siècles), Roma 1998

Risaliti, R., Gli slavi e l'Italia: viaggi e rapporti dal Quattrocento al Novecento, Moncalieri 1996

Risaliti, R., Storia problematica della Russia, 8 voll. Firenze 2002-2004

Risaliti, R., Sull'Eurasia e dintorni. A proposito del dibattito russo sui modelli di civiltà, in «Religioni e società», XXXIV (1999), pp. 98-104

Roemer, H.R., Timur in Iran, in , in The Cambridge History of Iran, Cambridge 1975, vol. 4, pp. $42-97$

Roux, J.P., Genghis Khan and the Mongol Empire, New York 2003

Roux, J.P., La mort chez les peuples altaiques anciens et médiévaux, Paris 1963

Roux, J.P., Les astres chez les Turcs et les Mongols, in «Révue de l'histoire des religions», CXCV (1979), pp. 153-192

Roux, J.P., Tamerlano, Milano 1995

Ryвакоv, B.A., Istorija kul'tury Drevnej Rusi. Domongol'skij period, 2 vol., Moskva-Leningrad 1948

Ryвакоv, B.A., Kievskaja Rus'i russkie knjažestva XII-XIII vv., Mokva 1993

Ryвina, E.A., Archeologičeskie Očerki Istorii Novgorodskoj Torgovli X-XIVvv., Moskva 1978

SACHarov, A.M., Goroda severo-vostočnoj Rusi XIV-XVvv., Moskva 1959 
Safargaliev, M.G., Raspad Zolotoj Ordy, Saransk, I960

Schmieder, F., Europa und die Fremden. Die Mongolen im Urteil des Abendlandes vom 13. bis in das 15 Jahrhundert, Sigmaringen 1994

Scholz, F., Nomadismus. Teorie und Wandel einer sozio-okologischen Kulturweise, Stuttgart 1995

Settia, A.A., Rapine, assedi, battaglie. La guerra nel Medioevo, Roma-Bari 2002

Setton, K., The Papacy and the Levant (1204-1511), Philadelphia 1976-1978

Shaube, A., Storia del commercio dei popoli latini del Mediterraneo sino alla fine delle crociate, Torino 1915

Sholtz, F., Nomadismus. Teorie und Wandel einer sozio-okologischen Kulturweise, Stoccarda 1995

Skržınskaja, E.Č., Rus', Italija i Vizantija v Srednevekov'e, Sankt Peterburg 2000

Skržınskaja, E.Č., Storia della Tana, in «Studi Veneziani», X (1968), pp. 3-47

Snisarenko, A.B., Evpatridy udaci. Tragedija aničnich morej, Leningrad 1990

Sokolov, N.P., A proposito del significato di "he Rosia" in alcuni documenti bizantini dei secoli XII e XIII, in Storici sovietici del Levante genovese, a cura di A. Prefumo, Genova 1985, pp. 89-97

Soranzo, G., Il Papato, l'Europa cristiana e i Tartari. Un secolo di penetrazione occidentale in Asia, Milano 1930

Sounders, J.J., The History of the Mongol Conquests, Philadelphia (PA) 2001

Sovetskaja Istoričeskaja Enciklopedija, 16 voll., Moskva 1961-1976

SpIneI V., The great migrations in the East and South East of Europe. From the Ninth to the Thirteenth Century, Cluj-Napoca 2003

Spuler, B., Die Goldene Horde. Die Mongolen in Russland, Wiesbaden, I965

Starr, J., Romania, The Jewries of the Levant after the Fourth Crusade, Paris 1949

STARR, J., The Jews in the Bizantine Empire, 641-1204, Atene 1939

SтӧкLY, D., Le systeme de l'Incanto des galées du marché à Venise (fin 13.e-milieu 15.e siècle), Leiden 1995

Šunurov, R.M., Tjurki na pravoslavnom Ponte v XIII-XV vv.: načal’nyj étap tjurkizacii?, in Pričernomor'e v srednie veka, a cura di S.P. Karpov, 2, Moskva 1995, pp. 68-103

Šunurov, R.M., Velikie Komniny i “sinopskij vopros" v 1254-1277 gg., Pričernomor'e v srednie veka, a cura di S.P. Karpov, 4, S. Pietroburgo 2000, pp. 177-208

Šunurov, R.M., Velikie Komniny i Vostok (1204-1461), Sankt Peterburg 2001 
Svanidze, A.A., Srednevekovyj gorod i rynok v Svezii XIII-XV vv., Moskva 1980

Sverdlov, M.B., Domongol'skaja Rus', S. Pietroburgo 2003

TANGHeroni, M., Commercio e navigazione nel Medioevo, Roma-Bari 1996

The Economic History of Byzantium: From the Seventh through the Fifteenth Century, a cura di A.

Laiou, 3 voll., Washington (DC) 2002

Thiriet, F., Etudes sur la Romanie gréco-vénitienne (Xe-Xve siècle), London 1977

Thiriet, F., La Romanie venitienne au moyen age : le developpement et l'exploitation du domaine colonial venitien (12.- 15. siecles), Paris 1959

Thompson, M.W., Novgorod the Great, New York-Washington DC 1967

Thomson, R.W., The Crusaders through Armenian Eyes, in The Crusaders from the perspective of Byzantium and the Muslim World, a cura di A.E. Laiou e R. Parviz Mottahedeh, Washington (DC) 2001

ToLočKo, P.P., Kočevye narody stepej i Kievskaja Rus', Sankt Peterburg 2003

Tosi, M., Dalla tribù all'impero. Riflessioni sul Caucaso, le steppe e i meccanismi dell'evoluzione sociale alla luce dei dati archeologici, in Il Caucaso: cerniera fra culture cit., I, pp. 246-270

Toumanoff, C., Caucasia and Byzantium, in «Traditio» 27 (1971), pp. 111-159

Toumanoff, C., Medieval Georgian Historical Literature (VIIth-XVth Centuries), «Traditio» 1 (1943), pp. 139-182

Toumanoff, C., Studies in Christian Caucasian History, Georgetown (TE) 1963

Toumanoff, C., The Bagratids of Iberia from the Eighth to the Eleventh Century, in "Le Muséon» 74 (1961). pp. 5-42

Treplavlov, V.V., Gosudarstvennyj stroj Mongol'skoj imperii XIII v., Moskva 1993

Uspenskij, F.I., Istoria Vizantijskoj imperii, Moskva 1948

UspenskiJ, F.I., Vydelenie Trapezunta iz sostava Vizantijskoj imperii, in «Seminarium Kondakovianum», I (1927), pp. 21-34

VÁsÁry, I., Cumans and Tatars. Oriental Military in the Pre-Ottoman Balkans, 1185-1365, Cambridge 2005

VAsiliev, A.A., The foundation of Trebizond, Cambridge (MS) 1936

Veruinden, C., Aspects de l'esclavage dans les colonies médiévaes italiennes, in Hommage à L. Febure, 2 voll., Paris, 1953, II, pp. 91-103

VerLINDEN, C., Esclaves alains en Italie et dans les colonies itliennes au XIVe siècle, in «Revue belge de Philologie et d'Histoire», 36 (1958), pp. 451-457 
Veruinden, C., Esclaves et ethnographie sur les bords de la mer Noire (XIIIe et XIVe siècles), in Miscellanea historica in honorem Leonis von der Essen, Bruxelles-Paris, 1947, pp. 287-298

Verlinden, C., L'esclavage dans l'Europe Medievale, 2 voll. Bruges-Gent 1955-1977

VerLinden, C., La colonie vénitienne de Tana centre de la traite des esclaves au XIVe et au début du XVe siècle, in Studi in onore di G. Luzzatto, Milano 1950, vol. 2, pp. 1-25

Verlinden, C., Le recrutement des esclaves à Genes, du milieu du XII siècle jusque vers 1275, in Fatti e idee di storia economica nei secoli XII-XX. Studi dedicati a F. Borlandi, Bologna 1976, pp. 37-57

Vernadsky, G., Kievan Russia, New Heaven-London 1948

Vernadsky, G., Political and Diplomatic History of Russia, London 1937

Vernadsky, G., Relations byzantino-russes au XII siècles, in «Byzantion», 4 (1928), pp. 269-276

Vernadsky, G., Russia at the Dawn of the Modern Age, in ID., A History of Russia, IV, New Heaven 1959

Vernadsky, G., The Mongols and Russia, New Heaven-London 1953

Vernadsky, G., The scape and content of Chingis Khan's Yasa, in «Harvard Journal of Asiatic Studies ", 3 (1938), pp. 337-360

Vladimircov, V.Ja., Le régime social des Mongols. Le féodalisme nomade, Paris 1948

Vladimircov, V.JA., Obščestvennyj stroj mongolov, Moskva-Leningrad 1934

Voronin, N.N., Pereslavl'-Zalesskij, Moskva 1948

Vyronis, S., Studies on Byzantium, Seljuks and Ottomans, Malibu $1981^{2}$

Vyros, S., The Decline of Medieval Hellenism in Asia Minor and the Process of Islamization from the Eleventh through the Fifteenth Century, Los Angeles, 1971

WaIda, M., Central Asian Mythology of the Origins of Death, in «Antropos», LXXVII (1982), pp. 663-702

WaIda, M., Myths of the Origin of Death: The Central Asian Type, in «Temenos», XVIII (1982), pp. $87-98$

Wickens, G.M., Nasir ad-Din Tusi on the Fall of Baghdad: a Further Study, in "Journal of Semitic Studies», 7 (1962), pp. 23-35

Widengren, G., Les Religions de l'Iran, Paris 1968

WolfF R.L., Studies in the Latin Empire of Constantinople, London 1976

XV Meždunarodnyj kongress vizantinistov v Afinach, in "Vizantijskij vremennik», XXXIX (1978), pp. $17-45$ 
ZaKIrov, S., Diplomatičeskie otnošenie Zolotoj Ordy s Egiptom, Moskva 1966

ZaLiznjaK, A.A., "Slovo o polku Igoreve": Vzgljad lingvista, Moskva 2004 
INDICE DEI NOMI 

Nell'indice che segue sono state omesse alcune voci che nel testo ricorrono con eccessiva frequenza. Mancano ad esempio i termini Caucasia, Mongoli, Tartari, Caucaso, Gengis khan, khan, khanato, ilkhan, ikhqanato, mar d'Azov e mancano i toponimi continentali.

A

'Ain Jālūt, 147, 157

Abaqa, Ilqan, 177

Abasgia, v. Abkhazia

Abkhazi, popolazione, 2n, 4, 7n, 21

Abkhazi, principi, 3, 4

Abkhazi, territori, 1

Abkhazia, 3, 4, 6, 13, 199

Abu Said, Ilqan, 163, 197

Abu'l Feda, 176

Abu-Bakr, emiro, 15n

Acri, 157

Adighé, popolazione, 51

Adigeia, 51

Adriatico, v. Mar Adriatico

Afanasij Nikitin, 189n

Afghanistan, 122

Ahmd Allah Qazvini, 199

Ahrwailer H., 39

Aktyubinsk, 25

Ala ad-Din Kaï-Qubad, sultano di Iconio, 130

Al-Achraf, sultano ayyubide, 130

Alamut, 154, 176

Albani, popolazione, 55

Albània, 4, 5, 25n, 56

Albertoni G., 86

Aleksandr Nevskij, 160, 160n

Aleppo, 71n, 150, 156, 164, 176, 176n

Alessandria d'Egitto, 110

Alessio III, imperatore di Trebisonda, 12

Alessio III, v. Angeli
Al-Juzjani, 114n, 118n, 121n, 138n

Allen W., X, 13, 56, 58

Allsen T., 147, 203

Al-Makrizi, 195

Almaligh, 116n

Al-Malik al-Ashraf, principe di Khilat, 124, 130n

Al-Musacim, califfo di Baghdad, 154

Alpi Transilvaniche, 37

Altaj, monti, 71

Altūntāsh, vicerè di Korazmia, 35n

Ambaqai-qagan, 77, 79

Amu-Darya, fiume, 156

Anatolia, 2, 8, 9, 10, 15, 16, 17, 19, 21, 22, $23,58,61,70,114,132,133,134,136$, $142,174,202$

Ancouria, 16

Anda, 78, 78n, 79, 79n, 82, 104, 170

Andrej Bogoljubskij, 62

Andrej, figlio di Vl. Monomach, 47n

Angeli,

Isacco II, 9, 9n, 16, 18, 19n, 42n

Alessio I, 15, 41, 43

Alessio III, 16

Ani, 14, 19, 19n, 37, 132, 145, 174, 181, 183

Ankara, 17

Anna Comnena, 28, 35, 37n, 40, 41, 44n

Antiochia, 19, 22, 156

Arabi, 3, 5, 5n, 37, 71n

Aras, v. Arasse 
Arasse, fiume, 129, 132, 199

Arban, 94, 99

Arcadiopoli, 16

Ardabil, 6n, 122

Arghun, funzionario fiscale mongolo, 150, 151, $151 \mathrm{n}, 152,154 \mathrm{n}, 184$

Arghun, Ilqan, 168, 177, 185

Armeni, popolazione, 153, 177, 181, 183

Armeni, territori, 182

Armenia, XI, XII, 2-8, 8n, 14, 16, 53, 56-57, $128,128 \mathrm{n}, 129,132,140,145,149,151$, 151n, 153, 174, 184, 197, 199, 200

Árpád, dinastia, 37

Arqai-Qasar, 92

Arran, 14n, 151n, 199

Arslan, Kilij, sultano, 15, 146

Arslan, re di Qayaligh, 62, 116n

Arsrunide, principato, 6-7

Arsrunudi, popolazione, 4-5

Artamonov M. I., 5n

Asen,

Giovanni (Ivan) II, 17,

Ashot II, principe armeno, 5

Ashtor E., 155n

Ashur-Ade, isola, 122n

Arsrunidi, dinastia degli, 4-5

Assassini, 154, 156, 176

Astrakhan, 126

Atabeg, 60,61,117, 125, 127, 133

Athos, v. monte Athos

Äträk, qan dei Cumani, 58-61

Attila, sovrano degli Unni, 29

Avesta, 179

Awfi, 172

Ayil, 80, 83-85, 89

Ayrarat, distretto,

Azak, 8

Azerbaijan, 3n, 6, 8, 14, 22, 60, 124, 125, $127,128,132,146,149-151,153,174$, 182,199

Aznaurni, 57

Azov, regione, 9, 10, 12, 22, 27, 28, 67, 112, $124,137,145,162,164,169,175,192$

B

Baghdad, 71, 117, 118, 125, 125n, 130, 141, $146,147,154,154 \mathrm{n}, 155-158,155 \mathrm{n}$, 157n, 164, 174, 176, 179, 183, 187,

Bagrat III, v. Bagratidi

Bagratide, dinastia, 4-6, 13, 18, 52, 57, 62
Bagratidi,

David II, 13, 15, 59, 61

Bagrat III, 1

Bagrat IV, 15

David III, 58

Giorgio III Lacha, 62, 124, 150n, 180

Tamara, 6, 13, 14, 18, 18n, 19, 19n, 21, 57, 61, 62, 62n, 124, 180, 180n, 196

Giorgio IV Lacha, 21

David Lacha, 146

Rasudan (Rasoudan), 128n, 132, 146, $150 \mathrm{n}$

Bahavi, 35n

Baihaq, 198

Baijkal, lago, 69, 72

Baïu, generale mongolo, 132, 134, 150n, 153, $155,182,183,197$

Bais M., 56

Balard M., 17n, 20,

Balcani, 9, 16, 17, 22, 109, 162, 188

Baldovino di Fiandra, 12, 22

Balin, 14n

Balkh, 121-122

Balkhash, lago, 116n

Baltico, v. Mar Baltico

Barda, 197

Bartchuq, re degli Uighuri, 116

Bartold V. V., 31n, 32n, 35n, 71, 113, 114n, 116,171

Basqaq, 123, 123n, 124, 188

Batčman, qan dei Cumani, 135

Batu, 27, 131, 133, 135-138, 145-148, 150n, $151,157-164,157 \mathrm{n}, 167 \mathrm{n}, 168,184-187$, 184n, 186n, 194

Batumi, 22, 58, 62

Bayburt, 8

Baylakan, 197

Bedrosian R., 8, 70n, 149, 197

Beki, 107, 171

Bela IV re di Ungheria, 134, 136

Belgrado, 26n

Beloozero, 195

Benedetto di Polonia (Polono), 30, 186n

Berend N., 134

Berendei, popolazione, 47-49, 48n

Berestovo, 47

Berke, khan dell'Orda d'oro, 163, 176, 187 188

Bibikov M. V., 37

Bisanzio, v. Costantinopoli

Bitinia, 16 
Bloch M., 86, 100-101, 101n

Bogol, 85, 89, 90, 100, 105-108, 107n

Bold B. O., 71n, 89n

Bonjak, khan dei Cumani, 41

Börte, 80

Bosforo, 9, 12, 19, 38

Bosporo Cimmerio, 1n, 26n, 51, 51n, 53

Boyle J. A., 34n, 156

Bratianu G. I., IX, 1, 52, 53, 91

Breslavia, 136

Browne E. G., 65-66

Bugha, funzionario mongolo, 149, 183-184

Bukaul, 160

Bukhara, 72, 98n, 115n, 118-119, 131

Bulgari del Volga, 44, 44n, 53, 135

Bulgari, 12, 16, 30, 42n, 188

Bulgaria, regno di, 35

C

Caffa, 20, 143, 143n, 162-163

Čagataï khan, 118-119, 123, 131, 145, 163

Čagataidi, 177, 187

Cahen C., 8

Cairo, 110

Califfato, 5n, 71, 125, 130, 146-147, 156, 174,179

Calonero, 16

Cappadocia, 6, 16

Carlo d'Angiò, 188

Carpazi, 37, 134

Caspio, v. Mar Caspio

Cazari, popolazione, 3n, 5n, 13, 26n, 36, 38, 52-54

Cazaro, impero, 4, 5n, 36, 44, 52

Čerbi, 91-92, 91n

Černigov, $167 \mathrm{n}$

Černye Klobuki, 47-49

Cesarea, 8, 153, 182

Chalwa, generale georgiano, 16

Chanet, popolazione di Lazi, 129, 129n

Charanis P., 6

Chehrkent, 118

Cherson, 26n, 51n, 186n

Chig-qutuqu, 123

Chin, dinastia cinese, 71, 113

Chio, $17 \mathrm{n}$

Chong Qing, 131

Cilicia, 6, 7, 15, 70n, 71n, 132, 150n, 151, $151 \mathrm{n}, 153,156,162,174,184,197$

Cina, 66, 68, 68n, 69, 69n, 71, 98, 112-115, 116n, 131, 133, 142-146, 153, 169, 173 n, 187, 189, 189n, 202-205

Cipro, 19

Circassi, 20-21, 51, 110, 125

Colchide, 3, 3n

Comneni,

Alessio I, 15, 41, 43

Alessio II, 16

Andronico I, 18, 18n

Manuele I, 9, 11, 18, 43

Comneni, dinastia, 11, 13, 18, 20, 21

Corea, 133

Čormaghan, 132, 141, 145, 153, 181-183, 197

Costantino IX, 7

Costantino VII Porfirogenito, 26

Costantinopoli, IX, XIII, 10, 12, 17, 19, 20, $22,48 \mathrm{n}, 53,130,204$

Cracovia, 136

Crimea, 1, 9, 10, 20-22, 26n, 27, 36, 50, 160, 160n, 162-164, 193-194, 202

Croati, 12

Cumani, v. Polovcy

Cumania Nera, 59

Cumania/Komania, 27

$\mathrm{D}$

Dabil, 197

Daffinà P., 97n, 167n

Daghestan, 129

Dalmazia, 5n, 110, 141, 150, 157, 164, 176, 176n, 183

Damasco, 5n, 141, 110, 157, 164, 176, 176n, 183

Damghan, 122

Danijl di Galizia, 160

Danubio, fiume, XI, 2, 25, 26n, 27, 32, 36-38, $40-45,44 n, 136,162,188$

Darial, passo caucasico, 3

Darqan, 108, 108n

Daruga, 123, 159, 160, 188

Darugačin, v. Da ruga

David Comneno figlio di Manuele I, 21

David II, v. Bagratidi

David III, v. Bagratidi

David Lasha, v. Bagratidi

David Narin, 146, 150

David Soslan, 16, 62

De Hartog L., 95

Degai, 91 
Delhi, 127

Derbent/Derbend, passo caucasico, 152

Desocializzazione, 102, 102n, 105

Dešt i-Kipčak, v. Orda d'Oro

Diaconu P., 32

Djezireh, 124

Dkébel, 124

Dnepr, fiume, XI, 25, 26, 32, 36, 44, 45, 47, $126,134,192,195$

Dnestr, fiume, XI, 26, 134

Dobrugia, 51n, 137, 162

Dodai-čerbi, 91

Don, fiume, VI, IX, XI, 1, 25, 26, 32, 36, 40n, $47,48,53,59,126,162,192,195,202$

Donec, fiume, 59, 61

Dristra, 40, 41

Ducas, Michele VII, 15

Romano IV Diogene, 8, 42

Ducas, dinastia, 18

Dunn J. L., 95

Dvin, $14,15 \mathrm{n}, 128$

Dvina, fiume, 47

Dyarbekir/Diyar-Bekr, 132, 151n

\section{E}

Ebrei, 20, 185

Eduardo I, re d'Inghilterra, 187

Egeo, v. Mar Egeo

Egitto, 110, 125n, 147, 155-156, 164, 177, $187,187 \mathrm{n}, 188,195,197-198$

Egrisi, 3

Elena, nipote di Romano III, 15

Enrico di Fiandra, 22

Enrico di Slesia, 136

Epiro, 17

Eraclio di Bisanzio, 3

Eristavni, 57

Erzindjan, 130, 132, 145, 153, 182

Erzrum, 16, 145, 153, 174, 196n

Etil/Itil, v. Volga

Eufrate, fiume, 38, 71n

Eurasia, 32n, 141, 194, 205

Eurasiatiche steppe, V, VI, 81, 202

Eurasismo, 75n

F

Fārāb, 32n

Faxium, 22
Federico I Barbarossa, 11n, 15-16

Federico II, 97

Feudalesimo, VII, 85-87, 90

Filippo II Augusto, re di Francia, 11n

Franchi, 12, 175n

Francia, 177

G

Galata, v. Pera

Galatea, 16

Galič, 136

Gallina M., 11

Gandzak, 14, 140, 140n, 180

Ganja, v. Gandzak

Gardizi, 31, 31-32n

Garman, principi di, 5

Garni, 184

Garni, fiume, 128, 128n

Gaza, 157

Gazaria,

Geikhatu, 177

Geluchelan, v. Mar Caspio

Genova, 9, 12, 17n, 202

Georgia, X-XII, 1-4, 6, 8, 10, 13-18, 20-21, 54, 58-62, 62n, 70, 124, 127-129, 128n, $129 \mathrm{n}, 130,132,134 \mathrm{n}, 140,145-147$, 149, 150n, 151, 151n, 153, 174, 180, 182, 192, 196, 197, 199

Georgiani, popolazione, 3-4, 13n, 14n, 15n, $16,16 \mathrm{n}, 58,61,125,125 \mathrm{n}, 127-128$, $128 \mathrm{n}, 129,141,150 \mathrm{n}, 153,154,156$, 175, 180-182, 184, 197

Georgiani, territori, 7, 59, 184

Gerusalemme, 11n, 156

Ghazan Ilqan, XIII, 152, 158, 158n, 168, 177 $179,185,197-198$

Ghazna, 123-124

Giorgio III, v. Bagratidi

Giorgio IV, v. Bagratidi

Giorgio V, v. Bagratidi

Giovanni Cinnamo, 28, 37n, 42n

Giovanni di Pian del Carpine, 30, 69, 91, 97-99, 115n, 146, 167, 167n, 171-173, 179,185

Giovanni Tzetze, $37 \mathrm{n}$

Giuči, qan, 112, 118, 131, 145, 158, 166

Giustiniano I, 7, 51

Gobi, deserto, 71, 73

Golfo Persico, 144

Gotia, 10 
Gozz, v. Guzi

Gran, 136

Grande Armenia, v. Armenia

Greci, 1, 3, 6, 20, 26, 42, 43, 43n, 51n, 71n, 188

Gregorio X, papa, 177

Gregorio di Akner, 70, 70n, 167, 179, 180

Grekov B. D., 47

Grousset R., 6, 73, 131, 147, 156, 186

Guaramidi, dinastia, 5

Güčügür, 91

Guglielmo di Rubruck, 30, 76, 97, 174

Gurgan, fiume, 122

Güyük, 115, 133, 145-146, 146n, 149-151, $150 \mathrm{n}, 168,175,183-184$

Guzi, popolazione, 2, 31

Gwari, 55-56, 106

$\mathrm{H}$

Halperin C., 45, 189, 189n

Hamadan, 124-125, 198

Hang-Tche Ou, capitale Song, 71

Herat/Hereth, 16, 198, 199

Hethum I, 132, 149, 150n, 151n, 156, 176, 184

Hethum, generale armeno, 27, 27n, 70, 177, 180

Heyd W., 10, 53

Höelün, 79, 104

Hülegü khan, 147-148, 153-158, 155n, 156n, $157 \mathrm{n}, 164,168,175,176-177,183,185$, 185 n, 187

I

Iberia, 3-7, 5n, 15, 20, 51, 54, 56

Ibn al-Athir, 115

Ibn Battuta, 144

Iconio/Konya, sultanato, 13, 16, 21, 22, 130, 133, 145, 146, 174, 182

Iconio/Konya, sultano, 16, 21, 132, 146

Igor' principe Rus' di Kiev, 45, 46n

Ilamish, fiume, 72

Iltoutmich sultano turco di Delhi, 127

Imerezi, popolazione, 16, 19

Imerezia, 4, 146

Impero Latino d'Oriente, 12, 17, 22, 153, 156

Inal-Khan, $117,118 \mathrm{n}$

India, XI, 69, 189n
Indo, fiume, 123

Ingur, $129 \mathrm{n}$

Innocenzo IV, papa, 91, 115

Iran, 3, 71, 121, 127, 147, 151n, 182

Irgän, $80,83,83 \mathrm{n}, 88$

Iris, flume, 21

Irtyh, fiume, 31, 71, 116, 124

Isauro, fiume, 16

Isfahan, 179

Isfara' in, 198

Ismaeliti, 154

Issiq-Kul, fiume, 71, 131

Italia, V, X, 30, 74, 86, 90, 175, 177, 205

Itil, v. Volga

Ivane, 129, 180-181

Iviron, 7

Izjaslav Mstislavovič, 48, 62

Izz ad-Din sultano di Iconio, 21

$\mathrm{J}$

Jaghun/djaghoun, 94, 99

Jakubovskij A. Ju., 75, 87n, 158n

Jalal ad-Din, sultano di Korazmia, 15, 113 , 122-124, 127-131, 148, 180,181, $181 \mathrm{n}$

Jarčut, tribù, 105

Jarligh/Jarlich, 157

Jaroslav, principe Rus' di Kiev, 44n, 47n, 52, 160,195

Jur'ev, 47

Jurij Dolgorukij, 48, 62n

Jurij II granduca di Suzdal', 135

Jurij Vsevolodovič, 160

Juvain, 122

Juvaini, Ata Malik, 17, 66n, 94, 98, 115-119, $128 \mathrm{n}, 131,133,138,140,143,151 \mathrm{n}$, $158,167,170,171,173,196$

K

Kachezia, 181, 184, 184n

Kadan generale mongolo, 136

Kagaria, 71

Kaidu, 136

Kai-Kavus II, 146

Kaï-Khosrau sultano di Iconio, 132

Kalets, v. Kalka

Kalka, fiume, 126

Kan-Sou, fiume, 71

Karatzas, 42 
Karkh, 155

Kars, 5, 7, 8, 14, 128, 132, 145, 183, 199

Kars, distretto di, 174

Kars, 5, 7, 8, 14, 128, 133, 145, 199

Kars, vescovato armeno, 183

Kart'li, v. Kartlia

Kartlia, 3-4, 16, 146, 151

Kartveli, popolazione, 3

Kassi, v. Russi

Kata figlia di David II, 15

Kay Khusraw sultano di Iconio, 17

Kazakistan, 25, 32n, 68, 69, 118, 150

Keler, v. Ungari

Kerait, 72, 73n, 79, 83, 85, 90, 92, 107, 108, 156n, 173

Kerč, regione, 52

Kerč, stretto di, 1, 26, 51, 51n, 53, 53n, 204

Kerč, vescovo di, 52

Kerimberi khan,

Kerman, 128-129

Kerulen, fiume, 73, 131

Kešigten, 92

Ket-Buqa, 155, 157

Khazaria, 1, 1n

Khilat, 124, 125, 130, 130n, 183

Khitai, 68

Khorassan, 122, 131

Khorazm-Shah, 122

Kiev, VI, 25, 34, 36, 45-48, 48n, 53, 136, 136n, 137, 160

Kimäk, popolazione, 31, 31n

Kin, v. Chin

Kipčaki, v. Polovcy

Kirghisi, 68, 68n

Kocho Tsaïdam, 73

Komanoi, v. Polovcy

Končak, 47, 47n

Konya, v. Iconio

Korazmia, 27, 53, 71, 72, 98n, 114-116, 120 , 121, 127, 129, 131, 163, 171, 192,193

Kortšin, 91

Kosroidi/Cosroidi, dinastia, 5

Kostantin, catholicos armeno, 183

Kotjan qan dei Cumani, 126

Kuban, 51-52, 52n

Kulikovo, VI

Kumys, 108

Kun, v. Polovcy

Kunik A., 33

Kura, fiume, 132

Kurdistan, 132
Kuren, 80-84, 80n, 83n

Kutaisi, v. Kutaysi

Kutan, v. Kotjan

Kutaysi, 4

L

La Copa, 53

Lago di Meozia, v. Mar d'Azov

Lascaris, Teodoro I, 22

Lascaris, dinastia, 21

Lazi, popolazione, 21, 125, 129n

Lazi, principi, 3

Lazica, 3, 4, 19

Lebunian, colle, 42

Leone II, principe abkazo, 4, 16

Liegniz, 136

Lituania, 193

Livonia, 23, 23n

Livonia, Ordine di, 193

Lo Vati, v. Faxium

Lopez R. S., 17

Lori, 5, 7, 62n, 128

Luigi IX, re di Francia, 76

Lukomor, 32

Lungarotti M. C., 69

M

Macedoni, Basilio II, 1, 52

Romano IV, 8, 42

Macedoni, dinastia, 42

Magiari, 36-37

Mamasakhlisi, 55

Mamelucchi, 110, 110n, 157, 164, 177, 188

Mamikonidi, dinastia, 4

Manzikert, 2, 9, 21, 31, 39

Mar Adriatico, 136

Mar Baltico, 9, 22, 47, 52, 53

Mar Caspio, XI, 1, 15, 25, 27, 36, 112, 115, $121,122,124,124 \mathrm{n}, 125,128,130,135$, $145,152,154,163,192$

Mar d'Azov, IX, XI, 20, 26n, 27, 51-53, 51n, 76, 100, 113, 126, 191

Mar Denha, patriarca nestoriano, 177

Mar di Reme, v. Mar d'Azov

Mar Egeo, 17

Mar Grande, v. Mar Nero

Mar Maggiore, v Mar Nero 
Mar Nero, VI, IX, XI, 1, 2, 10, 10n, 12, 13, $15,17,19,22,25,27,36,38,45,51 \mathrm{n}$, $109,110,129 \mathrm{n}, 130,132,137,145$, $162,188,192$

Maraga, 125

Marco Polo, 144, 145

Marsiglia,

Marta, figlia di Bagrat IV, 15

Mashhad, 122

Mason Smith J., 95

Matracha, v. Matrega

Matrega/Matracha, 1, 9, 52, 53

Maurokastron, 1

Medio Oriente, V, 15, 23, 147, 155, 185

Melik al-Ashraf, 124, 130n

Melitene, 8, 71n

Mengü, 133

Meng-wu, popolazione, 70

Meozi, popolazione, 51

Mërkit, 72, 79-80, 82n, 83, 83n, 85, 91, 107n, $140,170,171$

Merv, 98n, 122, 131, 199

Mesembria,

Mesopotamia, 16, 147, 155n

Michajl Vsevolodovič, 167

Michele Psello, 37n

Miklosich F., 33

Milani P., 103

Mingghan/Minggan, 92, 94, 99, 160

Mingrelia, 4

Minyak, impero, 113

Mohammed, di Korazmia, 117, 122n, 127

Monastras, 42

Möngke, 135, 135n, 145-148, 150n, 151, 153, 154, 156n, 157, 168, 184, 185, 187,197

Monte Athos, 7, 19

Moravia, 186

Mosca, VI, XIII, 46n, 135, 162, 195, 195

Mosul, 125, 132, 151n

Moxoene, principato di, 5, 7

Mstislav di Černigov, 114

Mstislavl di Galič, 126, 126n

Murwan Kru, sultano omayyade, 3

Myriokephalon, 39

$\mathrm{N}$

Naiman, 71-72, 71n, 85, 90, 92, 99, 172, $173,185 n$

Namakiyya, 31n
Narsete catholicos di Aghbania, 182n

Nasawi, 116, 117, 117n, 121n, 122n, 123n, $128 \mathrm{n}$

Nasir Yusuf sultano ayybide di Siria, 156

Nawruz emiro, 178

Neijiang, 131

Neman, 47

Nepradva, fiume,

Neva, fiume, 47

Nicea, 22

Nicea, impero greco di, 17, 22, 130, 174

Niceta Coniate, 28, 37n, 42n, 43, 99n

Nicola Maurokatakalon, 42

Nishapur, 121, 122, 122n, 123, 199

Nogaï, 162, 188

Nökör/Nököd, 88-100, 89n

Nor, 180

Norvegia, 53

Novgorod Velikij, 48, 53, 159, 159n, 160, 193

Noyon, 81, 81n, 85, 88, 88n, 89, 92-94, 100, $107,111,119,120,123,132,153,155$, $157,159,160,171,183,197$

$\mathrm{O}$

Oboq/Oboh, 55, 76, 77, 78, 80, 81, 85, 88, 99, 103-107, 111, 112n, 148, 153, 158, 159

Oby, fiume, 31

Ogödeï, qan, 34, 112, 118, 119, 123, 124, 131$133,137,141,145,146 n, 168$

Oguzi, v. Guzi

Oïrat, tribù, 171

Olgoviči, 48

Oljeitu, 178

Ommayyadi, 3

Onon, fiume, 131

Orda Azzurra, 74

Orda Blu, 33

Orda d'Oro, VI, XIII, 27, 33, 50, 66, 74, 74n, $75,131,137,142,143,145,148,152$, 157-160, 161n, 162-164, 168, 186-189, 188n, 192, 194-198, 202

Ordos, fiume, 71

Orel, fiume, 32

Oronte, fiume, 15

Ostikan, 151, 151n, 152, 154n, 184

Ottone di Frisinga, 28, 34

Ouza, 42 
P

Paflagonia, 16, 18n

Palestina, 11n, 157, 176

Panbak, monti, 128

Patronqmoba, 57

Patzinaki, v. Peceneghi

Peceneghi, 2, 30, 33n, 35-43, 40n, 44n, 45, $47,48 \mathrm{n}, 53$

Pechino, 71, 113, 114n

Pelliot P., 73, 81n

Perevoloka, 45

Persia, 20, 22, 31, 51-52, 66, 112, 127, 131, $132,133,134,142,147,147 \mathrm{n}, 151 \mathrm{n}$, $156,157,158 \mathrm{n}, 163,168,174,175$, $177,202,205$

Perwan, 123

Pesočev, 45

Pest, 136, 136n

Petruševskij I.P., 196

Piccola Armenia, v. Cilicia

Pizunda, 26n

Pletneva S.A., 48

Pohl W., 30, 31n

Polacchi, 136

Polonia, 136, 186, 193,

Polovcy, 13, 26-37, 29n, 39-49, 40n, 41n, 43n, $44 \mathrm{n}, 47 \mathrm{n}, 48 \mathrm{n}, 58,62,68,71,125,126$, $127,133,134,135,145,158,187,193$

Ponto, 9, 11, 18n, 22, 23, 39, 51n, 70

Poroz', 48, 48n

Priluk, 45

Pritsak O., 32n

Provero L., 86

Pseudo Costantino Diogene, 42

Q

Qabul-qagan, 77

Qaraču, 85, 105

Qara-Kitai, 71, 72

Qaraqorum/Karakorum, 131, 147, 148, 150

Qarluc, popolazione, 116n

Qasar, 91

Qayaligh, 116, 116n

Qipčiaki, v. Kipčaki (Polovcy)

Qmani, 57

Qotlogh-Khan, 117

Qubchur, 152

Qubi, 89

Qubilaj qan, 153, 168, 177
Quda, 80, 103

Quriltai, 90, 131, 133, 135, 137, 159

Qutlu Arslan, 62

$\mathrm{R}$

Rabban Ata, 182, 182n, 183

Rabban Marco, 177

Rabban Sauma, 177, 177n

Rashid ad-Din, 33, 34, 34n, 66, 77, 78, 79, 80, $82,85,88 n, 90 n, 104 n, 106,108,128 n$, $135 \mathrm{n}, 136,143,146 \mathrm{n}, 154 \mathrm{n}, 156,157 \mathrm{n}$, $158,167,171,178,185,185 n, 186,198$

Rasudan, v. Bagratidi

Regensburg, 34

Reidentificazione, 26n, 141, 203

Rjazan', 186n

Roberto di Clari, 28, 28n, 48n

Roma, 3, 56

Roman Nezdilovič, 48

Romania, 59n

Romano IV Diogene, v. Ducas

Rosia, 9

Rostov, 126

Rudhravar, 198

Rukn ad-Din capo degli Assassini, 154

Rum, 21, 129, 138, 151n, 157, 183

Rus', XI, 9, 10, 13, 14, 27, 33, 34, 36, 37, 40, $44,46,47,48,49,50,52,59,61,62,89 n$, $114,133,134,135,136,141,142,158$, $159,160,162,163,169,186,190,192$, 193, 193n, 194, 195, 196, 202

Russi, VI, XIII, 30, 35, 36, 45, 46, 47, 48, 49, $51,52,53,74,110,126,126 \mathrm{n}, 127 \mathrm{n}, 135$. 135n, 145, 159, 160, 161, 162, 163, 164, $174,183,186,188,189,193,194,195$

Russia, VI, X, XIV, 25, 30, 40, 66, 74, 75, 95 , $112,126,159$ n, 162, 189n, 190, 202

S

Sagwareulo, 55, 56, 106

Sajo, fiume, 136

Saladino, 11n, 15, 130n, 157

Salturid, 16

Samara, fiume, 32

Samarcanda, 72, 98n, 117, 119, 120, 121, 124 , $131,192,200$

Saraj, VII, XIII, 27, 160, 162, 163, 188, 188n, 200

Sarir, Avari del Daghestan, 129, 129n 
Sarkel, 36

Sarmati, popolazione, 25

Sartach qan, 168, 184, 186, 187

Sasun, principato, 7, 153

Savastopoli, 22, 58

Sciti, VII, 37, 37n, 40, 40n, 41, 42, 42n, 43n

Selenga, fiume, 68, 73

Selgiuchidi, 2, 2n, 8, 13, 14-17, 19, 22, 31, $32 \mathrm{n}, 53,54,58,59,61,130,149,174$, 179,188

Semnan, 122

Seneca, 103

Serbi, 12, 16

Settia A., 67n

Sevan, lago, 3, 128, 174

Shahna, 122, 122n, 124n

Shamakha, 197

She-wei, 70

Shiraz, 179

Shirvan passo caucasico, 14, 125, 127n, 199

Sighnāq, 32, 117, 118

Si-Hia, impero cinese, 71, 131

Siria, 7, 129, 138, 147, 154n, 156, 157, 176

Sit, fiume, 135

Siunia, principato, 4, 7

Siunidi, dinastia, 4

Sivas, 2, 8, 132, 153

Skopie, 16

Skržinskaja E.Č., 34, 45

Smbat III, Giovanni, principe armeno, 6

Smisso, 10

Smolensk, 48

Sobolevskij A.B., 33

Sofia, 16

Soldadea, v. Soldaïa

Soldaïa, 10n, 143, 163

Solimano, figlio di Kilij Arslan, 16, 17

Song, dinastia cinese, 71

Soslan, v. David Soslan

Sotirioupolis, v. Pizunda

Sozopoli, 43

Spalato, 136

Stati Uniti, X, 67n, 74n

Strabone, 51n, 52n, 54, 55

Strigonia, 136

Subcaucasia, 148

Sübeetai-Bagatur, 92

Subeteï generale mongolo, 119, 121, 122, 124, $125,126,135,136$

Sugrov, $14 \mathrm{n}$

Sukhumi, 3
Sung, dinastia, 113, 133, 144

Suzdal', 135, 160

Svan, popolazione, $129,129 \mathrm{n}$

Svjatopolk, 41n, 47, 47n

Svjatoslav Vsevolodič, 48, 52

Syr-Darya, fiume, 32n, 118

Syspiritis, 5

\section{$\mathrm{T}$}

Tabriz, 20, 22, 150, 182, 185n, 200

Tacito, 25

Tagudar Ilqan, 168, 177

Talas, 124, 150

Taman, penisola di, $51 \mathrm{n}, 52$

Tamara, v. Bagratidi

Tamerlano, VII, IX, XII, XIII, 96, 163, 188, 198

Tamgha, 182, 182n

Tangut, 68, 71

Tao', ducato del, 4n, 6

Taraun, 4n, 5

Tarkhat, 94

Tashir, 128

Tatu, 40

Tayk, v. Tao'

Tbilisi, XIII, 51, 62, 199

Tchu, 71, 124

Tenggri, 175

Teodoro I, v. Lascaris

Tessalonica, 17, 26n

Teutonici, cavalieri, 136

Teutonico, Ordine, 193

Theiss, fiume, 136

Thermodon, fiume, 21

Tibet, XI, 144

Tigri, flume, 125, 132

Timur, v. Tamerlano

Tmutorakan'/Tmutarakan, 36, 52, 52n, 53

Toghachar/Toquchar, 122, 123, 123n

Togha-Timur, 198

Toqtaï qan, 162

Tor, fiume, 32

Törägän, 146

Torki, 47, 48n

Tosi M., XI, 28, 201

Toumanoff C., 4, 6

Tourgokan, 41

Transcaucasia, 38, 54, 146, 148, 157

Transoxiana, 71, 117, 121, 124, 129, 187, 189 , 196, 199, 202, 203 
Trebisonda, 18-21, 19n, 53, 70, 130, 202, 204

Trebisonda, impero di, 17-22, 18n, 114, 130, 174

Tripoli, 156

Trubez, fiume, 47

Tuda qan, 188

Tulabuqa qan, 162

Tuli/Tului/Toli, 118, 122, 123, 131, 135, 145,146

Tulidi, 145,151

Tümen, 82, 83, 93, 93n, 94, 94n, 99, 100, 108, $118,153,160,197$

Turchia, 8, 130, 132

Turkestan, 53, 66, 70, 72n, 109, 113, 114, $116,117,123 \mathrm{n}, 131,138,140,142$, 144,163

Turkmeni, 8, 121, 149

Turkmenistan, 121

Tus, 122, 122n

Tver', 136, 189n, 195

Tyari, 177

$\mathrm{U}$

Uduid, tribù Merkit, 79

Uighur, v. Uighuri

Uighuri, 68, 68n, 71n, 116n, 131

Ukraina, 25

Ulus Giuči, 74, 158n, 196

Ulus, 71n, 76, 77, 77n, 81, 89, 90, 91, 99, 107, $107 \mathrm{n}, 111,112,112 \mathrm{n}, 113,124,131$, $137,140,147,148,152,158,158 \mathrm{n}, 160$, $161,162,163,164$

Unagan Bogol, 90, 100, 106, 107, 107n, 108

Ungari, 133

Ungheria, 43, 47, 53, 59, 134, 135, 136, 157 , $162,164,186,193$

Unione Sovietica, 87

Unni, 29n, 54

Ural, fiume, 25, 36

Urali, 25, 31

Urghench, 98n, 120, 121, 123, 192, 200

Urmiah, 8

Utrar, 115, 115n, 116, 117, 118, 119, 187

Uwas, tribù Merkit, 79

Uzbek atabeg di Azerbaijan, 124, 127

Uzi, 2

V

Van, lago, 8, 130, 132
Van-qan, 72, 79, 83, 156n

Vardapet, 140, 141, 180, 181, 181n, 184

Varjaghi, 1

Vaspurakan, 4, 5, 6, 8, 8n, 153

Veče, $160 n$

Venceslao di Boemia, 136

Vernadsky G., 75

Viatka, 195

Vilayet/vilayat, 153, 153n, 197, 198

Vistola, fiume, 136

Vladimir Monomach, 47n, 48, 61

Vladimir' I, principe di Rus', 45

Vladimircov V. Ja., 75, 75n, 78, 79n, 81, 85, $87 \mathrm{n}, 171$

Volga, fiume, XI, 1, 5n, 25, 27, 32, 36, 42, $44,45,47,48,52,54,59,61,135$, $135 \mathrm{n}, 143,160,163,188,188 \mathrm{n}, 192$, 195,204

Vsevolod Jurevič, 47n

W

Wassaf, 144

Weber M., 87

Y

Yenissei, fiume, 68n

Yesügai Bagatur, 76n, 78n, 79

Yüan, dinastia, 142, 143, 144, 189, 203, 205

Z

Zakaria generale georgiano, 16, 181

Žamuka, 78, 79, 79n, 82, 170

Zaporoze, 32

Žend, 32n, 98n, 117, 118

Zichi, v. Circassi

Zichia, 1, 51, 52

Žirgin, tribù, 107, 108 


\section{INDICE}

Marcello Garzaniti, Presentazione V V

INTRODUZIONE IX IX I

I. LE PREMESSE 1

1.1 Fino a Manzikert. La Caucasia e il fallimento bizantino. 2

1.2 Le cause della crisi 9

1.3 Le conseguenze della crociata. L'impero di Trebisonda 17

2. LE STEPPE DEI POLOVCY COME ESITO TRANSITORIO 25

2.1 Sull'origine dei Polovcy/Cumani 30

2.2 Le prime incursioni 35

3.3 Alcune considerazioni sui rapporti coi vicini: Bisanzio e la Rus' 40

3. La Caucasia fra nOmadismo e immigrazione 51

3.1 Il bosporo cimmerio fra Bisanzio e la seconda ondata nomade $\quad 51$

3.2 Le infiltrazioni nomadi in Georgia e le loro conseguenze 54

3.2.1 Popoli di Georgia e organizzazione sociale $\quad 54$

3.2.2 I Polovcy in Georgia $\quad 58$

3.2.3 Il nomadismo in Georgia: contrasto e integrazione $\quad 61$

4. LA COSTRUZIONE DI UN POPOLO 65

4.1 I primi gruppi altaici. Acquisizioni e problematiche aperte 65

4.2 Etnogenesi e nomadismo: i primi mutamenti 74

4.3 Alla base dell'unificazione $\quad 82$

4.4 I mutamenti successivi: è lecito parlare di "feudalesimo nomade"? 85

4.5 Lo strumento dell'esercito e la realizzazione delle solidarietà 91

4.6 Nomadismo e schiavitù 100

5. L'opera Di TIMUČIn E L’ESPANSIONE FRA CAUCASO E AZOV 111

5.1 Tempi e modi dell'invasione ad Ovest 113

5.2 La prima campagna contro il sultano di Korazmia e l'invasione
del Turkestan

5.3 La prima incursione fra Caucaso e Azov (1220-1221) 124 
5.4 La seconda incursione mongola a Ovest e la conquista definitiva della Caucasia (1230-1236)

5.5 La conquista del Dešt i-Qipčak (1236-1242)

6. Gli efFetTi Politici delle CONQUiste E LA SEPARAZione CAUCASICA FRA ORDA D'ORO E ILKHANATO

6.1 Dal nomadismo alla formazione dello stato

6.1.1 La razzia come prima risposta

139

6.1.2 I processi organizzativi

6.2 La riorganizzazione del territorio e la nuova geografia fra Caucaso e Azov

6.3 La Caucasia e l'Ilkhanato 148

6.4 Nell'Orda d'Oro

7. Il FATTORE RELIGIOSO E IL PROBLEMA DELL'INTEGRAZIONE DOPO LA CONQUISTA

7.2 I Mongoli, l'Islam e il Cristianesimo armeno-georgiano fino alla scelta di Ghazan (1220-1295)

170

7.3 L'Orda d'Oro, l'Azov e una riconsiderazione delle influenze

174

186

8. Popolazione e convivenza. L'aspetto demografico fra CONQUISTA E RICOSTRUZIONE

8.1 Alcune considerazioni sulle conseguenze demografiche delle conquiste

8.2 Nell'Orda d'Oro e nella regione dell'Azov (1237-1395)

192

8.3 Nella Caucasia

196

9. ConClusioni. Risultati E Prospettive 201

$\begin{array}{ll}\text { Cartine } & 207\end{array}$

$\begin{array}{ll}\text { Bibliografia } & 213\end{array}$

$\begin{array}{ll}\text { INDICE DEI NOMI } & 241\end{array}$ 